\title{
Treatments for Acute Pain: A Systematic Review
}




\section{Comparative Effectiveness Review}

Number 240

\section{Treatments for Acute Pain: A Systematic Review}

\section{Prepared for:}

Agency for Healthcare Research and Quality

U.S. Department of Health and Human Services

5600 Fishers Lane

Rockville, MD 20857

www.ahrq.gov

Contract No. 290-2015-00009-I

Prepared by:

Pacific Northwest Evidence-based Practice Center

Portland, OR

\section{Investigators:}

Roger Chou, M.D.

Jesse Wagner, M.A.

Azrah Y. Ahmed, B.A.

Ian Blazina, M.P.H.

Erika Brodt, B.S.

David I. Buckley, M.D., M.P.H.

Tamara P. Cheney, M.D.

Esther Choo, M.D., M.P.H.

Tracy Dana, M.L.S.

Debra Gordon, R.N., D.N.P., FANN

Saurabh Khandelwal, M.D.

Shelby Kantner, B.A.

Marian S. McDonagh, Pharm.D.

Christine Sedgley, M.D.S., M.D.Sc., FRACDS, MRACDS (ENDO), FACD, Ph.D.

Andrea C. Skelly, Ph.D., M.P.H.

AHRQ Publication No. 20(21)-EHC006

December 2020 
This report is based on research conducted by the Pacific Northwest Evidence-based Practice Center (EPC) under contract to the Agency for Healthcare Research and Quality (AHRQ), Rockville, MD (Contract No. 290-2015-00009-I). The findings and conclusions in this document are those of the authors, who are responsible for its contents; the findings and conclusions do not necessarily represent the views of AHRQ. Therefore, no statement in this report should be construed as an official position of AHRQ or of the U.S. Department of Health and Human Services.

\section{None of the investigators have any affiliations or financial involvement that conflicts with the material presented in this report.}

The information in this report is intended to help healthcare decision makers-patients and clinicians, health system leaders, and policymakers, among others-make well-informed decisions and thereby improve the quality of healthcare services. This report is not intended to be a substitute for the application of clinical judgment. Anyone who makes decisions concerning the provision of clinical care should consider this report in the same way as any medical reference and in conjunction with all other pertinent information, i.e., in the context of available resources and circumstances presented by individual patients.

This report is made available to the public under the terms of a licensing agreement between the author and the Agency for Healthcare Research and Quality. This report may be used and reprinted without permission except those copyrighted materials that are clearly noted in the report. Further reproduction of those copyrighted materials is prohibited without the express permission of copyright holders.

AHRQ or U.S. Department of Health and Human Services endorsement of any derivative products that may be developed from this report, such as clinical practice guidelines, other quality enhancement tools, or reimbursement or coverage policies, may not be stated or implied.

AHRQ appreciates appropriate acknowledgment and citation of its work. Suggested language for acknowledgment: This work was based on an evidence report, Treatments for Acute Pain: A Systematic Review, by the Evidence-based Practice Center Program at the Agency for Healthcare Research and Quality (AHRQ).

Suggested citation: Chou R, Wagner J, Ahmed AY, Blazina I, Brodt E, Buckley DI, Cheney TP, Choo E, Dana T, Gordon D, Khandelwal S, Kantner S, McDonagh MS, Sedgley C, Skelly AC. Treatments for Acute Pain: A Systematic Review. Comparative Effectiveness Review No. 240. (Prepared by the Pacific Northwest Evidence-based Practice Center under Contract No. 2902015-00009-I.) AHRQ Publication No. 20(21)-EHC006. Rockville, MD: Agency for Healthcare Research and Quality; December 2020. DOI: https://doi.org/10.23970/AHRQEPCCER240. Posted final reports are located on the Effective Health Care Program search page. 


\section{Preface}

The Agency for Healthcare Research and Quality (AHRQ), through its Evidence-based Practice Centers (EPCs), sponsors the development of evidence reports and technology assessments to assist public- and private-sector organizations in their efforts to improve the quality of healthcare in the United States.

The Centers for Disease Control and Prevention requested this report from the EPC Program at AHRQ. AHRQ assigned this report to the Pacific Northwest Evidence-based Practice Center (Contract Number: 290-2015-00009-I).

The reports and assessments provide organizations with comprehensive, evidence-based information on common medical conditions and new healthcare technologies and strategies. They also identify research gaps in the selected scientific area, identify methodological and scientific weaknesses, suggest research needs, and move the field forward through an unbiased, evidence-based assessment of the available literature. The EPCs systematically review the relevant scientific literature on topics assigned to them by AHRQ and conduct additional analyses when appropriate prior to developing their reports and assessments.

To bring the broadest range of experts into the development of evidence reports and health technology assessments, AHRQ encourages the EPCs to form partnerships and enter into collaborations with other medical and research organizations. The EPCs work with these partner organizations to ensure that the evidence reports and technology assessments they produce will become building blocks for healthcare quality improvement projects throughout the Nation. The reports undergo peer review and public comment prior to their release as a final report.

AHRQ expects that the EPC evidence reports and technology assessments, when appropriate, will inform individual health plans, providers, and purchasers as well as the healthcare system as a whole by providing important information to help improve healthcare quality.

If you have comments on this systematic review, they may be sent by mail to the Task Order Officer named below at: Agency for Healthcare Research and Quality, 5600 Fishers Lane, Rockville, MD 20857, or by email to epc@ahrq.hhs.gov.

Gopal Khanna, M.B.A.

Director

Agency for Healthcare Research and Quality

Christine Chang, M.D., M.P.H.

Acting Director

Evidence-based Practice Center Program

Center for Evidence and Practice Improvement Agency for Healthcare Research and Quality
Arlene S. Bierman, M.D., M.S.

Director

Center for Evidence and Practice

Improvement

Agency for Healthcare Research and Quality

Suchitra Iyer, Ph.D.

Task Order Officer

Center for Evidence and Practice

Improvement

Agency for Healthcare Research and Quality 


\section{Acknowledgments}

The authors gratefully acknowledge the following individuals for their contributions to this project: research associate, Shaun Ramirez, M.P.H., senior research assistant, Erica Hart, M.S.T., research assistant, Melanie Timmins, B.S., and program manager, Elaine Graham, M.L.S., all from Oregon Health \& Science University; Associate Editor, Margaret Maglione, M.P.P., at the RAND Southern California EPC; and Task Order Officer Suchitra Iyer, Ph.D., at AHRQ.

\section{Technical Expert Panel}

In designing the study questions and methodology at the outset of this report, the EPC consulted several technical and content experts. Broad expertise and perspectives were sought. Divergent and conflicted opinions are common and perceived as healthy scientific discourse that results in a thoughtful, relevant systematic review. Therefore, in the end, study questions, design, methodologic approaches, and/or conclusions do not necessarily represent the views of individual technical and content experts.

Technical Experts must disclose any financial conflicts of interest greater than \$5,000 and any other relevant business or professional conflicts of interest. Because of their unique clinical or content expertise, individuals with potential conflicts may be retained. The TOO and the EPC work to balance, manage, or mitigate any potential conflicts of interest identified.

The list of Technical Experts who provided input to this report follows:

G. Caleb Alexander, M.D., M.S.

Professor of Epidemiology

Johns Hopkins Bloomberg School of Public Health

Baltimore, MD

Monika Asnani, M.B.B.S., D.M., Ph.D.*

Professor of Family Medicine and Epidemiology

Caribbean Institute for Health Research

The University of the West Indies, Mona Campus

Kingston, Jamaica

Brian Bateman, M.D., M.Sc.*

Associate Professor of Anesthesia

Harvard Medical School

Boston, MA

Chad Brummett, M.D.

Associate Professor, Anesthesiology

Director, Anesthesia Clinical Research

Director, Division of Pain Research

University of Michigan

Ann Arbor, MI 
Andrew Chang, M.D., M.S.*

Vice Chair of Research and Academic Affairs

Professor of Emergency Medicine

Albany Medical College

Albany, NY

Thomas Dodson, D.M.D., M.P.H.*

Professor \& Chair, Oral and Maxillofacial Surgery

University of Washington

Seattle, WA

Sharon Hertz, M.D. ${ }^{\dagger}$

Director, Division of Anesthesiology, Addiction Medicine, and Pain Medicine

Food and Drug Administration

Silver Spring, MD

Kurt Kroenke, M.D., MACP

Professor of Medicine

Indiana University

VA HSR\&D Center for Health Information and Communication

Indianapolis, IN

Richard Nahin, Ph.D., M.P.H*

Lead Epidemiologist

National Center for Complementary and Integrative Health

Bethesda, MD

*Provided input on Draft Report.

${ }^{\dagger}$ No longer at the Food and Drug Administration.

\section{Peer Reviewers}

Prior to publication of the final evidence report, EPCs sought input from independent Peer Reviewers without financial conflicts of interest. However, the conclusions and synthesis of the scientific literature presented in this report do not necessarily represent the views of individual reviewers.

Peer Reviewers must disclose any financial conflicts of interest greater than $\$ 5,000$ and any other relevant business or professional conflicts of interest. Because of their unique clinical or content expertise, individuals with potential nonfinancial conflicts may be retained. The TOO and the EPC work to balance, manage, or mitigate any potential nonfinancial conflicts of interest identified.

The list of Peer Reviewers follows:

Benjamin Friedman, M.D., M.S.

Professor, Emergency Medicine 
Albert Einstein College of Medicine

Bronx, NY

Paul Moore, D.M.D., Ph.D., M.P.H.

Professor, School of Dental Medicine, Department of Dental Public Health University of Pittsburgh

Pittsburgh, PA

Jennifer Waljee, M.D., M.P.H., M.S.

Associate Professor in the Section of Plastic and Reconstructive Surgery

University of Michigan

Ann Arbor, MI 


\section{Treatments for Acute Pain: A Systematic Review}

\section{Structured Abstract}

Objectives. To evaluate the effectiveness and comparative effectiveness of opioid, nonopioid pharmacologic, and nonpharmacologic therapy in patients with specific types of acute pain, including effects on pain, function, quality of life, adverse events, and long-term use of opioids.

Data sources. Electronic databases $\left(\right.$ Ovid $^{\circledR}$ MEDLINE $^{\circledR}$, PsycINFO $^{\circledR}$, Embase $^{\circledR}$, the Cochrane Central Register of Controlled Trials, and the Cochrane Database of Systematic Reviews) to August 2020, reference lists, and a Federal Register notice.

Review methods. Using predefined criteria and dual review, we selected randomized controlled trials (RCTs) of outpatient therapies for eight acute pain conditions: low back pain, neck pain, other musculoskeletal pain, neuropathic pain, postoperative pain following discharge, dental pain (surgical or nonsurgical), pain due to kidney stones, and pain due to sickle cell disease. Metaanalyses were conducted on pharmacologic therapy for dental pain and kidney stone pain, and likelihood of repeat or rescue medication use and adverse events. The magnitude of effects was classified as small, moderate, or large using previously defined criteria, and strength of evidence was assessed.

Results. One hundred eighty-three RCTs on the comparative effectiveness of therapies for acute pain were included. Opioid therapy was probably less effective than nonsteroidal antiinflammatory drugs (NSAIDs) for surgical dental pain and kidney stones, and might be similarly effective as NSAIDs for low back pain. Opioids and NSAIDs were more effective than acetaminophen for surgical dental pain, but opioids were less effective than acetaminophen for kidney stone pain. For postoperative pain, opioids were associated with increased likelihood of repeat or rescue analgesic use, but effects on pain intensity were inconsistent. Being prescribed an opioid for acute low back pain or postoperative pain was associated with increased likelihood of use of opioids at long-term followup versus not being prescribed, based on observational studies. Heat therapy was probably effective for acute low back pain, spinal manipulation might be effective for acute back pain with radiculopathy, acupressure might be effective for acute musculoskeletal pain, an opioid might be effective for acute neuropathic pain, massage might be effective for some types of postoperative pain, and a cervical collar or exercise might be effective for acute neck pain with radiculopathy. Most studies had methodological limitations. Effect sizes were primarily small to moderate for pain, the most commonly evaluated outcome. Opioids were associated with increased risk of short-term adverse events versus NSAIDs or acetaminophen, including any adverse event, nausea, dizziness, and somnolence. Serious adverse events were uncommon for all interventions, but studies were not designed to assess risk of overdose, opioid use disorder, or long-term harms. Evidence on how benefits or harms varied in subgroups was lacking.

Conclusions. Opioid therapy was associated with decreased or similar effectiveness as an NSAID for some acute pain conditions, but with increased risk of short-term adverse events. Evidence on nonpharmacological therapies was limited, but heat therapy, spinal manipulation, massage, acupuncture, acupressure, a cervical collar, and exercise were effective for specific 
acute pain conditions. Research is needed to determine the comparative effectiveness of therapies for sickle cell pain, acute neuropathic pain, neck pain, and management of postoperative pain following discharge; effects of therapies for acute pain on non-pain outcomes; effects of therapies on long-term outcomes, including long-term opioid use; and how benefits and harms of therapies vary in subgroups. 


\section{Contents}

Evidence Summary ............................................................................................................................... 1

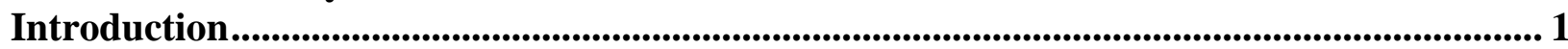

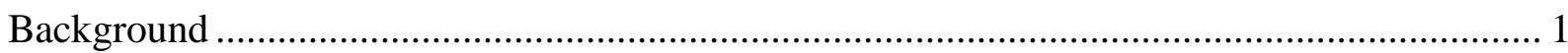

Purpose and Scope of the Systematic Review …………...................................................... 2

Methods............................................................................................................................................ 3

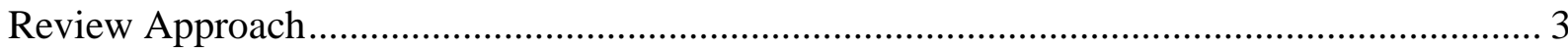

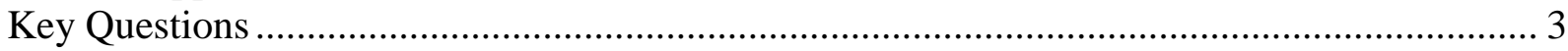

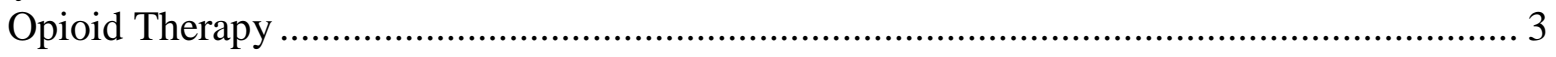

Nonopioid Pharmacologic Therapy ……............................................................................ 4

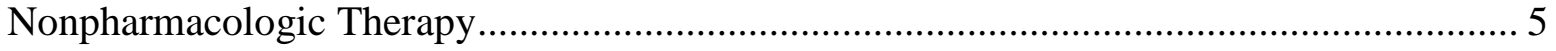

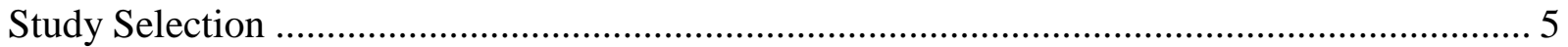

Data Extraction and Risk of Bias Assessment ........................................................................ 6

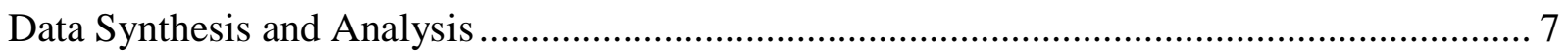

Grading the Strength of the Body of Evidence ........................................................................ 7

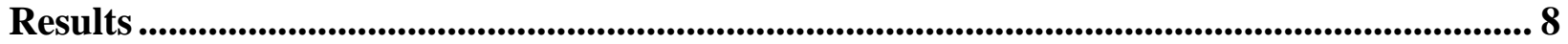

Key Question (KQ) 1. Acute Back Pain (Including Back Pain With Radiculopathy)................. 9

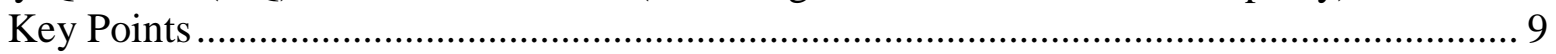

Summary of Findings........................................................................................................... 12

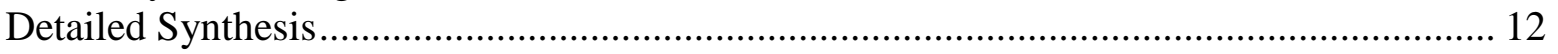

KQ 2. Acute Neck Pain (Including Neck Pain With Radiculopathy)........................................ 38

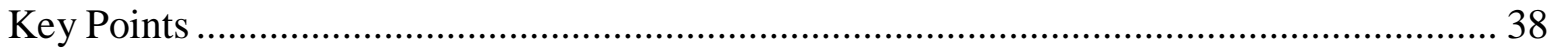

Summary of Findings.................................................................................................... 39

Detailed Synthesis....................................................................................................... 39

KQ 3. Musculoskeletal Pain Not Otherwise Included in KQ1 or KQ2 (Including Fractures) . 42

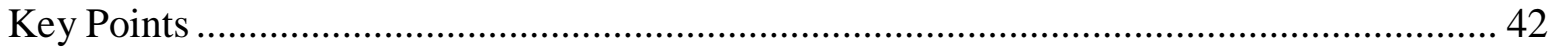

Summary of Findings....................................................................................................... 43

Detailed Synthesis.................................................................................................................... 43

KQ 4. Peripheral Neuropathic Pain (Related to Herpes Zoster and Trigeminal Neuralgia).... 58

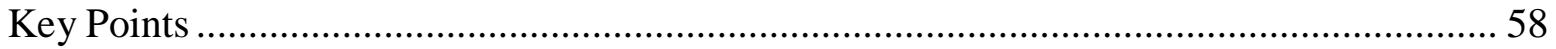

Summary of Findings................................................................................................. 58

Detailed Synthesis.............................................................................................................. 58

KQ 5. Postoperative Pain (Excluding Inpatient Management of Pain Following Major Surgical

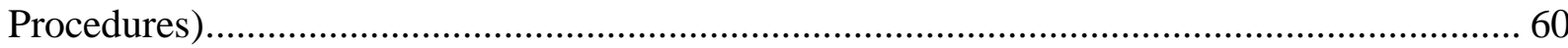

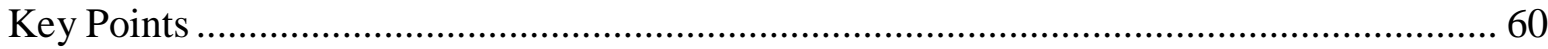

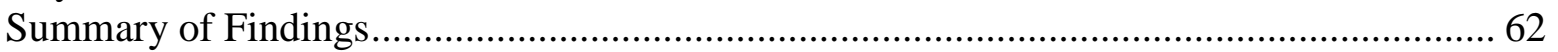

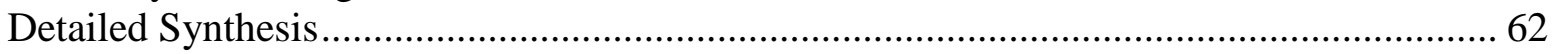

KQ 6. Dental Pain (Surgical and Nonsurgical After Discharge) ............................................... 85

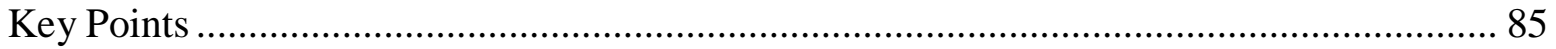

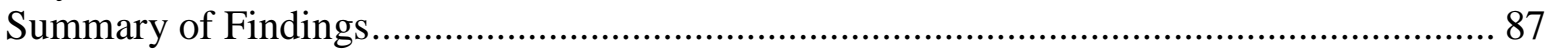

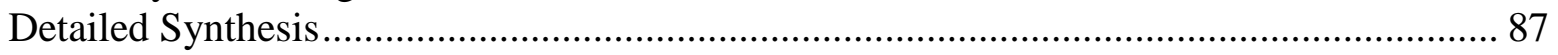

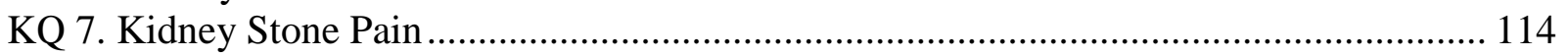

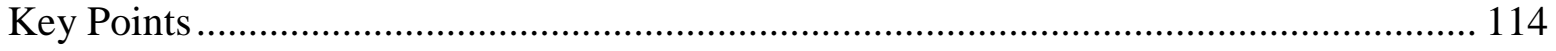

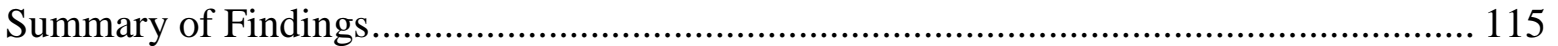

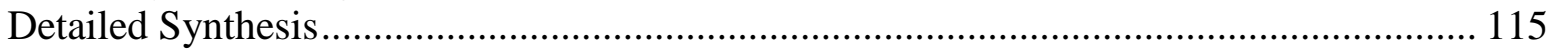

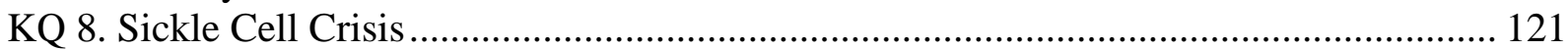




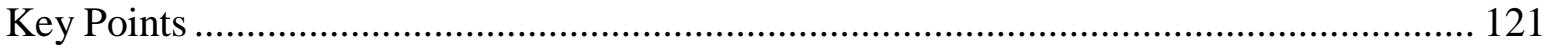

Summary of Findings................................................................................................ 121

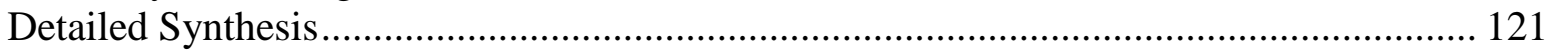

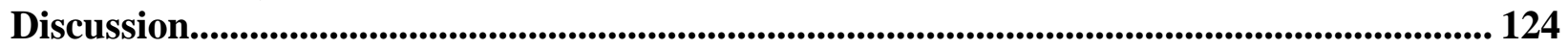

Key Findings and Strength of Evidence ………………................................................... 124

Findings in Relationship to What Is Already Known .......................................................... 130

Applicability .................................................................................................................... 131

Implications for Clinical and Policy Decision Making.......................................................... 131

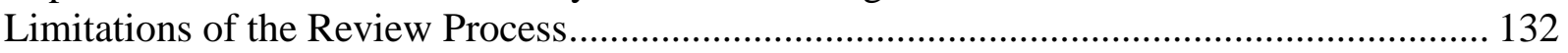

Limitations of the Evidence Base ..................................................................................... 133

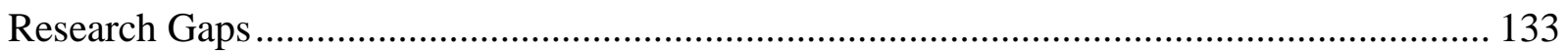

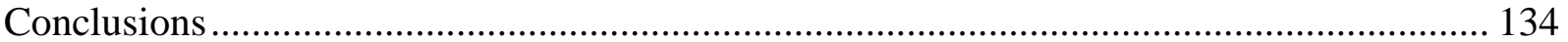

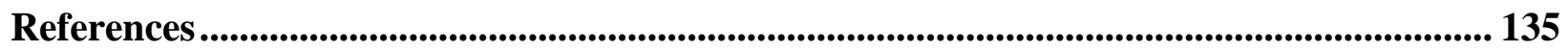

Abbreviations and Acronyms ........................................................................................................ 153

Tables

Table 1. Opioid versus NSAID or muscle relaxant for back pain .................................................. 13

Table 2. Opioid agonists versus mixed agents for back pain........................................................ 15

Table 3. Nonopioid pharmacologic therapy versus other nonopioid pharmacologic therapy for

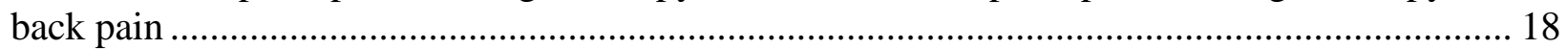

Table 4. Nonopioid pharmacologic therapy versus manipulation for back pain ........................... 20

Table 5. Nonopioid pharmacologic therapy versus acupuncture for back pain............................. 21

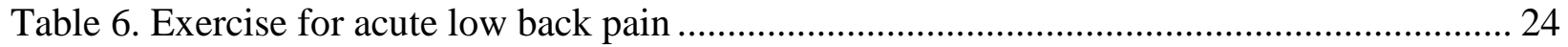

Table 7. Acupuncture versus sham or usual care for acute back pain .............................................. 28

Table 8. Braces or belts versus no braces or belts for acute low back pain.................................... 29

Table 9. Heat versus usual care, placebo, or cold therapy for acute low back pain ....................... 31

Table 10. Manipulation versus usual care, placebo, and other nonpharmacologic interventions for

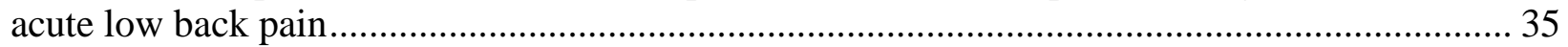

Table 11. Nonpharmacologic interventions for acute neck pain .................................................... 41

Table 12. Opioid therapy versus NSAIDs or mixed agent opioids................................................. 45

Table 13. NSAID versus other pharmacologic treatments for musculoskeletal pain ..................... 48

Table 14. Cold therapy versus other nonpharmacologic therapies for musculoskeletal pain........ 53

Table 15. Ultrasound versus no ultrasound or sham ultrasound for musculoskeletal pain............ 53

Table 16. Other nonpharmacologic therapies for musculoskeletal pain.......................................... 56

Table 17. Opioid versus gabapentin for herpes zoster-related pain ................................................ 59

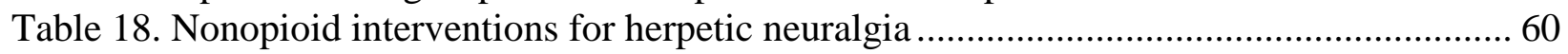

Table 19. Opioid therapy versus nonsteroidal anti-inflammatory drugs for postoperative pain .. 64

Table 20. Opioid therapy versus acetaminophen for postoperative pain ...........................................6 66

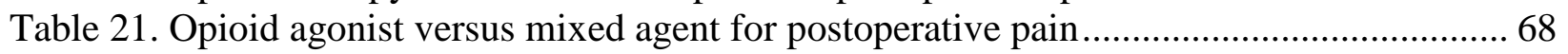

Table 22. NSAIDs versus acetaminophen or aspirin for postoperative pain ................................. 72

Table 23. Acupuncture or acupressure versus sham or usual care .................................................. 74

Table 24. Cold therapies versus no cold therapy or sham for postoperative pain ......................... 77

Table 25. Massage versus no massage for postoperative pain........................................................... 80

Table 26. Music therapy versus silence, usual care, or relaxation for postoperative pain.............. 83

Table 27. Neck stretching exercises versus no exercise for postoperative pain ............................. 84

Table 28. Transcutaneous electrical nerve stimulation or neuromuscular stimulation versus no

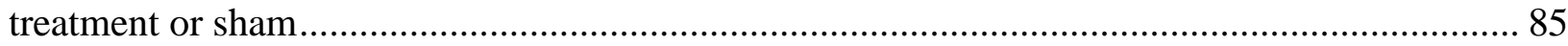


Table 29. Opioid therapy versus NSAIDs for dental pain ..................................................... 91

Table 30. Opioid therapy versus acetaminophen for dental pain........................................... 98

Table 31. Opioid agonists versus mixed agents for dental pain ........................................... 101

Table 32. NSAIDs versus acetaminophen for acute dental pain .......................................... 105

Table 33. Miscellaneous nonopioid pharmacologic interventions versus NSAIDs for acute dental

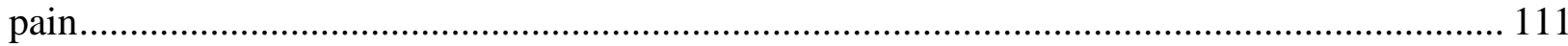

Table 34. Cold therapy versus no cold therapy for dental pain .......................................... 113

Table 35. Opioid versus NSAIDs for acute renal colic ..................................................... 116

Table 36. Opioid versus acetaminophen and opioid agonist versus partial agonist for acute renal

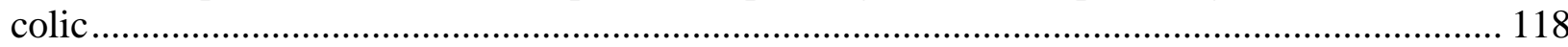

Table 37. NSAIDs versus acetaminophen for acute renal colic ......................................... 119

Table 38. Acupuncture versus NSAID or acetaminophen for acute renal colic....................... 120

Table 39. Opioid agonist or partial agonist versus mixed agent for sickle cell pain ................. 122

Table 40. Nonpharmacologic interventions for sickle cell pain .......................................... 123

Table 41. Summary of evidence of treatments for acute pain: pain ...................................... 125

Figures

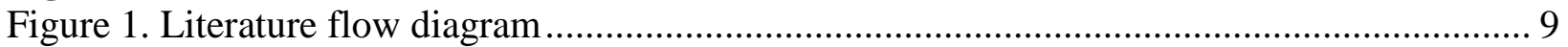

\section{Appendixes}

Appendix A. Literature Search Strategies

Appendix B. Methods

Appendix C. Included Studies List

Appendix D. Forest Plots

Appendix E. Evidence Tables

Appendix F. Risk of Bias Assessment

Appendix G. Details on Strength of Evidence

Appendix H. Excluded Studies List 


\section{Evidence Summary}

\section{Main Points}

- Opioids are probably less effective than nonsteroidal anti-inflammatory drugs (NSAIDs) for surgical dental pain and kidney stone pain and might be similarly effective to NSAIDs for low back pain.

- Opioids might be and NSAIDs are probably more effective than acetaminophen for surgical dental pain, but opioids are probably less effective than acetaminophen for kidney stone pain.

- An opioid might be more effective than gabapentin for acute neuropathic pain.

- Opioids are probably associated with increased risk of short-term adverse events versus nonopioid pharmacologic therapy for acute pain, including any adverse event, study withdrawal due to adverse events, nausea, dizziness, and somnolence, but serious adverse events are uncommon in randomized trials.

- Being prescribed an opioid for acute low back pain or postoperative pain might be associated with increased likelihood of use of opioids at long-term followup versus not being prescribed.

- Heat therapy is probably effective for acute low back pain, spinal manipulation might be effective for acute back pain with radiculopathy, massage might be effective for postoperative pain, and a cervical collar or exercise might be effective for acute neck pain with radiculopathy.

- Research is very limited on the comparative effectiveness of therapies for sickle cell pain, acute neuropathic pain, neck pain, and management of postoperative pain following discharge.

\section{Background and Purpose}

The purpose of this review is to evaluate the effectiveness and comparative effectiveness of opioid, nonopioid pharmacologic, and nonpharmacologic therapy in patients with specific types of acute pain, including effects on pain, function, quality of life, adverse events, and long-term use of opioids.

\section{Methods}

Electronic databases (Ovid ${ }^{\circledR}$ MEDLINE ${ }^{\circledR}$, PsycINFO ${ }^{\circledR}$, Embase $^{\circledR}$, the Cochrane Central Register of Controlled Trials, and the Cochrane Database of Systematic Reviews) were searched through August 5, 2020 for relevant publications. Searches were supplemented by reviewing reference lists and a Federal Register Notice.

Randomized controlled trials (RCTs) of opioid therapy versus nonopioid pharmacologic or nonpharmacologic therapy, nonopioid therapy versus nonpharmacologic therapy, nonpharmacologic therapy versus inactive controls (placebo, sham therapy, attention control, or a minimal intervention), and head-to-head trials of nonopioid pharmacologic and nonpharmacologic therapy were selected using predefined criteria and dual review. Observational studies on the association between being prescribed opioids for acute pain versus no opioids and on factors influencing opioid prescribing for acute pain conditions were also included. This review focused on eight acute pain conditions: low back pain, neck pain, other 
musculoskeletal pain, neuropathic pain, postoperative pain (excluding inpatient management of pain after major surgical procedures), dental pain, pain due to kidney stones, and pain due to sickle cell disease. The review focused on outpatient management or therapy initiated shortly before discharge (e.g., after surgery or in emergency department). Outcomes were analyzed at $<1$ day, 1 day to $<1$ week, 1 week to $<2$ weeks, 2 to $<4$ weeks, and $\geq 4$ weeks. Meta-analyses were conducted on pharmacologic therapy for dental pain and kidney stone pain and likelihood of repeat or rescue medication use and adverse events. Otherwise, meta-analyses were not conducted due to small number of studies, methodological limitations and study heterogeneity. The magnitude of effects was classified as small, moderate or large using previously defined criteria, and strength of evidence was assessed.

\section{Results}

The review included 183 RCTs on the comparative effectiveness of therapies for acute pain. Most studies had methodological limitations. Evidence did not suggest an increased risk of serious harms for any intervention, though studies were not designed to assess serious harms. Effect sizes were primarily small to moderate for pain, the most commonly evaluated outcome. Evidence on how benefits or harms varied in subgroups was lacking. Main findings (focusing on effects on pain) are summarized by acute pain condition.

Low back pain (38 trials): Evidence indicated that there might be no difference between an opioid versus an NSAID and there might be no difference versus a muscle relaxant. Opioids were associated with increased risk of short-term adverse events (any adverse event, study withdrawal due to adverse events, dizziness and nausea) for both comparisons. Serious adverse events were uncommon, but studies were not designed to assess risk of overdose, opioid use disorder, or long-term harms. Being prescribed opioids might be associated with increased risk of long-term use versus not being prescribed, based on observational studies. Muscle relaxants might be associated with small to moderate improvement versus benzodiazepines. There might be no difference between an NSAID or muscle relaxant versus manipulation at 1 to $<2$ weeks, 2 to $<4$ weeks, or $\geq 4$ weeks. Acupuncture might be associated with moderate decrease in pain and improved function at 2 to $<4$ weeks and at 4 weeks. Exercise might be associated with similar effects compared with usual care and probably associated with similar effects compared with bed rest at 1 week to 52 weeks, but might be associated with fewer sick days compared with bed rest at 3 and 12 weeks. Effects of traditional acupuncture varied depending on the type of sham control evaluated. There might be no difference between a brace versus no brace for acute pain associated with osteoporotic compression fracture at 2 to $<4$ weeks and at $\geq 4$ weeks. Heat therapy was probably associated with moderate decrease in pain versus usual care or placebo at 1 day to $<1$ week and 2 to $<4$ weeks. There might be no difference between manipulation versus inactive controls at 1 day to $<1$ week and $\geq 4$ weeks. Manipulation might be associated with increased likelihood of improvement in pain in patients with radiculopathy versus sham at 2 to $<4$ weeks and $\geq 4$ weeks.

Neck pain (5 trials): No trial evaluated pharmacologic therapy for acute neck pain. For neck pain with radiculopathy, a cervical collar or exercise might be associated with moderate to large decreases in pain versus usual activity at 2 to $<4$ weeks and at $\geq 4$ weeks; effects of a collar and exercise were similar. For whiplash neck sprain, there might be no difference between ultrasound versus sham at 1 to $<2$ weeks, and there might be no difference between a cervical collar versus usual activity or exercise at $\geq 4$ weeks. Evidence on other nonpharmacologic therapies for nonradicular neck pain was lacking. 
Other musculoskeletal pain (30 trials): Evidence on opioids versus NSAIDs was insufficient. An NSAID and acetaminophen were probably associated with similar effects for acute musculoskeletal injury (sprains, strains, minor trauma) at $<1$ day, at 1 day to $<1$ week, at 1 to $<2$ weeks, and at $\geq 4$ weeks. There might be no difference between ultrasound versus sham for ankle sprain. Acupressure might be associated with moderate to large decreases in pain at 1 day to $<1$ week and with small effects at 4 weeks compared with sham acupressure or usual care. Evidence on other nonpharmacologic therapy was insufficient.

Peripheral neuropathic pain (2 trials): An opioid might be associated with increased likelihood of improvement in pain versus gabapentin for acute herpes zoster at 1 to $<2$ weeks and at $\geq 4$ weeks, and increased likelihood of constipation. Evidence for acute neuropathic pain was otherwise lacking.

Postoperative pain (47 trials): Trials of postoperative pain focused on treatment in the immediate postoperative period, usually prior to discharge. A multidose course of opioids was probably associated with increased likelihood of repeat or rescue medication use versus an NSAID at $<1$ day. Opioids might be associated with increased risk of study withdrawal due to adverse events versus acetaminophen. Being prescribed an opioid for elective or minor surgery might be associated with increased likelihood of long-term use versus not being prescribed. Legislation mandating the use of prescription drug monitoring data and an opioid risk assessment tool might not decrease opioid prescribing. Auricular acupressure might be associated with decreased pain medication use versus sham at $<1$ day and 1 day to $<1$ week. There might be no difference between cold therapy versus sham in pain at 1 day to $<1$ week, 2 to $<4$ weeks or $\geq 4$ weeks, though cold therapy was associated with decreased pain medication use at $<1$ weeks. Massage might be associated with moderate to large decrease in pain intensity at $<1$ day and decreased pain medication use versus no massage. Single trials suggest that exercise or transcutaneous electrical nerve stimulation (TENS) following specific surgical procedures might reduce pain versus no exercise or sham TENS.

Dental pain (46 trials): A single dose of an opioid plus acetaminophen might be associated with decreased pain and decreased likelihood of rescue or repeat medication use at $<1$ day versus acetaminophen, and probably is associated with increased risk of adverse events. An opioid plus acetaminophen or NSAID is probably associated with a small to moderate increase in pain intensity versus an NSAID at $<1$ day and increased likelihood of rescue or repeat medication use (NSAID doses were lower in the opioid arm than the NSAID-only arm in some trials); opioids were probably associated with increased likelihood of adverse events. NSAIDs are probably associated with moderate to large decrease in pain versus acetaminophen at $<1$ day, decreased likelihood of rescue or repeat medication use, and decreased risk of adverse events. Evidence on nonpharmacologic therapy was insufficient.

Kidney stone pain (12 trials): A single dose of morphine is probably associated with increased likelihood of persistent pain at $<1$ day, decreased likelihood of pain relief, increased likelihood of rescue medication use, and increased likelihood of adverse events versus an NSAID. Findings were similar for a single dose of meperidine, though this medication is discouraged due to concerns about adverse events. NSAIDs might be associated with decreased likelihood of rescue medication use versus acetaminophen. Acupuncture might be associated with moderately increased pain intensity versus a single dose of an NSAID or acetaminophen at $<1$ day.

Sickle cell pain (3 trials): Evidence was insufficient from three small trials with methodological limitations. 


\section{Limitations}

We excluded non-English language articles and did not search for studies published only as abstracts. We did not conduct statistical and graphical methods for assessing for small sample effects (a potential marker for publication bias) due to small numbers of trials and heterogeneity in study design methods, patient populations, and outcomes.

The evidence base had important limitations. First, there was very little evidence for sickle cell pain, acute neuropathic pain, and neck pain. Evidence was also limited for musculoskeletal pain other than low back pain and kidney stone pain. Additionally, trials were not designed to evaluate how benefits and harms varied in subgroups. Patients with a history of substance use disorder, who represent an important clinical challenge, were often excluded from trials, and trials were not designed to evaluate how benefits and harms varied according to opioid dose or amount prescribed. Evidence on the accuracy and effectiveness of risk assessment instruments was unavailable, and evidence on how risk mitigation strategies, patient education, and other factors impact prescribing of opioids was very limited.

\section{Implications and Conclusions}

Opioid therapy was associated with decreased or similar effectiveness for pain versus an NSAID for surgical dental pain, kidney stone pain, and low back pain. Opioids and NSAIDs were more effective than acetaminophen for pain for surgical dental pain, but opioids were less effective than acetaminophen for kidney stone pain. Being prescribed an opioid for acute low back pain or postoperative pain was associated with increased likelihood of use of opioids at long-term followup versus not being prescribed, based on observational studies. With regard to nonpharmacological therapies, heat therapy is probably effective for acute low back pain, spinal manipulation is probably effective for acute back pain with radiculopathy, acupuncture might be effective for acute low back pain, massage might be effective for postoperative pain, acupressure might be effective for acute musculoskeletal pain, and a cervical collar or exercise might be effective for acute neck pain with radiculopathy. Research is needed to determine the comparative effectiveness of therapies for sickle cell pain, acute neuropathic pain, neck pain, and management of postoperative pain following discharge; effects of therapies for acute pain on non-pain outcomes; effects of therapies on long-term outcomes; and how benefits and harms of therapies vary in subgroups. 


\section{Introduction}

\section{Background}

Pain is nearly universal, contributing substantially to morbidity, mortality, disability, and healthcare system burdens. ${ }^{1,2}$ Acute pain has been defined as "the physiologic response and experience to noxious stimuli that can become pathologic, is normally sudden in onset, time limited, and motivates behaviors to avoid actual or potential tissue injuries."3 Acute pain usually lasts for less than 7 days but often extends up to 30 days; ${ }^{4}$ for some conditions, acute pain episodes may recur periodically. In some patients, acute pain persists to become chronic. Acute pain is expected and ubiquitous following surgery. ${ }^{5}$ Pain is the most common reason for emergency department visits and is commonly encountered in primary care, other outpatient, and inpatient settings. ${ }^{1,6,7}$

The key decisional dilemma in acute pain management involves selection of interventions to provide adequate pain relief, in order to improve quality of life, improve function, and facilitate recovery, while minimizing adverse effects and avoiding overprescribing of opioids. ${ }^{8}$ Evidence also suggests that adequate acute pain treatment may mitigate factors that promote the transition to chronic pain. ${ }^{3,9,10}$ However, shortcomings in acute pain care have been documented. ${ }^{11,12}$ In addition to the underlying cause of pain, patient factors that impact acute pain management include age, sex, race/ethnicity, pain severity, comorbidities (including mental health and substance use), genetic factors, pregnancy, or breastfeeding status. ${ }^{13-16}$ Timing of presentation and clinical setting can also influence acute pain management. For example, postoperative pain occurs at a specific point in time and is often managed with multimodal strategies in a monitored setting prior to discharge, whereas in outpatient clinic settings, timing of presentation of acute pain is variable, and assessing treatment response is often not feasible. Additionally, access and care options may vary. ${ }^{2,8}$ Different acute pain conditions (e.g., musculoskeletal pain, neuropathic pain, or visceral pain) may respond differently to treatments. Therefore, a treatment that is effective for one acute pain condition and patient in a particular setting may not be effective in others.

Opioids, traditionally considered the most potent analgesics, are frequently used for acute pain. Therefore, acute pain management must be considered within the context of the current opioid crisis. Opioid prescribing quadrupled from 1999 to 2010; concurrently, the number of opioid analgesics deaths and opioid use disorder cases similarly rose sharply. ${ }^{17}$ In 2017, an estimated 47,600 Americans died from opioid overdose (approximately 17,000 from prescription opioids ${ }^{18}$ ). Until recently, policy efforts have focused on opioids for chronic pain, but attention has increasingly shifted to use for acute pain. Recent data suggest an association between use of opioids for acute pain and persistent long-term use, with some evidence of a dose and durationresponse relationship. ${ }^{17,19-24}$ In addition, some studies indicate that opioids may not be more effective than nonopioid therapies for some acute pain conditions, ${ }^{25-29}$ and use of opioids may negatively affect recovery and function. ${ }^{30,31}$ Opioids prescribed for surgery and other acute pain conditions often go unused, a potential source for diversion and misuse. ${ }^{32-34}$ The 2016 Centers for Disease Control and Prevention (CDC) guideline focused on chronic pain, but included one recommendation to limit opioids for acute pain in most cases to 3 to 7 days. This recommendation was based on evidence indicating an association between use of opioids for acute pain and long-term use. ${ }^{35}$ In the last several years, over 25 states have passed laws restricting prescribing of opioids for pain; nearly half of the states with limits specify that they apply to acute pain. ${ }^{20,36}$ Although data indicate some effects of policies in reducing opioid 
prescribing, studies on clinical outcomes are lacking. Concerns include the effectiveness, availability, and insurance coverage of nonopioid treatment alternatives, potential undertreatment of acute pain, and other unintended consequences. ${ }^{37,38}$ A draft Agency for Healthcare Research and Quality Technical Brief (Treatment for Acute Pain: Evidence Map) identified a number of acute pain conditions for which evidence (from systematic reviews and original research) to inform treatment decisions is available, however it also noted that few reviews were sufficiently rigorous and comprehensive and that an up-to-date comprehensive systematic review would provide valuable information. ${ }^{39}$

\section{Purpose and Scope of the Systematic Review}

This systematic review will assess the comparative effectiveness of treatments and harms of opioid and nonopioid treatments for surgical and nonsurgical pain related to eight acute pain conditions (back pain, neck pain, other musculoskeletal pain, neuropathic pain, postoperative pain [excluding inpatient management of pain following major surgical procedures], dental pain, kidney stones, and sickle cell crisis). The intended audience includes the CDC, policy and decision makers, and clinicians who treat acute pain. A concurrent review addresses treatments for acute pain related to episodic migraines. 


\section{Methods}

\section{Review Approach}

This Comparative Effectiveness Review follows the methods suggested in the Agency for Healthcare Research and Quality (AHRQ) Methods Guide for Effectiveness and Comparative Effectiveness Reviews ${ }^{40}$ (hereafter “AHRQ Methods Guide”). All methods were determined a priori, and a protocol was published on the AHRQ website (https://effectivehealthcare.ahrq.gov/products/treatments-acute-pain/protocol) and registered with the PROSPERO systematic reviews registry (CRD42020165677). Below is a summary of the methods used in this review. Search strategies appear in Appendix A, and detailed methods are described in Appendix B.

\section{Key Questions}

Each Key Question (KQ) for this review focuses on a specific acute pain condition. The conditions and related subquestions are listed below:

KQ1: Acute back pain (including back pain with radiculopathy)

KQ2: Acute neck pain (including neck pain with radiculopathy)

KQ3: Musculoskeletal pain not otherwise included in KQ1 or KQ2 (including fractures)

KQ4: Peripheral neuropathic pain (related to herpes zoster and trigeminal neuralgia)

KQ5: Postoperative pain (excluding inpatient management of pain

following major surgical procedures)

KQ6: Dental pain (surgical and nonsurgical)

KQ7: Kidney stones (including inpatient management)

KQ8: Sickle cell crisis (episodic pain)

For each condition above, we addressed the following subquestions:

\section{Opioid Therapy}

a. What is the comparative effectiveness of opioid therapy versus: (1) nonopioid pharmacologic therapy (e.g., acetaminophen, nonsteroidal anti-inflammatory drugs [NSAIDs], antidepressants, anticonvulsants) or (2) nonpharmacologic therapy (e.g., exercise, cognitive behavioral therapy, acupuncture) for outcomes related to pain, function, pain relief satisfaction, and quality of life and after followup at the following intervals: less than 1 day, 1 day to less than 1 week, 1 week to less than 2 weeks, 2 weeks to less than 4 weeks, 4 weeks or longer?

b. How does effectiveness of opioid therapy vary depending on: (1) patient demographics (e.g., age, race, ethnicity, gender); (2) patient medical or psychiatric comorbidities; (3) dose of opioids; (4) duration of opioid therapy, including number of 
opioid prescription refills and quantity of pills used; (5) opioid use history; (6) substance use history; (7) use of concomitant therapies?

c. What are the harms of opioid therapy versus nonopioid pharmacologic therapy or nonpharmacologic therapy with respect to: (1) misuse, opioid use disorder, and related outcomes; (2) overdose; (3) other harms, including gastrointestinal-related harms, falls, fractures, motor vehicle accidents, endocrinological harms, infections, cardiovascular events, cognitive harms, and psychological harms (e.g., depression)?

d. How do harms vary depending on: (1) patient demographics (e.g., age, gender); (2) patient medical or psychiatric comorbidities; (3) the dose of opioid used; (4) the duration of opioid therapy; (5) opioid use history; or (6) substance use history?

e. What are the effects of prescribing opioid therapy versus not prescribing opioid therapy for acute pain on (1) short-term ( $<3$ months) continued need for prescription pain relief, such as need for opioid refills, and (2) long-term opioid use (3 months or greater)?

f. For patients with acute pain being considered for opioid therapy, what is the accuracy of instruments for predicting risk of opioid misuse, opioid use disorder, or overdose?

g. For patients with acute pain being considered for opioid therapy, what is the effectiveness of instruments for predicting risk of opioid misuse, opioid use disorder, or overdose?

h. For patients with acute pain being considered for opioid therapy, what is the effect of the following factors on the decision to prescribe opioids: (1) existing opioid management plans; (2) patient education; (3) clinician and patient values and preferences related to opioids; (4) urine drug screening; (5) use of prescription drug monitoring program data; (6) availability of close followup?

\section{Nonopioid Pharmacologic Therapy}

i. What is the comparative effectiveness of nonopioid pharmacologic therapy (e.g., acetaminophen, NSAIDs, antidepressants, anticonvulsants) versus: (1) other nonopioid pharmacologic treatments, such as those in a different medication class, or (2) nonpharmacologic therapy for outcomes related to pain, function, pain relief satisfaction, and quality of life after followup at the following intervals: less than 1 day, 1 day to less than 1 week, 1 week to less than 2 weeks, 2 weeks to less than 4 weeks, 4 weeks or longer?

j. How does effectiveness of nonopioid pharmacologic therapy vary depending on:

(1) patient demographics (e.g., age, race, ethnicity, gender); (2) patient medical and psychiatric comorbidities; (3) the type of nonopioid medication; (4) dose of medication; (5) duration of treatment?

k. What are the harms of nonopioid pharmacologic therapy versus other nonopioid pharmacologic therapy or nonpharmacologic therapy with respect to: (1) misuse; (2) overdose; (3) other harms, including gastrointestinal-related harms, cardiovascularrelated harms, kidney-related harms, falls, fractures, motor vehicle accidents, 
endocrinological harms, infections, cognitive harms, and psychological harms (e.g., depression)?

I. How do harms vary depending on: (1) patient demographics (e.g., age, gender); (2) patient medical comorbidities; (3) the type of nonopioid medication; (4) dose of medication; (5) the duration of therapy?

\section{Nonpharmacologic Therapy}

$\mathrm{m}$. What is the comparative effectiveness of nonpharmacologic therapy versus sham treatment, waitlist, usual care, attention control, and no treatment after followup at the following intervals: less than 1 day, 1 day to less than 1 week, 1 week to less than 2 weeks, 2 weeks to less than 4 weeks, 4 weeks or longer?

$\mathrm{n}$. What is the comparative effectiveness of nonpharmacologic treatments (e.g., exercise, cognitive behavioral therapy, acupuncture) for outcomes related to pain, function, pain relief satisfaction, and quality of life after followup at the following intervals: less than 1 day, 1 day to less than 1 week, 1 week to less than 2 weeks, 2 weeks to less than 4 weeks, 4 weeks or longer?

o. How does effectiveness of nonpharmacologic therapy vary depending on: (1) patient demographics (e.g., age, gender); (2) patient medical and psychiatric comorbidities?

p. How do harms vary depending on: (1) patient demographics (e.g., age, gender); (2) patient medical and psychiatric comorbidities; (3) the type of treatment used; (4) the frequency of therapy; (5) the duration of therapy?

\section{Study Selection}

Electronic searches for evidence were conducted in August 2020 on Ovid $^{\circledR}$ MEDLINE ${ }^{\circledR}$, PsycINFO $^{\circledR}$, Embase ${ }^{\circledR}$, the Cochrane Central Register of Controlled Trials, and the Cochrane Database of Systematic Reviews from the inception of each database. Search strategies are available in Appendix A. Electronic searches were supplemented with review of reference lists of relevant studies. A Federal Register Notice was posted and a Supplemental Evidence And Data for Systematic review (SEADS) portal was available for submission of unpublished studies.

Studies were selected for inclusion using pre-established criteria based on the KQs and populations, interventions, comparators, outcomes, timing, and settings (PICOTS) of interest (Appendix B, Table B-1). ${ }^{40}$ Briefly, this report focused on the eight acute pain (defined as pain $<4$ weeks in duration) conditions described above. The focus of the report was on outpatient management of acute pain; therefore, it excluded studies on inpatient management of acute pain, including inpatient management of pain following major surgical procedures. However, because acute pain is often initially managed in emergency department and acute care settings, studies in which therapy for included acute pain conditions was initiated in such settings in patients who were then discharged within one day or expected to be discharged soon were included. Studies of therapies initiated in postoperative or dental care settings were also included. In some cases (e.g., single dose studies of pharmacologic therapies or single session of nonpharmacologic therapy) therapy was not continued as an outpatient; we did not exclude such studies. Because few studies on outpatient management of sickle cell pain were expected, inpatient studies were included. 
For pharmacologic therapy (opioid or nonopioid), this report focused on studies of comparative effectiveness, to address decisional dilemmas regarding the choice of pharmacologic therapy. The efficacy of pharmacologic therapy versus placebo has been addressed in other reviews. For nonpharmacologic therapy, there is more uncertainty about efficacy for acute pain. Therefore, studies comparing nonpharmacologic therapy versus inactive therapies (placebo, sham therapy, no therapy, attention control, or minimal intervention) were included, as well as head-to-head studies of comparative effectiveness. Interventions that involved combinations of different therapy were excluded, with the exception of an opioid plus acetaminophen or NSAID, since these medications are commonly administered together; this combination was classified as an opioid and analyzed separately from an opioid alone if sufficient data were available. Studies in which all patients received background therapy (i.e., not involved in the analyzed comparison) were included, as long as the background therapy could reasonably be considered usual care (e.g., non-opioid analgesics, education, or standard home exercises).

Outcomes were pain, function, quality of life (including mood and sleep), and harms. Outcomes were assessed at prespecified time periods: $<1$ day, 1 day to $<1$ week, 1 week to $<2$ weeks, 2 weeks to $<4$ weeks, and $\geq 4$ weeks.

For KQs related to effectiveness and comparative effectiveness, inclusion was restricted to randomized controlled trials. Controlled observational studies (cohort, case-control, and beforeafter studies) were eligible to assess effects of opioid prescribing versus no prescribing on longterm use, accuracy and effectiveness of risk prediction instruments, and factors influencing prescribing.

All citations were reviewed by one investigator for potential inclusion and full text review. Excluded abstracts were reviewed by a second investigator to confirm the exclusion decision. Each full-text article was independently reviewed for eligibility by two team members. Disagreements were resolved by consensus.

Searches will be updated for new publication while the draft report is posted for public comment. Literature identified during the update search will be assessed using the process described above for the original search. Any pertinent new literature identified in the update search will be incorporated into the report prior to finalization.

\section{Data Extraction and Risk of Bias Assessment}

Data were extracted from included studies into standardized Excel spreadsheets, including study characteristics, inclusion criteria, patient characteristics, and results. Some studies reported summary data based on multiple pain measurements over a specified time period (e.g., average pain or sum of pain intensity differences). When available, we reported outcomes reported at specific time points; when necessary, data were estimated from figures provided in the studies. We extracted continuous as well as dichotomous results. We prioritized results for pain intensity in the following order: 1) mean difference in pain intensity at followup; 2) mean difference in pain intensity change from baseline; 3) mean difference in pain relief at followup; 4) other outcomes for pain intensity. Effects on pain intensity were converted when necessary to a 0 to 10 scale to facilitate interpretation across studies using different scales. Unadjusted relative risks and mean differences with 95 percent confidence intervals were calculated if necessary, using an online calculator (MedCalc).

Predefined criteria were used to assess the quality of individual controlled trials, in conjunction with the approach recommended in the chapter, AHRQ Methods Guide. ${ }^{40}$ 


\section{Data Synthesis and Analysis}

Evidence tables were created to show detailed study characteristic and results, and summary tables were created to highlight the main findings. Meta-analyses were not appropriate for most comparisons and outcomes due to the small number of studies for each comparison and outcome, methodological limitations in the studies, and variability in the studies, including methods for measuring outcomes. ${ }^{41}$ Comparisons and outcomes for which meta-analyses could be conducted were limited to opioids versus NSAIDs or acetaminophen for the outcomes rescue or repeat medication use and selected harms for dental pain and kidney stone pain. Meta-analyses were conducted using Review Manager 5.3 (The Nordic Cochrane Centre, the Cochrane Collaboration, 2014).

The magnitude of effects for pain and function was classified as small, moderate, or large using the same system applied in other recent AHRQ-funded, pain-related systematic reviews conducted at our Evidence-based Practice Center (EPC). ${ }^{42-45}$

\section{Grading the Strength of the Body of Evidence}

The strength of evidence for each KQ/body of evidence was initially assessed by one researcher for each clinical outcome (see PICOTS) by using the approach described in the AHRQ Methods Guide (see Appendix B). ${ }^{40}$ To ensure the consistency and validity of the assessment, the strength of evidence grade was reviewed by the entire team of investigators prior to assigning a final grade. The strength of evidence grades of high, moderate, low, or insufficient was based on the assessment of the following factors:

- Study limitations (low, medium, or high level of study limitations)

- Consistency (consistent, inconsistent, or unknown/not applicable)

- Directness (direct or indirect)

- Precision (precise or imprecise)

- Reporting bias (suspected or undetected) 


\section{Results}

A total of 22,499 abstracts were reviewed, including 22,045 from electronic database searches and 454 from reviewing studies included in prior Evidence-based Practice Center reports and other systematic reviews. After dual review of titles and abstracts, 2,072 were selected for full-text review, of which 183 randomized controlled trials (RCTs) assessing treatments, 5 observational studies on risk of long-term opioid use, and 2 observational studies assessing effects on prescribing rates were included in this review. By pain condition, low back pain was addressed in 38 trials, neck pain in 5 trials, other musculoskeletal pain in 30 trials, neuropathic pain in 2 trials, postoperative pain in 47 trials, dental pain in 46 trials, kidney stone pain in 12 trials, and sickle cell pain in 3 trials. The search results and selection of studies are summarized in the literature flow diagram (Figure 1). Appendix C provides a list of all included studies.

Appendix D contains additional results for pooled data from studies where data were available. Detailed evidence tables for included studies and quality assessments are available in Appendixes E and F. Appendix G contains details on the strength of evidence, and Appendix $\mathrm{H}$ lists excluded studies along with reasons for exclusion. 
Figure 1. Literature flow diagram

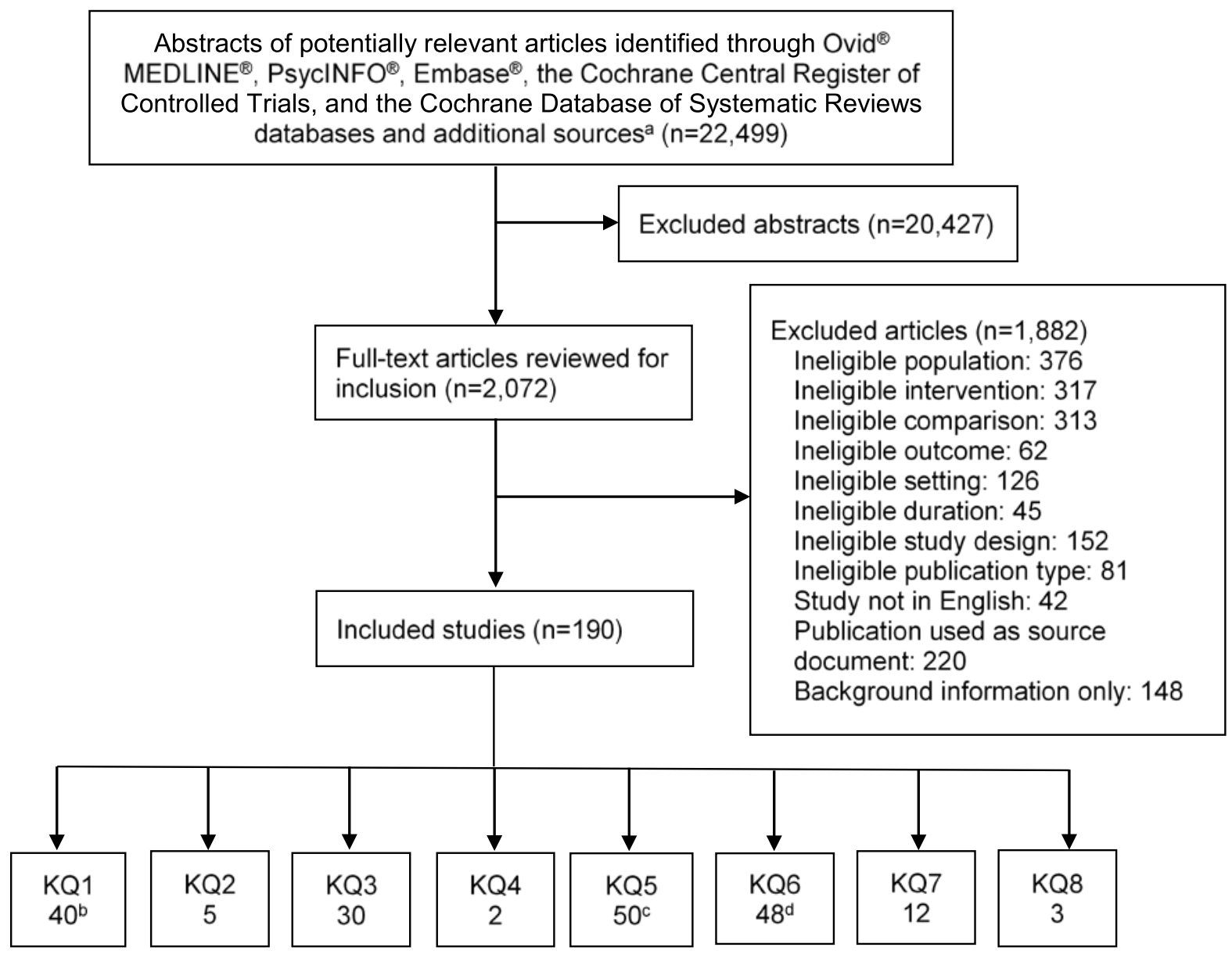

Abbreviations: KQ = Key Question

${ }^{a}$ Additional sources include suggested references, reference lists, etc.

b 38 randomized controlled trials of treatments and 2 observational studies of long-term use.

c 47 randomized controlled trials of treatments, 2 observational studies of long-term use, and 1 observational study assessing effects on prescribing rates.

d 46 randomized controlled trials of treatments, 1 observational study of long-term use, and 1 observational study assessing effects on prescribing rates.

\section{Key Question (KQ) 1. Acute Back Pain (Including Back Pain With Radiculopathy)}

\section{Key Points}

- Opioid vs. nonsteroidal anti-inflammatory drug (NSAID)

0 No difference in pain intensity at $<1$ day, pain relief at 1 day to $<1$ week, or functional impairment at 1 day to $<1$ week, based on 1 trial (strength of evidence [SOE]: low).

o Opioid associated with increased risk of serious adverse events (relative risk [RR] 5.25, 95\% confidence interval [CI] 1.20 to 22.98), study withdrawal due to adverse events ( $11.9 \%$ vs. $0 \%, \mathrm{p}=0.005)$, and any adverse event (64\% vs. 34\%, RR 1.90, 95\% CI 1.28 to 2.83), based on one trial (SOE: low). 
- Opioid vs. muscle relaxant

o No difference in likelihood of pain response, likelihood of functional improvement, or severity of functional impairment at 1 week or 3 months, based on one trial (SOE: low).

o Opioid associated with increased likelihood of dizziness (15\% vs. 3\%, RR 5.33, $95 \%$ CI 1.60 to 17.78 ) and nausea or vomiting (18\% vs. $4 \%$, RR 4.75 , 95\% CI 1.67 to 13.50), based on one trial (SOE: moderate).

- Opioid agonist vs. mixed agent, low back pain due to vertebral compression fracture

0 No difference in pain intensity at 1 day to $<1$ week or at 1 to $<2$ weeks, based on one trial (SOE: low).

0 Estimates for adverse events were imprecise (SOE: insufficient).

- Opioid agonist vs. mixed agent, low back pain with radiculopathy

0 No difference in pain intensity at 1 to $<2$ weeks or likelihood of pain improvement at 1 day to $<1$ week or at 1 to $<2$ weeks, based on one trial (SOE: low).

o Estimates for adverse events were imprecise (SOE: insufficient).

- Opioid prescribing for low back pain versus no prescribing and long-term use

0 Prescribing of opioids for low back pain associated with no difference in opioid use at 3 months in one trial, but increased risk of long-term use (through 1 to 2 years) in two observational studies (SOE: low).

- NSAID vs. acetaminophen

o Insufficient evidence from one poor quality trial (SOE: insufficient).

- Muscle relaxant vs. benzodiazepine

o Muscle relaxant associated with small to moderate decrease in pain at 1 day to $<1$ week, based on two trials (SOE: low).

o Effects on function were inconsistent and imprecise (SOE: insufficient).

o Carisoprodol associated with decreased likelihood of sleep impairment, based on one trial (SOE: low).

- Nonopioid pharmacologic therapy (NSAID or muscle relaxant) vs. manipulation

o Possibly no differences in pain intensity or function at 1 to $<2$ weeks, 2 to $<4$ weeks, or $\geq 4$ weeks, but some inconsistency at 1 to $<2$ weeks, based on three trials (SOE: low).

o Sparse data for adverse events (SOE: insufficient).

- NSAID (single dose) vs. acupuncture

0 Acupuncture associated with moderate decrease in pain and improvement in function at 2 to $<4$ weeks and at 4 weeks, based on one trial (SOE: low).

- Acetaminophen vs. electroacupuncture

o Insufficient evidence from one poor quality trial (SOE: insufficient).

- Exercise vs. usual care

o No differences in pain intensity, likelihood of improvement in pain, or likelihood functional improvement at 1 week to 52 weeks, based on two trials (SOE: low).

o Adverse events were not reported (SOE: insufficient).

- Exercise vs. bed rest

o No differences in pain at 1 week to 52 weeks or function at 3 weeks to 6 months, based on three trials (SOE: moderate). 
o Exercise associated with fewer sick days at 3 weeks and 12 weeks, based on one trial (SOE: low).

o Sparse data for adverse events (SOE: insufficient).

- Exercise vs. advice to remain active

o Evidence limited and somewhat inconsistent, based on two trials (SOE: insufficient).

- Exercise vs. manipulation

o Evidence sparse and with methodological limitations, based on two trials (SOE: insufficient).

- Acupuncture vs. sham or usual care

o Traditional Chinese acupuncture associated with decreased likelihood of persistent low back pain at 2 to $<4$ weeks versus nonpenetrating sham acupuncture (46.9\% vs. $70.3 \%$, RR $0.67,95 \%$ CI 0.49 to 0.90 ) or usual care (46.9\% vs. $72.1 \%$, RR $0.65,95 \%$ CI 0.48 to 0.88 ), but not needle sham acupuncture, based on one trial. No differences between traditional Chinese acupuncture and controls at 3 months (SOE: low).

o Traditional Chinese acupuncture associated with increased likelihood of functional improvement at 2 to $<4$ weeks versus usual care (74\% vs. $44 \%$, RR 1.66, 95\% CI 1.23 to 2.24) and nonpenetrating sham acupuncture (98\% vs. 80\%, RR 1.22, 95\% CI 1.06 to 1.40), but not needle sham acupuncture, based on one trial. No differences between traditional Chinese acupuncture and controls at 3 months (SOE: low).

o In one trial, no differences between scalp acupuncture versus nonpenetrating sham acupuncture in pain or function at 1 day to $<1$ week and 1 to $<2$ weeks; scalp acupuncture associated with moderate reduction in pain intensity and moderate improvement in function at 2 to $<4$ weeks and at 4 weeks (SOE: low)

o No serious adverse events or study withdrawals due to adverse events were reported (SOE: low).

- Brace vs. no brace, osteoporotic compression fracture

0 No difference between brace versus no brace in pain or function at 2 to $<4$ weeks and at $\geq 4$ weeks, based on 1 trial (SOE: low).

- Heat therapy vs. usual care or placebo

o Heat therapy associated with moderate decrease in pain intensity at 1 day to $<1$ week ( 6 trials), 1 to $<2$ weeks ( 2 trials), and 2 to $<4$ weeks ( 2 trials) and moderate improvement in function at 1 day to $<1$ week ( 3 trials), 1 to $<2$ weeks ( 1 trial), and 2 to $<4$ weeks ( 1 trial) (SOE: low to moderate).

o No serious adverse events and few non-serious adverse events (SOE: low).

- Heat therapy vs. cold therapy

o Heat therapy associated with decreased pain intensity at 1 day to $<1$ week, 1 to $<2$ weeks, and 2 to $<4$ weeks, based on one trial (SOE: low).

- Manipulation vs. sham therapy, usual care, placebo, or no treatment; no radiculopathy

0 No differences in pain or function at 1 day to $<1$ week, 1 to $<2$ weeks, 2 to $<4$ weeks, or $\geq 4$ weeks, based on six trials (SOE: low to moderate).

o Reporting of adverse events limited, with few or no serious adverse events reported (SOE: low).

- Manipulation vs. sham manipulation; radiculopathy 
0 Manipulation associated with increased likelihood of reduction in back pain at day 15 (86\% vs. 61\%, RR 1.41, 95\% CI 1.10 to 1.81), reduction in leg pain at days 15, 30 and 180 (RRs 1.20 to 1.55), being back pain free at day 180 (28\% vs. 6\%, RR 5.00, 95\% CI 1.55 to 16.16), and being leg pain free at day 180 (55\% vs. 20\%, RR 2.90, 95\% CI 1.60 to 5.27), based on one trial (SOE: low).

o No adverse events were reported in either group in one trial (SOE: low).

\section{Summary of Findings}

Thirty-eight trials $(\mathrm{N}=4,289)$ evaluated interventions for low back pain (Appendix E, Table E-1). ${ }^{26,46-82}$ Five trials ${ }^{26,51,53,68,82}$ evaluated opioid therapy (KQ 1a and 1c), 11 trials ${ }^{48,50,52,56,59,62,64,66,71,75,81}$ evaluated a nonopioid medication versus nonpharmacologic treatment or another nonopioid (KQ $1 \mathrm{i}$ and 1j), and 22 trials $^{46,47,49,54,55,57,58,60,61,63,65,67,69,70,72-74,76-80}$ (KQ $1 \mathrm{~m}$ and 1o) evaluated nonpharmacologic treatment versus an inactive control or another nonpharmacologic treatment. Fifteen trials ${ }^{26,46,58,61-63,66,68,69,73,74,77-79,82}$ excluded patients with radiculopathy, 3 trials ${ }^{51,65,76}$ were restricted to patients with radiculopathy, 7 trials ${ }^{48,49,57,71,72,75,80}$ enrolled a mixed population, and $13^{47,50,52-56,59,60,64,67,70,81}$ trials did not specify inclusion or exclusion of patients with radiculopathy. The trials did not enroll patients with specific causes of low back pain such as cancer, inflammatory conditions, or infection, with the exception of two trials ${ }^{47,51}$ of patients with vertebral compression fracture. The duration of pain was $<7$ days in 15 trials, ${ }^{26,46,48-50,52,53,57-59,68,69,71,72,80} \geq 7$ days in 11 trials, ${ }^{54,55,60-66,75,79}$ and not described in 12 trials. ${ }^{47,51,56,67,70,73,74,76-78,81,82}$ The duration of treatment ranged from 15 minutes to 12 weeks. The duration of followup was less than 1 week in 7 trials, ${ }^{56,68,70,71,73,74,77} 1$ week to less than 4 weeks in 17 trials, ${ }^{46-54,58,60,61,64,69,78,81,82}$ and more than 4 weeks in 17 trials. $^{26,47-49,55,57,59,62,63,65-}$ 67,72,75,76,79,80 Twelve trials were conducted in the United States, ${ }^{26,51-53,55,60,66,73,74,77,78,82} 15$ trials in Europe, ${ }^{46,49,58,59,61,64,65,69,70,72,75,76,79,80}$ and 11 trials elsewhere. ${ }^{47,48,50,54,56,57,62,63,67,68,71}$ The mean age was 70 years in both trials of patients with vertebral compression fracture. ${ }^{47,51}$ In the other trials, the mean age ranged from 29 to 57. Few trials reported race or ethnicity. Five trials excluded patients with a history of substance use disorder, ${ }^{52,73,74,77,81} 17$ trials excluded pregnant or breastfeeding patients, ${ }^{48,52,53,57,64,66,68-70,73-75,77,78,80,81} 2$ trials excluded patients with psychiatric illness, ${ }^{52,75}$ and 1 trial excluded patients with other comorbidities. ${ }^{68}$ Five trials were rated good quality, ${ }^{26,62,63,76,80} 22$ trials fair quality, ${ }^{47,48,51,52,54,55,57,58,60,61,64-66,68,69,72-74,77,79,81,82}$ and 11 trials poor quality ${ }^{46,49,50,53,56,59,67,70,71,75,78}$ (Appendix F, Table F-1). Methodological limitations in the fair and poor quality trials included failure to report adequate randomization or allocation concealment methods, unblinded design, failure to report attrition, high attrition, and no intention to treat analysis.

\section{Detailed Synthesis}

\section{Opioid Therapy}

KQs 1a and 1c address the comparative effectiveness and harms of opioid therapy versus: 1 ) nonopioid pharmacologic therapy (e.g., acetaminophen, nonsteroidal anti-inflammatory drugs [NSAIDs], antidepressants, anticonvulsants) or 2) nonpharmacologic therapy (e.g., exercise, cognitive behavioral therapy, acupuncture).

Five trials evaluated opioids (with or without acetaminophen) for acute back pain (Appendix E, Table E-1). Two trials compared an opioid agonist versus tapentadol ${ }^{51,82}$ and three trials compared an opioid versus a nonopioid medication (NSAID or muscle relaxant). ${ }^{26,53,68}$ Three 
trials ${ }^{26,53,68}$ excluded patients with radiculopathy or did not describe whether patients with radiculopathy were included or excluded, one trial ${ }^{51}$ was restricted to patients with radiculopathy, and one trial ${ }^{82}$ enrolled patients with acute low back pain due to vertebral compression fracture. Four trials blinded patients and caregivers to trial medications. ${ }^{26,51,68,82}$ One trial was rated good quality, ${ }^{26}$ three trials were rated fair quality, ${ }^{51,68,82}$ and one trial was rated poor quality (Appendix F, Table F-1)..$^{53}$

\section{Opioid Versus NSAID}

One fair quality $(n=113)^{68}$ and one poor quality $(n=40)^{53}$ trial compared codeine plus acetaminophen versus an NSAID for acute low back pain without radiculopathy (Table 1). The fair quality trial found no difference between codeine plus acetaminophen (60/600 mg every 4 to 6 hours as needed) versus ketorolac promethazine (10 mg every 4 to 6 hours as needed, maximum $40 \mathrm{mg}$ daily) in pain intensity at 4 or 6 hours or the sum of pain intensity differences through 6 hours. ${ }^{68}$ There were also no differences in the likelihood of experiencing pain relief or improved function at 4 days. The opioid plus acetaminophen combination was associated with increased risk of serious adverse events (17\% vs. 3\%, RR 5.25, 95\% CI 1.20 to 22.98), study withdrawal due to adverse events $(11.9 \%$ vs. $0 \%, \mathrm{p}=0.005)$, and any adverse event (64\% vs. $34 \%$, RR 1.90, 95\% CI 1.28 to 2.83). The smaller, poor quality trial found no differences in pain intensity at 5 or 7 days. ${ }^{53}$ At 12 days, pain intensity was lower in the opioid arm (mean 0.8 vs. 2.2 on a 0 to 10 scale) but at 15 days it was lower in the NSAID arm (mean 2.5 vs. 1.0 on a 0 to 10 scale). However, results are difficult to interpret because the statistical significance of differences was not reported. Estimates for adverse events were imprecise.

\section{Opioid Versus Muscle Relaxant}

One good quality trial $(n=216)$ compared oxycodone plus acetaminophen (5/326 1 to 2 days every 8 hours as needed) versus cyclobenzaprine (5 to $10 \mathrm{mg}$ every 8 hours) for acute nonradicular low back pain (Table 1 ). ${ }^{26}$ There were no differences in likelihood of pain response (worst pain, none or mild), likelihood of functional improvement (Roland Morris Disability Questionnaire score improved $\geq 5$ points), or mean Roland Morris Disability Questionnaire score at 1 week or 3 months. There was also no difference in time to return to work, or measures of healthcare utilization. Adverse events that were serious or that led to study withdrawal were not reported. Oxycodone plus acetaminophen was associated with increased likelihood of dizziness (15\% vs. 3\%, RR 5.33, 95\% CI 1.60 to 17.78 ) and nausea or vomiting (18\% vs. 4\%, RR 4.75, 95\% CI 1.67 to 13.50 ); serious adverse events were not reported.

Table 1. Opioid versus NSAID or muscle relaxant for back pain

\begin{tabular}{|c|c|c|c|c|}
\hline $\begin{array}{l}\text { Author, } \\
\text { Year } \\
\text { Quality }\end{array}$ & Interventions & $\begin{array}{l}\text { Type of Back } \\
\text { Pain; } \\
\text { Followup Duration } \\
\text { Sample Size }\end{array}$ & Pain Intensity Results & $\begin{array}{l}\text { Other Pain and Function } \\
\text { Results }\end{array}$ \\
\hline $\begin{array}{l}\text { Brown, } \\
1986^{53} \\
\text { Poor }\end{array}$ & $\begin{array}{l}\text { A. Codeine } 60 \mathrm{mg} \\
+ \text { acetaminophen } \\
600 \mathrm{mg} \text { loading } \\
\text { dose, } 30 / 300 \mathrm{mg} \\
\text { every } 4 \text { hours } \\
\text { B. Diflunisal } 1000 \\
\text { mg loading dose, } \\
500 \mathrm{mg} \text { every } 12 \\
\text { hours }\end{array}$ & $\begin{array}{l}\text { Low back pain } \\
5,7,12 \text {, and } 15 \\
\text { days } \\
n=40\end{array}$ & $\begin{array}{l}\text { Pain intensity (mean [SD } \\
\text { NR] } 0 \text { to } 4 \text { categorical } \\
\text { scale converted to } 0 \text { to } \\
10): 2.0 \text { vs. } 2.5 \text { at day } 5 \text {, } \\
2.5 \text { vs. } 2.2 \text { at day } 7,0.8 \\
\text { vs. } 2.2 \text { at day } 12,2.5 \text { vs. } \\
1.0 \text { at day } 15, p=N R\end{array}$ & NR \\
\hline
\end{tabular}




\begin{tabular}{|c|c|c|c|c|}
\hline $\begin{array}{l}\text { Author, } \\
\text { Year } \\
\text { Quality }\end{array}$ & Interventions & $\begin{array}{l}\text { Type of Back } \\
\text { Pain; } \\
\text { Followup Duration } \\
\text { Sample Size }\end{array}$ & Pain Intensity Results & $\begin{array}{l}\text { Other Pain and Function } \\
\text { Results }\end{array}$ \\
\hline $\begin{array}{l}\text { Innes, } \\
1998^{68} \\
\text { Fair }\end{array}$ & $\begin{array}{l}\text { A. Codeine } 60 \mathrm{mg} \\
+ \text { Acetaminophen } \\
600 \mathrm{mg} \text { every } 4 \text { to } \\
6 \text { hours } \\
\text { B. Ketorolac } 10 \mathrm{mg} \\
\text { every } 4 \text { to } 6 \text { hours }\end{array}$ & $\begin{array}{l}\text { Low back pain; } \\
4 \text { and } 6 \text { hours for } \\
\text { pain intensity; } 4 \\
\text { days for other } \\
\text { outcomes } \\
n=113\end{array}$ & $\begin{array}{l}\text { Pain intensity (mean [SD], } \\
0 \text { to } 100 \text { VAS converted to } \\
0 \text { to } 10 \text { scale): } 5.24(2.46) \\
\text { vs. } 5.25(2.08), \mathrm{MD}-0.01 \\
(95 \% \mathrm{Cl}-0.86 \text { to } 0.84) \text { at } \\
4 \text { hours; } 6.21(2.32) \text { vs. } \\
6.16(2.31), \mathrm{MD} 0.05(95 \% \\
\mathrm{Cl}-0.81 \text { to } 0.91) \text { at } 6 \\
\text { hours }\end{array}$ & $\begin{array}{l}\text { Proportion with "a lot" or } \\
\text { "complete" relief of pain } \\
4 \text { days: } 55 \%(95 \% \mathrm{Cl} 42 \% \\
\text { to } 68 \%) \text { vs. } 53 \%(95 \% \mathrm{Cl} \\
40 \% \text { to } 66 \%)\end{array}$ \\
\hline $\begin{array}{l}\text { Friedman, } \\
2015^{26} \\
\text { Good }\end{array}$ & $\begin{array}{l}\text { A. Oxycodone } 5 \\
\text { mg }+ \\
\text { acetaminophen } \\
325 \text { mg every } 8 \\
\text { hours } \\
\text { B. } \\
\text { Cyclobenzaprine } 5 \\
\text { mg every } 8 \text { hours } \\
\text { Both groups } \\
\text { received naproxen } \\
500 \text { mg every } 12 \\
\text { hours }\end{array}$ & $\begin{array}{l}\text { Low back pain; } \\
1 \text { week and } 3 \\
\text { months } \\
n=216\end{array}$ & $\begin{array}{l}\text { Worst pain in } 24 \text { hours } \\
\text { mild or none: } 65 \% \\
\text { ( } 70 / 108) \text { vs. } 60 \%(65 / 108) \\
\text { at } 1 \text { week, RR } 1.08(95 \% \\
\mathrm{Cl} 0.88 \text { to } 1.32) ; 81 \% \\
(87 / 108) \text { vs. } 75 \%(81 / 108) \\
\text { at } 3 \text { months, RR } 1.07 \\
(95 \% \mathrm{Cl} 0.93 \text { to } 1.24)\end{array}$ & $\begin{array}{l}\text { RMDQ score (mean [SD], } 0 \\
\text { to } 24 \text { scale): } 7.8 \text { ( } 8.5) \text { vs. } \\
8.2(8.5) \text { at } 1 \text { week, MD } \\
-0.4(95 \% \mathrm{Cl}-2.7 \text { to } 1.2) \text {; } \\
4.6(7.7) \text { vs. } 4.5(7.7) \text { at } 3 \\
\text { months, MD } 0.2(95 \% \mathrm{Cl} \\
-0.9 \text { to } 2.2) \\
\text { RMDQ score improved } \geq 5 \\
\text { points: } 11.1 \%(12 / 108) \text { vs. } \\
10.1 \%(11 / 108) \text { at } 1 \text { week, } \\
\text { ARD } 0.9 \%(95 \% \mathrm{Cl}-2.1 \text { to } \\
3.9)\end{array}$ \\
\hline
\end{tabular}

Abbreviations: $\mathrm{ARD}$ = absolute risk difference; $\mathrm{CI}$ = confidence interval; $\mathrm{MD}$ = mean difference; $\mathrm{NR}$ = not reported; RMDQ = Roland Morris Disability Questionnaire; RR = relative risk; VAS = visual analog scale

\section{Opioid Agonist Versus Mixed Agent}

Two fair quality trials compared an opioid agonist versus a mixed mechanism agent (tapentadol) for acute low back pain with radiculopathy ${ }^{51}$ or due to vertebral compression fracture (Table 2) ${ }^{82}$ Compared with patients in the radiculopathy trial, patients in the compression fracture trial were older (mean age 70 versus 45 years) and a higher proportion was female (71\% versus 50\%), consistent with the population affected by this condition. ${ }^{82}$ The vertebral compression fracture trial was terminated early after enrolling 108 of a planned 625 patients, due to slow enrollment, resulting in insufficient statistical power for statistical analysis. $^{82}$ It found no differences between immediate-release oxycodone (30 to $90 \mathrm{mg}$ daily) versus tapentadol (200 to $450 \mathrm{mg}$ daily) in the sum of pain intensity differences at 5 or 10 days. The trial of patients with radiculopathy ${ }^{51}$ found no differences between immediate-release oxycodone (20 to $30 \mathrm{mg}$ ) versus tapentadol (200 to $300 \mathrm{mg}$ ) in pain intensity at 10 days or likelihood of $\geq 30$ percent or $\geq 50$ percent improvement in pain intensity at 5 or 10 days. The trials reported few serious adverse events (total five cases). Estimates for study withdrawal due to adverse events and specific adverse events were imprecise. 
Table 2. Opioid agonists versus mixed agents for back pain

\begin{tabular}{|c|c|c|c|c|}
\hline $\begin{array}{l}\text { Author, Year } \\
\text { Quality }\end{array}$ & $\begin{array}{l}\text { Interventions } \\
\text { Treatment } \\
\text { Duration }\end{array}$ & $\begin{array}{l}\text { Type of } \\
\text { Back Pain; } \\
\text { Followup } \\
\text { Duration } \\
\text { Sample Size }\end{array}$ & Pain Intensity Results & $\begin{array}{l}\text { Other Pain and Function } \\
\text { Results }\end{array}$ \\
\hline $\begin{array}{l}\text { Biondi, } 2013^{51} \\
\text { Fair }\end{array}$ & $\begin{array}{l}\text { A: Oxycodone } \\
20 \text { to } 30 \mathrm{mg} \\
\text { daily, titrated } \\
\text { up to } 90 \mathrm{mg} \\
\text { daily } \\
\text { B: Tapentadol } \\
200 \text { to } 300 \mathrm{mg} \\
\text { daily, titrated } \\
\text { up to } 600 \mathrm{mg} \\
\text { daily } \\
10 \text { days }\end{array}$ & $\begin{array}{l}\text { Low back } \\
\text { pain; } \\
10 \text { days } \\
n=613\end{array}$ & 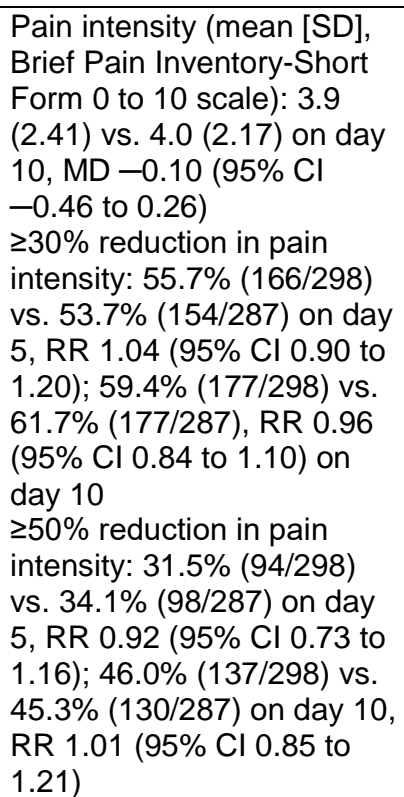 & $\begin{array}{l}\text { Global impression "much } \\
\text { improved" or "very much } \\
\text { improved": } 67.9 \% \text { (205/302) } \\
\text { vs. } 66.2 \% \text { (206/311), RR } 1.02 \\
\text { (95\% Cl } 0.92 \text { to } 1.15)\end{array}$ \\
\hline $\begin{array}{l}\text { Vorsanger, } \\
2013^{82} \\
\text { Fair }\end{array}$ & $\begin{array}{l}\text { A: Oxycodone } \\
5 \text { to } 10 \mathrm{mg} \\
\text { every } 4 \text { to } 6 \\
\text { B: Tapentadol: } \\
50 \text { to } 75 \mathrm{mg} \\
\text { every } 4 \text { to } 6 \\
\text { hours } \\
\text { As needed for } \\
10 \text { days }\end{array}$ & $\begin{array}{l}\text { Low back } \\
\text { pain due to } \\
\text { vertebral } \\
\text { compression } \\
\text { fracture; } \\
5 \text { days and } \\
10 \text { days } \\
n=87\end{array}$ & $\begin{array}{l}\text { Sum of pain intensity } \\
\text { difference (mean [SD], } 0 \text { to } \\
10 \text { scale): } 252.7(208.91) \\
\text { vs. } 227.1(200.66) \text { at day } 5 \text {, } \\
\text { MD } 25.60(95 \% \mathrm{Cl}-61.75 \\
\text { to } 112.95) ; 505.0(373.00) \\
\text { vs. } 422.9(382.78) \text { at day } \\
10, \text { MD } 82.10(95 \% \mathrm{Cl} \\
-79.01 \text { to } 243.21) \text { to } 40.77) \\
\text { at day } 10\end{array}$ & NR \\
\hline
\end{tabular}

Abbreviations: $\mathrm{CI}$ = confidence interval; $\mathrm{MD}$ = mean difference; $\mathrm{NR}$ = not reported; $\mathrm{RR}$ = relative risk; $\mathrm{SD}=$ standard deviation

KQs $1 \mathrm{~b}$ and 1d ask how the comparative effectiveness and harms of opioid therapy vary depending on: (1) patient demographics (e.g., age, race, ethnicity, gender); (2) patient medical or psychiatric comorbidities; (3) dose of opioids; (4) duration of opioid therapy, including number of opioid prescription refills and quantity of pills used; (5) opioid use history; (6) substance use history; (7) use of concomitant therapies.

Evidence on how comparative effectiveness and harms of opioid therapy varied in patients with acute low back pain according to patient and prescribing factors was very limited, due to small numbers of trials for each comparison, small sample sizes, and exclusion or lack of information regarding subgroups of interest, including persons with medical or psychiatric comorbidities or substance use history. No study conducted within-study or across-study evaluations of subgroup effects. Two trials of an opioid agonist versus the mixed agent tapentadol that evaluated different populations (vertebral compression fracture, $71 \%$ female, and mean age 70 years vs. radiculopathy, $50 \%$ female, and mean age 45 years) reported consistent findings of no difference in effectiveness. ${ }^{51,82}$ Evidence was too limited to determine effects of different opioid doses on comparative effectiveness and harms. No trial permitted opioid refills, 
and the duration of treatment was up to 15 days; the trials did not evaluate how effectiveness varied according to the amount of opioid used.

KQ 1e concerns the effects of prescribing opioid therapy versus not prescribing opioid therapy for acute pain on 1 ) short-term ( $<3$ months) continued need for prescription pain relief, such as need for opioid refills, and 2) long-term opioid use (3 months or greater).

One good quality trial of patients presenting to the emergency department with acute low back pain found that at 3 months, rates of opioid use were similarly low in 215 persons randomized to either an opioid plus acetaminophen or placebo (2\% vs. 3\%, absolute risk difference [ARD] $-1 \%, 95 \%$ CI $-5 \%$ to 3\%). Patients in both groups received usual care with naproxen. $^{26}$

Two retrospective, fair quality cohort studies of patients with worker's compensation claims for acute low back pain found an association between prescribing opioid therapy for acute low back pain and continued opioid use (Appendix E, Table E-2). One study $(n=8,443)$ found early opioid use (defined as within 15 days following onset) associated with an increased likelihood of late opioid use (defined as 5 or more opioid prescriptions from 30 to 730 days following onset) versus non-use, ${ }^{31}$ with a dose-response relationship observed between higher early opioid exposure and increased risk. Versus no early opioid use, the adjusted odds ratio (OR) was 2.08 (95\% CI 1.55 to 2.78) for 1 to $140 \mathrm{mg}$ morphine equivalents and increased to 6.14 (95\% CI 4.92 to 7.66$)$ for $\geq 450 \mathrm{mg}$. Another study $(n=2,887)$ of acute low back pain initially evaluated in the emergency department (ED) found being prescribed an opioid within 2 days of the visit associated with increased likelihood of long-term use, defined as $\geq 3$ opioid prescriptions filled between 4 days and 12 months from injury onset (adjusted RR 1.29, 95\% CI 1.05 to 1.58). ${ }^{83}$ It did not evaluate how risk of long-term use varied by opioid dose.

KQs $1 \mathrm{f}$ and $1 \mathrm{~g}$ concern the accuracy and effectiveness of instruments for predicting risk of opioid misuse, opioid use disorder, or overdose in patients with acute pain being considered for opioid therapy.

No study evaluated the accuracy and effectiveness of instruments for predicting risk of opioid misuse, opioid use disorder, or overdose in patients with acute low back pain.

KQ $1 \mathrm{~h}$ addresses the effect of the following factors on the decision to prescribe opioids for patients with acute pain being considered for opioid therapy: (1) existing opioid management plans; (2) patient education; (3) clinician and patient values and preferences related to opioids; (4) urine drug screening; (5) use of prescription drug monitoring program data; (6) availability of close followup.

Substantial variations between providers in rates of prescribing opioids for acute low back pain have been described. ${ }^{84,85}$ However, evidence on the effects of patient education, use of risk mitigation strategies, clinician and patient values and preferences, or availability of followup on decisions to prescribe opioids for acute low back pain is lacking. One study found no difference in rates or amount of opioid prescribing in EDs before versus after a legislative requirement for provider prescription drug monitoring review was enacted. ${ }^{86}$ However, the study did not meet inclusion criteria because the duration of low back pain was not specified; in addition, 39 percent of patients were not opioid-naïve. Another study found no effect of automated prescription drug monitoring program queries on rates or amounts of opioids prescribing in EDs, but also did not meet inclusion criteria because patients with back pain comprised less than 10 percent of the study sample and the duration of symptoms was not specified. ${ }^{87}$ 


\section{Nonopioid Pharmacologic Therapy}

KQs $1 \mathrm{i}$ and $1 \mathrm{k}$ address the comparative effectiveness and harms of nonopioid pharmacologic therapy versus: (1) nonopioid pharmacologic therapy or (2) nonpharmacologic therapy.

Eleven trials evaluated nonopioid pharmacologic therapy for acute back pain (Appendix E, Table E-1). ${ }^{48,50,52,56,59,62,64,66,71,75,81}$ Two trials excluded patients with radiculopathy, ${ }^{62,66}$ three trials included patients with or without radiculopathy, ${ }^{48,71,75}$ and the other trials did not specify inclusion or exclusion of patients with radiculopathy. The nonopioid medication evaluated was an NSAID (diclofenac, indomethacin, mefenamic acid, or diflunisal) in seven trials, ${ }^{48,50,56,62,71,75,81}$ a muscle relaxant (cyclobenzaprine, carisoprodol, or tizanidine, or methocarbamol) in four trials, ${ }^{50,52,64,66}$ acetaminophen in two trials, ${ }^{56,59}$ and a benzodiazepine (diazepam) in two trials. ${ }^{52,64}$ Four trials compared one nonopioid medication versus another nonopioid medication, ${ }^{50,52,56,64}$ and seven trials compared a nonopioid medication versus nonpharmacologic therapy (electroacupuncture or manipulation). ${ }^{48,59,62,66,71,75,81}$ One trial was rated good quality ${ }^{62}$ five trials fair quality, ${ }^{48,52,64,66,81}$ and five trials poor quality (Appendix F, Table F-1). ${ }^{50,56,59,71,75}$ Four head-to-head trials of nonopioid medications blinded patients and caregivers to treatments, ${ }^{50,52,62,64}$ and five trials of nonopioid medications versus nonpharmacologic therapy utilized placebo and sham treatments. . $2,59,62,66,75,81$

\section{NSAID Versus Acetaminophen}

One small $(n=30)$ poor quality crossover trial compared various NSAIDs (indomethacin, mefenamic acid, phenylbutazone, or aspirin) versus acetaminophen for acute low back pain (presence of radiculopathy not specified) (Table 3). ${ }^{56}$ Pain intensity at 1 week was slightly lower with NSAIDs versus acetaminophen (range 4.6 to 5.0 vs. 5.5 on a 0 to 10 scale), but differences were not statistically significant. Estimates were similar for non-aspirin NSAIDs versus aspirin. Estimates for adverse events were imprecise.

\section{Muscle Relaxant Versus NSAID}

One poor quality trial $(\mathrm{n}=87)$ compared cyclobenzaprine ( $10 \mathrm{mg}$ daily) versus diflunisal (1000 mg daily) for acute low back or neck pain (presence of radiculopathy not specified) ${ }^{50}$ (Table 3). The likelihood of a global rating of moderate or marked improvement favored cyclobenzaprine at day 2 ( $30 \%$ vs. $43 \%$, RR $0.70,95 \%$ CI 0.40 to 1.2 ) but the difference was not statistically significant; there was no difference at day 7 to 10 (84\% vs. $79 \%$, RR $1.06,95 \%$ CI 0.86 to 1.30 ). Other outcomes and adverse events were not reported.

\section{Muscle Relaxant Versus Benzodiazepine}

Two fair quality trials ( $\mathrm{n}=80$ and 30$)$ compared a muscle relaxant versus the benzodiazepine diazepam for acute low back pain (presence of radiculopathy not specified) (Table 3). ${ }^{52,64}$ One of the trials also included patients with thoracic back pain. ${ }^{52}$ The muscle relaxant was carisoprodol (1400 mg daily) in one trial ${ }^{52}$ and tizanidine (12 mg daily) in the other. ${ }^{64}$ The dose of diazepam ranged from 12 to $30 \mathrm{mg}$ daily in both trials.

One trial found carisoprodol associated with larger improvement in pain intensity from baseline at 7 days (mean improvement 5.5 vs. 4.6 on a 0 to 10 scale at day 7, p not reported) though effects on pain intensity at day 5 were very similar (mean improvement 4.4 vs. 4.2 ). ${ }^{52}$ Carisoprodol was also associated with greater likelihood of having slight or no pain (52\% vs. $39 \%$ at day $5,65 \%$ vs. $42 \%$ at day $7, \mathrm{p}>0.05$ ) and activity impairment ( $59 \%$ vs. $31 \%$ at day 5 , $71 \%$ vs. $42 \%$ at day 7 ), but the differences were not statistically significant. However, 
carisoprodol was associated with a statistically significant increase in likelihood of having slight or no sleep impairment at day 7 (85\% vs. 61\%, p $\leq 0.05)$. Although results favored carisoprodol for likelihood of any adverse event, central nervous system adverse events, and drowsiness, estimates were imprecise and differences were not statistically significant.

In the other trial, tizanidine was associated with lower pain intensity versus diazepam (mean 4.3 vs. 5.7 on a 0 to 10 scale at 3 days and 2.0 vs. 3.7 at 7 days), though the statistical significance of differences was not reported. ${ }^{64}$ The likelihood of pain improvement was higher with tizanidine (87\% vs. 53\% at day 3, RR 1.62, 95\% CI 0.97 to 2.72 and $93 \%$ vs. $73 \%$ at day 7 , RR 1.27, 95\% CI 0.90 to 1.78), but the differences were not statistically significant. Effects on function were similar between the two medications. The trial only reported one adverse event in a patient randomized to tizanidine.

Table 3. Nonopioid pharmacologic therapy versus other nonopioid pharmacologic therapy for back pain

\begin{tabular}{|c|c|c|c|c|}
\hline $\begin{array}{l}\text { Author, } \\
\text { Year } \\
\text { Quality }\end{array}$ & Interventions & $\begin{array}{l}\text { Type of Back } \\
\text { Pain; } \\
\text { Followup } \\
\text { Duration } \\
\text { Sample Size }\end{array}$ & Pain Intensity Results & $\begin{array}{l}\text { Other Pain and } \\
\text { Function Results }\end{array}$ \\
\hline $\begin{array}{l}\text { Basmajian, } \\
1989^{50} \\
\text { Poor }\end{array}$ & $\begin{array}{l}\text { A. Cyclobenzaprine } \\
10 \text { mg daily for } 7 \text { to } \\
10 \text { days } \\
\text { B. Diflunisal } 1000 \mathrm{mg} \\
\text { daily for } 7 \text { to } 10 \text { days }\end{array}$ & $\begin{array}{l}\text { Mixed back } \\
\text { and neck pain } \\
2 \text { days and } 7 \\
\text { to } 10 \text { days } \\
\mathrm{n}=87\end{array}$ & NR & $\begin{array}{l}\text { Global rating moderate or } \\
\text { marked improvement: } \\
30 \%(13 / 43) \text { vs. } 43 \% \\
(19 / 44) \text { on day } 2 \text {, RR } 0.70 \\
(95 \% \text { Cl } 0.40 \text { to } 1.23) ; \\
84 \%(37 / 44) \text { vs. } 79 \% \\
\text { (31/390) on days } 7 \text { to } 10 \text {, } \\
\text { RR } 1.06 \text { (95\% Cl } 0.86 \text { to } \\
1.30)\end{array}$ \\
\hline $\begin{array}{l}\text { Boyles, } \\
1983^{52} \\
\text { Fair }\end{array}$ & $\begin{array}{l}\text { A. Carisoprodol } 1400 \\
\text { mg daily for } 7 \text { days } \\
\text { B. Diazepam } 20 \mathrm{mg} \\
\text { daily for } 7 \text { days }\end{array}$ & $\begin{array}{l}\text { Lower and } \\
\text { thoracic back } \\
\text { pain; } \\
5 \text { days and } 7 \\
\text { days } \\
n=80\end{array}$ & $\begin{array}{l}\text { Pain intensity difference } \\
\text { (mean improvement from } \\
\text { baseline [SD NR], } 0 \text { to } 100 \\
\text { VAS converted to } 0 \text { to } 10 \\
\text { scale): } 4.4 \text { vs. } 4.2 \text { on day } 5 \text {, } \\
p=N S ; 5.5 \text { vs. } 4.6 \text { on day } 7 \text {, } \\
p=N S \\
\text { Pain slight or none: } 52 \% \text { vs. } \\
39 \% \text { on day } 5, p=N S ; 65 \% \\
\text { vs. } 42 \% \text { on day } 7, p=N S\end{array}$ & 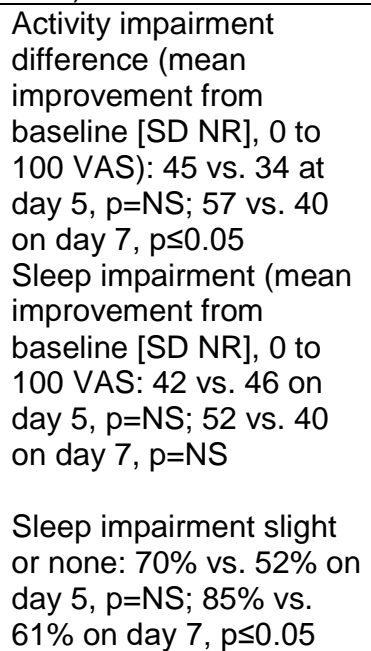 \\
\hline $\begin{array}{l}\text { Evans, } \\
1980^{56} \\
\text { Poor }\end{array}$ & $\begin{array}{l}\text { A: Indomethacin } 150 \\
\text { mg } \times 7 \text { days } \\
\text { B: Mefenamic acid } \\
1500 \text { mg } \times 7 \text { days } \\
\text { C: Phenylbutazone } \\
300 \text { mg } \times 7 \text { days } \\
\text { D: Aspirin } 3600 \mathrm{mg} \times \\
7 \text { days } \\
\text { E: Acetaminophen } \\
4000 \text { mg } \times 7 \text { days }\end{array}$ & $\begin{array}{l}\text { Low back pain; } \\
7 \text { days } \\
\mathrm{n}=30 \\
\text { (crossover) }\end{array}$ & $\begin{array}{l}\text { Pain intensity (mean [SD } \\
\text { NR], } 0 \text { to } 3 \text { scale converted } \\
\text { to } 0 \text { to } 10 \text { ): } 5.0 \text { vs. } 4.6 \text { vs. } \\
4.8 \text { vs. } 4.8 \text { vs. } 5.5, p=N S \text { for } \\
\text { all comparisons }\end{array}$ & NR \\
\hline
\end{tabular}




\begin{tabular}{|c|c|c|c|c|}
\hline $\begin{array}{l}\text { Author, } \\
\text { Year } \\
\text { Quality }\end{array}$ & Interventions & $\begin{array}{l}\text { Type of Back } \\
\text { Pain; } \\
\text { Followup } \\
\text { Duration } \\
\text { Sample Size }\end{array}$ & Pain Intensity Results & $\begin{array}{l}\text { Other Pain and } \\
\text { Function Results }\end{array}$ \\
\hline $\begin{array}{l}\text { Hennies, } \\
1981^{64} \\
\text { Fair }\end{array}$ & $\begin{array}{l}\text { A. Tizanidine } 12 \mathrm{mg} x \\
7 \text { days } \\
\text { B. Diazepam } 12 \text { to } 30 \\
\mathrm{mg} \times 7 \text { days }\end{array}$ & $\begin{array}{l}\text { Nonspecific } \\
\text { back pain; } \\
3 \text { days and } 7 \\
\text { days } \\
\mathrm{n}=30\end{array}$ & $\begin{array}{l}\text { Pain intensity (mean [SD } \\
\text { NR], } 0 \text { to } 3 \text { categorical scale } \\
\text { converted to } 0 \text { to } 10 \text { ) } \\
4.3 \text { vs. } 5.7, p=N R \text { on day } 3 ; \\
2.0 \text { vs. } 3.7, p=N R \text { on day } 7\end{array}$ & $\begin{array}{l}\text { Pain improved: } 86.7 \% \\
\text { (13/15) vs. } 53.3 \%(8 / 15) \\
\text { on day 3, RR } 1.62(95 \% \\
\text { Cl 0.97 to } 2.72) ; 92.9 \% \\
\text { (13/14) vs. } 73.3 \%(11 / 15) \\
\text { on day } 7, \text { RR } 1.27(95 \% \\
\text { Cl } 0.90 \text { to } 1.78) \\
\\
\text { Activities of daily living } \\
\text { (mean [SD NR], scale } \\
\text { NR): } 0.4 \text { vs } 0.8, M D-0.4 \\
\text { (95\% CI NR); p=NR on } \\
\text { day } 7\end{array}$ \\
\hline
\end{tabular}

Abbreviations: $\mathrm{CI}$ = confidence interval; $\mathrm{MD}$ = mean difference; NR = not reported; NS = not significant; RR = relative risk; SD = standard deviation; VAS = visual analog scale

\section{Nonopioid Pharmacologic Therapy Versus Manipulation}

Four trials ( $\mathrm{N}=389)$ compared manipulation versus nonopioid pharmacologic therapy for acute low back pain (Table 4). ${ }^{62,66,75,81}$ One trial was rated good quality, ${ }^{62}$ two trials fair quality, ${ }^{66,81}$ and one trial poor quality. ${ }^{75}$ Two trials excluded patients with radiculopathy, ${ }^{62,66}$ one trial reported results separately for patients with and without radiculopathy, ${ }^{75}$ and one trial did not specify inclusion or exclusion of patients with radiculopathy. ${ }^{81}$ In three trials the nonopioid was the NSAID diclofenac (dose 100 to $150 \mathrm{mg}$ daily or "full dose"); the other trial ${ }^{66}$ evaluated various muscle relaxants (carisoprodol, cyclobenzaprine, or methocarbamol). The manipulation intervention ranged from 3 to 6 sessions over 3 days to 21 sessions over 3 weeks. When described, the manipulation method was usually use of high velocity low amplitude thrust techniques.

The good quality trial $(n=179)$ compared manipulation 12 sessions over 4 weeks versus diclofenac $100 \mathrm{mg}$ daily. ${ }^{62}$ Patients in the diclofenac group received sham therapy with detuned (inactive) ultrasound. There were no differences between manipulation versus diclofenac in pain intensity or function at $1,2,4$, or 12 weeks, or in time to resolution of pain. A fair quality trial $(n=71)$ found manipulation ( 3 to 6 sessions over 3 days) associated with greater effects on function versus diclofenac (150 mg daily) at 7 to 9 days, based on the Roland Morris Disability Questionnaire (mean improvement from baseline 7.71 vs. 4.75 on a 0 to 24 scale, $p=0.01$ ) ${ }^{81}$ The trial also stated that manipulation was associated with greater effects on pain intensity and the 36-Item Short-Form Survey (SF-36) physical component summary score, but did not provide data. There were no statistically significant differences in rescue medication use (mean 2.22 vs. 6.41 tablets, $\mathrm{p}>0.05$ ) or time off work (1.24 vs. 1.80 days). The poor quality trial $(\mathrm{n}=69)$ found manipulation (21 sessions over 3 weeks) associated with better outcomes versus diclofenac (150 mg daily) in patients with or without radiculopathy, but it evaluated outcomes using a nonvalidated composite outcome and results were poorly reported. ${ }^{75}$

One fair quality trial $(n=70)$ found no differences between manipulation (8 sessions over 2 weeks) versus various muscle relaxants in pain intensity, function, depression, or analgesic use at 2 or 4 weeks. $^{66}$

One trial ${ }^{81}$ reported no adverse events, and adverse events were not reported in the other trials. 
Table 4. Nonopioid pharmacologic therapy versus manipulation for back pain

\begin{tabular}{|c|c|c|c|c|}
\hline $\begin{array}{l}\text { Author, } \\
\text { Year } \\
\text { Quality }\end{array}$ & Interventions & $\begin{array}{l}\text { Type of Back } \\
\text { Pain; } \\
\text { Followup } \\
\text { Duration } \\
\text { Sample Size }\end{array}$ & Pain Intensity Results & $\begin{array}{l}\text { Other Pain and Function } \\
\text { Results }\end{array}$ \\
\hline $\begin{array}{l}\text { Hancock, } \\
2007^{62} \\
\text { Good }\end{array}$ & $\begin{array}{l}\text { A: Manipulation, } 12 \\
\text { treatments for } 4 \\
\text { weeks, plus placebo } \\
\text { medication } \\
\text { B: Diclofenac } 100 \mathrm{mg} \\
\text { daily plus detuned } \\
\text { (inactive) pulsed } \\
\text { ultrasound } \\
\text { C: No treatment, } \\
\text { placebo diclofenac } \\
\text { and detuned } \\
\text { ultrasound }\end{array}$ & $\begin{array}{l}\text { Lower back } \\
\text { pain; } \\
2 \text { and } 12 \\
\text { weeks } \\
n=179\end{array}$ & $\begin{array}{l}\text { A vs. B } \\
\text { Pain intensity difference } \\
\text { (mean change from } \\
\text { baseline versus placebo } \\
\text { [95\% Cl], } 0 \text { to } 10 \text { NRS): } \\
0.2(-0.3 \text { to } 0.7) \text { vs. }-0.2 \\
(-0.7 \text { to } 0.3) \text { at week } 1 \text {, } \\
0.4(-1.0 \text { to } 0.1) \text { vs. }-0.1 \\
(-0.7 \text { to } 0.4) \text { at week } 2 \text {, } \\
-0.2(-0.7 \text { to } 0.3) \text { vs. }-0.1 \\
(-0.6 \text { to } 0.4) \text { at week } 4, \\
-0.2(-0.7 \text { to } 0.3) \text { vs. } 0.0 \\
(-0.5 \text { to } 0.4) \text { at week } 12\end{array}$ & $\begin{array}{l}\text { A vs. B } \\
\text { RMDQ difference (mean } \\
\text { change from baseline versus } \\
\text { placebo }[95 \% \mathrm{Cl}], 0 \text { to } 24 \\
\text { scale): }-0.7(-2.1 \text { to } 0.6) \text { vs. } \\
0.5(-0.8 \text { to } 1.8) \text { at week } 1 \text {, } \\
-1.4(-2.7 \text { to } 0.1) \text { vs. }-0.6 \\
(-1.9 \text { to } 0.8) \text { at week } 2,-1.0 \\
(-2.1 \text { to } 0.1) \text { vs. }-0.7(-1.8 \\
\text { to } 0.4) \text { at week } 4,-0.5(-1.7 \\
\text { to } 0.7) \text { vs. }-0.1(-1.3 \text { to } 1.1) \\
\text { at week } 12\end{array}$ \\
\hline $\begin{array}{l}\text { Hoiriis, } \\
2004^{66} \\
\text { Fair }\end{array}$ & $\begin{array}{l}\text { A: Manipulation, } \\
\text { Chiropractic } \\
\text { adjustment + oral } \\
\text { placebo, } 8 \text { visits over } \\
2 \text { weeks } \\
\text { B: Muscle relaxants } \\
\text { + sham chiropractic } \\
\text { adjustment }\end{array}$ & $\begin{array}{l}\text { Lower back } \\
\text { pain; } \\
2 \text { and } 4 \\
\text { weeks } \\
n=70\end{array}$ & $\begin{array}{l}\text { Pain intensity (mean [SD], } \\
0 \text { to } 10 \text { VAS): } 2.44(2.22) \\
\text { vs. } 2.73(2.15), \mathrm{MD}-0.29 \\
(95 \% \mathrm{Cl}-1.33 \text { to } 0.75) \text { at } \\
\text { week } 2 ; 1.71(1.88) \text { vs. } \\
2.24(2.23), \mathrm{MD}-0.53 \\
(95 \% \mathrm{Cl}-1.52 \text { to } 0.46) \text { at } \\
\text { week } 4\end{array}$ & $\begin{array}{l}\text { Oswestry Low Back Pain } \\
\text { Disability Questionnaire } \\
\text { (mean, [SD], } 0 \text { to } 100 \text { scale): } \\
17.02(13.75) \text { vs. } 16.99 \\
(12.18), \text { MD } 0.03(95 \% \mathrm{Cl} \\
-5.32 \text { to } 5.38) \text { at week } 2 ; \\
11.94(11.93) \text { vs. } 16.04 \\
(16.12), \mathrm{MD}-4.10(95 \% \mathrm{Cl} \\
-9.95 \text { to } 1.75) \text { at week } 4\end{array}$ \\
\hline $\begin{array}{l}\text { Postachhini, } \\
1988^{75} \\
\text { Poor }\end{array}$ & $\begin{array}{l}\text { A. Manipulation, daily } \\
\text { for } 1 \text { week, then twice } \\
\text { daily for } 2 \text { weeks } \\
\text { B: Diclofenac, } 150 \mathrm{mg} \\
\text { per day }\end{array}$ & $\begin{array}{l}\text { Low back } \\
\text { pain with and } \\
\text { without } \\
\text { radiculopathy; } \\
3 \text { weeks, } 2 \\
\text { months, and } 6 \\
\text { months } \\
n=69\end{array}$ & NR & $\begin{array}{l}\text { No radiculopathy group } \\
\text { Composite outcome for pain, } \\
\text { function, and flexion-strength } \\
\text { measurements (mean } \\
\text { change from baseline [SD } \\
\text { NR], } 5 \text { to } 32 \text { scale, higher } \\
\text { score indicates better } \\
\text { status): } 24.7 \text { vs. } 17.2 \text { at } \\
\text { week } 3 ; 26.9 \text { vs. } 24.9, \mathrm{p}=\mathrm{NS} \\
\text { at month } 2 ; 29.5 \text { vs. } 28.2 \text {, } \\
\text { p=NS at month } 6 \\
\text { Radiculopathy group } \\
\text { Composite outcome as } \\
\text { above (mean change from } \\
\text { baseline [SD NR]): } 6.3 \text { vs. } \\
4.7, p<0.05 \text { at week } 3 ; 9.2 \\
\text { vs. } 8.7 \text { at month } 2,12.1 \text { vs. } \\
10.9, p=N S \text { at month } 6\end{array}$ \\
\hline $\begin{array}{l}\text { von } \\
\text { Heyman, } \\
2013^{81} \\
\text { Fair }\end{array}$ & $\begin{array}{l}\text { A: Manipulation, high } \\
\text { velocity, low } \\
\text { amplitude (1 to } 2 \\
\text { sessions) plus } \\
\text { placebo diclofenac for } \\
3 \text { days } \\
\text { B: Diclofenac, } 50 \mathrm{mg} \\
\text { three times per day + } \\
\text { sham manipulation } \\
\text { for } 3 \text { days }\end{array}$ & $\begin{array}{l}\text { Low back } \\
\text { pain; } \\
7 \text { to } 9 \text { days } \\
n=71\end{array}$ & $\begin{array}{l}\text { Results "similar" to RMDQ } \\
\text { results, data NR }\end{array}$ & $\begin{array}{l}\text { RMDQ difference (mean } \\
\text { improvement from baseline } \\
\text { [SD], } 0 \text { to } 24 \text { scale): } 7.71 \\
\text { (4.88) vs. } 4.75 \text { (4.93), } \\
\text { p=0.01 } \\
\text { Rescue medication (mean } \\
\text { [SD], number of tablets): } \\
2.22 \text { (3.73) vs. } 6.41 \text { (10.67), } \\
p=N S\end{array}$ \\
\hline
\end{tabular}

Abbreviations: $\mathrm{CI}$ = confidence interval; $\mathrm{MD}$ = mean difference; $\mathrm{NR}=$ not reported; NRS = numeric rating scale; NS = not significant; RMDQ = Roland Morris Disability Questionnaire; SD = standard deviation; VAS = visual analog scale;

${ }^{\text {a }}$ Cyclobenzaprine, carisoprodol, or methocarbamol 


\section{NSAID Versus Acupuncture}

Two trials compared acupuncture versus diclofenac for acute low back pain with or without radiculopathy (proportion with radiculopathy not reported). ${ }^{48,71}$ One fair quality trial $(\mathrm{n}=58)$ compared a single 20 minute session of motion-style acupuncture versus a single intramuscular injection of diclofenac $75 \mathrm{mg}$ in patients with acute low back pain, with or without radiculopathy. ${ }^{48}$ At 2 to 4 weeks, acupuncture was associated with greater improvement from baseline in pain intensity (differences 1.50 and 1.66 points on a 0 to 10 scale), no differences in leg pain intensity, and greater improvement in function (differences 16.9 to 20.1 points on the 0 to 100 Oswestry Disability Index). However, at 24 weeks there were no statistically significant differences in back pain or function, though acupuncture was associated with lower leg pain intensity (difference $-1.85,95 \%$ CI -3.47 to -0.22 ). A small ( $n=44$ ), poor quality trial found no differences between acupuncture (one session daily for 5 days) versus diclofenac $100 \mathrm{mg}$ daily in pain intensity or function at 5 days. ${ }^{71}$ There were no differences in pain intensity or function at 5 days. One trial ${ }^{48}$ reported no adverse events, and in the other trial, ${ }^{71}$ adverse events were not reported.

\section{Acetaminophen Versus Electroacupuncture}

One poor quality trial ( $n=41)$ compared electroacupuncture (two 15-minute sessions) versus acetaminophen (every 4 hours, dose not reported) for acute low back pain (inclusion of patients with radiculopathy not specified $)^{59}$ (Table 5). There was no difference between electroacupuncture versus acetaminophen in pain intensity or function (mobility score) at 1 or 2 weeks, though electroacupuncture was associated with lower pain intensity (mean 0.33 vs. 1.37 on a 0 to 10 scale at 6 weeks, $\mathrm{p}<0.05$ ) and better mobility (mean 1.9 vs. 15.8 on a 0 to 100 scale, $\mathrm{p}<0.05)$ at 6 weeks. Adverse events were not reported.

Table 5. Nonopioid pharmacologic therapy versus acupuncture for back pain

\begin{tabular}{|c|c|c|c|c|}
\hline $\begin{array}{l}\text { Author, } \\
\text { Year } \\
\text { Quality }\end{array}$ & Interventions & $\begin{array}{l}\text { Type of Back } \\
\text { Pain; } \\
\text { Followup } \\
\text { Duration } \\
\text { Sample Size }\end{array}$ & Pain Intensity Results & $\begin{array}{l}\text { Other Pain and Function } \\
\text { Results }\end{array}$ \\
\hline $\begin{array}{l}\text { Hackett, } \\
1988^{59} \\
\text { Poor }\end{array}$ & $\begin{array}{l}\text { A: Electroacupuncture, } \\
\text { two 15-minute } \\
\text { treatments + placebo } \\
\text { tablet } \\
\text { B: Acetaminophen } \\
\text { Every } 4 \text { hours (dose } \\
\text { NR) + placebo } \\
\text { electroacupuncture (no } \\
\text { current) }\end{array}$ & $\begin{array}{l}\text { Low back } \\
\text { pain; } \\
1,2 \text {, and } 6 \\
\text { weeks } \\
n=41\end{array}$ & $\begin{array}{l}\text { Pain intensity (mean [SD } \\
\text { NR], } 0 \text { to } 100 \text { VAS } \\
\text { converted to } 0 \text { to } 10): \\
2.32 \text { vs. } 2.34 \text { at } 1 \text { week, } \\
\text { p=NS; } 1.83 \text { vs. } 2.20 \text { at } 2 \\
\text { weeks, p=NS; } 0.33 \text { vs. } \\
1.37 \text { at } 6 \text { weeks, p<0.05 }\end{array}$ & $\begin{array}{l}\text { Mobility Score (mean [SD } \\
\text { NR], } 0 \text { to } 100 \text { VAS): } 26.5 \text { vs. } \\
25.2 \text { at week } 1, p=N S ; 17.8 \\
\text { vs. } 17.0 \text { at week } 2, p=N S, \\
1.9 \text { vs. } 15.8 \text { at week } 6, \\
\text { p<0.05 }\end{array}$ \\
\hline $\begin{array}{l}\text { Liu, } 2010^{71} \\
\text { Poor }\end{array}$ & $\begin{array}{l}\text { A: Acupuncture, } \\
\text { needles inserted for } 30 \\
\text { minutes with } \\
\text { manipulation every } 10 \\
\text { minutes, once daily for } \\
5 \text { days } \\
\text { B: Diclofenac } 100 \mathrm{mg} \\
\text { daily for } 5 \text { days }\end{array}$ & $\begin{array}{l}\text { Low back } \\
\text { pain with and } \\
\text { without } \\
\text { radiculopathy; } \\
5 \text { days } \\
\mathrm{n}=44\end{array}$ & $\begin{array}{l}\text { Pain intensity (mean [SD], } \\
\text { NRS } 0 \text { to } 10 \text { scale): } 2.65 \\
(1.22) \text { vs. } 3.02(1.56), \mathrm{MD} \\
-0.37(95 \% \mathrm{Cl}-1.22 \text { to } \\
0.48)\end{array}$ & $\begin{array}{l}\text { RMDQ (mean [SD], } 0 \text { to } 24 \\
\text { scale): } 6.45 \text { (2.44) vs. } 6.25 \\
\text { (2.99), MD } 0.20 \text { (95\% Cl } \\
-1.45 \text { to } 1.85)\end{array}$ \\
\hline
\end{tabular}




\begin{tabular}{|c|c|c|c|c|}
\hline $\begin{array}{l}\text { Author, } \\
\text { Year } \\
\text { Quality }\end{array}$ & Interventions & $\begin{array}{l}\text { Type of Back } \\
\text { Pain; } \\
\text { Followup } \\
\text { Duration } \\
\text { Sample Size }\end{array}$ & Pain Intensity Results & $\begin{array}{l}\text { Other Pain and Function } \\
\text { Results }\end{array}$ \\
\hline $\begin{array}{l}\text { Shin, } 2013^{48} \\
\text { Fair }\end{array}$ & $\begin{array}{l}\text { A: Motion style } \\
\text { acupuncture; single } \\
\text { session, approximately } \\
20 \text { minutes } \\
\text { B: Diclofenac: single } \\
75 \text { mg IM injection }\end{array}$ & $\begin{array}{l}\text { Low back } \\
\text { pain with and } \\
\text { without } \\
\text { radiculopathy; } \\
2,4, \text { and } 24 \\
\text { weeks } \\
\mathrm{n}=58\end{array}$ & $\begin{array}{l}\text { Pain intensity difference, } \\
\text { back (mean [SD], } 0 \text { to } 10 \\
\text { NRS): } 5.83 \text { (2.61) vs. } 4.17 \\
\text { (3.05), MD } 1.66 \text { ( } 95 \% \mathrm{Cl} \\
0.17 \text { to } 3.15) \text { at } 2 \text { weeks; } \\
6.41(2.45) \text { vs. } 4.91 \\
\text { (2.94), MD } 1.5(95 \% \mathrm{Cl} \\
0.08 \text { to } 2.92) \text { at } 4 \text { weeks; } \\
6.64(2.47) \text { vs. } 6.84(1.9) \text {, } \\
\text { MD }-0.21 \text { (95\% Cl }-1.37 \\
\text { to } 0.95) \text { at } 24 \text { weeks } \\
\\
\text { Pain intensity difference, } \\
\text { leg (mean [SD] } 0 \text { to } 10 \\
\text { NRS): } 1.57 \text { (2.7) vs } 1.83 \\
\text { (2.66), MD }-0.26(95 \% \mathrm{Cl} \\
-1.67 \text { to } 1.15) \text { at } 2 \text { weeks; } \\
1.59(2.78) \text { vs. } 2.33 \\
\text { (3.06), MD }-0.74(95 \% \mathrm{Cl} \\
-2.28 \text { to } 0.8) \text { at } 4 \text { weeks; } \\
1.64(2.46) \text { vs. } 3.48 \\
\text { (3.62), MD }-1.85(95 \% \mathrm{Cl} \\
-3.47 \text { to }-0.22) \text { at } 24 \\
\text { weeks }\end{array}$ & $\begin{array}{l}\text { ODI, improvement from } \\
\text { baseline (mean [SD], } 0 \text { to } \\
100 \text { scale): } 56.41 \text { (24.86) vs. } \\
36.34 \text { (29.1), MD } 20.07 \\
(95 \% \mathrm{Cl} 5.83 \text { to } 34.31) \text { at } 2 \\
\text { weeks; } 67.72(21.88) \text { vs. } \\
45.84(29.58), \text { MD } 16.88 \\
(95 \% \mathrm{Cl} 3.19 \text { to } 30.57) \text { at } 4 \\
\text { weeks; } 73.23(20.24) \text { vs. } \\
80.83(13.58), \mathrm{MD}-7.6 \\
(95 \% \text { Cl }-16.67 \text { to } 1.47) \text { at } \\
24 \text { weeks }\end{array}$ \\
\hline
\end{tabular}

Abbreviations: $\mathrm{CI}$ = confidence interval; ODI = Oswestry Disability Index; $\mathrm{MD}$ = mean difference; $\mathrm{NR}=$ not reported; NRS = numeric rating scale; NS = not significant; RMDQ = Roland Morris Disability Questionnaire; SD = standard deviation; VAS = visual analog scale

KQs $1 \mathrm{~b}$ and 1d ask how the comparative effectiveness and harms of nonopioid pharmacologic therapy vary depending on: (1) patient demographics (e.g., age, race, ethnicity, gender); (2) patient medical and psychiatric comorbidities; (3) the type of nonopioid medication; (4) dose of medication; (5) duration of treatment.

Evidence was too limited to evaluate how comparative effectiveness and harms of nonopioid therapy for acute low back pain varied in subgroups, due to few trials for each comparison, small sample sizes, methodological limitations, and exclusion or lack of information regarding relevant subgroups. No study conducted within-study or across-study evaluations of subgroup effects. Details regarding the nonopioid medications prescribed, dose, and duration of treatment are described above.

\section{Nonpharmacologic Therapy}

KQs $1 \mathrm{~m}$ and $1 \mathrm{n}$ address the comparative effectiveness of nonopioid pharmacologic therapy versus: (1) inactive controls or (2) other nonpharmacologic therapies.

Twenty-six trials evaluated nonpharmacologic treatment versus an inactive control or another nonpharmacologic treatment for acute back pain (Appendix E, Table E-1). ${ }^{46,47,49,54,55,57,58,60-63,65-}$ 67,69,70,72-81 Twelve trials excluded patients with radiculopathy, 46,58,61-63,66,69,73,74,77-79 six trials included patients with or without radiculopathy, $49,57,65,72,75,76,80$ and the other trials did not specify inclusion or exclusion of patients with radiculopathy. ${ }^{47,54,55,60,67,70,81}$ The nonpharmacologic therapy was heat therapy in 6 trials, ${ }^{54,70,73,74,77,78}$ cold therapy in one trial, ${ }^{54}$ exercise therapy in 6 trials, ${ }^{55,57,65,67,72,79}$ bed rest in 4 trials, ${ }^{49,57,65,72}$ manipulation in 11 trials, ${ }^{55,58,60-62,66,67,69,75,76,81}$ 
mobilization in 1 trial, ${ }^{60,65,69,72}$ acupuncture in 2 trials, ${ }^{63,80}$ braces or belts in 2 trials, ${ }^{46,47}$ and advice to remain active in 2 trials. ${ }^{65,72}$ Eleven trials compared nonpharmacologic treatment versus an inactive control (no treatment, usual care, or minimal intervention), ${ }^{46,47,49,54,57,61,62,65,69,70,72,78-80}$ and four trials compared one nonpharmacologic treatment versus another. ${ }^{47,54,55,60,67}$ Six trials compared nonpharmacologic treatment to sham therapy (designed to look or feel similar to the active nonpharmacologic treatment, but without therapeutic effects). ${ }^{58,63,66,76,80,81}$ Four trials were rated good quality, ${ }^{62,63,76,80} 16$ trials fair quality, ${ }^{47,54,55,57,58,60,61,65,66,69,72-74,77,79,81}$ and 6 trials poor quality ${ }^{46,49,67,70,75,78}$ (Appendix F, Table F-1).

\section{Exercise Versus Usual Care}

Two fair quality trials $(\mathrm{n}=127$ and 67$)$ compared exercise therapy versus usual care in patients with acute low back pain (Table 6). ${ }^{57,79}$ One trial ${ }^{57}$ enrolled a mixed population of patients with and without radiculopathy (proportion with radiculopathy 31\%), and one trial 79 excluded patients with radiculopathy. The exercise intervention was one-on-one instruction in isometric flexion exercises followed by home exercises (3 times daily) in one triall ${ }^{57}$ and group instruction in extension (McKenzie) exercises in the other trial. ${ }^{79}$ Both trials found no differences between exercise therapy versus usual care in pain intensity or the likelihood of improvement in pain at one to 52 weeks. There were also no differences in severity of functional limitations for likelihood of functional improvement at one to 52 weeks, other than increased likelihood of achieving an Oswestry Disability Index score $<20$ (0 to 100 scale) at 1 week in one trial (71\% vs. 47\%, RR 1.52, 95\% CI 1.01 to 2.29$).{ }^{79}$ Adverse events were not reported in either trial.

\section{Exercise Versus Bed Rest}

Three fair quality trials (sample sizes 100 to 162, N=384) compared exercise therapy versus bed rest (Table 6). ${ }^{57,65,72}$ Two trials ${ }^{57,72}$ evaluated mixed populations of patients with and without radiculopathy (proportion with radiculopathy $13 \%$ and $31 \%$ ), and one trial ${ }^{65}$ restricted inclusion to patients with radiculopathy. The exercise intervention was one-on-one instruction in flexion exercise followed by home exercises (three times daily) in one trial, ${ }^{57}$ two sessions weekly of various techniques (segmental mobilization, disc unloading and unloading, and hydrotherapy) in one trial, ${ }^{65}$ and back mobilization exercises (one face-to-face session followed by home exercises) in one trial. ${ }^{72}$ Bed rest ranged from 2 to 7 days. Across the trials, there were no differences between exercise therapy versus bed rest in pain intensity or likelihood of improvement in pain at one to 52 weeks. There were also no difference in function at 3 weeks to 6 months. ${ }^{57,65,72}$ One trial found no differences in health-related quality of life, satisfaction with treatment, or healthcare utilization at 3 or 12 weeks, though exercise therapy was associated with fewer sick days at 3 weeks (mean 5.7 vs. 7.5 days, p not reported) and at 12 weeks (mean 7.2 vs. 9.2 days, p not reported). ${ }^{72}$ The trial of patients with radiculopathy reported one case of cauda equina syndrome (bed rest group), one pulmonary embolism (bed rest group), and 10 cases of recurrent radiculopathy (not reported by group). ${ }^{65}$ Otherwise, the trials did not report adverse events.

\section{Exercise Versus Advice To Remain Active}

Two fair quality trials (sample sizes 163 and 103) compared exercise therapy versus advice to remain active. ${ }^{65,72}$ One trial of patients with radiculopathy found no differences between exercise (segmental mobilization, disc unloading and unloading, and hydrotherapy) versus 
advice to remain active in pain intensity or function at 1 or 6 months. ${ }^{65}$ The other trial $(n=103$; $13 \%$ with radiculopathy) found exercise (back mobilization) associated with worse function at 3 weeks (adjusted mean difference 6.6 points on the 0 to 100 Oswestry Disability Index, 95\% CI 2.0 to 11.1 ) at 3 weeks and more sick days at 3 weeks (adjusted mean difference 1.8 days, 95\% CI 0.1 to 3.5 ) and at 12 weeks (adjusted mean difference 2.5 days, 95\% CI 0.2 to 4.9 ) versus advice to remain active. ${ }^{72}$ Effects on pain intensity favored exercise, but the difference was not statistically significant. There were no differences in health-related quality of life or healthcare utilization, except exercise therapy was associated with more doctor visits (mean difference 0.5, 95\% CI 0.1 to 0.9$).{ }^{72}$ Adverse events were not reported.

\section{Advice To Remain Active Versus Bed Rest}

One poor quality trial $(n=34)$ found advice to remain active associated with no statistically significant differences versus 48 hours of bed rest at 7 or 28 days, though effects favored advice to remain active (differences 3.7 to 9.6 points on a 0 the 0 to 100 Oswestry Disability Index). ${ }^{49}$ There was no difference in likelihood of improvement in the Oswestry Disability Index at 7 or 28 days, though estimates were imprecise.

\section{Exercise Versus Manipulation}

A small ( $n=24)$, fair quality trial (inclusion of patients with radiculopathy not specified) found extension-oriented (McKenzie) exercises associated with decreased likelihood of symptom resolution at 1 week ( $17 \%$ vs. 75\%, RR 0.22 , 95\% CI 0.06 to 0.82 ) and worse function at 5 days (mean 25 vs. 7 on the 0 to 100 Oswestry Disability Index, mean difference 18.0, 95\% CI 8.5 to 27.5). ${ }^{55}$ The other trial $(n=60)$ found exercise associated with improved likelihood of favorable outcomes for pain and function, but had serious methodological limitations and was rated poor quality. ${ }^{67}$ Adverse events were not reported by either trial.

Table 6. Exercise for acute low back pain

\begin{tabular}{|c|c|c|c|c|}
\hline $\begin{array}{l}\text { Author, } \\
\text { Year } \\
\text { Quality }\end{array}$ & Intervention & $\begin{array}{l}\text { Type of } \\
\text { Back Pain; } \\
\text { Followup } \\
\text { Duration } \\
\text { Sample } \\
\text { Size }\end{array}$ & Pain Intensity Results & $\begin{array}{l}\text { Other Pain and Function } \\
\text { Results }\end{array}$ \\
\hline $\begin{array}{l}\text { Erhard, } \\
1994^{55} \\
\text { Fair }\end{array}$ & $\begin{array}{l}\text { A: Exercise therapy, } \\
\text { extension-oriented } \\
\text { (McKenzie) exercises, } 3 \\
\text { times over } 1 \text { week } \\
\text { B: Manipulation, thrust } \\
\text { manipulation and hand-heel } \\
\text { rocking once or twice per } \\
\text { session, } 3 \text { times over } 1 \\
\text { week }\end{array}$ & $\begin{array}{l}\text { Low back } \\
\text { pain; } \\
3 \text { and } 5 \text { days } \\
n=24\end{array}$ & NR & $\begin{array}{l}\text { ODI (mean [SD], } 0 \text { to } 100 \\
\text { scale): } 35(13) \text { vs. } 20 \text { (12), } \\
\text { MD } 15.0(95 \% \text { Cl } 4.4 \text { to } 25.6) \\
\text { on day } 3 ; 25(13) \text { vs. } 7(9), \\
\text { MD } 18.0(95 \% \text { Cl } 8.5 \text { to } 27.5) \\
\text { on day } 5\end{array}$ \\
\hline
\end{tabular}




\begin{tabular}{|c|c|c|c|c|}
\hline $\begin{array}{l}\text { Author, } \\
\text { Year } \\
\text { Quality }\end{array}$ & Intervention & $\begin{array}{l}\text { Type of } \\
\text { Back Pain; } \\
\text { Followup } \\
\text { Duration } \\
\text { Sample } \\
\text { Size }\end{array}$ & Pain Intensity Results & $\begin{array}{l}\text { Other Pain and Function } \\
\text { Results }\end{array}$ \\
\hline $\begin{array}{l}\text { Gilbert, } \\
1985^{57} \\
\text { Fair }\end{array}$ & $\begin{array}{l}\text { A: Exercise therapy, } \\
\text { isometric flexion exercises } \\
\text { three times daily } \\
\text { B: Bed rest, stay in bed for } \geq \\
4 \text { days with written } \\
\text { instructions depicting } \\
\text { appropriate positions } \\
\text { C: Usual care, received } \\
\text { same analgesics as other } \\
\text { groups, no other treatment } \\
\text { or instruction }\end{array}$ & $\begin{array}{l}\text { Low back } \\
\text { pain; } \\
10 \text { days, } 6 \\
\text { weeks, and } \\
12 \text { weeks } \\
n=187\end{array}$ & $\begin{array}{l}\text { Sum of pain intensity scores } \\
\text { through } 10 \text { days (mean } \\
\text { [SD], scale unclear): } 25.94 \\
(7.47) \text { vs. } 24.15 \text { ( }(7.12) \text { vs. } \\
22.88 \text { (5.88), mean } \\
\text { difference } 1.79(95 \% \mathrm{Cl} \\
-1.30 \text { to } 4.88 \text { ) for A vs. B, } \\
3.06 \text { ( } 95 \% \mathrm{Cl} 0.25 \text { to } 5.87) \\
\text { for A vs. C, and } 1.27 \text { (95\% } \\
\mathrm{Cl}-1.38 \text { to } 3.93 \text { ) for B vs. C }\end{array}$ & 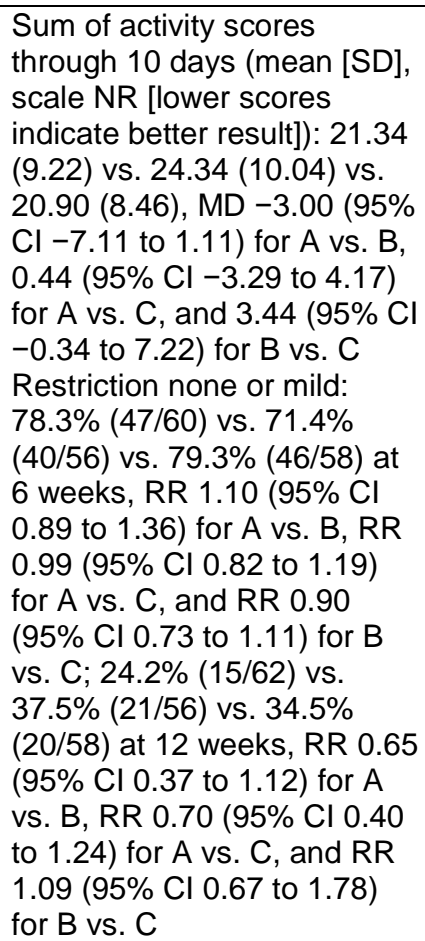 \\
\hline $\begin{array}{l}\text { Hofstee, } \\
2002^{65} \\
\text { Fair }\end{array}$ & $\begin{array}{l}\text { A: Exercise therapy, } \\
\text { instruction and advice, } \\
\text { segmental mobilization, disc } \\
\text { unloading and loading, and } \\
\text { hydrotherapy; } 2 \text { sessions } \\
\text { each week for } 4 \text { to } 8 \text { weeks } \\
\text { B: Bed rest, remain in bed } \\
\text { for } 7 \text { days except to use } \\
\text { bathroom and shower } \\
\text { C: Remain active, continue } \\
\text { usual activities, adjusted } \\
\text { according to pain }\end{array}$ & $\begin{array}{l}\text { Low back } \\
\text { pain; } \\
1 \text { and } 6 \\
\text { months } \\
n=245\end{array}$ & $\begin{array}{l}\text { Pain intensity difference } \\
\text { (mean change from } \\
\text { baseline [SD NR], } 0 \text { to } 100 \\
\text { scale converted to } 0 \text { to } 10 \\
\text { scale): } 2.42 \text { vs. } 2.59 \text { vs. } \\
2.34, \mathrm{MD} 0.08 \text { ( } 95 \% \mathrm{Cl} \\
-0.82 \text { to } 0.98 \text { ) for } \mathrm{A} \text { vs. C } \\
\text { and } 0.25(95 \% \mathrm{Cl}-0.64 \text { to } \\
1.14 \text { ) for } \mathrm{B} \text { vs. } \mathrm{C} \text { at } 1 \text { month; } \\
4.68 \text { vs. } 4.82 \text { vs. } 4.78, \mathrm{MD} \\
-0.10 \text { ( } 95 \% \mathrm{Cl}-1.00 \text { to } \\
0.80 \text { ) for } \mathrm{A} \text { vs. C and } 0.05 \\
\text { (95\% Cl } 0.84 \text { to } 0.93 \text { ) for B } \\
\text { vs. C at } 6 \text { months }\end{array}$ & $\begin{array}{l}\text { Quebec Disability Scale } \\
\text { (mean change from baseline } \\
\text { [SD NR], } 0 \text { to } 100 \text { scale): } \\
15.7 \text { vs. } 11.4 \text { vs. } 16.2, \mathrm{MD} \\
-0.5(95 \% \mathrm{Cl}-6.3 \text { to } 5.3) \text { for } \\
\mathrm{A} \text { vs. } \mathrm{C} \text { and }-4.8(95 \% \mathrm{Cl} \\
-10.6 \text { to } 0.9) \text { for } \mathrm{B} \text { vs. C at } 1 \\
\text { month; } 34.6 \text { vs. } 32.7 \text { vs. } \\
35.4, \mathrm{MD}-0.7(95 \% \mathrm{Cl}-8.4 \\
\text { to } 6.9) \text { for } \mathrm{A} \text { vs. } \mathrm{C} \text { and }-2.7 \\
(95 \% \mathrm{Cl}-10.2 \text { to } 4.8) \text { for B } \\
\text { vs. } \mathrm{C} \text { at } 6 \text { months }\end{array}$ \\
\hline $\begin{array}{l}\text { Hussain, } \\
2013^{67} \\
\text { Poor }\end{array}$ & $\begin{array}{l}\text { A: Manipulation, } 2 \text { to } 3 \\
\text { treatments per week over } 4 \\
\text { weeks } \\
\text { B: Exercise, McKenzie back } \\
\text { extension, William flexion, } \\
\text { raising head in crook lying } \\
\text { position, twice daily for } 4 \\
\text { weeks }\end{array}$ & $\begin{array}{l}\text { Low back } \\
\text { pain; } \\
4 \text { weeks } \\
n=60\end{array}$ & $\begin{array}{l}\text { Pain intensity } 0 \text { to } 2 \text { (0 to } 5 \\
\text { scale): } 96.7 \%(29 / 30) \text { vs. } \\
46.7 \%(14 / 30) \text { RR } 2.07 \\
\text { (95\% Cl } 1.40 \text { to } 3.05)\end{array}$ & $\begin{array}{l}\text { ODI (mean [SD NR], scoring } \\
\text { unclear): } 244 \text { vs. } 388 \text { at } 4 \\
\text { weeks, } p=0.001\end{array}$ \\
\hline
\end{tabular}




\begin{tabular}{|c|c|c|c|c|}
\hline $\begin{array}{l}\text { Author, } \\
\text { Year } \\
\text { Quality }\end{array}$ & Intervention & $\begin{array}{l}\text { Type of } \\
\text { Back Pain; } \\
\text { Followup } \\
\text { Duration } \\
\text { Sample } \\
\text { Size }\end{array}$ & Pain Intensity Results & $\begin{array}{l}\text { Other Pain and Function } \\
\text { Results }\end{array}$ \\
\hline $\begin{array}{l}\text { Malmivaara, } \\
1995^{72} \\
\text { Fair }\end{array}$ & $\begin{array}{l}\text { A. Exercise, back } \\
\text { mobilization, } 1 \text { individual, } \\
\text { physiotherapy-led session; } \\
\text { written instructions for home } \\
\text { exercise every other hour } \\
\text { during the day } \\
\text { B. Bed rest, } 2 \text { full days, then } \\
\text { return to routine activities as } \\
\text { tolerated } \\
\text { C. Usual activity as } \\
\text { tolerated; avoid bed rest }\end{array}$ & $\begin{array}{l}\text { Low back } \\
\text { pain; } \\
3 \text { and } 12 \\
\text { weeks } \\
n=162\end{array}$ & $\begin{array}{l}\text { Pain intensity (mean [SD } \\
\text { NR], } 0 \text { to } 10 \text { NRS): } 3.1 \text { vs. } \\
2.4 \text { vs. } 1.9 \text { at } 3 \text { weeks, } \\
\text { adjusted } \mathrm{MD} 0.9 \text { ( } 95 \% \mathrm{Cl} \\
-0.001 \text { to } 1.7) \text { for } \mathrm{A} \text { vs. C } \\
\text { and } 0.3(95 \% \mathrm{Cl}-0.4 \text { to } \\
0.9) \text { for } \mathrm{B} \text { vs. } \mathrm{C} ; 1.8 \text { vs. } 2.1 \\
\text { vs. } 1.3 \text { at } 12 \text { weeks, } \\
\text { adjusted } \mathrm{MD} 0.2 \text { ( } 95 \% \mathrm{Cl} \\
-0.5 \text { to } 1.0 \text { ) for } \mathrm{A} \text { vs. } \mathrm{C} \text { and } \\
0.7 \text { (95\% } \mathrm{Cl} 0.03 \text { to } 1.4 \text { ) for } \\
\text { B vs. C }\end{array}$ & $\begin{array}{l}\text { ODI (mean [SD NR], } 0 \text { to } 100 \\
\text { scale): } 18.6 \text { vs. } 16.0 \text { vs. } 10.0 \\
\text { at } 3 \text { weeks, adjusted MD } 6.6 \\
\text { ( } 95 \% \mathrm{Cl} 2.0 \text { to } 11.1 \text { ) for } \mathrm{A} \text { vs. } \\
\mathrm{C} \text { and } 3.9 \text { ( } 95 \% \mathrm{Cl}-0.2 \text { to } \\
\text { 8.0) for B vs. C; } 10.8 \text { vs. } 11.8 \\
\text { vs. } 7.4 \text { at } 12 \text { weeks, adjusted } \\
\text { MD } 2.6 \text { ( } 95 \% \mathrm{Cl}-1.6 \text { to } 6.7) \\
\text { for } \mathrm{A} \text { vs. } \mathrm{C} \text { and } 3.8 \text { (95\% Cl } \\
0.1 \text { to } 7.5 \text { ) for B vs. C }\end{array}$ \\
\hline $\begin{array}{l}\text { Underwood, } \\
1998^{79} \\
\text { Fair }\end{array}$ & $\begin{array}{l}\text { A. McKenzie exercises plus } \\
\text { usual care, single 1-hour, } \\
\text { physiotherapist-led session } \\
\text { of passive back extension } \\
\text { exercises; could be } \\
\text { repeated once } \\
\text { B. Usual care, not specified }\end{array}$ & $\begin{array}{l}\text { Low back } \\
\text { pain; } \\
1,2,4 \text {, and } \\
52 \text { weeks } \\
n=67\end{array}$ & $\begin{array}{l}\text { Pain intensity (mean } \\
\text { decrease from baseline [SD } \\
\text { NR], } 0 \text { to } 100 \text { VAS } \\
\text { converted to } 0 \text { to } 10 \text { scale: } \\
1.51 \text { vs. } 1.26 \text { at } 1 \text { week, MD } \\
0.25(95 \% \mathrm{Cl}-0.83 \text { to } \\
1.32) ; 2.43 \text { vs. } 1.98 \text { at } 2 \\
\text { weeks, MD } 0.44(95 \% \mathrm{Cl} \\
-1.05 \text { to } 1.93) ; 2.64 \text { vs. } \\
2.70 \text { at } 4 \text { weeks, MD }-0.06 \\
(95 \% \mathrm{Cl}-1.48 \text { to } 1.36) ; \\
3.58 \text { vs. } 3.67 \text { at } 52 \text { weeks, } \\
\text { MD }-0.09(95 \% \mathrm{Cl}-1.97 \text { to } \\
1.79)\end{array}$ & $\begin{array}{l}\text { ODI (mean decrease from } \\
\text { baseline [SD NR], } 0 \text { to } 100 \\
\text { scale): } 8.4 \text { vs. } 9.9 \text { at week } 1 \text {, } \\
\text { MD }-1.5 \text { ( } 95 \% \mathrm{Cl}-9.9 \text { to } \\
6.8) ; 14.1 \text { vs. } 18.2 \text { at week } 2 \text {, } \\
\text { MD }-4.1(95 \% \mathrm{Cl}-14.3 \text { to } \\
6.0 \text { ) at week } 2 ; 18.6 \text { vs. } 24.1 \\
\text { at week } 4, \mathrm{MD}-5.5(95 \% \mathrm{Cl} \\
-15.4 \text { to } 4.2) ; 21.5 \text { vs. } 27.6 \text { at } \\
\text { week } 52, \mathrm{MD}-6.1(95 \% \mathrm{Cl} \\
-18.0 \text { to } 5.8)\end{array}$ \\
\hline
\end{tabular}

Abbreviations: CI = confidence interval; $\mathrm{MD}$ = mean difference; $\mathrm{NR}=$ not reported; NRS = Numeric Rating Scale; ODI = Oswestry Disability Index; RMDQ = Roland Morris Disability Questionnaire; SD = standard deviation; VAS = Visual Analog Scale.

\section{Acupuncture Versus Sham or Usual Care}

Two good-quality trials ${ }^{63,80}$ compared needle acupuncture versus sham acupuncture or usual care for acute low back pain (Table 7). One trial ${ }^{63}$ excluded patients with radiculopathy and the other trial ${ }^{80}$ enrolled a mixed population of patients with and without radiculopathy (60\% had radiculopathy). One trial ${ }^{80}$ used traditional Chinese acupuncture techniques, and the other trial ${ }^{63}$ used scalp acupuncture.

The trial $(n=261)^{80}$ of traditional Chinese acupuncture evaluated five 20-minute sessions over 14 days versus two different types of sham acupuncture (needling at nonspecific acupuncture points or use of a nonpenetrating/semiblunted needle) or usual care only (standard pharmacologic therapies, posture and exercise/activity recommendations). Acupuncture was associated with decreased likelihood of persistent low back pain at 3 weeks versus nonpenetrating sham (46.9\% vs. $70.3 \%$, RR $0.67,95 \%$ CI 0.49 to 0.90 ) or usual care ( $46.9 \%$ vs. $72.1 \%$, RR 0.65 , 95\% CI 0.48 to 0.88 ), with no difference versus needle (nonspecific acupoints) sham. There were no differences between acupuncture and controls in likelihood of persistent pain at 3 months. At 12 months, acupuncture was associated with decreased likelihood of persistent pain versus needle sham, though the proportion of patients with persistent pain was low in all groups ( $0 \%$ vs. $13 \%, p=0.01)$. For function, acupuncture was associated with increased likelihood of functional improvement (Roland Morris Disability Questionnaire improved $\geq 35 \%$ ) versus usual care at 3 weeks (74\% vs. 44\%, RR 1.66, 95\% CI 1.23 to 2.24 ) and versus 
nonpenetrating acupuncture at 3 months (98\% vs. 80\%; RR 1.22, 95\% CI 1.06 to 1.40). No differences between groups were reported at 12 months. There were no differences in the likelihood of occupational incapacity due to low back pain. No serious adverse events or withdrawals due to adverse events were reported.

The trial $(n=80)^{63}$ of scalp acupuncture compared five 30 minute sessions of Yamamoto's new scalp acupuncture versus sham acupuncture (nonpenetrating using the handle only) over 28 days; usual care in both groups included diclofenac. There were no differences between acupuncture versus sham acupuncture in pain intensity or severity of functional impairment at days 3 or 7 . At 21 and 28 days, acupuncture was associated with decreased pain intensity (mean differences 1.69 and 1.40 points on a 0 to 10 scale) and better function (mean differences -4.10 and -4.40 points on a 0 to 24 scale). Acupuncture was also associated with better scores for SF36 functional capacity, limitation in physical aspects, pain, and vitality subscales (differences 10.80 to 23.00 points on a 0 to 100 scale). No adverse events were reported. 
Table 7. Acupuncture versus sham or usual care for acute back pain

\begin{tabular}{|c|c|c|c|c|}
\hline $\begin{array}{l}\text { Author, } \\
\text { Year } \\
\text { Quality }\end{array}$ & Interventions & $\begin{array}{l}\text { Type of Back } \\
\text { Pain; } \\
\text { Followup } \\
\text { Duration } \\
\text { Sample Size }\end{array}$ & Pain Results & Function Results \\
\hline $\begin{array}{l}\text { Hasagawa, } \\
2014^{63} \\
\text { Good }\end{array}$ & $\begin{array}{l}\text { A: Acupuncture (Yamamoto's new } \\
\text { scalp acupuncture), five } 30 \text {-minute } \\
\text { sessions }+50 \mathrm{mg} \text { diclofenac every } \\
8 \text { hours as needed } \\
\text { B: Sham acupuncture, five } 30- \\
\text { minute sessions }\end{array}$ & $\begin{array}{l}\text { Nonspecific } \\
\text { back pain; } \\
3,7,21 \text { and } 28 \\
\text { days } \\
\mathrm{n}=80\end{array}$ & $\begin{array}{l}\text { Pain intensity (mean [SD], VAS } 0 \\
\text { to10 scale): } 4.63 \text { ( } 2.23) \text { vs. } 5.13 \\
\text { (2.21) on day } 3, \mathrm{MD}-0.5(95 \% \\
\mathrm{Cl}-1.49 \text { to } 0.49) ; 3.83(2.61) \text { vs. } \\
4.40(2.09) \text { on day } 7, \mathrm{MD}-0.57 \\
(95 \% \mathrm{Cl}-1.62 \text { to } 0.48) ; 2.49 \\
(2.40) \text { vs. } 4.18(2.52) \text { on day } 21 \\
\mathrm{MD}-1.69(95 \% \mathrm{Cl}-2.79 \text { to } \\
-0.59) ; 1.98(2.12) \text { vs. } 3.38 \\
\text { (2.26) on day } 28, \mathrm{MD}-1.40 \text { ( } 95 \% \\
\mathrm{Cl}-2.38 \text { to }-0.42)\end{array}$ & $\begin{array}{l}\text { RMDQ (mean [SD], } 0 \text { to } 24 \text { scale): } 10.30(5.40) \text { vs. } \\
12.40 \text { (4.50) at day } 3, \mathrm{MD}-2.10(95 \% \mathrm{Cl}-4.31 \text { to } \\
0.11) ; 8.10(5.50) \text { vs. } 10.20(5.30) \text { at day } 7, \mathrm{MD} \\
-2.1(-4.50 \text { to } 0.30), \mathrm{p}=0.09 ; 5.30(4.60) \text { vs. } 8.90 \\
(5.2) \text { at day } 14, \mathrm{MD}-3.60(95 \% \mathrm{Cl}-5.78 \text { to }-1.41) \\
4.40(4.40) \text { vs. } 8.50(6.20) \text { at day } 21, \mathrm{MD}-4.10 \\
(95 \% \mathrm{Cl}-6.49 \text { to }-1.71) ; 4.10(4.40) \text { vs. } 8.50(6.20) \\
\text { at day } 28, \mathrm{MD}-4.40(95 \% \mathrm{Cl}-6.79 \text { to }-2.01)\end{array}$ \\
\hline $\begin{array}{l}\text { Vas, } 2012^{80} \\
\text { Good }\end{array}$ & $\begin{array}{l}\text { A: Traditional Chinese } \\
\text { acupuncture + usual care, } 5 \text { 20- } \\
\text { minute sessions over } 2 \text { weeks } \\
\text { B: Placebo acupuncture + usual } \\
\text { care, nonpenetrating } 5 \text { 20-minute } \\
\text { sessions over } 2 \text { weeks } \\
\text { C: Sham acupuncture + usual } \\
\text { care, needling nonspecific } \\
\text { acupuncture points, } 5 \text { 20-minute } \\
\text { sessions over } 2 \text { weeks } \\
\text { D: Usual care, no acupuncture or } \\
\text { sham acupuncture }\end{array}$ & $\begin{array}{l}\text { Low back pain; } \\
3 \text { weeks, } 3 \\
\text { months, } 12 \\
\text { months } \\
n=261\end{array}$ & $\begin{array}{l}\text { Continuing pain: } 46.9 \%(30 / 64) \\
\text { vs. } 70.3 \%(45 / 64) \text { vs. } 50.8 \% \\
(33 / 65) \text { vs. } 72.1 \%(49 / 68) \\
p=0.01 \text { for } A \text { vs. } B, p=0.79 \text { for } A \\
\text { vs. } C \text {, and } p=0.01 \text { for } A \text { vs. } D \text { at } 3 \\
\text { weeks; } 9.8 \%(5 / 51) \text { vs } 21.6 \% \\
(11 / 51) \text { vs. } 13.8 \%(8 / 58) \text { vs. } \\
16.7 \%(10 / 60), p=0.17 \text { for } A \text { vs. } \\
\text { B, } p=0.73 \text { for } A \text { vs. } C \text {, and } p=0.44 \\
\text { for } A \text { vs. } D \text { at } 3 \text { months; } 0 \%(0 / \\
51) \text { vs } 6.1 \%(3 / 49) \text { vs. } 13.2 \% \\
\text { (7/53) vs. } 0 \%(0 / 57), p=0.11 \text { for } A \\
\text { vs. B and } p=0.01 \text { for A vs. C }\end{array}$ & 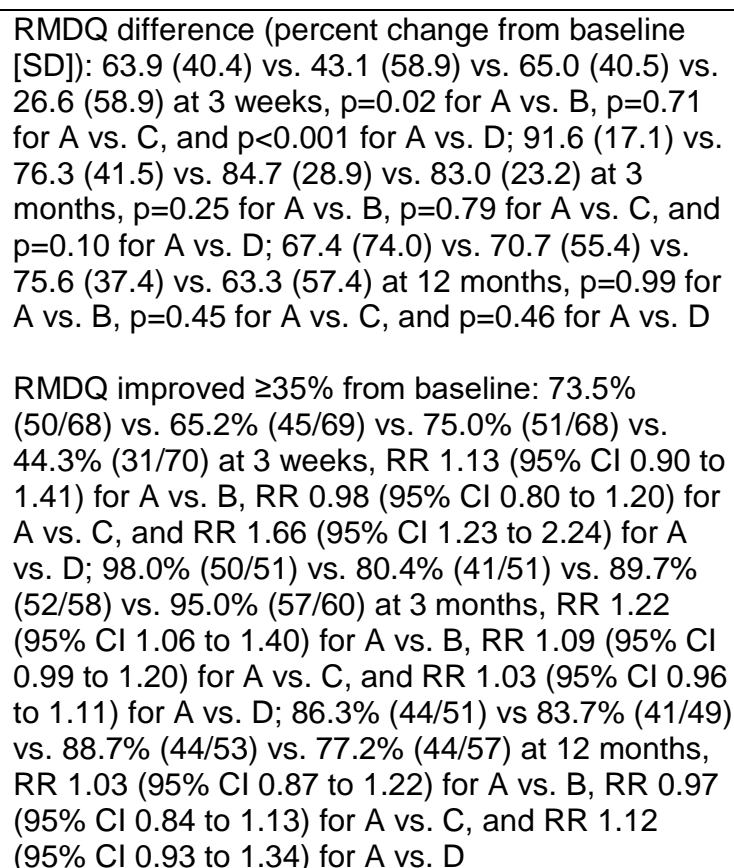 \\
\hline
\end{tabular}

Short-Form Survey; VAS = visual analog Scale. 


\section{Brace or Belt Versus No Brace or Belt}

Two trials compared a back belt or brace versus no brace or belt (Table 8). ${ }^{46,47}$ For acute back pain due to osteoporotic compression fracture, one fair quality trial $(n=60)$ found no differences between a rigid or soft brace versus no brace in pain intensity, function or opioid use at 2, 6, or 12 weeks. ${ }^{47}$ Adverse events were not reported, other than one death thought unrelated to the interventions. For acute low back pain (no specific cause), a poor quality trial $(n=36)$ found no differences between an abdominal elastic lumbar support belt versus no belt in pain or function at 1 or 3 weeks. ${ }^{46}$

Table 8. Braces or belts versus no braces or belts for acute low back pain

\begin{tabular}{|c|c|c|c|c|}
\hline $\begin{array}{l}\text { Author, } \\
\text { Year } \\
\text { Quality }\end{array}$ & Interventions & $\begin{array}{l}\text { Type of Back } \\
\text { Pain; } \\
\text { Followup } \\
\text { Duration } \\
\text { Sample Size }\end{array}$ & Pain Results & Function Results \\
\hline $\begin{array}{l}\text { Anders, } \\
2019^{46} \\
\text { Poor }\end{array}$ & $\begin{array}{l}\text { A: Lumbar support belt: } \\
\text { Lumbotrain abdominal } \\
\text { elastic lumbar support } \\
\text { belt to be worn at least } 4 \\
\text { hours daily } \\
\text { B: No belt }\end{array}$ & $\begin{array}{l}\text { Low back } \\
\text { pain; } \\
1 \text { and } 3 \\
\text { weeks } \\
n=36\end{array}$ & $\begin{array}{l}\text { Pain: No significant } \\
\text { overall effect }(p=0.21)\end{array}$ & $\begin{array}{l}\text { ODI, baseline-adjusted (mean } \\
\text { [95\% CI], converted to } 0 \text { to } 100 \\
\text { point scale): } 33.54(23.79 \text { to } \\
43.29) \text { vs. } 37.83 \text { ( } 26.77 \text { to } 48.90) \\
\text { vs. } 35.95(25.42 \text { to } 46.47), p=N S ; \\
\text { MD ( } 95 \% \text { Cl) } 2.41 \text { ( }-7.86 \text { to } 9.27) \\
\text { for A vs. C, }-1.88 \text { (-7.02 to } 9.38) \\
\text { for B vs. C }\end{array}$ \\
\hline $\begin{array}{l}\text { Kim, } 2014^{47} \\
\text { Fair }\end{array}$ & $\begin{array}{l}\text { A: Rigid brace: } \\
\text { thoracolumbar sacral } \\
\text { orthosis worn at all times } \\
\text { (except when lying } \\
\text { down) for } 8 \text { weeks } \\
\text { followed by 2-week } \\
\text { weaning period } \\
\text { B: Soft brace: soft back } \\
\text { brace worn at all times } \\
\text { (except when lying } \\
\text { down) for } 8 \text { weeks } \\
\text { followed by 2-week } \\
\text { weaning period } \\
\text { C: No brace: instructed } \\
\text { to walk without brace as } \\
\text { long as comfortable }\end{array}$ & $\begin{array}{l}\text { Low back pain } \\
\text { due to acute } \\
\text { osteoporotic } \\
\text { thoracolumbar } \\
\text { compression } \\
\text { fracture; } \\
2,6 \text {, and } 12 \\
\text { weeks } \\
n=60\end{array}$ & $\begin{array}{l}\text { Pain (mean [SD NR], } 0 \\
\text { to } 10 \text { VAS): } 5.7 \text { vs. } 7.1 \\
\text { vs. } 6.0 \text { at } 2 \text { weeks; } 5.1 \\
\text { vs. } 6.0 \text { vs. } 4.3 \text { at } 6 \\
\text { weeks; } 3.7 \text { vs. } 4.0 \text { vs. } \\
4.3 \text { at } 12 \text { weeks; no } \\
\text { significant differences, } \\
\text { treatment } x \text { time } \\
\text { interaction } p=0.292\end{array}$ & $\begin{array}{l}\text { RMDQ (mean [SD], } 0 \text { to } 24 \text { scale): } \\
9.7(19.9) \text { vs. } 5.3(5.7), p<0.05 \text { at } \\
\text { day } 7 ; 5.9 \text { (5.6) vs. } 3.2(4.0), \\
\text { p=NS at day } 28 \\
\text { RMDQ (percent with improvement } \\
\text { from day } 1): 73 \%(11 / 15) \text { vs. } 83 \% \\
(15 / 18), p=N S \text { at day } 7 ; 86 \% \\
(12 / 14) \text { vs. } 95 \%(19 / 20), p=N S \text { at } \\
\text { day } 28 \\
\text { ODI (mean [SD], } 0 \text { to } 100 \text { scale): } \\
36.0(19.9) \text { vs. } 26.4(21.1), p=N S \\
\text { at day } 7 ; 22.9(21.6) \text { vs. } 19.2 \\
(15.3), p=N S \text { at day } 28 \\
\text { ODI (percent with improvement } \\
\text { from day } 1): 87 \%(13 / 15) \text { vs. } 83 \% \\
(15 / 18), p=N S \text { at day } 7 ; 79 \% \\
\text { (11/14) vs. } 95 \%(19 / 20), p=N S \text { at } \\
\text { day } 28\end{array}$ \\
\hline
\end{tabular}

Abbreviations: CI = confidence interval; ODI = Oswestry Disability Index; MD = mean difference; NR = not reported; NS = not significant; RMDQ = Roland Morris Disability Questionnaire; SD = standard deviation; VAS = visual analog scale

\section{Heat Therapy Versus Usual Care or Placebo}

Six trials (sample sizes 30 to $180, \mathrm{~N}=425)^{54,70,73,74,77,78}$ compared heat therapy versus usual care or placebo tablets for acute low back pain (Table 9).$^{72,79}$ Four trials ${ }^{73,74,77,78}$ excluded patients with radiculopathy, and two trials ${ }^{54,70}$ did not specify inclusion or exclusion of patients with radiculopathy. Heat therapy interventions varied in terms of length of sessions and number of days (e.g., 20 minutes twice daily for 7 days, 8 hours for one day, 8 hours for 3 days), but was applied using a heat wrap in all trials except for one, ${ }^{54}$ which used a hot water bag. Four trials were rated fair quality ${ }^{54,73,74,77}$ and two trials poor quality. ${ }^{70,78}$

Heat therapy was consistently associated with decreased pain intensity versus usual care or placebo. At 1 day to $<1$ week, differences were 1.6 to 2.0 points on a 0 to 10 scale for pain intensity ( 2 trials), ${ }^{70,78} 0.94$ to 1.5 points on a 0 to 5 scale for pain relief ( 3 trials), ${ }^{73,74,77}$ and 2.58 
points on the McGill Pain Questionnaire in one trial ${ }^{54}$ (scoring method and scale unclear). At 1 to 2 weeks and 2 to 4 weeks, differences in pain intensity were 1.6 to 1.8 points on a 0 to 10 scale $(1 \text { trial })^{78}$ and 4.0 to 4.8 points on the McGill Pain Questionnaire in one trial. ${ }^{54}$ Heat therapy was also associated with better functional status versus placebo or usual care. At 1 day to $<1$ week, differences ranged from 2.1 to 2.4 points on the 0 to 24 Roland Morris Disability Questionnaire ( 3 trials).$^{73,74,78}$ At 1 week to $<4$ weeks, the difference was 4.0 to 4.6 points in one trial. ${ }^{78}$ One trial found heat therapy associated with better sleep quality at 2 to 4 days (mean difference 0.39 on a 0 to 5 scale, mean difference $0.39,95 \%$ CI 0.01 to 0.77$),{ }^{73}$ and one trial found heat therapy associated with decreased likelihood of being woken up at night due to pain ( $0 \%$ vs. $58 \%,=0.001$ ). Three trials reported no serious adverse events; there were a total of two nonserious adverse events. ${ }^{73,74,77}$

\section{Heat Therapy Versus Cold Therapy}

One fair quality trial $(n=58)$ compared heat versus cold therapy (Table 9). ${ }^{54}$ The therapies were administered twice daily for twenty minutes, for seven days. Heat therapy was associated with better scores on the McGill Pain Questionnaire at day 3, 8, and 15 (mean difference -1.99 to -1.38 points). However, the scoring method for the McGill Pain Questionnaire and scale were unclear. Other outcomes and adverse events were not reported. 
Table 9. Heat versus usual care, placebo, or cold therapy for acute low back pain

\begin{tabular}{|c|c|c|c|c|}
\hline $\begin{array}{l}\text { Author, Year } \\
\text { Quality }\end{array}$ & Interventions & $\begin{array}{l}\text { Type of } \\
\text { Back } \\
\text { Pain; } \\
\text { Followup } \\
\text { Duration } \\
\text { Sample } \\
\text { Size } \\
\end{array}$ & Pain Results & Function Results \\
\hline $\begin{array}{l}\text { Dehghan, } 2014^{54} \\
\text { Fair }\end{array}$ & $\begin{array}{l}\text { A: Heat therapy, hot water bag } \\
\text { twice daily for twenty minutes for } 7 \\
\text { days } \\
\text { B: Cold therapy, ice pack twice } \\
\text { daily for twenty minutes for } 7 \text { days } \\
\text { C: Usual care, included naproxen } \\
1000 \text { mg per day for } 7 \text { days (all } \\
\text { groups) }\end{array}$ & $\begin{array}{l}\text { Low back } \\
\text { pain; } \\
3,8 \text { and } 15 \\
\text { days } \\
\mathrm{n}=87\end{array}$ & $\begin{array}{l}\text { MPQ, Overall Pain Score (mean [SD], scale NR): } \\
7.28(3.18) \text { vs } 9.27(2.67) \text { vs. } 9.86(2.26) \text { at day } 3 \text {, } \\
\text { MD }-1.99(95 \% \mathrm{Cl}-3.53 \text { to }-0.44) \text { for A vs. B and } \\
-2.58(95 \% \mathrm{Cl}-4.03 \text { to }-1.13) \text { for A vs. C; } 3.72 \\
(2.37) \text { vs. } 5.10(2.30) \text { vs. } 7.72(2.51) \text { at day } 8, \mathrm{MD} \\
-1.38(95 \% \mathrm{Cl}-2.61 \text { to }-0.15) \text { for A vs. B and } \\
-4.00(95 \% \mathrm{Cl}-5.28 \text { to }-2.27) \text { for A vs. C; } 0.76 \\
(0.38) \text { vs. } 2.20(2.12) \text { vs. } 5.59(2.01) \text { at day } 15, \mathrm{MD} \\
-1.44(95 \% \mathrm{Cl}-2.24 \text { to }-0.63) \text { for A vs. B and } \\
-4.83(95 \% \mathrm{Cl}-5.59 \text { to }-4.07) \text { for A vs. C }\end{array}$ & NR \\
\hline $\begin{array}{l}\text { Kettenmann, } \\
2007^{70} \\
\text { Poor }\end{array}$ & $\begin{array}{l}\text { A: Heat wrap } \geq 4 \text { hours per day for } 4 \\
\text { days } \\
\text { B: Usual care, analgesics as } \\
\text { needed }\end{array}$ & $\begin{array}{l}\text { Low back } \\
\text { pain; } \\
1 \text { and } 4 \\
\text { days } \\
\mathrm{n}=30\end{array}$ & $\begin{array}{l}\text { Pain severity (mean [SD], } 0 \text { to } 100 \text { scale } \\
\text { converted to } 0 \text { to } 10 \text { scale): } 3.95(1.94) \text { vs } 5.00 \\
(2.71) \text { at day } 1, \mathrm{MD}-1.05(95 \% \mathrm{Cl}-2.81 \text { to } 0.71) \\
2.70(2.32) \text { vs } 4.70(2.71) \text { at day } 4, \mathrm{MD}-2.00 \\
(95 \% \mathrm{Cl}-38.9 \text { to }-1.1)\end{array}$ & NR \\
\hline $\begin{array}{l}\text { Nadler, 2003a } \\
\text { Fair }\end{array}$ & $\begin{array}{l}\text { A: Heat wrap, } 8 \text { consecutive hours } \\
\text { daily for } 3 \text { days } \\
\text { B: Oral placebo tablet }\end{array}$ & $\begin{array}{l}\text { Low back } \\
\text { pain; } \\
2 \text { days, } 4 \\
\text { to } 5 \text { days } \\
\mathrm{n}=63\end{array}$ & $\begin{array}{l}\text { Pain relief (mean [SD], } 0 \text { to } 5 \text { scale): } 2.36(2.00) \\
\text { vs. } 1.28(1.36) \text { at day } 2, \mathrm{MD} 1.11 \text { (95\% Cl } 0.25 \text { to } \\
1.97) ; 2.90(1.62) \text { vs. } 1.60(1.53) \text { at day } 4 \text { to } 5, \mathrm{MD} \\
1.30(95 \% \mathrm{Cl} 0.51 \text { to } 2.09)\end{array}$ & $\begin{array}{l}\text { RMDQ (mean [SD] } 0 \text { to } 24 \\
\text { scale): } 3.60(3.90) \text { vs } 5.80(3.96) \\
\text { at day } 4, \mathrm{MD}-2.20(95 \% \mathrm{Cl} \\
-4.18 \text { to }-0.22)\end{array}$ \\
\hline $\begin{array}{l}\text { Nadler, 2003b } \\
\text { Fair }\end{array}$ & $\begin{array}{l}\text { A: Heat wrap, } 8 \text { consecutive hours } \\
\text { daily for } 3 \text { days } \\
\text { B: Oral placebo tablet }\end{array}$ & $\begin{array}{l}\text { Low back } \\
\text { pain; } \\
1 \text { day, } 3 \\
\text { days, and } \\
4 \text { to } 5 \text { days } \\
\mathrm{n}=180\end{array}$ & $\begin{array}{l}\text { Pain relief (mean [SD], } 0 \text { to } 5 \text { scale): } 1.76(0.96) \text { vs } \\
1.05(1.04) \text { at day } 1, \mathrm{MD} 0.71(95 \% \mathrm{Cl} 0.41 \text { to } \\
1.00) ; 2.50(1.53) \text { vs } 1.56(1.69) \text { at days } 4 \text { to } 5, \mathrm{MD} \\
0.94(95 \% \mathrm{Cl} 0.47 \text { to } 1.41) \\
\text { Pain relief complete: } 15.4 \% \text { vs. } 5.5 \% \text { at day } 5, \text { OR } \\
2.89 \text { (p=0.04) }\end{array}$ & $\begin{array}{l}\text { RMDQ (mean }[S D] 0 \text { to } 24 \\
\text { scale): } 5.3 \text { vs. } 7.4 \text { at day } 3 \\
p<0.0002 ; 4.6 \text { vs. } 6.7 \text { at day } 5 \text {, } \\
p<0.001\end{array}$ \\
\hline $\begin{array}{l}\text { Stark, } 2014^{77} \\
\text { Fair }\end{array}$ & $\begin{array}{l}\text { A: Heat wrap for } 8 \text { continuous } \\
\text { hours } \\
\text { B: Oral placebo }\end{array}$ & $\begin{array}{l}\text { Low back } \\
\text { pain; } \\
8 \text { hours } \\
n=51\end{array}$ & $\begin{array}{l}\text { Pain relief (mean [SD NR], } 0 \text { to } 5 \text { scale, higher } \\
\text { score=greater relief): } 3.2 \text { vs. } 1.7, p<0.001 \\
\text { Total pain relief (mean [SD NR], sum of pain relief } \\
\text { scores on } 0 \text { to } 5 \text { scale from } 2 \text { to } 8 \text { hours): } 22.0 \text { vs. } \\
11.5, p<0.001\end{array}$ & NR \\
\hline
\end{tabular}




\begin{tabular}{|c|c|c|c|c|}
\hline $\begin{array}{l}\text { Author, Year } \\
\text { Quality }\end{array}$ & Interventions & $\begin{array}{l}\text { Type of } \\
\text { Back } \\
\text { Pain; } \\
\text { Followup } \\
\text { Duration } \\
\text { Sample } \\
\text { Size }\end{array}$ & Pain Results & Function Results \\
\hline $\begin{array}{l}\text { Tao, 2005a78 } \\
\text { Poor }\end{array}$ & $\begin{array}{l}\text { A: Heat wrap during daytime hours } \\
\text { for } 3 \text { days } \\
\text { B: Usual care, minimal education } \\
\text { intervention (both groups) }\end{array}$ & $\begin{array}{l}\text { Low back } \\
\text { pain; } \\
4,7 \text { and } 14 \\
\text { days } \\
\mathrm{n}=43\end{array}$ & $\begin{array}{l}\text { Pain intensity difference (mean change from } \\
\text { baseline [SD], } 0 \text { to10 NRS): }-3.24 \text { vs. }-1.61 \text { at day } \\
4, \mathrm{MD}-1.63(95 \% \mathrm{Cl}-2.99 \text { to }-0.28) ;-3.44 \text { vs. } \\
-1.78 \text { at day } 7, \mathrm{MD}-1.66(95 \% \mathrm{Cl}-2.97 \text { to } \\
-0.37) ;-3.85 \text { vs. }-2.22 \text { at day } 14, \mathrm{MD}-1.63(95 \% \\
\mathrm{Cl}-2.92 \text { to }-0.34)\end{array}$ & $\begin{array}{l}\text { RMDQ difference (mean change } \\
\text { from baseline [SD], } 0 \text { to } 24 \\
\text { Scale): }-2.88 \text { vs. }-0.50 \text { at day } 4 \text {, } \\
\text { MD }-2.38(95 \% \mathrm{Cl}-5.62 \text { to } \\
0.85) ;-5.32 \text { vs. }-0.72 \text { at day } 7 \text {, } \\
\text { MD }-4.60(95 \% \mathrm{Cl}-8.27 \text { to } \\
-0.94) ;-6.55 \text { vs. }-2.53 \text { at day } \\
14, \mathrm{MD}-4.02(95 \% \mathrm{Cl}-7.82 \text { to } \\
-0.24)\end{array}$ \\
\hline
\end{tabular}

Abbreviations: CI = confidence interval; MD = mean difference; MPQ = McGill Pain Questionnaire; NR = not reported; NRS = numeric rating scale; RMDQ = Roland Morris Disability Questionnaire; RR = risk ratio; $\mathrm{SD}=$ standard deviation

a Pain intensity, Pain relief, and RMDQ adjusted for sex, age, baseline pain intensity, and pain medication 


\section{Spinal Manipulation Versus Sham Therapy, Usual Care, Placebo, or No Treatment}

Eight trials compared spinal manipulation versus inactive treatments (sham therapy, usual care, placebo, or no treatment) for acute low back pain low back pain (Table 10). ${ }^{58,61,62,66,69,75,76,81}$ Five trials excluded patients with radiculopathy, ${ }^{58,61,62,66,69}$ one trial was restricted to patients with radiculopathy, ${ }^{76}$ one trial enrolled a mixed population of patients with and without radiculopathy, ${ }^{75}$ and one trial did not specify whether patients with radiculopathy were included or excluded. ${ }^{81}$ The manipulation intervention ranged from a single 15 -minute session to up to 20 sessions over 4 weeks. Details of manipulation techniques were limited, but when described usually involved high velocity low amplitude thrusts. Three trials compared spinal manipulation against sham manipulation, which consisted of simulated manipulation that was similar to active manipulation but did not follow specific patterns or involve rapid thrusts, ${ }^{76}$ placement of the hand on the paravertebral musculature with light pressure, ${ }^{66}$ or a high velocity low amplitude maneuver applied to the sacroiliac joint area. ${ }^{81}$ Two trials were rated good quality, ${ }^{62,76}$ four trials fair quality, ${ }^{58,61,66,81}$ and one trial poor quality ${ }^{75}$ (Appendix F, Table F-1).

In six trials of manipulation versus inactive controls that did not include patients with radiculopathy, effects on pain were inconsistent and differences were small, with no trial reporting a statistically significant difference. ${ }^{58,61,62,66,69,81}$ For pain intensity, at 1 day to $<1$ week, one trial found manipulation associated with higher pain intensity (difference 0.75 point on a 0 to 10 scale) ${ }^{69}$ At 1 week to $<2$ weeks, effects of manipulation and inactive controls on pain intensity were very similar in two trials (difference 0.2 point in both trials). ${ }^{62,69}$ At 2 to $<4$ weeks, differences ranged from 0.74 point in favor of manipulation to 0.5 point in favor of inactive treatment in four trials. ${ }^{61,62,66,69} \mathrm{At} \geq 4$ weeks, differences were 0.2 and 0.5 point in favor of manipulation in two trials. ${ }^{62,69}$ There was also no difference between spinal manipulation versus inactive controls in likelihood of experiencing improvement in pain at 1 day to $<1$ week (1 trial), ${ }^{58} 2$ to $<4$ weeks, ${ }^{69}$ or $\geq 4$ weeks. ${ }^{69}$ For function, results favored manipulation versus inactive treatments at 1 to $<2$ weeks in two trials ${ }^{62,81}$ (difference -0.7 and -2.7 points on the 0 to 24 Roland Morris Disability Questionnaire), but results were inconsistent at 2 to $<4$ weeks in four trials, ${ }^{61,62,66,69}$ and at $\geq 4$ weeks in two trials. ${ }^{62,66}$ One trial found no effect of manipulation on depression severity at 2 or 4 weeks. ${ }^{66}$ There were also no differences in analgesic (NSAID) use in three trials, though estimates favored manipulation. ${ }^{66,69,81}$ Reporting of adverse events was very limited. One trial reported no difference between manipulation versus usual care in risk of serious adverse events ( $2 \%$ vs. $2 \%) ;{ }^{69}$ otherwise no serious adverse events were reported.

One good quality trial ( $\mathrm{n}=102$ ) compared manipulation (up to 20 sessions over 4 weeks) versus sham manipulation (maneuvers resembling manipulation but not following a specific pattern and without rapid thrusts) in patients with radiculopathy. ${ }^{76}$ Manipulation was associated with increased likelihood of experiencing a reduction in back pain at day 15 (86\% vs. 61\%, RR $1.41,95 \%$ CI 1.10 to 1.81 ), with no differences at day 30 or day 180 . Manipulation was also associated with increased likelihood of reduction in leg pain at day 15 (82\% vs. 53\%, RR 1.55, 95\% CI 1.16 to 2.08 ), day 30 (94\% vs. 77\%, RR 1.22 , 95\% CI 1.03 to 1.44 ), and day 180 (100\% vs. $83 \%$, RR 1.20, 95\% CI 1.06 to 1.36), increased likelihood at 180 days of being back pain free at day 180 (28\% vs. $6 \%$, RR 5.00, 95\% CI 1.55 to 16.16$)$ and increased likelihood of being leg pain free at day 180 (55\% vs. $20 \%$, RR 2.90, 95\% CI 1.60 to 5.27). There were no differences between manipulation versus sham in SF-36 subscales of the Kellner Rating Scale for anxiety or depression, and no statistically significant difference in NSAID use (mean difference in number of days $-1.9,95 \%$ CI -4.0 to 0.2 ). No adverse events were reported in either group. 
One other trial evaluated ${ }^{75}$ a mixed population of patient with and without radiculopathy, but had serious methodological limitations and only reported results using a nonvalidated composite outcome for pain, function, and flexion-strength measurements.

\section{Manipulation Versus Mobilization}

One fair quality trial $(\mathrm{n}=54)$ compared a single session of manipulation versus mobilization for acute low back pain (Table 10). ${ }^{60}$ The inclusion or exclusion of patients with radiculopathy was not specified. Analyses were stratified according to duration of pain $\leq 2$ weeks or 2 to 4 weeks. In patients with pain $\leq 2$ weeks, there were no difference between mobilization versus manipulation on the Roland Morris Disability Questionnaire at 6 or 12 days. In patients with pain for 2 to 4 weeks, mobilization was associated with worse function at day 6 (6.0 vs. 3.75, p not reported), but there was no difference at day 12. Effects on pain were not reported, but there was no difference in the likelihood of reporting the back felt "much better." Adverse events were not reported. 


\begin{tabular}{|c|c|c|c|c|}
\hline $\begin{array}{l}\text { Author, Year } \\
\text { Quality }\end{array}$ & Interventions & $\begin{array}{l}\text { Type of Back } \\
\text { Pain; } \\
\text { Followup } \\
\text { Duration } \\
\text { Sample Size }\end{array}$ & Pain Results & Function Results \\
\hline $\begin{array}{l}\text { Glover, } 1974^{58} \\
\text { Fair }\end{array}$ & $\begin{array}{l}\text { A: Manipulation, one 15-minute } \\
\text { session + } 4 \text { 15-minute sham } \\
\text { diathermy sessions } \\
\text { B: Sham diathermy, five 15- } \\
\text { minute sessions }\end{array}$ & $\begin{array}{l}\text { Low back pain; } \\
3 \text { and } 7 \text { days } \\
n=84\end{array}$ & $\begin{array}{l}\text { Percent pain relief: } 50 \% \text { vs. } 56 \% \\
\text { at day } 3, p>0.05 ; 75 \% \text { vs. } 80 \% \text { at } \\
\text { day } 7, p>0.05\end{array}$ & NR \\
\hline $\begin{array}{l}\text { Hadler, } 1987^{60} \\
\text { Fair }\end{array}$ & $\begin{array}{l}\text { A: Spinal mobilization, single } \\
\text { session } \\
\text { B: Spinal manipulation, single } \\
\text { session }\end{array}$ & $\begin{array}{l}\text { Low back pain; } \\
6 \text { and } 12 \text { days } \\
n=54\end{array}$ & NR & $\begin{array}{l}\text { RMDQ (mean [SD NR], } 0 \text { to } 24 \text { scale): } \\
\text { Patients with pain for } \leq 2 \text { weeks: } 4.10 \text { vs. } \\
4.50 \text {, at day } 6, p>0.05 ; 2.10 \text { vs. } 2.0 \text { at day } \\
12, p>0.05 \\
\text { Patients with pain for } 2 \text { to } 4 \text { weeks: } 6.0 \text { vs. } \\
3.75 \text { at day } 6 . p=N R ; 5.0 \text { vs. } 4.50 \text { at day } \\
12, p=N R\end{array}$ \\
\hline $\begin{array}{l}\text { Hallegraeff, } \\
2009^{61} \\
\text { Fair }\end{array}$ & $\begin{array}{l}\text { A: Manipulation, } 4 \text { HVLA } \\
\text { treatments over } 2.5 \text { weeks }+ \\
\text { usual care light exercise } \\
\text { B: Usual care, } 5 \text { minutes of low } \\
\text { intensity, low load endurance } \\
\text { exercises twice daily }\end{array}$ & $\begin{array}{l}\text { Low back pain; } \\
2.5 \text { weeks } \\
n=64\end{array}$ & $\begin{array}{l}\text { Pain intensity (mean [SD], } 0 \text { to } \\
100 \text { VAS converted to } 0 \text { to } 10 \\
\text { scale): } 1.90 \text { (1.69) vs. } 2.48 \\
(2.01), \mathrm{MD}-0.58(95 \% \mathrm{Cl}-1.51 \\
\text { to } 0.35)\end{array}$ & $\begin{array}{l}\text { ODI (mean [SD], } 0 \text { to } 50 \text { scale): } 0.14 \\
(0.17) \text { vs. } 0.14(0.12), p=0.38\end{array}$ \\
\hline $\begin{array}{l}\text { Hancock, } 2007^{62} \\
\text { Good }\end{array}$ & $\begin{array}{l}\text { A: Manipulation, } 12 \text { treatments } \\
\text { for } 4 \text { weeks, plus placebo } \\
\text { medication } \\
\text { B: Diclofenac: } 100 \text { mg daily plus } \\
\text { detuned (inactive) pulsed } \\
\text { ultrasound } \\
\text { C: No treatment, placebo } \\
\text { diclofenac and detuned } \\
\text { ultrasound }\end{array}$ & $\begin{array}{l}\text { Low back pain; } \\
1,2,4 \text {, and } 12 \\
\text { weeks } \\
n=179\end{array}$ & $\begin{array}{l}\text { A vs. B } \\
\text { Pain intensity difference (mean } \\
\text { change from baseline vs. } \\
\text { placebo }[95 \% \text { Cl], } 0 \text { to } 10 \text { NRS): } \\
0.2(-0.3 \text { to } 0.7) \text { vs. }-0.2(-0.7 \text { to } \\
0.3) \text { at week } 1,0.4(-1.0 \text { to } 0.1) \\
\text { vs. }-0.1(-0.7 \text { to } 0.4) \text { at week } 2 \text {, } \\
-0.2(-0.7 \text { to } 0.3) \text { vs. }-0.1(-0.6 \\
\text { to } 0.4) \text { at week } 4,-0.2(-0.7 \text { to } \\
0.3) \text { vs. } 0.0(-0.5 \text { to } 0.4) \text { at week } \\
12\end{array}$ & $\begin{array}{l}\text { A vs. B } \\
\text { RMDQ difference (mean change from } \\
\text { baseline vs. placebo [95\% Cl], } 0 \text { to } 24 \\
\text { scale): }-0.7(-2.1 \text { to } 0.6) \text { vs. } 0.5 \text { ( }-0.8 \text { to } \\
1.8) \text { at week } 1,-1.4(-2.7 \text { to } 0.1) \text { vs. }-0.6 \\
(-1.9 \text { to } 0.8) \text { at week } 2,-1.0(-2.1 \text { to } 0.1) \\
\text { vs. }-0.7(-1.8 \text { to } 0.4) \text { at week } 4,-0.5 \\
(-1.7 \text { to } 0.7) \text { vs. }-0.1(-1.3 \text { to } 1.1) \text { at week } \\
12\end{array}$ \\
\hline $\begin{array}{l}\text { Hoiriis, 200466 } \\
\text { Fair }\end{array}$ & $\begin{array}{l}\text { A: Manipulation, chiropractic } \\
\text { adjustment }+ \text { oral placebo, } 8 \\
\text { visits over } 2 \text { weeks } \\
\text { B: Sham chiropractic adjustment } \\
+ \text { oral placebo }\end{array}$ & $\begin{array}{l}\text { Low back pain; } \\
2 \text { and } 4 \text { weeks } \\
n=74\end{array}$ & $\begin{array}{l}\text { Pain intensity (mean [SD], } 0 \text { to } \\
10 \text { VAS): } 2.44(2.22) \text { vs. } 3.18 \\
(2.4) \text { at } 2 \text { weeks, MD }-0.74(95 \% \\
\mathrm{Cl}-1.82 \text { to } 0.34) ; 1.71(1.88) \text { vs. } \\
2.21(2.02) \text { at } 4 \text { weeks, MD } \\
-0.50(95 \% \mathrm{Cl}-1.43 \text { to } 0.43)\end{array}$ & $\begin{array}{l}\text { ODI (mean, [SD], } 0 \text { to } 100 \text { scale): } 17.02 \\
\text { (13.75) vs. } 19.35 \text { (13.70) at } 2 \text { weeks, MD } \\
-2.33(95 \% \mathrm{Cl}-7.95 \text { to } 3.29) ; 11.94 \\
(11.93) \text { vs. } 16.32(12.95) \text { at } 4 \text { weeks, MD } \\
-4.38(95 \% \mathrm{Cl}-9.49 \text { to } 0.73)\end{array}$ \\
\hline
\end{tabular}




\begin{tabular}{|c|c|c|c|c|}
\hline $\begin{array}{l}\text { Author, Year } \\
\text { Quality }\end{array}$ & Interventions & $\begin{array}{l}\text { Type of Back } \\
\text { Pain; } \\
\text { Followup } \\
\text { Duration } \\
\text { Sample Size } \\
\end{array}$ & Pain Results & Function Results \\
\hline $\begin{array}{l}\text { Juni, } 2009^{69} \\
\text { Fair }\end{array}$ & $\begin{array}{l}\text { A: Manipulation, HVLA thrust, } \\
\text { mobilization, and muscle energy } \\
\text { technique, up to } 5 \text { sessions over } \\
2 \text { weeks } \\
\text { B: Usual care, advice and } \\
\text { analgesics }\end{array}$ & $\begin{array}{l}\text { Low back pain; } \\
6,13,14 \text { days } \\
\text { and } 6 \text { months } \\
n=97\end{array}$ & $\begin{array}{l}\text { Pain intensity (mean [SD NR], } 0 \\
\text { to } 10 \text { scale): } 3.00 \text { vs. } 2.25 \text { at day } \\
6, p=N R ; 1.95 \text { vs. } 1.75 \text { at day } 13 \text {, } \\
\text { p=NR; } 1.95 \text { vs. } 1.45 \text { at day } 14, \\
\text { MD } 0.5(95 \% \mathrm{Cl}-0.2 \text { to } 1.2) \\
\text { Pain intensity (mean difference } \\
\text { between groups, } 95 \% \mathrm{Cl}): 0.6 \\
(-0.4 \text { to } 1.6 \text { ) at } 6 \text { months }\end{array}$ & $\begin{array}{l}\text { RMDQ (mean [SD], } 0 \text { to } 24 \text { scale): } 5.8 \text { vs. } \\
5.2 \text { at day } 14 \text {, adjusted MD } 0.8(95 \% \mathrm{Cl} \\
-1.5 \text { to } 3.2)\end{array}$ \\
\hline $\begin{array}{l}\text { Postachhini, } \\
1988^{75} \\
\text { Poor }\end{array}$ & $\begin{array}{l}\text { A: Manipulation, daily for } 1 \text { week, } \\
\text { then twice daily for } 2 \text { weeks } \\
\text { B: Oral placebo }\end{array}$ & $\begin{array}{l}\text { Low back pain; } \\
3 \text { weeks and } 2 \\
\text { and } 6 \text { months } \\
n=65\end{array}$ & NR & $\begin{array}{l}\text { No radiculopathy group } \\
\text { Composite outcome for pain, function, and } \\
\text { flexion-strength measurements (mean } \\
\text { change from baseline [SD NR], } 5 \text { to } 32 \\
\text { scale, higher score indicates better } \\
\text { status): } 24.7 \text { vs. } 16.5 \text { at week } 3, p<0.01 ; \\
26.9 \text { vs. vs. } 23.8 \text { at month } 2, p=N S ; 29.5 \\
\text { vs. } 27.5 \text { at month } 6, p=N S \\
\text { Radiculopathy group } \\
\text { Composite outcome as above (mean } \\
\text { change from baseline [SD NR], } 5 \text { to } 32 \\
\text { scale): } 6.3 \text { vs. } 2.2 \text { at week } 3, p<0.05 ; 9.2 \\
\text { vs. } 5.1 \text { at month } 2,12.1 \text { vs. } 9.8 \text { at month } \\
6, p=N S\end{array}$ \\
\hline $\begin{array}{l}\text { Santilli, } 2006^{76} \\
\text { Good }\end{array}$ & $\begin{array}{l}\text { A: Manipulation, rapid thrusts } \\
\text { and specific patterns, } 5 \text { days per } \\
\text { week, maximum } 20 \text { sessions } \\
\text { B: Sham manipulation, soft } \\
\text { muscle pressing similar to } \\
\text { manipulation but not following } \\
\text { any specific pattern and no rapid } \\
\text { thrusts, } 5 \text { days per week, } \\
\text { maximum } 20 \text { sessions }\end{array}$ & $\begin{array}{l}\text { Low back pain } \\
\text { with } \\
\text { radiculopathy; } \\
15,30 \text {, and } 180 \\
\text { days } \\
n=102\end{array}$ & $\begin{array}{l}\text { Pain intensity, back (mean [SD], } \\
0 \text { to } 10 \text { VAS): } 4.4 \text { vs } 5.6 \text { at day } \\
15,3.3 \text { vs. } 4.6 \text { at day } 30,2.2 \text { vs. } \\
3.3 \text { at day } 180 ; p=N R \\
\text { Pain intensity, radiating pain } \\
\text { (mean [SD], } 0 \text { to } 10 \text { VAS): } 3.6 \text { vs. } \\
4.1 \text { at day } 15,2.2 \text { vs. } 3.7 \text { at day } \\
30,1.3 \text { vs. } 2.5 \text { at day } 180 ; p=N R \\
\text { Reduction in back pain: } 86 \% \\
\text { (44/51) vs. } 61 \%(30 / 49) \text { at day } \\
15, \text { RR } 1.41 \text { ( } 95 \% \mathrm{Cl} 1.10 \text { to } \\
1.81) ; 94 \%(47 / 50) \text { vs. } 85 \% \\
\text { (41/48) at day } 30, \mathrm{RR} 1.10 \text { (95\% } \\
\mathrm{Cl} 0.96 \text { to } 1.26) ; 98 \%(47 / 48) \text { vs. } \\
94 \%(45 / 48) \text { at day } 180, \mathrm{RR} 1.04 \\
(95 \% \mathrm{Cl} 0.96 \text { to } 1.14)\end{array}$ & NR \\
\hline
\end{tabular}




\begin{tabular}{|c|c|c|c|c|}
\hline $\begin{array}{l}\text { Author, Year } \\
\text { Quality }\end{array}$ & Interventions & $\begin{array}{l}\text { Type of Back } \\
\text { Pain; } \\
\text { Followup } \\
\text { Duration } \\
\text { Sample Size }\end{array}$ & Pain Results & Function Results \\
\hline $\begin{array}{l}\text { von Heymann, } \\
2013^{81} \\
\text { Fair }\end{array}$ & $\begin{array}{l}\text { A: Manipulation, HVLA thrust ( } 1 \\
\text { to } 2 \text { sessions) + placebo } \\
\text { diclofenac for } 3 \text { days } \\
\text { B: Sham manipulation ( } 1 \text { to } 2 \\
\text { sessions) + placebo diclofenac } \\
\text { for } 3 \text { days }\end{array}$ & $\begin{array}{l}\text { Low back pain; } \\
7 \text { to } 9 \text { days } \\
\mathrm{n}=57\end{array}$ & $\begin{array}{l}\text { Results "similar" to RMDQ, data } \\
\text { NR }\end{array}$ & $\begin{array}{l}\text { RMDQ (mean improvement from baseline } \\
\text { [SD], } 0 \text { to } 24 \text { scale): } 7.71 \text { (4.88) vs. } 10 \\
\text { (5.0) at } 7 \text { to } 9 \text { days, } p=0.01\end{array}$ \\
\hline
\end{tabular}


KQs 10 and 1p ask how the comparative effectiveness and harms of nonpharmacologic therapy vary depending on: (1) patient demographics (e.g., age, gender); (2) patient medical and psychiatric comorbidities; (3) the type of treatment used; (4) the frequency of therapy; (5) the duration of therapy.

Evidence on how the comparative effectiveness and harms of nonpharmacologic therapy varied based on patient and intervention factors was very limited. One study of manipulation versus usual care reported no subgroup effects when results were stratified by sex, age, occupation (manual vs. non-manual), pain duration ( $<7$ or $\geq 7$ days), pain intensity ( $<7$ or $\geq 7$ ), baseline Roland Morris Disability score $(<14$ or $\geq 4)$, or healthcare setting (ED or primary care). ${ }^{69}$ Manipulation was associated with beneficial effects on pain and function in a trial ${ }^{76}$ that restricted inclusion to patients with radiculopathy, but not in non-radiculopathy trials. However, it was not possible to draw strong conclusions about differential effects of manipulation by presence of radiculopathy from an indirect (between-studies) comparison involving a single radiculopathy trial.

\section{KQ 2. Acute Neck Pain (Including Neck Pain With Radiculopathy)}

\section{Key Points}

- Cervical collar vs. usual activity, neck pain with radiculopathy

o A semi-hard cervical collar was associated with moderate to large decrease in neck pain intensity versus usual activity at 2 to $<4$ weeks and at $\geq 4$ weeks, with no difference in disability, based on one trial (SOE: low).

- Cervical collar vs. exercise, neck pain with radiculopathy

o A semi-hard cervical collar and exercise therapy were associated with similar effects on neck pain intensity and disability at 2 to $<4$ weeks and at $\geq 4$ weeks, based on one trial (SOE: low).

- Exercise vs. usual activity, neck pain with radiculopathy

o Exercise was associated with moderate decrease in neck pain intensity versus activity as usual at 2 to $<4$ weeks and at $\geq 4$ weeks, with no difference in disability, based on one trial (SOE: low).

- Ultrasound vs. sham ultrasound, whiplash neck sprain

0 No difference in pain intensity at 1 to $<2$ weeks, though ultrasound was associated with a small increase in pain intensity at 2 to $<4$ weeks, based on one trial (SOE: low).

- Cervical collar vs. usual activity, whiplash neck sprain

o No difference between a semi-hard cervical collar versus usual activity in pain or health status at $\geq 4$ weeks, based on one trial (SOE: low).

- Cervical collar vs. exercise, whiplash neck sprain

o No difference between a semi-hard cervical hard collar and exercise in pain or health status at $\geq 4$ weeks, based on one trial (SOE: low).

- Exercise vs. usual activity, whiplash neck sprain.

0 No difference between exercise therapy versus usual activity in pain or health status at $\geq 4$ weeks, based on one trial (SOE: low). 


\section{Summary of Findings}

Five trials $(\mathrm{N}=1,020)^{88-92}$ evaluated interventions for acute neck pain (Appendix E, Table E3 ). All of the trials compared one nonpharmacologic treatment to another (KQ $2 \mathrm{~m}$ and $2 \mathrm{n}$ ). Four trials ${ }^{88,89,91,92}$ evaluated patients with acute whiplash neck sprain, and one trial ${ }^{90}$ evaluated patients with acute cervical radiculopathy. The duration of pain was $\geq 7$ days in two trials, ${ }^{89,90}$ and not described in three trials. ${ }^{88,91,92}$ The duration of treatment ranged from 1 to 6 weeks. The duration of followup was 1 week to $<4$ weeks in one trial, ${ }^{91}$ and $\geq 4$ weeks in four trials. ${ }^{88-90,92}$ All five trials were conducted in Europe. The mean age ranged from 29 to 47 . Few trials reported race or ethnicity. One trial excluded patients with a history of substance use disorder, ${ }^{88}$ one trial excluded pregnant or breastfeeding patients, ${ }^{92}$ two trials excluded patients with psychiatric illness, ${ }^{88,91}$ and one trial excluded patients with fractures or dislocations of the cervical spine. ${ }^{88}$ Three trials $^{88,90,91}$ were fair quality, and two trials ${ }^{89,92}$ poor quality (Appendix F, Table F-1). Methodological limitations included failure to report adequate randomization or allocation concealment methods, failure to report attrition, high attrition, and no intention to treat analysis. It was not possible to blind patients and caregivers to study interventions.

\section{Detailed Synthesis}

\section{Opioid Therapy}

No evidence was found for opioid therapy for neck pain.

\section{Nonopioid Pharmacologic Therapy}

No evidence was found for opioid therapy for neck pain.

\section{Nonpharmacologic Therapy}

KQs $2 \mathrm{~m}$ and $2 \mathrm{n}$ address the comparative effectiveness of nonpharmacologic therapy versus: (1) inactive controls or (2) other nonpharmacologic therapies.

Five trials assessed nonpharmacologic therapy for acute neck pain ${ }^{88-92}$ (Appendix E, Table E3). Four trials ${ }^{88-90,92}$ compared a cervical collar versus exercise or usual activity, and one trial ${ }^{91}$ compared ultrasound versus sham ultrasound.

\section{Cervical Collar Versus Usual Activity}

Two trials compared a cervical collar versus usual activity for acute neck pain ${ }^{89,90}$ (Table 11). One fair quality trial ( $\mathrm{n}=135$ for this comparison) compared a semi-hard cervical collar (worn during the day for 3 weeks, then weaned off over 3 weeks) versus usual activity in patients with recent onset (within 1 month) cervical radiculopathy. ${ }^{90}$ The cervical collar was associated with lower neck pain intensity versus usual activity at 3 weeks (mean 3.80 vs. 5.50 on a 0 to 10 scale $\mathrm{p}=0.001$ ) and 6 weeks (mean 3.10 vs. $5.11, \mathrm{p}=0.0002$ ). The collar was also associated with lower arm pain intensity at 3 weeks (5.03 vs. 5.91 on a 0 to 10 scale, $p=0.061$ ) and 6 weeks (mean 3.35 vs. 4.86, $\mathrm{p}=0.006$ ), but the difference was only statistically significant at 6 weeks. Neck Disability Index scores were similar at 3 and 6 weeks and there was no difference in patient satisfaction with treatment.

Two trials evaluated patients with acute whiplash injury ( $\mathrm{N}=504) .{ }^{88,89}$ A fair quality trial ( $n=303$ for this comparison) found no differences between a semi-hard cervical collar versus usual activity in pain, headache severity, or SF-36 physical health summary score at 3, 6, or 12 months. ${ }^{88}$ A poor quality trial $(n=201)$ found no statistically significant difference in mean pain 
scores between soft collar immobilization (2 hours on and 2 hours off during the day and continuous use at night) versus usual care at 6 weeks (mean 2.97 vs. 3.29 on a 0 to 10 visual analog scale [VAS], $\mathrm{p}=0.49$ ) or at 6 months (mean 3.11 vs. 2.66 on a 0 to 10 VAS, $\mathrm{p}=0.29$ ). ${ }^{89}$ There was also no difference in self-reported global improvement at 6 months.

Harms were not reported by any trial.

\section{Cervical Collar Versus Exercise}

Three trials compared a cervical collar versus exercise for acute neck pain (Table 11). ${ }^{88,90,92}$ For acute cervical radiculopathy, one fair quality trial described above ( $n=139$ for this comparison) compared a semi-hard cervical collar versus exercise therapy (face-to-face instruction and home exercise daily). ${ }^{90}$ Differences in neck pain intensity were small and not statistically significant at 3 weeks (3.80 vs. 4.45 on a 0 to 10 scale, $p=0.212$ ) or 6 weeks ( 3.10 vs. 3.62, $\mathrm{p}=0.321$ ). Results were similar for arm pain. Neck Disability Index scores were very similar at 3 and 6 weeks and there was no difference in patient satisfaction with treatment. Two trials evaluated patients with acute whiplash injury. ${ }^{88,92}$ A fair quality trial $(n=297$ for this comparison) found no difference between a semi-hard cervical collar versus exercise (active mobilization) in pain, neck disability, SF-36 physical or mental summary scores, or headache intensity at 3, 6, or 12 months. ${ }^{88}$ A poor quality trial $(n=150)$ compared a soft cervical collar (continuous for 1 week) versus exercise (face-to-face instruction on home mobilization exercises) for acute whiplash injury. ${ }^{92}$ The collar was associated with higher pain intensity versus exercise at 6 weeks (mean 1.60 vs. 1.04 on a 0 to 10 scale, $\mathrm{p}=0.047$ ) and greater self-received disability (mean 1.56 vs. 0.92 on a 0 to 10 scale, $p=0.042$ ). Harms were not reported by any trial.

\section{Exercise Therapy Versus Usual Activity}

Two trials described above compared exercise therapy versus usual activity (Table 11) activity. ${ }^{88,90}$ For acute cervical radiculopathy, a fair quality trial ( $\mathrm{n}=136$ for this comparison) found exercise therapy associated with lower neck pain intensity versus usual activity at 3 weeks (mean 4.45 vs. 5.50 on a 0 to 10 scale, $p=0.059$ ) and 6 weeks (mean 3.62 vs. 5.11, respectively, $\mathrm{p}=0.007$ ), though the difference was of borderline statistical significance at 3 weeks. ${ }^{90}$ Results were similar for arm pain intensity. There were no differences in Neck Disability Index scores or patient satisfaction with treatment. A fair quality trial ( $n=296$ for this comparison) found no difference between exercise therapy (active mobilization) versus usual activity in pain, neck disability, SF-36 36 physical or mental summary scores, or headache intensity at 3, 6, or 12 months. ${ }^{88}$ Harms were not reported in either trial.

\section{Ultrasound Versus Sham Ultrasound}

One fair quality trial $(n=54)$ compared ultrasound ( 5 days of pulsed ultrasound followed by 5 days of continuous ultrasound) versus sham ultrasound for whiplash neck sprain (Table 11). ${ }^{91}$ Usual care in both groups included massage and exercise. There was no difference in mean pain intensity between ultrasound versus sham ultrasound at the end of treatment (mean 3.18 vs. 3.01 on a 0 to 10 scale, $\mathrm{p}=0.742$ ), though ultrasound was associated with higher mean neck pain intensity at 25 days (mean 6.16 vs. 5.24 on a 0 to 10 scale, p=0.040). Harms were not reported. 
Table 11. Nonpharmacologic interventions for acute neck pain

\begin{tabular}{|c|c|c|c|c|}
\hline $\begin{array}{l}\text { Author, Year } \\
\text { Quality }\end{array}$ & Interventions & $\begin{array}{l}\text { Type of Neck } \\
\text { Pain; } \\
\text { Followup } \\
\text { Duration } \\
\text { Sample Size }\end{array}$ & Pain Intensity Results & $\begin{array}{l}\text { Other Pain and Function } \\
\text { Results }\end{array}$ \\
\hline $\begin{array}{l}\text { Borchgrevink, } \\
1998^{89} \\
\text { Poor }\end{array}$ & $\begin{array}{l}\text { A: Soft collar } \\
\text { immobilization } \\
\text { and sick leave } \\
\text { for } 14 \text { days } \\
\text { B: Usual activity } \\
\text { for } 14 \text { days }\end{array}$ & $\begin{array}{l}\text { Whiplash } \\
\text { neck sprain; } \\
6 \text { weeks and } \\
6 \text { months } \\
n=163\end{array}$ & $\begin{array}{l}\text { Neck pain intensity (mean } \\
\text { [SD], } 0 \text { to } 100 \text { VAS } \\
\text { converted to } 0 \text { to } 10 \text { scale): } \\
2.97(2.65) \text { vs. } 3.29(3.53) \\
\text { at } 6 \text { weeks, MD }-0.32 \\
(95 \% \mathrm{Cl}-1.24 \text { to } 0.60) ; \\
3.11(2.16) \text { vs. } 2.66(2.35) \\
\text { at } 6 \text { months, MD } 0.45(95 \% \\
\mathrm{Cl}-0.22 \text { to } 1.12)\end{array}$ & $\begin{array}{l}\text { Pain during daily activities } \\
\text { (mean [SD], } 0 \text { to } 4 \text { scale): } \\
1.38(0.39) \text { vs. } 1.41(0.44) \text { at } \\
2 \text { weeks, MD }-0.03(95 \% \mathrm{Cl} \\
-0.15 \text { to } 0.09) ; 1.48(0.45) \\
\text { vs. } 1.34(0.47) \text { at } 6 \text { months, } \\
\text { MD } 0.14(95 \% \text { Cl } 0.004 \text { to } \\
0.28)\end{array}$ \\
\hline $\begin{array}{l}\text { Kongsted, } \\
2007^{88} \\
\text { Fair }\end{array}$ & $\begin{array}{l}\text { A: Semi-hard } \\
\text { cervical collar } \\
\text { during waking } \\
\text { hours for } 2 \\
\text { weeks followed } \\
\text { by active } \\
\text { mobilization for } 4 \\
\text { weeks } \\
\text { (maximum } 2 \\
\text { sessions weekly) } \\
\text { B: Active } \\
\text { mobilization: } \\
\text { physiotherapist- } \\
\text { led sessions in } \\
\text { addition to home } \\
\text { exercises for six } \\
\text { weeks } \\
\text { (maximum } 2 \\
\text { sessions weekly) } \\
\text { C: Usual care } 1 \\
\text { hour educational } \\
\text { session }\end{array}$ & $\begin{array}{l}\text { Whiplash } \\
\text { neck sprain; } \\
3,6 \text {, and } 12 \\
\text { months } \\
n=448\end{array}$ & $\begin{array}{l}\text { Neck pain (median [IQR], } 0 \\
\text { to } 10 \text { VAS): } 3.8 \text { ( } 1.2 \text { to } 6.1 \text { ) } \\
\text { vs. } 3.1(1.2 \text { to } 6.1 \text { ) vs. } 4.2 \\
\text { ( } 1.2 \text { to } 7.0), p=0.5 \text { at } 3 \\
\text { months; } 4.1 \text { ( } 1.2 \text { To } 7.0) \text { vs. } \\
3.0(0.2 \text { to } 6.1) \text { vs. } 4.1 \text { ( } 2.1 \\
\text { to } 6.1), p=0.2 \text { at } 6 \text { months; } \\
3.0(1 \text { to } 7) \text { vs. } 3.0 \text { (0 to } 6) \\
\text { vs. } 4.5(0 \text { to } 8), p=0.1 \text { at } 12 \\
\text { months } \\
\text { Headache (median [IQR], } 0 \\
\text { to } 10 \text { VAS): } 4.0(0.1 \text { to } 6.0) \\
\text { vs. } 3.0(0.1 \text { to } 6.9) \text { vs. } 3.0 \\
(1.0 \text { to } 8.0), p=0.6 \text { at } 3 \\
\text { months; } 4.0(1.0 \text { to } 7.0) \text { vs. } \\
2.0(0.5 \text { to } 6.0) \text { vs. } 4.0(1.0 \\
\text { to } 7.0), p=0.1 \text { at } 6 \text { months; } \\
4.0(0.3 \text { to } 6.9) \text { vs. } 2.0(0.3 \\
\text { to } 6.9) \text { vs. } 3.7(0.3 \text { to } 6.9), \\
\text { p=0.3 at } 12 \text { months }\end{array}$ & 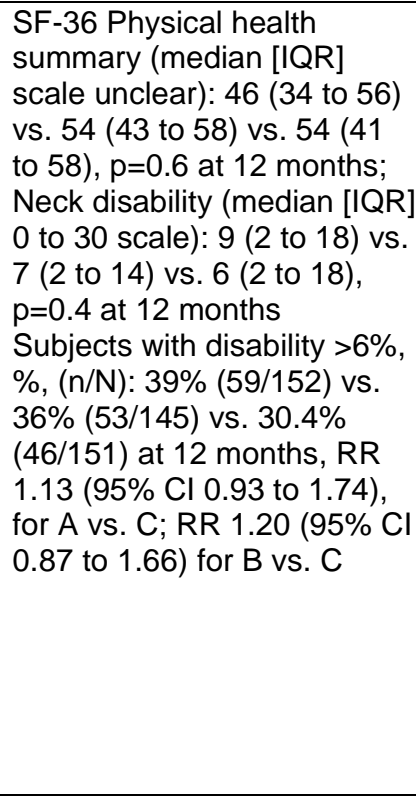 \\
\hline $\begin{array}{l}\text { Kuijper, } 2009^{90} \\
\text { Fair }\end{array}$ & $\begin{array}{l}\text { A: Semi-hard } \\
\text { cervical collar } \\
\text { during the day } \\
\text { for } 3 \text { weeks, } \\
\text { weaned off at } 6 \\
\text { weeks } \\
\text { B: Exercise with } \\
\text { focus on } \\
\text { mobilizing and } \\
\text { stabilizing } \\
\text { cervical spine } \\
\text { twice a week for } \\
6 \text { weeks } \\
\text { C: No treatment }\end{array}$ & $\begin{array}{l}\text { Cervical } \\
\text { radiculopathy; } \\
3 \text { and } 6 \\
\text { weeks and } 6 \\
\text { months } \\
\mathrm{n}=205\end{array}$ & 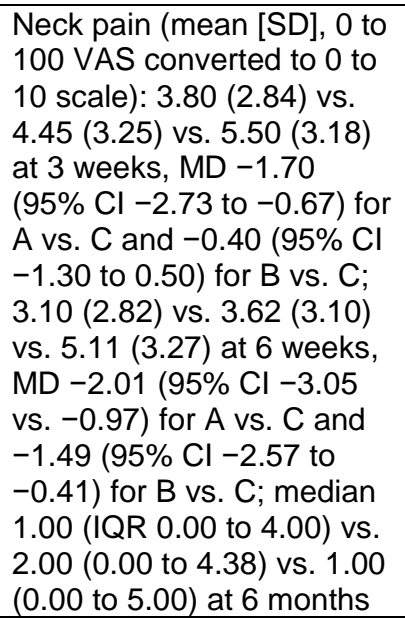 & $\begin{array}{l}\text { Neck disability index (mean } \\
\text { [SD], } 0 \text { to } 100 \text { scale): } 33.8 \\
(18.7) \text { vs. } 34.6(16.1) \text { vs. } \\
34.3(18.8) \text { at } 3 \text { weeks, MD } \\
-0.50(95 \% \mathrm{Cl}-6.90 \text { to } 5.90) \\
\text { for } \mathrm{A} \text { vs. } \mathrm{C} \text { and } 0.30(95 \% \mathrm{Cl} \\
0.30(95 \% \mathrm{Cl}-5.63 \text { to } 6.23) \\
\text { for B vs. C; } 25.9(19.1) \text { vs. } \\
27.8(17.7) \text { vs. } 29.9(20.0) \text { at } \\
6 \text { weeks, MD }-4.0(95 \% \mathrm{Cl} \\
-10.66 \text { to } 2.66) \text { for A vs. C } \\
\text { and }-2.10(95 \% \mathrm{Cl}-8.50 \text { to } \\
4.30) \text { for B vs. C; median } \\
\text { (IQR) } 8.0(0 \text { to } 26.0) \text { vs. } 10.0 \\
\text { (2.0 to } 29.2) \text { vs. } 8.0 \text { (0 to } 26 .) \\
\text { at } 6 \text { months }\end{array}$ \\
\hline
\end{tabular}




\begin{tabular}{|c|c|c|c|c|}
\hline $\begin{array}{l}\text { Author, Year } \\
\text { Quality }\end{array}$ & Interventions & $\begin{array}{l}\text { Type of Neck } \\
\text { Pain; } \\
\text { Followup } \\
\text { Duration } \\
\text { Sample Size }\end{array}$ & Pain Intensity Results & $\begin{array}{l}\text { Other Pain and Function } \\
\text { Results }\end{array}$ \\
\hline $\begin{array}{l}\text { Ruiz-Molinero, } \\
2014^{91} \\
\text { Fair }\end{array}$ & $\begin{array}{l}\text { A: Ultrasound, } \\
\text { pulsed and } \\
\text { continuous, } 1 \\
\text { session per day } \\
\text { for } 10 \text { days } \\
\text { B: Sham } \\
\text { ultrasound, } 1 \\
\text { session per day } \\
\text { for } 10 \text { days }\end{array}$ & $\begin{array}{l}\text { Whiplash } \\
\text { neck sprain; } \\
10 \text { and } 25 \\
\text { days } \\
n=54\end{array}$ & $\begin{array}{l}\text { Pain intensity (mean [SD], } \\
0 \text { to } 10 \text { VAS): } 3.18 \text { (2.12) } \\
\text { vs. } 3.01 \text { (1.62) at day } 10 \text {, } \\
\text { MD } 0.17(95 \% \mathrm{Cl}-0.86 \text { to } \\
1.20) ; 6.16(1.89) \text { vs. } 5.24 \\
(1.25) \text { at day } 25, \text { MD: } 0.92 \\
(95 \% \mathrm{Cl} 0.04 \text { to } 1.80)\end{array}$ & NR \\
\hline $\begin{array}{l}\text { Schnabel, } \\
2004^{92} \\
\text { Poor }\end{array}$ & $\begin{array}{l}\text { A: Soft cervical } \\
\text { collar, day and } \\
\text { night for } 1 \text { week } \\
\text { B: Exercise } \\
\text { therapy, } \\
\text { mobilization of } \\
\text { affected body } \\
\text { regions, } 2 \text { to } 5 \\
\text { sessions in first } \\
\text { week, } 3 \text { sessions } \\
\text { daily for } 6 \text { weeks }\end{array}$ & $\begin{array}{l}\text { Whiplash } \\
\text { neck sprain; } \\
6 \text { weeks } \\
n=150\end{array}$ & $\begin{array}{l}\text { Pain intensity (mean [SD], } \\
0 \text { to } 10 \text { VAS): } 1.60(2.15) \\
\text { vs. } 1.04(1.81), \text { MD } 0.56 \\
(p=0.047)\end{array}$ & $\begin{array}{l}\text { Perceived disability for } \\
\text { normal activities (mean [SD], } \\
0 \text { to } 10 \text { VAS): } 1.56(2.22) \text { vs. } \\
0.92(1.70) \text {, MD } 0.64 \\
(p=0.042)\end{array}$ \\
\hline
\end{tabular}

Abbreviations: $\mathrm{CI}$ = confidence interval; IQR = interquartile range; $\mathrm{MD}=$ mean difference; $\mathrm{NR}$ = not reported; $\mathrm{SD}=$ standard deviation; VAS = visual analog scale.

KQs 20 and 2p ask how the comparative effectiveness and harms of nonpharmacologic therapy vary depending on: (1) patient demographics (e.g., age, gender); (2) patient medical and psychiatric comorbidities; (3) the type of treatment used; (4) the frequency of therapy; (5) the duration of therapy.

It was not possible to determine how the comparative effectiveness and harms of nonpharmacologic therapy for acute neck pain varied based on patient and intervention factors, due to small numbers of trials and methodological limitations in the trials. No trial conducted subgroup analyses.

\section{KQ 3. Musculoskeletal Pain Not Otherwise Included in KQ1 or KQ2 (Including Fractures)}

\section{Key Points}

- Opioid vs. NSAID

o Insufficient evidence from four poor quality trials (SOE: insufficient).

- Opioid agonist vs. mixed agent

0 Inconsistent effects on pain intensity at $<1$ day ( 3 trials), no difference in pain intensity at 1 day to $<1$ week ( 1 trial) or improvement in function at 1 day to $<1$ week based on one trial) (SOE: low for outcomes at 1 day to $<1$ week).

- NSAID vs. acetaminophen, acute musculoskeletal injury

o Similar effects on pain intensity at $<1$ day ( 4 trials), 1 day to $<1$ week ( 8 trials), 1 to $<2$ weeks ( 6 trials), and $\geq 4$ weeks ( 2 trials) (SOE: moderate).

- Non-aspirin NSAID vs. NSAID

o Insufficient evidence from four poor quality trials (SOE: insufficient). 
- Ultrasound vs. sham ultrasound, ankle sprain

o No difference in pain or other outcomes at 1 day to $<1$ week or $\geq 4$ weeks, based on three trials (SOE: low).

- Acupressure vs. sham acupressure or usual care

o Acupressure associated with moderate to large decrease in pain intensity versus sham acupressure or standard treatment at 1 day to $<1$ week, with small effects at 4 weeks (SOE: low).

- Cold therapy, transcutaneous electrical nerve stimulation, or relaxation

o Insufficient evidence from poor quality trials (SOE: insufficient).

\section{Summary of Findings}

Thirty trials $(\mathrm{N}=2,866)^{93-122}$ evaluated interventions for acute musculoskeletal pain, excluding low back and neck pain (Appendix E, Table E-4). Seven trials $97,98,105,109,112,116,118$ evaluated opioid therapy (KQ 3a and 3c), 13 trials ${ }^{93-96,99,101-103,107,108,111,120,122 ~ e v a l u a t e d ~ a ~}$ nonopioid medication versus nonpharmacologic treatment or another nonopioid (KQ $3 \mathrm{i}$ and $3 \mathrm{j}$ ), and 10 trials ${ }^{100,104,106,110,113-115,117,119,121}$ (KQ 3m and 3n) evaluated nonpharmacologic treatment versus an inactive control or another nonpharmacologic treatment. Twelve trials ${ }^{95,99,101,104-}$ 106,109,110,113,117,119,121 evaluated patients with ankle sprain, 3 trials $^{98,100,115}$ fractures, 1 trial $^{93}$ acute rotator cuff tear, and 14 trials ${ }^{94,96,97,102,103,107,108,111,112,114,116,118,120,122}$ various musculoskeletal pain conditions (e.g., fracture, trauma, and/or sprains/strains). The duration of pain was $<7$ days in 12 trials s $93,95,96,99,101-104,109,110,117,119,121,122$ was $<14$ days in 2 trials, ${ }^{94,97}$ and not described in 14 trials. $^{98,100,105-108,111-116,118,120}$ The duration of treatment ranged from a single dose or treatment session ${ }^{98,107,117,118}$ to 8 weeks. ${ }^{104}$ The duration of followup was less than 1 week in 16 trials,,${ }^{95,98-}$ $105,107,109-111,116,118,1201$ week to $<4$ weeks in 15 trials, 94-97,99-101,106,108,112,113,117,119,121,122 and $\geq 4$ weeks in 5 trials..$^{93,99,104,114,115}$ Ten trials ${ }^{95,96,100,105,107,109,110,112,114,118}$ were conducted in the United States, 11 trials $^{94,101,103,106,111,113,116,117,119,121,122}$ in Europe, and 8 trials $^{93,98,99,102,104,108,115,120}$ elsewhere. The mean age ranged from 23 to 50 . Few trials reported race or ethnicity. Four trials ${ }^{98,101,102,120}$ excluded patients with a history of substance use disorder, 16 trials ${ }^{93-96,98,99,101-}$ 103,105,108,112,114,116,120,122 excluded pregnant or breastfeeding patients, no trials excluded patients with psychiatric illness, and 5 trials excluded patients with other comorbidities. ${ }^{94,96,98,104,121}$ Three trials ${ }^{102,107,120}$ were rated good quality, 12 trials $^{93,95,98,99,101,103,104,109,113,115,118,122}$ fair quality, and 15 trials $^{94,96,97,100,105,106,108,110-112,114,116,117,119,121}$ poor quality (Appendix F, Table F-1).

Methodological limitations in the fair and poor quality trials included failure to report adequate randomization or allocation concealment methods, unblinded design, failure to report attrition, high attrition, and no intention to treat analysis.

\section{Detailed Synthesis}

\section{Opioid Therapy}

KQs 3a and 3c address the comparative effectiveness and harms of opioid therapy versus: (1) nonopioid pharmacologic therapy (e.g., acetaminophen, NSAIDs, antidepressants, anticonvulsants) or (2) nonpharmacologic therapy (e.g., exercise, cognitive behavioral therapy, acupuncture).

Seven trials ${ }^{97,98,105,109,112,116,118}$ evaluated opioid therapy for acute musculoskeletal pain (Appendix E, Table E-4). Four trials compared an opioid versus an NSAID, ${ }^{97,105,112,116}$ and three trials $^{98,109,118}$ compared an opioid agonist versus a mixed agent (buprenorphine, tramadol or 
tapentadol). Three trials blinded patients to treatments. ${ }^{98,109,118}$ Three trials ${ }^{98,109,118}$ were rated fair quality, and four trials ${ }^{97,105,112,116}$ were rated poor quality (Appendix F, Table F-1).

\section{Opioid Versus NSAID}

Four poor quality trials compared an opioid (alone or in combination with acetaminophen) versus an NSAID for acute musculoskeletal pain (primarily sprains, strains, or fractures; or mixed musculoskeletal pain) (Table 12) ${ }^{97,105,112,116}$ Treatment was initiated in the ED in one trial $^{105}$ and in outpatient settings in three trials. ${ }^{97,112,116}$ The sample size for the opioid versus NSAID comparisons ranged from 35 to $113(\mathrm{~N}=237)$. The duration of treatment ranged from 4 to up to 7 days. Three trials ${ }^{97,105,112}$ compared the combination of codeine plus acetaminophen (30 to $60 \mathrm{mg}$ plus $300 \mathrm{mg}$ every four to six hours; daily morphine equivalent dose 18 to $36 \mathrm{mg}$ ) versus diflunisal (1000 mg loading dose, then $500 \mathrm{mg}$ twice to three times daily), and the third trial $^{116}$ compared tramadol (200 to $300 \mathrm{mg} /$ day) versus diclofenac (200 to $300 \mathrm{mg} /$ day).

One trial found no statistically significant difference in pain intensity between an opioid versus an NSAID at 6 hours (mean difference -0.40 on a 0 to 10 scale, $95 \%$ CI -1.10 to 0.30 ). ${ }^{116}$ At five to seven days, two trials found opioids associated with a small to moderate decrease in pain intensity versus an NSAID in two trials (mean difference -0.7 and -1.0 point), ${ }^{112,116}$ but the difference was statistically significant in only one ${ }^{116}$ of the trials. A third trial found a small difference in pain intensity favoring an opioid versus an NSAID at 5 days, but did not report statistical differences. ${ }^{97}$ Both groups had no pain at 7 days. A fourth trial found no difference between an opioid versus an NSAID in the proportion reporting no or mild pain at 1 to 7 days (90.5\% to $94.7 \%$, RR $0.96,95 \%$ CI 0.80 to 1.14 ), though patients randomized to the opioid were less likely to rate pain relief as very good or excellent (38\% vs. 84\%, RR 0.45, 95\% CI 0.25 to 0.81). ${ }^{105}$ None of the trials found differences between an opioid versus NSAID in function. Evidence on adverse events was limited. Serious adverse events were not reported and withdrawal due to adverse events were few; opioids were associated with increased likelihood of any adverse event in one trial, but the difference was not statistically significant (65\% vs. 28\%, $\mathrm{p}=0.06) .{ }^{112}$ Estimates for specific adverse events were imprecise.

\section{Opioid Agonist Versus Mixed Agent}

Three fair-quality trials compared an opioid agonist versus the mixed agent tramadol or versus buprenorphine for acute musculoskeletal pain (Table 12). ${ }^{98,109,118}$ One fair-quality trial $(n=62)$ of patients with fracture, sprain/strain, contusion, or tendon rupture found a single dose of hydrocodone plus acetaminophen 5/500 mg (5 mg morphine equivalent dose) associated with markedly lower pain intensity at 3 hours versus tramadol $100 \mathrm{mg}$ (10 mg morphine equivalent dose) (mean 2.3 vs. 5.1 on a 0 to 10 scale, $\mathrm{p}<0.01$ ). ${ }^{118}$ However, a larger multidose trial of patients with ankle sprain $(n=346)$ found no differences between hydrocodone plus acetaminophen versus tramadol plus acetaminophen (median 26.1 vs. 32.2 morphine equivalent dose) in pain intensity at 4 hours or 5 days, or in likelihood of experiencing $\geq 30$ percent or $\geq 50$ percent improvement; outcomes were nearly identical in the two groups. ${ }^{109}$ There was also no difference in the likelihood of no mild or activity impairment at 5 days (73.8\% vs. $65.2 \%$, RR $1.13,95 \%$ CI 0.99 to 1.29 ), and the risk of adverse events was similar, with no statistically significant differences. The third trial, which evaluated patients with extremity fracture $(n=89)$, found no difference between a single dose of intravenous (IV) morphine (5 mg) versus sublingual buprenorphine $\left(0.4 \mathrm{mg}\right.$, or $15.5 \mathrm{mg}$ morphine equivalents) in pain at 60 minutes. ${ }^{98}$ No serious adverse events were reported in the trials. 
Table 12. Opioid therapy versus NSAIDs or mixed agent opioids

\begin{tabular}{|c|c|c|c|c|}
\hline $\begin{array}{l}\text { Author, Year } \\
\text { Quality }\end{array}$ & Interventions & $\begin{array}{l}\text { Pain Condition; } \\
\text { Followup } \\
\text { Duration } \\
\text { Sample Size }\end{array}$ & Pain Intensity Results & $\begin{array}{l}\text { Other Pain and } \\
\text { Function Results }\end{array}$ \\
\hline $\begin{array}{l}\text { Aghababian, } \\
1986^{105} \\
\text { Poor }\end{array}$ & $\begin{array}{l}\text { A. Codeine } 30 \mathrm{mg}+ \\
\text { acetaminophen } 300 \\
\text { mg } 1 \text { to } 2 \text { tablets } \\
\text { every } 4 \text { hours as } \\
\text { needed for pain } \\
\text { B. Diflunisal } 1000 \mathrm{mg} \\
\text { loading dose, then } \\
500 \text { mg every } 12 \\
\text { hours as needed for } \\
\text { pain } \\
\text { For mean of } 4 \text { days }\end{array}$ & $\begin{array}{l}\text { Grade } 2 \text { ankle } \\
\text { sprain } \\
1 \text { to } 7 \text { days } \\
n=40\end{array}$ & $\begin{array}{l}\text { No or mild pain: } 90.5 \% \\
\text { (19/21) vs. } 94.7 \%(18 / 19) \text {; } \\
\text { RR } 0.96 \text { ( } 95 \% \text { Cl } 0.80 \text { to } \\
1.14)\end{array}$ & $\begin{array}{l}\text { Medication efficacy } \\
\text { and tolerability "very } \\
\text { good" or "excellent": } \\
43 \%(9 / 21) \text { vs. } 89 \% \\
(17 / 19) ; \text { RR } 0.4895 \% \\
\text { CI } 0.28 \text { to } 0.80) \\
\\
\text { Pain relief 'very good' } \\
\text { or 'excellent', \% (n/N) } \\
38 \%(8 / 21) \text { vs. } 84 \% \\
(16 / 19) ; \text { RR } 0.45(95 \% \\
\text { CI } 0.25 \text { to } 0.81)\end{array}$ \\
\hline $\begin{array}{l}\text { Hewitt, } \\
2007^{109} \\
\text { Fair }\end{array}$ & $\begin{array}{l}\text { A. Hydrocodone } 7.5 \\
\text { mg + } \\
\text { acetaminophen } 650 \\
\text { mg up to } 4 \text { times a } \\
\text { day for } 5 \text { days } \\
\text { B. Tramadol } 75 \text { mg } \\
+ \text { acetaminophen } \\
650 \text { mg up to } 4 \\
\text { times a day for } 5 \\
\text { days }\end{array}$ & $\begin{array}{l}\text { Ankle sprain with } \\
\text { partial ligament } \\
\text { tear } \\
4 \text { hours and up to } \\
5 \text { days } \\
n=346\end{array}$ & $\begin{array}{l}\text { Pain intensity (mean [SD], } \\
0 \text { to } 3 \text { NRS converted to } 0 \\
\text { to } 10 \text { scale) at day } 5: 3.33 \\
\text { (3.63) vs. } 3.33 \text { (3.50), MD } \\
-0.00 \text { ( }-0.15 \text { to } 0.16) \\
\text { Pain intensity difference } \\
\text { (mean [SD], } 0 \text { to } 3 \text { NRS } \\
\text { converted to } 0 \text { to } 10 \text { scale) } \\
\text { at } 4 \text { hours: } 3.33 \text { (2.33) vs. } \\
3.33 \text { (2.33) } \\
\text { Sum of pain intensity } \\
\text { differences (mean [SDI], } \\
\text { sum of hourly differences): } \\
3.2 \text { (2.5) vs. } 3.3 \text { (2.1), MD } \\
0.64 \text { (-0.78 to } 2.05 \text { ) }\end{array}$ & $\begin{array}{l}\geq 30 \% \text { improvement in } \\
\text { pain intensity at } 4 \\
\text { hours: } 61.7 \% \\
(117 / 190) \text { vs. } 63.7 \% \\
(128 / 201), \text { MD }-2.0 \% \\
(-11.9 \% \text { to } 7.9 \%) \\
\geq 50 \% \text { improvement in } \\
\text { pain intensity at } 4 \\
\text { hours: } 39.9 \%(77 / 201) \\
\text { vs. } 38.3 \%(76 / 190) \text {, } \\
\text { MD } 1.6 \%(-8.4 \% \text { to } \\
11.6 \%) \\
\text { No or mild activity } \\
\text { impairment: } 73.8 \% \\
(144 / 195) \text { vs. } 65.2 \% \\
(122 / 187), \text { RR } 1.13 \\
(95 \% \text { Cl } 0.99 \text { to } 1.29)\end{array}$ \\
\hline $\begin{array}{l}\text { Indelicato, } \\
1986^{97} \\
\text { Poor }\end{array}$ & $\begin{array}{l}\text { A: Codeine } 30 \mathrm{mg}+ \\
\text { acetaminophen } 300 \\
\text { mg, } 1 \text { to } 2 \text { tablets } \\
\text { every } 4 \text { to } 6 \text { hours } \\
\text { for } 7 \text { days } \\
\text { B: Diflunisal } 1000 \\
\text { mg loading dose + } \\
500 \text { mg every } 12 \\
\text { hours for } 7 \text { days }\end{array}$ & $\begin{array}{l}\text { Acute strains and } \\
\text { sprains; } \\
1,5 \text {, and } 7 \text { days } \\
n=49\end{array}$ & $\begin{array}{l}\text { Pain (mean [SD NR], } 0 \text { to } 3 \\
\text { converted to } 0 \text { to } 10 \text { scale): } \\
6.7 \text { vs. } 6.3 \text { at day } 1 ; 1.3 \text { vs. } \\
2.0 \text { at day } 5 ; 0 \text { vs. } 0 \text { at day } \\
7\end{array}$ & $\begin{array}{l}\text { Patient-reported } \\
\text { efficacy }(\%[\mathrm{n} / \mathrm{N}] \text { rated } \\
\text { "very good", } \\
\text { "excellent": } 28 \%(7 / 25) \\
\text { vs. } 38 \%(9 / 24), \mathrm{RR} \\
0.68(95 \% \mathrm{Cl} 0.31 \text { to } \\
1.53)\end{array}$ \\
\hline $\begin{array}{l}\text { Jalili, } 2012^{98} \\
\text { Fair }\end{array}$ & $\begin{array}{l}\text { A: Morphine sulfate: } \\
5 \text { mg IV plus } \\
\text { sublingual placebo, } \\
\text { single dose } \\
\text { B: Buprenorphine: } \\
0.4 \text { mg sublingual } \\
\text { plus } 5 \mathrm{~mL} \text { sterile } \\
\text { water IV placebo, } \\
\text { single dose }\end{array}$ & $\begin{array}{l}\text { Extremity } \\
\text { fracture; } \\
60 \text { minutes } \\
n=89\end{array}$ & $\begin{array}{l}\text { Pain (mean [SD], } 0 \text { to } 10 \\
\text { NRS): } 2.2(1.7) \text { vs. } 2.2 \\
(0.7), p=0.9 ; \mathrm{MD}, 0.0(95 \% \\
\mathrm{Cl}-0.55 \text { to } 0.55)\end{array}$ & NR \\
\hline
\end{tabular}




\begin{tabular}{|c|c|c|c|c|}
\hline $\begin{array}{l}\text { Author, Year } \\
\text { Quality }\end{array}$ & Interventions & $\begin{array}{l}\text { Pain Condition; } \\
\text { Followup } \\
\text { Duration } \\
\text { Sample Size }\end{array}$ & Pain Intensity Results & $\begin{array}{l}\text { Other Pain and } \\
\text { Function Results }\end{array}$ \\
\hline $\begin{array}{l}\text { Muncie, } \\
1986^{112} \\
\text { Poor }\end{array}$ & $\begin{array}{l}\text { A. Codeine } 60 \mathrm{mg}+ \\
\text { acetaminophen } 650 \\
\text { mg every } 4 \text { hours as } \\
\text { needed for } 7 \text { days } \\
\text { B. Diflunisal } 1000 \\
\text { mg loading dose, } \\
\text { then } 500 \text { mg every } \\
12 \text { hours as needed } \\
\text { for } 7 \text { days }\end{array}$ & $\begin{array}{l}\text { Mild to moderate } \\
\text { sprain or strain, } \\
\text { or low back pain } \\
\text { Up to } 7 \text { days } \\
n=35\end{array}$ & $\begin{array}{l}\text { Pain (mean [SD], } 0 \text { to } 4 \\
\text { scale converted to } 0 \text { to } 10 \\
\text { scale): } 3.3(2.8) \text { vs. } 4.0 \\
(3.8), p=N S\end{array}$ & $\begin{array}{l}\text { Limitation of function } \\
\text { (mean [SD]), } 0 \text { to } 4 \\
\text { scale: } 1.5(1.3) \text { vs. } 1.9 \\
(1.8), p=N R\end{array}$ \\
\hline $\begin{array}{l}\text { Pagliara, } \\
1997^{116} \\
\text { Poor }\end{array}$ & $\begin{array}{l}\text { A. Tramadol } 200 \text { to } \\
300 \mathrm{mg} \text { per day for } 5 \\
\text { to } 7 \text { days } \\
\text { B. Diclofenac } 200 \text { to } \\
300 \mathrm{mg} \text { per day for } 5 \\
\text { to } 7 \text { days }\end{array}$ & $\begin{array}{l}\text { Musculoskeletal } \\
\text { pain due to } \\
\text { trauma; } \\
6 \text { hours and } 5 \text { to } \\
7 \text { days } \\
n=113\end{array}$ & $\begin{array}{l}\text { Pain intensity (mean [SD] } 0 \\
\text { to } 100 \text { VAS converted to } 0 \\
\text { to } 10 \text { scale) } \\
5.7(1.5) \text { vs. } 6.1(2.2) \text {, at } 6 \\
\text { hours, MD }-0.40(95 \% \mathrm{Cl} \\
-1.10 \text { to } 0.30) ; 1.5(1.5) \text { vs. } \\
2.5(2.2) \text {, at } 5 \text { to } 7 \text { days, } \\
\text { MD }-1.0(95 \% \mathrm{Cl}-1.70 \text { to } \\
-0.30)\end{array}$ & $\begin{array}{l}\text { Patient rated efficacy } \\
\text { (mean [SD], } 0 \text { to } 100 \\
\text { VAS converted to } 0 \text { to } \\
10 \text { scale): } 9.02(1.20) \\
\text { vs. } 7.43(2.11), \text { MD } \\
1.59(95 \% \mathrm{Cl} 0.95 \text { to } \\
2.23)\end{array}$ \\
\hline $\begin{array}{l}\text { Turturro, } \\
1998^{118} \\
\text { Fair }\end{array}$ & $\begin{array}{l}\text { A. Hydrocodone } 5 \\
\text { mg }+ \\
\text { acetaminophen } 500 \\
\text { mg } \times 1 \\
\text { B. Tramadol } 100 \text { mg } \\
\times 1\end{array}$ & $\begin{array}{l}\text { Trauma (fracture, } \\
\text { sprain/strain, } \\
\text { contusion, } \\
\text { tendon rupture) } \\
3 \text { hours } \\
\mathrm{n}=62\end{array}$ & $\begin{array}{l}\text { Pain intensity (mean [SD], } \\
0 \text { to } 100 \text { VAS converted to } \\
0 \text { to } 10 \text { scale): } 2.3(2.2) \text { vs. } \\
5.1(2.9), p<0.01\end{array}$ & NR \\
\hline
\end{tabular}

Abbreviations: AE = adverse event; CI = confidence interval; IV = intravenous; MD = mean difference; NR = not reported; NRS = numeric rating scale; $\mathrm{NS}=$ not significant; $\mathrm{RR}=$ relative risk; $\mathrm{SD}=$ standard deviation; $\mathrm{VAS}=$ visual analog scale

KQs 3b and 3d ask how the comparative effectiveness and harms of opioid therapy vary depending on: (1) patient demographics (e.g., age, race, ethnicity, gender); (2) patient medical or psychiatric comorbidities; (3) dose of opioids; (4) duration of opioid therapy, including number of opioid prescription refills and quantity of pills used; (5) opioid use history; (6) substance use history; (7) use of concomitant therapies.

Evidence on how comparative effectiveness and harms of opioid therapy for acute musculoskeletal pain vary according to patient and prescribing factors was lacking. The number of trials was small for each comparisons and most trials had small sample sizes. In addition, trials excluded patients with subgroup characteristics of interest, such as substance use history or presence of medical or psychiatric comorbidities, or did not provide information regarding these factors. No study conducted within-study or across-study evaluations of subgroup effects.

KQ 3e concerns the effects of prescribing opioid therapy versus not prescribing opioid therapy for acute pain on (1) short-term (<3 months) continued need for prescription pain relief, such as need for opioid refills, and (2) long-term opioid use (3 months or greater).

No evidence was found for KQ 3 e.

KQs $3 f$ and $3 g$ concern the accuracy and effectiveness of instruments for predicting risk of opioid misuse, opioid use disorder, or overdose in patients with acute pain being considered for opioid therapy.

No study evaluated the accuracy or effectiveness of instruments for predicting risk of opioid misuse, opioid use disorder, or overdose in patients with acute musculoskeletal pain.

KQ $3 \mathrm{~h}$ addresses the effect of the following factors on the decision to prescribe opioids for patients with acute pain being considered for opioid therapy: (1) existing opioid management plans; (2) patient education; (3) clinician and patient values and preferences related to opioids; 
(4) urine drug screening; (5) use of prescription drug monitoring program data; (6) availability of close followup.

Evidence on the effect of patient education, use of risk mitigation strategies, clinician and patient values and preferences, or availability of followup on decisions to prescribe opioids for postoperative pain was not available.

\section{Nonopioid Pharmacologic Therapy}

KQs $3 \mathrm{i}$ and $3 \mathrm{k}$ address the comparative effectiveness and harms of nonopioid pharmacologic therapy versus: (1) nonopioid pharmacologic therapy or (2) nonpharmacologic therapy.

Thirteen trials evaluated nonopioid pharmacologic therapy for acute musculoskeletal pain (Appendix E, Table E-4; Table 13). ${ }^{93-96,99,101-103,107,108,111,120}$ Eight trials compared an NSAID versus acetaminophen ${ }^{93,95,99,101-103,107,120}$ four trials compared a non-aspirin NSAID versus aspirin, ${ }^{94,96,108,111}$ and one trial compared a topical NSAID to an oral NSAID. ${ }^{122}$ Three trials ${ }^{102,107,120}$ were rated good quality, six trials fair quality, ${ }^{93,95,99,101,103,122}$ and four trials $^{94,96,108,111}$ poor quality (Appendix F, Table F-1).

\section{NSAID Versus Acetaminophen}

Three good quality RCTs ${ }^{102,107,120}$ and five fair quality trials ${ }^{93,95,99,101,103}(\mathrm{~N}=1,139)$ compared an NSAID versus acetaminophen for acute musculoskeletal injury (sprains or strains, musculoskeletal injury or trauma, or rotator cuff injury). In most trials, differences between NSAIDs and acetaminophen were small and not statistically significant at $<1$ day ( 4 trials, mean difference on 0 to 10 pain scale ranged from -0.5 to -0.1 point), ${ }^{102,103,107,120} 1$ day to 1 week (8 trials, mean differences ranged from -0.9 to 1.2 points), ${ }^{94,95,99,101-103,111,120} 1$ to $<2$ weeks ( 6 trials, mean difference ranged from -0.8 to 0.5 point), ${ }^{94-96,99,101,102}$ and at $\geq 4$ weeks (mean difference 0.2 point in two trials). ${ }^{93,99}$ Findings were similar when focusing on the good quality RCTs. One good quality trial $(n=60)$ of a single dose of ibuprofen $800 \mathrm{mg}$ versus acetaminophen $1000 \mathrm{mg}$ found similar pain intensity at 1 hour ( 3.9 vs. 4.3 on a 0 to 10 scale) and no difference in rescue medication use. ${ }^{107}$ Another good quality trial ( $\left.\mathrm{n}=201\right)$ compared diclofenac (25 mg every 8 hours) or indomethacin (25 mg every 8 hours) versus acetaminophen (1000 mg every 6 ours) for 3 days in patients with sprain, contusion, wound, crush, or fracture. ${ }^{120}$ Effects on pain intensity were similar at 2 hours and 3 days; differences in mean change from baseline were 0.1 to 0.2 point on at 0 to 10 scale. The third good quality trial found no difference between a single dose of ibuprofen $800 \mathrm{mg}$ versus acetaminophen $1000 \mathrm{mg}$ in pain intensity at 60 minutes in patients with acute musculoskeletal injury (mean difference $-0.40,95 \%$ CI -1.9 to 1.1 ). ${ }^{107}$ No severe adverse events were reported and there were no differences in nonsevere adverse events, though estimates were imprecise and reporting of adverse events was suboptimal.

\section{Non-Aspirin NSAID Versus Aspirin}

Four poor quality trials compared an NSAID (piroxicam $40 \mathrm{mg}$ followed by $20 \mathrm{mg}$ daily, flurbiprofen $150 \mathrm{mg}$ daily, naproxen $750 \mathrm{mg}$ daily, or diclofenac $150 \mathrm{mg}$ daily) versus aspirin (2,000 to 4,000 mg daily). ${ }^{94,96,108,111}$ Trials were rated poor quality due to inadequate reporting of methods. In both studies, results favored the non-aspirin NSAID, though differences were not always statistically significant. One trial of professional soccer players with lower limb injuries $(n=51)$ found no difference between flurbiprofen versus aspirin in pain at 1 day (mean 4.1 vs. 4.2, $\mathrm{p}=\mathrm{NS}$; scale not reported) but flurbiprofen was associated with decreased pain at day 6 (0.3 vs. 1.2, $\mathrm{p}<0.01) .{ }^{111}$ Flurbiprofen was also associated with decreased time to training fitness and 
match fitness (differences 0.9 to 1.2 days). The second trial $(n=69)$ found piroxicam associated with increased likelihood of being able to accomplish activity within 16 days, though the difference was not statistically significant (80\% vs. 65\%, RR 1.24, 95\% CI 0.92 to 1.67). ${ }^{108}$ Effects on pain intensity were not reported. Piroxicam was also associated with decreased risk of any adverse event or gastrointestinal adverse events, though the difference were not statistically significant.

Table 13. NSAID versus other pharmacologic treatments for musculoskeletal pain

\begin{tabular}{|c|c|c|c|c|}
\hline $\begin{array}{l}\text { Author, } \\
\text { Year } \\
\text { Quality }\end{array}$ & Interventions & $\begin{array}{l}\text { Pain Condition; } \\
\text { Followup Duration } \\
\text { Sample Size }\end{array}$ & Pain Intensity Results & $\begin{array}{l}\text { Other Pain and } \\
\text { Function Results }\end{array}$ \\
\hline $\begin{array}{l}\text { AlRuthia, } \\
2019^{93} \\
\text { Fair }\end{array}$ & $\begin{array}{l}\text { A: Ibuprofen, } 400 \text { to } \\
800 \text { mg every } 6 \text { to } \\
8 \text { hours as needed. } \\
\text { Patients may } \\
\text { increase dose to } \\
<3200 \text { mg per day } \\
\text { B: Acetaminophen, } \\
500 \text { mg every } 6 \text { to } \\
8 \text { hours as needed; } \\
\text { for patients whose } \\
\text { pain is not } \\
\text { controlled by this } \\
\text { dose: } 1000 \text { mg } \\
\text { every } 6 \text { hours as } \\
\text { needed }\end{array}$ & $\begin{array}{l}\text { Acute rotator cuff } \\
\text { disease; } \\
6 \text { weeks } \\
n=33\end{array}$ & $\begin{array}{l}\text { Mean difference in pain } \\
\text { from baseline (SD), } 100 \\
\text { point SPADI } \\
\text { questionnaire converted } \\
\text { to } 0 \text { to } 10 \text { scale: }-1.91 \\
(2.96) \text { vs. }-2.11(3.49)\end{array}$ & $\begin{array}{l}\text { Mean difference in } \\
\text { disability from baseline } \\
\text { (SD), } 100 \text { point SPADI } \\
\text { questionnaire: }-17.30 \\
\text { (20.54) vs. }-16.35 \\
\text { (39.84) } \\
\text { Mean difference in } \\
\text { Quick-DASH score } \\
\text { (SD), } 100 \text { point scale: } \\
\text {-13.41 (17.49) vs. } \\
-15.04(24.91)\end{array}$ \\
\hline $\begin{array}{l}\text { Andersen, } \\
1984^{94} \\
\text { Poor }\end{array}$ & $\begin{array}{l}\text { A: Naproxen, } 250 \\
\text { mg in the morning } \\
\text { and } 500 \text { mg in the } \\
\text { evening for } 7 \text { days } \\
\text { B: Aspirin: } 500 \text { mg } \\
\text { in the morning and } \\
\text { at noon and } 1000 \\
\text { mg in the evening }\end{array}$ & $\begin{array}{l}\text { Soft tissue injuries } \\
7 \text { days } \\
n=63\end{array}$ & $\begin{array}{l}\text { Pain on movement } \\
\text { (mean [SD NR], } 0 \text { to } 100 \\
\text { VAS converted to } 0 \text { to } 10 \\
\text { scale): } 5.3 \text { vs. } 5.0 \text { at day } \\
1 ; 4.0 \text { vs. } 4.2 \text { at day } 3 ; \\
3.0 \text { vs. } 2.7 \text { at day } 7 ; \text { all } \\
\text { comparisons, p=NS }\end{array}$ & $\begin{array}{l}\text { A vs. B } \\
\text { Functional capacity } \\
\text { (mean [SD NR], } 1 \text { to } 4 \\
\text { scale): } 2.5 \text { vs. } 2.7 \text { at } \\
\text { day } 1 ; 2.0 \text { vs. } 2.5 \text { at day } \\
3 ; 2.0 \text { vs. } 2.2 \text { at day } 7, \\
\text { p=NS for all } \\
\text { comparisons }\end{array}$ \\
\hline $\begin{array}{l}\text { Bondarsky, } \\
2013^{107} \\
\text { Good }\end{array}$ & $\begin{array}{l}\text { A. Ibuprofen } 800 \\
\text { mg } \times 1 \\
\text { B. Acetaminophen } \\
1000 \mathrm{mg} \times 1\end{array}$ & $\begin{array}{l}\text { Acute } \\
\text { musculoskeletal } \\
\text { injury; } \\
60 \text { minutes } \\
\mathrm{n}=60\end{array}$ & $\begin{array}{l}\text { Mean (SD) (0 to } 10 \\
\text { scale): } 3.9 \text { (3.07) vs. } 4.3 \\
(2.79), \mathrm{MD}-0.40 \text { (95\% } \\
\mathrm{Cl}-1.92 \text { to } 1.12)\end{array}$ & $\begin{array}{l}\text { Rescue medication use: } \\
36.7 \%(11 / 30) \text { vs. } \\
33.3 \%(10 / 30) \text {, RR } 1.10 \\
(95 \% \mathrm{Cl} 0.55 \text { to } 2.20)\end{array}$ \\
\hline $\begin{array}{l}\text { Dalton, } \\
2006^{95} \\
\text { Fair }\end{array}$ & $\begin{array}{l}\text { A. Ibuprofen, } 400 \\
\text { mg/ } 3 \text { times daily } \\
\text { for } 9 \text { days } \\
\text { B. Extended } \\
\text { release } \\
\text { acetaminophen, } \\
1,300 \text { mg } 3 \text { times } \\
\text { daily for } 9 \text { days }\end{array}$ & $\begin{array}{l}\text { Grade } 1 \text { or } 2 \text { lateral } \\
\text { ankle sprain; } \\
4 \text { and } 9 \text { days } \\
n=255\end{array}$ & $\begin{array}{l}\text { Pain intensity on walking } \\
\text { (least squares mean } \\
\text { change from baseline), } 0 \\
\text { to } 100 \text { VAS converted to } \\
0 \text { to } 10 \text { scale: }-3.5 \text { vs. } \\
-3.7, p=0.24 \text { at day } 4 ; \\
-5.7 \text { vs. }-5.6, p=0.73 \text { at } \\
\text { day } 9 .\end{array}$ & $\begin{array}{l}\text { Satisfaction with } \\
\text { treatment (Least } \\
\text { squares mean change } \\
\text { from baseline), } 0 \text { to } 100 \\
\text { VAS (higher=more } \\
\text { satisfied): } 7.7 \text { vs. } 7.9, \\
p=0.22 \text { at day } 4 ; 8.4 \text { vs. } \\
8.8, p=0.03 \text { at day } 9\end{array}$ \\
\hline
\end{tabular}




\begin{tabular}{|c|c|c|c|c|}
\hline $\begin{array}{l}\text { Author, } \\
\text { Year } \\
\text { Quality }\end{array}$ & Interventions & $\begin{array}{l}\text { Pain Condition; } \\
\text { Followup Duration } \\
\text { Sample Size }\end{array}$ & Pain Intensity Results & $\begin{array}{l}\text { Other Pain and } \\
\text { Function Results }\end{array}$ \\
\hline $\begin{array}{l}\text { Duncan, } \\
1988^{96} \\
\text { Poor }\end{array}$ & $\begin{array}{l}\text { A: Diclofenac: } 75 \\
\text { mg twice daily ( } 150 \\
\text { mg daily) plus } 2 \\
\text { placebo tablets } \\
\text { three times daily, } \\
\text { for } 3 \text { to } 10 \text { days } \\
\text { B: Aspirin: } 2600 \\
\text { mg tablets three } \\
\text { times daily ( } 3.6 \mathrm{~g} \\
\text { daily) plus two } \\
\text { placebo tablets } \\
\text { twice daily, for } 3 \text { to } \\
10 \text { days }\end{array}$ & $\begin{array}{l}\text { Acute sprains and/or } \\
\text { strains of the knee } \\
\text { or ankle } \\
\text { Up to } 10 \text { days (final } \\
\text { visit) } \\
n=96\end{array}$ & $\begin{array}{l}\text { Pain on active motion } \\
\text { (mean difference [SEM], } \\
\text { difference baseline to } \\
\text { final visit, } 0 \text { to } 3 \text { scale): } \\
-1.96(0.10) \text { vs. }-1.82 \\
(0.10)\end{array}$ & NR \\
\hline $\begin{array}{l}\text { Heere, } \\
1998^{108} \\
\text { Poor }\end{array}$ & $\begin{array}{l}\text { A. Piroxicam } 40 \mathrm{mg} \\
\text { once daily for } 2 \\
\text { days, then } 20 \mathrm{mg} \\
\text { once daily } \\
\text { B. Aspirin } 4000 \mathrm{mg} \\
\text { daily }\end{array}$ & $\begin{array}{l}\text { Sprains and } \\
\text { tendonitis; } \\
16 \text { days } \\
n=69\end{array}$ & NR & $\begin{array}{l}\text { Able to accomplish } \\
\text { activity within } 16 \text { days: } \\
80 \% \text { (28/35) vs. } 65 \% \\
\text { (22/34), RR } 1.24 \text { ( } 95 \% \\
\text { Cl } 0.92 \text { to } 1.67) \\
\\
\text { Overall impression of } \\
\text { efficacy good or } \\
\text { excellent: } 94.3 \% \\
\text { (33/35) vs. } 82.4 \% \\
(28 / 34), \text { RR } 1.14(95 \% \\
\text { Cl } 0.96 \text { to } 1.36)\end{array}$ \\
\hline $\begin{array}{l}\text { Kayali, } \\
2007^{99} \\
\text { Fair }\end{array}$ & $\begin{array}{l}\text { A: Diclofenac } \\
\text { sodium, } 75 \mathrm{mg} \\
\text { twice daily for } 5 \\
\text { days } \\
\text { B: Acetaminophen, } \\
500 \mathrm{mg} 3 \text { times } \\
\text { daily for } 5 \text { days }\end{array}$ & $\begin{array}{l}\text { Grade } 1 \text { or } 2 \text { lateral } \\
\text { ankle sprain; } \\
2 \text { days, } 10 \text { days, and } \\
6 \text { weeks } \\
n=100\end{array}$ & $\begin{array}{l}\text { Pain intensity (mean [SD } \\
\text { NR], } 0 \text { to } 100 \text { VAS } \\
\text { converted to a } 0 \text { to } 10 \\
\text { scale): } 2.1 \text { vs. } 1.2 \text { at } 2 \\
\text { days, MD } 0.9 ; 0.98 \text { vs. } \\
0.63 \text { at } 10 \text { days, MD } \\
0.35 ; 0.46 \text { vs. } 0.30 \text { at } 6 \\
\text { weeks, MD } 0.16 \\
\text { B vs. A MD }(95 \% \mathrm{Cl}): \\
-0.88(-1.4 \text { to }-0.35) \text { at } \\
2 \text { days; }-0.37(-0.68 \text { to } \\
-0.05) \text { at } 10 \text { days; }-0.16 \\
(-0.40 \text { to } 0.08) \text { at } 6 \\
\text { weeks }\end{array}$ & NR \\
\hline $\begin{array}{l}\text { Lyrtzis, } \\
2011^{101} \\
\text { Fair }\end{array}$ & $\begin{array}{l}\text { A: Diclofenac, } 75 \\
\text { mg } 2 \text { times daily for } \\
10 \text { days } \\
\text { B: Acetaminophen, } \\
500 \text { mg } 3 \text { times } \\
\text { daily for } 10 \text { days }\end{array}$ & $\begin{array}{l}\text { Grade } 2 \text { ankle sprain } \\
\text { of the lateral } \\
\text { collateral ligaments; } \\
3 \text { and } 10 \text { days } \\
n=86\end{array}$ & $\begin{array}{l}\text { Pain intensity (mean } \\
\text { [SD], } 0 \text { to } 100 \text { VAS } \\
\text { converted to a } 0 \text { to } 10 \\
\text { scale): } 2.21(1.28) \text { vs. } \\
2.23(1.44) \text { at day } 3, M D \\
-0.02(95 \% \mathrm{Cl}-0.6 \text { to } \\
0.6) ; 0.69(0.83) \text { vs. } 0.51 \\
(0.68) \text { at day } 10, \mathrm{MD} \\
0.18(95 \% \mathrm{Cl}-0.1 \text { to } 0.5) \\
\end{array}$ & NR \\
\hline
\end{tabular}




\begin{tabular}{|c|c|c|c|c|}
\hline $\begin{array}{l}\text { Author, } \\
\text { Year } \\
\text { Quality }\end{array}$ & Interventions & $\begin{array}{l}\text { Pain Condition; } \\
\text { Followup Duration } \\
\text { Sample Size }\end{array}$ & Pain Intensity Results & $\begin{array}{l}\text { Other Pain and } \\
\text { Function Results }\end{array}$ \\
\hline $\begin{array}{l}\text { Man, 2004 }{ }^{102} \\
\text { Good }\end{array}$ & $\begin{array}{l}\text { A: Diclofenac, } 25 \\
\text { mg } 3 \text { times a day } \\
\text { for } 3 \text { days } \\
\text { B: Indomethacin, } 25 \\
\text { mg } 3 \text { times a day } \\
\text { for } 3 \text { days } \\
\text { C. Acetaminophen } \\
1000 \text { mg } 4 \text { times a } \\
\text { day for } 3 \text { days }\end{array}$ & $\begin{array}{l}\text { Isolated soft tissue } \\
\text { limb injury } \\
2 \text { hours, } 1 \text { day, and } \\
3 \text { days } \\
n=39\end{array}$ & $\begin{array}{l}\text { Pain intensity at rest } \\
\text { (mean [SD], } 0 \text { to } 100 \\
\text { VAS converted to a } 0 \text { to } \\
10 \text { scale: } 3.0(2.0) \text { vs. } 1.0 \\
\text { (1.5) vs. } 1.8(2.2) \text { at day } \\
1, \mathrm{MD} 1.2(95 \% \mathrm{Cl}-0.5 \\
\text { to } 2.9) \text { for } \mathrm{A} \text { vs. } \mathrm{C} \text { and } \\
-0.9 \text { (95\% } \mathrm{Cl}-2.5 \text { to } 0.7) \\
\text { for } \mathrm{B} \text { vs. C } \\
\text { Change from baseline in } \\
\text { pain intensity at rest } \\
\text { (mean, } 0 \text { to } 100 \text { scale } \\
\text { converted to } 0 \text { to } 10 \\
\text { scale): }-0.9(95 \% \mathrm{Cl} \\
-1.3 \text { to }-0.4) \text { vs. }-0.9 \\
(95 \% \mathrm{Cl}-1.3 \text { to }-0.4) \text { vs. } \\
-0.9(95 \% \mathrm{Cl}-1.3 \text { to } \\
-0.5) \text { at } 2 \text { hours, } \mathrm{MD} 0.1 \\
(95 \% \mathrm{Cl}-0.8 \text { to } 1.0) \text { for } \\
\mathrm{A} \text { vs. } \mathrm{C} \text { and }-0.1(95 \% \\
\mathrm{Cl}-0.8 \text { to } 1.0) \text { for } \mathrm{B} \text { vs. } \\
\mathrm{C} ;-1.6(95 \% \mathrm{Cl}-2.2 \text { to } \\
-1.0) \text { vs. }-0.4(95 \% \mathrm{Cl} \\
-0.9 \text { to } 0.2) \text { vs. }-0.6 \\
(95 \% \mathrm{Cl}-1.0 \text { to }-0.1) \text { at } \\
3 \text { days, } \mathrm{MD}-0.8(95 \% \mathrm{Cl} \\
-1.9 \text { to } 0.4) \text { for } \mathrm{A} \text { vs. C } \\
\text { and } 0.5(95 \% \mathrm{Cl}-0.6 \text { to } \\
1.6) \text { for } \mathrm{B} \text { vs. C }\end{array}$ & $\begin{array}{l}\text { Requested additional } \\
\text { analgesic after } 3 \text { days, } \\
\%(\mathrm{n} / \mathrm{N}): 11 \%(1 / 9) \text { vs. } \\
30 \%(3 / 10) \text { vs. } 0 \% \\
(0 / 14)\end{array}$ \\
\hline $\begin{array}{l}\text { Muckle, } \\
1974^{111} \\
\text { Poor }\end{array}$ & $\begin{array}{l}\text { A. Flurbiprofen } 150 \\
\mathrm{mg} \text { for } 6 \text { days } \\
\text { B. Aspirin } 1200 \mathrm{mg} \\
3 \text { times daily for } 6 \\
\text { days }\end{array}$ & $\begin{array}{l}\text { Soft tissue trauma to } \\
\text { lower limb; } \\
1 \text { and } 6 \text { days } \\
n=51\end{array}$ & $\begin{array}{l}\text { Pain (mean [SD NR], } \\
\text { total daily score, scale } \\
\text { not reported): } 4.1 \text { vs. } 4.2 \\
\text { at day } 1, p=N R ; 0.3 \text { vs. } \\
1.2 \text { at day } 6, p<0.01\end{array}$ & $\begin{array}{l}\text { Days to training fitness } \\
\text { (mean [SD NR]): } 3.38 \\
\text { vs. } 4.27, p<0.05 \\
\text { Days to match fitness } \\
\text { (mean [SD NR]): } 4.79 \\
\text { vs. } 6.04, p<0.05\end{array}$ \\
\hline
\end{tabular}




\begin{tabular}{|c|c|c|c|c|}
\hline $\begin{array}{l}\text { Author, } \\
\text { Year } \\
\text { Quality }\end{array}$ & Interventions & $\begin{array}{l}\text { Pain Condition; } \\
\text { Followup Duration } \\
\text { Sample Size }\end{array}$ & Pain Intensity Results & $\begin{array}{l}\text { Other Pain and } \\
\text { Function Results }\end{array}$ \\
\hline $\begin{array}{l}\text { Ridderikhof, } \\
2018^{103} \\
\text { Fair }\end{array}$ & $\begin{array}{l}\text { A. Diclofenac, } 75 \\
\text { mg } 3 \text { times daily for } \\
3 \text { days } \\
\text { B: Acetaminophen, } \\
1000 \text { mg } 4 \text { times } \\
\text { daily for } 3 \text { days }\end{array}$ & $\begin{array}{l}\text { Minor } \\
\text { musculoskeletal } \\
\text { trauma of an } \\
\text { extremity; } \\
90 \text { minutes and } 3 \\
\text { days } \\
\mathrm{n}=365\end{array}$ & 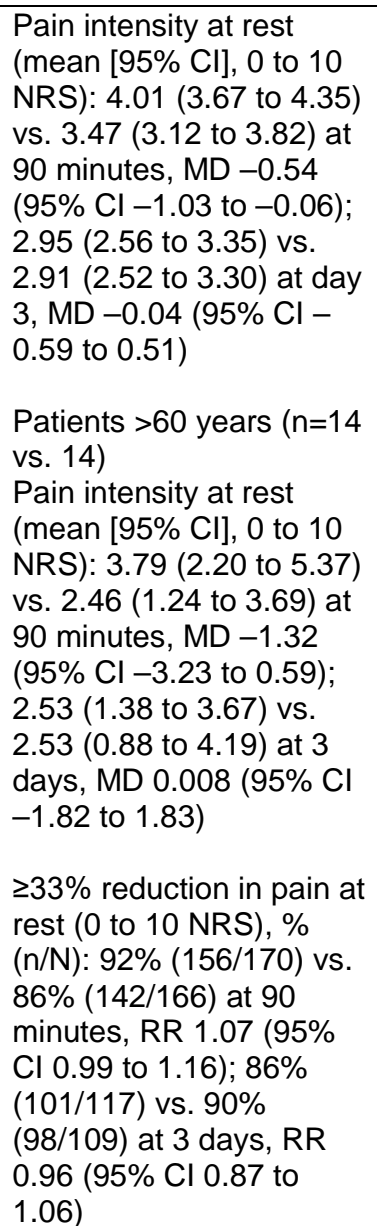 & $\begin{array}{l}\text { Proportion "satisfied" or } \\
\text { "very satisfied" with } \\
\text { pain relief, } \%(\mathrm{n} / \mathrm{N}) \text { : } \\
55 \%(92 / 166) \text { vs } 57 \% \\
\text { (98/171) at } 90 \text { minutes, } \\
\text { RR } 0.97(95 \% \mathrm{Cl} 0.80 \\
\text { to } 1.17) ; 74 \%(99 / 134) \\
\text { vs. } 67 \%(83 / 123) \text { at } 3 \\
\text { days, RR } 1.09 \text { ( } 95 \% \mathrm{Cl} \\
0.93 \text { to } 1.28) \\
\text { Additional opioid } \\
\text { analgesics, } \%(\mathrm{n} / \mathrm{N}): \\
0.5 \%(1 / 182) \text { vs. } 0 \% \\
(0 / 183) \text { at } 90 \text { minutes; } \\
9 \%(16 / 182) \text { vs. } 4 \% \\
(8 / 183) \text { at } 3 \text { days, RR } \\
2.01(95 \% \mathrm{Cl} 0.88 \text { to } \\
4.58)\end{array}$ \\
\hline $\begin{array}{l}\text { Woo, } \\
2005^{120} \\
\text { Good }\end{array}$ & $\begin{array}{l}\text { A. Diclofenac } 25 \\
\mathrm{mg} \text { every } 8 \text { hours } \\
\text { for } 3 \text { days } \\
\text { B. Indomethacin } 25 \\
\mathrm{mg} \text { every } 8 \text { hours } \\
\text { for } 3 \text { days } \\
\text { A. Acetaminophen } \\
1000 \text { mg every } 6 \\
\text { hours for } 3 \text { days }\end{array}$ & $\begin{array}{l}\text { Limb injury after } \\
\text { trauma (sprain, } \\
\text { contusion, wound, } \\
\text { crush, fracture); } \\
2 \text { hours and } 3 \text { days } \\
\mathrm{n}=201\end{array}$ & $\begin{array}{l}\text { Pain intensity difference } \\
\text { (mean change from } \\
\text { baseline, } 0 \text { to } 100 \text { VAS } \\
\text { converted to } 0 \text { to } 10 \\
\text { scale): } 0.6 \text { vs. } 0.9 \text { vs. } 0.9 \\
\text { at } 2 \text { hours, MD in change } \\
\text { from baseline, }-0.1 \text { ( } 95 \% \\
\mathrm{Cl}-0.4 \text { to } 0.2 \text { ) for } \mathrm{A} \text { vs. } \\
\mathrm{C} \text { and }-0.1(95 \% \mathrm{Cl}-0.3 \\
\text { to } 0.4 \text { ) for } \mathrm{B} \text { vs. C; } 0.7 \mathrm{vs.} \\
0.9 \text { vs. } 0.5 \text { at } 3 \text { days, MD } \\
0.1 \text { ( } 95 \% \mathrm{Cl}-0.6 \text { to } 0.4) \\
\text { for } \mathrm{A} \text { vs. } \mathrm{C} \text { and }-0.2 \\
(95 \% \mathrm{Cl} 0.7 \text { to }-0.4) \text { for } \\
\text { B vs. C }\end{array}$ & NR \\
\hline
\end{tabular}

Abbreviations: $\mathrm{CI}=$ confidence interval; DASH = Disabilities of the Arm, Shoulder, and Hand; MD = mean difference; NR = not reported; NS = not significant; RR = relative risk; $S D=$ standard deviation; $S E M$ = standard error of the mean; SPADI = Shoulder Pain and Disability Index; VAS = visual analog scale

\section{Oral NSAID Versus Topical NSAID}

One fair quality trial $(n=100)$ based in the United Kingdom compared topical ibuprofen versus oral ibuprofen $\left(400 \mathrm{mg}\right.$ ) for acute soft tissue injuries. ${ }^{122}$ There were no differences in 
median pain intensity at rest or with movement at 1 or 2 days, and the time to improvement in pain was similar in both groups.

KQs $3 \mathrm{j}$ and $3 \mathrm{l}$ ask how the comparative effectiveness and harms of nonopioid pharmacologic therapy vary depending on: (1) patient demographics (e.g., age, race, ethnicity, gender); (2) patient medical and psychiatric comorbidities; (3) the type of nonopioid medication; (4) dose of medication; (5) duration of treatment.

Evidence was too limited to evaluate how comparative effectiveness and harms of nonopioid therapy for acute musculoskeletal pain varied in subgroups, due to few trials, small sample sizes, methodological limitations, and exclusion of relevant subgroups or lack of information about them. One trial found no statistically significant differences between an NSAID versus acetaminophen in pain intensity in a subgroup analysis of patients over 60 years of age, but the number of patients was small, and estimates were imprecise. ${ }^{103}$ No other study conducted withinstudy or across-study evaluations of subgroup effects. Details regarding the nonopioid medications prescribed, dose, and duration of treatment are described above.

\section{Nonpharmacologic Therapy}

KQs $3 m$ and $3 n$ address the comparative effectiveness of nonpharmacologic therapy versus: (1) inactive controls or (2) other nonpharmacologic therapies.

Ten trials evaluated nonpharmacologic therapy for acute musculoskeletal pain (Appendix E, Table E-4). ${ }^{100,104,106,110,113-115,117,119,121}$ Three trials ${ }^{106,110,117}$ evaluated cold therapy, one trial ${ }^{110}$ heat therapy, three trials ${ }^{113,119,121}$ ultrasound, one trial ${ }^{114}$ transcutaneous electrical nerve stimulation, one trial acupressure, ${ }^{104}$ one trial a rib belt, ${ }^{100}$ and one trial ${ }^{115}$ relaxation. Three trials ${ }^{104,113,115}$ were rated fair quality, and seven trials ${ }^{100,106,110,114,117,119,121}$ poor quality (Appendix F, Table F-1).

\section{Cold Therapy Versus No Cold Therapy or Sham}

Two trials compared cold therapy versus no cold therapy or sham for ankle sprain (Table 14). ${ }^{106,117}$ It was not possible to blind patients to use of cold, though one of the trials used a sham (non-inflated anklet without use of cold material). The trials were rated poor quality, primarily due to inadequate reporting of methods. In addition, it was unclear if one of the trials ${ }^{106}$ was randomized. In that trial $(n=60), 48$ hours of therapy with a cold pack gel was associated with increased likelihood of recovery at 2 and 7 days versus no cold therapy (absolute difference $13.0 \%$ and $23.6 \%$, respectively), but statistical significance testing was not reported and not calculable. At 14 days, the difference favored cold therapy but was small (8.0\%). Cold therapy was also associated with decreased period of disability (9.7 vs. 14.8) of unknown statistical significance. In the other trial $(n=116)$, there was no difference between cold therapy administered in an inflated anklet versus sham in pain relief (data not reported) or in likelihood of improving $\geq 2$ points on a 5 point injury severity scale ( $88 \%$ vs. $79 \%, p=0.15) .{ }^{117}$ Adverse events were not reported in either trial.

\section{Cold Therapy Versus Heat Therapy}

One trial ( $n=37$ ) found cold therapy (ice whirlpool or ice pack three times daily for 3 days) associated with shorter time to achieve functional milestones (walk without pain, stand without pain, climb stars without pain, or run and jump without pain) than heat therapy (warm soak or heating pad 1 to 3 times daily for 3 days) (Table 14). ${ }^{110}$ However, the trial was rated poor quality, and it was not clear if it was truly randomized. In addition, results for cold therapy were 
reported only stratified by time to initiation and by severity of sprain (not by overall randomization), with no evaluation of statistical significance. Adverse events were not reported.

Table 14. Cold therapy versus other nonpharmacologic therapies for musculoskeletal pain

\begin{tabular}{|c|c|c|c|c|}
\hline $\begin{array}{l}\text { Author, } \\
\text { Year } \\
\text { Quality }\end{array}$ & Interventions & $\begin{array}{l}\text { Pain Condition; } \\
\text { Followup Duration } \\
\text { Sample Size }\end{array}$ & $\begin{array}{l}\text { Pain Intensity } \\
\text { Results }\end{array}$ & $\begin{array}{l}\text { Other Pain and } \\
\text { Function Results }\end{array}$ \\
\hline $\begin{array}{l}\text { Basur, } \\
1976^{106} \\
\text { Poor }\end{array}$ & $\begin{array}{l}\text { A: Cold gel pack for } \\
48 \text { hours } \\
\text { B: No cold therapy }\end{array}$ & $\begin{array}{l}\text { Ankle sprain; } \\
2,7, \text { and } 14 \text { days } \\
n=60\end{array}$ & $\begin{array}{l}\text { Recovery (not defined) } \\
\text { (n/N NR): } 42.1 \% \text { vs. } \\
29.1 \% \text { at day } 2,84.2 \% \\
\text { vs. } 60.6 \% \text { at day } 7, \\
\text { and } 96.2 \% \text { vs. } 88.2 \% \\
\text { at day } 14, \mathrm{p}=\mathrm{NR}\end{array}$ & $\begin{array}{l}\text { Period of disability (mean } \\
\text { days [SD NR]): } 9.7 \text { vs. } \\
14.8, p=N R\end{array}$ \\
\hline $\begin{array}{l}\text { Hocutt, } \\
1983^{110} \\
\text { Poor }\end{array}$ & $\begin{array}{l}\text { A: Cryotherapy, ice } \\
\text { whirlpool for } 12 \text { to } \\
20 \text { minutes or ice } \\
\text { pack for } 15 \text { to } 20 \\
\text { minutes } 1 \text { to } 3 \\
\text { times daily } 3 \text { days } \\
\text { B: Heat therapy, } \\
\text { warm soak or } \\
\text { heating pad for } 15 \\
\text { minutes } 1 \text { to } 3 \\
\text { times daily for } \geq 3 \\
\text { days }\end{array}$ & $\begin{array}{l}\text { Grade } 3 \text { or } 4 \text { ankle } \\
\text { sprain; } \\
\text { Outcomes reported as } \\
\text { time to milestones } \\
n=37\end{array}$ & NR & $\begin{array}{l}\text { A (initiating on day } 0 \text { or } \\
\text { 1) vs. A (initiated on day } \\
\text { 2) vs. B } \\
\text { Walk without pain (mean } \\
\text { [SD NR], days): } 2.6 \text { vs. } \\
5.2 \text { vs. } 7.8 \text { for class } 3 \\
\text { sprain, } 4.2 \text { vs. } 12.0 \text { vs. } \\
9.7 \text { for class } 4 \text { sprain } \\
\text { Stand without pain (Mean } \\
\text { [SD NR], days): } 2.7 \text { vs. } \\
6.2 \text { vs. } 5.7 \text { for class } 4 \\
\text { sprain }\end{array}$ \\
\hline $\begin{array}{l}\text { Sloan, } \\
1989^{117} \\
\text { Poor }\end{array}$ & $\begin{array}{l}\text { A: Inflated cooling } \\
\text { anklet for } 30 \\
\text { minutes while ankle } \\
\text { elevated } \\
\text { B: Sham therapy, } \\
\text { non-inflated anklet } \\
\text { without elevation }\end{array}$ & $\begin{array}{l}\text { Ankle sprain; } \\
7 \text { days } \\
n=116\end{array}$ & $\begin{array}{l}\text { Pain improved } \geq 2 \\
\text { points on a } 5 \text { point } \\
\text { scale: } 88 \% \text { vs. } 79 \% \text {, } \\
p=0.15\end{array}$ & $\begin{array}{l}\text { Pain relief: No difference } \\
\text { (data not provided) }\end{array}$ \\
\hline
\end{tabular}

Abbreviations: $\mathrm{NR}=$ not reported; $\mathrm{SD}=$ standard deviation

\section{Ultrasound Versus No Ultrasound or Sham Ultrasound}

Three trials $(\mathrm{N}=190)$ compared ultrasound versus no ultrasound or sham ultrasound in patients with ankle sprains (Table 15). ${ }^{113,119,121}$ One trial ${ }^{113}$ was rated fair quality, and two trials ${ }^{119,121}$ were rated poor quality. The fair quality trial $(n=51)$ found no differences between ultrasound (10 minutes daily for three days) versus sham ultrasound in pain or ability to bear body weight at 3 or 14 days. ${ }^{113}$ The poor quality trials $(n=29$ and $n=110)$ found no differences between ultrasound versus sham ultrasound or no ultrasound on pain intensity or a composite outcome (swelling, discomfort, limp, and pain) at 1 to 4 weeks. ${ }^{119,121}$ Adverse events were not reported in any of the trials.

Table 15. Ultrasound versus no ultrasound or sham ultrasound for musculoskeletal pain

\begin{tabular}{|c|c|c|c|c|}
\hline $\begin{array}{l}\text { Author, } \\
\text { Year } \\
\text { Quality }\end{array}$ & Interventions & $\begin{array}{l}\text { Pain Condition; } \\
\text { Followup } \\
\text { Duration } \\
\text { Sample Size }\end{array}$ & $\begin{array}{l}\text { Pain Intensity } \\
\text { Results }\end{array}$ & $\begin{array}{l}\text { Other Pain and Function } \\
\text { Results }\end{array}$ \\
\hline $\begin{array}{l}\text { Nyanzi, } \\
1999^{113} \\
\text { Fair }\end{array}$ & $\begin{array}{l}\text { A: Ultrasound, 10- } \\
\text { minute sessions } \\
\text { daily for } 3 \text { days } \\
\text { B: Sham } \\
\text { ultrasound, same } \\
\text { as above, but } \\
\text { probes disabled }\end{array}$ & $\begin{array}{l}\text { Lateral ankle } \\
\text { ligament sprain; } \\
3 \text { and } 14 \text { days } \\
\mathrm{n}=51\end{array}$ & $\begin{array}{l}\text { Pain (mean [SD], } 0 \\
\text { to } 10 \text { VAS): } 1.9 \\
(2.5) \text { vs. } 2.2(2.1) \text { at } \\
\text { day } 3,0.9(1.4) \text { vs. } \\
0.7(1.4) \text { at day } 14, \\
\text { p=NR at both time } \\
\text { points }\end{array}$ & $\begin{array}{l}\text { Percent of body weight in affected } \\
\text { leg (SD): } 42.8(5.8) \text { vs. } 43.0(6.0) \\
\text { at day } 3,44.7(5.6) \text { vs. } 45.1(4.6) \\
\text { at day } 14\end{array}$ \\
\hline
\end{tabular}




\begin{tabular}{|c|c|c|c|c|}
\hline $\begin{array}{l}\text { Author, } \\
\text { Year } \\
\text { Quality }\end{array}$ & Interventions & $\begin{array}{l}\text { Pain Condition; } \\
\text { Followup } \\
\text { Duration } \\
\text { Sample Size }\end{array}$ & $\begin{array}{l}\text { Pain Intensity } \\
\text { Results }\end{array}$ & $\begin{array}{l}\text { Other Pain and Function } \\
\text { Results }\end{array}$ \\
\hline $\begin{array}{l}\text { Williamson, } \\
1983^{119} \\
\text { Poor }\end{array}$ & $\begin{array}{l}\text { A: Ultrasound, } \\
\text { session every other } \\
\text { day } \\
\text { B: No ultrasound }\end{array}$ & $\begin{array}{l}\text { Lateral ankle } \\
\text { ligament sprain; } \\
1,2,3 \text {, and } 4 \\
\text { weeks } \\
n=110\end{array}$ & NR & $\begin{array}{l}\text { Overall score for subjective } \\
\text { assessment of swelling, patient } \\
\text { discomfort, degree of limp, pain } \\
\text { on inversion, pain on } \\
\text { plantarflexion (median [IQR NR], } \\
0 \text { to } 15 \text { scale): } 1.8 \text { vs. } 1.2 \text { at week } \\
1,1.0 \text { vs. } 1.0 \text { at week } 2,0.5 \text { vs. } \\
0.9 \text { at week } 3,0 \text { vs. } 0.6 \text { at week } 4 \text {; } \\
\text { p=NR } \\
\text { Proportion with overall score of } 0 \\
\text { or } 1: 52 \% \text { vs. } 53 \% \text { at week } 1,90 \% \\
\text { vs. } 84 \% \text { at week } 2,100 \% \text { vs. } 95 \% \\
\text { at week } 3,100 \% \text { vs. } 99 \% \text { at week } \\
4 ; p=N R\end{array}$ \\
\hline $\begin{array}{l}\text { Zammit, } \\
2005^{121} \\
\text { Poor }\end{array}$ & $\begin{array}{l}\text { A: Ultrasound, } 1 \\
\text { 10- minute session } \\
\text { every other day for } \\
2 \text { weeks, and } 1 \\
\text { followup session on } \\
\text { day } 21 \\
\text { B: Sham } \\
\text { ultrasound, as } \\
\text { above, but } \\
\text { ultrasound not } \\
\text { turned on } \\
\text { C: No ultrasound }\end{array}$ & $\begin{array}{l}\text { Lateral ankle } \\
\text { ligament sprain; } \\
7,14, \text { and } 21 \\
\text { days } \\
n=29\end{array}$ & $\begin{array}{l}\text { Pain intensity } \\
\text { (mean [SD NR], } 0 \\
\text { to } 10 \text { VAS): } 1.8 \text { vs. } \\
2.1 \text { vs. } 2.9 \text { at day } 7 \\
0.8 \text { vs. } 1.2 \text { vs. } 1.7 \\
\text { at day } 14,0.3 \text { vs. } \\
0.7 \text { vs. } 0.7 \text { at day } \\
21 ; \text { p>0.05 }\end{array}$ & NR \\
\hline
\end{tabular}

Abbreviations: IQR = interquartile range; NR = not reported; SD = standard deviation; VAS = visual analog scale

\section{Transcutaneous Electrical Nerve Stimulation (TENS) Versus Sham TENS}

One poor-quality trial $(n=100)$ compared TENS versus sham TENS, in patients with acute musculoskeletal injuries (sprain, laceration, fracture, or hematoma/contusion) (Table 16). ${ }^{114}$ Patients were randomized to four groups: TENS or sham TENS with or without the addition of codeine plus acetaminophen. TENS was worn at all times or as needed for pain for 3 days; for sham TENS the unit was not turned on. At two days, TENS was associated with decreased pain intensity versus sham TENS when each was administered without codeine plus acetaminophen (mean difference -2.44, 95\% CI -3.89 to -0.99), but there was no difference between TENS versus sham TENS with codeine plus acetaminophen (mean difference $0.00,95 \% \mathrm{CI}-1.33$ to 1.33). There was no difference between TENS versus sham TENS at 1 month, with or without the opioid. The only adverse event reported was mild tingling in 20 percent of overall patients (not reported by group).

\section{Relaxation Versus Usual Care}

One small ( $\mathrm{n}=30)$, fair quality trial of patients with nonoperatively-treated, simple long or short-bone fractures found prana energization (a relaxation technique based on yoga principles) associated with decreased pain intensity at 4 weeks (mean difference -3.23 on a 0 to 10 scale, 95\% CI -4.13 to -2.32) (Table 16). ${ }^{115}$ The intervention consisted of 30-minute sessions daily for 7 days, followed by home sessions twice daily for 3 weeks. Adverse events were not reported. 


\section{Acupressure Versus Sham Acupressure or Usual Care}

One fair quality trial $(n=62)$ compared a single session of acupressure versus sham acupressure or standard treatment without acupressure (rest ice, compression, and evaluation) for ankle sprain (Table 16). ${ }^{104}$ It found acupressure associated with a moderate decrease in pain intensity at 3 days versus sham acupressure (difference -1.7 points on a 0 to 10 scale, $\mathrm{p}<0.01$ ) and large decrease versus standard treatment (difference -2.1 points, $\mathrm{p}<.01$ ). At 4 weeks, effects also favored acupressure but were smaller (differences -0.79 and $-0.90, \mathrm{p}<0.01$ ), with no statistically significant differences at 8 weeks (differences -0.16 and -0.31 ). Acupressure was also associated with small beneficial effects on the SF-36 physical and mental component summary scores at 4 weeks that were not statistically significant (differences 7.22 to 8.43 points on a 0 to 100 scale), with little difference on these summary scores at 8 weeks (differences 0.9 to 3.9 points).

\section{Rib Belt Versus No Rib Belt}

A small $(n=20)$, poor quality trial found a rib belt associated with a pain intensity difference at day 3 versus no rib belt that was small and not statistically significant (4.4 vs. 5.2, p>0.20) (Table 16). ${ }^{100}$ At 2 weeks, adverse events in the belt group included one case of bloody pleural effusion, two cases of asymptomatic atelectasis, and one case of contact dermatitis; there were no adverse events in the non-belt group. 


\begin{tabular}{|c|c|c|c|c|}
\hline $\begin{array}{l}\text { Author, Year } \\
\text { Quality }\end{array}$ & Interventions & $\begin{array}{l}\text { Pain Condition; } \\
\text { Followup Duration } \\
\text { Sample Size }\end{array}$ & Pain Intensity Results & Other Pain and Function Results \\
\hline $\begin{array}{l}\text { Lazcano, } \\
1989^{100} \\
\text { Poor }\end{array}$ & $\begin{array}{l}\text { A: Wear rib belt at all times } \\
\text { except bathing; duration NR } \\
\text { B: No rib belt }\end{array}$ & $\begin{array}{l}\text { Rib fracture; } \\
3 \text { days and } 2 \text { weeks } \\
n=20\end{array}$ & $\begin{array}{l}\text { Pain (mean [SD NR], } 0 \text { to } 10 \text { NRS): } \\
4.4 \text { vs. } 5.2 \text { at day } 3, p>0.20\end{array}$ & NR \\
\hline $\begin{array}{l}\text { Ordog, } 1987^{114} \\
\text { Poor }\end{array}$ & $\begin{array}{l}\text { A: TENS, worn at all times or } \\
\text { as needed for pain in area of } \\
\text { injury } \\
\text { B: TENS + codeine and } \\
\text { acetaminophen } 30 / 300 \mathrm{mg} \text {, } \\
\text { TENS as above }+1 \text { to } 2 \\
\text { tablets every } 4 \text { hours as } \\
\text { needed } \\
\text { C: Sham TENS, worn at all } \\
\text { times or as needed for pain in } \\
\text { area of injury } \\
\text { D: Sham TENS + codeine } \\
\text { and acetaminophen } 30 / 300 \\
\text { mg; as above }\end{array}$ & $\begin{array}{l}\text { Sprain, laceration, fracture, } \\
\text { hematoma/contusion; } \\
2 \text { days and } 1 \text { month } \\
n=100\end{array}$ & $\begin{array}{l}\text { Pain intensity (mean [SD], } 0 \text { to } 10 \\
\text { NRS): } 3.04(2.6) \text { vs. } 3.84(2.3) \text { vs. } \\
5.48(2.5) \text { vs. } 3.84(2.38) \text { at } 2 \text { days, } \\
\mathrm{MD}-2.44(95 \% \mathrm{Cl}-3.89 \text { to }-0.99) \\
\text { for } \mathrm{A} \text { vs. } \mathrm{C} \text { and } 0.00(95 \% \mathrm{Cl}-1.33 \\
\text { to } 1.33) \text { for B vs. } \mathrm{D} ; 0.28(0.54) \text { vs. } \\
0.52(0.96) \text { vs. } 0.44(0.65) \text { vs. } 0.52 \\
(1.0) \text { at } 1 \text { month, MD }-0.16(95 \% \mathrm{Cl} \\
-0.50 \text { to } 0.18) \text { for } \mathrm{A} \text { vs. C and } 0.00 \\
(95 \% \mathrm{Cl}-0.56 \text { to } 0.56) \text { for B vs. D }\end{array}$ & NR \\
\hline $\begin{array}{l}\text { Oswal, } 2011^{115} \\
\text { Fair }\end{array}$ & $\begin{array}{l}\text { A: Yoga relaxation technique } \\
\text { (Prana energization), } 30 \\
\text { minutes daily for } 7 \text { days, then } \\
\text { twice daily for } 3 \text { weeks } \\
\text { B: Usual care }\end{array}$ & $\begin{array}{l}\text { Simple long or short-bone } \\
\text { fractures } \\
4 \text { weeks } \\
n=30\end{array}$ & $\begin{array}{l}\text { Pain intensity (mean [SD], } 0 \text { to } 10 \\
\text { NRS): } 0.50(0.75) \text { vs. } 3.73(1.48) \\
M D-3.23(95 \% \mathrm{Cl}-4.13 \text { to }-2.32)\end{array}$ & NR \\
\hline
\end{tabular}




\begin{tabular}{|c|c|c|c|c|}
\hline $\begin{array}{l}\text { Author, Year } \\
\text { Quality }\end{array}$ & Interventions & $\begin{array}{l}\text { Pain Condition; } \\
\text { Followup Duration } \\
\text { Sample Size }\end{array}$ & Pain Intensity Results & Other Pain and Function Results \\
\hline $\begin{array}{l}\text { Zhao, 2018 } \\
\text { Fair }\end{array}$ & $\begin{array}{l}\text { A: Acupressure plus standard } \\
\text { treatment: pressure applied to } \\
\text { the Yongquan point of injured } \\
\text { foot for } 10 \text { minutes along with } \\
\text { pressure to tenderness point } \\
\text { on contralateral hand + } \\
\text { standard treatment for } \geq 3 \\
\text { sessions } \\
\text { B: Sham acupressure: } \\
\text { pressure applied to the } \\
\text { Yongquan point of non-injured } \\
\text { foot and tenderness point of } \\
\text { contralateral hand + standard } \\
\text { treatment for } \geq 3 \text { sessions } \\
\text { C: Standard treatment: rest, } \\
\text { ice (20 minutes in } 48 \text { hours), } \\
\text { compression (Tubigrip } \\
\text { compression stocking for } 6 \\
\text { weeks), and elevation }\end{array}$ & $\begin{array}{l}\text { Grade } 1 \text { or } 2 \text { ankle sprain; } \\
1,3,28 \text {, and } 56 \text { days } \\
n=62\end{array}$ & 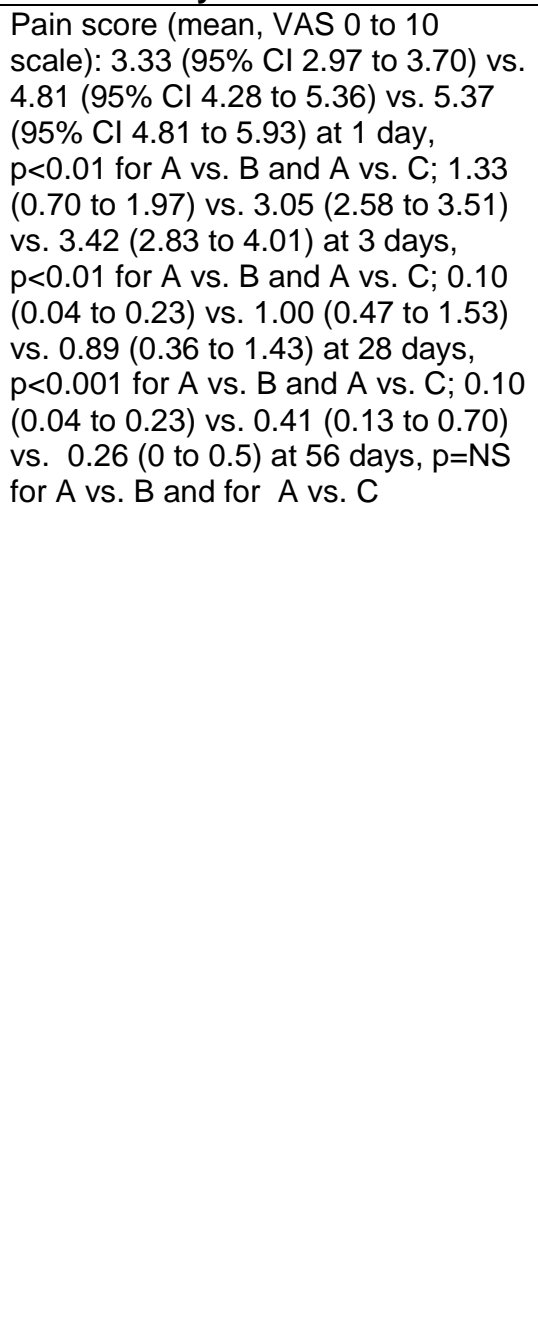 & 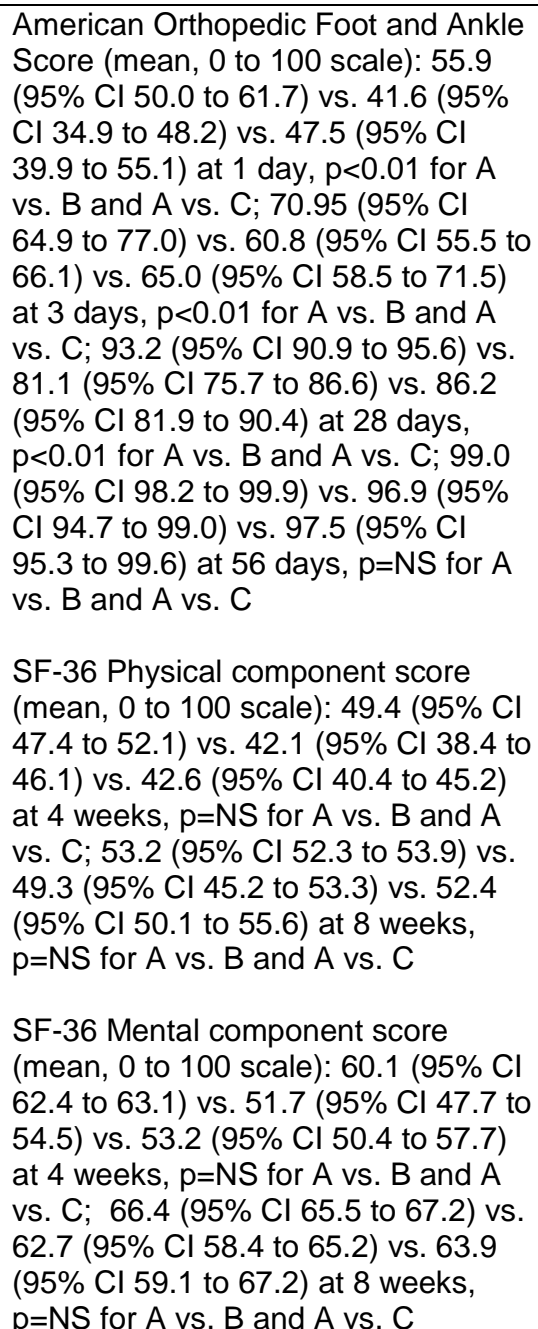 \\
\hline
\end{tabular}

Abbreviations: $\mathrm{CI}$ = confidence interval; $\mathrm{MD}$ = mean difference; $\mathrm{NR}$ = not reported; $\mathrm{NS}$ = not significant; $\mathrm{NRS}$ = numeric rating scale; $\mathrm{SD}$ = standard deviation; $\mathrm{SF}$ = short form; TENS = transcutaneous electrical nerve stimulation 
KQs $3 o$ and 3p ask if the comparative effectiveness and harms of nonpharmacologic therapy vary depending on: (1) patient demographics (e.g., age, gender); (2) patient medical and psychiatric comorbidities; (3) the type of treatment used; (4) the frequency of therapy; (5) the duration of therapy.

Evidence on how the comparative effectiveness and harms of nonpharmacologic therapy for acute musculoskeletal pain varied based on patient and intervention factors was not available. The number of trials and sample sizes for each comparison were too small for between-study comparisons to be informative and no trial conducted within-study subgroup analyses.

\section{KQ 4. Peripheral Neuropathic Pain (Related to Herpes Zoster and Trigeminal Neuralgia)}

\section{Key Points}

- Opioid vs. gabapentin, herpes zoster

o Oxycodone associated with increased likelihood of improvement in pain at 1 to $<2$ weeks ( $79.3 \%$ vs. $55.2 \%$, RR $1.4,95 \%$ CI 1.0 to 2.1$)$ and at $\geq 4$ weeks ( $86.2 \%$ vs. 62.1\%, RR 1.5, 95\% CI 1.1 to 2.0) (SOE: low).

o Oxycodone associated with increased likelihood of constipation (RR 2.50, 95\% CI 1.13 to 5.53), based on one trial (SOE: low).

- Topical vs. oral aspirin, herpes zoster

o Insufficient evidence from one poor quality trial (SOE: insufficient).

- No trials of nonopioid pharmacologic therapy for acute peripheral neuropathic pain.

\section{Summary of Findings}

Evidence on the comparative effectiveness of interventions for peripheral neuropathic pain was limited to two small ( $\mathrm{n}=45$ and 30) trials (Appendix E, Table E-5). ${ }^{123,124}$ One trial evaluated opioid therapy (KQ 4a and 4c), and one trial evaluated nonopioid pharmacologic therapy (KQ 4i and $4 \mathrm{j}$ ). Both trials evaluated patients with acute herpes zoster, and the duration of pain was $\geq 7$ days in both trials. The mean age of patients was 49 and 66 years, and the proportion female was 30 percent and 66 percent. The trial of opioid therapy excluded patients with alcohol or drug abuse history within the last 5 years. ${ }^{124}$ Otherwise the trials did not describe psychiatric or medical comorbidities. One trial ${ }^{124}$ was rated fair quality, and one trial ${ }^{123}$ was rated poor quality (Appendix F, Table F-1). The poor quality trial was open-label, did not report randomization and allocation concealment methods, had high attrition, and did not perform intention to treat analysis.

\section{Detailed Synthesis}

\section{Opioid Therapy}

KQs 4a and 4c address the comparative effectiveness and harms of opioid therapy versus: (1) nonopioid pharmacologic therapy (e.g., acetaminophen, NSAIDs, antidepressants, anticonvulsants) or (2) nonpharmacologic therapy (e.g., exercise, cognitive behavioral therapy, acupuncture). 


\section{Opioid Versus Gabapentin}

One fair quality trial $(\mathrm{n}=45)$ compared oxycodone sustained-release (titrated up to $60 \mathrm{mg}$ twice daily) versus gabapentin (titrated up to $600 \mathrm{mg}$ three times a day) for acute herpes zoster ${ }^{124}$ (Table 17). Oxycodone was associated with decreased average pain intensity versus gabapentin at day 1 to 8 , day 1 to 14 , and day 1 to 28 , though differences were small ( -0.5 to -0.8 point on a 0 to 10 point scale) and not statistically significant. Oxycodone was associated with increased likelihood of $\geq 30 \%$ improvement in pain at day 14 (79.3\% vs. 55.2\%, RR 1.4 , 95\% CI 1.0 to 2.1) and at day 28 (86.2\% vs. 62.1\%, RR 1.5, 95\% CI 1.1 to 2.0) and increased likelihood of $\geq 50 \%$ improvement in pain at day 28 (72.4\% vs. 48.3\%, RR 1.6, 95\% CI 1.0 to 2.3). Oxycodone was also associated with slightly decreased rescue medication use, though the difference was not statistically significant. There was no difference between oxycodone versus gabapentin in function. Oxycodone was associated with increased likelihood of constipation versus gabapentin (RR 2.50, 95\% CI 1.13 to 5.53); there were no differences in serious adverse events, withdrawal due to adverse events, or other specific adverse events, but there were few events and estimates were imprecise.

Table 17. Opioid versus gabapentin for herpes zoster-related pain

\begin{tabular}{|c|c|c|c|c|}
\hline $\begin{array}{l}\text { Author, } \\
\text { Year } \\
\text { Quality }\end{array}$ & Interventions & $\begin{array}{l}\text { Followup } \\
\text { Duration } \\
\text { Sample } \\
\text { Size }\end{array}$ & $\begin{array}{l}\text { Pain Intensity } \\
\text { Results }\end{array}$ & Other Pain Results \\
\hline $\begin{array}{l}\text { Dworkin, } \\
2009^{124} \\
\text { Fair }\end{array}$ & $\begin{array}{l}\text { A: Oxycodone, } 10 \\
\text { mg } 1 \text { or } 2 \text { times } \\
\text { daily titrated to up } \\
\text { to } 60 \text { mg every } 12 \\
\text { hours } \\
\text { B: Gabapentin, } \\
300 \text { mg every } \\
\text { night titrated to up } \\
\text { to } 600 \text { mg every } 8 \\
\text { hours }\end{array}$ & $\begin{array}{l}\text { Days } 1 \text { to } 14 \\
\text { and days } 1 \\
\text { to } 28 ; 8,14 \\
\text { and } 15 \text { days } \\
n=45\end{array}$ & $\begin{array}{l}\text { Average pain intensity } \\
\text { (adjusted mean [SD], } 0 \\
\text { to } 10 \text { VAS): } 3.5(8.6) \\
\text { vs. } 4.0(8.6) \text { at day } 1 \text { to } \\
\text { 8, MD }-0.5(95 \% \mathrm{Cl} \\
-5.0 \text { to } 4.0) ; 2.5(8.6) \\
\text { vs. } 3.3(8.6) \text { at day } 1 \text { to } \\
\text { 14, MD }-0.8(95 \% \mathrm{Cl} \\
-5.3 \text { to } 3.7) ; 0.6(9.2) \\
\text { vs. } 1.4(9.2) \text { at day } 1 \text { to } \\
28, \mathrm{MD}-0.8(95 \% \mathrm{Cl} \\
-5.6 \text { to } 4.0)\end{array}$ & 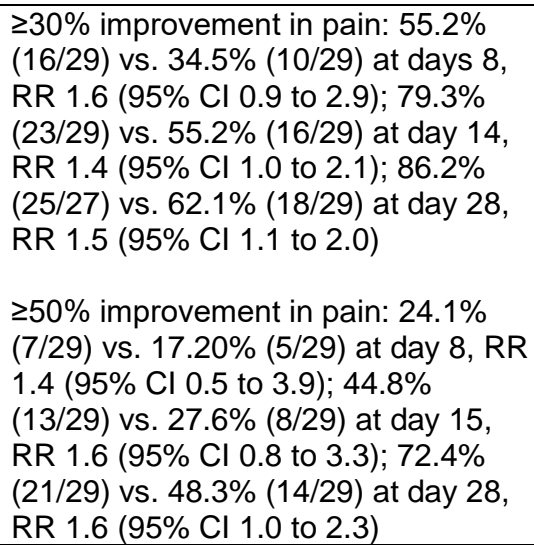 \\
\hline
\end{tabular}

Abbreviations: $\mathrm{CI}$ = confidence interval; $\mathrm{MD}=$ mean difference; $\mathrm{RR}=$ relative risk; $\mathrm{SD}=$ standard deviation; VAS = visual analog scale

No evidence was found for other subquestions (KQs $4 \mathrm{~b}$ and $4 \mathrm{~d}$ to $4 \mathrm{~h}$ ) related to opioid therapy for acute peripheral neuropathic pain.

\section{Nonopioid Pharmacologic Therapy}

KQs 4i and 4k address the comparative effectiveness of nonopioid pharmacologic therapy versus: (1) nonopioid pharmacologic therapy and (2) nonpharmacologic therapy.

\section{Topical Versus Oral Aspirin}

One small $(\mathrm{n}=30)$, poor quality trial compared topical versus oral aspirin for acute herpes zoster $^{123}$ (Table 18). Oral aspirin was dosed at 375 to $750 \mathrm{mg}$ daily and topical aspirin was administered as a solution three times daily. The topical aspirin was associated with decreased pain intensity at 3 hours, 1 week, and 3 weeks (differences ranged from -1.6 to -3.6 points on a 0 to 10 point pain scale), though oral aspirin was associated with decreased pain intensity at 1 day 
(difference 0.9 point). Topical aspirin was associated with markedly increased likelihood of experiencing >50\% improvement in pain (100\% vs. 6.7\%, RR 15.00, 95\% CI 2.26 to 99.64).

Table 18. Nonopioid interventions for herpetic neuralgia

\begin{tabular}{|c|c|c|c|c|}
\hline $\begin{array}{l}\text { Author, Year } \\
\text { Quality }\end{array}$ & Interventions & $\begin{array}{l}\text { Followup } \\
\text { Duration } \\
\text { Sample } \\
\text { Size }\end{array}$ & Pain Intensity Results & Other Pain Results \\
\hline $\begin{array}{l}\text { Balakrishnan, } \\
2001^{123} \\
\text { Poor }\end{array}$ & $\begin{array}{l}\text { A: Topical } \\
\text { aspirin, } 75 \mathrm{mg} \\
\text { aspirin/1 mL } \\
\text { moisturizer } \\
\text { solution, up to } \\
10 \mathrm{~mL} \text { of } \\
\text { solution } \\
\text { applied } 3 \text { times } \\
\text { daily } \\
\text { B: Oral aspirin, } \\
375 \text { to } 750 \\
\text { mg, }^{\text {a } 3} \text { times a } \\
\text { daily }\end{array}$ & $\begin{array}{l}3 \text { hours, } 1 \\
\text { week, and } 3 \\
\text { weeks } \\
n=30\end{array}$ & $\begin{array}{l}\text { Pain intensity (mean [SD], } 0 \text { to } \\
100 \text { VAS converted to } 0 \text { to } 10 \\
\text { scale): } 1.35(0.30) \text { vs. } 3.69(0.65) \\
\text { at } 3 \text { hours, MD }-2.34(95 \% \mathrm{Cl} \\
-2.72 \text { to }-1.96) ; 7.75(0.37) \text { vs. } \\
6.82(0.61) \text { at } 1 \text { day, MD } 0.93 \\
\text { (95\% Cl } 0.55 \text { to } 1.31) ; 3.38 \\
(0.67) \text { vs. } 5.0(0.90) \text { at } 1 \text { week, } \\
\text { MD: }-1.62(95 \% \mathrm{Cl}-2.21 \text { to } \\
-1.03) ; 0.68(0.30) \text { vs. } 4.31 \\
(0.87) \text { at } 3 \text { weeks, MD }-3.63 \\
(95 \% \mathrm{Cl}-4.12 \text { to }-3.14)\end{array}$ & $\begin{array}{l}\text { Pain improved >50\%: } \\
100 \%(15 / 15) \text { vs. } 6.7 \% \\
(1 / 15), \text { RR } 15.00(95 \% \mathrm{Cl} \\
2.26 \text { to } 99.640\end{array}$ \\
\hline
\end{tabular}

Abbreviations: $\mathrm{CI}$ = confidence interval; $\mathrm{MD}$ = mean difference; $\mathrm{SD}$ = standard deviation; $\mathrm{RR}$ = relative risk; VAS = visual analogue scale

${ }^{\text {a }}$ Dosage depending on patients' body mass index.

KQs $4 \mathrm{j}$ and $4 \mathrm{l}$ ask how the comparative effectiveness and harms of nonopioid pharmacologic therapy vary depending on: (1) patient demographics (e.g., age, race, ethnicity, gender); (2) patient medical and psychiatric comorbidities; (3) the type of nonopioid medication; (4) dose of medication; (5) duration of treatment.

No evidence was found for KQ $4 \mathrm{j}$ and $4 \mathrm{l}$.

\section{Nonpharmacologic Therapy}

KQ $4 \mathrm{~m}$ and $4 \mathrm{n}$ address the comparative effectiveness of nonopioid pharmacologic therapy versus: (1) inactive controls or (2) other nonpharmacologic therapies.

No evidence was found for KQ $4 \mathrm{~m}$ and $4 \mathrm{n}$.

\section{KQ 5. Postoperative Pain (Excluding Inpatient Management of Pain Following Major Surgical Procedures)}

\section{Key Points}

- Opioid vs. NSAID, single dose

0 No differences in pain or rescue medication use at $<1$ day, based on two trials (SOE: low).

- Opioid vs. NSAID, multidose course

o Opioid associated with increased likelihood of repeat or rescue medication use at 1 day to 1 week (RRs ranged from 1.22 to 2.04), based on four trials; evidence on pain intensity insufficient due to inconsistency (SOE: moderate for repeat or rescue medication use, insufficient for pain intensity).

- Opioid vs. acetaminophen, single dose

0 No difference in pain intensity at $<1$ day and no difference in need for re-medication, based on one trial (SOE: low). 
- Opioid vs. acetaminophen, multidose course

o Evidence on comparative effectiveness limited and imprecise (SOE: insufficient).

o Opioid associated with increased risk of withdrawal due to severe nausea and vomiting versus acetaminophen in one trial and increased risk of withdrawal due to any adverse event in two trials (SOE: low).

- Opioid agonist vs. mixed agent

o No difference in pain at $<1$ day ( 1 trial), 1 day to $<1$ week ( 6 trials), or 1 to $<2$ weeks (1 trial) (SOE: low to moderate).

- Opioid prescribing following a minor surgical procedure or elective surgery was associated with increased likelihood of opioid use at long-term followup versus no opioid prescribing, based on two observational studies (SOE: low).

- NSAID vs. acetaminophen, single dose

o Evidence on pain intensity and use of analgesics inconsistent, based on two trials (SOE: insufficient).

- NSAID vs. acetaminophen, multidose course

o Evidence insufficient from one poor quality trial (SOE: insufficient).

- Acupuncture vs. sham acupuncture

o Evidence on pain intensity inconsistent, based on two trials (SOE: insufficient).

- Acupressure vs. sham acupressure

o Acupressure associated with decreased pain medication use versus sham acupressure at $<1$ day and 1 day to $<1$ week, based on two trials, but effects on pain intensity were small and not statistically significant (SOE: low for pain medication use, insufficient for pain intensity).

- Cold therapy vs. sham therapy

o No difference between cold therapy versus sham therapy or no cold therapy in pain intensity at 1 day to $<1$ week, based on three fair quality trials (SOE: low).

0 No difference between cold therapy versus sham therapy in pain intensity, function, or quality of life at 2 to $<4$ weeks or $\geq 4$ weeks, based on one good quality trial, though cold therapy was associated with decreased pain medication use in the first 4 days (SOE: low).

- Massage vs. no massage

o Massage associated with moderate to large decrease in pain intensity at $<1$ day (6 trials), decreased pain medication use (3 trials), and decreased anxiety (3 trials) (SOE: low).

- Music therapy vs. no music therapy

o Music therapy was associated with moderate decrease in pain intensity versus no music therapy at $<1$ day ( 1 trial) and small to moderate decrease in pain intensity at 1 day to $<1$ week ( 1 trial) (SOE: low).

- Exercise vs. no exercise

o One trial found exercise therapy (stretching) associated with large improvements on the Neck and Disability Index following thyroid surgery at 1 week, but there was no difference at 1 month (SOE: low).

- TENS vs. sham TENS

o TENS associated with moderate to large decease in pain intensity versus sham TENS following liposuction at 12 hours and at 24 hours and decreased analgesic use (1 trial) (SOE: low). 


\section{Summary of Findings}

Forty-seven trials $(\mathrm{N}=6,078)$ evaluated interventions for postoperative pain, ${ }^{125-171}$ excluding inpatient management of pain after major surgical procedures (Appendix E, Table E-6). Fourteen trials $126,128,130,141,145,150,153,159,160,164,167,169-171$ evaluated opioid therapy (KQ 5a and 5c), 5 trials ${ }^{131,140,152,157,163}$ evaluated a nonopioid medication versus nonpharmacologic treatment or another nonopioid (KQ $5 \mathrm{i}$ and 5j), and 28 trials ${ }^{125,127,129,132-139,142-144,146-149,151,154-}$

156,158,161,162,165,166,168 (KQ 5m and 5n) evaluated nonpharmacologic treatment versus an inactive control or another nonpharmacologic treatment. The most common surgical procedures were cesarean sections, anterior cruciate ligament (ACL) reconstructions, knee arthroplasties, and cholecystectomies. The duration of treatment ranged from a single dose or treatment session to 6 weeks. The duration of followup was less than 1 week in 35 trials, ${ }^{125-128,131-135,137,139,140,143-147,149-~}$ 152,154-158,160-165,167,169,171 1 week to $<4$ weeks in six trials, ${ }^{129,130,136,153,166,170}$ and $\geq 4$ weeks in seven trials. ${ }^{129,138,141,142,148,159,168}$ Sixteen trials ${ }^{126,130,131,139,142,144,145,150,152,157,161,163,166,169,170}$ were conducted in the United States, 14 trials in Europe, ${ }^{128,129,134,135,140,141,146,147,153,154,158,160,168,171}$ and 17 trials elsewhere. ${ }^{125,127,132,133,136-138,143,148,149,151,155,156,159,162,165,167}$ The mean age ranged from 22 to 70 . Few trials reported race or ethnicity. Five trials $143,145,160,167,169$ excluded patients with a history of substance use disorder, 12 trials $^{128,135,143,145,150,152,153,159,160,167-169}$ excluded pregnant or breastfeeding patients, 4 trials ${ }^{127,160,167,169}$ excluded patients with psychiatric illness, and 10 trials ${ }^{126,128,141,145,152,153,159,160,162,169}$ excluded patients with other comorbidities. Two trials ${ }^{143,168}$ were rated good quality, 28 trials ${ }^{126,128,130,132-135,138,141,142,145,147,149-153,157-160,162-164,167,169-171}$ fair quality, and 17 trials $^{125,127,129,131,136,137,139,140,144,146,148,154-156,161,165,166}$ poor quality (Appendix F, Table F-1). Methodological limitations in the fair and poor quality trials included failure to report adequate randomization or allocation concealment methods, unblinded design, failure to report attrition, high attrition, and no intention to treat analysis.

\section{Detailed Synthesis}

\section{Opioid Therapy}

KQs 5a and 5c address the comparative effectiveness of opioid therapy versus: (1) nonopioid pharmacologic therapy (e.g., acetaminophen, NSAIDs, antidepressants, anticonvulsants) or (2) nonpharmacologic therapy (e.g., exercise, cognitive behavioral therapy, acupuncture).

Fourteen trials evaluated opioid therapy for acute postoperative pain following various surgical procedures (Appendix E, Table E-6). ${ }^{126,128,130,141,145,150,153,159,160,164,167,169-171}$ Five trials compared an opioid versus an NSAID, ${ }^{130,141,150,159,167}$ four trials compared an opioid versus acetaminophen, ${ }^{153,160,164,167}$ and six trials compared an opioid agonist versus a mixed agent (tramadol or tapentadol). ${ }^{126,128,145,169-171}$ Nine trials blinded patients and caregivers to the study medications. All of the trials were rated fair quality (Appendix F, Table F1). ${ }^{126,128,130,145,150,159,160,164,167,169,171}$

\section{Opioid Versus NSAID}

Five fair-quality trials compared an opioid (with or without acetaminophen) versus an NSAID for postoperative pain (Table 19). ${ }^{130,141,150,159,167}$ The surgery was arthroscopic rotator cuff repair in one trial, ${ }^{159}$ cesarean section in one trial, ${ }^{167}$ various orthopedic surgeries in one trial, ${ }^{150}$ herniotomy in one trial, ${ }^{141}$ and otolaryngologic surgery in one trial. ${ }^{130}$ Sample sizes ranged from 108 to $366(\mathrm{~N}=1,090)$. The trials were conducted in the United States, ${ }^{130,150}$ Venezuela, ${ }^{167}$ Italy, ${ }^{141}$ and South Korea. ${ }^{159}$ Two of the studies evaluated a single dose of 
medication followed by re-randomization and evaluation of a 2 or 5 day multidose course of therapy. ${ }^{150,167}$ The duration of treatment in the other trials was 4 days, up to 7 days, and 2 weeks. ${ }^{130,141,159}$ The opioid was hydrocodone in two trials (10 $\mathrm{mg}$ single dose, then up to $30 \mathrm{mg}$ daily in one ${ }^{150}$ and $5 \mathrm{mg}$ every six hours in the other), ${ }^{130}$ oxycodone in one trial (10 mg single dose, then up to $30 \mathrm{mg}$ daily), ${ }^{167}$ and tramadol in two trials (100 to $112 \mathrm{mg}$ daily). ${ }^{141,159}$ Hydrocodone and oxycodone were administered with acetaminophen. The NSAID was ketorolac (30 mg daily), celecoxib (up to $600 \mathrm{mg}$ daily), ibuprofen (1,155 to 2,400 $\mathrm{mg}$ daily) or ketoprofen (600 mg daily).

Two studies found no differences between a single dose of an opioid versus an NSAID in pain or rescue medication use at $<1$ day followup. ${ }^{150,167}$ At 1 day to $<1$ week in multidose trials, effects of an opioid versus an NSAID on pain intensity were inconsistent (four trials, differences ranged from 0.1 to 1.6 points on a 0 to 10 scale in favor of the NSAID), ${ }^{130,141,150,159}$ though opioids were associated with increased likelihood of repeat or rescue medication use (4 trials, RRs ranged from 1.22 to 2.04). ${ }^{130,150,159,167}$ Also at 1 day to $<1$ week, one trial ${ }^{141}$ found no difference between an opioid versus an NSAID in likelihood of pain intensity $\geq 4$ on a 0 to 10 scale and one trial ${ }^{150}$ found an opioid associated with better function (general activity, walking ability) and quality of life (mood, relations with others, sleep, and enjoyment of life); differences were about 1 point on a 0 to 10 scale. At 1 week, one trial found an opioid plus NSAID associated with higher pain intensity versus ibuprofen, though the difference was small and not statistically significant ( 3.5 vs. 2.8 on a 0 to 10 scale, $p=0.12$ ); the opioid plus NSAID was also associated with increased rescue medication use. ${ }^{130}$ At 2 weeks and 24 months, one trial found no difference between an opioid versus an NSAID in pain intensity or rescue medication use. ${ }^{159}$ In this trial, tramadol was associated with decreased risk of rotator cuff re-tear versus celecoxib ( $4.0 \%$ vs. $36.7 \%$, RR $0.11,95 \%$ CI 0.02 to 0.79 ) but the rate of re-tears for tramadol and ibuprofen were similar ( $4.0 \%$ vs. $7.4 \%$, RR $0.54,95 \%$ CI 0.05 to 5.59$)$. Serious adverse events were otherwise not reported. One trial found no difference between an opioid versus an NSAID in likelihood of withdrawal due to adverse events. ${ }^{150}$ Opioids were associated with increased risk of any adverse event in three trials (RRs ranged from 1.33 to 1.96 ). ${ }^{141,150,167}$ Two trials ${ }^{150,159}$ found opioids associated with increased likelihood of nausea, though the difference was small and not statistically significant in one of the trials. ${ }^{159}$ 
Table 19. Opioid therapy versus nonsteroidal anti-inflammatory drugs for postoperative pain

\begin{tabular}{|c|c|c|c|c|}
\hline $\begin{array}{l}\text { Study, Year } \\
\text { Quality }\end{array}$ & Interventions & $\begin{array}{l}\text { Procedure; } \\
\text { Followup Duration } \\
\text { Sample Size }\end{array}$ & Pain Intensity Results & Other Results \\
\hline $\begin{array}{l}\text { Bugada, } \\
2015^{141} \\
\text { Fair }\end{array}$ & $\begin{array}{l}\text { A. Tramadol } 100 \mathrm{mg} \text { IV every } 8 \\
\text { hours for first } 24 \text { hours, then } \\
\text { tramadol } 37.5 \\
\text { mg/acetaminophen } 325 \mathrm{mg} \\
\text { orally every } 8 \text { hours after } \\
\text { discharge for } 3 \text { days } \\
\text { B: Ketorolac } 30 \mathrm{mg} \text { IV every } 8 \\
\text { hours for first } 24 \text { hours, then } \\
\text { ketorolac } 10 \text { mg orally every } 8 \\
\text { hours after discharge for } 3 \text { days }\end{array}$ & $\begin{array}{l}\text { Herniotomy; } \\
\text { Up to } 5 \text { days, } 1 \\
\text { month, and } 3 \\
\text { months } \\
n=194\end{array}$ & $\begin{array}{l}\text { Pain intensity (mean [SD], } 0 \text { to } 10 \mathrm{NRS} \text { ): } \\
2.74 \text { (2.04) vs. } 2.62 \text { (1.90) at up to } 5 \text { days, } \\
\text { MD } 0.14 \text { (95\% Cl }-0.44 \text { to } 0.67) \\
\text { Pain intensity } \geq 4 \text { ( } 0 \text { to } 10 \text { NRS): } 1.1 \% \\
\text { (1/98) vs. } 1.0 \%(1 / 96) \text { at } 1 \text { month; } 0 \% \text { vs. } \\
0 \% \text { at } 3 \text { months }\end{array}$ & NR \\
\hline $\begin{array}{l}\text { Gimbel, } 2001^{150} \\
\text { Single dose } \\
\text { component } \\
\text { Fair }\end{array}$ & $\begin{array}{l}\text { A. Hydrocodone } 10 \mathrm{mg}+ \\
\text { acetaminophen } 1000 \mathrm{mg} \times 1 \\
\text { B. Celecoxib } 200 \mathrm{mg} \times 1\end{array}$ & $\begin{array}{l}\text { Orthopedic surgery; } \\
4 \text { and } 8 \text { hours } \\
n=277\end{array}$ & $\begin{array}{l}\text { Pain intensity difference (mean [SD NR], } 0 \\
\text { to } 3 \text { VAS converted to } 0 \text { to } 10 \text { scale): } 2.6 \\
\text { vs. } 2.7 \text { at } 4 \text { hours, } p=N S, 0.9 \text { vs. } 2.0 \text { at } 8 \\
\text { hours; } p<0.05 \\
\text { Sum of pain intensity differences (mean } \\
\text { [SD NR], sum of } 0 \text { to } 3 \text { VAS from } 0.25 \text { to } 8 \\
\text { hours): } 7.1 \text { vs. } 7.8, p<0.001\end{array}$ & $\begin{array}{l}\text { Rescue medication use: } 51 \%(69 / 136) \\
\text { vs. } 44 \%(62 / 144) \text {, RR } 1.18(95 \% \mathrm{Cl} \\
0.92 \text { to } 1.51)\end{array}$ \\
\hline $\begin{array}{l}\text { Gimbel, } 2001^{150} \\
\text { Multidose } \\
\text { component } \\
\text { Fair }\end{array}$ & $\begin{array}{l}\text { A. Hydrocodone } 10 \mathrm{mg}+ \\
\text { acetaminophen } 1000 \mathrm{mg} \\
\text { B. Celecoxib } 200 \mathrm{mg}\end{array}$ & $\begin{array}{l}\text { Orthopedic surgery; } \\
2 \text { and } 5 \text { days } \\
n=366\end{array}$ & $\begin{array}{l}\text { Maximum pain intensity (mean [SD NR], } 0 \\
\text { to } 3 \text { VAS converted to } 0 \text { to } 10 \text { scale): } 7.0 \\
\text { vs. } 5.7 \text { at day } 2, p<0.001 ; 5.3 \text { vs. } 3.7 \text { at } \\
\text { day } 5, p<0.001\end{array}$ & $\begin{array}{l}\text { No medication required, day 5: } 20 \% \\
\text { (36/181) vs. } 41 \%(76 / 185), \text { RR } 0.48 \\
(95 \% \mathrm{Cl} 0.34 \text { to } 0.68)\end{array}$ \\
\hline $\begin{array}{l}\text { Nguyen, } \\
2019^{130} \\
\text { Fair }\end{array}$ & $\begin{array}{l}\text { A: Hydrocodone }+ \\
\text { acetaminophen: } 5 / 325 \mathrm{mg} \text { every } \\
6 \text { hours as needed for up to } 7 \\
\text { days } \\
\text { B: Ibuprofen: } 600 \text { mg every } 6 \\
\text { hours as needed for up to } 7 \\
\text { days }\end{array}$ & $\begin{array}{l}\text { Otolaryngology } \\
\text { surgery (not } \\
\text { including } \\
\text { tonsillectomy); } \\
1 \text { week } \\
\mathrm{n}=108\end{array}$ & $\begin{array}{l}\text { Pain, overall (mean, } 0 \text { to } 10 \mathrm{NRS}): 3.5 \\
(2.8 \text { to } 4.1) \text { vs. } 2.8(95 \% \mathrm{Cl} 2.2 \text { to } 3.4) \\
\text { p=0.12 } \\
\text { Pain, maximum (mean, } 0 \text { to } 10 \mathrm{NRS}): 5.7 \\
(95 \% \mathrm{Cl} 5.0 \text { to } 6.5) \text { vs. } 5.3(95 \% \mathrm{Cl} 4.6 \text { to } \\
6.1), p=0.49\end{array}$ & $\begin{array}{l}\text { Rescue medication, total pills (mean): } \\
4.5(95 \% \mathrm{Cl} 3.2 \text { to } 5.7) \text { vs. } 2.0(95 \% \mathrm{Cl} \\
0.9 \text { to } 3.1), p=0.004\end{array}$ \\
\hline
\end{tabular}




\begin{tabular}{|c|c|c|c|c|}
\hline $\begin{array}{l}\text { Study, Year } \\
\text { Quality }\end{array}$ & Interventions & $\begin{array}{l}\text { Procedure; } \\
\text { Followup Duration } \\
\text { Sample Size }\end{array}$ & Pain Intensity Results & Other Results \\
\hline $\begin{array}{l}\text { Oh, } 2018^{159} \\
\text { Fair }\end{array}$ & $\begin{array}{l}\text { A: Tramadol, } 50 \mathrm{mg} \text { twice daily } \\
\text { for } 2 \text { weeks } \\
\text { B: Celecoxib, } 200 \mathrm{mg} \text { twice daily } \\
\text { for } 2 \text { weeks } \\
\text { C: Ibuprofen, } 385 \mathrm{mg} \text { three } \\
\text { times daily for } 2 \text { weeks }\end{array}$ & $\begin{array}{l}\text { Rotator cuff repair; } \\
3 \text { days, } 2 \text { weeks, } \\
\text { and mean of } 24 \\
\text { months } \\
n=162\end{array}$ & $\begin{array}{l}\text { Pain intensity (mean [SD], } 0 \text { to } 10 \text { VAS): } \\
4.1(1.7) \text { vs. } 3.4(1.6) \text { vs. } 3.9(1.9) \text { at } 3 \\
\text { days, MD } 0.70(95 \% \mathrm{Cl} 0.07 \text { to } 1.33) \text { for } \mathrm{A} \\
\text { vs. B and } 0.20(95 \% \mathrm{Cl}-0.49 \text { to } 0.89) \text { for } \\
\mathrm{A} \text { vs. C; } 3.2(1.9) \text { vs. } 2.8(2.1) \text { vs. } 3.2(1.9) \\
\text { at } 2 \text { weeks, MD } 0.40(95 \% \mathrm{Cl}-0.37 \text { to } \\
1.17) \text { for A vs. B, and } 0.00(95 \% \mathrm{Cl}-0.72 \\
\text { to } 0.72) \text { for } \mathrm{A} \text { vs. } \mathrm{C} ; 0.8(1.5) \text { vs. } 0.9(1.9) \\
\text { vs. } 0.6(1.1) \text { at } 24 \text { months; MD }-0.10 \\
(95 \% \mathrm{Cl}-1.04 \text { to } 0.84) \text { for } \mathrm{A} \text { vs. B and } \\
0.20(95 \% \mathrm{Cl}-0.53 \text { to } 0.93) \text { for } \mathrm{A} \text { vs. C }\end{array}$ & $\begin{array}{l}\text { Rescue medication use: } 66.7 \%(36 / 54) \\
\text { vs. } 50.9 \%(27 / 53) \text { vs. } 54.5 \%(30 / 55) \text { at } \\
3 \text { days, RR } 1.31 \text { ( } 95 \% \mathrm{Cl} 0.95 \text { to } 1.81) \\
\text { for A vs. B and RR } 1.22(95 \% \mathrm{Cl} 0.90 \\
\text { to } 1.66) \text { for A vs. C; } 38.9 \%(21 / 54) \text { vs. } \\
28.3 \%(15 / 53) \text { vs. } 30.9 \%(17 / 55) \text { at } 2 \\
\text { weeks, RR } 1.26(95 \% \mathrm{Cl} 0.75 \text { to } 2.11) \\
\text { for A vs. B and RR } 1.37(95 \% \mathrm{Cl} 0.80 \\
\text { to } 2.37) \text { for A vs. C }\end{array}$ \\
\hline $\begin{array}{l}\text { Sunshine, } \\
1993^{167} \\
\text { Single-dose } \\
\text { component } \\
\text { Fair }\end{array}$ & $\begin{array}{l}\text { A. Oxycodone } 10 \mathrm{mg}+ \\
\text { acetaminophen } 650 \mathrm{mg} \times 1 \\
\text { B. Ketoprofen } 50 \mathrm{mg} \mathrm{x} 1 \\
\text { C. Ketoprofen } 100 \mathrm{mg} \times 1\end{array}$ & $\begin{array}{l}\text { Cesarean section; } \\
8 \text { hours } \\
n=144\end{array}$ & $\begin{array}{l}\text { Pain intensity difference (mean [SD NR], } 0 \\
\text { to } 3 \text { NRS converted to } 0 \text { to } 10 \text { scale): } 4.7 \\
\text { vs. } 5.0 \text { vs. } 5.7 \text { at } 4 \text { hours, } p=N S \text { for A vs. } \\
\text { B, or C; } 1.3 \text { vs. } 2.0 \text { vs. } 3.3 \text { at } 8 \text { hours, } \\
\text { p=NS for A vs. B or } \\
\text { Sum of pain intensity differences (mean } \\
\text { [SD NR], sum of differences on } 0 \text { to } 30 \\
\text { scale from } 1 \text { to } 8 \text { hours): } 8.73 \text { vs. } 8.34 \text { vs. } \\
10.34, p<0.05 \text { for B vs. C }\end{array}$ & $\begin{array}{l}\text { Global rating (mean [SD NR], } 0 \text { to } 3 \\
\text { scale): } 2.04 \text { vs. } 1.90 \text { vs. } 2.25, p=N S\end{array}$ \\
\hline $\begin{array}{l}\text { Sunshine, } \\
1993^{167} \\
\text { Multi-dose } \\
\text { component } \\
\text { Fair }\end{array}$ & $\begin{array}{l}\text { A: Oxycodone } 5 \mathrm{mg}+ \\
\text { acetaminophen } 325 \mathrm{mg} \text { every } 4 \\
\text { hours for up to } 7 \text { days } \\
\text { B: Ketoprofen } 50 \mathrm{mg} \text { every } 4 \\
\text { hours as needed for up to } 7 \\
\text { days } \\
\text { C: Ketoprofen } 100 \mathrm{mg} \text { every } 4 \\
\text { hours for up to } 7 \text { days }\end{array}$ & $\begin{array}{l}\text { Cesarean section; } \\
2 \text { days } \\
n=224\end{array}$ & $\begin{array}{l}\text { Pain severe } 2 \text { hours after first dose on day } \\
2: 8 \%(6 / 76) \text { vs. } 3 \%(2 / 74) \text { vs. } 7 \%(5 / 74)\end{array}$ & $\begin{array}{l}\text { Global assessment "very good" or } \\
\text { "excellent": } 39 \%(29 / 76) \text { vs. } 64 \% \\
\text { (47/74) vs. } 60 \%(45 / 74), \text { RR } 0.60(95 \% \\
\mathrm{Cl} 0.43 \text { to } 0.84) \text { for A vs. B and RR } \\
0.63(95 \% \mathrm{Cl} 0.45 \text { to } 0.88) \text { for A vs. C }\end{array}$ \\
\hline
\end{tabular}

$=$ visual analog scale 


\section{Opioid Versus Acetaminophen}

Four fair quality trials compared an opioid versus acetaminophen (Table 20). ${ }^{153,160,164,167}$ One trial $(n=96)$ compared a single dose of oxycodone plus acetaminophen (10/650 mg) versus acetaminophen (650 mg) following cesarean section. ${ }^{167}$ The opioid was associated with a greater sum of pain intensity differences versus acetaminophen at 8 hours; however, the differences at 4 and 8 hours were not statistically significant. The difference in need for re-medication was also not statistically significant (72.9\% vs. $87.5 \%)$.

Three trials evaluated multidose courses of an opioid versus acetaminophen. One trial each enrolled patients who underwent extremity fracture surgery, ${ }^{153}$ hand surgery, ${ }^{160}$ or Moh's surgery. ${ }^{164}$ Sample sizes were 39,73 , and $140(\mathrm{~N}=252)$. The opioid was tramadol (50 mg every 8 hours) plus acetaminophen (1000 mg every 6 hours) in one trial, ${ }^{153}$ tramadol (100 mg every 8 hours) in one trial, ${ }^{160}$ and codeine plus acetaminophen (30/325 every 4 hours) in one trial. ${ }^{164}$ At $<1$ day, one trial found no difference between an opioid versus acetaminophen in pain intensity or use of additional doses. ${ }^{164}$ At 1 day to $<1$ week, one trial found no difference between an opioid versus acetaminophen in pain intensity (mean difference $0.30,95 \%$ CI -0.53 to 1.13 ) or likelihood of rescue medication use. ${ }^{160}$ At 2 weeks to $<4$ weeks, one trial found tramadol plus acetaminophen associated with decreased pain intensity versus acetaminophen, but the difference was not statistically significant (mean difference -0.9 on a 0 to 10 scale, $95 \%$ CI -2.00 to 0.06). ${ }^{153}$ Effects on function also favored tramadol plus acetaminophen, but the differences were small and not statistically significant. Opioids were associated with increased risk of withdrawal due to severe nausea and vomiting in one trial (17.5\% vs. $0 \%$, RR 12.81 , $95 \%$ CI 0.75 to $217.60)^{160}$ and increased risk of withdrawal due to any adverse event in two trials (RR 5.40, 95\% CI 1.31 to 22.3 and RR 6.00, 95\% CI 1.39 to 25.83). ${ }^{153,164}$

Table 20. Opioid therapy versus acetaminophen for postoperative pain

\begin{tabular}{|c|c|c|c|c|}
\hline $\begin{array}{l}\text { Study, Year } \\
\text { Quality }\end{array}$ & Interventions & $\begin{array}{l}\text { Procedure; } \\
\text { Followup } \\
\text { Duration } \\
\text { Sample Size }\end{array}$ & Pain Intensity Results & Other Results \\
\hline $\begin{array}{l}\text { Helmerhorst, } \\
2017^{153} \\
\text { Fair }\end{array}$ & $\begin{array}{l}\text { A. Tramadol } 50 \text { mg } \\
\text { every } 8 \text { hours }+ \\
\text { acetaminophen } \\
1000 \text { mg every } 6 \\
\text { hours for } 2 \text { weeks } \\
\text { B. Acetaminophen } \\
1000 \text { mg every } 6 \\
\text { hours for } 2 \text { weeks }\end{array}$ & $\begin{array}{l}\text { Fracture; } \\
2 \text { weeks } \\
n=39\end{array}$ & $\begin{array}{l}\text { Pain intensity (mean [SD } \\
\text { NR], } 0 \text { to } 10 \text { VAS): } 3.0 \text { vs. } \\
3.9, \mathrm{MD}-0.9(95 \% \mathrm{Cl} \\
-2.00 \text { to } 0.06) \text { at } 2 \text { weeks }\end{array}$ & $\begin{array}{l}\text { SMFA, function index } \\
\text { (mean [SD NR], } 0 \text { to } 100 \\
\text { scale, higher score=worse } \\
\text { outcome): } 37.7 \text { vs. } 44.7, \\
\text { p=0.12; bothersome index } \\
\text { (mean [SD NR], } 0 \text { to } 100 \\
\text { scale): } 35.7 \text { vs. } 44.8, \\
p=0.14 \\
\text { Rescue medication use: } 0 \% \\
\text { (0/25) vs. } 7.4 \%(2 / 27)\end{array}$ \\
\hline $\begin{array}{l}\text { Rawal, } \\
2001^{160} \\
\text { Fair }\end{array}$ & $\begin{array}{l}\text { A. Tramadol } 100 \mathrm{mg} \\
\text { every } 6 \text { hours for } 2 \\
\text { days } \\
\text { B. Acetaminophen } \\
1000 \text { mg every } 6 \\
\text { hours for } 2 \text { days }\end{array}$ & $\begin{array}{l}\text { Hand surgery; } \\
1 \text { and } 2 \text { days } \\
n=73\end{array}$ & $\begin{array}{l}\text { Pain intensity (mean [SD], } \\
0 \text { to } 10 \text { VAS): } 2.0(2.4) \mathrm{vs} . \\
3.0(2.7) \text { at day } 1 \text { at } \\
\text { bedtime, MD }-1.0(95 \% \mathrm{Cl} \\
-2.14 \text { to } 0.14) ; 1.8(1.7) \mathrm{vs} \text {. } \\
1.5(2.0) \text { at day } 2 \text { at } \\
\text { bedtime, MD } 0.30(95 \% \mathrm{Cl} \\
-0.53 \text { to } 1.13)\end{array}$ & $\begin{array}{l}\text { Took rescue medication: } \\
23 \%(8 / 34) \text { vs. } 42 \%(16 / 38) \\
\text { at day } 1,20 \%(7 / 34) \text { vs. } \\
24 \%(9 / 38) \text { at day } 2, p=N S\end{array}$ \\
\hline
\end{tabular}




\begin{tabular}{|c|c|c|c|c|}
\hline $\begin{array}{l}\text { Study, Year } \\
\text { Quality }\end{array}$ & Interventions & $\begin{array}{l}\text { Procedure; } \\
\text { Followup } \\
\text { Duration } \\
\text { Sample Size }\end{array}$ & Pain Intensity Results & Other Results \\
\hline $\begin{array}{l}\text { Sniezek, } \\
2001^{164} \\
\text { Fair }\end{array}$ & $\begin{array}{l}\text { A. Codeine } 30 \mathrm{mg}+ \\
\text { acetaminophen } 650 \\
\text { mg every } 4 \text { hours up } \\
\text { to } 4 \text { doses } \\
\text { B. Acetaminophen } \\
1000 \text { mg every } 4 \\
\text { hours up to } 4 \text { doses }\end{array}$ & $\begin{array}{l}\text { Mohs surgery; } \\
4 \text { and } 12 \text { hours } \\
n=140\end{array}$ & $\begin{array}{l}\text { Pain intensity change from } \\
\text { baseline (mean [SD], } 0 \text { to } \\
100 \text { VAS converted to } 0 \text { to } \\
10 \text { scale): } 2.17(2.23) \text { vs. } \\
1.82(2.25) \text { at } 4 \text { hours, } \\
p=0.20 ; 0.97 \text { ( } 1.83) \text { vs. } 0.84 \\
(1.37) \text { at } 12 \text { hours, } p=0.82\end{array}$ & $\begin{array}{l}\text { Additional dose used: } 70 \% \\
\text { (59/70) vs. } 60 \% \text { (42/70), } \\
\text { RR } 1.40(95 \% \mathrm{Cl} 1.13 \text { to } \\
1.74)\end{array}$ \\
\hline $\begin{array}{l}\text { Sunshine, } \\
1993^{167} \\
\text { Single dose } \\
\text { component } \\
\text { Fair }\end{array}$ & $\begin{array}{l}\text { A. Oxycodone } 10 \\
\mathrm{mg}+ \\
\text { acetaminophen } 650 \\
\mathrm{mg} \times 1 \\
\text { B. Acetaminophen } \\
650 \mathrm{mg} \times 1\end{array}$ & $\begin{array}{l}\text { Cesarean } \\
\text { section } \\
<1 \text { day (8 } \\
\text { hours) } \\
n=96\end{array}$ & $\begin{array}{l}\text { Pain intensity difference } \\
\text { (mean [SD NR], } 0 \text { to } 3 \text { NRS } \\
\text { converted to } 0 \text { to } 10 \text { scale): } \\
4.7 \text { vs. } 5.0 \text { vs. } 5.7 \text { vs. } 3.7 \text { at } \\
4 \text { hours, } p<0.05 \text { for B or C } \\
\text { vs. D and } p=N S \text { for A vs. B, } \\
\text { C, or D; } 1.3 \text { vs. } 2.0 \text { vs. } 3.3 \\
\text { vs. } 0.7 \text { at } 8 \text { hours, } p<0.05 \\
\text { for B or C vs. D and p=NS } \\
\text { for A vs. B, C, or D } \\
\text { Sum of pain intensity } \\
\text { differences (mean [SD NR], } \\
\text { sum of differences on } 0 \text { to } \\
30 \text { scale from } 1 \text { to } 8 \text { hours): } \\
8.73 \text { vs. } 8.34 \text { vs. } 10.34 \text { vs. } \\
6.54, p<0.05 \text { for A vs. D, B } \\
\text { vs. C, and C vs. D }\end{array}$ & $\begin{array}{l}\text { Re-medication: } 72.9 \% \\
\text { (35/48) vs. } 68.8 \%(33 / 48) \\
\text { vs. } 57.9 \%(22 / 38) \text { vs. } \\
87.5 \%(42 / 48), \text { RR } 0.66 \\
(95 \% \text { Cl } 0.49 \text { to } 0.89) \text { for C } \\
\text { vs. D, otherwise } \mathrm{p}=\mathrm{NS}\end{array}$ \\
\hline
\end{tabular}

Abbreviations: $\mathrm{CI}$ = confidence interval; NR = not reported; NRS = numeric rating scale; NS = not significant; RR = relative risk; SD = standard deviation; SMFA=Short Musculoskeletal Function Assessment; VAS = visual analog scale

\section{Opioid Agonist Versus Mixed Agent}

Six fair quality trials compared an opioid agonist versus a mixed agent (tramadol or tapentadol) for postoperative pain (Table 21). ${ }^{126,128,145,169-171}$ The surgery was bunionectomy in three trials, ${ }^{126,145,169}$ arthroscopic shoulder surgery in one trial, ${ }^{170}$ cesarean section in one trial, ${ }^{128}$ and various orthopedic surgeries in one trial. ${ }^{171}$ Sample sizes ranged from 57 to $755(\mathrm{~N}=1,462)$. The duration of treatment and followup ranged from two to seven days. ${ }^{126,128,145,169-171}$ The opioid agonist was oxycodone in five trials (dose ranged from 20 to $60 \mathrm{mg}$ daily) 126,128,145,170,171 and morphine in one trial (120 to $180 \mathrm{mg}$ daily). ${ }^{169}$ The mixed agent was tapentadol (dose ranged from 100 to $450 \mathrm{mg}$ daily) in five trials $\mathrm{s}^{126,128,145,169,170}$ and tramadol (100 mg twice daily) in one trial. ${ }^{171}$ Two trials ${ }^{128,171}$ compared sustained-release medications, and the others evaluated immediate-release medications. In two trials ${ }^{126,170}$ the daily dose in morphine equivalents was lower for the opioid agonist (30 to $90 \mathrm{mg}$ or 90 to $135 \mathrm{mg}$ ) compared to the mixed agent (80 to $240 \mathrm{mg}$ ). In the other trials the doses of the opioid agonist and mixed agent were similar in daily morphine equivalents (range 30 to $180 \mathrm{mg}$ ).

There were no differences between an opioid agonist versus mixed agent in pain related outcomes (mean pain intensity, likelihood of improvement, or use of repeat or rescue medications) at 12 hours ( 1 trial), ${ }^{169} 2$ to 3 days (6 trials), ${ }^{126,128,145,169-171}$ or 7 days ( 1 trial). ${ }^{170}$ One trial found oxycodone associated with increased likelihood of pain interference with sleep, but the difference was not statistically significant (26.5\% vs. $17.7 \%$, RR 1.50, 95\% 0.98 to 2.30 ). ${ }^{170}$ Otherwise, outcomes related to function or quality of life were not reported. There was no difference in risk of withdrawal due to adverse events and serious adverse events were not reported. Effects of an opioid agonist versus mixed agent on nausea were inconsistent; there were no differences in risk of other specific adverse events. 
Table 21. Opioid agonist versus mixed agent for postoperative pain

\begin{tabular}{|c|c|c|c|c|}
\hline $\begin{array}{l}\text { Study, } \\
\text { Year } \\
\text { Quality }\end{array}$ & Interventions & $\begin{array}{l}\text { Procedure; } \\
\text { Followup } \\
\text { Duration } \\
\text { Sample Size }\end{array}$ & Pain Intensity Results & Other Results \\
\hline $\begin{array}{l}\text { Daniels, } \\
2009 a^{145} \\
\text { Fair }\end{array}$ & $\begin{array}{l}\text { A: Oxycodone } \\
10 \text { mg every } 4 \\
\text { to } 6 \text { hours up to } \\
72 \text { hours } \\
\text { B: Tapentadol } \\
50 \text { mg every } 4 \\
\text { to } 6 \text { hours up to } \\
72 \text { hours } \\
\text { C: Tapentadol } \\
75 \text { mg every } 4 \\
\text { to } 6 \text { hours up to } \\
72 \text { hours }\end{array}$ & $\begin{array}{l}\text { Bunionectomy; } \\
48 \text { and } 72 \\
\text { hours } \\
n=755\end{array}$ & $\begin{array}{l}\text { Sum of pain intensity differences (least } \\
\text { squares mean [95\% Cl], sum of } \\
\text { differences from } 0.5 \text { to } 72 \text { hours on a } 0 \text { to } \\
10 \text { scale): } 119.9(83.52 \text { to } 156.21) \text { vs. } \\
89.3 \text { ( } 52.93 \text { to } 125.70) \text { vs. } 120.0 \text { ( } 83.64 \\
\text { to } 156.33 \text { ); differences of A vs. B or C } \\
\text { within noninferiority margin } \\
\geq 30 \% \text { improvement in pain intensity: } \\
75.2 \% \text { vs. } 77.5 \% \text { vs. } 76.5 \% \text { at } 48 \text { hours } \\
\geq 50 \% \text { improvement in pain intensity: } \\
64.4 \% \text { vs. } 64.7 \% \text { vs. } 64.0 \% \text { at } 48 \text { hours }\end{array}$ & $\begin{array}{l}\text { Use of rescue } \\
\text { medication: } 3.2 \% \\
\text { vs. } 6.2 \% \text { vs. } 1.4 \%\end{array}$ \\
\hline $\begin{array}{l}\text { Daniels, } \\
\text { 2009b } \\
\text { Fair }\end{array}$ & $\begin{array}{l}\text { A: Oxycodone } \\
\text { HCL } 15 \text { mg } \\
\text { B: Tapentadol } \\
\text { IR } 50 \text { mg; } \\
\text { C: Tapentadol } \\
\text { IR } 75 \text { mg } \\
\text { D: Tapentadol } \\
\text { IR } 100 \text { mg } \\
\text { Day 1: } 4 \text { to } 7 \\
\text { doses could be } \\
\text { administered; } \\
\text { Days } 2 \text { and 3: } 4 \\
\text { to } 6 \text { doses could } \\
\text { be administered }\end{array}$ & $\begin{array}{l}\text { Bunionectomy; } \\
12,24,48 \text {, and } \\
72 \text { hours } \\
n=482\end{array}$ & $\begin{array}{l}\text { Sum of pain intensity differences (mean } \\
\text { [SD] sum of differences through } 72 \text { hours } \\
\text { on a } 0 \text { to } 10 \text { scale): } 288 \text { (170.67) vs. } \\
207.9 \text { ( } 207.57) \text { vs. } 230.5 \text { (189.36) vs. } \\
271.1 \text { (154.57) } \\
\geq 30 \% \text { improvement in pain intensity at } 48 \\
\text { hours: } 78.4 \% \text { vs. } 64.7 \% \text { vs. } 68.3 \% \text { vs. } \\
78.8 \% \\
\geq 50 \% \text { improvement in pain intensity at } 48 \\
\text { hours: } 72.8 \% \text { vs. } 58.0 \% \text { vs. } 56.7 \% \text { v. } \\
70.3 \%\end{array}$ & $\begin{array}{l}\text { Rescue } \\
\text { medication use: } \\
9 \% \text { vs. } 19 \% \text { vs. } \\
14 \% \text { vs. } 10 \% \\
\text { Percent reporting } \\
\text { "much improved" } \\
\text { or "very much } \\
\text { improved" overall } \\
\text { status on } 1 \text { to } 7 \\
\text { point VRS: } 88 \% \\
\text { vs. } 67 \% \text { vs. } 77 \% \\
\text { vs. } 89 \%\end{array}$ \\
\hline $\begin{array}{l}\text { Ffrench- } \\
\text { O'Carrol, } \\
2019^{128} \\
\text { Fair }\end{array}$ & $\begin{array}{l}\text { A. Oxycodone } \\
\text { controlled } \\
\text { release: } 10 \mathrm{mg} \\
\text { every } 12 \text { hours } \\
\text { for } 2 \text { days } \\
\text { B. Tapentadol } \\
\text { controlled } \\
\text { release: } 50 \mathrm{mg} \\
\text { every } 12 \text { hours } \\
\text { for } 2 \text { days }\end{array}$ & $\begin{array}{l}\text { Cesarean } \\
\text { section; } \\
36 \text { hours and } 2 \\
\text { days } \\
n=68\end{array}$ & $\begin{array}{l}\text { Sum of pain intensity differences (mean } \\
\text { [SD] sum of differences through } 72 \text { hours } \\
\text { on a } 0 \text { to } 10 \text { scale } \times 24): 65.14(70.23) \\
\text { vs. } 74.54(77.97), M D-11.45(95 \% \mathrm{Cl} \\
-35.35 \text { to } 12.45)\end{array}$ & $\begin{array}{l}\text { Satisfaction score } \\
\text { (mean [SD] on } 1 \\
\text { to } 5 \text { scale), at } 2 \\
\text { days: } 4.14(0.84) \\
\text { vs. } 4.34(1.21) ; \\
\text { MD } 0.032(95 \% \mathrm{Cl} \\
-0.47 \text { to } 0.53)\end{array}$ \\
\hline $\begin{array}{l}\text { Viscusi, } \\
2019^{169} \\
\text { Fair }\end{array}$ & $\begin{array}{l}\text { A: Morphine: } 30 \\
\text { mg every } 4 \text { to } 6 \\
\text { hours for } 3 \text { days } \\
\text { B: Tapentadol: } \\
75 \text { mg every } 4 \\
\text { to } 6 \text { hours for } 3 \\
\text { days }\end{array}$ & $\begin{array}{l}\text { Bunionectomy; } \\
12 \text { hours and } 3 \\
\text { days } \\
n=189\end{array}$ & $\begin{array}{l}\text { Sum of pain intensity differences (least } \\
\text { squares mean [SD], sum of difference on } \\
0 \text { to } 10 \text { NRS from } 0.5 \text { hours): } 8.1 \text { (18.2) } \\
\text { vs. } 9.9(18.5) \text { at } 12 \text { hours, MD }-1.80 \\
\text { (95\% Cl }-7.07 \text { to } 3.47) ; 177.1(279.7) \text { vs. } \\
108.2(281.4) \text { at } 3 \text { days, MD } 68.90(95 \% \\
\mathrm{Cl}-11.63 \text { to } 149.43)\end{array}$ & $\begin{array}{l}\text { Use of rescue } \\
\text { medication: } 64.6 \% \\
(62 / 96) \text { vs. } 49.5 \% \\
(46 / 93) ; \text { RR } 1.31 \\
(95 \% \text { Cl } 1.01 \text { to } \\
1.68) \\
\text { Global impression } \\
\text { much improved or } \\
\text { very much } \\
\text { improved: } 88.2 \% \\
(82 / 93) \text { vs. } 84.4 \% \\
(81 / 96)\end{array}$ \\
\hline
\end{tabular}




\begin{tabular}{|c|c|c|c|c|}
\hline $\begin{array}{l}\text { Study, } \\
\text { Year } \\
\text { Quality }\end{array}$ & Interventions & $\begin{array}{l}\text { Procedure; } \\
\text { Followup } \\
\text { Duration } \\
\text { Sample Size }\end{array}$ & Pain Intensity Results & Other Results \\
\hline $\begin{array}{l}\text { Vorsanger, } \\
2013^{170} \\
\text { Fair }\end{array}$ & $\begin{array}{l}\text { A: Oxycodone } 5 \\
\text { mg, then } 5 \text { to } 10 \\
\text { mg every } 4 \text { to } 6 \\
\text { hours up to } 7 \\
\text { days } \\
\text { B: Tapentadol } \\
50 \mathrm{mg} \text {, then } 50 \\
\text { to } 100 \mathrm{mg} \text { every } \\
4 \text { to } 6 \text { hours for } \\
\text { up to } 7 \text { days }\end{array}$ & $\begin{array}{l}\text { Arthroscopic } \\
\text { shoulder } \\
\text { surgery; } \\
3 \text { and } 7 \text { days } \\
\mathrm{n}=378\end{array}$ & $\begin{array}{l}\text { Sum of pain intensity differences (mean } \\
\text { [SD], sum of differences on } 0 \text { to } 10 \\
\text { scale): } 41.1 \text { (10.32) vs. } 32.1(10.19) \text { at } 3 \\
\text { days, least squares MD } 9.0(95 \% \mathrm{Cl} \\
-18.9 \text { to } 36.9) ; 121.3(20.43) \text { vs. } 130.6 \\
(20.20) \text { at } 7 \text { days, least squares MD }-9.3 \\
(-64.7,46.0) \\
\text { Pain intensity improved } \geq 30 \%: 34.4 \% \\
(53 / 154) \text { vs. } 33.5 \%(53 / 158) \text { at day } 3 \text {, } \\
42.2 \%(65 / 154) \text { vs. } 48.1 \%(76 / 158) \text { at } \\
\text { day } 7\end{array}$ & $\begin{array}{l}\text { Patient global } \\
\text { impression much } \\
\text { or very much } \\
\text { improved: } 69 \% \\
(125 / 181) \text { vs. } 75 \% \\
(144 / 192)\end{array}$ \\
\hline $\begin{array}{l}\text { Wirz, } \\
2005^{171} \\
\text { Fair }\end{array}$ & $\begin{array}{l}\text { A: Oxycodone } \\
10 \text { mg twice } \\
\text { daily for } 3 \text { days } \\
\text { B: Tramadol } \\
100 \text { mg twice } \\
\text { daily for } 3 \text { days }\end{array}$ & $\begin{array}{l}\text { Orthopedic } \\
\text { surgery; } \\
2 \text { and } 3 \text { days } \\
n=57\end{array}$ & $\begin{array}{l}\text { Pain intensity at rest (mean [SD NR], } 0 \text { to } \\
100 \text { VAS converted to } 0 \text { to } 10 \text { scale: } 2.10 \\
(1.64) \text { vs. } 1.71(1.45) \text { at day } 2, p=0.32 ; \\
1.63(1.54) \text { vs. } 1.06 \text { (1.39) at day } 3, \\
p=0.16\end{array}$ & $\begin{array}{l}\text { Rescue } \\
\text { medication use: } \\
30.8 \%(8 / 26) \text { vs. } \\
35.5 \%(11 / 31)\end{array}$ \\
\hline
\end{tabular}

Abbreviations: $\mathrm{CI}$ = confidence interval; $\mathrm{MD}$ = mean difference; $\mathrm{NR}$ = not reported; NRS = numeric SD = standard deviation; VAS = visual analog scale; VRS = verbal rating scale

KQs 5b and 5d ask how the comparative effectiveness and harms of opioid therapy vary depending on: (1) patient demographics (e.g., age, race, ethnicity, gender); (2) patient medical or psychiatric comorbidities; (3) dose of opioids; (4) duration of opioid therapy, including number of opioid prescription refills and quantity of pills used; (5) opioid use history; (6) substance use history; (7) use of concomitant therapies.

Evidence on how comparative effectiveness and harms of opioid therapy for postoperative pain vary according to patient and prescribing factors was lacking. The number of trials was small for each comparison and most trials had small sample sizes. In addition, trials excluded patients with subgroup characteristics of interest, such as substance use history or presence of medical or psychiatric comorbidities, or did not provide information regarding these factors. No study conducted within-study or across-study evaluations of subgroup effects. Evidence was too limited to determine effects of different opioid doses (converted into morphine milligram equivalents) on comparative effectiveness and harms. No trial permitted opioid refills, and the duration of treatment was up 15 days; the trials did not evaluate how effectiveness varied in subgroups defined according to the amount of opioid used.

KQ 5e concerns the effects of prescribing opioid therapy versus not prescribing opioid therapy for acute pain on (1) short-term ( $<3$ months) continued need for prescription pain relief, such as need for opioid refills, and (2) long-term opioid use (3 months or greater).

Two fair quality ${ }^{172,173}$ retrospective cohort studies found patients who received opioids after surgery were at increased likelihood of opioid use at longer-term followup (Appendix E, Table E-7; Appendix F, Table F-2). Both studies evaluated patients who were opioid-naïve prior to surgery. One study $(n=391,139)$ evaluated patients who had undergone low-risk surgery (defined as cataract surgery, laparoscopic cholecystectomy, transurethral resection of the prostate, or varicose vein stripping). ${ }^{172}$ The analysis was based on administrative claims data and statistical adjustment was performed on age, sex, Charlson comorbidity index, socioeconomic stats, residence in long-term care facility, and hospital type. Opioids were prescribed within 7 days of surgery in 7.1 percent of patients, and opioids were prescribed at 1 year from surgery in 7.7 
percent. Having opioids prescribed within 7 days of surgery was associated with increased risk of use at 1 year (adjusted OR 1.44, 95\% CI 1.39 to 1.50). Estimates were similar when findings were stratified by the specific surgical procedure (adjusted ORs ranged from 1.33 to 1.62). The study was not able to adjust for factors not available in administrative claims, such as pain severity, functional status, level of psychiatric distress, or other measures of clinical status following surgery.

The other study ( $n=444,764)$ evaluated patients who underwent 20 selected elective surgeries associated with a range of expected postoperative pain. ${ }^{173}$ The most common surgeries were laparoscopic cholecystectomy, knee arthroscopy with meniscectomy, open inguinal hernia repair, and total knee arthroplasty. Opioids were prescribed at discharge in 80.4 percent of patients. The study evaluated three definitions of opioid use at followup ("prolonged use," "persistent use," and the CONSORT definition). The proportion of patients with opioid fills 90 to 180 days after surgery ("prolonged use") was 7.1 percent, the proportion with opioid use lasting 90 or more days in the period from 180 days after surgery ("persistent use") was 1.0 percent, and the proportion meeting the CONSORT definition for persistent use (opioid use lasting 90 or more days and either 10 or more opioid fills or 120 or more days' supply) was 0.46 percent. Having an opioid prescribed at discharge was associated with increased risk of opioid use at followup compared with no opioid prescribed, though findings were based on crude event rates without adjustment for confounders. In addition, the likelihood of persistent use increased at higher opioid doses. Prolonged opioid use was observed in 4.4 percent of patients with no opioid fill, and the proportion ranged from 6.8 percent in patients prescribed 1 to $199 \mathrm{mg}$ morphine equivalent dose to 10.4 percent among those prescribed $\geq 500 \mathrm{mg}$. The CONSORT definition for persistent use was met by $0.2 \%$ of patients with no opioid fill and the proportion ranged from $0.27 \%$ at 1 to $199 \mathrm{mg}$ morphine equivalent dose to $1.30 \%$ at $\geq 500 \mathrm{mg}$ MED. The study was primarily designed to assess the risk of persistent opioid use for tramadol and long-acting opioids, relative to other (non-tramadol) short acting opioids. It found tramadol associated with increased risk of prolonged use (adjusted OR 1.06, 95\% CI 1.00 to 1.13), persistent use (adjusted OR 1.47, 95\% CI 1.25 to 1.69), and the CONSORT definition (adjusted OR 1.41, 95\% CI 1.08 to 1.75), after adjustment for year, surgery, sex, race/ethnicity, socioeconomic factors, age, opioid dose, and comorbidities. Long acting opioids were associated with increased risk of persistent use (adjusted OR 1.18, 95\% CI 1.02 to 1.35) and the CONSORT definition (adjusted OR 1.69, 95\% CI 1.36 to 2.02), with no association with prolonged use (adjusted OR 0.95, 95\% CI 0.87 to 1.03).

KQs $5 f$ and $5 g$ address the accuracy and effectiveness of instruments for predicting risk of opioid misuse, opioid use disorder, or overdose for patients with acute pain being considered for opioid therapy.

No study evaluated the accuracy or effectiveness of instruments for predicting risk of opioid misuse, opioid use disorder, or overdose in patients with postoperative pain.

KQ $5 \mathrm{~h}$ addresses the effect of the following factors on the decision to prescribe opioids for patients with acute pain being considered for opioid therapy: (1) existing opioid management plans; (2) patient education; (3) clinician and patient values and preferences related to opioids; (4) urine drug screening; (5) use of prescription drug monitoring program data; (6) availability of close followup.

Evidence on the effect of patient education, use of risk mitigation strategies, clinician and patient values and preferences, or availability of followup on decisions to prescribe opioids for postoperative pain was limited. One study of 1,057 who underwent elective surgery in New 
Hampshire found no difference in the rate of opioid prescribing before compared with after legislation mandating the use of a prescription drug monitoring program and an opioid risk assessment tool ( $80 \%$ vs. $77 \%$, p=0.29) (Appendix E, Table E-7). ${ }^{174}$ No high risk patient not subsequently prescribed opioids was identified. There was also no difference in the number of opioid pills prescribed, which had been decreasing prior to the legislation taking effect.

\section{Nonopioid Pharmacologic Therapy}

KQs $5 \mathrm{i}$ and 5k address the comparative effectiveness and harms of nonopioid pharmacologic therapy versus: (1) nonopioid pharmacologic therapy or (2) nonpharmacologic therapy.

Five trials evaluated nonopioid pharmacologic therapy for acute postoperative pain (Appendix E, Table E-6; Table 22). ${ }^{131,140,152,157,163}$ The surgery was knee arthoscopy, ${ }^{140}$ strabismus surgery, ${ }^{157}$ episiotomy, ${ }^{163}$ photorefractive keratectomy, ${ }^{131}$ and gynecological surgery $^{152}$ in one trial each. Three trials compared an NSAID versus acetaminophen ${ }^{140,157,163}$ one trial compared a non-aspirin NSAID versus aspirin, ${ }^{152}$ and one trial compared an oral NSAID versus a topical NSAID. ${ }^{131}$ Three trials ${ }^{152,157,163}$ were rated fair quality and two trials ${ }^{131,140}$ poor quality (Appendix F, Table F-1).

\section{NSAID Versus Acetaminophen}

Three trials compared an NSAID versus acetaminophen for postoperative pain. ${ }^{140,157,163}$ The surgery was knee arthroscopy, ${ }^{140}$ strabismus surgery, ${ }^{157}$ and episiotomy. ${ }^{163}$ Sample sizes were 40, 45, and 73. Two trials evaluated a single dose of ibuprofen (400 or $650 \mathrm{mg}$ ) versus acetaminophen (650 or $1000 \mathrm{mg}$ ). ${ }^{157,163}$ The third trial evaluated ketoprofen (50 mg every 8 hours) or dexketoprofen (25 mg every 8 hours) versus acetaminophen (500 mg every 6 hours) for 24 hours. ${ }^{140}$ Two trials were rated fair quality, ${ }^{157,163}$ and one trial was rated poor quality. ${ }^{140}$

The two single dose trials reported inconsistent effects of an NSAID versus acetaminophen on pain intensity and use of analgesics. ${ }^{157,163}$ One trial ${ }^{163}$ reported no adverse events, and the other trial ${ }^{157}$ found no difference in likelihood of nausea.

The other, poor quality trial $(n=45)$ found NSAIDs associated with decreased pain intensity at discharge versus acetaminophen (difference 0.7 to 1.1 points on a 0 to 10 scale, $p<0.05$ ). ${ }^{140}$ Few patients randomized to an NSAID or acetaminophen reported maximum pain as "severe" or "worst” or required medication use after discharge. Adverse events were not reported.

\section{Non-Aspirin NSAID Versus Aspirin}

One fair quality trial $(n=157)$ compared a single dose of diclofenac potassium $50 \mathrm{mg}$ or 100 mg versus aspirin $650 \mathrm{mg}$ following gynecological surgery. ${ }^{152}$ Effects on pain intensity favored diclofenac potassium (differences 0.7 to 1.7 points on a 0 to 10 scale at 4 or 8 hours) but the statistical significance of the difference was not reported. The difference in the sum of pain intensity differences was not statistically significant, but diclofenac was associated with decreased likelihood of repeat medication use (58\% to 60\% vs. 85\%, RR 0.70, 95\% CI 0.55 to 0.90 for $50 \mathrm{mg}$ dose and RR 0.68, 95\% CI 0.52 to 0.88 for $100 \mathrm{mg}$ dose). Estimates for adverse events were imprecise.

\section{Oral NSAID Versus Topical NSAID}

One poor quality trial ( $\mathrm{n}=135$ ) of patients undergoing photorefractive keratectomy found no difference between topical ketorolac $0.4 \%$ every 12 hours versus oral naproxen $440 \mathrm{mg}$ daily for 
3 days in pain intensity or of additional analgesic medication (Table 22). ${ }^{131}$ No adverse events were reported in either group.

Table 22. NSAIDs versus acetaminophen or aspirin for postoperative pain

\begin{tabular}{|c|c|c|c|c|}
\hline $\begin{array}{l}\text { Study, Year } \\
\text { Quality }\end{array}$ & Interventions & $\begin{array}{l}\text { Procedure; } \\
\text { Followup } \\
\text { Duration } \\
\text { Sample Size }\end{array}$ & Pain Intensity Results & Other Results \\
\hline $\begin{array}{l}\text { Berti, } 2000^{140} \\
\text { Poor }\end{array}$ & $\begin{array}{l}\text { A: Ketoprofen: } 50 \\
\text { mg every } 8 \text { hours } \\
\text { for } 24 \text { hours } \\
\text { B: Dexketoprofen } \\
25 \text { mg every } 8 \\
\text { hours for } 24 \text { hours } \\
\text { C: } \\
\text { Acetaminophen } \\
500 \text { mg every } 6 \\
\text { hours for } 24 \text { hours }\end{array}$ & $\begin{array}{l}\text { Knee } \\
\text { arthroscopy; } \\
\text { At discharge } \\
\text { (median } \sim 4 \\
\text { hours) and } 1 \\
\text { day } \\
\mathrm{n}=45\end{array}$ & $\begin{array}{l}\text { Pain intensity at } \\
\text { discharge (mean [SD } \\
\text { NR], } 0 \text { to } 100 \text { VAS } \\
\text { converted to } 0 \text { to } 10 \\
\text { scale): } 1.7 \text { vs. } 1.3 \text { vs. } \\
2.4, p<0.05 \text { for }\end{array}$ & $\begin{array}{l}\text { Rescue medication use after } \\
\text { discharge: } 6.7 \%(1 / 15) \text { vs. } \\
0 \%(0 / 15) \text { vs. } 6.7 \%(1 / 15) \\
\text { Maximum pain "severe" or } \\
\text { "worst" in first } 24 \text { hours after } \\
\text { discharge: } 6.7 \%(1 / 15) \text { vs. } \\
0 \%(0 / 15) \text { vs. } 13.3 \%(2 / 15)\end{array}$ \\
\hline $\begin{array}{l}\text { Hebertson, } \\
1995^{152} \\
\text { Fair }\end{array}$ & $\begin{array}{l}\text { A: Diclofenac } \\
\text { potassium } 50 \mathrm{mg} \\
\text { x } 1 \\
\text { B: Diclofenac } \\
\text { potassium } 100 \\
\mathrm{mg} \times 1 \\
\mathrm{C}: \text { Aspirin } 650 \mathrm{mg} \\
\text { x } 1\end{array}$ & $\begin{array}{l}\text { Gynecological } \\
\text { surgery; } \\
8 \text { hours } \\
n=157\end{array}$ & $\begin{array}{l}\text { Pain intensity difference } \\
\text { (mean [SD NR], } 0 \text { to } 3 \\
\text { NRS converted to } 0 \text { to } 10 \\
\text { scale): } 3.3 \text { vs. } 3.0 \text { vs. } 2.3 \\
\text { at } 4 \text { hours, } 1.7 \text { vs. } 2.0 \text { vs. } \\
0.3 \text { at } 8 \text { hours, p=NR } \\
\text { Sum of pain intensity } \\
\text { differences (mean [SD } \\
\text { NR], sum of differences } \\
\text { on } 0 \text { to } 3 \text { scale from } 0.5 \\
\text { to } 8 \text { hours): } 6.2 \text { vs. } 6.3 \\
\text { vs. } 4.8 ; p=N R \text { for A or B } \\
\text { vs. C }\end{array}$ & $\begin{array}{l}\text { Global evaluation (mean [SD } \\
\text { NR], } 0 \text { to } 4 \text { scale): } 2.33 \text { vs. } \\
2.25 \text { vs. } 1.94 ; p=N S \\
\text { Repeat medication use: } 60 \% \\
\text { (31/52) vs. } 58 \%(30 / 52) \text { vs. } \\
85 \%(45 / 53), \text { RR } 0.70 \text { (95\% } \\
\text { Cl } 0.55 \text { to } 0.90) \text { for A vs. C } \\
\text { and } 0.68(95 \% \text { Cl } 0.52 \text { to } \\
0.88) \text { for B vs. C }\end{array}$ \\
\hline $\begin{array}{l}\text { Morrison, } \\
1994^{157} \\
\text { Fair }\end{array}$ & $\begin{array}{l}\text { A: Ibuprofen } 600 \\
\mathrm{mg} \times 1 \\
\mathrm{~B}: \\
\text { Acetaminophen } \\
650 \mathrm{mg} \times 1\end{array}$ & $\begin{array}{l}\text { Strabismus } \\
\text { surgery; } \\
5 \text { hours } \\
n=40\end{array}$ & $\begin{array}{l}\text { Pain intensity (mean [SD } \\
\text { NR], } 0 \text { to } 100 \text { VAS } \\
\text { converted to } 0 \text { to } 10 \\
\text { scale): } 5.0 \text { vs. } 4.3 \text { at } 2 \\
\text { hours, } 4.4 \text { vs. } 3.9 \text { at } 5 \\
\text { hours; p=NR }\end{array}$ & $\begin{array}{l}\text { Use of analgesic medication: } \\
30 \%(6 / 20) \text { vs. } 30 \%(6 / 20)\end{array}$ \\
\hline $\begin{array}{l}\text { Ripa, } 2020^{131} \\
\text { Poor }\end{array}$ & $\begin{array}{l}\text { A. Topical } \\
\text { ketorolac } 0.4 \% \\
\text { every } 12 \text { hours for } \\
3 \text { days } \\
\text { B. Oral naproxen } \\
\text { sodium, } 440 \\
\text { mg/day for } 3 \text { days }\end{array}$ & $\begin{array}{l}\text { Photorefractive } \\
\text { keratectomy; } \\
5 \text { days } \\
n=135\end{array}$ & $\begin{array}{l}\text { Pain intensity (mean } \\
\text { [SD], } 0 \text { to } 10 \text { NRS): } 0.66 \\
\text { (1.39) vs. } 0.41(0.83) \text { at } \\
\text { day 5, MD } 0.25(95 \% \mathrm{Cl} \\
-0.14 \text { to } 0.64) ; \mathrm{MD} 0.26 \\
\text { (95\% } \mathrm{Cl}-0.14 \text { to } 0.65) \\
\text { adjusted for hydrocodone } \\
+ \text { acetaminophen use }\end{array}$ & $\begin{array}{l}\text { Supplemental analgesic use: } \\
25.6 \%(11 / 43) \text { vs. } 35 \% \\
(14 / 40) \text {, RR } 0.73(95 \% \mathrm{Cl} \\
0.38 \text { to } 1.4)\end{array}$ \\
\hline $\begin{array}{l}\text { Schachtel, } \\
1989^{163} \\
\text { Fair }\end{array}$ & $\begin{array}{l}\text { A: Ibuprofen } 400 \\
\mathrm{mg} \times 1 \\
\mathrm{~B}: \\
\text { Acetaminophen } \\
1000 \mathrm{mg} \times 1\end{array}$ & $\begin{array}{l}\text { Episiotomy } \\
4 \text { hours } \\
n=73\end{array}$ & $\begin{array}{l}\text { Pain intensity difference } \\
\text { (mean [SD NR], } 0 \text { to } 3 \\
\text { NRS converted to } 0 \text { to } 10 \\
\text { scale): } 3.3 \text { vs. } 2.0 \text { at } 4 \\
\text { hours, p } \leq 0.05 \\
\text { Sum of pain intensity } \\
\text { differences (mean [SD], } \\
\text { sum of differences on } 0 \\
\text { to } 3 \text { scale from } 0.5 \text { to } 4 \\
\text { hours): } 4.4(3.1 \text { ) vs. } 2.9 \\
93.2 \text { ), p } \leq 0.05\end{array}$ & $\begin{array}{l}\text { Supplemental analgesic use: } \\
22 \%(8 / 36) \text { vs. } 36 \%(15 / 37) \text {, } \\
\text { p } \leq 0.05 \\
\text { Overall assessment (mean } \\
\text { [SD], } 1 \text { to } 5 \text { scale, } \\
5=e x c e l l e n t): 3.3(1.3) \text { vs. } \\
2.9(1.4), p<0.001\end{array}$ \\
\hline
\end{tabular}

Abbreviations: $\mathrm{CI}$ = confidence interval; $\mathrm{MD}$ = mean difference; $\mathrm{NR}$ = not reported; NRS = numeric rating scale; NS = not significant; RR = relative risk; $\mathrm{SD}$ = standard deviation; VAS = visual analog scale 
KQs 5j and $5 l$ ask how the comparative effectiveness and harms of nonopioid pharmacologic therapy vary depending on: (1) patient demographics (e.g., age, race, ethnicity, gender); (2) patient medical and psychiatric comorbidities; (3) the type of nonopioid medication; (4) dose of medication; (5) duration of treatment.

Evidence was too limited to evaluate how comparative effectiveness and harms of nonopioid therapy for postoperative pain varied in subgroups, due to few trials, small sample sizes, methodological limitations, and exclusion of relevant subgroups or lack of information about them. No study conducted within-study or across-study evaluations of subgroup effects. Details regarding the nonopioid medications prescribed, dose, and duration of treatment are described above.

\section{Nonpharmacologic Therapy}

KQs $5 \mathrm{~m}$ and $5 \mathrm{n}$ address the comparative effectiveness of nonopioid pharmacologic therapy versus: (1) inactive controls or (2) other nonpharmacologic therapies.

Twenty-eight trials evaluated nonpharmacologic therapy for acute postoperative pain following various surgical procedures (Appendix E, Table E-6). ${ }^{125,127,129,132-139,142-144,146-149,151,154-}$ 156,158,161,162,165,166,168 Two trials evaluated acupuncture, ${ }^{135,149}$ two trials acupressure, ${ }^{146,151}$ eleven trials cold therapy, ${ }^{129,132-134,136,137,139,144,155,166,168}$ seven trials massage, ${ }^{125,127,148,154,156,162,165}$ two trials music therapy, ${ }^{147,158}$ one trial exercise therapy, ${ }^{138}$ two trials transcutaneous electrical nerve stimulation, ${ }^{143,161}$ and one trial neuromuscular stimulation. ${ }^{142}$ Two trials were rated good quality, ${ }^{143,168} 11$ trials fair quality, ${ }^{132-135,138,142,147,149,151,158,162}$ and 15 trials poor quality (Appendix F, Table F-1). ${ }^{125,127,129,136,137,139,144,146,148,154-156,161,165,166}$

\section{Acupuncture Versus Sham Acupuncture}

Two small ( $\mathrm{n}=33$ and 56), fair quality trials compared acupuncture versus sham acupuncture for postoperative pain (Table 23). ${ }^{135,149}$ One small $(n=33)$ trial compared acupuncture twice per day with sham acupuncture after stapled hemorrhoidopexy. ${ }^{135}$ Both the acupuncture and sham acupuncture groups also received usual care with traditional analgesia (diclofenac $50 \mathrm{mg}$ three times a day and metamizol $500 \mathrm{mg}$ four times a day). Sham acupuncture consisted of needle insertion in nonacupuncture points. Another small $(n=56)$ trial compared a single 20-minute acupuncture session at two points versus sham acupuncture following cesarean section. ${ }^{149}$ Sham acupuncture utilized a cotton pad to prevent contact between the acupuncture needle and skin. Effects on pain were inconsistent. In one trial, ${ }^{135}$ acupuncture was associated with decreased pain intensity on postoperative day one ( 2.7 vs. 4.0 on a 0 to 10 scale, $p=0.007$ ), though pain intensity was similar on postoperative day two (1.6 vs. 2.1, $\mathrm{p}>0.05)$. In the other trial, ${ }^{149}$ pain intensity was greater in the acupuncture arm at 24 hours (mean 3 vs. 0 on a 0 to 10 scale, $p=0.71$ ) and at 48 hours (mean 2 vs. $0, \mathrm{p}=0.03$ ), though the difference was only statistically significant at 48 hours. Effects on rescue analgesia use were also inconsistent. One trial ${ }^{135}$ reported no nausea, vomiting, acupuncture site reactions, or other harms; the other trial ${ }^{149}$ did not report adverse events.

\section{Acupuncture Versus Usual Care}

One of the fair quality acupuncture versus sham acupuncture trials described above also compared acupuncture versus usual care $(n=34)$ in patients following stapled hemorrhoidopexy (Table 23). ${ }^{135}$ The difference between acupuncture versus usual care was not statistically significant in the afternoon of postoperative day one ( 2.7 vs. 4.1 on a 0 to 10 scale, $\mathrm{p}=0.06$ ) or day two (1.6 vs. 2.1, p=NS). Acupuncture was associated with decreased rescue analgesic use on 
the afternoon of day 1 ( $6 \%$ vs. $53 \%, \mathrm{p}=0.03$ ), but the difference on day 2 was smaller and not statically significant ( $0 \%$ vs. $13 \%, \mathrm{p}=0.35)$.

\section{Acupressure Versus Sham Acupressure}

Two trials compared acupressure versus sham acupressure for postoperative pain (Table 23). ${ }^{146,151}$ One fair quality trial $(n=90)^{151}$ compared auricular acupressure (3 minutes, four times per day for 7 days) versus sham acupuncture (pressure applied to nonacupoints) following total knee arthroplasty. At 12 hours and 5 days, differences in pain intensity were small and not statistically significant, though acupressure was associated with decreased patient controlled analgesia use through 48 hours (mean 222.60 vs. 266.62 ml, mean difference -44.02 , 95\% CI -71.62 to -16.42$)$. At 7 days, the difference was slightly greater and statistically significant (mean difference -0.65 on a 0 to 10 scale, $95 \%$ CI -1.09 to -0.21 ). Acupressure was associated with better scores on the Hospital for Special Surgery Knee Rating Scale at 2 weeks (mean difference $-6.80,95 \%$ CI -11.30 to -2.30 on a 0 to 100 scale), with no difference on this outcome at 3 months. Acupressure was associated with decreased risk of nausea or vomiting (17.8\% vs. $71.1 \%$, RR 0.25 , 95\% CI 0.13 to 0.48$)$, dizziness or drowsiness $(4.4 \%$ vs. $24.4 \%$, RR $0.18,95 \%$ CI 0.04 to 0.77 ), and urinary retention (6.7\% vs. $26.7 \%$, RR 0.25 , $95 \%$ CI 0.08 to 0.83).

A small ( $n=40)$, poor quality trial compared acupressure to points on the body (one 30 minute session) versus sham acupressure (light stimulation to nonacupoints) following knee arthroscopy (Table 23). ${ }^{151}$ Acupressure was associated with lower pain intensity at 1 hour (mean 1.2 vs. 2.0 on a 0 to 10 scale, $\mathrm{p}<0.05$ ) and at 1 day (mean 0.5 vs. $2.3, \mathrm{p}<0.0001$ ). One patient randomized to acupressure experienced bradycardia of less than 2 minutes duration; adverse events were otherwise not reported.

Table 23. Acupuncture or acupressure versus sham or usual care

\begin{tabular}{|c|c|c|c|c|}
\hline $\begin{array}{l}\text { Author, Year } \\
\text { Quality }\end{array}$ & Interventions & $\begin{array}{l}\text { Procedure; } \\
\text { Followup Duration } \\
\text { Sample Size }\end{array}$ & $\begin{array}{l}\text { Pain } \\
\text { Intensity }\end{array}$ & Other Results \\
\hline $\begin{array}{l}\text { Gamermann, } \\
2014^{149} \\
\text { Fair }\end{array}$ & $\begin{array}{l}\text { A: Acupuncture at } \\
\text { P6 and L14 for a } \\
\text { 20-minute session } \\
\text { B: Sham } \\
\text { acupuncture } \\
\text { (Cotton pad under } \\
\text { needle) for a 20- } \\
\text { minute session }\end{array}$ & $\begin{array}{l}\text { Cesarean section; } \\
24 \text { and } 48 \text { hours } \\
n=56\end{array}$ & $\begin{array}{l}\text { Pain at rest } \\
\text { (mean [SD } \\
\text { NR], } 0 \text { to } 10 \\
\text { VAS): } 3 \text { vs. } 0 \\
\text { at } 24 \text { hours, } \\
p=0.71 \text { at } 24 \\
\text { hours; } 2 \text { vs. } 0 \\
\text { at } 48 \text { hours, } \\
p=0.03\end{array}$ & $\begin{array}{l}\text { Satisfaction with pain } \\
\text { relief (mean [SD NR], } \\
0 \text { to } 10 \text { NRS): } 10.00 \\
\text { vs. } 10.00 \text { at } 24 \text { hours, } \\
p=0.62 ; 10.00 \text { vs. } \\
10.00 \text { at } 48 \text { hours, } \\
p=0.79\end{array}$ \\
\hline
\end{tabular}




\begin{tabular}{|c|c|c|c|c|}
\hline $\begin{array}{l}\text { Author, Year } \\
\text { Quality }\end{array}$ & Interventions & $\begin{array}{l}\text { Procedure; } \\
\text { Followup Duration } \\
\text { Sample Size }\end{array}$ & $\begin{array}{l}\text { Pain } \\
\text { Intensity }\end{array}$ & Other Results \\
\hline $\begin{array}{l}\text { Langenbach, } \\
2012^{135} \\
\text { Fair }\end{array}$ & $\begin{array}{l}\text { A: Verum } \\
\text { acupuncture at } \\
\text { Du2, Du20, Bi30, } \\
\text { Bi57, Ma44, and } \\
\text { Pe6 twice per day } \\
\text { B: Sham } \\
\text { acupuncture } \\
\text { C: Traditional } \\
\text { analgesia } \\
\text { (diclofenac at } 50 \\
\text { mg orally three } \\
\text { times a day and } \\
\text { metamizol } 500 \text { mg } \\
\text { orally four times a } \\
\text { day) }\end{array}$ & $\begin{array}{l}\text { Stapled hemorrhoidopexy; } \\
\text { Postoperative days } 1 \text { and } 2 \\
n=50\end{array}$ & $\begin{array}{l}\text { Pain (mean } \\
\text { [SD], } 0 \text { to } 10 \\
\text { NRS): } 2.7 \\
(1.5) \text { vs. } 4.0 \\
(1.0) \text { vs. } 4.1 \\
\text { (1.9) at } \\
\text { afternoon of } \\
\text { postoperative } \\
\text { day } 1 \text {, } \\
p=0.057 \text { for } \\
\text { A vs C, } \\
p=0.007 \text { for } \\
\text { A vs. B }\end{array}$ & $\begin{array}{l}\text { Proportion using } \\
\text { rescue analgesia: } 6 \% \\
(1 / 17) \text { vs. } 38 \%(6 / 16) \\
\text { vs. } 53 \%(9 / 17) \text { at } \\
\text { afternoon of } \\
\text { postoperative day } 1 \text {, } \\
\text { RR } 0.16(95 \% \mathrm{Cl} \\
0.02 \text { to } 1.16) \text { for } \mathrm{A} \text { vs. } \\
\text { B, RR } 0.11 \text { (95\% Cl } \\
0.016 \text { to } 0.78), \\
\text { p=0.0275 for A vs. C; } \\
0 \%(0 / 17) \text { vs. } 13 \% \\
(2 / 16) \text { vs. } 18 \%(3 / 17) \\
\text { at afternoon of } \\
\text { postoperative day } 2 \text {, } \\
\text { RR } 0.19 \text { (95\% Cl } \\
0.01 \text { to } 3.66), \\
\text { p=0.270 for A vs. B, } \\
\text { RR } 0.14 \text { (95\% Cl } \\
0.01 \text { to } 2.57) \text {, } \\
\text { p=0.187 for A vs. C }\end{array}$ \\
\hline $\begin{array}{l}\text { Felhendler, } \\
1996^{146} \\
\text { Poor }\end{array}$ & $\begin{array}{l}\text { A: Acupressure, } \\
\text { stimulation to } 15 \\
\text { classical acupoints } \\
\text { B: Sham } \\
\text { acupressure, light } \\
\text { stimulation to } 15 \\
\text { nonacupoints } \\
30 \text { minutes after } \\
\text { patients awoke } \\
\text { from anesthesia for } \\
\text { each group }\end{array}$ & $\begin{array}{l}\text { Knee arthroscopy; } \\
1 \text { hour and } 1 \text { day } \\
n=40\end{array}$ & $\begin{array}{l}\text { Pain intensity } \\
\text { (median } \\
\text { [IQR], } 0 \text { to } 10 \\
\text { VAS): } 1.2 \\
(0.6 \text { to } 2.4) \\
\text { vs. } 2.0(1.5 \\
\text { to } 3.6) \text { at } 1 \\
\text { hour, p<0.05; } \\
0.5(0.2 \text { to } \\
1.1) \text { vs. } 2.3 \\
(1.4 \text { to } 2.8) \text { at } \\
1 \text { day, } \\
\text { p<0.0001 }\end{array}$ & NR \\
\hline $\begin{array}{l}\text { He, } \\
2013^{151} \\
\text { Fair }\end{array}$ & $\begin{array}{l}\text { A: Auricular } \\
\text { acupressure, } \\
\text { pressure on } \\
\text { auricular acupoints } \\
\text { at } 4 \text { acupressure } \\
\text { points } \\
\text { B: Sham } \\
\text { acupressure, } \\
\text { Pressure on } \\
\text { acupoints at } 4 \\
\text { sham sites } \\
3 \text { minutes per } \\
\text { point, four times } \\
\text { per day, for } 7 \text { days } \\
\text { for each group }\end{array}$ & $\begin{array}{l}\text { Knee arthroplasty; } \\
12 \text { hours, } 5 \text { days, and } 7 \text { days } \\
n=90\end{array}$ & $\begin{array}{l}\text { Pain intensity } \\
\text { (mean [SD], } \\
0 \text { to } 10 \text { VAS): } \\
4.78 \text { (1.66) } \\
\text { vs. } 4.85 \\
(1.55) \text { at } 12 \\
\text { hours; } 2.91 \\
(1.12) \text { vs. } \\
3.51 \text { (1.16) at } \\
5 \text { days, MD } \\
-0.60 \text { (95\% } \\
\text { Cl }-1.08 \text { to } \\
-0.12) ; 2.22 \\
(1.02) \text { vs. } \\
2.87(1.08) \text { at } \\
7 \text { days, MD } \\
-0.65(95 \% \\
\text { Cl }-1.09 \text { to } \\
-0.21)\end{array}$ & $\begin{array}{l}\text { HSS Knee Rating } \\
\text { Scale (mean [SD], } 0 \\
\text { to } 100 \text { scale): } 80.24 \\
(10.68) \text { vs. } 73.44 \\
(10.80) \text { at } 2 \text { weeks, } \\
\text { MD }-6.80 \text { (95\% Cl } \\
-11.30 \text { to }-2.30) ; \\
92.89 \text { (6.99) vs. } \\
91.91 \text { (7.13) at } 3 \\
\text { months, MD -0.98 } \\
\text { (95\% Cl }-3.94 \text { to } \\
1.97) \\
\text { PCA used through } 48 \\
\text { hours (mean [SD], } \\
\text { ml): } 222.60(62.59) \\
\text { vs. } 266.62(69.03), \\
\text { MD }-44.02 \text { (95\% Cl } \\
-71.62 \text { to }-16.42)\end{array}$ \\
\hline
\end{tabular}

Abbreviations: $\mathrm{CI}=$ confidence interval; IQR = interquartile range; $\mathrm{MD}=$ mean difference; $\mathrm{NR}=$ not reported; NRS = numeric rating scale; HSS = Hospital for Special Surgery score; PCA = patient-controlled analgesia; $\mathrm{SD}=$ standard deviation; VAS = visual analog scale 


\section{Cold Therapy Versus No Cold Therapy or Sham}

Eleven trials evaluated cold therapy versus no cold therapy or a sham treatment for postoperative pain. Sample sizes ranged from 19 to 131 (total $N=655)^{129,132-}$

134,136,137,139,144,155,166,168 (Appendix E, Table E-6; Table 24). The surgery was ACL repair ${ }^{132-}$ $134,137,139,144$ in six trials, arthroscopic knee surgery in two trials, ${ }^{129,136}$ and shoulder surgery, ${ }^{166}$ herniotomy, ${ }^{155}$ and total knee arthroplasty ${ }^{168}$ in one trial each. Cold therapy varied from a cold compress for 20 minutes to continuous application using a circulating device for up to 7 days; some devices for delivering cold therapy enabled precise temperature control. One trial was rated good quality. ${ }^{168}$ It compared cold therapy using a device that maintained a consistent temperature (10 to 12 degrees Celsius) versus sham therapy using the device without cooling (21 degrees Celsius). Three trials ${ }^{132-134}$ were rated fair quality, and seven trials were rated poor quality (Appendix F, Table F-1). ${ }^{129,136,137,139,144,155,166,168}$

The good quality trial $(n=60)$, which evaluated patients who had undergone knee arthroplasty, found no difference between cold therapy (4 hours or more daily) versus sham therapy in pain intensity at 2 weeks (mean difference -0.30 on a 0 to 10 scale, $95 \%$ CI -1.33 to 0.73), 6 weeks (mean difference $-0.40,95 \%$ CI -1.18 to 0.38 ), or 12 weeks (mean difference $0.10,95 \%$ CI -0.97 to 1.17$).{ }^{168}$ There were also no differences in outcomes based on the Western Ontario and McMaster Universities Osteoarthritis Index, the Oxford Knee Scale, or the EQ-5D. However, cold therapy was associated with less tramadol use versus sham in the first 4 days (47 tablets [2350 mg] vs. 83 tablets [4150 mg]). Three fair quality trials ( $\mathrm{n}=19,78$, and 71 ) found no differences between cold therapy versus no cold therapy or sham in pain at 1 day to $<1$ week. ${ }^{132-}$ 134 Differences ranged from -1.3 points on a 0 to 10 scale in favor of cold therapy to 0.50 points in favor of sham cold therapy. Two of the trials also found no differences in analgesic medication use. ${ }^{133,134}$

Evidence from seven poor quality trials of cold therapy versus no cold therapy was difficult to interpret due to the methodological limitations, differences in the surgeries treated, differences in methods for administering cold therapy, and some inconsistency in results. ${ }^{129,136,137,139,144,155,166}$ At $<1$ day, cold therapy was associated with decreased pain intensity versus no cold therapy in two trials (mean differences -1.1 and -1.3 points on a 0 to 10 scale). ${ }^{139,155}$ At 1 day to $<1$ week, differences ranged from 3.1 points in favor of cold therapy to 4.2 points in favor of no cold therapy. ${ }^{129,137,139,144,155,166}$ At 1 week to $<2$ weeks effects on pain intensity ranged from 1.4 points in favor of cold therapy to no difference (0 points). ${ }^{129,136,166}$ One trial found no difference between cryotherapy versus no cold therapy at 6 weeks in pain at rest or on motion. ${ }^{129}$ One trial found cold therapy associated with decreased likelihood of pain medication use at $<1$ day (28.2\% vs. $91.5 \%$, RR 0.22 [95\% CI 0.05 to 0.90$]$ ]) ${ }^{155}$ Effects on analgesic medication use at 1 day to $<1$ week were inconsistent, with two trials ${ }^{129,137}$ finding cold therapy associated with decreased analgesic medication use versus no cold therapy and two trials ${ }^{139,144}$ finding no difference. Harms were not well reported, though no serious adverse events were described. 
Table 24. Cold therapies versus no cold therapy or sham for postoperative pain

\begin{tabular}{|c|c|c|c|c|}
\hline $\begin{array}{l}\text { Author, Year } \\
\text { Quality }\end{array}$ & Interventions & $\begin{array}{l}\text { Procedure; } \\
\text { Followup } \\
\text { Duration } \\
\text { Sample Size }\end{array}$ & Pain Intensity & Other Results \\
\hline $\begin{array}{l}\text { Barber, } \\
1998^{139} \\
\text { Poor }\end{array}$ & $\begin{array}{l}\text { A: Continuous cold- } \\
\text { flow therapy using } \\
\text { a constant flow unit } \\
\text { for } 3 \text { days, then as } \\
\text { needed for } 4 \text { days } \\
\text { B: No cold therapy }\end{array}$ & $\begin{array}{l}\mathrm{ACL} \\
\text { reconstruction; } \\
6 \text { and } 7 \text { days } \\
\mathrm{n}=100\end{array}$ & $\begin{array}{l}\text { Pain intensity (mean [SD } \\
\text { NR], } 0 \text { to } 10 \text { VAS): } 4.10 \\
\text { vs. } 5.22 \text { at } 8 \text { hours, } 5.33 \\
\text { vs. } 4.39 \text { at } 6 \text { days; } p=N R\end{array}$ & $\begin{array}{l}\text { Rescue medication use } \\
\text { (mean [SD], number of pills): } \\
1.73 \text { vs. } 1.82 \text { at } 7 \text { days, } \\
\text { p=NR }\end{array}$ \\
\hline $\begin{array}{l}\text { Daniel, } \\
1994^{144} \\
\text { Poor }\end{array}$ & $\begin{array}{l}\text { A: Cold therapy } \\
\text { pad, } 40 \text { degrees F } \\
\text { B: Cold therapy } \\
\text { pad, } 45 \text { degrees F } \\
\text { C: Cold therapy } \\
\text { pad, } 55 \text { degrees F } \\
\text { D: Cold therapy } \\
\text { pad, } 70 \text { degrees F } \\
\text { E: No cold therapy } \\
\text { For cold therapy } \\
\text { groups: continuous } \\
\text { use during } \\
\text { hospitalization, } \\
\text { except } 3 \text { hours } \\
\text { twice a day to use } \\
\text { passive-motion } \\
\text { machine }\end{array}$ & $\begin{array}{l}\text { ACL } \\
\text { reconstruction; } \\
\text { Day } 3 \text { or } \\
\text { discharge if } \\
\text { earlier } \\
n=131\end{array}$ & $\begin{array}{l}\text { Pain (mean [SD], } 0 \text { to } 10 \\
\text { VAS): } 4.1 \text { (2.1) vs. } 4.6 \\
\text { (1.7) vs. } 5.7(2.0) \text { vs. } 4.9 \\
\text { (2.4) vs. } 4.6(2.0), p=N S\end{array}$ & $\begin{array}{l}\text { Use of parenteral } \\
\text { meperidine (mean [SD], } \\
\text { number of } 75 \text { mg doses): } 5.5 \\
\text { (4.1) vs. } 5.3(4.3) \text { vs. } 4.8 \\
\text { (3.8) vs. } 4.4(2.6) \text { vs. } 4.8 \\
\text { (2.9), p=NS } \\
\text { Use of oral codeine (mean } \\
\text { [SD], number of } 60 \text { mg } \\
\text { doses): } 8.8(3.0) \text { vs. } 8.6 \\
\text { (3.6) vs. } 9.4(3.4) \text { vs. } 9.9 \\
\text { (4.0) vs. } 10.2(4.5), p=N S\end{array}$ \\
\hline $\begin{array}{l}\text { Dambros, } \\
2012^{132} \\
\text { Fair }\end{array}$ & $\begin{array}{l}\text { A: Cold therapy, ice } \\
\text { pack, } 20 \text { minutes } \\
\text { twice daily } \\
\text { B: No cold therapy } \\
\text { Both groups } \\
\text { received } 2 \text { physical } \\
\text { therapy sessions, } \\
\text { one in the morning, } \\
\text { and one in the } \\
\text { afternoon }\end{array}$ & $\begin{array}{l}\mathrm{ACL} \\
\text { reconstruction; } \\
\text { After each } \\
\text { physical } \\
\text { therapy } \\
\text { session on } \\
\text { first day after } \\
\text { surgery } \\
\mathrm{n}=19\end{array}$ & $\begin{array}{l}\text { Pain (mean [SD], } 0 \text { to } 10 \\
\text { VAS): } 2.2(2.0) \text { vs. } 2.4 \\
(2.4) \text { after first treatment, } \\
\text { MD }-0.20(95 \% \mathrm{Cl}-2.33 \\
\text { to } 1.93) ; 1.19(1.8) \text { vs. } \\
2.5(2.0) \text { after second } \\
\text { treatment, MD }-1.31 \\
(95 \% \mathrm{Cl}-3.15 \text { to } 0.53)\end{array}$ & NR \\
\hline $\begin{array}{l}\text { Dervin, } 1998^{133} \\
\text { Fair }\end{array}$ & $\begin{array}{l}\text { A: Cold therapy, } \\
\text { Cryocuff with ice } \\
\text { water } \\
\text { B: Sham cold } \\
\text { therapy, Cryocuff } \\
\text { with room } \\
\text { temperature water }\end{array}$ & $\begin{array}{l}\mathrm{ACL} \\
\text { reconstruction; } \\
24 \text { hours } \\
n=78\end{array}$ & $\begin{array}{l}\text { Pain (mean [SD], } 0 \text { to } \\
100 \text { VAS converted to } 0 \\
\text { to } 10) \text { at } 24 \text { hours: } 3.0 \\
(1.7) \text { vs. } 2.5(1.3), p=N S \text {, } \\
\text { MD } 0.50(95 \% \mathrm{Cl}-0.18 \\
\text { to } 1.18)\end{array}$ & $\begin{array}{l}\text { Morphine use (mean [SD] } \\
\text { total infused } \mathrm{mg} / \mathrm{kg}): 0.37 \\
(0.23) \text { vs. } 0.35(0.21), \mathrm{p}=\mathrm{NS} \\
\text { Codeine use (mean [SD] } \\
\text { number of } 30 \mathrm{mg} \text { tablets): } \\
\text { 3.86 (2.72) vs. } 3.44(2.1) \\
\text { p=NS }\end{array}$ \\
\hline $\begin{array}{l}\text { Edwards, } \\
1996^{134} \\
\text { Fair }\end{array}$ & $\begin{array}{l}\text { A: Cryotherapy, } \\
\text { Cryocuff with ice } \\
\text { water } \\
\text { B: Sham } \\
\text { cryotherapy, } \\
\text { Cryocuff with room } \\
\text { temperature water } \\
\text { C: No cryotherapy, } \\
\text { no Cryocuff }\end{array}$ & $\begin{array}{l}\mathrm{ACL} \\
\text { reconstruction; } \\
3 \text { days } \\
\mathrm{n}=71\end{array}$ & $\begin{array}{l}\text { Pain (mean [SD NR], } \\
\text { VAS } 0 \text { to } 10 \text { ) at day } 3 \text { : } \\
1.2 \text { vs. } 1.8 \text { vs. } 1.7, p=\mathrm{N}\end{array}$ & $\begin{array}{l}\text { Morphine use (mean, mg/kg } \\
\text { of body weight) during } \\
\text { inpatient stay: } 0.65 \text { vs. } 0.60 \\
\text { vs. } 0.65, \mathrm{p}=\mathrm{NS} \\
\text { Codeine use (mean, } \mathrm{mg} / \mathrm{kg} \\
\text { of body weight) during } \\
\text { inpatient stay: } 4.14 \text { vs. } 3.91 \\
\text { vs. } 4.31, \mathrm{p}=\mathrm{NS} \\
\text { Acetaminophen use (mean, } \\
\text { mg/kg of body weight) } \\
\text { during inpatient stay: } 73.8 \\
\text { vs. } 85.2 \text { vs. } 70.6, \mathrm{p}=\mathrm{NS}\end{array}$ \\
\hline
\end{tabular}




\begin{tabular}{|c|c|c|c|c|}
\hline $\begin{array}{l}\text { Author, Year } \\
\text { Quality }\end{array}$ & Interventions & $\begin{array}{l}\text { Procedure; } \\
\text { Followup } \\
\text { Duration } \\
\text { Sample Size } \\
\end{array}$ & Pain Intensity & Other Results \\
\hline $\begin{array}{l}\text { Holmstrom, } \\
2005^{129} \\
\text { Poor }\end{array}$ & $\begin{array}{l}\text { A: Cryotherapy, } \\
\text { Cryofcuff with ice } \\
\text { water: continuous } \\
\text { chilling (10 to } 15 \\
\text { degrees Celsius) } \\
\text { and pulsing } \\
\text { compression for } 48 \\
\text { hours } \\
\text { B: No cold therapy }\end{array}$ & $\begin{array}{l}\text { Arthroscopic } \\
\text { knee surgery; } \\
\text { Day } 1 \text {, day } 7 \text {, } \\
\text { and week } 6 \\
\mathrm{n}=40\end{array}$ & $\begin{array}{l}\text { Pain at rest (mean [SD } \\
\text { NR], } 0 \text { to } 10 \text { VAS): } 3.2 \\
\text { vs. } 3.0 \text { at day } 1 ; 1.0 \text { vs. } \\
1.3 \text { at day } 7 ; 0.5 \text { vs } 0.5 \\
\text { at } 6 \text { weeks, } p=N S \text { for all } \\
\text { time points } \\
\text { Pain on motion (mean } \\
\text { [SD NR], } 0 \text { to } 10 \text { VAS): } \\
5.7 \text { vs. } 5.8 \text { at day } 1 ; 2.0 \\
\text { vs. } 3.5 \text { at day } 7 ; 1.8 \text { vs. } \\
1.8 \text { at } 6 \text { weeks, p=NS for } \\
\text { all time points }\end{array}$ & $\begin{array}{l}\text { Morphine consumption } \\
\text { (mean [SD NR] mg): } 13.4 \\
\text { vs. } 20.8, p=0.028 \text { at } 24 \\
\text { hours } \\
\text { Mean over first } 3 \\
\text { postoperative days: } 18.7 \text { vs. } \\
\text { 28.4, p=NS }\end{array}$ \\
\hline $\begin{array}{l}\text { Koc, } \\
20066^{155} \\
\text { Poor }\end{array}$ & $\begin{array}{l}\text { A: Cold compress, } \\
20 \text { minutes after } \\
\text { surgery } \\
\text { B: Room } \\
\text { temperature } \\
\text { compress, } 20 \\
\text { minutes after } \\
\text { surgery }\end{array}$ & $\begin{array}{l}\text { Inguinal } \\
\text { hernia; } \\
6 \text { and } 24 \\
\text { hours } \\
n=40\end{array}$ & $\begin{array}{l}\text { Pain intensity (mean } \\
\text { [SD], } 0 \text { to } 10 \text { VAS): } 4.0 \\
(0.3) \text { vs. } 5.3(0.4) \text { at } 6 \\
\text { hours, } p=0.02 ; 2.4(0.2) \\
\text { vs. } 3.6(0.3) \text { at } 24 \text { hours, } \\
p=0.007\end{array}$ & $\begin{array}{l}\text { Analgesic medication use in } \\
\text { first } 24 \text { hours: } 10 \%(2 / 20) \text { vs. } \\
45 \%(9 / 20), \text { RR } 0.22(95 \% \\
\text { Cl } 0.05 \text { to } 0.90)\end{array}$ \\
\hline $\begin{array}{l}\text { Lessard, } \\
1997^{136} \\
\text { Poor }\end{array}$ & $\begin{array}{l}\text { A: Cryotherapy } \\
\text { (cold gel packs), } 20 \\
\text { minutes four times } \\
\text { per day, followed } \\
\text { by exercises } \\
\text { B: No cryotherapy, } \\
\text { exercises only }\end{array}$ & $\begin{array}{l}\text { Minor } \\
\text { arthroscopic } \\
\text { knee surgery; } \\
7 \text { days } \\
n=45\end{array}$ & $\begin{array}{l}\text { Pain (mean [SD], McGill } \\
\text { pain questionnaire total } \\
\text { pain rating score, } 0 \text { to } 78 \\
\text { scale): } 8.78 \text { ( } 7.08 \text { ) vs. } \\
10.45(6.97), \mathrm{MD}-1.67 \\
\text { ( } 95 \% \mathrm{Cl}-5.6 \text { to } 2.3 \text { ) } \\
\text { Most pain in the day } \\
\text { (mean [SD NR], VAS } 0 \\
\text { to } 100 \text { converted to } 0 \text { to } \\
10 \text { scale): } 2.4 \text { vs. } 2.7, \\
\text { p=NS }\end{array}$ & NR \\
\hline $\begin{array}{l}\text { Ohkoshi, } \\
\text { 1999137 } \\
\text { Poor }\end{array}$ & $\begin{array}{l}\text { A: Cryotherapy } \\
\text { using Icing System } \\
2000 \text { at } 5 \text { degrees } \\
\text { Celsius } \\
\text { B: Cryotherapy } \\
\text { using Icing System } \\
2000 \text { at } 10 \text { degrees } \\
\text { Celcius } \\
\text { C: No cold therapy }\end{array}$ & $\begin{array}{l}\mathrm{ACL} \\
\text { reconstruction; } \\
2 \text { days } \\
\mathrm{n}=21\end{array}$ & $\begin{array}{l}\text { Pain (mean [SD], } 0 \text { to } \\
100 \text { VAS converted to } 0 \\
\text { to } 10 \text { scale): } 7.67(1.51) \\
\text { vs. } 3.47(2.98) \text { vs. } 6.57 \\
(2.05) ; A \text { vs. B and B vs. } \\
\text { C, p<0.05; MD } 4.22 \\
\text { ( } 95 \% \mathrm{Cl} 1.47 \text { to } 6.97) \text { for } \\
\text { A vs. B and MD }-3.1 \\
(95 \% \text { Cl }-6.08 \text { to }-0.12) \\
\text { for B vs. C }\end{array}$ & $\begin{array}{l}\text { Number of analgesic doses } \\
\text { (mean [SD], } 25 \mathrm{mg} \text { of } \\
\text { diclofenac sodium } \\
\text { suppositories): } 1.25(0.4) \text { vs. } \\
0.7 \text { ( } 0.8 \text { ) vs. } 1.5(1.0) \text {, } \\
\mathrm{p}=<0.05 \text { for B vs. C }\end{array}$ \\
\hline
\end{tabular}




\begin{tabular}{|c|c|c|c|c|}
\hline $\begin{array}{l}\text { Author, Year } \\
\text { Quality }\end{array}$ & Interventions & $\begin{array}{l}\text { Procedure; } \\
\text { Followup } \\
\text { Duration } \\
\text { Sample Size }\end{array}$ & Pain Intensity & Other Results \\
\hline $\begin{array}{l}\text { Thijs, } \\
\text { 2019168 } \\
\text { Good }\end{array}$ & $\begin{array}{l}\text { A: Computer- } \\
\text { assisted cold } \\
\text { therapy, } 10 \text { to } 12 \\
\text { degrees Celsius, } \\
\text { varying time and } \\
\text { temperature } \\
\text { through } 7 \text { days } \\
\text { B: Sham } \\
\text { cryotherapy: Same } \\
\text { as intervention, } \\
\text { except at } 21 \\
\text { degrees Celsius }\end{array}$ & $\begin{array}{l}\text { Total knee } \\
\text { arthroplasty; } \\
2,6 \text {, and } 12 \\
\text { weeks } \\
n=60\end{array}$ & $\begin{array}{l}\text { Pain intensity (mean } \\
\text { [SD], } 0 \text { to } 10 \text { NRS): } 2.8 \\
(1.5) \text { vs. } 3.1(2.4) \text { at } 2 \\
\text { weeks, MD }-0.30(95 \% \\
\mathrm{Cl}-1.33 \text { to } 0.73) ; 1.7 \\
(1.3) \text { vs. } 2.1(1.7) \text { at } 6 \\
\text { weeks, MD }-0.40(95 \% \\
\mathrm{Cl}-1.18 \text { to } 0.38) ; 2.0 \\
(2.3) \text { vs. } 1.9(1.8) \text { at } 12 \\
\text { weeks, MD } 0.10(95 \% \mathrm{Cl} \\
-0.97 \text { to } 1.17)\end{array}$ & 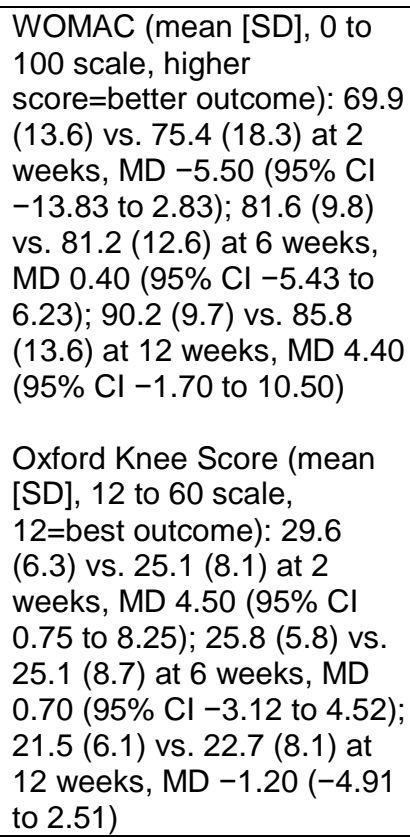 \\
\hline $\begin{array}{l}\text { Speer, } \\
1996^{166} \\
\text { Poor }\end{array}$ & $\begin{array}{l}\text { A: Cold therapy, } \\
\text { Cryo/Cuff bladder } \\
\text { with ice water } \\
\text { every } 1 \text { to } 2 \text { hours } \\
\text { while awake for } 24 \\
\text { hours; then ice } 4 \text { to } \\
6 \text { times daily and } \\
\text { as needed for } 10 \\
\text { days } \\
\text { B: No cryotherapy }\end{array}$ & $\begin{array}{l}\text { Shoulder } \\
\text { surgery; } \\
1 \text { and } 10 \text { days } \\
n=50\end{array}$ & $\begin{array}{l}\text { Worst pain intensity } \\
\text { (mean [SD] } 0 \text { to } 100 \\
\text { VAS converted to } 0 \text { to } \\
10 \text { scale): } 3.1 \text { vs. } 5.6 \text { at } \\
\text { day } 1, p=0.001 ; 3.3 \text { vs. } \\
4.7 \text { at day } 10, p=0.03\end{array}$ & NR \\
\hline
\end{tabular}

Abbreviations: ACL = anterior cruciate ligament; $\mathrm{CI}$ = confidence interval; IQR = interquartile range; $\mathrm{MD}=$ mean difference; $\mathrm{NR}=$ not reported; NRS = numeric rating scale; $\mathrm{SD}=$ standard deviation; VAS = visual analog scale; WOMAC = Western Ontario and McMaster Universities Osteoarthritis Index

\section{Massage Versus No Massage}

Seven trials compared massage versus no massage for postoperative pain (Appendix E, Table E-6; Table 25). ${ }^{125,127,148,154,156,162,165}$ Sample sizes ranged from 59 to 196 (total N=792). The surgery was a laparoscopic procedure in three trials, ${ }^{154,156,165}$ cesarean section in three trials, ${ }^{125,127,162}$ and lymph node dissection in one trial. ${ }^{148}$ In six trials, the intervention consisted of a single massage session to the hand or foot for 5 to 10 minutes. ${ }^{125,127,154,156,162,165}$ In the seventh trial, patients received 10 minute arm massages as often as needed for 3 days following lymph node dissection. ${ }^{148}$ Due to the nature of the intervention, the trials could not be blinded. One trial was rated fair quality ${ }^{162}$ and the others were poor quality (Appendix F, Table F-1).

Five trials found a single massage session associated with decreased pain intensity versus no massage at $<1$ day (90 minutes to 3 hours or time to discharge); mean differences ranged from 1.4 to 2.6 points on a 0 to 10 scale. ${ }^{125,127,156,162,165}$ One other trial found no difference in pain intensity. ${ }^{154}$ Three trials ${ }^{125,156,165}$ found a single session of massage associated with decreased pain medication use (28.2\% vs. 91.5\%, RR 0.31, 95\% CI 0.22 to $0.44 ; 31.7 \%$ [foot massage] or $21.5 \%$ [foot and hand massage] vs. $89.7 \%$, RR $0.35,95 \%$ CI 0.24 to 0.51 and RR $0.28,95 \%$ CI 
0.18 to 0.45 ; and $15 \%$ vs. $70 \%$, RR 0.21 , $95 \%$ CI 0.10 to 0.46 ). Two trials ${ }^{156,165}$ found single session massage associated with decreased anxiety at $<1$ day (differences about 22 points on the 20 to 80 State and Continuous Anxiety Inventory, state anxiety scale), but one other trial ${ }^{162}$ found difference between massage versus no massage in anxiety.

A small $(n=59)$ trial of multiple arm massage sessions after lymph node dissection reported effects on pain poorly. ${ }^{148}$ Massage was associated with better function as measured on the Shoulder Pain and Disability Index versus no massage at 2 weeks (differences 1.7 to 2.0 points on a 0 to 10 scale for various activities).

Adverse events were not reported in the massage trials.

Table 25. Massage versus no massage for postoperative pain

\begin{tabular}{|c|c|c|c|c|}
\hline $\begin{array}{l}\text { Author, } \\
\text { Year } \\
\text { Quality }\end{array}$ & Interventions & $\begin{array}{l}\text { Procedure; } \\
\text { Followup } \\
\text { Duration } \\
\text { Sample Size }\end{array}$ & Pain Intensity & $\begin{array}{l}\text { Other Pain and Function } \\
\text { Results }\end{array}$ \\
\hline $\begin{array}{l}\text { Abbaspoor, } \\
2014^{125} \\
\text { Poor }\end{array}$ & $\begin{array}{l}\text { A: Hand and foot } \\
\text { massage (5 } \\
\text { minutes each, } 20 \\
\text { minutes total) } \\
\text { B: Attention } \\
\text { control: } \\
\text { Investigator talked } \\
\text { with patient for } 20 \\
\text { minutes + usual } \\
\text { care }\end{array}$ & $\begin{array}{l}\text { Cesarean } \\
\text { section; } \\
1.5 \text { hours after } \\
\text { treatment } \\
n=80\end{array}$ & $\begin{array}{l}\text { Pain intensity (mean [SD], } \\
0 \text { to } 10 \text { NRS): } 3.58(0.64) \\
\text { vs. } 6.23(0.68), \text { MD }-2.65 \\
(95 \% \mathrm{Cl}-2.94 \text { to }-2.36)\end{array}$ & $\begin{array}{l}\text { Medication use during the } \\
1.5 \mathrm{~h} \text { after intervention } \\
\text { Diclofenac: } 15 \%(6 / 40) \text { vs. } \\
70 \%(28 / 40) \mathrm{RR} 0.21 \text { ( } 95 \% \\
\mathrm{Cl} 0.10 \text { to } 0.46) \\
\text { Meperidine: } 0 \% \text { vs. } 7.5 \% \\
(3 / 40) \\
\text { No analgesic medication: } \\
85 \% \text { (34/40) vs. } 22.5 \% \\
(9 / 40), \text { RR } 3.78995 \% \mathrm{Cl} \\
2.90 \text { to } 6.81)\end{array}$ \\
\hline $\begin{array}{l}\text { Degirmen, } \\
2010^{127} \\
\text { Poor }\end{array}$ & $\begin{array}{l}\text { A: Foot and hand } \\
\text { massage: } \\
\text { petrissage, } \\
\text { kneading, and } \\
\text { friction applied to } \\
\text { the patient's } \\
\text { hands and feet } \\
\text { with classical } \\
\text { massage } \\
\text { techniques, } 5 \\
\text { minutes per } \\
\text { hand/foot for total } \\
\text { of } 20 \text { minutes } \\
\text { massage } \\
\text { B: Foot massage: } \\
\text { petrissage as } \\
\text { above, } 5 \text { minutes } \\
\text { per foot for total of } \\
10 \text { minutes } \\
\text { massage } \\
\text { C: Control: no } \\
\text { massage }\end{array}$ & $\begin{array}{l}\text { Cesarean section } \\
60 \text { minutes } \\
n=75\end{array}$ & $\begin{array}{l}\text { Pain intensity (mean [SD], } \\
0 \text { to } 10 \text { NRS): } 3.64(1.22) \\
\text { vs. } 3.76(1.20) \text { vs. } 5.20 \\
\text { (1.11); MD }-0.12 \text { ( } 95 \% \mathrm{Cl} \\
-0.81 \text { to } 0.57) \text { for A vs. B; } \\
\text { MD }-1.56 \text { ( } 95 \% \mathrm{Cl}-2.22 \\
\text { to }-0.90) \text { for A vs. C; MD } \\
-1.44 \text { (95\% Cl }-2.10 \text { to } \\
-0.78 \text { ) for B vs. C }\end{array}$ & NR \\
\hline
\end{tabular}




\begin{tabular}{|c|c|c|c|c|}
\hline $\begin{array}{l}\text { Author, } \\
\text { Year } \\
\text { Quality }\end{array}$ & Interventions & $\begin{array}{l}\text { Procedure; } \\
\text { Followup } \\
\text { Duration } \\
\text { Sample Size }\end{array}$ & Pain Intensity & $\begin{array}{l}\text { Other Pain and Function } \\
\text { Results }\end{array}$ \\
\hline $\begin{array}{l}\text { Forchuk, } \\
2004^{148} \\
\text { Poor }\end{array}$ & $\begin{array}{l}\text { A: Arm massage, } \\
\text { suggested 10- } \\
\text { minute sessions } \\
\text { as needed for } 3 \\
\text { days } \\
\text { B: No massage }\end{array}$ & $\begin{array}{l}\text { Breast cancer } \\
\text { surgery; } \\
1 \text { and } 3 \text { days } \\
n=59\end{array}$ & $\begin{array}{l}\text { Pain control from } \\
\text { massage }^{\mathrm{C}} \text { (mean [SD], \%): } \\
54.4(37.3) \text { vs. NR at day } \\
1 ; 83.18(28.64) \text { vs. NR at } \\
\text { day } 3 \\
\text { Pain control from } \\
\text { medication }^{\mathrm{c}} \text { (mean [SD], } \\
\% \text { ): } 83.18(28.64) \text { vs. } \\
84.74(16.45) \text { at day } 1 ; \\
87.81(13.29) \text { vs. } 80.31 \\
\text { (12.44) at day } 3\end{array}$ & $\begin{array}{l}\text { Shoulder Pain and Disability } \\
\text { Index (mean [SD], } 0 \text { to } 10 \\
\text { scale) at } 2 \text { weeks } \\
\text { Wash back: } 2.86(2.98) \text { vs. } \\
4.89 \text { (3.48), MD }-2.03 \text { ( } 95 \% \\
\mathrm{Cl}-3.72 \text { to }-0.34 \text { ) } \\
\text { Put on undershirt or } \\
\text { pullover: } 2.2(3.0) \text { vs. } 4.04 \\
\text { (3.36), MD }-1.84 \text { (95\% Cl } \\
-3.50 \text { to }-0.18) \\
\text { Place object on high shelf: } \\
3.03(3.40) \text { vs. } 5.0 \text { (3.56), } \\
\text { MD }-1.97 \text { (95\% } \mathrm{Cl}-3.78 \text { to } \\
-0.16) \\
\text { Remove object on high } \\
\text { shelf: } 1.23(2.32) \text { vs. } 2.94 \\
\text { (3.04), MD }-1.71 \text { (95\% Cl } \\
-3.11 \text { to }-0.30)\end{array}$ \\
\hline $\begin{array}{l}\text { Hulme, } \\
1999154 \\
\text { Poor }\end{array}$ & $\begin{array}{l}\text { A: Foot massage } \\
\text { with grapeseed oil } \\
\text { for } 5 \text { minutes } \\
\text { B: Usual care } \\
\text { (standard } \\
\text { analgesia): dose } \\
\text { not reported }\end{array}$ & $\begin{array}{l}\text { Laparoscopic } \\
\text { sterilization; } \\
\text { On discharge } \\
n=59\end{array}$ & $\begin{array}{l}\text { Pain (mean [SD NR], } 0 \text { to } \\
10 \text { NRS): } 3.1 \text { vs. } 3.3, \\
\text { p }>0.05\end{array}$ & $\begin{array}{l}>3 \text { doses of analgesia from } \\
\text { discharge to post-operative } \\
\text { day 1: } 40.0 \%(12 / 30) \text { vs. } \\
48.3 \%(14 / 29), \text { RR } 0.83 \\
\text { (95\% Cl } 0.46 \text { to } 1.48) \\
\\
\text { Pain relief "no pain" or "very } \\
\text { effective": } 30.0 \%(9 / 30) \text { vs. } \\
27.6 \%(8 / 29)\end{array}$ \\
\hline $\begin{array}{l}\text { Koras, } \\
2019^{156} \\
\text { Poor }\end{array}$ & $\begin{array}{l}\text { A: Foot massage, } \\
20 \text { minutes when } \\
\text { pain }>4 \text { on } 0 \text { to } 10 \\
\text { VAS } \\
\text { B: Usual care } \\
\text { (standard } \\
\text { analgesia) } \\
\end{array}$ & $\begin{array}{l}\text { Laparoscopic } \\
\text { cholecystectomy; } \\
2 \text { hours } \\
n=167\end{array}$ & $\begin{array}{l}\text { Pain (mean [SD], } 0 \text { to } 10 \\
\text { VAS): } 1.3 \text { vs. } 3.6, \mathrm{MD} \text { : } \\
-2.3(95 \% \mathrm{Cl}-2.7 \text { to } \\
-1.9)\end{array}$ & $\begin{array}{l}\text { Pain medication use: } 28.2 \% \\
\text { (24/85) vs. } 91.5 \%(75 / 82), \\
\text { RR 0.31 (95\% Cl } 0.22 \text { to } \\
0.44)\end{array}$ \\
\hline $\begin{array}{l}\text { Saatsaz, } \\
2016^{162} \\
\text { Fair }\end{array}$ & $\begin{array}{l}\text { A: Foot massage, } \\
5 \text { minutes each } \\
\text { limb, single } \\
\text { session } \\
\text { B: Hand and foot } \\
\text { massage, } 5 \\
\text { minutes each } \\
\text { limb, single } \\
\text { session } \\
\text { C: Usual care }\end{array}$ & $\begin{array}{l}\text { Cesarean } \\
\text { section; } \\
90 \text { minutes } \\
\mathrm{n}=156\end{array}$ & $\begin{array}{l}\text { Pain (mean [SD], } 0 \text { to } 10 \\
\text { VAS): Post-massage: } \\
3.62(0.69) \text { vs } 3.56(0.57) \\
\text { vs. } 6.17(0.58), \mathrm{MD}:-2.55 \\
\text { (95\% } \mathrm{Cl}-2.80 \text { to }-2.30) \\
\text { for A vs. C and }-2.61 \\
\text { ( } 95 \% \mathrm{Cl}-2.83 \text { to }-2.39) \\
\text { for B vs. C }\end{array}$ & $\mathrm{NR}$ \\
\hline $\begin{array}{l}\text { Sozen, } \\
\text { 2019165 } \\
\text { Poor }\end{array}$ & $\begin{array}{l}\text { A: Foot massage, } \\
20 \text { minutes, single } \\
\text { session } \\
\text { B: Hand massage, } \\
20 \text { minutes, single } \\
\text { session } \\
\text { C: No massage }\end{array}$ & $\begin{array}{l}\text { Laparoscopic } \\
\text { cholecystectomy; } \\
2.5 \text { hours } \\
n=196\end{array}$ & $\begin{array}{l}\text { Pain intensity (mean [SD], } \\
0 \text { to } 10 \text { VAS): } 1.43(1.20) \\
\text { vs. } 1.61 \text { (1.40) vs. } 3.44 \\
(1.43), \mathrm{MD}-2.01(95 \% \mathrm{Cl} \\
-2.47 \text { to }-1.55) \mathrm{A} \text { vs. C } \\
\text { and }-1.83(95 \% \mathrm{Cl}-2.31 \\
\text { to }-1.34 \text { ) for B vs. C }\end{array}$ & $\begin{array}{l}\text { Analgesic use: } 31.7 \% \\
\text { (20/63) vs. } 21.5 \%(14 / 65) \\
\text { vs. } 89.7 \%(61 / 68), \text { RR } 0.35 \\
\text { ( } 95 \% \mathrm{Cl} 0.24 \text { to } 0.51 \text { ) for A } \\
\text { vs. C and RR } 0.28 \text { (95\% Cl } \\
0.18 \text { to } 0.45 \text { ) for B vs. C }\end{array}$ \\
\hline
\end{tabular}

Abbreviations: $\mathrm{CI}$ = confidence interval; $\mathrm{NR}$ = not reported; $\mathrm{MD}=$ mean difference; $\mathrm{RR}$ = relative risk; $\mathrm{SD}=$ standard deviation; VAS = visual analog scale

${ }^{c}$ Unclear how patients distinguished pain control from massage and pain control from medication 


\section{Music Therapy}

Two fair quality trials compared music therapy versus no music therapy for postoperative pain (Table 26). ${ }^{147,158}$ One trial $(\mathrm{n}=50)$ found music administered in the recovery unit associated with moderate effects on pain intensity versus no music at 1 hour (mean difference -1.70 on a 0 to 10 scale, $95 \%$ CI -2.6 vs. -0.73 ) and decreased anxiety (mean difference -0.9 point on a 0 to 10 scale, $95 \%$ CI -1.7 to -0.1$).{ }^{158}$ Music was also associated with decreased morphine use, but the difference was small (mean difference $-1.9 \mathrm{mg}, 95 \% \mathrm{CI}-3.34$ to -0.46 ). The other trial $(\mathrm{n}=98)$ evaluated four different types of music administered for 12 to 15 minutes for 3 days versus no music. ${ }^{147}$ Effects on pain intensity favored the music intervention on day 1 (mean difference 0.68 to 1.69 points on a 0 to 10 scale) but there were no differences on day 3 . There was also no difference in scores on the Profile of Mood States scale (data not provided). Harms were not reported, though not expected with this intervention. 
Table 26. Music therapy versus silence, usual care, or relaxation for postoperative pain

\begin{tabular}{|c|c|c|c|c|}
\hline $\begin{array}{l}\text { Author, Year } \\
\text { Quality }\end{array}$ & Interventions & $\begin{array}{l}\text { Procedure; } \\
\text { Followup Duration } \\
\text { Sample Size }\end{array}$ & Pain Intensity & Other Results \\
\hline $\begin{array}{l}\text { Finlay } \\
2016^{147} \\
\text { Fair }\end{array}$ & $\begin{array}{l}\text { A: High harmonicity and } \\
\text { rhythmicity music } \\
\text { B: Low harmonicity and low } \\
\text { rhythmicity music } \\
\text { C: Low harmonicity and high } \\
\text { rhythmicity music } \\
\text { D: High harmonicity and low } \\
\text { rhythmicity music } \\
\text { E: Control, silence using } \\
\text { noise-canceling headphones } \\
\text { All interventions used } 12 \text { to } 15 \\
\text { minutes per day for } 3 \text { days }\end{array}$ & $\begin{array}{l}\text { Knee arthroplasty; } \\
1 \text { and } 3 \text { days } \\
n=98\end{array}$ & 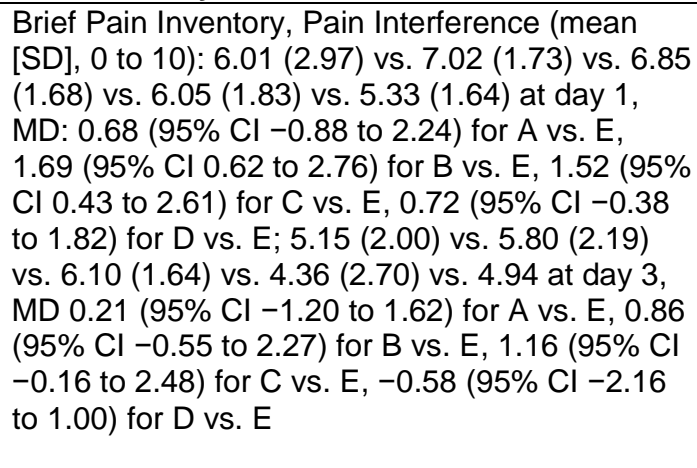 & NR \\
\hline $\begin{array}{l}\text { Nilsson } \\
2005^{158} \\
\text { Fair }\end{array}$ & $\begin{array}{l}\text { A: Music CD upon arrival in } \\
\text { PACU and for } 1 \text { hour after } \\
\text { B: Sham CD player (numbers } \\
\text { shown, no sound) }\end{array}$ & $\begin{array}{l}\text { Hernia repair; } \\
1 \text { hour } \\
n=50\end{array}$ & $\begin{array}{l}\text { Pain intensity (mean [SD], } 0 \text { to } 10 \mathrm{NRS}): 2.1 \\
(1.5) \text { vs. } 3.8(1.9) \text { at } 1 \text { hour, } \mathrm{MD}-1.70(95 \% \mathrm{Cl} \\
-2.6 \text { to }-0.73)\end{array}$ & $\begin{array}{l}\text { Use of analgesic } \\
\text { medication: } 30 \%(6 / 20) \\
\text { vs. } 30 \%(6 / 20)\end{array}$ \\
\hline
\end{tabular}

Abbreviations: $\mathrm{CI}$ = confidence interval; $\mathrm{MD}$ = mean difference; $\mathrm{NR}$ = not reported; $\mathrm{NRS}$ = numeric rating scale; $\mathrm{SD}$ = standard deviation 


\section{Exercise Versus No Exercise}

One fair quality trial $(n=80)$ compared neck stretching exercises versus no exercise following thyroidectomy patients (Table 27). ${ }^{138}$ Five repetitions of eight exercises were performed three times daily for 1 month. There was no difference between exercise versus no exercise in pain with neck movement at 1 week or 1 month, but pain intensity was very low in both groups. Exercise was associated with better Neck and Disability Score at 1 week (mean difference -2.15 on a 0 to 10 scale, $95 \%$ CI -2.69 to -1.61 ), but there was no effect at 1 month (mean difference: $-0.18,95 \%$ CI -0.40 to 0.04$)$.

Table 27. Neck stretching exercises versus no exercise for postoperative pain

\begin{tabular}{|c|c|c|c|}
\hline $\begin{array}{l}\text { Author, Year } \\
\text { Quality }\end{array}$ & Interventions & $\begin{array}{l}\text { Procedure; } \\
\text { Followup } \\
\text { Duration } \\
\text { Sample Size }\end{array}$ & Pain Intensity \\
\hline $\begin{array}{l}\text { Ayhan, } \\
2016^{138} \\
\text { Fair }\end{array}$ & $\begin{array}{l}\text { A: } 5 \text { sets, } 8 \text { different neck } \\
\text { exercises, three times a } \\
\text { day for one month } \\
\text { B: Resume pre-op neck } \\
\text { motion }\end{array}$ & $\begin{array}{l}\text { Thyroidectomy; } \\
1 \text { week and } 1 \\
\text { month } \\
n=80\end{array}$ & $\begin{array}{l}\text { Neck Pain and Disability Score (mean [SD], } 0 \text { to } \\
100 \text { scale converted to } 0 \text { to } 10): 0.88(1.22) \text { vs. } \\
3.03(1.21) \text { at } 1 \text { week, } \mathrm{MD}-2.15(95 \% \mathrm{Cl}-2.69 \text { to } \\
-1.61) ; 0.17(0.27) \text { vs. } 0.35(0.66) \text { at } 1 \text { month, MD } \\
-0.18(95 \% \mathrm{Cl}-0.40 \text { to } 0.04) \\
\text { Pain with neck movement (median [IQR], } 0 \text { to } 14 \\
\text { scale): } 0(0.20) \text { vs. } 0.35(0.58) \text { at } 1 \text { week, } 0(0.0) \\
\text { vs. } 0(1.8) \text { at } 1 \text { month }\end{array}$ \\
\hline
\end{tabular}

Abbreviations: $\mathrm{CI}$ = confidence interval; IQR = interquartile range; $\mathrm{MD}$ = mean difference; $\mathrm{SD}$ = standard deviation;

\section{TENS Versus Sham or No TENS}

Two small trials ( $\mathrm{n}=12$ and 42) evaluated transcutaneous electrical nerve stimulation for postoperative pain (Table 28). ${ }^{143,161}$ One fair quality trial $(n=42)$ found TENS associated with decreased pain intensity versus sham TENS following liposuction at 12 hours (median 0 vs. 3.0 on a 0 to 10 scale, $\mathrm{p}=0.001$ ) and at 24 hours (median 0 vs. 2.0, $\mathrm{p}=0.001$ ), though pain scores were low in both groups. ${ }^{143}$ TENS was also associated with decreased analgesic use (19.0\% vs. $100 \%$ at 24 hours, RR $0.19,95 \%$ CI 0.08 to 0.46$)$. A very small $(n=12)$, poor quality trial of patients following cholecystectomy found TENS associated with decreased morphine use versus no TENS, but estimates were imprecise and differences were not statistically significant. ${ }^{161}$ One trial $^{161}$ reported minor skin reactions at the TENS electrode sites and one trial ${ }^{143}$ reported drowsiness and nausea in patients randomized to sham TENS. No serious adverse events were reported.

\section{Neuromuscular Stimulation Versus Sham Neuromuscular Stimulation}

One small $(\mathrm{n}=29)$ trial compared neuromuscular stimulation (two 20-minute sessions daily) versus sham stimulation following total hip arthroplasty (Appendix E, Table E-6; Table 28). ${ }^{142}$ It was rated poor quality; methodological limitations included high loss to followup and lack of intention to treat analysis (Appendix F, Table F-1). Neuromuscular stimulation was not associated with decreased pain intensity at discharge or at 6 weeks, less use of opioids, or improved function versus sham stimulation. Device malfunctions occurred in 7.5 percent (3/40) of patients randomized to neuromuscular stimulation. 
Table 28. Transcutaneous electrical nerve stimulation or neuromuscular stimulation versus no treatment or sham

\begin{tabular}{|c|c|c|c|c|}
\hline $\begin{array}{l}\text { Author, } \\
\text { Year } \\
\text { Quality }\end{array}$ & Interventions & $\begin{array}{l}\text { Procedure; } \\
\text { Followup } \\
\text { Duration } \\
\text { Sample Size }\end{array}$ & Pain Intensity & Other Results \\
\hline $\begin{array}{l}\text { Castellano, } \\
2016^{142} \\
\text { Poor }\end{array}$ & $\begin{array}{l}\text { A: NMES, } 2 \text { 20- } \\
\text { minute sessions } \\
\text { daily } \\
\text { B: Sham } \\
\text { stimulation, } \\
\text { subclinical level } \\
\text { of stimulation } \\
\text { for } 2 \text { 20-minute } \\
\text { sessions daily }\end{array}$ & $\begin{array}{l}\text { Total hip } \\
\text { arthroplasty; } \\
\text { At discharge and } \\
6 \text { weeks } \\
n=29\end{array}$ & $\begin{array}{l}\text { Pain intensity change } \\
\text { from baseline (mean } \\
\text { [SD NR], } 0 \text { to } 10 \\
\text { VAS): } 0.07 \text { vs. }-0.21 \\
\text { at discharge, } p>0.05 ; \\
-1.73 \text { vs. }-2.21 \text { at } 6 \\
\text { weeks, } p>0.05\end{array}$ & $\begin{array}{l}\text { Use of opioids (mean [SD NR], } \\
\text { days): } 3.13 \text { vs. } 3.36, p=N S\end{array}$ \\
\hline $\begin{array}{l}\text { da Silva, } \\
2015^{143} \\
\text { Fair }\end{array}$ & $\begin{array}{l}\text { A: TENS, one } \\
\text { 30-minute } \\
\text { session } \\
\text { B: Sham TENS, } \\
\text { one 30-minute } \\
\text { session with no } \\
\text { current }\end{array}$ & $\begin{array}{l}\text { Liposuction; } \\
12 \text { and } 24 \text { hours } \\
n=42\end{array}$ & $\begin{array}{l}\text { Pain intensity: } \\
\text { (median [IQR], } 0 \text { to } \\
10 \text { VAS): } 0 \text { (0 to } 0) \\
\text { vs. } 3.0(3.0 \text { to } 5.0) \text { at } \\
12 \text { hours, } p=0.001 ; 0 \\
\text { ( } 0 \text { to } 0) \text { vs. } 2.0(2.0 \text { to } \\
3.0) \text { at } 24 \text { hours, } \\
p=0.001\end{array}$ & $\begin{array}{l}\text { Request for analgesics: } 8.7 \% \\
(2 / 21) \text { vs. } 95.2 \%(20 / 21) \text { at } 12 \\
\text { hours, RR } 0.25(95 \% \text { Cl } 0.06 \text { to } \\
1.04) ; 19.0 \%(4 / 21) \text { vs. } 100 \% \\
(21 / 21) \text { at } 24 \text { hours, RR } 0.19 \\
(95 \% \text { Cl } 0.08 \text { to } 0.46)\end{array}$ \\
\hline $\begin{array}{l}\text { Rosenberg, } \\
1978^{161} \\
\text { Poor }\end{array}$ & $\begin{array}{l}\text { A: TENS, } \\
\text { duration and } \\
\text { number of } \\
\text { sessions per } \\
\text { patient } \\
\text { B: No TENS }\end{array}$ & $\begin{array}{l}\text { Cholecystectomy } \\
; \\
\text { Day of surgery } \\
\text { and } 2 \text { days after } \\
\text { surgery } \\
n=12\end{array}$ & NR & $\begin{array}{l}\text { Opioid use (mean [SD], } \\
\text { morphine equivalents/day): } 8.5 \\
\text { (2.4) vs. } 11.5 \text { ( } 15.9 \text { ) day of } \\
\text { surgery, MD }-3.00 \text { ( } 95 \% \mathrm{Cl} \\
-17.65 \text { to } 11.65) ; 5.5 \text { (15.9) vs. } \\
19.0 \text { (36.7) postoperative day } 2 \text {, } \\
\text { MD }-13.50 \text { (95\% } \mathrm{Cl}-49.93 \text { to } \\
22.93) ; 7.5(7.3) \text { vs. } 19.0(17.1) \\
\text { total, MD }-11.50 \text { (95\% Cl }-28.47 \\
\text { to } 5.47)\end{array}$ \\
\hline
\end{tabular}

Abbreviations: ACL = anterior cruciate ligament; $\mathrm{CI}$ = confidence interval; IQR = interquartile range; $\mathrm{MD}=$ mean difference; NMES = neuromuscular electrical stimulation; $\mathrm{NR}=$ not reported; $\mathrm{RR}=$ relative risk; $\mathrm{SD}=$ standard deviation; TENS = Transcutaneous Electrical Nerve Stimulation; VAS = visual analog scale

KQs 50 and 5p ask how the comparative effectiveness and harms of nonpharmacologic therapy vary depending on: (1) patient demographics (e.g., age, gender); (2) patient medical and psychiatric comorbidities; (3) the type of treatment used; (4) the frequency of therapy; (5) the duration of therapy.

Evidence on how the comparative effectiveness and harms of nonpharmacologic therapy for postoperative pain varied based on patient and intervention factors was lacking. The number of trials and sample sizes for each comparison were too small for between study comparisons to be informative and no trial conducted within-study subgroup analyses.

\section{KQ 6. Dental Pain (Surgical and Nonsurgical After Discharge)}

\section{Key Points}

- Opioid plus acetaminophen or NSAID vs. NSAID, primarily surgical pain

o A single dose of an opioid plus acetaminophen or NSAID was associated with small to moderate increase in pain intensity versus an NSAID at $<1$ day (12 trials) and increased likelihood of rescue or repeat medication use (9 trials, RR 1.35, 95\% CI 1.23 to 1.48) (SOE: low for pain intensity, moderate for rescue or repeat medication use). 
o No difference between a multidose course of therapy with an opioid plus acetaminophen or an NSAID versus an NSAID in pain intensity at 1 day to $<1$ week, based on 3 trials (SOE: low).

- Opioid versus NSAID, surgical or nonsurgical dental pain

o Insufficient evidence due to inconsistent results from six trials (SOE: insufficient).

- Opioid (with or without acetaminophen) versus NSAID, primarily surgical pain

- Opioid associated with increased likelihood of any adverse event (11 trials, pooled RR 1.72, 95\% CI 1.29 to 2.28), nausea (12 trials, pooled RR 2.72, 95\% CI 1.84 to 4.01), dizziness ( 10 trials, pooled RR 2.97, 95\% CI 1.59 to 5.54), and drowsiness (9 trials, pooled RR 1.76, 95\% CI 1.00 to 3.10) (SOE: moderate).

- Opioid plus acetaminophen vs. acetaminophen, single dose, surgical pain

o Inconsistent effects of opioid plus acetaminophen versus acetaminophen on pain intensity at $<1$ day (11 trials), but opioid plus acetaminophen associated with larger sum of pain intensity differences at $<1$ day (10 trials) and decreased likelihood of rescue or repeat medication use ( 7 trials, pooled RR $0.81,95 \%$ CI 0.67 to 0.97 ) (SOE: moderate for sum of pain intensity differences and rescue or repeat medication use).

- Opioid vs. acetaminophen, single dose, surgical pain

o Similar effects on pain intensity at $<1$ day (2 trials) and likelihood of rescue medication use based on one trial (SOE: low).

- Opioid (with or without acetaminophen) vs. acetaminophen, surgical pain

o Opioid may be associated with increased likelihood of any adverse event (8 trials), nausea (8 trials), drowsiness (6 trials), and dizziness ( 5 trials), but differences were not statistically significant, and estimates were imprecise (SOE: low).

- Opioid agonist versus tramadol, surgical or nonsurgical pain

o Insufficient evidence from two trials with inconsistent results (SOE: insufficient).

- NSAID versus acetaminophen, single dose, surgical pain

o NSAIDs were associated with moderate to large decrease in pain intensity versus acetaminophen at $<1$ day (14 trials) and decreased likelihood of rescue or repeat medication use ( 11 trials, pooled RR $0.64,95 \%$ CI 0.58 to 0.71 ) (SOE: moderate).

0 NSAIDS may be associated with slightly decreased risk of any adverse event versus acetaminophen (12 trials, pooled RR $0.85,95 \%$ CI 0.72 to 1.00) (SOE: low).

- Pregabalin versus NSAID, surgical pain

o Insufficient evidence to determine effects on pain intensity, based on 1 trial (SOE: insufficient).

o Pregabalin $300 \mathrm{mg}$ associated with increased risk of any adverse event versus ibuprofen (RR 5.55, 95\% CI 2.56 to 12.03) (SOE: low).

- Non-aspirin NSAID versus aspirin, surgical pain

o Non-aspirin NSAID associated with a moderate to large decrease in pain intensity versus aspirin at $<1$ day, based on two trials (SOE: low).

- Acupuncture versus sham acupuncture, surgical pain

o Insufficient evidence from one poor quality trial (SOE: insufficient).

- Cold therapy versus no cold therapy, surgical pain

o Insufficient evidence on pain intensity at 1 day to $<1$ week, based on four trials (SOE: insufficient). 


\section{Summary of Findings}

Forty-six trials $(\mathrm{N}=7,119)$ evaluated interventions for dental pain (Appendix E, Table E8). ${ }^{175-218}$ Twenty-five trials evaluated opioid therapy (KQ 6a and 6c), ${ }^{175,177,179,181,184-}$

192,194,195,201,205,207,210-213,215,218 15 trials evaluated a nonopioid medication versus nonpharmacologic treatment or another nonopioid (KQ 6i and 6j), ${ }^{176,178,180,182,193,197,198,202-}$ 204,206,208,209,216,219 and 6 trials (KQ 6m and 6n) evaluated nonpharmacologic treatment versus an inactive control or another nonpharmacologic treatment. ${ }^{183,196,199,200,214,217}$ The most common dental procedure was third molar extraction (40 trials). ${ }^{175-178,180-203,207-209,212-219}$ Other procedures were root canals, ${ }^{179,205}$ apical periodontitis, ${ }^{210}$ periradicular abscess surgery, ${ }^{211}$ and various oral surgery procedures. ${ }^{204}$ For dental conditions associated with pain prior to surgery, the duration of pain was not reported. Thirty-five trials evaluated single doses of medications. ${ }^{176-178,180-}$ $182,184,186,188-195,197,198,201-205,207-210,212,213,215,216,219$ In the other trials, the duration of treatment ranged from 45 minutes to 4 days. The duration of followup was less than one week in 44 trials $^{175-182,184-199,201-219}$ and 1 week to $<4$ weeks in 2 trials. ${ }^{183,200}$ Twenty-eight trials were conducted in the United States, ${ }^{177-180,182,187-195,197,200-204,207-209,213,215,216,219} 7$ trials in Europe, ${ }^{176,181,185,186,196,198,212}$ and 11 trials elsewhere. ${ }^{175,183,184,199,205,206,210,211,214,217,218}$ The mean age ranged from 18 to 43 years. Few trials reported race or ethnicity. Eleven trials excluded patients with a history of substance use disorder, ${ }^{175,178,190,194,201,203,207,209,213,215,218} 28$ trials excluded pregnant or breastfeeding patients, ${ }^{175,177-181,183,187,190,194-198,200,201,203,206-211,213-215,218} 1$ trial excluded patients with psychiatric illness, ${ }^{178,215}$ and 11 trials excluded patients with other comorbidities. ${ }^{176,178,182,190,194,201,203,206,208,211,218}$ Three trials were rated good quality, ${ }^{181,186,202} 33$ trials fair quality, ${ }^{175-180,182,184,186-189,192,193,196-198,201,203-205,207-210,212-216,218-220}$ and 10 trials poor quality (Appendix F, Table F-1). ${ }^{183,190,191,194,195,199,200,206,211,217}$ Methodological limitations in the fair and poor quality trials included failure to report adequate randomization or allocation concealment methods, unblinded design, failure to report attrition, high attrition, and no intention to treat analysis. In most trials, results for pain intensity and pain intensity differences at 4 hours and at 6 to 8 hours were estimated from graphs; the statistical significance of differences were usually not reported or calculable (e.g., standard deviations not provided).

\section{Detailed Synthesis}

\section{Opioid Therapy}

KQs 6a and 6c address the comparative effectiveness and harms of opioid therapy versus: (1) nonopioid pharmacologic therapy (e.g., acetaminophen, NSAIDs, antidepressants, anticonvulsants) or (2) nonpharmacologic therapy (e.g., exercise, cognitive behavioral therapy, acupuncture).

Twenty-five trials evaluated opioid therapy for acute dental pain (Appendix E, Table E8). ${ }^{175,177,179,181,184-192,194,195,201,205,207,210-213,215,218} 11$ trials compared an opioid versus acetaminophen, ${ }^{184-186,188,190-192,194,195,212,213} 18$ trials an opioid versus an NSAID, ${ }^{175,177,179,181,186,187,189-191,194,195,201,205,210,213,215,218 ~ a n d ~} 2$ trials an opioid agonist versus a mixed agent (tramadol). ${ }^{207,211} 23$ trials blinded patients and caregivers to the study medications. ${ }^{175,177,179,181,190,194,195,205,207,210-213,215,218}$ Two trials were rated good quality, ${ }^{181,186} 18$ trials fair quality, ${ }^{175,177,179,184,185,187-189,192,201,205,207,210,212,213,215,218}$ and 5 trials poor quality (Appendix F, Table F-1). ${ }^{190,191,194,195,211}$ 


\section{Opioid Versus NSAID}

Eighteen trials compared an opioid (alone or in combination with acetaminophen or an NSAID) versus an NSAID for acute dental pain (Table 29). ${ }^{175,177,179,181,186,187,189-}$

$191,194,195,201,205,210,213,215,218$ Dental pain was due to third molar extraction in fifteen trials, ${ }^{175,177,181,186,187,189-191,194,195,201,213,215,218}$ root canal instrumentation for irreversible pulpitis in two trials, ${ }^{179,205}$ and acute apical periodontitis in one trial. ${ }^{210}$ The sample size for the opioid

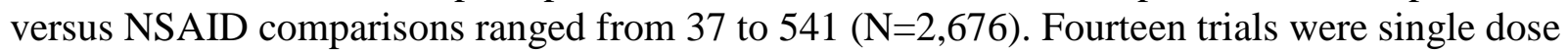
studies, and four trials ${ }^{175,179,187,218}$ evaluated three or four days of therapy. In the single dose trials, the duration of followup ranged from ninety minutes to 24 hours. Eight trials evaluated multiple opioid or NSAID arms. ${ }^{179,181,189,191,194,195,213,215}$ The opioid was codeine in 13 trials (dose $60 \mathrm{mg}$ in 11 trials, ${ }^{177,186,187,189-191,194,195,201,213} 50 \mathrm{mg}$ in 1 trial, ${ }^{218}$ and $15 \mathrm{mg}$ in 1 trial $^{210}$ ), oxycodone in 1 trial (5 mg), ${ }^{215}$ and tramadol in 4 trials ${ }^{175,179,181,205}$ (100 mg). In 11 trials, the opioid was administered with acetaminophen (dose 325 to 1000 mg), ${ }^{177,186,187,190,191,194,195,201,210,213 ~ f i v e ~ t r i a l s ~ e v a l u a t e d ~ t h e ~ o p i o i d ~ a d m i n i s t e r e d ~ w i t h ~ a n ~ N S A I D ~}$ (ibuprofen $400 \mathrm{mg}$, aspirin $650 \mathrm{mg}$, diclofenac $50 \mathrm{mg}$, flurbiprofen $50 \mathrm{mg}$ [after $100 \mathrm{mg}$ loading dose], and dexketoprofen trometamol 12.5 or $25 \mathrm{mg}),{ }^{179,181,189,215,218}$ and six trials evaluated an opioid alone. ${ }^{175,179,181,189,205,215}$ The opioid was compared with the NSAID ibuprofen in five trials (400 or $600 \mathrm{mg}),{ }^{181,187,189,195,215}$ naproxen or naproxen sodium in four trials (440, 500 or 550 $\mathrm{mg}),{ }^{177,201,205}$ diclofenac in two trials (50 or $100 \mathrm{mg}$ ), ${ }^{186,218}$ flurbiprofen in four trials (50 or 100 $\mathrm{mg}),{ }^{179,191,194,213}$ and aspirin (650 mg), ${ }^{189}$ meclofenamate sodium (100 mg), ${ }^{190}$ ketorolac or ketorolac tromethamine (10 or $20 \mathrm{mg}),{ }^{195,210}$ or dexketoprofen trometamol (12.5 or $\left.25 \mathrm{mg}\right)^{181}$ in one trial each.

All of the trials used a blinded design with matching placebo, with the exception of one that blinded without using a placebo ${ }^{175}$ and two trials ${ }^{191,201}$ in which blinding status was unclear. One trial $^{218}$ used a crossover design, and the others were parallel group trials. Two trials was rated good quality, ${ }^{181,186}$ twelve trials fair quality, ${ }^{175,177,179,187,189,201,205,210,213,215,218}$ and four trials poor quality (Appendix F, Table F-1). ${ }^{190,191,194,195}$

Fifteen trials evaluated effects of an opioid plus acetaminophen or NSAID versus an NSAID alone on pain intensity (based on the pain intensity difference [PID] or mean pain intensity). ${ }^{177,179,181,186,187,189-191,194,195,210,213,215}$ The trials varied in timing of outcome assessment. One trial of patients with apical periodontitis $(n=63)$ found codeine plus acetaminophen associated with a large reduction in pain intensity versus ketorolac tromethamine at 90 minutes (mean 4.34 vs. 1.00 on a 0 to 10 scale, $p=0.05$ ); ${ }^{210}$ longer-term followup was not reported. Nine trials $(\mathrm{N}=1,556)$ evaluated pain intensity at 4 hours in patients who underwent third molar extraction. In six trials, an opioid plus acetaminophen or an NSAID was associated with lesser effects on pain intensity than an NSAID alone (differences ranged from 0.2 to 2.1 points on a 0 to 10 scale). ${ }^{189-191,194,195,213}$ In one trial, oxycodone plus ibuprofen combination therapy was associated with lesser effects on pain intensity at 4 hours than an opioid alone by 4.0 points, but only by 0.5 points compared with ibuprofen alone. ${ }^{215}$ Another trial found that differences between an opioid plus NSAID versus an NSAID alone in pain intensity at 4 hours varied between various dose combinations of tramadol (37.5 or $75 \mathrm{mg}$ ) and dexketoprofen trometamol (12.5 or $25 \mathrm{mg}$ ) versus dexketoprofen (12.5 or $25 \mathrm{mg}$ ) or ibuprofen (400 mg) (differences ranged from -3.0 to 2.0 points on a 0 to 10 scale).$^{181}$ In the ninth trial, opioid combination therapy was favored by 1.0 point. ${ }^{186}$ Three trials $(\mathrm{N}=465)$ did not report effects on pain intensity but found codeine plus acetaminophen associated with less pain relief versus naproxen sodium at 4 hours (mean 0.8 to 3.8, p values not reported). ${ }^{177,201}$ Four trials $(\mathrm{N}=375)$ found single dose codeine plus 
acetaminophen associated with lesser effects on pain intensity at 6 hours (after analgesia had peaked) versus an NSAID alone (differences 0.1 to 2.7 points on a 0 to 10 scale). ${ }^{191,194,195,213}$ One trial $(n=25)$ found tramadol plus an NSAID associated with lower pain intensity versus an NSAID alone at 6 hours (2.3 vs. 3.8 on a 0 to 10 scale). ${ }^{179}$ One trial $(n=45)$ found little difference between single dose codeine plus acetaminophen versus diclofenac in pain intensity at 8 hours (3.3 versus 3.5, p not reported). ${ }^{186}$ Two trials $(\mathrm{N}=300)$ of patients who underwent third molar extraction found no difference between a course of therapy with codeine plus acetaminophen or an NSAID versus an NSAID alone in pain intensity at 3 to 4 days; ${ }^{187,218}$ one of the trials found codeine plus acetaminophen associated with lesser effects on pain relief at $<1$ day. ${ }^{220}$ One trial $(n=25)$ of patients with endodontic pain following a root canal found a course of tramadol plus flurbiprofen associated with decreased pain intensity versus flurbiprofen alone at 2 days (0 vs. 0.8 on a 0 to 10 scale). ${ }^{179}$

Ten trials ${ }^{177,189-191,194,195,201,213,215}(\mathrm{~N}=1,550)$ of patients who underwent third molar extraction tended to find lesser effects on the sum of pain intensity differences (SPID) for an opioid plus acetaminophen or an NSAID versus an NSAID alone, though there was some inconsistency. One $\operatorname{trial}^{186}(\mathrm{n}=45)$ found codeine plus acetaminophen associated with lower average pain intensity (mean of all measurements) versus diclofenac ( $2.5 \mathrm{vs.} 3.8$ on a 0 to 10 scale, $p=0.02$ ). An opioid plus acetaminophen or an NSAID was associated with increased likelihood of rescue or repeat medication use versus an NSAID (9 trials, $\mathrm{N}=1,180$, pooled RR 1.35, 95\% CI 1.23 to 1.48, $\mathrm{I}^{2}=0 \%$; ARD 15\%, 95\% CI 9\% to 22\%; Appendix D, Figure D-1). ${ }^{177,186,187,190,191,195,201,213}$ Effects on the likelihood of being rated "very good" or "excellent" favored opioids, but the difference was of borderline statistical significance ( 7 trials, $\mathrm{N}=983$, pooled RR $0.76,95 \%$ CI 0.57 to 1.00 , $\mathrm{I}^{2}=74 \%$; Appendix D, Figure D-2). ${ }^{177,187,190,191,201,218}$ Findings for rescue or repeat medication use and likelihood of being rated "very good" or "excellent” were similar when poor quality trials were excluded from the pooled analyses. One trial $(n=136)$ found single dose codeine plus acetaminophen associated with decreased likelihood of 50\% pain relief versus an NSAID (13\% vs. 36\%, RR 0.37 , 95\% CI 0.16 to 0.87$).{ }^{195}$ Quality of life and function were not reported in the trials.

Six trials $(\mathrm{N}=1,011)$ compared an opioid alone versus an NSAID. ${ }^{175,179,181,189,205,215}$ One trial $(n=50)$ of patients who underwent root canal instrumentation found a single dose of tramadol associated with higher pain intensity than naproxen at 6 hours (mean difference 2.4 on a 0 to 10 scale, $95 \%$ CI 1.2 to 3.6) and 24 hours (mean difference $1.5,95 \%$ CI 0.5 to 2.5 ). ${ }^{205}$ Another small trial $(n=24)$ of patients with endodontic pain following root canal found a course of tramadol and flurbiprofen associated with similar pain intensity at 6 hours and 2 days. ${ }^{179}$ Three trials compared a single dose of opioid alone versus an NSAID in patients who underwent third molar extraction. ${ }^{181,189,215}$ Results for pain intensity were somewhat inconsistent. One trial $(n=298)$ that compared different doses of tramadol (37.5 or $75 \mathrm{mg}$ ) versus dexketoprofen trometamol (12.5 mg or $25 \mathrm{mg}$ ) or ibuprofen (400 mg) found that differences in pain intensity at 4 hours ranged from -2.7 to 0.3 points on a 0 to 10 scale. ${ }^{181}$ One trial $(n=117)$ found a single dose of codeine associated with a larger PID than ibuprofen or aspirin (differences 0.4 to 0.8 on a 0 to 10 scale, $p$ not reported), though the difference was small. ${ }^{189}$ The third trial $(n=435)$ found a single $5 \mathrm{mg}$ dose of oxycodone associated with a smaller PID than a single dose of ibuprofen $\left(-0.4\right.$ vs. 2.6 at 6 hours, $p$ not reported). ${ }^{215}$ One other trial $(n=87)$ that evaluated a course of therapy after third molar extraction found tramadol associated with higher pain intensity at 4 hours (5.2 vs. 2.4 on a 0 to 10 scale, $\mathrm{p}=0.001$ ), but differences were smaller and not statistically significant at 8 hours (3.2 vs. 2.3) or 2 days (0.8 vs. 0.4$).{ }^{175}$ 
Opioids (with or without acetaminophen or an NSAID) were associated with increased risk of any adverse event versus an NSAID alone ( 11 trials, N=1,900, pooled RR 1.72, 95\% CI 1.29 to $2.28, \mathrm{I}^{2}=47 \%$; ARD $14 \%$, 95\% CI $7 \%$ to $22 \%$ Appendix D, Figure D-

3), ${ }^{175,177,187,189,191,194,195,201,213,215}$ nausea (12 trials, $\mathrm{N}=2,405$, pooled RR 2.72, 95\% CI 1.84 to 4.01, $\mathrm{I}^{2}=0 \%$; ARD 5\%, 95\% CI 2\% to 7\%; Appendix D, Figure D4), ${ }^{175,177,181,186,187,189,191,194,195,201,215}$ dizziness (10 trials, $N=2,286$, pooled RR 2.97, 95\% CI 1.59 to 5.54, $\mathrm{I}^{2}=0 \%$; ARD 3\%, 95\% CI 1\% to 6\%; Appendix D, Figure D-5), ${ }^{175,177,181,187,189,191,195,201,215}$ and drowsiness ( 9 trials, $\mathrm{N}=1,643$, pooled RR 1.76, 95\% CI 1.00 to $3.10, \mathrm{I}^{2}=29 \%$; ARD 3\%, 95\% CI -1\% to 8\%, Appendix D, Figure D-6). ${ }^{175,177,181,186,189,191,195,201}$ The estimate for headache was imprecise ( 9 trials, $\mathrm{N}=1,832$, pooled RR 0.99 , 95\% CI 0.46 to $2.11, \mathrm{I}^{2}=24 \%$ Appendix D, Figure D-7). ${ }^{177,181,186,189,191,194,195,201}$ The number of adverse events in most trials was small, and all trials except for two were single dose studies. ${ }^{175,187}$ The larger multidose trial $(\mathrm{n}=254)$, which evaluated dosing every 6 hours for three days, found codeine plus acetaminophen associated with increased risk of headache (6.5\% vs. $0.5 \%$, RR $12.41,95 \%$ CI 1.41 to 108.77$)$, nausea (9.7\% vs. $2.1 \%$, RR 4.65 , 95\% CI 1.35 to 15.93 ), and vomiting (8.1\% vs. $0.5 \%$, RR $15.48,95 \%$ CI 1.84 to 130.02) versus ibuprofen. ${ }^{187}$ In this trial, the difference in risk of study withdrawal due to adverse events was not statistically significant (4.8\% vs. $0.5 \%$, RR 9.29, 95\% CI 0.98 to 87.71 ). 


\begin{tabular}{|c|c|c|c|c|}
\hline $\begin{array}{l}\text { Study, Year } \\
\text { Quality }\end{array}$ & Interventions & $\begin{array}{l}\text { Procedure; } \\
\text { Followup } \\
\text { Duration } \\
\text { Sample Size }\end{array}$ & Pain Intensity Results & Other Results \\
\hline $\begin{array}{l}\text { Akinbade, } 2019^{175} \\
\text { Fair }\end{array}$ & $\begin{array}{l}\text { A. Tramadol } 100 \mathrm{mg} \\
\text { every } 8 \text { hours for } 48 \\
\text { hours } \\
\text { B. Celecoxib } 400 \mathrm{mg} \\
\text { followed by } 200 \mathrm{mg} \text { every } \\
12 \text { hours for } 48 \text { hours }\end{array}$ & $\begin{array}{l}\text { Third molar } \\
\text { extraction; } \\
<1 \text { day }(4,8, \\
\text { and } 16 \text { hours }) \\
<1 \text { week ( } 1 \\
\text { and } 2 \text { days) } \\
\mathrm{n}=87\end{array}$ & $\begin{array}{l}\text { Pain, (median [range], } 0 \text { to } 100 \text { VAS converted } \\
\text { to } 0 \text { to } 10 \text { scale: } 5.2(0.5 \text { to } 10.0) \text { vs. } 2.4 \text { ( } 0 \text { to } \\
0.97) \text { at } 4 \text { hours ( } p=0.001) ; 3.2(0 \text { to } 9.8) \text { vs. } \\
2.3(0 \text { to } 8.3) \text { at } 8 \text { hours }(p=0.12) ; 1.6(0 \text { to } 7.8) \\
\text { vs. } 1.5(0 \text { to } 9.8) \text { at } 16 \text { hours }(p=0.63) ; 1.0(0 \text { to } \\
7.9) \text { vs. } 0.7(0 \text { to } 9.8) \text { at } 1 \text { day }(p=0.21) ; 0.8(0 \\
\text { to } 8.0) \text { vs. } 0.4(0 \text { to } 8.9) \text { at day } 2(p=0.19)\end{array}$ & NR \\
\hline $\begin{array}{l}\text { Breivik, 1999186 } \\
\text { Good }\end{array}$ & $\begin{array}{l}\text { A: Codeine } 60 \mathrm{mg}+ \\
\text { acetaminophen } 1000 \mathrm{mg} \\
\times 1 \\
\text { B: Diclofenac } 100 \mathrm{mg} \times 1\end{array}$ & $\begin{array}{l}\text { Third molar } \\
\text { extraction; } \\
<1 \text { day ( } 8 \\
\text { hours) } \\
\mathrm{n}=45\end{array}$ & $\begin{array}{l}\text { Pain (mean [SD NR], } 0 \text { to } 100 \text { VAS converted } \\
\text { to } 0 \text { to } 10 \text { scale): } 2.1 \text { vs. } 3.1 \text { at } 4 \text { hours, } 3.3 \text { vs. } \\
3.5 \text { at } 8 \text { hours, } p=N R \\
\text { Average pain (mean [SD], of all values from } \\
0.5 \text { to } 8 \text { hours, } 0 \text { to } 100 \text { VAS converted to } 0 \text { to } \\
10 \text { scale): } 2.5(1.8) \text { vs. } 3.8(1.8), p=0.02 \text { for A } \\
\text { vs. B }\end{array}$ & $\begin{array}{l}\text { Rescue medication use (codeine }+ \\
\text { acetaminophen): } 35 \%(8 / 23) \text { vs. } 36 \% \\
(8 / 22) \text { vs. } 45 \%(10 / 22), \text { RR } 0.96(95 \% \mathrm{Cl} \\
0.44 \text { to } 2.10)\end{array}$ \\
\hline $\begin{array}{l}\text { Brown, 2013 } \\
\text { Daniels, } 2011^{220} \\
\text { Fair }\end{array}$ & $\begin{array}{l}\text { A: Codeine } 60 \mathrm{mg}+ \\
\text { acetaminophen } 600 \mathrm{mg} \\
\text { every } 6 \text { hours } \times 1 \text { day, } \\
\text { then every } 6 \text { hours as } \\
\text { needed } \times 2 \text { days } \\
\text { B: Ibuprofen } 600 \text { mg q } 6 \\
\text { hours } \times 1 \text { day, then every } \\
6 \text { hours as needed } \times 2 \\
\text { days }\end{array}$ & $\begin{array}{l}\text { Third molar } \\
\text { extraction; } \\
3 \text { days } \\
n=254\end{array}$ & $\begin{array}{l}\text { Average pain, difference versus placebo (least } \\
\text { square mean [95\% Cl], } 0 \text { to } 10 \mathrm{NRS}):-0.87 \\
(-1.63 \text { to }-0.11) \text { vs. }-1.11(-1.75 \text { to }-0.48) \text { at } \\
\text { day } 2, \mathrm{MD} 0.24(95 \% \mathrm{Cl}-0.96 \text { to } 1.44) ;-0.45 \\
(-1.18 \text { to } 0.29) \text { vs. }-0.28(-0.89 \text { to } 0.33) \text { at day } \\
3, \mathrm{MD}-0.17(95 \% \mathrm{Cl}-1.33 \text { to } 0.99) \\
\text { Pain relief (mean [SD NR], } 0 \text { to } 4 \text { categorical } \\
\text { scale converted to } 0 \text { to } 10): 5 \text { vs. } 7.5 \text { at } 4 \\
\text { hours; } 4.6 \text { vs. } 6.8 \text { at } 6 \text { hours; } 5.4 \text { vs. } 6.2 \text { at } 24 \\
\text { hours }\end{array}$ & $\begin{array}{l}\text { Rescue medication use (acetaminophen): } \\
23 \%(14 / 62) \text { vs. } 23 \% \text { (44/192) day } 2, \mathrm{RR} \\
0.99(95 \% \mathrm{Cl} 0.58 \text { to } 1.67) ; 20 \%(12 / 62) \\
\text { vs. } 18 \%(34 / 192) \text { day } 3, \mathrm{RR} 1.09(95 \% \mathrm{Cl} \\
0.60 \text { to } 1.98) \\
\text { Global assessment "very good" or } \\
\text { "excellent": } 47 \% \text { (29/62) vs. } 64 \% \\
\text { (123/192) at day 2, RR } 0.73(95 \% \mathrm{Cl} 0.55 \\
\text { to } 0.97) ; 47 \%(29 / 62) \text { vs. } 60 \%(115 / 192) \\
\text { at day 3, RR } 0.78(95 \% \mathrm{Cl} 0.58 \text { to } 1.04)\end{array}$ \\
\hline $\begin{array}{l}\text { Cattry, } 2020- \\
\text { Study } 1^{177} \\
\text { Fair }\end{array}$ & $\begin{array}{l}\text { A: Codeine } 60 \mathrm{mg}+ \\
\text { acetaminophen } 600 \mathrm{mg} x \\
1 \\
\mathrm{~B} \text { : Naproxen sodium } 440 \\
\mathrm{mg} \times 1\end{array}$ & $\begin{array}{l}\text { Third molar } \\
\text { extraction; } \\
<1 \text { day ( } 8 \\
\text { hours) } \\
n=181\end{array}$ & $\begin{array}{l}\text { Sum of pain intensity differences (least square } \\
\text { mean [SD] sum of differences on } 0 \text { to } 3 \\
\text { categorical scale from } 0.5 \text { to } 8 \text { hours):): } 2.1 \\
(6.0) \text { vs. } 4.3(6.0), p<0.05 \\
\text { Pain relief (mean [SD NR], } 0 \text { to } 4 \text { categorical } \\
\text { scale converted to } 0 \text { to } 10): 1.7 \text { vs. } 0.9 \text { at } 4 \\
\text { hours ( } p<0.05 \text { ); } 2.5 \text { vs. } 0.8 \text { at } 8 \text { hours, } p<0.05\end{array}$ & $\begin{array}{l}\text { Rescue medication use (not specified): } \\
85 \%(77 / 91) \text { vs. } 62 \%(56 / 90) \text {, RR } 1.36 \\
(95 \% \mathrm{Cl} 1.13 \text { to } 1.63) \\
\text { Global assessment "very good" or } \\
\text { "excellent": } 23 \%(21 / 91) \text { vs. } 41 \% \text { (37/90), } \\
\text { RR } 0.56 \text { (95\% Cl } 0.36 \text { to } 0.88)\end{array}$ \\
\hline
\end{tabular}




\begin{tabular}{|c|c|c|c|c|}
\hline $\begin{array}{l}\text { Study, Year } \\
\text { Quality }\end{array}$ & Interventions & $\begin{array}{l}\text { Procedure; } \\
\text { Followup } \\
\text { Duration } \\
\text { Sample Size }\end{array}$ & Pain Intensity Results & Other Results \\
\hline $\begin{array}{l}\text { Cattry, } 2020- \\
\text { Study } 2^{177} \\
\text { Fair }\end{array}$ & $\begin{array}{l}\text { A: Codeine } 60 \mathrm{mg}+ \\
\text { acetaminophen } 600 \mathrm{mg} \text { x } \\
1 \\
\text { B: Naproxen sodium } 440 \\
\mathrm{mg} \times 1\end{array}$ & $\begin{array}{l}\text { Third molar } \\
\text { extraction; } \\
<1 \text { day ( } 8 \\
\text { hours) } \\
\mathrm{n}=183\end{array}$ & $\begin{array}{l}\text { Sum of pain intensity differences (least square } \\
\text { mean [SD] sum of differences on } 0 \text { to } 3 \\
\text { categorical scale from } 0.5 \text { to } 8 \text { hours): } 2.0(4.9) \\
\text { vs. } 2.8(6.0), p>0.05 \\
\text { Pain relief (mean [SD NR], } 0 \text { to } 4 \text { categorical } \\
\text { scale converted to } 0 \text { to } 10 \text { ): } 4.0 \text { vs. } 2.5 \text { at } 4 \\
\text { hours ( } p<0.05) ; 2.8 \text { vs. } 1.2 \text { at } 8 \text { hours, } p<0.05\end{array}$ & $\begin{array}{l}\text { Rescue medication use (not specified): } \\
77 \%(70 / 91) \text { vs. } 55 \% \text { (51/92), RR } 1.39 \\
(95 \% \mathrm{Cl} 1.12 \text { to } 1.72) \\
\text { Global assessment "very good" or } \\
\text { "excellent": } 35 \%(32 / 91) \text { vs. } 42 \% \text { (39/92), } \\
\text { RR } 0.83 \text { (95\% Cl } 0.57 \text { to } 1.20)\end{array}$ \\
\hline $\begin{array}{l}\text { Cooper, } 1982^{189} \\
\text { Fair }\end{array}$ & $\begin{array}{l}\text { A: Codeine } 60 \mathrm{mg} \times 1 \\
\text { B: Codeine } 60 \mathrm{mg}+ \\
\text { ibuprofen } 400 \mathrm{mg} \times 1 \\
\text { C: Codeine } 60 \mathrm{mg}+ \\
\text { aspirin } 650 \mathrm{mg} \times 1 \\
\text { D: Ibuprofen } 400 \mathrm{mg} \times 1 \\
\text { E: Aspirin } 650 \mathrm{mg} \times 1\end{array}$ & $\begin{array}{l}\text { Third molar } \\
\text { extraction; } \\
<1 \text { day ( } 4 \\
\text { hours) } \\
n=203\end{array}$ & $\begin{array}{l}\text { Pain intensity difference (mean [SD NR], } 0 \text { to } 3 \\
\text { categorical scale converted to } 0 \text { to } 10 \text { scale) at } \\
4 \text { hours: } 0.2 \text { vs. } 1.2 \text { vs. } 0.7 \text { vs. } 1.0 \text { vs. } 0.6 \text {, } \\
\text { p=NR } \\
\text { Sum of pain intensity differences (mean [SD } \\
\text { NR], sum of differences on } 0 \text { to } 3 \text { categorical } \\
\text { scale from } 1 \text { to } 4 \text { hours): } 0.95 \text { vs. } 4.71 \text { vs. } 3.33 \\
\text { vs. } 3.76 \text { vs. } 1.76, p=N R\end{array}$ & NR \\
\hline $\begin{array}{l}\text { Cooper, } 1988^{190} \\
\text { Poor }\end{array}$ & $\begin{array}{l}\text { A: Codeine } 60 \mathrm{mg}+ \\
\text { acetaminophen } 600 \mathrm{mg} x \\
1 \\
\text { B: Meclofenamate } \\
\text { sodium } 100 \mathrm{mg} \mathrm{x} 1\end{array}$ & $\begin{array}{l}\text { Third molar } \\
\text { extraction; } \\
<1 \text { day ( } 6 \\
\text { hours) } \\
n=67\end{array}$ & $\begin{array}{l}\text { Pain intensity difference (mean [SD NR], } 0 \text { to } 3 \\
\text { NRS converted to } 0 \text { to } 10 \text { scale): } 2.8 \text { vs. } 3.4 \text { at } \\
4 \text { hours, } 1.7 \text { vs. } 1.8 \text { at } 6 \text { hours } p=N R \\
\text { Sum of pain intensity differences (mean [SD } \\
\text { NR], sum of differences on } 0 \text { to } 3 \text { NRS from } \\
0.5 \text { to } 6 \text { hours): } 5.26 \text { vs. } 5.61, p=N R\end{array}$ & $\begin{array}{l}\text { Repeat medication use: } 58 \% \text { (18/31) vs. } \\
53 \%(19 / 36) \text {; RR } 1.10 \text { ( } 95 \% \text { Cl } 0.72 \text { to } \\
1.69) \\
\text { Medication rated 'very good' or 'excellent': } \\
49 \%(15 / 31) \text { vs. } 47 \%(17 / 36) \text {; RR } 1.02 \\
\text { (95\% Cl } 0.62 \text { to } 1.69)\end{array}$ \\
\hline $\begin{array}{l}\text { Cooper, } 1991^{191} \\
\text { Poor }\end{array}$ & $\begin{array}{l}\text { A: Codeine } 60 \mathrm{mg}+ \\
\text { acetaminophen } 650 \mathrm{mg} \mathrm{x} \\
1 \\
\text { B: Flurbiprofen } 50 \mathrm{mg} \mathrm{x} 1 \\
\text { C: Flurbiprofen } 100 \mathrm{mg} \mathrm{x} \\
1\end{array}$ & $\begin{array}{l}\text { Third molar } \\
\text { extraction; } \\
<1 \text { day ( } 6 \\
\text { hours) } \\
n=122\end{array}$ & $\begin{array}{l}\text { Pain intensity difference (mean [SD NR], } 0 \text { to } 3 \\
\text { categorical converted to } 0 \text { to } 10 \text { ): } 2.3 \text { vs. } 2.5 \\
\text { vs. } 3.3 \text { at } 4 \text { hours, } p=N R ; 1.6 \text { vs. } 1.7 \text { vs. } 3.0 \text { at } \\
6 \text { hours, } p=N R \\
\text { Sum of pain intensity differences (mean [SD } \\
\text { NR], sum of differences on } 0 \text { to } 3 \text { scale from } 1 \\
\text { to } 6 \text { hours): } 4.54 \text { vs. } 4.33 \text { vs. } 6.37, p=N S\end{array}$ & $\begin{array}{l}\text { Repeat medication use: } 79.5 \%(31 / 39) \text { vs. } \\
69.0 \%(29 / 42) \text { vs. } 58.5 \%(24 / 41) \\
\text { Medication rated "very good" or } \\
\text { "excellent": } 35.8 \%(14 / 39) \text { vs. } 40.5 \% \\
\text { (17/42) vs. } 53.6 \%(22 / 41)\end{array}$ \\
\hline $\begin{array}{l}\text { Doroschak, } \\
\text { 1999179 } \\
\text { Fair }\end{array}$ & $\begin{array}{l}\text { A. Tramadol } 100 \mathrm{mg} \\
\text { every } 6 \text { hours }+ \\
\text { flurbiprofen } 100 \mathrm{mg} \text { then } \\
\text { B. Tramadol } 100 \mathrm{mg} \\
\text { every } 6 \text { hours } \\
50 \mathrm{mg} \text { every } 6 \text { hours } \\
\text { C. Flurbiprofen } 100 \mathrm{mg} \\
\text { then } 50 \text { mg every } 6 \text { hours }\end{array}$ & $\begin{array}{l}\text { Endodontic } \\
\text { pain following } \\
\text { a root canal; } \\
<1 \text { day ( } 6 \\
\text { hours), }<1 \\
\text { week ( } 2 \text { days) } \\
n=37\end{array}$ & $\begin{array}{l}\text { Pain intensity (mean [SD NR], } 0 \text { to } 100 \text { VAS } \\
\text { converted to a } 0 \text { to } 10 \text { scale: } 2.3 \text { vs. } 3.7 \text { vs. } 3.8 \\
\text { at } 6 \text { hours, and } 0 \text { vs. } 0.3 \text { vs. } 0.8 \text { at day } 2\end{array}$ & NR \\
\hline
\end{tabular}




\begin{tabular}{|c|c|c|c|c|}
\hline $\begin{array}{l}\text { Study, Year } \\
\text { Quality }\end{array}$ & Interventions & $\begin{array}{l}\text { Procedure; } \\
\text { Followup } \\
\text { Duration } \\
\text { Sample Size }\end{array}$ & Pain Intensity Results & Other Results \\
\hline $\begin{array}{l}\text { Dionne, } 1994^{194} \\
\text { Poor }\end{array}$ & $\begin{array}{l}\text { A: Codeine } 60 \mathrm{mg}+ \\
\text { acetaminophen } 650 \mathrm{mg} \mathrm{x} \\
1 \\
\text { B: Flurbiprofen } 50 \mathrm{mg} \mathrm{x} 1 \\
\text { C: Flurbiprofen } 100 \mathrm{mg} \times \\
1\end{array}$ & $\begin{array}{l}\text { Third molar } \\
\text { extraction; } \\
<1 \text { day ( } 6 \\
\text { hours) } \\
\mathrm{n}=72\end{array}$ & $\begin{array}{l}\text { Pain intensity difference (mean [SD NR], } 0 \text { to } 3 \\
\text { categorical converted to } 0 \text { to } 10 \text { ): } 3.7 \text { vs. } 5.2 \\
\text { vs. } 6.0 \text { at } 4 \text { hours, } p<0.05 \text { for B vs. C, } p=N S \\
\text { for A vs. C; } 2.8 \text { vs. } 4.7 \text { vs. } 5.5 \text { at } 6 \text { hours, } \\
\text { p<0.05 for B vs. C, } p=N S \text { for A vs. C } \\
\text { Sum of pain intensity differences (mean [SD } \\
\text { NR], sum of differences on } 0 \text { to } 3 \text { scale from } 1 \\
\text { to } 6 \text { hours): } 6.1 \text { vs. } 8.4 \text { vs. } 9.4, p<0.05 \text { for A or } \\
\text { B vs. C }\end{array}$ & NR \\
\hline $\begin{array}{l}\text { Forbes, } 1990^{195} \\
\text { Poor }\end{array}$ & $\begin{array}{l}\text { A: Codeine } 60 \mathrm{mg}+ \\
\text { acetaminophen } 600 \mathrm{mg} \times \\
1 \\
\text { B: Ketorolac } 10 \mathrm{mg} \times 1 \\
\text { C: Ketorolac } 20 \mathrm{mg} \times 1 \\
\text { D: Ibuprofen } 400 \mathrm{mg} \times 1\end{array}$ & $\begin{array}{l}\text { Third molar } \\
\text { extraction; } \\
<1 \text { day ( } 6 \\
\text { hours) } \\
\mathrm{n}=136\end{array}$ & $\begin{array}{l}\text { Pain intensity difference (mean [SD NR], } 0 \text { to } 3 \\
\text { categorical scale converted to } 0 \text { to } 10 \text { ): } 1.40 \\
\text { vs. } 3.33 \text { vs. } 3.03 \text { vs. } 3.53 \text { at } 4 \text { hours, } p \leq 0.01 \\
\text { for A vs. B, C, or D; } 0.80 \text { vs. } 1.83 \text { vs. } 2.56 \text { vs. } \\
1.86 \text { at } 6 \text { hours, } p<0.01 \text { for A vs. C, } p=N S \text { for A } \\
\text { vs. B or D } \\
\text { Sum of pain intensity differences (mean [SD } \\
\text { NR], sum of differences on } 0 \text { to } 3 \text { scale from } 1 \\
\text { to } 6 \text { hours): } 2.84 \text { vs. } 5.84 \text { vs. } 5.69 \text { vs. } 5.31 \text {, } \\
\text { p=NS }\end{array}$ & $\begin{array}{l}50 \% \text { pain relief: } 21.0 \%(8 / 38) \text { vs. } 64.5 \% \\
(20 / 31) \text { vs. } 68.6 \%(24 / 35) \text { vs. } 59.4 \% \\
(19 / 32) \text { at } 4 \text { hours, } p<0.01 \text { for A vs. B, C } \\
\text { or D; } 13.2 \%(5 / 38) \text { vs. } 32.3 \%(10 / 31) \text { vs. } \\
42.9 \%(15 / 35) \text { vs. } 31.2 \%(10 / 32) \text { at } 6 \\
\text { hours, } p<0.01 \text { for A vs. C p=NS for A or B } \\
\text { or D } \\
\text { Repeat medication use: } 84.2 \%(32 / 38) \text { vs. } \\
51.6 \%(16 / 31) \text { vs. } 57.1 \%(20 / 35) \text { vs. } \\
62.5 \%(20 / 32)\end{array}$ \\
\hline $\begin{array}{l}\text { Malmstrom, } \\
2004^{201} \\
\text { Fair }\end{array}$ & $\begin{array}{l}\text { A: Codeine } 60 \mathrm{mg}+ \\
\text { acetaminophen } 600 \mathrm{mg} x \\
1 \\
\mathrm{~B}: \text { Naproxen sodium } 550 \\
\mathrm{mg} \times 1\end{array}$ & $\begin{array}{l}\text { Third molar } \\
\text { extraction; } \\
1 \text { day } \\
n=101\end{array}$ & $\begin{array}{l}\text { Pain relief (mean [SD NR], } 0 \text { to } 4 \text { categorical } \\
\text { scale converted to } 0 \text { to } 10 \text { scale): } 3.2 \text { vs. } 7.0 \text { at } \\
4 \text { hours, } 3.0 \text { vs. } 5.5 \text { at } 20 \text { hours, } 3.0 \text { vs. } 5.2 \text { at } \\
1 \text { day; p=NR } \\
\text { Sum of pain intensity differences (mean [SD], } \\
\text { sum of differences on } 0 \text { to } 4 \text { scale at } 0.25 \text { to } 8 \\
\text { hours): } 5.1(6.01) \text { vs. } 11.7 \text { (6.0), MD }-6.60 \\
\text { ( } 95 \% \mathrm{Cl}-9.00 \text { to }-4.20 \text { ) }\end{array}$ & $\begin{array}{l}\text { Perceptible pain relief (not defined): } 60 \% \\
\text { (30/50) vs. } 90.2 \% \text { ( } 46 / 51) \text {, RR } 0.65 \text { ( } 95 \% \\
\text { Cl } 0.51 \text { to } 0.83) \\
\text { Rescue medication use (hydrocodone } 5 \\
\text { mg + acetaminophen } 500 \mathrm{mg}): 76.0 \% \\
\text { (38/50) vs. } 52.9 \% \text { ( } 27 / 51) \text {, RR } 1.44 \text { (95\% } \\
\mathrm{Cl} 1.06 \text { to } 1.94) \\
\text { Response 'good,' 'very good' or 'excellent': } \\
48 \%(24 / 50) \text { vs. } 84 \%(43 / 51) \text { at } 8 \text { hours, } \\
\text { RR } 0.57(95 \% \mathrm{Cl} 0.42 \text { to } 0.78)\end{array}$ \\
\hline $\begin{array}{l}\text { Mehrvarzfar, } \\
2012^{205} \\
\text { Fair }\end{array}$ & $\begin{array}{l}\text { A: Tramadol } 100 \mathrm{mg} \times 1 \\
\text { B: Naproxen } 500 \mathrm{mg} \times 1\end{array}$ & $\begin{array}{l}\text { Root canal } \\
\text { instrumentation } \\
\text { for irreversible } \\
\text { pulpitis; } \\
1 \text { day } \\
\mathrm{n}=50\end{array}$ & $\begin{array}{l}\text { Pain intensity (mean [SD], } 0 \text { to } 10 \text { VAS): } 3.2 \\
(2.6) \text { vs. } 0.8(1.1) \text { at } 6 \text { hours, MD } 2.4(95 \% \mathrm{Cl} \\
1.2 \text { to } 3.6) ; \text { and } 2.1 \text { (1.4) vs. } 0.5(0.5) \text { at } 12 \\
\text { hours, MD } 1.6(1.0 \text { to } 2.2) ; 2.2(2.2) \text { vs. } 0.7 \\
\text { (1.0) at } 24 \text { hours, MD } 1.5 \text { ( } 95 \% \mathrm{Cl} 0.5 \text { to } 2.5)\end{array}$ & NR \\
\hline
\end{tabular}




\begin{tabular}{|c|c|c|c|c|}
\hline $\begin{array}{l}\text { Study, Year } \\
\text { Quality }\end{array}$ & Interventions & $\begin{array}{l}\text { Procedure; } \\
\text { Followup } \\
\text { Duration } \\
\text { Sample Size }\end{array}$ & Pain Intensity Results & Other Results \\
\hline $\begin{array}{l}\text { Moore, } 2015^{181} \\
\text { Good }\end{array}$ & $\begin{array}{l}\text { A: Tramadol } \\
\text { hydrochloride } 37.5 \mathrm{mg}+ \\
\text { dexketoprofen } \\
\text { trometamol } 12.5 \mathrm{mg} \mathrm{\times} 1 \\
\text { B: Tramadol } \\
\text { hydrochloride } 75 \mathrm{mg} \mathrm{+} \\
\text { dexketoprofen } \\
\text { trometamol } 12.5 \mathrm{mg} \mathrm{x} 1 \\
\text { C: Tramadol } \\
\text { hydrochloride } 37.5 \mathrm{mg}+ \\
\text { dexketoprofen } \\
\text { trometamol } 25 \mathrm{mg} \mathrm{x} 1 \\
\text { D: Tramadol } \\
\text { hydrochloride } 75 \mathrm{mg}+ \\
\text { dexketoprofen } \\
\text { trometamol } 25 \mathrm{mg} \mathrm{x} 1 \\
\text { E: Tramadol } \\
\text { hydrochloride } 37.5 \mathrm{mg} \times \\
\text { 1 } \\
\text { F: Tramadol } \\
\text { hydrochloride } 75 \mathrm{mg} \times 1 \\
\text { G: Dexketoprofen } \\
\text { trometamol } 12.5 \mathrm{mg} \times 1 \\
\text { H: Dexketoprofen } \\
\text { trometamol } 25 \mathrm{mg} \times 1 \\
\text { I: Ibuprofen } 400 \mathrm{mg} \mathrm{x} 1\end{array}$ & $\begin{array}{l}\text { Third molar } \\
\text { extraction; } \\
<1 \text { day }(4,6,8, \\
\text { and } 24 \text { hours); } \\
1 \text { day to }<1 \\
\text { week ( } 24 \\
\text { hours) } \\
n=544\end{array}$ & $\begin{array}{l}\text { A vs. B vs. C vs. D vs. E vs. F vs. G. vs. H. vs. } \\
\text { I } \\
\text { Pain intensity (mean [SD NR], } 0 \text { to } 3 \\
\text { categorical scale converted to } 0 \text { to } 10): 5.3 \text { vs. } \\
3.7 \text { vs. } 6.7 \text { vs. } 4.3 \text { vs } 4.0 \text { vs. } 5.0 \text { vs. } 6.7 \text { vs. } 6.7 \\
\text { vs. } 4.7 \text { at } 4 \text { hours, } 6.7 \text { vs. } 5.3 \text { vs. } 7.3 \text { vs. } 5.7 \text { vs. } \\
5.7 \text { vs. } 6.7 \text { vs. } 7.7 \text { vs. } 7.0 \text { vs. } 5.0 \text { at } 6 \text { hours, } \\
7.0 \text { vs. } 6.0 \text { vs. } 7.3 \text { vs. } 5.7 \text { vs. } 6.3 \text { vs. } 7.0 \text { vs. } 7.3 \\
\text { vs. } 7.0 \text { vs. } 6.0 \text { at } 8 \text { hours, } 7.3 \text { vs. } 6.3 \text { vs. } 7.3 \\
\text { vs. } 6.0 \text { vs. } 6.7 \text { vs. } 6.7 \text { vs. } 7.3 \text { vs. } 7.0 \text { vs. } 7.0 \text { at } \\
24 \text { hours } \\
\text { Sum of Pain Intensity Difference (mean [SD], } \\
\text { sum of difference on } 0 \text { to } 3 \text { scale from } 4 \text { to } 12 \\
\text { hours): } 5.6(6.1) \text { vs. } 9.0(8.8) \text { vs. } 8.6(7.6) \text { vs. } \\
10.1(8.4 \text { vs. } 2.4(4.6) \text { vs. } 3.8(6.3) \text { vs. } 4.8 \\
\text { (6.2) vs. } 6.0(7.0) \text { vs. } 7.4(9.3)\end{array}$ & $\begin{array}{l}\text { A vs. B vs. C vs. D vs. E vs. F vs. G. vs. } \\
\text { H. vs. I } \\
\text { Experienced } \geq 50 \% \text { maximum total pain } \\
\text { relief: } 12 \%(7 / 60) \text { vs. } 36 \%(22 / 62) \text { vs. } 29 \% \\
(18 / 63) \text { vs. } 38 \%(23 / 61) \text { vs. } 5.1 \%(3 / 59) \\
\text { vs. } 15 \%(9 / 59) \text { vs.10\% (6/60) vs. } 13 \% \\
(8 / 60) \text { vs. } 25 \%(15 / 60) \\
\text { Proportion of patients rating the study } \\
\text { medication as 'very good' or 'excellent', } \% \\
\text { (n/N): } 27 \%(16 / 60) \text { vs. } 47 \%(29 / 62) \text { vs. } \\
46 \%(29 / 63) \text { vs. } 51 \%(31 / 61) 25 \%(15 / 59) \\
\text { vs. } 14 \%(8 / 59) \text { vs. } 33 \%(20 / 60) \text { vs.28\% } \\
(17 / 60) \text { vs. } 33 \%(20 / 60) \\
\text { Rescue medication use (paracetamol) at } \\
24 \text { hours: } 75 \%(45 / 60) \text { vs. } 58 \%(36 / 62) \text { vs. } \\
49 \%(31 / 63) \text { vs. } 54 \%(33 / 61) \text { vs. } 73 \% \\
(43 / 59) \text { vs. } 66 \%(39 / 59) \text { vs. } 65 \%(39 / 60) \\
\text { vs. } 62 \%(37 / 60) \text { vs. } 65 \%(39 / 60)\end{array}$ \\
\hline $\begin{array}{l}\text { Sadeghein, } \\
1999^{210} \\
\text { Fair }\end{array}$ & $\begin{array}{l}\text { A: Codeine } 15 \mathrm{mg}+ \\
\text { acetaminophen } 325 \mathrm{mg} \mathrm{x} \\
1 \\
\text { B: Ketorolac } \\
\text { tromethamine } 10 \mathrm{mg} \mathrm{x} 1\end{array}$ & $\begin{array}{l}\text { Acute apical } \\
\text { periodontitis; } \\
<1 \text { day ( } 90 \\
\text { minutes) } \\
\mathrm{n}=63\end{array}$ & $\begin{array}{l}\text { Pain intensity (mean [SD NR], } 0 \text { to } 10 \text { VAS): } \\
4.34 \text { vs } 1.00, p=0.05\end{array}$ & NR \\
\hline
\end{tabular}




\begin{tabular}{|c|c|c|c|c|}
\hline $\begin{array}{l}\text { Study, Year } \\
\text { Quality }\end{array}$ & Interventions & $\begin{array}{l}\text { Procedure; } \\
\text { Followup } \\
\text { Duration } \\
\text { Sample Size }\end{array}$ & Pain Intensity Results & Other Results \\
\hline $\begin{array}{l}\text { Sunshine, } 1986^{213} \\
\text { Fair }\end{array}$ & $\begin{array}{l}\text { A: Codeine } 60 \mathrm{mg}+ \\
\text { acetaminophen } 650 \mathrm{mg} \mathrm{x} \\
1 \\
\text { B: Flurbiprofen } 50 \mathrm{mg} \mathrm{x} 1 \\
\text { C: Flurbiprofen } 100 \mathrm{mg} \mathrm{x} \\
1\end{array}$ & $\begin{array}{l}\text { Third molar } \\
\text { extraction; } \\
<1 \text { day ( } 6 \\
\text { hours) } \\
\mathrm{n}=91\end{array}$ & $\begin{array}{l}\text { Pain intensity difference (mean [SD NR], } 0 \text { to } 3 \\
\text { categorical scale transformed to } 0 \text { to } 10 \text { ): } 2.03 \\
\text { vs. } 3.76 \text { vs. } 3.90 \text { at } 4 \text { hours, p=NS for A vs. B } \\
\text { or C; } 1.30 \text { vs. } 3.33 \text { vs. } 3.56 \text { at } 6 \text { hours, } p=N S \\
\text { for A vs. B or C } \\
\text { Sum of pain intensity differences (mean [SD } \\
\text { NR], sum of differences on } 0 \text { to } 3 \text { scale from } \\
0.5 \text { to } 6 \text { hours): } 4.66 \text { vs. } 7.01 \text { vs. } 6.85, p=N S \\
\text { for A vs. B or C }\end{array}$ & $\begin{array}{l}\text { Rescue medication use: } 92.0 \% \text { (9/31) vs. } \\
19.4 \%(6 / 31) \text { vs. } 13.8 \%(4 / 29)\end{array}$ \\
\hline $\begin{array}{l}\text { Van Dyke, } 2004^{215} \\
\text { Fair }\end{array}$ & $\begin{array}{l}\text { A: Oxycodone } 5 \mathrm{mg}+ \\
\text { ibuprofen } 400 \mathrm{mg} \times 1 \\
\text { B: Oxycodone } 5 \mathrm{mg} \\
\text { C: Ibuprofen } 400 \mathrm{mg} \times 1\end{array}$ & $\begin{array}{l}\text { Third molar } \\
\text { extraction } \\
<1 \text { day }(6 \\
\text { hours) } \\
\mathrm{n}=435\end{array}$ & $\begin{array}{l}\text { Pain intensity difference (mean [SD NR], } 0 \text { to } 3 \\
\text { categorical scale converted to } 0 \text { to } 10 \text { scale): } \\
3.6 \text { vs. }-0.4 \text { vs. } 3.1 \text { at } 4 \text { hours, } 3.0 \text { vs. }-0.4 \text { vs. } \\
2.6 \text { at } 6 \text { hours; } p=N R \\
\text { Sum of pain intensity differences (mean [SD], } \\
\text { sum of differences on } 0 \text { to } 3 \text { scale from } 0.25 \text { to } \\
6 \text { hours): } 6.54 \text { (0.42) vs. } 0.14(0.60 \text { ) vs. } 5.41 \\
\text { ( } 0.44) ; p=0.002 \text { for A vs. C, p=NS for B vs. C }\end{array}$ & $\begin{array}{l}\text { Pain improved } \geq 50 \% \text { after } 1 \text { hour: } 74.9 \% \\
(140 / 186) \text { vs. } 19.0 \%(12 / 63) \text { vs. } 59.1 \% \\
(110 / 186), p<0.05 \text { for A vs. C and } p<0.001 \\
\text { for B vs. C } \\
\text { Global evaluation (mean [SD], } 0 \text { to } 4 \\
\text { scale): } 2.63(0.10) \text { vs. } 0.66(0.16) \text { vs. } 2.26 \\
\text { (0.10); } p<0.01 \text { for A vs. } C \text { and } p<0.001 \text { for } \\
\text { B vs. C }\end{array}$ \\
\hline $\begin{array}{l}\text { Zupelari- } \\
\text { Goncalves, } \\
2017^{218} \\
\text { Fair }\end{array}$ & $\begin{array}{l}\text { A: Codeine } 50 \mathrm{mg}+ \\
\text { diclofenac } 50 \mathrm{mg} \text { every } 8 \\
\text { hours } \times 4 \text { days } \\
\text { B: Diclofenac } 50 \mathrm{mg} \times 1 \\
\text { every } 8 \text { hours } \times 4 \text { days }\end{array}$ & $\begin{array}{l}\text { Third molar } \\
\text { extraction; } \\
4 \text { days } \\
\mathrm{n}=46\end{array}$ & $\begin{array}{l}\text { Pain (median [IQR], } 0 \text { to } 100 \text { VAS converted to } \\
0 \text { to } 10 \text { scale): } 0 \text { ( } 0 \text { to } 0) \text { vs. } 1.8(0 \text { to } 4.7) \text { at } 18 \\
\text { hours, } 1.0(0 \text { to } 2.5) \text { vs. } 1.0(0 \text { to } 2.5) \text { at } 4 \\
\text { days, } p=N S\end{array}$ & $\begin{array}{l}\text { Rescue medication use (mean [SD], } \\
\text { tablets): } 3.0 \text { (3.3) vs. } 5.0 \text { (3.4), p } \leq 0.05 \\
\text { Global efficacy "very good" or "excellent": } \\
65 \% \text { (30/46) vs. } 37 \% \text { (17/46), RR } 1.76 \\
(95 \% \mathrm{Cl} 1.15 \text { to } 2.72)\end{array}$ \\
\hline
\end{tabular}

VAS = visual analog scale;

Note: Pain intensity difference=pain at followup minus pain at baseline 


\section{Opioid Versus Acetaminophen}

Eleven trials compared an opioid (alone or in combination with acetaminophen) versus acetaminophen for acute dental pain (Appendix E, Table E-8; Table 30). ${ }^{184-186,188,190-}$

$192,194,195,212,213$ Dental pain was due to third molar extraction in all of the trials; one of the trials ${ }^{184}$ also included patients undergoing other extractions and procedures. The sample size for the opioid versus acetaminophen arms ranged from 20 to $209(\mathrm{~N}=891)$. All trials were single dose studies, with the exception of one trial ${ }^{185}$ in which three doses were taken over six hours. The duration of followup ranged from four to eight hours. All trials evaluated an opioid in combination with acetaminophen; two trials also evaluated an opioid alone. ${ }^{184,192}$ The opioid was codeine in 10 trials $^{184-186,188,190,191,194,195,212,213}$ (dose $60 \mathrm{mg}$ ) and oxycodone in 1 trial $^{192}$ (dose 5 or $10 \mathrm{mg}$ ). The dose of acetaminophen administered with an opioid was $1000 \mathrm{mg}$ in five trials ${ }^{184-}$ 186,192,212 and 500 to $650 \mathrm{mg}$ in seven trials ${ }^{188,190-192,194,195,213}$ (one of the trials ${ }^{192}$ evaluated acetaminophen 500 and $1000 \mathrm{mg}$ ). The dose of acetaminophen in the non-opioid arm was 1000 $\mathrm{mg}$ in 5 trials, ${ }^{184-186,192,212} 600$ or $650 \mathrm{mg}$ in six trials; ${ }^{188,190,191,194,195,213}$ one of the $1000 \mathrm{mg}$ dose trials $^{212}$ also had a $2000 \mathrm{mg}$ arm.

All of the trials used a parallel group design and used a blinded design, except for one trial ${ }^{191}$ in which blinding status was unclear. One trial was rated good quality, ${ }^{186}$ six trials fair quality, ${ }^{184,185,188,192,212,213}$ and four trials ${ }^{190,191,194,195}$ poor quality (Appendix F, Table F-1).

Eleven trials $(\mathrm{N}=828)$ evaluated effects of an opioid plus acetaminophen versus acetaminophen alone on pain intensity at 4 hours, based on the PID or mean pain intensity. ${ }^{184-}$ $186,188,190-192,194,195,212,213$ In seven of the trials, an opioid plus acetaminophen was associated with greater reduction in pain intensity versus acetaminophen alone; in most of the trials, the magnitude of the effect was moderate (range 0.8 to 2.9 points on a 0 to 10 scale). ${ }^{184-186,190-192,194}$ In the other four trials, effects of an opioid plus acetaminophen and acetaminophen alone on pain intensity were similar (range -0.2 to 0.3 point). ${ }^{188,195,212,213}$ The trial that evaluated three doses over six hours reported the largest effects in favor of the opioid combination on pain intensity; the difference in this trial was statistically significant ( $\mathrm{n}=20$, PID 4.8 vs. 2.0, $\mathrm{p}<0.01$ ). ${ }^{185}$ Eight trials $(\mathrm{N}=546)$ evaluated effects of an opioid plus acetaminophen versus acetaminophen alone on pain intensity at 6 to 8 hours ${ }^{185,186,190,191,194,195,212,213}$ (subsequent to peak analgesic effects in most single dose trials). Results were inconsistent, with differences ranging from 3 points on a 0 to 10 scale in favor of acetaminophen to 2.5 points in favor of an opioid plus acetaminophen. An opioid plus acetaminophen was associated with larger SPID versus acetaminophen in 10 trials (N=898), ${ }^{184,185,188,190-192,194,195,212,213}$ though the difference was statistically significant in only one trial. ${ }^{185}$ An opioid plus acetaminophen was also associated with less average pain (mean of all assessments) ( 1 trial, $n=45,2.5$ vs. 3.7 on a 0 to 10 scale, $p=0.03),{ }^{186}$ and decreased likelihood of rescue or repeat medication use ( 7 trials, $\mathrm{N}=484$, pooled RR $0.81,95 \%$ CI 0.67 to $0.97, \mathrm{I}^{2}=39 \%$; ARD -12\%, 95\% CI -19\% to -5\%; Appendix D, Figure D-8). ${ }^{184,186,188,190,191,195,213}$ Effects on likelihood of rescue or repeat medication use were similar when poor quality trials were excluded (4 trials, $\mathrm{N}=267$, pooled RR 0.62 , $95 \%$ CI 0.45 to $0.86, \mathrm{I}^{2}=0 \%$ ). ${ }^{184,186,188,213}$ One trial ${ }^{195}$ $(n=74)$ found no difference in the likelihood of 50 percent pain relief, though two trials ${ }^{190,191}$ ( $n=143$ ) found an opioid plus acetaminophen more likely to be rated "very good" or "excellent" (49\% vs. $28 \%$, RR 0.45 , $95 \%$ CI 0.09 to 2.20 and 36\% vs. $8.1 \%$, RR 4.42, 95\% CI 1.38 to 14.17). Quality of life and function were not reported in the trials.

Two trials ( $\mathrm{N}=149)$ compared an opioid alone versus acetaminophen. ${ }^{184,192}$ Effects on pain intensity at 4 hours were below the threshold for small (differences ranged from 0.23 point on a 0 to 10 scale favoring the opioid to 0.1 point favoring acetaminophen). Differences in the SPID 
were also small or not statistically significant. One trial $(n=62)$ found no difference between an opioid versus acetaminophen in likelihood of rescue medication use (RR 1.08, 95\% CI 0.77 to 1.54). ${ }^{184}$

There were no statistically significant differences between an opioid (alone or in combination with acetaminophen) versus acetaminophen alone in risk of any adverse event (8 trials, $\mathrm{N}=774$, pooled RR 1.43, 95\% CI 0.87 to 2.37, I²=65\%; Appendix D, Figure D-9), ${ }^{184,188,191,192,194,195,212,213}$ nausea (8 trials, $\mathrm{N}=669$, pooled RR 1.55 , 95\% CI 0.75 to 3.18, $\mathrm{I}^{2}=0 \%$; Appendix D, Figure D10), ${ }^{185,186,188,191,192,194,195,212}$ drowsiness (6 trials, $\mathrm{N}=512$, pooled RR 2.03, 95\% CI 0.70 to 5.93, $\mathrm{I}^{2}=53 \%$; Appendix D, Figure D-11), ${ }^{185,186,188,191,192,195}$ dizziness (5 trials, N=465, pooled RR 2.49, 95\% CI 0.66 to 9.49, I ${ }^{2}=27 \%$; Appendix D, Figure D-12), ${ }^{185,188,191,192,195}$ or headache (4 trials, $\mathrm{N}=415$, pooled RR 0.67, 95\% CI 0.20 to 2.18, $\mathrm{I}^{2}=0 \%$; Appendix D, Figure D-13). ${ }^{188,191,192,194}$ However, most estimates favored acetaminophen, the number of adverse events in most trials was small, estimates were imprecise, and statistical heterogeneity was large for some outcomes. In addition, all trials except for one were single dose studies, and the single multiple dose trial was very small $(n=20) .{ }^{185}$ Serious adverse events were not reported. 


\begin{tabular}{|c|c|c|c|c|}
\hline $\begin{array}{l}\text { Study, Year } \\
\text { Quality }\end{array}$ & Interventions & $\begin{array}{l}\text { Procedure; } \\
\text { Followup } \\
\text { Duration } \\
\text { Sample Size }\end{array}$ & Pain Intensity Results & Other Results \\
\hline $\begin{array}{l}\text { Bentley, } \\
1987^{184} \\
\text { Fair }\end{array}$ & $\begin{array}{l}\text { A. Codeine } 60 \mathrm{mg} \times 1 \\
\text { B. Codeine } 60 \mathrm{mg}+ \\
\text { acetaminophen } 1000 \mathrm{mg} \times 1 \\
\text { C. Acetaminophen } 1000 \mathrm{mg} \times 1\end{array}$ & $\begin{array}{l}\text { Extraction or } \\
\text { other } \\
\text { procedure } \\
\text { involving } \\
\text { reflection of the } \\
\text { mucoperiosteal } \\
\text { flap and } \\
\text { removal of } \\
\text { bone; } \\
<1 \text { day (5 } \\
\text { hours) } \\
\mathrm{n}=103\end{array}$ & $\begin{array}{l}\text { Pain intensity (mean [SD NR], } 0 \text { to } 9 \text { NRS converted } \\
\text { to } 0 \text { to } 10 \text { scale): } 1.1 \text { vs. } 1.8 \text { vs. } 1.0 \text { at } 4 \text { hours, } 1.1 \\
\text { vs. } 1.5 \text { vs. } 0.8 \text { at } 5 \text { hours, p=NR } \\
\text { Sum of pain intensity differences (mean [SD] of } \\
\text { differences on } 0 \text { to } 9 \text { NRS at } 1 \text { to } 5 \text { hours): } 4.33 \\
(11.80) \text { vs. } 9.71 \text { ( } 10.49) \text { vs. } 6.17 \text { ( } 8.48) ; \text { MD }-5.38 \\
(95 \% \mathrm{Cl}-11.25 \text { to } 0.49) \text { for A vs. B, MD }-1.84(95 \% \\
\mathrm{Cl}-7.05 \text { to } 3.37 \text { ) for A vs. C, MD } 3.54 \text { ( } 95 \% \mathrm{Cl}-0.65 \\
\text { to } 7.73 \text { ) for B vs. C }\end{array}$ & $\begin{array}{l}\text { Rescue medication use through } \\
4 \text { hours: } 70 \%(15 / 21) \text { vs. } 38 \% \\
\text { (16/41) vs. } 67 \%(27 / 41), \text { RR } \\
1.83 \text { (95\% Cl } 1.15 \text { to } 2.92) \text { for A } \\
\text { vs. B, RR } 1.08 \text { (95\% Cl } 0.77 \text { to } \\
1.54) \text { for A vs. C, and RR } 0.59 \\
\text { (95\% Cl } 0.38 \text { to } 0.92) \text { for B vs. } \\
\text { C }\end{array}$ \\
\hline $\begin{array}{l}\text { Breivik, } 1998^{185} \\
\text { Fair }\end{array}$ & $\begin{array}{l}\text { A. Codeine + acetaminophen } \\
60 / 1000 \mathrm{mg} \text { every } 3 \text { hours } \times 3 \\
\text { days } \\
\text { B. Acetaminophen } 1000 \mathrm{mg} \\
\text { every } 3 \text { hours } \times 3 \text { days }\end{array}$ & $\begin{array}{l}\text { Third molar } \\
\text { extraction; } \\
<1 \text { day ( } 8 \\
\text { hours) } \\
n=20\end{array}$ & $\begin{array}{l}\text { Pain intensity (mean [SD NR], } 0 \text { to } 100 \text { VAS } \\
\text { converted to } 0 \text { to } 10 \text { scale): } 1.6 \text { vs. } 4.5 \text { at } 4 \text { hours, } \\
p<0.01 ; 1.0 \text { vs. } 4.8 \text { at } 8 \text { hours, } p<0.005 \\
\text { Pain intensity difference (mean [SD NR], } 0 \text { to } 100 \\
\text { VAS converted to } 0 \text { to } 10 \text { scale): } 4.8 \text { vs. } 2.0 \text { at } 4 \\
\text { hours, } p<0.01 ; 2.1 \text { vs. } 5.1 \text { at } 8 \text { hours, } p<0.05 \\
\text { Sum of pain intensity differences (median [IQR], sum } \\
\text { of differences on } 0 \text { to } 100 \text { VAS from } 0 \text { to } 8 \text { hours): } \\
551 \text { (148) vs. } 318 \text { ( } 252 \text { ), p=0.01 }\end{array}$ & NR \\
\hline $\begin{array}{l}\text { Breivik, 1999186 } \\
\text { Good }\end{array}$ & $\begin{array}{l}\text { A. Codeine } 60 \mathrm{mg}+ \\
\text { acetaminophen } 1000 \mathrm{mg} \times 1 \\
\text { B. Acetaminophen } 1000 \mathrm{mg} \times 1\end{array}$ & $\begin{array}{l}\text { Third molar } \\
\text { extraction; } \\
<1 \text { day ( } 8 \\
\text { hours) } \\
n=45\end{array}$ & $\begin{array}{l}\text { Pain intensity (mean [SD NR], } 0 \text { to } 100 \text { VAS } \\
\text { converted to } 0 \text { to } 10 \text { scale): } 2.1 \text { vs. } 3.1 \text { at } 4 \text { hours; } 3.3 \\
\text { vs. } 3.5 \text { vs. } 5.8 \text { at } 8 \text { hours, p=NR } \\
\text { Average pain (mean [SD] of all values from } 0.5 \text { to } 8 \\
\text { hours, } 0 \text { to } 100 \text { VAS converted to } 0 \text { to } 10 \text { scale): } 2.5 \\
\text { (1.8) vs. } 3.7 \text { (2.4), p=0.03 }\end{array}$ & $\begin{array}{l}\text { Rescue medication use: } 35 \% \\
\text { (8/23) vs. } 45 \%(10 / 22) \text {, RR } 0.77 \\
\text { ( } 95 \% \text { Cl } 0.37 \text { to } 1.58)\end{array}$ \\
\hline $\begin{array}{l}\text { Cooper, } \\
1980^{192} \\
\text { Fair }\end{array}$ & $\begin{array}{l}\text { A. Oxycodone } 5 \mathrm{mg} \times 1 \\
\text { B. Oxycodone } 5 \mathrm{mg}+ \\
\text { acetaminophen } 500 \mathrm{mg} \times 1 \\
\text { C. Oxycodone } 5 \mathrm{mg}+ \\
\text { acetaminophen } 1000 \mathrm{mg} \times 1 \\
\text { D. Oxycodone } 10 \mathrm{mg}+ \\
\text { acetaminophen } 1000 \mathrm{mg} \times 1 \\
\text { E. Acetaminophen } 1000 \mathrm{mg} \times 1\end{array}$ & $\begin{array}{l}\text { Third molar } \\
\text { extraction } \\
<1 \text { day ( } 4 \\
\text { hours) } \\
n=209\end{array}$ & $\begin{array}{l}\text { Pain intensity difference (mean [SD NR], } 0 \text { to } 3 \\
\text { categorical scale converted to } 0 \text { to } 10 \text { scale) at } 4 \\
\text { hours: } 1.03 \text { vs. } 1.93 \text { vs. } 2.56 \text { vs. } 3.26 \text { vs. } 0.80, p=N R \\
\text { Sum of pain intensity differences (mean [SD NR], } \\
\text { sum of differences on } 0 \text { to } 3 \text { categorical scale from } 1 \\
\text { to } 4 \text { hours): } 1.38 \text { vs. } 3.00 \text { vs. } 3.55 \text { vs. } 4.49 \text { vs. } 1.49 \text {, } \\
\text { p=NR }\end{array}$ & $\begin{array}{l}\text { Observations with } 50 \% \text { pain } \\
\text { relief (mean [SD NR]): } 1.12 \text { vs. } \\
2.00 \text { vs. } 2.15 \text { vs. } 2.56 \text { vs. } 0.95 \text {, } \\
\text { p=NR }\end{array}$ \\
\hline
\end{tabular}




\begin{tabular}{|c|c|c|c|c|}
\hline $\begin{array}{l}\text { Study, Year } \\
\text { Quality }\end{array}$ & Interventions & $\begin{array}{l}\text { Procedure; } \\
\text { Followup } \\
\text { Duration } \\
\text { Sample Size }\end{array}$ & Pain Intensity Results & Other Results \\
\hline $\begin{array}{l}\text { Cooper, } \\
1981^{188} \\
\text { Fair }\end{array}$ & $\begin{array}{l}\text { A. Codeine } 60 \mathrm{mg}+ \\
\text { acetaminophen } 650 \mathrm{mg} \times 1 \\
\text { B. Acetaminophen } 650 \mathrm{mg} \times 1\end{array}$ & $\begin{array}{l}\text { Third molar } \\
\text { extraction; } \\
<1 \text { day ( } 4 \\
\text { hours) } \\
\mathrm{n}=79\end{array}$ & $\begin{array}{l}\text { Pain intensity difference (mean [SD NR], } 0 \text { to } 3 \text { NRS } \\
\text { converted to } 0 \text { to } 10 \text { scale) at } 4 \text { hours: } 1.60 \text { vs. } 1.90 \text {, } \\
\text { p=NR } \\
\text { Sum of pain intensity differences (mean [SD NR], } \\
\text { sum of differences on } 0 \text { to } 3 \text { NRS from } 1 \text { to } 4 \text { hours): } \\
3.05 \text { vs. } 2.84, p=N R\end{array}$ & $\begin{array}{l}\text { Observations with } 50 \% \text { relief: } \\
58 \% \text { vs. } 55 \%, p=N R \\
\text { Repeat use of medication: } 5 \% \\
\text { (2/37) vs. } 12 \%(5 / 42) \text {, RR } 0.45 \\
(95 \% \text { Cl } 0.09 \text { to } 2.20)\end{array}$ \\
\hline $\begin{array}{l}\text { Cooper, } \\
1988^{190} \\
\text { Poor }\end{array}$ & $\begin{array}{l}\text { A. Codeine } 60 \mathrm{mg}+ \\
\text { acetaminophen } 600 \mathrm{mg} \times 1 \\
\text { B. Acetaminophen } 600 \mathrm{mg} \times 1\end{array}$ & $\begin{array}{l}\text { Third molar } \\
\text { extraction; } \\
<1 \text { day ( } 6 \\
\text { hours) } \\
\mathrm{n}=67\end{array}$ & $\begin{array}{l}\text { Pain intensity difference (mean [SD NR, } 0 \text { to } 3 \text { NRS } \\
\text { converted to } 0 \text { to } 10 \text { scale): } 2.8 \text { vs. } 1.5 \text { at } 4 \text { hours, } 1.7 \\
\text { vs. } 0.3 \text { at } 6 \text { hours } p=N R \\
\text { Sum of pain intensity differences (mean [SD NR] } \\
\text { sum of differences on } 0 \text { to } 3 \text { NRS from } 0.5 \text { to } 6 \\
\text { hours): } 5.26 \text { vs. } 2.86, p=N R\end{array}$ & $\begin{array}{l}\text { Repeat medication use: } 58 \% \\
\text { (18/31) vs. } 78 \%(28 / 36), \mathrm{RR} \\
0.75 \text { (95\% Cl } 0.53 \text { to } 1.06) \\
\text { Medication rated "very good" or } \\
\text { "excellent": } 49 \%(15 / 31) \text { vs. } \\
28 \%(10 / 36), \mathrm{RR} 1.74(95 \% \mathrm{Cl} \\
0.92 \text { to } 3.30)\end{array}$ \\
\hline $\begin{array}{l}\text { Cooper, } \\
1991^{191} \\
\text { Poor }\end{array}$ & $\begin{array}{l}\text { A: Codeine } 60 \mathrm{mg}+ \\
\text { acetaminophen } 650 \mathrm{mg} \times 1 \\
\text { B: Acetaminophen } 650 \mathrm{mg} \times 1\end{array}$ & $\begin{array}{l}\text { Third molar } \\
\text { extraction; } \\
<1 \text { day ( } 6 \\
\text { hours) } \\
\mathrm{n}=76\end{array}$ & $\begin{array}{l}\text { Pain intensity difference (mean [SD NR], } 0 \text { to } 3 \\
\text { categorical converted to } 0 \text { to } 10 \text { ): } 2.3 \text { vs. } 1.2 \text { at } 4 \\
\text { hours, } p=N R ; 1.6 \text { vs. } 0.7 \text { at } 6 \text { hours, } p=N R \\
\text { Sum of pain intensity differences (mean [SD NR], } \\
\text { sum of differences on } 0 \text { to } 3 \text { scale from } 1 \text { to } 6 \text { hours): } \\
4.54 \text { vs. } 2.41, p=N S\end{array}$ & $\begin{array}{l}\text { Repeat medication use: } 79.5 \% \\
\text { (31/39) vs. } 94.6 \%(35 / 37) \\
\text { Medication rated "very good" or } \\
\text { "excellent": } 35.8 \%(14 / 39) \text { vs. } \\
8.1 \%(3 / 37), \text { RR } 4.42(95 \% \mathrm{Cl} \\
1.38 \text { to } 14.17)\end{array}$ \\
\hline $\begin{array}{l}\text { Dionne, } 1994^{194} \\
\text { Poor }\end{array}$ & $\begin{array}{l}\text { A: Codeine } 60 \mathrm{mg}+ \\
\text { acetaminophen } 650 \mathrm{mg} \times 1 \\
\text { B: Acetaminophen } 650 \mathrm{mg} \times 1\end{array}$ & $\begin{array}{l}\text { Third molar } \\
\text { extraction; } \\
<1 \text { day ( } 6 \\
\text { hours) } \\
\mathrm{n}=51\end{array}$ & $\begin{array}{l}\text { Pain intensity difference (mean [SD NR], } 0 \text { to } 3 \\
\text { categorical converted to } 0 \text { to } 10 \text { ): } 3.7 \text { vs. } 2.7 \text { at } 4 \\
\text { hours, } p=N S ; 2.8 \text { vs. } 2.7 \text { at } 6 \text { hours, } p=N S \\
\text { Sum of pain intensity differences (mean [SD NR], } \\
\text { sum of differences on } 0 \text { to } 3 \text { scale from } 1 \text { to } 6 \text { hours): } \\
6.1 \text { vs. } 5.4, p=N S\end{array}$ & NR \\
\hline $\begin{array}{l}\text { Forbes, } 1990^{195} \\
\text { Poor }\end{array}$ & $\begin{array}{l}\text { A: Codeine } 60 \mathrm{mg}+ \\
\text { acetaminophen } 600 \mathrm{mg} \times 1 \\
\text { B: Acetaminophen } 600 \mathrm{mg} \mathrm{x}\end{array}$ & $\begin{array}{l}\text { Third molar } \\
\text { extraction; } \\
<1 \text { day ( } 6 \\
\text { hours) } \\
\mathrm{n}=74\end{array}$ & $\begin{array}{l}\text { Pain intensity difference (mean [SD NR], } 0 \text { to } 3 \\
\text { categorical scale converted to } 0 \text { to } 10 \text { ): } 1.40 \text { vs. } 1.20 \\
\text { at } 4 \text { hours, } p=N S ; 0.80 \text { vs. } 0.47 \text { at } 6 \text { hours, } p=N S \\
\text { Sum of pain intensity differences (mean [SD NR], } \\
\text { sum of differences on } 0 \text { to } 3 \text { scale from } 1 \text { to } 6 \text { hours): } \\
2.84 \text { vs. } 2.47, p=N S\end{array}$ & $\begin{array}{l}50 \% \text { pain relief: } 21.0 \%(8 / 38) \\
\text { vs. } 27.8 \%(10 / 36) \text { at } 4 \text { hours, } \\
\text { p=NS; } 16.7 \%(6 / 36) \text { vs. } 13.2 \% \\
\text { (5/38) at } 6 \text { hours, p=NS } \\
\text { Repeat medication use: } 84.2 \% \\
(32 / 38) \text { vs. } 83.3 \%(30 / 36)\end{array}$ \\
\hline
\end{tabular}




\begin{tabular}{|c|c|c|c|c|}
\hline $\begin{array}{l}\text { Study, Year } \\
\text { Quality }\end{array}$ & Interventions & $\begin{array}{l}\text { Procedure; } \\
\text { Followup } \\
\text { Duration } \\
\text { Sample Size } \\
\end{array}$ & Pain Intensity Results & Other Results \\
\hline $\begin{array}{l}\text { Skoglund, } \\
1991^{212} \\
\text { Fair }\end{array}$ & $\begin{array}{l}\text { A. Codeine } 60 \mathrm{mg}+ \\
\text { acetaminophen } 1000 \mathrm{mg} \times 1 \\
\text { B. Acetaminophen } 1000 \mathrm{mg} \times 1 \\
\text { C. Acetaminophen } 2000 \mathrm{mg} \times 1\end{array}$ & $\begin{array}{l}\text { Third molar } \\
\text { extraction; } \\
<1 \text { day ( } 6 \\
\text { hours) } \\
\mathrm{n}=106\end{array}$ & $\begin{array}{l}\text { Pain intensity difference (mean [SD NR], } 0 \text { to } 100 \\
\text { VAS converted to } 0 \text { to } 10 \text { scale): } 2.1 \text { vs. } 2.3 \text { vs. } 1.8 \text { at } \\
4 \text { hours, } 1.3 \text { vs. } 1.1 \text { vs. } 1.4 \text { at } 6 \text { hours, p=NR } \\
\text { Sum of pain intensity differences (mean [SD], sum of } \\
\text { differences on } 0 \text { to } 100 \text { VAS from } 1 \text { to } 6 \text { hours): } \\
132.6(147.8 \text { ) vs. } 124.5 \text { (169.4) vs. } 95.1 \text { (167.5); } \\
\text { p<0.001 for A vs. B, p=NS for A vs. C and B vs. C }\end{array}$ & NR \\
\hline $\begin{array}{l}\text { Sunshine, } \\
1986^{213} \\
\text { Fair }\end{array}$ & $\begin{array}{l}\text { A: Codeine } 60 \mathrm{mg}+ \\
\text { acetaminophen } 650 \mathrm{mg} \times 1 \\
\text { B: Acetaminophen } 650 \mathrm{mg} \times 1\end{array}$ & $\begin{array}{l}\text { Third molar } \\
\text { extraction; } \\
<1 \text { day ( } 6 \\
\text { hours) } \\
\mathrm{n}=61\end{array}$ & $\begin{array}{l}\text { Pain intensity difference (mean [SD NR], } 0 \text { to } 3 \\
\text { categorical scale transformed to } 0 \text { to } 10 \text { ): } 2.03 \text { vs. } \\
2.23 \text { at } 4 \text { hours, } p=N S ; 1.30 \text { vs. } 0.67 \text { at } 6 \text { hours, } \\
p=N S \\
\text { Sum of pain intensity differences (mean [SD NR], } \\
\text { sum of differences on } 0 \text { to } 3 \text { scale from } 0.5 \text { to } 6 \\
\text { hours): } 4.66 \text { vs. } 4.23, p=N S\end{array}$ & $\begin{array}{l}\text { Rescue medication use: } 29.0 \% \\
\text { (9/31) vs. } 46.7 \%(14 / 30)\end{array}$ \\
\hline
\end{tabular}

Abbreviations: $\mathrm{CI}$ = confidence interval; IQR = interquartile range; $\mathrm{MD}$ = mean difference; $\mathrm{NR}$ = not reported; NRS = numeric rating scale; $\mathrm{NS}$ = not significant; RR = relative risk; $\mathrm{SD}$ = standard deviation; VAS = visual analog scale 


\section{Opioid Agonist Versus Mixed Agent}

Two trials compared a pure opioid agonist versus a mixed agent (tramadol) in patients with dental pain (Appendix E, Table E-8; Table 31). ${ }^{207,211}$ One fair-quality trial $(n=172)$ of patients who underwent third molar extraction found single dose codeine $60 \mathrm{mg}$ associated with smaller effects on pain intensity versus tramadol 50 or $100 \mathrm{mg}$ (difference 0.6 and 1.3 points, respectively, on a 0 to 10 scale; p not reported) and a smaller SPID through 6 hours. $^{207}$ Differences in rescue medication use were not statistically significant (80\% vs. 69\% vs. 73\%; RR 1.15, 95\% CI 0.89 to 1.49 for codeine versus tramadol $50 \mathrm{mg}$ and RR 1.10, 95\% CI 0.86 to 1.41 for codeine versus tramadol $100 \mathrm{mg}$ ). This trial also evaluated codeine plus acetaminophen and found similar effects compared with tramadol 50 or $100 \mathrm{mg}$. A small $(\mathrm{n}=20)$, poor quality trial of patients with periradicular abscess found no difference between codeine (pure opioid agonist) plus acetaminophen versus tramadol (mixed agent) plus acetaminophen in pain intensity (median 1.0 vs 1.0 on a 0 to 10 scale at 6 hours, $p=0.59 ; 0.6$ vs. 0.6 at 12 hours, $p=0.54$, and 0 vs. 0 at 3 days, $\mathrm{p}=0.43) .{ }^{211}$ In both trials, estimates for adverse events did not indicate differences.

\section{Table 31. Opioid agonists versus mixed agents for dental pain}

\begin{tabular}{|c|c|c|c|c|}
\hline $\begin{array}{l}\text { Study, } \\
\text { Year } \\
\text { Quality }\end{array}$ & Interventions & $\begin{array}{l}\text { Procedure; } \\
\text { Followup } \\
\text { Duration } \\
\text { Sample } \\
\text { Size }\end{array}$ & Pain Intensity Results & Other Results \\
\hline $\begin{array}{l}\text { Moore, } \\
1998^{207} \\
\text { Fair }\end{array}$ & $\begin{array}{l}\text { A: Codeine } 60 \mathrm{mg}+ \\
\text { aspirin } 650 \mathrm{mg} \times 1 \\
\text { B: Codeine } 60 \mathrm{mg} \times 1 \\
\text { C: Tramadol } 50 \mathrm{mg} \times 1 \\
\text { D: Tramadol } 100 \mathrm{mg} \times 1\end{array}$ & $\begin{array}{l}\text { Third molar } \\
\text { extraction; } \\
<1 \text { day ( } 4 \\
\text { and } 8 \\
\text { hours) } \\
\mathrm{n}=172\end{array}$ & $\begin{array}{l}\text { Pain intensity difference (mean } \\
\text { [SD NR], } 0 \text { to } 3 \text { categorical scale } \\
\text { converted to } 0 \text { to } 10 \text { scale): } 1.1 \\
\text { vs. } 0.3 \text { vs. } 0.9 \text { vs. } 1.6 \text { at } 4 \text { hours, } \\
\text { p=NR; } 0.7 \text { vs. } 0.5 \text { vs. } 1.1 \text { vs. } 1.4 \\
\text { at } 8 \text { hours, p=NR } \\
\text { Sum of pain intensity differences } \\
\text { (mean [SD], sum of difference on } \\
0 \text { to } 3 \text { scale from } 0.5 \text { to } 6 \text { hours): } \\
2.2(0.45) \text { vs. } 0.6(0.31) \text { vs. } 1.5 \\
\text { (0.47) vs. } 2.3(0.61), p<0.05 \text { for A } \\
\text { and D vs. placebo, p=NS for B } \\
\text { and C vs. placebo }\end{array}$ & $\begin{array}{l}\text { Rescue medication } \\
\text { use: } 73.7 \%(23 / 38) \text { vs. } \\
80.0 \%(24 / 30) \text { vs. } \\
69.4 \%(34 / 49) \text { vs. } \\
72.9 \%(35 / 48) ; \text { RR } \\
1.15(95 \% \mathrm{Cl} 0.89 \text { to } \\
1.49) \text { for B vs. C and } \\
\text { RR } 1.10(95 \% \text { Cl } 0.86 \\
\text { to } 1.41) \text { for B vs. D }\end{array}$ \\
\hline $\begin{array}{l}\text { Santini, } \\
2017^{211} \\
\text { Poor }\end{array}$ & $\begin{array}{l}\text { A: Codeine } 30 \mathrm{mg}+ \\
\text { acetaminophen } 500 \mathrm{mg} \\
\text { every } 4 \text { hours } \times 3 \text { days } \\
\text { B: Tramadol } 37.5 \mathrm{mg}+ \\
\text { acetaminophen } 500 \mathrm{mg} \\
\text { every } 4 \text { hours } \times 3 \text { days }\end{array}$ & $\begin{array}{l}\text { Third molar } \\
\text { extraction; } \\
12 \text { hours } \\
\text { and } 3 \text { days } \\
n=20\end{array}$ & $\begin{array}{l}\text { Pain (median [IQR], } 0 \text { to } 10 \\
\text { VAS): } 1.0 \text { (0 to } 6.0) \text { vs. } 1.0(0.5 \\
\text { to } 6.2) \text { at } 6 \text { hours, } p=0.59 ; 0.6(0 \\
\text { to } 3.6) \text { vs. } 0.6(0 \text { to } 6.2) \text { at } 12 \\
\text { hours, } p=0.54,0 \text { ( } 0 \text { to } 0.4) \text { vs. } 0 \\
(0 \text { to } 2.0) \text { at } 3 \text { days, } p=0.43\end{array}$ & NR \\
\hline
\end{tabular}

Abbreviations: $\mathrm{CI}$ = confidence interval; IQR = interquartile range; NR = not reported; NS = not significant; RR = relative risk; $\mathrm{SD}=$ standard deviation; VAS = visual analog scale

KQs 6b and 6d ask how the comparative effectiveness and harms of opioid therapy vary depending on: (1) patient demographics (e.g., age, race, ethnicity, gender); (2) patient medical or psychiatric comorbidities; (3) dose of opioids; (4) duration of opioid therapy, including number of opioid prescription refills and quantity of pills used; (5) opioid use history; (6) substance use history; (7) use of concomitant therapies.

Evidence on how comparative effectiveness and harms of opioid therapy for dental pain vary according to patient and prescribing factors was lacking. The trials did not describe substance use history or presence of medical or psychiatric comorbidities. Most trials were conducted in populations of younger persons undergoing third molar surgery. This surgery is typically 
performed for third molar impaction, a condition that usually is not painful. Two trials evaluated older populations of patients with painful dental conditions (irreversible pulpitis or acute apical periodontitis), ${ }^{205,210}$ but there was insufficient evidence to determine how presence of nonsurgical pain, antecedent pain or age impacted findings. Evidence was too limited to determine effects of different opioid doses (converted into morphine milligram equivalents) on comparative effectiveness and harms. Most trials evaluated single doses of opioids, and no trial permitted opioid refills.

KQ 6e concerns the effects of prescribing opioid therapy versus not prescribing opioid therapy for acute pain on (1) short-term ( $<3$ months) continued need for prescription pain relief, such as need for opioid refills, and (2) long-term opioid use (3 months or greater).

One retrospective cohort study $(n=70,942)$ of opioid-naïve patients who underwent third molar extraction found a filled perioperative opioid prescription (from 7 days prior to 3 days after the surgery) associated with increased risk of persistent opioid use (adjusted OR 2.69, 95\% CI 2.10 to 3.44) (Appendix E and F; Tables E-9 and F-2). ${ }^{221}$ The analysis adjusted for age, sex, insurance status, income, chronic conditions, perioperative medications, and third molar impaction status. Persistent use was defined as at least one opioid prescription filled during postprocedure days 4 to 90 and 91 to 365.

KQs $6 f$ and $6 g$ address the accuracy and effectiveness of instruments for predicting risk of opioid misuse, opioid use disorder, or overdose for patients with acute pain being considered for opioid therapy.

No study evaluated the accuracy or effectiveness of instruments for predicting risk of opioid misuse, opioid use disorder, or overdose in patients with acute dental pain.

KQ $6 \mathrm{~h}$ addresses the effect of the following factors on the decision to prescribe opioids for patients with acute pain being considered for opioid therapy: (1) existing opioid management plans; (2) patient education; (3) clinician and patient values and preferences related to opioids; (4) urine drug screening; (5) use of prescription drug monitoring program data; (6) availability of close followup.

Evidence on the effect of patient education, use of risk mitigation strategies, clinician and patient values and preferences, or availability of followup on decisions to prescribe opioids for postoperative pain was limited. One before-after study $(n=6,204)$ found that among patients who received pain medication, the likelihood of receiving opioid analgesics was decreased in two assessment periods following implementation of mandatory use of prescription drug monitoring data prior to prescribing a controlled substance, compared with before implementation (OR 0.37, 95\% CI 0.31 to 0.45 and OR 0.24 , 95\% CI 0.20 to 0.30 ) (Appendix E, Table E-9). ${ }^{222}$ In addition to use of a before-after study design, the study did not control for potential confounders.

\section{Nonopioid Pharmacologic Therapy}

KQs $6 \mathrm{i}$ and 6k address the comparative effectiveness and harms of nonopioid pharmacologic therapy versus: (1) nonopioid pharmacologic therapy or (2) nonpharmacologic therapy?

Seventeen trials evaluated nonopioid pharmacologic therapy for acute dental pain (Appendix E, Table E-8). ${ }^{176,178,180,182,186,190,191,193-195,197,198,202-204,206,208,209,213,216,219 ~ D e n t a l ~ p a i n ~ w a s ~ r e l a t e d ~ t o ~}$ third molar extraction in 19 trials, ${ }^{176,178,180,182,186,190,191,193-195,197,198,202,203,208,209,213,216,219}$ various oral surgeries in 1 trial, ${ }^{204}$ and mouth flap surgery for periodontitis in 1 trial. $^{206}$ Seventeen trials compared an NSAID versus acetaminophen, ${ }^{176,178,180,182,186,190,191,193-195,197,202,204,209,213,216,219 ~ o n e ~}$ trial pregabalin versus an NSAID, ${ }^{198}$ two trials a non-aspirin versus aspirin NSAID, ${ }^{203,208}$ and one trial an oral versus topical NSAID. ${ }^{206}$ Two trials were rated good quality, ${ }^{186,202}$ fourteen trials 
were rated fair quality ${ }^{176,178,180,182,193,197,198,203,204,208,209,213,216,219}$ and five trials poor quality (Appendix F, Table F-1). ${ }^{190,191,194,195,206}$

\section{NSAID Versus Acetaminophen}

Seventeen trials compared an NSAID versus acetaminophen for acute dental pain (Table 32). ${ }^{186,190,191,193-195,197,202,204,209,213,216}$ Dental pain was due to third molar extraction in all trials except for one, ${ }^{204}$ which evaluated dental pain related to extractions and other dental procedures. The sample size ranged from 38 to $612(\mathrm{~N}=3,152)$. All trials were single dose studies with duration of followup that ranged from of 6 to 12 hours. Fourteen trials were conducted in the United States, and two trials in Europe. The NSAID was ibuprofen in nine trials $^{178,182,193,195,197,202,204,209,219}$ (200 mg in one trial, $250 \mathrm{mg}$ in one trial, $400 \mathrm{mg}$ in five trials), flurbiprofen in three trials (50 or $100 \mathrm{mg}$ ), ${ }^{191,194,213}$ ketoprofen in two trials (25 or $\left.100 \mathrm{mg}\right),{ }^{176,209}$ and ketorolac (10 or $20 \mathrm{mg}),{ }^{195}$ diclofenac (100 mg), ${ }^{186}$ meclofenamate (100 mg), ${ }^{190}$ aspirin $(1000 \mathrm{mg})^{216}$ and naproxen sodium $(440 \mathrm{mg})^{180}$ in one trial each (two trials evaluated two different types of NSAIDs). Two trials of ibuprofen used a solubilized ("liquigel”) formulation rather than a standard solid tablet, ${ }^{197,209}$ and one trial evaluated ibuprofen sodium and ibuprofen with poloxamer. ${ }^{178}$ The dose of acetaminophen was $1000 \mathrm{mg}$ in twelve trials ${ }^{176,178,180,182,186,193,197,202,204,209,216,219}$ and 600 to $650 \mathrm{mg}$ in five trials. ${ }^{190,191,194,195,213}$

All of the trials used a parallel group design and were blinded using matching placebo, with the exception of one trial ${ }^{191}$ in which blinding was unclear. Two trials were rated good quality, ${ }^{186,202} 11$ trials fair quality, ${ }^{176,178,180,182,193,197,204,209,213,216,219}$ and four trials poor quality (Appendix F, Table F-1). ${ }^{190,191,194,195}$ Methodological limitations in the fair and poor quality trials included failure to report randomization and allocation concealment methods or unclear blinding of outcome assessors; the poor quality trials also did not conduct intention to treat analysis. In most trials, results for pain intensity and PID at 4 hours and at 6 to 8 hours had to be estimated from graphs; the statistical significance of differences were usually not reported or calculable (e.g., standard deviations not provided).

Fifteen trials $(\mathrm{N}=2,506)$ evaluated effects of an NSAID versus acetaminophen on pain intensity at 4 hours, based on the PID or mean pain intensity. ${ }^{176,178,180,182,186,190,191,193-}$

$195,197,202,204,213,219$ In thirteen trials, effects on pain intensity favored the NSAID, with differences that ranged from moderate to large (1.2 to 3.3 points on a 0 to 10 scale). ${ }^{176,178,180,182,190,191,193-}$ $195,197,204,213,219$ In the other two trials, NSAIDs and acetaminophen were associated with similar effects on pain intensity (differences 0 and 0.2 points). ${ }^{186,202}$ The largest trial $(n=612)$, which evaluated patients undergoing extractions and other dental procedures, found NSAIDs associated with moderate effects versus acetaminophen on pain intensity (mean difference in PID 1.33 points on a 0 to 10 scale, $95 \%$ CI 0.87 to 1.79$).{ }^{204}$ Fourteen trials (N=2,503) also evaluated effects of an NSAID versus acetaminophen on pain intensity at 6 to 8 hours (subsequent to the typical peak analgesic effect of a single dose). ${ }^{178,180,182,186,190,191,193-195,197,202,204,213,219}$ Thirteen of the trials found that effects on pain intensity favored NSAIDs at 6 to 8 hours (differences 1.0 to 2.8 on a 0 to 10 scale); ${ }^{186,190,191,193-195,197,204,213,219}$ the other trial ${ }^{202}$ found NSAIDs and acetaminophen associated with similar effects on pain intensity at 8 hours. One trial $(n=200)$ did not report pain intensity but reported pain relief. ${ }^{209}$ It found ibuprofen liquigel and ketoprofen associated with greater pain relief versus acetaminophen at 4 hours (differences 0.9 and 0.4 on a 0 to 4 scale, respectively; p not reported). Ibuprofen, but not ketoprofen, was associated with greater pain relief than acetaminophen at 6 hours (differences 0.7 and 0.1, respectively; p not reported). 
Fifteen trials $(\mathrm{N}=2,868)$ evaluated effects of an NSAID versus acetaminophen on the SPID. ${ }^{176,180,182,190,191,193-195,197,202,204,209,213,216,219}$ The SPID is difficult to interpret and not directly comparable across trials, because it varies depending on the pain intensity scale used, the number and timing of measurements, and the duration of followup. NSAIDs were associated with larger SPID versus acetaminophen in 13 trials; most trials ${ }^{176,180,182,190,193,197,204,209,219}$ found that the

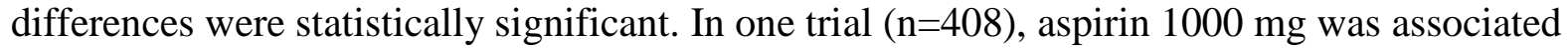
with a smaller SPID than acetaminophen $1000 \mathrm{mg}$ (5.9 [SD 5.1] vs. 7.9 [SD 4.9], p<0.001). ${ }^{216}$ One other trial ${ }^{202}(n=103)$ found no statistically significant difference between ibuprofen versus acetaminophen in the sum of pain relief plus pain intensity differences through 8 hours, and one $\operatorname{trial}^{186}(\mathrm{n}=44)$ found no difference between diclofenac versus acetaminophen in average pain (mean of all measurements) through 8 hours (3.8 vs. 3.7, $\mathrm{p}>0.05$ ). NSAIDs were associated with decreased likelihood of rescue or repeat medication use versus acetaminophen (11 trials, $\mathrm{N}=2,014$, pooled RR $0.64,95 \%$ CI 0.58 to 0.71 , I²=15\%; ARD $-22 \%$, 95\% CI $-27 \%$ to $-17 \%$; Figure D-14) 178,186,190,191,193,195,197,204,209,213,219 and increased likelihood of being rated "very good" or "excellent" (6 trials, N=936, pooled RR 1.61, 95\% CI 1.37 to 1.88, I²=21\%; ARD 25\%, 95\% CI 18\% to 32\%; Figure D-15). ${ }^{17,190,191,193,197,219}$ Three trials ${ }^{178,195,204}$ (N=926) found NSAIDs associated with greater likelihood of more than 50 percent pain improvement (45\% vs. 30\%, RR $1.50,95 \%$ CI 1.21 to 1.85 , 36\% vs. $17 \%$, RR 2.14, 95\% CI 0.98 to 4.66 , and $91 \%$ vs. $75 \%$, RR 1.22, 95\% CI 1.06 to 1.39$)$, and one trial ${ }^{209}(\mathrm{n}=200)$ found ibuprofen liquigel associated with greater likelihood of complete pain relief (73\% vs. 48\%, RR 1.51, 95\% CI 1.13 to 2.01). One trial $(n=333)$ found ibuprofen and acetaminophen associated with similar likelihood of experiencing meaningful pain relief as defined by the patient (79\% vs. $72 \%$, RR 1.11, 95\% CI 0.98 to 1.26). ${ }^{182}$ Effects on quality of life or function were not reported, other than one trial that found ibuprofen associated with less pain interference with daily activities, based on the Rainier scale (difference 5.3 to 7.3 points, scale not reported). ${ }^{178}$ 
Table 32. NSAIDs versus acetaminophen for acute dental pain

\begin{tabular}{|c|c|c|c|c|}
\hline $\begin{array}{l}\text { Author, } \\
\text { Year } \\
\text { Quality }\end{array}$ & Interventions & $\begin{array}{l}\text { Procedure; } \\
\text { Followup Duration } \\
\text { Sample Size }\end{array}$ & Pain Intensity & Other Results \\
\hline $\begin{array}{l}\text { Akural, } \\
2009^{176} \\
\text { Fair }\end{array}$ & $\begin{array}{l}\text { A. Ketoprofen, } 100 \mathrm{mg} x \\
1 \\
\text { B. Acetaminophen, } 1000 \\
\mathrm{mg} \times 1\end{array}$ & $\begin{array}{l}\text { Third molar } \\
\text { extraction; } \\
<1 \text { day (10 hours) } \\
n=38\end{array}$ & $\begin{array}{l}\text { Pain intensity difference at rest (mean [SD NR], } \\
0 \text { to } 10 \text { NRS): } 1.9 \text { vs. } 0.7 \text { at } 4 \text { hours } \\
\text { Sum of pain intensity difference at rest (mean } \\
\text { [SD], sum of differences on } 0 \text { to } 10 \text { NRS from } \\
0.25 \text { to } 10 \text { hours): } 14.91 \text { (19.50) vs. }-4.22 \\
(19.92), p<0.05\end{array}$ & NR \\
\hline $\begin{array}{l}\text { Breivik, } \\
1999^{186} \\
\text { Good }\end{array}$ & $\begin{array}{l}\text { A: Diclofenac } 100 \mathrm{mg} x \\
1 \\
\text { B: Acetaminophen } 1000 \\
\mathrm{mg} \times 1\end{array}$ & $\begin{array}{l}\text { Third molar } \\
\text { extraction; } \\
<1 \text { day ( } 8 \text { hours) } \\
n=44\end{array}$ & $\begin{array}{l}\text { Pain (mean [SD NR], } 0 \text { to } 100 \text { VAS converted } \\
\text { to } 0 \text { to } 10 \text { scale): } 3.1 \text { vs. } 3.1 \text { at } 4 \text { hours, } 3.5 \text { vs. } \\
5.8 \text { at } 6 \text { hours, } p=N R \\
\text { Average pain (mean [SD] of all values from } 0.5 \\
\text { to } 8 \text { hours, } 0 \text { to } 100 \text { VAS converted to } 0 \text { to } 10 \\
\text { scale): } 3.8(1.8) \text { vs. } 3.7(2.4), p>0.05\end{array}$ & $\begin{array}{l}\text { Rescue medication use: } 36 \% \text { (8/22) vs. } 45 \% \\
\text { (10/22), RR } 0.80 \text { (95\% Cl } 0.39 \text { to } 1.64)\end{array}$ \\
\hline $\begin{array}{l}\text { Cooper, } \\
1988^{190} \\
\text { Poor }\end{array}$ & $\begin{array}{l}\text { A: Meclofenamate } \\
\text { sodium } 100 \mathrm{mg} \times 1 \\
\mathrm{~B}: \text { Acetaminophen } 600 \\
\mathrm{mg} \times 1\end{array}$ & $\begin{array}{l}\text { Third molar } \\
\text { extraction; } \\
<1 \text { day ( } 6 \text { hours) } \\
\mathrm{n}=72\end{array}$ & $\begin{array}{l}\text { Pain intensity difference (mean [SD NR], } 0 \text { to } 3 \\
\text { NRS converted to } 0 \text { to } 10 \text { scale): } 3.4 \text { vs. } 1.5 \text { at } \\
4 \text { hours, } 1.8 \text { vs. } 0.3 \text { at } 6 \text { hours; } p=N R \\
\text { Sum of pain intensity differences (mean [SD } \\
\text { NR] sum of differences on } 0 \text { to } 3 \text { NRS from } 0.5 \\
\text { to } 6 \text { hours): } 5.61 \text { vs. } 2.86, p<0.05\end{array}$ & $\begin{array}{l}\text { Medication rated "very good" or "excellent": } \\
47 \%(17 / 36) \text { vs. } 28 \%(10 / 36), \text { RR } 1.70 \text { (95\% } \\
\text { Cl } 0.91 \text { to } 3.19) \\
\text { Repeat medication use: } 53 \%(19 / 36) \text { vs. } \\
78 \%(28 / 36), \text { RR } 0.68(0.48 \text { to } 0.97)\end{array}$ \\
\hline $\begin{array}{l}\text { Cooper, } \\
1989^{193} \\
\text { Fair }\end{array}$ & $\begin{array}{l}\text { A: Ibuprofen } 400 \text { mg x } 1 \\
\text { B: Acetaminophen } 1000 \\
m g \times 1\end{array}$ & $\begin{array}{l}\text { Third molar } \\
\text { extraction; } \\
<1 \text { day ( } 6 \text { hours) } \\
\mathrm{n}=120\end{array}$ & $\begin{array}{l}\text { Pain intensity difference (mean [SD NR, } 0 \text { to } 3 \\
\text { NRS converted to } 0 \text { to } 10 \text { scale): } 3.0 \text { vs. } 1.5 \text { at } \\
4 \text { hours, } 2.1 \text { vs. } 0.5 \text { at } 6 \text { hours, } p=N R \\
\text { Sum of pain intensity differences (mean [SD] } \\
\text { sum of difference from } 0.5 \text { to } 6 \text { hours on } 0 \text { to } 3 \\
\text { NRS): } 5.7(0.7) \text { vs. } 3.4(0.7), p<0.05\end{array}$ & $\begin{array}{l}\text { Medication rated "excellent" or "very good": } \\
52 \%(32 / 61) \text { vs. } 27 \%(16 / 59), \text { RR } 1.94(95 \% \\
\text { Cl } 1.19 \text { to } 3.13) \\
\text { Repeat medication use within } 4 \text { hours: } 30 \% \\
(18 / 61) \text { vs. } 51 \%(30 / 59), \text { RR } 0.58(95 \% \mathrm{Cl} \\
0.37 \text { to } 0.92)\end{array}$ \\
\hline $\begin{array}{l}\text { Cooper, } \\
1991^{191} \\
\text { Poor }\end{array}$ & $\begin{array}{l}\text { A: Flurbiprofen } 50 \mathrm{mg} \\
\text { B: Flurbiprofen } 100 \mathrm{mg} \\
\text { C: Acetaminophen } 650 \\
\text { mg }\end{array}$ & $\begin{array}{l}\text { Third molar } \\
\text { extraction; } \\
<1 \text { day ( } 6 \text { hours) } \\
n=120\end{array}$ & $\begin{array}{l}\text { Pain intensity difference (mean [SD NR], } 0 \text { to } 3 \\
\text { categorical converted to } 0 \text { to } 10 \text { ): } 2.5 \text { vs. } 3.3 \text { vs. } \\
1.2 \text { at } 4 \text { hours, } p=N R ; 1.7 \text { vs. } 3.0 \text { vs. } 0.7 \text { at } 6 \\
\text { hours, } p=N R \\
\text { Sum of pain intensity differences (mean [SD } \\
\text { NR], sum of differences on } 0 \text { to } 3 \text { scale from } 1 \\
\text { to } 6 \text { hours): } 4.33 \text { vs. } 6.37 \text { vs. } 2.41, p<0.05 \text { for B } \\
\text { vs. C }\end{array}$ & $\begin{array}{l}\text { Repeat medication use: } 69.0 \%(29 / 42) \text { vs. } \\
58.5 \%(24 / 41) \text { vs. } 94.6 \%(35 / 37) \\
\text { Medication rated "very good" or "excellent": } \\
40.5 \%(17 / 42) \text { vs. } 53.6 \%(22 / 41) \text { vs. } 35.8 \% \\
(14 / 39)\end{array}$ \\
\hline
\end{tabular}




\begin{tabular}{|c|c|c|c|c|}
\hline $\begin{array}{l}\text { Author, } \\
\text { Year } \\
\text { Quality }\end{array}$ & Interventions & $\begin{array}{l}\text { Procedure; } \\
\text { Followup Duration } \\
\text { Sample Size }\end{array}$ & Pain Intensity & Other Results \\
\hline $\begin{array}{l}\text { Daniels, } \\
2009 c^{178} \\
\text { Fair }\end{array}$ & $\begin{array}{l}\text { A: Ibuprofen } 512 \mathrm{mg} \mathrm{x} 1 \\
\text { B: Ibuprofen } 400 \mathrm{mg}+ \\
\text { poloxamer } 407120 \mathrm{mg} \mathrm{x} \\
1 \\
\text { C: Acetaminophen: } 1000 \\
\text { mg x } 1\end{array}$ & $\begin{array}{l}\text { Third molar } \\
\text { extraction; } \\
<1 \text { day ( } 6 \text { hours) } \\
\mathrm{n}=240\end{array}$ & $\begin{array}{l}\text { Pain intensity difference, (mean [SD NR], } 0 \text { to } 3 \\
\text { categorical scale converted to } 0 \text { to } 10 \text { scale): } \\
3.8 \text { vs. } 4.0 \text { vs. } 2.2 \text { at } 4 \text { hours, } 3.0 \text { vs. } 3.0 \text { vs. } 1.8 \\
\text { at } 6 \text { hours }\end{array}$ & 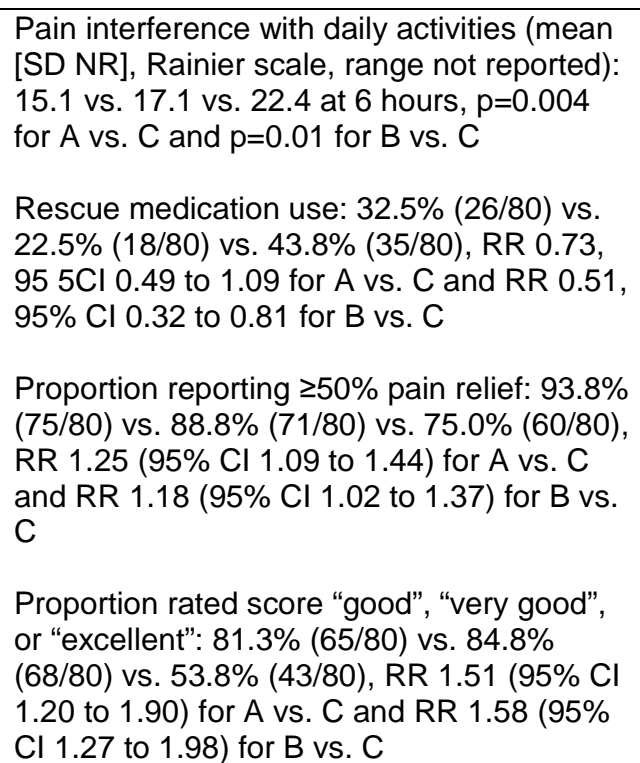 \\
\hline $\begin{array}{l}\text { Dionne, } \\
\text { 1994 } \\
\text { Poor }\end{array}$ & $\begin{array}{l}\text { A: Flurbiprofen } 50 \mathrm{mg} \times \\
1 \\
\mathrm{~B} \text { : Flurbiprofen } 100 \mathrm{mg} \times \\
1 \\
\mathrm{C} \text { : Acetaminophen } 650 \\
\mathrm{mg} \times 1\end{array}$ & $\begin{array}{l}\text { Third molar } \\
\text { extraction; } \\
<1 \text { day ( } 6 \text { hours) } \\
\mathrm{n}=75\end{array}$ & $\begin{array}{l}\text { Pain intensity difference (mean [SD NR], } 0 \text { to } 3 \\
\text { categorical converted to } 0 \text { to } 10 \text { ): } 5.2 \text { vs. } 6.0 \text { vs. } \\
2.7 \text { at } 4 \text { hours, } p<0.05 \text { for A or B vs. C; } 4.7 \text { vs. } \\
5.5 \text { vs. } 2.7 \text { at } 6 \text { hours, } p<0.05 \text { for A or B vs. C } \\
\text { Sum of pain intensity differences (mean [SD } \\
\text { NR], sum of differences on } 0 \text { to } 3 \text { scale from } 1 \\
\text { to } 6 \text { hours): } 8.4 \text { vs. } 9.4 \text { vs. } 5.4, p<0.05 \text { for A or } \\
\text { B vs. C }\end{array}$ & NR \\
\hline $\begin{array}{l}\text { Forbes, } \\
1990^{195} \\
\text { Poor }\end{array}$ & $\begin{array}{l}\text { A: Ketorolac } 10 \mathrm{mg} \times 1 \\
\text { B: Ketorolac } 20 \mathrm{mg} \times 1 \\
\text { C: Ibuprofen } 400 \mathrm{mg} \times 1 \\
\text { D: Acetaminophen } 600 \\
\mathrm{mg} \times 1\end{array}$ & $\begin{array}{l}\text { Third molar } \\
\text { extraction; } \\
<1 \text { day (6 hours) } \\
\mathrm{n}=134\end{array}$ & $\begin{array}{l}\text { Pain intensity difference (mean [SD NR], } 0 \text { to } 3 \\
\text { categorical converted to } 0 \text { to } 10 \text { ): } 3.33 \text { vs. } 3.03 \\
\text { vs. } 3.53 \text { vs. } 1.20 \text { at } 4 \text { hours, } p<0.01 \text { for } A, B \text {, or } \\
\text { C vs. D; } 1.83 \text { vs. } 2.56 \text { vs. } 1.86 \text { vs. } 0.47 \text { at } 6 \\
\text { hours, } p<0.01 \text { for B vs. D, } p=N S \text { for } A \text { or } C \text { vs. } \\
\text { D } \\
\text { Sum of pain intensity differences (mean [SD } \\
\text { NR], sum of differences on } 0 \text { to } 3 \text { scale from } 1 \\
\text { to } 6 \text { hours): } 5.84 \text { vs. } 5.69 \text { vs. } 5.31 \text { vs. } 2.47 \text {, } \\
p<0.01 \text { for } A, B \text {, or } C \text { vs. D }\end{array}$ & $\begin{array}{l}\geq 50 \% \text { pain relief: } 64.5 \%(20 / 31) \text { vs. } 68.6 \% \\
(24 / 35) \text { vs. } 59.4 \%(19 / 32) \text { vs. } 27.8 \%(10 / 36) \\
\text { at } 4 \text { hours, } p<0.01 \text { for A, B or C vs. D; } \\
32.3 \%(10 / 31) \text { vs. } 42.9 \%(15 / 35) \text { vs. } 31.2 \% \\
(10 / 32) \text { vs. } 16.7 \%(6 / 36) \text { at } 6 \text { hours, } p<0.01 \\
\text { for B vs. D, p=NS for A or C vs. D } \\
\text { Repeat medication use: } 51.6 \%(16 / 31) \text { vs. } \\
57.1 \%(20 / 35) \text { vs. } 62.5 \%(20 / 32) \text { vs. } 83.3 \% \\
(30 / 36)\end{array}$ \\
\hline
\end{tabular}




\begin{tabular}{|c|c|c|c|c|}
\hline $\begin{array}{l}\text { Author, } \\
\text { Year } \\
\text { Quality }\end{array}$ & Interventions & $\begin{array}{l}\text { Procedure; } \\
\text { Followup Duration } \\
\text { Sample Size }\end{array}$ & Pain Intensity & Other Results \\
\hline $\begin{array}{l}\text { Hersh, } \\
2000^{197} \\
\text { Fair }\end{array}$ & $\begin{array}{l}\text { A: Ibuprofen liquigel } 200 \\
\text { mg } \times 1 \\
\text { B: Ibuprofen liquigel } 400 \\
\text { mg } \times 1 \\
\text { C: Acetaminophen } 1000 \\
\text { mg } \times 1\end{array}$ & $\begin{array}{l}\text { Third molar } \\
\text { extraction; } \\
<1 \text { day ( } 6 \text { hours) } \\
n=183\end{array}$ & $\begin{array}{l}\text { Pain intensity difference (mean [SD NR, } 0 \text { to } 3 \\
\text { NRS converted to } 0 \text { to } 10 \text { scale): } 3.7 \text { vs. } 4.7 \text { vs. } \\
2.3 \text { at } 4 \text { hours, } p<0.01 \text { for A vs. C and } p<0.001 \\
\text { for B vs. C; } 2.7 \text { vs. } 3.3 \text { vs. } 1.3 \text { at } 6 \text { hours, } \\
\text { p<0.01 for A vs. C and } p<0.001 \text { for B vs. C } \\
\text { Sum of pain intensity differences (mean [SD], } \\
\text { sum of differences on } 0 \text { to } 3 \text { NRS from } 0.25 \text { to } 6 \\
\text { hours): } 6.93 \text { ( } 4.61 \text { ) vs. } 8.07 \text { ( } 3.84 \text { ) vs. } 5.05 \\
(4.60), p<0.001 \text { for A or B vs. C }\end{array}$ & $\begin{array}{l}\text { Medication rated "very good" or "excellent": } \\
63 \%(38 / 61) \text { vs. } 79 \%(47 / 59) \text { vs. } 52 \% \\
\text { (33/63); RR } 0.78(95 \% \text { Cl } 0.62 \text { to } 0.99) \text { for A } \\
\text { vs. B, RR } 1.19(95 \% \text { Cl } 0.88 \text { to } 1.61) \text { for A } \\
\text { vs. C, RR } 1.52(95 \% \text { Cl } 1.16 \text { to } 1.99) \\
\text { Rescue medication use: } 31 \%(19 / 61) \text { vs. } \\
23 \%(14 / 59) \text { vs. } 51 \%(32 / 63) \text {; RR } 1.31(95 \% \\
\text { Cl } 0.73 \text { to } 2.37) \text { for A vs. B, RR } 0.61(95 \% \\
\mathrm{Cl} 0.39 \text { to } 0.96) \text { for A vs. C, RR } 0.47(95 \% \\
\text { Cl } 0.28 \text { to } 0.78) \text { for B vs. C }\end{array}$ \\
\hline $\begin{array}{l}\text { Kiersch, } \\
1994^{180} \\
\text { Fair }\end{array}$ & $\begin{array}{l}\text { A: Naproxen sodium } 440 \\
\text { mg x } 1 \\
\text { B: Acetaminophen } 1000 \\
\text { mg x } 1\end{array}$ & $\begin{array}{l}\text { Third molar } \\
\text { extraction; } \\
<1 \text { day (12 hours) } \\
\mathrm{n}=181\end{array}$ & $\begin{array}{l}\text { Pain intensity difference (mean [SD NR], } 0 \text { to } \\
100 \text { VAS converted to } 0 \text { to } 10 \text { scale): } 2.4 \text { vs. } 0.6 \\
\text { at } 4 \text { hours, } 2.2 \text { vs. }-0.1 \text { at } 6 \text { hours, } 1.3 \text { vs. }-0.7 \\
\text { at } 12 \text { hours } \\
\text { Sum of pain intensity difference (mean [SD } \\
\text { NR], sum of differences on } 0 \text { to } 3 \text { NRS from } 4 \\
\text { to } 12 \text { hours): } 5.7 \text { vs. }-0.06, p<0.001 \\
\text { Sum of pain intensity difference (mean [SD], } \\
\text { sum of differences on } 0 \text { to } 100 \text { VAS from } 4 \text { to } \\
12 \text { hours): } 224.7 \text { vs. } 11.7, p<0.001 \text { at } 12 \text { hours }\end{array}$ & NR \\
\hline $\begin{array}{l}\text { Mehlisch, } \\
1990^{204} \\
\text { Fair }\end{array}$ & $\begin{array}{l}\text { A: Ibuprofen } 400 \text { mg x } 1 \\
\text { B: Acetaminophen } 1000 \\
\text { mg x } 1\end{array}$ & $\begin{array}{l}\text { Various oral } \\
\text { surgery } \\
\text { procedures; } \\
<1 \text { day ( } 6 \text { hours) } \\
\mathrm{n}=612\end{array}$ & $\begin{array}{l}\text { Pain intensity difference (mean [SD], } 1 \text { to } 4 \\
\text { NRS converted to } 0 \text { to } 10 \text { scale): } 3.26 \text { ( } 2.90) \text { vs. } \\
1.93(2.90) \text { at } 4 \text { hours, MD } 1.33 \text { ( } 95 \% \mathrm{Cl} 0.87 \text { to } \\
1.79) ; 2.40 \text { ( } 3.50) \text { vs. } 0.13 \text { (3.50) at } 6 \text { hours, MD } \\
2.27 \text { ( } 95 \% \mathrm{Cl} 1.71 \text { to } 2.83) \\
\text { Sum of pain intensity differences (mean [SD], } \\
\text { sum of differences on } 1 \text { to } 4 \text { scale from } 0.5 \text { to } 6 \\
\text { hours): } 5.84(4.37) \text { vs. } 4.14 \text { ( } 2.97), \mathrm{MD}-1.70 \\
(95 \% \mathrm{Cl}-2.29 \text { to }-1.11 \text { ) }\end{array}$ & $\begin{array}{l}\text { Pain improved >50\%: } 45 \%(138 / 306) \text { vs. } \\
30 \%(92 / 306), \text { RR } 1.50(95 \% \mathrm{Cl} 1.21 \text { to } \\
1.85) \\
\text { Repeat medication use: } 41 \%(125 / 306) \text { vs. } \\
57 \%(174 / 306), \text { RR } 0.72(95 \% \mathrm{Cl} 0.61 \text { to } \\
0.85)\end{array}$ \\
\hline $\begin{array}{l}\text { Mehlisch, } \\
\text { 1995b } \\
\text { Fair }\end{array}$ & $\begin{array}{l}\text { A: Ibuprofen lysine } 400 \\
\text { mg x } 1 \\
\text { B: Acetaminophen } 1000 \\
\text { mg x } 1\end{array}$ & $\begin{array}{l}\text { Third molar } \\
\text { extraction; } \\
<1 \text { day ( } 6 \text { hours) } \\
n=199\end{array}$ & $\begin{array}{l}\text { Pain intensity differences (mean [SD NR], } 0 \text { to } \\
3 \text { categorical scale converted to } 0 \text { to } 10 \text { scale): } \\
4.2 \text { vs. } 2.5 \text { at } 4 \text { hours, } p \leq 0.05 ; 3.3 \text { vs. } 2.0 \text {, at } 6 \\
\text { hours, } p \leq 0.05 \\
\text { Sum of pain intensity differences (mean [SD] } \\
\text { sum of } 0 \text { to } 3 \text { pain intensity from } 0.25 \text { to } 6 \\
\text { hours) }: 6.46(3.72) \text { vs. } 3.95(4.47), p<0.05\end{array}$ & $\begin{array}{l}\text { Medication rated 'very good' or 'excellent': } \\
66 \%(65 / 98) \text { vs. } 31 \%(31 / 101) \text {, RR } 2.16 \\
(95 \% \mathrm{Cl} 1.56 \text { to } 2.99) \\
\text { Repeat medication: } 26 \%(25 / 98) \text { vs. } 60 \% \\
(61 / 101) \text {, RR } 0.42(95 \% \mathrm{Cl} 0.29 \text { to } 0.61)\end{array}$ \\
\hline
\end{tabular}




\begin{tabular}{|c|c|c|c|c|}
\hline $\begin{array}{l}\text { Author, } \\
\text { Year } \\
\text { Quality }\end{array}$ & Interventions & $\begin{array}{l}\text { Procedure; } \\
\text { Followup Duration } \\
\text { Sample Size }\end{array}$ & Pain Intensity & Other Results \\
\hline $\begin{array}{l}\text { Mehlisch, } \\
2010^{202} \\
\text { Good }\end{array}$ & $\begin{array}{l}\text { A: Ibuprofen } 400 \text { mg x } 1 \\
\text { B: Acetaminophen } 1000 \\
m g \times 1\end{array}$ & $\begin{array}{l}\text { Third molar } \\
\text { extraction; } \\
<1 \text { day ( } 8 \text { hours) } \\
\mathrm{n}=103\end{array}$ & $\begin{array}{l}\text { Pain intensity difference (mean [SD NR], } 0 \text { to } 3 \\
\text { NRS converted to } 0 \text { to } 10 \text { scale): } 2.8 \text { vs. } 2.2 \text { at } \\
4 \text { hours, p=NS; } 1.5 \text { vs. } 1.6 \text { at } 8 \text { hours, p=NS } \\
\text { Sum of pain relief and intensity differences } \\
\text { (mean [SD] sum of } 0 \text { to } 3 \text { pain intensity and } \\
\text { pain relief (scale NR) differences from } 0.25 \text { to } 8 \\
\text { hours): } 18.0 \text { (14.1) vs. } 15.5 \text { (15.0), p=NS }\end{array}$ & NR \\
\hline $\begin{array}{l}\text { Olson, } \\
2001^{209} \\
\text { Fair }\end{array}$ & $\begin{array}{l}\text { A: Ibuprofen liquigel } 400 \\
\text { mg x } 1 \\
\text { B: Ketoprofen } 25 \text { mg x } 1 \\
\text { C: Acetaminophen } 1000 \\
\text { mg x } 1\end{array}$ & $\begin{array}{l}\text { Third molar } \\
\text { extraction; } \\
<1 \text { day ( } 6 \text { hours) } \\
\mathrm{n}=200\end{array}$ & $\begin{array}{l}\text { Sum of pain intensity differences (mean [SD] } \\
\text { sum of } 0 \text { to } 3 \text { NRS from } 0.25 \text { to } 6 \text { hours): } 11.77 \\
(4.2) \text { vs. } 9.64 \text { (4.4) vs. } 8.36 \text { (4.7); MD } 3.41 \\
(95 \% \mathrm{Cl} 1.88 \text { to } 4.94) \text { for A vs. C and MD } 1.28 \\
(95 \% \mathrm{Cl}-0.28 \text { to } 2.84 \text { ) for B vs. C } \\
\text { Pain relief (mean [SD NR] on } 0 \text { to } 4 \text { scale, } \\
4=\text { complete relief): } 3.0 \text { vs. } 2.5 \text { vs. } 2.1 \text { at } 4 \\
\text { hours, } 2.6 \text { vs. } 2.0 \text { vs. } 1.9 \text { at } 6 \text { hours, p=NR }\end{array}$ & 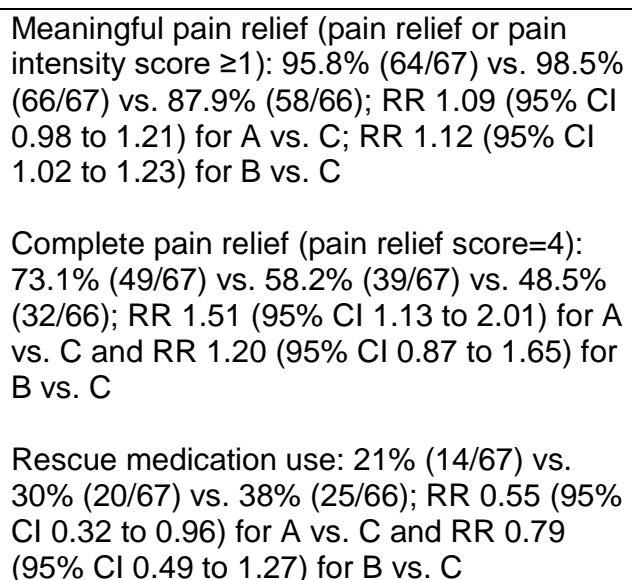 \\
\hline $\begin{array}{l}\text { Searle, } \\
2020^{182} \\
\text { Fair }\end{array}$ & $\begin{array}{l}\text { A: Ibuprofen } 250 \mathrm{mg} \times 1 \\
\mathrm{~B}: \text { Acetaminophen } 650 \\
\mathrm{mg} \times 1\end{array}$ & $\begin{array}{l}\text { Third molar } \\
\text { extraction; } \\
<1 \text { day ( } 8 \text { hours) } \\
\mathrm{n}=333\end{array}$ & $\begin{array}{l}\text { Pain intensity difference (mean [SD], } 0 \text { to } 10 \\
\text { NRS): } 4.2 \text { vs. } 2.7 \text { at } 4 \text { hours, } p<0.05 ; 3.7 \text { vs. } 2.1 \\
\text { at } 6 \text { hours, } 1.7 \text { vs. } 1.3 \text { at } 12 \text { hours, } p<0.05 \\
\text { Sum of pain intensity differences (least square } \\
\text { mean [SE] using analysis of covariance model, } \\
\text { sum of differences on } 0 \text { to } 10 \text { scale from } 0.25 \text { to } \\
8 \text { hours): } 28.7(1.5) \text { vs. } 19.6(1.6), p<0.001\end{array}$ & $\begin{array}{l}\text { Meaningful pain relief as defined by patient): } \\
79.4 \%(139 / 175) \text { vs. } 71.5 \%(118 / 165) ; \mathrm{RR} \\
1.11(95 \% \mathrm{Cl} 0.98 \text { to } 1.26) \\
\text { Treatment failure (rescue medication use or } \\
\text { dropout due to adverse event or lack of } \\
\text { efficacy) at } 8 \text { hours: } 60.0 \%(105 / 175) \text { vs. } \\
63.6 \%(105 / 165), \text { RR } 0.94(95 \% \mathrm{Cl} 0.80 \text { to } \\
1.11)\end{array}$ \\
\hline
\end{tabular}




\begin{tabular}{|c|c|c|c|c|}
\hline $\begin{array}{l}\text { Author, } \\
\text { Year } \\
\text { Quality }\end{array}$ & Interventions & $\begin{array}{l}\text { Procedure; } \\
\text { Followup Duration } \\
\text { Sample Size }\end{array}$ & Pain Intensity & Other Results \\
\hline $\begin{array}{l}\text { Sunshine, } \\
1986^{213} \\
\text { Fair }\end{array}$ & $\begin{array}{l}\text { A: Flurbiprofen } 50 \mathrm{mg} \mathrm{x} \\
1 \\
\text { B: Flurbiprofen } 100 \mathrm{mg} x \\
1 \\
\text { C: Acetaminophen } 1000 \\
\mathrm{mg} \times 1\end{array}$ & $\begin{array}{l}\text { Third molar } \\
\text { extraction; } \\
<1 \text { day ( } 6 \text { hours) } \\
\mathrm{n}=90\end{array}$ & $\begin{array}{l}\text { Pain intensity difference (mean [SD NR], } 0 \text { to } 3 \\
\text { categorical scale converted to } 0 \text { to } 10 \text { ): } 3.76 \text { vs. } \\
3.90 \text { vs. } 2.23 \text { at } 4 \text { hours, p=NS for A or B vs. C; } \\
3.33 \text { vs. } 3.56 \text { vs. } 0.67 \text { at } 6 \text { hours, p } \leq 0.05 \text { for A } \\
\text { or B vs. C } \\
\text { Sum of pain intensity differences (mean [SD } \\
\text { NR], sum of differences on } 0 \text { to } 3 \text { scale from } 0.5 \\
\text { to } 6 \text { hours): } 7.01 \text { vs. } 6.85 \text { vs. } 4.23 \text {, p=NS for A } \\
\text { or B vs. C }\end{array}$ & $\begin{array}{l}\text { Rescue medication use: } 19.4 \% \text { (6/31) vs. } \\
13.8 \%(4 / 29) \text { vs. } 46.7 \%(14 / 30)\end{array}$ \\
\hline $\begin{array}{l}\text { Voelker, } \\
2016^{216} \\
\text { Fair }\end{array}$ & $\begin{array}{l}\text { A: Aspirin } 1000 \mathrm{mg} \times 1 \\
\mathrm{~B}: \text { Acetaminophen } 1000 \\
\mathrm{mg} \times 1\end{array}$ & $\begin{array}{l}\text { Third molar } \\
\text { extraction; } \\
<1 \text { day ( } 6 \text { hours) } \\
\mathrm{n}=408\end{array}$ & $\begin{array}{l}\text { Sum of pain intensity difference (mean [SD] } \\
\text { sum of } 0 \text { to } 10 \text { NRS from } 5 \text { minutes to } 6 \text { hours): } \\
5.9(5.1) \text { vs. } 7.9(4.9), p<0.001\end{array}$ & NR \\
\hline
\end{tabular}

Abbreviations: $\mathrm{CI}$ = confidence interval; $\mathrm{MD}$ = mean difference; $\mathrm{NR}=$ not reported; NRS = numeric rating scale; NS = not significant; RR = relative risk; $\mathrm{SD}$ = standard deviation; VAS = visual analog scale

Note: Pain intensity difference $=$ pain at followup minus pain at baseline. 
NSAIDs were associated with decreased risk of any adverse event versus acetaminophen (12 trials, $\mathrm{N}=2,512$, pooled RR 0.85, 95\% CI 0.72 to 1.00 , $\mathrm{I}^{2}=0 \%$; Figure D-16). ${ }^{178,180,191,193-}$ 195,197,202,204,213,216,219 NSAIDs were also associated with decreased risk of nausea versus acetaminophen (13 trials, $\mathrm{N}=2,230$, pooled RR 0.75 , 95\% CI 0.57 to $0.98, \mathrm{I}^{2}=0 \%$; ARD $-2 \%$, 95\% CI -4\% to 0\%; Figure D-17), ${ }^{176,178,180,182,185,191,193-195,197,202,209,216}$ There was no statistically significant difference in risk of drowsiness ( 7 trials, $\mathrm{N}=1,459$, pooled RR $1.17,95 \%$ CI 0.60 to 2.30, $\mathrm{I}^{2}=28 \%$; Figure D-18), ${ }^{180,186,191,193,195,204,209}$ dizziness (8 trials, $\mathrm{N}=1,750$, pooled RR 0.59, 95\% CI 0.33 to $1.05, \mathrm{I}^{2}=6 \%$; Figure D-19), ${ }^{178,180,182,193,195,197,202,216}$ or headache (12 trials, $\mathrm{N}=2,762$, pooled RR 0.96, 95\% CI 0.65 to $1.42, \mathrm{I}^{2}=0 \%$; Figure D-20). ${ }^{178,180,182,191,193-}$

195,197,202,204,209,216 However, pooled estimates favored NSAIDs for drowsiness and dizziness, the number of adverse events in most trials was small, estimates were imprecise, and all of the trials were single dose studies. The largest trial $(\mathrm{n}=617)$ found no difference between ibuprofen versus acetaminophen in risk of any adverse event (10\% vs. 10\%, RR 1.04, 95\% CI 0.65 to 1.66), gastrointestinal adverse events (4\% vs. 5\%, RR 0.82, 95\% CI 0.40 to 1.68), sleepiness (3\% vs. 2\%, RR 1.68, 95\% CI 0.62 to 4.57 ), or headache (1\% vs. $1 \%$, RR 2.02, 95\% CI 0.37 to 10.95$).{ }^{204}$ One trial found no difference between an NSAID versus acetaminophen in risk of severe adverse events (28\% vs. $35 \%$, RR $0.78,95 \%$ CI 0.43 to 1.41$).{ }^{201}$ One trial ${ }^{180}$ reported NSAIDs and acetaminophen associated with low rates of discontinuation from participation due to adverse events (1.1\% vs. $2.2 \%)$, and one trial ${ }^{182}$ reported no discontinuations due to adverse events. Serious adverse events were not reported.

\section{Pregabalin Versus NSAID}

One fair-quality trial ( $\mathrm{n}=148)$ compared single dose pregabalin $50 \mathrm{mg}$, pregabalin $300 \mathrm{mg}$, and ibuprofen $400 \mathrm{mg}$ in patients who underwent third molar extraction ${ }^{198}$ (Table 33). Pregabalin $50 \mathrm{mg}$ was not associated with improved pain intensity at any time point through 12 hours. Pregabalin $300 \mathrm{mg}$ and ibuprofen were associated with slightly smaller PID at 4 hours and slightly larger PID at 12 hours (difference $~ 0.1$ point), but the scale was not reported. Pregabalin $300 \mathrm{mg}$ was associated with increased risk of any adverse event versus ibuprofen (68\% vs. $12 \%$, RR 5.55, 95\% CI 2.56 to 12.03); specific adverse events were not reported.

\section{Non-Aspirin Versus Aspirin NSAID}

Two fair quality trials ( $\mathrm{n}=156$ and 204) compared a single dose of a non-aspirin NSAID (diclofenac) versus aspirin following third molar extraction (Appendixes E and F, Tables E-8 and F-1; Table 33). ${ }^{203,208}$ In both trials, a single dose of diclofenac (25 to $100 \mathrm{mg}$ ) was associated with larger reductions in pain intensity than aspirin $(650 \mathrm{mg}$ ) at 4 hours (differences 1.8 to 3.5 on a 0 to 10 scale) and larger SPIDs. However, there was no difference in repeat medication use between diclofenac 25,50 , or $100 \mathrm{mg}$ versus aspirin in one of the trials ( $61 \% \mathrm{vs} .47 \% \mathrm{vs}$. $38 \%$ vs. $51 \%$, respectively; RR 0.73 , 95\% CI 0.47 to 1.14 for diclofenac 100 mg versus aspirin). Adverse events were similar between diclofenac and aspirin.

\section{Oral Versus Topical NSAID}

One small $(n=30)$, poor quality trial of patients who underwent third molar extraction found no difference between oral diclofenac versus diclofenac mouthwash in pain intensity (mean difference $-0.50,95 \%$ CI -1.70 to 0.70 on a 0 to 10 scale at day 1 ; mean difference $-0.37,95 \%$ CI -1.29 to 0.55 at day 2) (Table 33). ${ }^{206}$ Other outcomes were not reported. 


\begin{tabular}{|c|c|c|c|c|}
\hline $\begin{array}{l}\text { Author, Year } \\
\text { Quality }\end{array}$ & Interventions & $\begin{array}{l}\text { Procedure; } \\
\text { Followup } \\
\text { Duration } \\
\text { Sample Size }\end{array}$ & Pain Intensity & Other Results \\
\hline $\begin{array}{l}\text { Hill, } 2001^{198} \\
\text { Fair }\end{array}$ & $\begin{array}{l}\text { A: Pregabalin } 50 \mathrm{mg} \times 1 \\
\mathrm{~B} \text { : Pregabalin } 300 \mathrm{mg} \times 1 \\
\mathrm{C} \text { : Ibuprofen } 400 \mathrm{mg} \times 1\end{array}$ & $\begin{array}{l}\text { Third molar } \\
\text { extraction; } \\
<1 \text { day ( } 4 \text { hours } \\
\text { and } 12 \text { hours) } \\
\mathrm{n}=148\end{array}$ & $\begin{array}{l}\text { Pain intensity difference (mean [SD NR], } \\
\text { scale NR): } 0 \text { vs. } 0.6 \text { vs. } 0.7 \text { at } 4 \text { hours, } \\
\text { p>0.05; }-0.1 \text { vs. } 0.1 \text { vs. } 0 \text { at } 12 \text { hours, } p>0.05\end{array}$ & $\begin{array}{l}\text { Patient global assessment (mean [SD], } 0 \\
\text { to } 4 \text { scale): } 2.25 \text { vs. } 3.50 \text { vs. } 3.25, p=N R\end{array}$ \\
\hline $\begin{array}{l}\text { Mehlisch, } \\
1995 a^{203} \\
\text { Fair }\end{array}$ & $\begin{array}{l}\text { A: Diclofenac } 50 \text { mg x } 1 \\
\text { B: Diclofenac } 100 \mathrm{mg} \times 1 \\
\text { C: Aspirin } 650 \mathrm{mg} \mathrm{x} 1\end{array}$ & $\begin{array}{l}\text { Third molar } \\
\text { extraction; } \\
<1 \text { day ( } 30 \\
\text { minutes and } \\
\text { hourly for } 8 \\
\text { hours) } \\
n=156\end{array}$ & $\begin{array}{l}\text { Pain intensity difference (mean [SD NR], } 0 \text { to } \\
3 \text { categorical scale converted to } 0 \text { to } 10 \\
\text { scale): } 3.7 \text { vs. } 4.7 \text { vs. } 1.7 \text { at } 4 \text { hours, } p<0.05 \\
\text { for B vs. C; } 1.0 \text { vs. } 2.3 \text { vs. }-0.7 \text { at } 8 \text { hours, } \\
\text { p<0.05 for B vs. C } \\
\text { Sum of pain intensity differences (mean [SD], } \\
0 \text { to } 3 \text { scale differences from } 0.5 \text { to } 8 \text { hours): } \\
6.3(2.2) \text { vs. } 8.1 \text { (2.2) vs. } 4.0 \text { ( } 2.9 \text { ); MD } 2.3 \\
(95 \% \mathrm{Cl} 1.3 \text { to } 3.3 \text { ) for A vs. C and MD } 4.10 \\
(95 \% \mathrm{Cl} 3.11 \text { to } 5.19 \text { ) for B vs. C }\end{array}$ & $\begin{array}{l}\text { Overall rating "very good" or "good": } 75 \% \\
\text { (40/53) vs. } 85 \%(44 / 52) \text { vs. } 53 \%(27 / 51) \text {; } \\
\text { RR } 1.4(95 \% \mathrm{Cl} 1.1 \text { to } 1.9) \text { for A vs. C and } \\
\text { RR } 1.6(95 \% \mathrm{Cl} 1.2 \text { to } 2.1) \text { for B vs. C }\end{array}$ \\
\hline $\begin{array}{l}\text { Nelson, } \\
1994^{208} \\
\text { Fair }\end{array}$ & $\begin{array}{l}\text { A: Diclofenac } 25 \text { mg x } 1 \\
\text { B: Diclofenac } 50 \text { mg x } 1 \\
\text { C: Diclofenac } 100 \text { mg x } 1 \\
\text { D: Aspirin } 650 \text { mg x } 1\end{array}$ & $\begin{array}{l}\text { Third molar } \\
\text { extraction; } \\
<1 \text { day ( } 30 \\
\text { minutes and } \\
\text { hourly for } 8 \\
\text { hours) } \\
\mathrm{n}=204\end{array}$ & $\begin{array}{l}\text { Pain intensity difference (mean [SD NR], } 0 \text { to } \\
3 \text { categorical rating scale converted to } 0 \text { to } 10 \\
\text { scale): } 2.3 \text { vs. } 2.7 \text { vs. } 4.0 \text { vs. } 0.5 \text { at } 4 \text { hours, } \\
\text { p=NS; } 1.0 \text { vs. } 0.7 \text { vs. } 2.7 \text { vs. } 0.7 \text { at } 8 \text { hours, } \\
\text { p<0.05 for C vs. D, p=NS for A or B vs. D } \\
\text { Sum of pain intensity differences (mean [SD], } \\
\text { sum of differences on } 0 \text { to } 3 \text { scale at } 0.5 \text { to } 8 \\
\text { hours): } 4.3 \text { vs. } 4.9 \text { vs. } 8.1 \text { vs. } 4.0, p<0.05 \text { for } \\
\text { C vs. D, } p=N S \text { for A or B vs. C }\end{array}$ & $\begin{array}{l}\text { Repeat medication use: } 61 \% \text { (31/51) vs. } \\
47 \%(24 / 51) \text { vs. } 38 \%(19 / 51) \text { vs. } 51 \% \\
\text { (26/51); RR } 0.73 \text { ( } 95 \% \text { Cl } 0.47 \text { to } 1.14) \text { for } \\
\text { C vs. D. }\end{array}$ \\
\hline $\begin{array}{l}\text { Mishra, } 2017^{206} \\
\text { Poor }\end{array}$ & $\begin{array}{l}\text { A: Diclofenac tablet } 50 \mathrm{mg} \\
\text { twice daily for } 3 \text { days } \\
\text { B: Diclofenac mouth } \\
\text { wash, rinse } 15 \mathrm{~mL} \\
0.074 \% \text { solution for } 30 \\
\text { seconds twice daily for } 3 \\
\text { days }\end{array}$ & $\begin{array}{l}\text { Mouth flap } \\
\text { surgery for } \\
\text { periodontitis; } \\
1 \text { and } 2 \text { days } \\
\mathrm{n}=30\end{array}$ & $\begin{array}{l}\text { Pain intensity (mean [SD], } 0 \text { to } 10 \text { VAS): } 1.50 \\
(1.51) \text { vs. } 2.00(1.69) \text { at day } 1, \mathrm{MD}-0.50 \\
(95 \% \mathrm{Cl}-1.70 \text { to } 0.70) ; 1.13(1.25) \text { vs. } 1.50 \\
(1.20) \text { at day } 2, \mathrm{MD}-0.37(95 \% \mathrm{Cl}-1.29 \text { to } \\
0.55)\end{array}$ & NR \\
\hline
\end{tabular}


KQs $6 \mathrm{j}$ and $6 \mathrm{l}$ ask how the comparative effectiveness and harms of nonopioid pharmacologic therapy vary depending on: (1) patient demographics (e.g., age, race, ethnicity, gender); (2) patient medical and psychiatric comorbidities; (3) the type of nonopioid medication; (4) dose of medication; (5) duration of treatment.

Evidence was too limited to evaluate how comparative effectiveness and harms of nonopioid therapy for acute dental pain varied in subgroups, due to few trials, small sample sizes, methodological limitations, and exclusion of relevant subgroups or lack of information about them. No study conducted within-study or across-study evaluations of subgroup effects. Details regarding the nonopioid medications prescribed, dose, and duration of treatment are described above.

\section{Nonpharmacologic Therapy}

KQs $6 \mathrm{~m}$ and $6 \mathrm{n}$ address the comparative effectiveness of nonopioid pharmacologic therapy versus: (1) inactive controls or (2) other nonpharmacologic therapies.

Six trials evaluated nonpharmacologic therapy for acute dental pain (Appendix E, Table E8). ${ }^{183,196,199,200,214,217}$ One trial evaluated acupuncture ${ }^{200}$ and five trials cold therapy. ${ }^{183,196,199,214,217}$

Two trials were rated fair quality, ${ }^{196,214}$ and four trials poor quality (Appendix F, Table F1). ${ }^{183,199,200,217}$

\section{Acupuncture Versus Sham Acupuncture}

One small ( $\mathrm{n}=19)$ trial found two sessions of acupuncture following third molar extraction associated with decreased pain intensity versus sham acupuncture at $5 .{ }^{200}$ However, the trial was rated poor quality, and the statistical significance of the difference was not reported. There was no difference in rescue medication use (mean 17.6 vs. 21.4 tablet, $\mathrm{p}>0.05$ ).

\section{Cold Therapy Versus No Cold Therapy}

Five trials evaluated cold therapy versus no cold therapy following third molar extraction (Table 34). The sample sizes ranged from 18 to 128 (N=331). ${ }^{183,196,199,214,217}$ Cold therapy consisted of cold compresses applied to the cheek overlying the surgical site. All trials compared a cold compress to no compress; one trial ${ }^{196}$ also included a non-cold compress comparison. The duration of cold therapy was 45 minutes in one trial ${ }^{196}$ and 24 to 48 hours in the others. Due to the nature of the intervention, patients could not be blinded. Two trials were rated fair quality ${ }^{196,214}$ and three trials poor quality. ${ }^{183,199,217}$ Methodological limitations included failure to report randomization and allocation concealment methods and no intention to treat analysis.

At one to three days following third molar extraction, differences in pain intensity ranged from -0.9 points on a 0 to 10 scale in favor of a cold compress to 0.3 points in favor of no compress, based on four trials. ${ }^{183,196,214,217}$ One of the trials found little difference in pain intensity between a cold compress versus a non-cold compress (1.9 vs. 2.2, $\mathrm{p}=0.64)$. ${ }^{196}$ At 6 to 7 days, two trials ${ }^{183,196}$ found inconsistent effects of a cold compress versus no compress or a noncold compress; in one other trial, ${ }^{217}$ pain had resolved in all patients. One other trial reported that a cold compress was associated with decreased pain throughout the 7 day followup period, but did not provide data. ${ }^{199}$

One trial found a cold compress associated with better quality of life versus no compress through postoperative day 7, but differences were small (1.2 to 2.4 points on the 14 to 56 Oral Health Impact Profile-14 score). ${ }^{199}$ One trial ${ }^{196}$ reported no adverse events, and one trial reported 
nausea but the number of events was small and findings imprecise. ${ }^{214}$ The other trials did not report adverse events.

Table 34. Cold therapy versus no cold therapy for dental pain

\begin{tabular}{|c|c|c|c|c|}
\hline $\begin{array}{l}\text { Author, } \\
\text { Year } \\
\text { Quality }\end{array}$ & Interventions & $\begin{array}{l}\text { Procedure; } \\
\text { Followup } \\
\text { Duration } \\
\text { Sample Size }\end{array}$ & Pain Intensity & Other Results \\
\hline $\begin{array}{l}\text { Altiparmak, } \\
2018^{183} \\
\text { Poor }\end{array}$ & $\begin{array}{l}\text { A: Cold compress for } \\
24 \text { hours except } \\
\text { during sleep } \\
\text { B: No cold compress }\end{array}$ & $\begin{array}{l}\text { Third molar } \\
\text { extraction; } \\
3 \text { and } 7 \text { days } \\
\mathrm{n}=18\end{array}$ & $\begin{array}{l}\text { Pain intensity (mean [SD], } \\
0 \text { to } 10 \text { VAS) } 1.9(3.3) \text { vs. } \\
2.2(3.8) \text { at day } 3, p=0.64 ; \\
1.2(0.2) \text { vs. } 1.4(0.3) \text { at } \\
\text { day } 7, p=0.06\end{array}$ & NR \\
\hline $\begin{array}{l}\text { Forouzanfar, } \\
2008^{196} \\
\text { Fair }\end{array}$ & $\begin{array}{l}\text { A: Cold compress for } \\
45 \text { minutes } \\
\text { immediately following } \\
\text { extraction } \\
\text { B: Compress without } \\
\text { ice for } 45 \text { minutes } \\
\text { immediately following } \\
\text { extraction } \\
\text { C: No compress }\end{array}$ & $\begin{array}{l}\text { Third molar } \\
\text { extraction; } \\
\text { Daily for } 6 \\
\text { days } \\
\mathrm{n}=95\end{array}$ & $\begin{array}{l}\text { Pain intensity (mean [SD], } \\
0 \text { to } 100 \text { VAS converted to } \\
0 \text { to } 10 \text { scale): } 2.2(1.6) \\
\text { vs. } 2.5(1.9) \text { vs. } 3.1(2.2) \\
\text { at day } 1, p=N S ; 1.3(1.8) \\
\text { vs. } 1.1(1.2) \text { vs. } 2.4(2.5) \\
\text { at day } 6, p<0.05 \text { for A vs. } \\
C, p=N S \text { for A vs. B }\end{array}$ & $\begin{array}{l}\text { Patient reported treatment } \\
\text { success (not defined): } \\
64.7 \%(22 / 34) \text { vs. } 71.0 \% \\
(22 / 31) \text { vs. } 43.3 \%(13 / 30) \\
\text { p>0.05 for A vs. B and A } \\
\text { vs. C } \\
\text { lbuprofen use mean, } \\
\text { mg/24 hour (SD): } 1038.2 \\
\text { (702.4) vs. } 1148.4(732.1) \\
\text { vs. } 1140.0(882.2) \text { at day } \\
1, p=N S ; 432.4(787.3) \text { vs. } \\
464.5(629.1) \text { vs. } 780.0 \\
(802.3) \text { at day } 6, p=N S\end{array}$ \\
\hline $\begin{array}{l}\text { Ibikunle, } \\
2016^{199} \\
\text { Poor }\end{array}$ & $\begin{array}{l}\text { A: Cold compress } \\
\text { intermittent } \\
\text { application for } 30 \\
\text { minutes every } 90 \\
\text { minutes for } 24 \text { hours } \\
\text { except during sleep } \\
\text { B: No cold compress }\end{array}$ & $\begin{array}{l}\text { Third molar } \\
\text { extraction; } \\
1,3 \text {, and } 7 \\
\text { days } \\
\mathrm{N}=128\end{array}$ & $\begin{array}{l}\text { Pain significantly lower in } \\
\text { A vs. B throughout } \\
\text { postoperative evaluation } \\
\text { period, } p<0.05 \text { (data not } \\
\text { provided) }\end{array}$ & $\begin{array}{l}\text { Oral Health Impact Profile- } \\
14 \text { score (mean [SD NR], } \\
14 \text { to } 56 \text { scale): } 20.4 \text { vs. } \\
21.6 \text { at day } 1, p=0.01 ; \\
37.5 \text { vs. } 39.9 \text { at day } 4, \\
p=0.002 ; 26.9 \text { vs. } 28.9 \text { at } \\
\text { day } 7, p=0.003 \\
\text { Quality of life affected (not } \\
\text { defined): } 93.9 \% \text { vs. } 96.8 \% \\
\text { at day } 1, p=0.40 ; 18.8 \% \\
\text { vs. } 59.4 \% \text { at day } 7, \\
p=0.001\end{array}$ \\
\hline $\begin{array}{l}\text { van der } \\
\text { Westhuijzen, } \\
2005^{214} \\
\text { Fair }\end{array}$ & $\begin{array}{l}\text { A: Cold compress for } \\
\text { first } 24 \text { hours } \\
\text { including during sleep } \\
\text { B: No cold compress }\end{array}$ & $\begin{array}{l}\text { Third molar } \\
\text { extraction; } \\
4 \text { hours, } \\
\text { evening of } \\
\text { surgery, } \\
\text { morning after } \\
\text { surgery } \\
n=60\end{array}$ & $\begin{array}{l}\text { Pain intensity (mean [SD } \\
\text { NR], } 0 \text { to } 10 \text { VAS): } 3.1 \text { vS. } \\
3.3 \text { at } 4 \text { hours, } p>0.05 ; 3.1 \\
\text { vs. } 2.8 \text { at morning after } \\
\text { surgery, } p>0.05\end{array}$ & $\begin{array}{l}\text { Symptom control excellent } \\
\text { or good: } 97 \%(28 / 29) \text { vs. } \\
90 \%(27 / 30), p>0.05\end{array}$ \\
\hline $\begin{array}{l}\text { Zandi, } \\
2016^{217} \\
\text { Poor }\end{array}$ & $\begin{array}{l}\text { A: Cold compress, } \\
\text { alternating on and off } \\
\text { every } 20 \text { minutes for } \\
24 \text { hours except } \\
\text { during sleep } \\
\text { B: No cold compress }\end{array}$ & $\begin{array}{l}\text { Third molar } \\
\text { extraction; } \\
2 \text { and } 7 \text { days } \\
\mathrm{n}=30\end{array}$ & $\begin{array}{l}\text { Pain (mean [SD], } 0 \text { to } 100 \\
\text { VAS converted to } 0 \text { to } 10 \\
\text { scale): } 3.83(2.33) \text { vs. } \\
4.43(2.09) \text { at day } 2 \\
\text { p=0.29; } 0 \text { vs. } 0 \text { at day } 7\end{array}$ & $\begin{array}{l}\text { Satisfaction (mean [SD], } 0 \\
\text { to } 10): 7.27(1.48) \text { vs. } 7.00 \\
(1.66), p=0.46\end{array}$ \\
\hline
\end{tabular}

Abbreviations: NR = not reported; NS = not significant; SD = standard deviation; VAS = visual analog scale

KQs 60 and 6p ask how the comparative effectiveness and harms of nonpharmacologic therapy vary depending on: (1) patient demographics (e.g., age, gender); (2) patient medical and 
psychiatric comorbidities; (3) the type of treatment used; (4) the frequency of therapy; (5) the duration of therapy.

No evidence was found for KQ 60 or $6 \mathrm{p}$.

\section{KQ 7. Kidney Stone Pain}

\section{Key Points}

- Opioid vs. NSAID, single dose

o Morphine was associated with increased likelihood of persistent pain versus an NSAID (38\% vs. 24\%, RR 1.57, 95\% CI 1.31 to 1.89), reduced likelihood of $\geq 50 \%$ pain relief (93\% vs. 97\%, RR 0.95, 95\% CI 0.93 to 0.98 ), and increased likelihood of rescue medication use ( $23 \%$ vs. $12 \%$, RR $1.99,95 \%$ CI 1.51 to 2.63 ) at 60 to 90 minutes, based on one trial (SOE: moderate).

o Morphine associated with increased likelihood of any adverse event versus an NSAID, though the frequency of adverse events was low (3\% vs. $1 \%$, RR $2.70,95 \%$ CI 1.15 to 6.38) (SOE: moderate).

o Meperidine was associated with moderate to large increase in pain intensity at 1 hour compared with an NSAID (4 trials), inconsistent effects on likelihood of pain relief (3 trials), and increased likelihood of rescue medication use (6 trials, RR 1.48, 95\% CI 1.02 to 2.14) (SOE: moderate for pain intensity and likelihood of rescue medication use, insufficient for likelihood of pain relief).

- Meperidine was associated with increased likelihood of any adverse event (5 trials, RR 1.71, 95\% CI 0.99 to 2.96), somnolence (4 trials, RR 1.98, 95\% 0.82 to 4.79 ), and nausea (5 trials, N=573, RR 1.84, 95\% CI 1.02 to 3.31) (SOE: low).

- Opioid vs. acetaminophen, single dose

- Morphine was associated with increased likelihood of persistent pain (pain $>2$ on a 0 to 10 scale) at 60 minutes ( $38 \%$ vs. $30 \%$, RR $1.28,95 \%$ CI 1.08 to 1.51 ), similar likelihood of $\geq 50 \%$ pain relief at 90 minutes (93\% vs. 92\%, RR 1.01, 95\% CI 0.97 to 1.04 ), and similar likelihood of rescue medication use (23\% vs. 20\%, RR 1.13, 95\% CI 0.90 to 1.42), based on one trial (SOE: moderate).

o Morphine was associated with increased likelihood of any adverse event versus acetaminophen, though the frequency of adverse events was low (3\% vs. $1 \%$, RR 2.71, 95\% CI 1.15 to 6.39 ) (SOE: moderate).

- Opioid agonist vs. partial agonist

o Insufficient evidence from one small trial (SOE: insufficient).

- NSAID vs. acetaminophen, single dose

o Inconsistent effects of NSAIDs versus acetaminophen on pain intensity (3 trials) and likelihood of pain relief (2 trials); NSAIDs associated with decreased likelihood of rescue medication use (2 trials) (SOE: low for rescue medication use; insufficient for pain intensity and likelihood of pain relief).

0 The frequency of adverse events was low with NSAIDs and acetaminophen, based on 3 trials (SOE: moderate).

- Acupuncture vs. NSAID or acetaminophen

o Acupuncture (single session) associated with moderately increased pain intensity versus a single dose of an NSAID or acetaminophen at 2 hours, based on 1 trial (SOE: low). 
o There were few adverse events in one trial (SOE: low).

\section{Summary of Findings}

Twelve trials $(\mathrm{N}=2,762)$ evaluated interventions for acute renal colic (Appendix E, Table E10). ${ }^{223-234}$ Nine trials evaluated opioid therapy (KQ 7a and 7c), ${ }^{223-226,229,230,232-234}$ Three trials evaluated nonopioid pharmacologic therapy (KQ 7i and 7j), ${ }^{227,228,231}$ and one trial evaluated nonpharmacologic therapy (KQ 7m and 7n). ${ }^{228}$ The mean age of patients ranged from 32 to 44 years, and the proportion female ranged from 15 to 59 percent. Three trials of opioid therapy excluded patients with alcohol or drug abuse history within the last 5 years. ${ }^{229,232,234}$ Otherwise the trials did not describe psychiatric or medical comorbidities. Pain duration was $<7$ days in one trial $^{230}$ and not described in the others. One trial was rated good quality, ${ }^{233}$ nine trials fair quality, ${ }^{224-229,231,232,234}$ and two trials poor quality (Appendix F, Table F-1). ${ }^{223,230}$ Methodological limitations in the fair and poor quality trials included open label design, failure to report adequate randomization and allocation concealment methods, high or unclear attrition, and no intention to treat analysis.

\section{Detailed Synthesis}

\section{Opioid Therapy}

KQs 7a and 7c address the comparative effectiveness and harms of opioid therapy versus: (1) nonopioid pharmacologic therapy (e.g., acetaminophen, NSAIDs, antidepressants, anticonvulsants) or (2) nonpharmacologic therapy (e.g., exercise, cognitive behavioral therapy, acupuncture).

Nine trials $(\mathrm{N}=2,491)$ evaluated opioids for acute renal colic (Appendix E, Table E-10). ${ }^{223-}$ 226,229,230,232-234 Eight trials compared an opioid versus an NSAID, ${ }^{223,224,226,229,230,232-234}$ one trial compared an opioid versus acetaminophen, ${ }^{233}$ and one trial compared an opioid agonist versus a partial agonist. ${ }^{225}$ All trials evaluated single dose of parenteral therapy and were not designed to assess subsequent pain management. In five trials the duration of followup was 1 to 2 hours, and in four trials the duration of followup was up to 6 to 24 hours. Outcomes assessed at less than one hour were not abstracted. One trial was rated good quality, ${ }^{233}$ six trials fair quality, ${ }^{224-}$ 226,229,232,234 and two trials poor quality (Appendix F, Table F-1). ${ }^{223,230}$ Methodological limitations in the fair and poor quality trial included failure to report adequate randomization or allocation concealment methods, unblinded design, high or unclear attrition, and no or unclear use of intention treat analysis.

\section{Opioid Versus NSAID}

Eight trials compared an opioid versus an NSAID for acute renal colic (Table $35){ }^{223,224,226,229,230,232-234}$ Sample sizes ranged from 50 to 1,097 $(\mathrm{N}=1,918)$. The trials were conducted in the United States, ${ }^{224,229}$ Europe, ${ }^{226,234}$ and other countries. ${ }^{22,230,232,233}$ All of the trials evaluated a single parenteral dose of medication. In one trial ${ }^{233}$ the opioid was morphine, and in the other trials the opioid was meperidine (pethidine), a drug which has become less widely used in the United States due to risk of adverse events including seizures, anticholinergic effects, and drug-drug interactions, including serotonin syndrome. ${ }^{235}$ The NSAID was indomethacin in one trial (100 mg), ${ }^{223}$ ketorolac in four trials (10 to $\left.90 \mathrm{mg}\right),{ }^{224,229,232,234}$ and diclofenac in three trials $(75 \mathrm{mg}) .{ }^{226,230,233}$ One trial was rated good quality, ${ }^{233}$ five trials fair quality, ${ }^{224,226,229,232,234}$ and two trials poor quality (Appendix F, Table F-1). ${ }^{223,230}$ 
The trial of morphine versus an NSAID was the largest ( $n=1,097$ for this comparison) and only good quality trial. It compared morphine $15 \mathrm{mg}$ intravenous (IV) versus diclofenac $75 \mathrm{mg}$ intramuscular injection (IM) (Table 35). ${ }^{233}$ Morphine was associated with increased likelihood of persistent pain (pain $>2$ on a 0 to 10 scale) at 60 minutes (38\% vs. 24\%, RR 1.57, 95\% CI 1.31 to 1.89), slightly decreased likelihood of $\geq 50 \%$ pain relief at 90 minutes ( $93 \%$ vs. $97 \%$, RR 0.95 , 95\% CI 0.93 to 0.98 ), and increased likelihood of rescue medication use (23\% vs. 12\%, RR 1.99, 95\% CI 1.51 to 2.63). At 90 minutes, median pain intensity was 0 in both groups. Morphine was associated with increased likelihood of any adverse event, though the frequency of adverse events was low (3\% vs. 1\%, RR 2.70, 95\% CI 1.15 to 6.38). No serious adverse events were reported. The likelihood of serum creatinine elevations was low in both groups; in those with creatinine elevations, normalization occurred within one week.

Seven trials evaluated meperidine versus an NSAID (Table 35). Sample sizes ranged from 50 to 234 ( $\mathrm{N}=821)$. Meperidine was associated with increased pain intensity at 1 hour compared with an NSAID in four trials (difference 0.3 to 3.2 points on a 0 to 10 scale), ${ }^{26,229,230,232}$ and one other trial ${ }^{234}$ found meperidine associated with a smaller sum of pain intensity differences at 1 hour. At 1 to 1.5 hours, one trial found meperidine associated with decreased likelihood of $\geq 50$ percent pain relief, ${ }^{227}$ but two other trials found high rates of pain relief for both meperidine and NSAIDs. ${ }^{223,232}$ One trial found meperidine associated with a smaller SPID versus NSAIDs at 6 hours. ${ }^{224}$ Meperidine was associated with increased likelihood of rescue medication use (5 trials, $\mathrm{N}=671$, RR 1.48, 95\% CI 1.02 to 2.14, $\mathrm{I}^{2}=71 \%$; Figure D-21). ${ }^{223,224,226,229,232,234}$ Meperidine was associated with increased likelihood of any adverse event (5 trials, N=471, RR 1.71, 95\% CI 0.99 to 2.96, $\mathrm{I}^{2}=62 \%$; Figure D-22), somnolence (4 trials, N=555, RR 1.98, 95\% 0.82 to 4.79, $\mathrm{I}^{2}=65 \%$; Figure D-23), and nausea (5 trials, $\mathrm{N}=573$, RR $1.84,95 \%$ CI 1.02 to $3.31, \mathrm{I}^{2}=42 \%$; Figure D-24), though only the estimate for nausea was statistically significant.

Table 35. Opioid versus NSAIDs for acute renal colic

\begin{tabular}{|c|c|c|c|c|}
\hline $\begin{array}{l}\text { Author, Year } \\
\text { Quality }\end{array}$ & Interventions & $\begin{array}{l}\text { Followup } \\
\text { Duration } \\
\text { Sample } \\
\text { Size }\end{array}$ & Pain Intensity & Other Results \\
\hline $\begin{array}{l}\text { al-Sahlawi, } \\
1996^{223} \\
\text { Poor }\end{array}$ & $\begin{array}{l}\text { A: Meperidine } \\
100 \mathrm{mg} \text { IV } \times 1 \\
\text { B: Indomethacin } \\
100 \mathrm{mg} \text { IV } \times 1\end{array}$ & $\begin{array}{l}60 \text { minutes } \\
n=100\end{array}$ & NR & $\begin{array}{l}\text { Percent with complete pain relief at } \\
60 \text { minutes } \\
100 \%(50 / 50) \text { vs. } 100 \%(50 / 50) \\
\text { Rescue medication use at } 30 \\
\text { minutes: } 0 \%(0 / 50) \text { vs. } 4 \%(2 / 50) \text {, } \\
\text { RR } 0.20(95 \% \mathrm{Cl} 0.01 \text { to } 4.06)\end{array}$ \\
\hline $\begin{array}{l}\text { Cordell, } 1996^{224} \\
\text { Fair }\end{array}$ & $\begin{array}{l}\text { A: Meperidine } 50 \\
\text { mg IV x } 1 \\
\text { B: Meperidine } 50 \\
\text { mg + Ketorolac } \\
60 \text { mg IV x } 1 \\
\text { C: Ketorolac } 60 \\
\text { mg IV } \times 1\end{array}$ & $\begin{array}{l}6 \text { hours } \\
N=106\end{array}$ & $\begin{array}{l}\text { Sum of pain intensity } \\
\text { difference (mean [SD], sum } \\
\text { of differences on } 0 \text { to } 100 \\
\text { VAS from } 15 \text { minutes to } 6 \\
\text { hours): } 267.8(26.0) \text { vs. } \\
319.3(26.2) \text { vs. } 409.1 \\
(24.7), p<0.001 \text { for } A \text { vs. C, } \\
\text { p=0.124 for A vs. B, } p=0.009 \\
\text { for B vs. C }\end{array}$ & $\begin{array}{l}\text { Rescue medication use: } 89 \% \\
(31 / 35) \text { vs. } 66 \%(23 / 35) \text { vs. } 64 \% \\
(23 / 36) \text {; RR } 1.35(95 \% \mathrm{Cl} 1.03 \text { to } \\
1.76) \text { for A vs. B, RR } 1.39(95 \% \mathrm{Cl} \\
1.06 \text { to } 1.82) \text { for A vs. C, RR } 1.03 \\
(95 \% \mathrm{Cl} 0.73 \text { to } 1.45) \text { for B vs. C } \\
\\
\text { Functional impairment at } 6 \text { hours } \\
\text { (mean [SD NR], } 0 \text { to } 4 \text { scale, } \\
4=\text { severe impairment) } \\
1.2 \text { vs. } 1.5 \text { vs. } 1.8, \mathrm{p}=0.003 \text { for A } \\
\text { vs. C, other comparisons NS }\end{array}$ \\
\hline $\begin{array}{l}\text { Garcća-Alonso, } \\
1991^{226} \\
\text { Fair }\end{array}$ & $\begin{array}{l}\text { A: Meperidine } \\
100 \text { mg IM x } 1 \\
\text { B: Diclofenac } 75 \\
\text { mg IM x } 1\end{array}$ & $\begin{array}{l}60 \text { minutes } \\
\mathrm{n}=234\end{array}$ & $\begin{array}{l}\text { Pain intensity (mean [SD } \\
\text { NR], } 0 \text { to } 10 \text { VAS, in patients } \\
\text { not requiring rescue } \\
\text { treatment: } 2.0 \text { vs. } 1.0 \text { at } 1 \\
\text { hour ( } p=N R)\end{array}$ & $\begin{array}{l}\text { Rescue medication use at } 30 \\
\text { minutes: } 20 \%(23 / 118) \text { vs. } 16 \% \\
(19 / 116) \text {, RR } 1.19(95 \% \text { Cl } 0.69 \text { to } \\
2.06)\end{array}$ \\
\hline
\end{tabular}




\begin{tabular}{|c|c|c|c|c|}
\hline $\begin{array}{l}\text { Author, Year } \\
\text { Quality }\end{array}$ & Interventions & $\begin{array}{l}\text { Followup } \\
\text { Duration } \\
\text { Sample } \\
\text { Size }\end{array}$ & Pain Intensity & Other Results \\
\hline $\begin{array}{l}\text { Larkin, 1999229 } \\
\text { Fair }\end{array}$ & $\begin{array}{l}\text { A: Meperidine } \\
100 \text { to } 150 \mathrm{mg} \\
\mathrm{IM} \times 1 \\
\mathrm{~B}: \text { Ketorolac } 60 \\
\mathrm{mg} \mathrm{IM} \times 1\end{array}$ & $\begin{array}{l}90 \text { minutes } \\
n=70\end{array}$ & $\begin{array}{l}\text { Pain intensity (mean [SD } \\
\text { NR], } 0 \text { to } 10 \text { VAS): } 4.0 \text { vs. } \\
1.8 \text { at } 90 \text { minutes, } p<0.05\end{array}$ & $\begin{array}{l}\text { Rescue medication use: } 43 \% \\
(16 / 37) \text { vs. } 33 \%(11 / 33), \text { RR } 1.30 \\
(95 \% \text { Cl } 0.71 \text { to } 2.38)\end{array}$ \\
\hline $\begin{array}{l}\text { Marthak, } 1991^{230} \\
\text { Poor }\end{array}$ & $\begin{array}{l}\text { A: Meperidine } 75 \\
\text { mg IM x } 1 \\
\text { B: Diclofenac } 75 \\
\text { mg IM x } 1\end{array}$ & $\begin{array}{l}60 \text { minutes } \\
n=50\end{array}$ & $\begin{array}{l}\text { Pain intensity difference } \\
\text { (mean improvement from } \\
\text { baseline [SD NR], } 0 \text { to } 100 \\
\text { VAS converted to } 0 \text { to } 10 \\
\text { scale): } 7.9 \text { vs. } 8.2 \text { at } 60 \\
\text { minutes, p=NS }\end{array}$ & $\begin{array}{l}\text { Physician rated treatment as good } \\
\text { to excellent: } 88 \%(22 / 25) \text { vs. } 100 \% \\
(25 / 25) \text {, RR } 0.88(95 \% \text { Cl } 0.76 \text { to } \\
1.02)\end{array}$ \\
\hline $\begin{array}{l}\text { Oosterlinck, } \\
1990^{232}\end{array}$ & $\begin{array}{l}\text { A. Meperidine } \\
100 \text { mg IM, } \\
\text { single treatment } \\
\text { B: Ketorolac } 10 \\
\text { mg IM, single } \\
\text { treatment } \\
\text { C: Ketorolac } 90 \\
\text { mg IM, single } \\
\text { treatment }\end{array}$ & $\begin{array}{l}1 \text { and } 10 \\
\text { hours } \\
n=121\end{array}$ & $\begin{array}{l}\text { Pain intensity difference } \\
\text { (mean improvement from } \\
\text { baseline [SD], } 0 \text { to } 100 \text { VAS } \\
\text { converted to } 0 \text { to } 10 \text { scale): } \\
5.7(2.6) \text { vs. } 5.4 \text { ( } 2.6 \text { ) vs. } 6.5 \\
\text { (1.8) at } 1 \text { hour, } p=0.4 \text { for A } \\
\text { vs. B; } p=0.12 \text { for A vs. C; } \\
\text { p=0.02 for B vs. C } \\
\text { Pain none or mild at } 60 \\
\text { minutes: } 84 \%(31 / 37) \text { vs. } \\
82 \%(32 / 39) \text { vs. } 86 \% \\
\text { (30/35); RR } 1.02 \text { ( } 95 \% \mathrm{Cl} \\
0.83 \text { to } 1.25) \text { for A vs. B, RR } \\
0.98 \text { (95\% Cl } 0.80 \text { to } 1.19) \\
\text { for A vs. C, RR } 0.96(95 \% \mathrm{Cl} \\
0.78 \text { to } 1.17) \text { for B vs. C }\end{array}$ & $\begin{array}{l}\text { Rescue medication use within } 10 \\
\text { hours: } 47 \%(18 / 38) \text { vs. } 39 \%(17 / 44) \\
\text { vs. } 17 \%(6 / 36) ; \text { RR } 1.23(95 \% \mathrm{Cl} \\
0.74 \text { to } 2.02) \text { for A vs. B, RR } 2.84 \\
\text { (95\% Cl } 1.27 \text { to } 6.35) \text { for A vs. C, } \\
\text { and RR } 2.31(95 \% \text { Cl } 1.02 \text { to } 5.26) \\
\text { for B vs. C }\end{array}$ \\
\hline $\begin{array}{l}\text { Pathan, } 2016^{233} \\
\text { Good }\end{array}$ & $\begin{array}{l}\text { A: Morphine } 15 \\
\text { mg IV x } 1 \\
\text { B: Diclofenac } 75 \\
\text { mg IM x } 1\end{array}$ & $\begin{array}{l}60 \text { and } 90 \\
\text { minutes } \\
n=1,097\end{array}$ & $\begin{array}{l}\text { Pain intensity (median [IQR], } \\
0 \text { to } 10 \text { scale): } 0 \text { (0 to } 2) \text { vs. } \\
0 \text { (0 to } 1) \text { at } 90 \text { minutes } \\
\text { Pain >2 on } 0 \text { to } 10 \text { scale at } \\
60 \text { minutes: } 38 \%(207 / 549) \\
\text { vs. } 24 \%(131 / 547), \text { RR } 1.57 \\
(95 \% \mathrm{Cl} 1.31 \text { to } 1.89)\end{array}$ & $\begin{array}{l}\text { Rescue medication use: } 23 \% \\
\text { (126/549) vs. } 12 \%(63 / 547), \mathrm{RR} \\
1.99 \text { (95\% Cl } 1.51 \text { to } 2.63) \\
\text { Pain intensity decreased } \geq 50 \% \text { at } \\
90 \text { minutes: } 93 \%(511 / 549) \mathrm{vs} \text {. } \\
97.4 \%(533 / 547), \mathrm{RR} 0.96(95 \% \mathrm{Cl} \\
0.93 \text { to } 0.98)\end{array}$ \\
\hline Sandhu, $1994^{234}$ & $\begin{array}{l}\text { A: Meperidine } \\
100 \text { mg IM x } 1 \\
\text { B: Ketorolac } 30 \\
\text { mg IM x } 1\end{array}$ & $\begin{array}{l}60 \text { minutes } \\
n=140\end{array}$ & $\begin{array}{l}\text { Sum of pain intensity } \\
\text { differences (mean [SD NR], } \\
0 \text { to } 10 \text { VAS from } 15 \text { to } 60 \\
\text { minutes): } 32.7 \text { vs. } 39.0, \\
\text { p=NS }\end{array}$ & $\begin{array}{l}\text { Rescue medication use: } 74 \% \\
\text { (53/72) vs. } 65 \% \text { (44/68), RR } 1.14 \\
\text { (95\% Cl } 0.91 \text { to } 1.42)\end{array}$ \\
\hline
\end{tabular}

Abbreviations: CI = confidence interval; IM = intramuscular; IQR = interquartile range; IV = intravenous; NR = not reported; RR = relative risk; $\mathrm{SD}=$ standard deviation; VAS = visual analog scale

\section{Opioid Versus Acetaminophen}

One good quality trial compared a single dose of morphine $15 \mathrm{mg}$ IV versus acetaminophen $1000 \mathrm{mg}$ IV was the largest ( $\mathrm{n}=1,096$ for this comparison) (Table 36). ${ }^{233}$ Morphine was associated with increased likelihood of persistent pain (pain $>2$ on a 0 to 10 scale) at 60 minutes (38\% vs. 30\%, RR 1.28, 95\% CI 1.08 to 1.51), similar likelihood of $\geq 50 \%$ pain relief at 90 minutes (93\% vs. 92\%, RR 1.01, 95\% CI 0.97 to 1.04), and similar likelihood of rescue medication use (23\% vs. 20\%, RR 1.13, 95\% CI 0.90 to 1.42). At 90 minutes, median pain intensity was 0 in both groups. Morphine was associated with increased likelihood of any adverse event, though the frequency of adverse events was low (3\% vs. 1\%, RR 2.71, 95\% CI 1.15 to 6.39). No serious adverse events were reported. The likelihood of serum creatinine 
elevations was low in both groups; in those with creatinine elevations, normalization occurred within 1 week.

\section{Opioid Agonist Versus Partial Agonist}

One small ( $\mathrm{n}=26)$, fair quality trial compared a single dose of meperidine $100 \mathrm{mg}$ (an opioid agonist) versus the partial agonist buprenorphine $0.3 \mathrm{mg} \cdot{ }^{225}$ Meperidine was associated with increased pain intensity at 12 hours (mean 4.2 vs. 1.2 on a 0 to 10 scale, mean difference 3.0, 95\% CI 2.8 to 3.2) and less time pain free (mean 4.5 vs. 9.0 hours, $\mathrm{p}<0.01$ ). Meperidine was also associated with increased likelihood of rescue medication use (92\% vs. 46\%, RR 2.00, 95\% CI 1.09 to 3.67). There was no difference in the likelihood of nausea and vomiting.

Table 36. Opioid versus acetaminophen and opioid agonist versus partial agonist for acute renal colic

\begin{tabular}{|c|c|c|c|c|}
\hline $\begin{array}{l}\text { Author, Year } \\
\text { Quality }\end{array}$ & Interventions & $\begin{array}{l}\text { Followup } \\
\text { Duration } \\
\text { Sample Size }\end{array}$ & Pain Intensity & Other Results \\
\hline $\begin{array}{l}\text { Pathan, } \\
2016^{233} \\
\text { Good }\end{array}$ & $\begin{array}{l}\text { A: Morphine } 15 \mathrm{mg} \\
\text { IV } \times 1 \\
\text { B: Acetaminophen } \\
1000 \mathrm{mg} \text { IV x } 1\end{array}$ & $\begin{array}{l}60 \text { and } 90 \\
\text { minutes } \\
n=1,096\end{array}$ & $\begin{array}{l}\text { Pain intensity (median [IQR], } \\
0 \text { to } 10 \text { scale): } 0 \text { (0 to } 2) \text { vs. } \\
0 \text { (0 to } 2 \text { ) at } 90 \text { minutes } \\
\text { Pain >2 on } 0 \text { to } 10 \text { scale at } \\
60 \text { minutes: } 38 \%(207 / 549) \\
\text { vs. } 30 \%(162 / 548), \text { RR } 1.28 \\
(95 \% \mathrm{Cl} 1.08 \text { to } 1.51)\end{array}$ & $\begin{array}{l}\text { Rescue medication use: } 23 \% \\
\text { (126/549) vs. } 20 \%(111 / 548), \mathrm{RR} \\
1.13 \text { (95\% Cl } 0.90 \text { to } 1.42) \\
\text { Pain intensity decreased } \geq 50 \% \text { at } \\
90 \text { minutes: } 93 \% \text { (511/549) vs. } \\
92.5 \%(507 / 548) \text {, RR } 1.01 \text { (95\% Cl } \\
0.97 \text { to } 1.04)\end{array}$ \\
\hline $\begin{array}{l}\text { Finlay, } \\
1982^{225} \\
\text { Fair }\end{array}$ & $\begin{array}{l}\text { A: Meperidine } 100 \\
\text { mg IM x } 1 \\
\text { B: Buprenorphine } \\
0.3 \text { mg IM x } 1\end{array}$ & $\begin{array}{l}12 \text { hours } \\
n=26\end{array}$ & $\begin{array}{l}\text { Pain intensity (mean [SD], } 0 \\
\text { to } 10 \text { VAS): } 4.16(0.28) \text { vs. } \\
1.16(0.12) \text { at } 12 \text { hours, MD } \\
3.0(95 \% \mathrm{Cl} 2.8 \text { to } 3.2)\end{array}$ & $\begin{array}{l}\text { Rescue medication use: } 92 \% \\
\text { (12/13) vs. } 46 \%(6 / 13), \text { RR } 2.00 \\
\text { (95\% Cl } 1.09 \text { to } 3.67) \\
\text { Period pain free in first } 12 \text { hours } \\
\text { (mean [SD], hours) } \\
4.47 \text { (1.0) vs. } 9.02(0.9), p<0.01\end{array}$ \\
\hline
\end{tabular}

Abbreviations: $\mathrm{CI}$ = confidence interval; IM = intramuscular; IV = intravenous; $\mathrm{MD}=$ mean difference; $\mathrm{RR}=$ relative risk; $\mathrm{SD}=$ standard deviation; VAS = visual analog scale

KQs 7b and 7d ask how the comparative effectiveness and harms of opioid therapy vary depending on: (1) patient demographics (e.g., age, race, ethnicity, gender); (2) patient medical or psychiatric comorbidities; (3) dose of opioids; (4) duration of opioid therapy, including number of opioid prescription refills and quantity of pills used; (5) opioid use history; (6) substance use history; (7) use of concomitant therapies.

Evidence on how comparative effectiveness and harms of opioid therapy for kidney stone pain vary according to patient and prescribing factors was lacking. Three trials excluded patients with a history of substance use disorder, ${ }^{229,232,234}$ and substance use disorder was not described in the others. Information on medical and psychiatric comorbidities was lacking. No study conducted within-study analyses of subgroup effects, with the exception of two trials that reported that results in the patients with ureteric calculi on imaging were similar compared with all patients presenting clinically with renal colic. ${ }^{233,234}$ Evidence was too limited to determine effects of different dose of meperidine on comparative effectiveness and harms. All trials evaluated single dose of opioids and did not address opioid use as an outpatient.

KQ 7e concerns the effects of prescribing opioid therapy versus not prescribing opioid therapy for acute pain on 1) short-term ( $<3$ months) continued need for prescription pain relief, such as need for opioid refills, and 2) long-term opioid use (3 months or greater). 
No study compared the association between prescribing of opioids for acute renal colic versus not prescribing and continued use of opioids. Two studies found a history of kidney stones associated with increased likelihood of opioid use, but did not meet inclusion criteria because they were cross-sectional and did not evaluate prescribing for acute episodes as a risk factor. $^{236,237}$

KQs $7 \mathrm{f}$ and $7 \mathrm{~g}$ address the accuracy and effectiveness of instruments for predicting risk of opioid misuse, opioid use disorder, or overdose for patients with acute pain being considered for opioid therapy.

No evidence was found for KQs $7 \mathrm{f}$ and $7 \mathrm{~g}$.

KQ 7h addresses the effect of the following factors on the decision to prescribe opioids for patients with acute pain being considered for opioid therapy, what is: (1) existing opioid management plans; (2) patient education; (3) clinician and patient values and preferences related to opioids; (4) urine drug screening; (5) use of prescription drug monitoring program data; (6) availability of close followup.

No evidence was found for KQ 7h.

\section{Nonopioid Pharmacologic Therapy}

KQ 7i and 7k address the comparative effectiveness and harms of nonopioid pharmacologic therapy versus: (1) nonopioid pharmacologic therapy or (2) nonpharmacologic therapy.

\section{NSAID Versus Acetaminophen}

Four trials $(n=1,325)$ compared a single dose of an NSAID versus acetaminophen for acute renal colic (Table 37). ${ }^{227,228,231,233}$ In all of the trials, the NSAID and acetaminophen were administered parenterally, except for one trial ${ }^{231}$ in which acetaminophen was administered by mouth. The NSAID was diclofenac in three trials $(75 \mathrm{mg})$ and piroxicam in one trial (20 mg). The dose of acetaminophen was $1000 \mathrm{mg}$. Outcomes were assessed at 1 to 2 hours. One trial was rated good quality, ${ }^{233}$ and three trials were rated fair quality.

The trials reported inconsistent effects of NSAIDs versus acetaminophen on pain intensity (three trials) 228,231,233 and likelihood of pain relief (two trials). ${ }^{227,233}$ Two trials found NSAIDs associated with decreased likelihood of rescue medication use (8\% vs. 24\%, RR 0.33, 95\% CI 0.07 to 1.50 and $12 \%$ vs. $20 \%$, RR $0.57,95 \%$ CI 0.43 to 0.76$).{ }^{231,233}$ Few adverse events were reported in three trials. ${ }^{227,228,233}$ One trial found similar likelihood of serum creatinine elevations that normalized within 1 week. ${ }^{233}$

Table 37. NSAIDs versus acetaminophen for acute renal colic

\begin{tabular}{|c|c|c|c|c|}
\hline $\begin{array}{l}\text { Author, } \\
\text { Year } \\
\text { Quality }\end{array}$ & Interventions & $\begin{array}{l}\text { Followup } \\
\text { Duration } \\
\text { Sample Size }\end{array}$ & Pain Intensity & Other Results \\
\hline $\begin{array}{l}\text { Grissa, } \\
2011^{227} \\
\text { Fair }\end{array}$ & $\begin{array}{l}\text { A: Piroxicam } 20 \\
\text { mg IM } \times 1 \\
\text { B: Acetaminophen } \\
1 \mathrm{~g} / 100 \mathrm{~mL} \mathrm{IV} \times 1\end{array}$ & $\begin{array}{l}90 \text { minutes } \\
n=100\end{array}$ & NR & $\begin{array}{l}\geq 50 \% \text { pain relief: } 48 \%(24 / 50) \text { vs. } \\
80 \%(40 / 50) \text { at } 90 \text { minutes, RR } 0.60 \\
(95 \% \mathrm{Cl} 0.44 \text { to } 0.83)\end{array}$ \\
\hline $\begin{array}{l}\text { Narci, } \\
2008^{231} \\
\text { Fair }\end{array}$ & $\begin{array}{l}\text { A: Diclofenac } 75 \\
\text { mg IM, x } 1+\text { oral } \\
\text { placebo } \\
\text { B: Acetaminophen } \\
1000 \text { mg oral x } 1 \\
+ \text { IM placebo }\end{array}$ & $\begin{array}{l}60 \text { minutes } \\
n=50\end{array}$ & $\begin{array}{l}\text { Pain intensity (mean [SD], } \\
0 \text { to } 100 \text { VAS converted to } \\
0 \text { to } 10 \text { scale): } 1.41(2.0) \\
\text { vs. } 2.71(1.69), \mathrm{MD}-1.30 \\
(95 \% \mathrm{Cl}-2.35 \text { to }-0.25)\end{array}$ & $\begin{array}{l}\text { Rescue medication use: } 8 \%(2 / 25) \\
\text { vs. } 24 \%(6 / 25) \text {, RR } 0.33(95 \% \mathrm{Cl} 0.07 \\
\text { to } 1.50)\end{array}$ \\
\hline
\end{tabular}




\begin{tabular}{|c|c|c|c|c|}
\hline $\begin{array}{l}\text { Author, } \\
\text { Year } \\
\text { Quality }\end{array}$ & Interventions & $\begin{array}{l}\text { Followup } \\
\text { Duration } \\
\text { Sample Size }\end{array}$ & Pain Intensity & Other Results \\
\hline $\begin{array}{l}\text { Kaynar, } \\
2015^{228} \\
\text { Fair }\end{array}$ & $\begin{array}{l}\text { A: Diclofenac } 75 \\
\text { mg IM } \times 1 \\
\text { B: Acetaminophen } \\
1 \mathrm{~g} / 100 \mathrm{~mL} \text { IV } \times 1\end{array}$ & $\begin{array}{l}2 \text { hours } \\
n=80\end{array}$ & $\begin{array}{l}\text { Pain intensity (mean [SD } \\
\text { NR], } 0 \text { to } 10 \text { VAS): } 2.75 \text { vs. } \\
2.10, p=0.49\end{array}$ & NR \\
\hline $\begin{array}{l}\text { Pathan, } \\
2016^{233} \\
\text { Good }\end{array}$ & $\begin{array}{l}\text { A: Diclofenac } 75 \\
\text { mg IM x } 1 \\
\text { C: Acetaminophen } \\
1000 \text { mg IV x } 1\end{array}$ & $\begin{array}{l}60 \text { and } 90 \\
\text { minutes } \\
n=1,095\end{array}$ & $\begin{array}{l}\text { Pain intensity (median } \\
\text { [IQR], } 0 \text { to } 10 \text { scale): } 0 \text { (0 to } \\
\text { 1) vs. } 0 \text { (0 to 2) at } 90 \\
\text { minutes } \\
\text { Pain >2 on } 0 \text { to } 10 \text { scale at } \\
60 \text { minutes: } 24 \%(131 / 547) \\
\text { vs. } 30 \%(162 / 548), \mathrm{RR} \\
0.81 \text { ( } 95 \% \mathrm{Cl} 0.66 \text { to } 0.99)\end{array}$ & $\begin{array}{l}\text { Rescue medication use: } 12 \% \\
\text { (63/547) vs. } 20 \%(111 / 548), \text { RR } 0.57 \\
\text { ( } 95 \% \text { Cl } 0.43 \text { to } 0.76) \\
\text { Pain intensity decreased } \geq 50 \% \text { at } 90 \\
\text { minutes: } 97.4 \%(533 / 547) \text { vs. } 92.5 \% \\
\text { (507/548), RR } 1.05(95 \% \mathrm{Cl} 1.02 \text { to } \\
1.08)\end{array}$ \\
\hline
\end{tabular}

Abbreviations: $\mathrm{CI}$ = confidence interval; IM = intramuscular; IQR = interquartile range; IV = intravenous; NR = not reported; RR = relative risk; $\mathrm{SD}=$ standard deviation; VAS = visual analog scale

KQs 7j and 7l ask how the comparative effectiveness and harms of nonopioid pharmacologic therapy vary depending on: (1) patient demographics (e.g., age, race, ethnicity, gender); (2) patient medical and psychiatric comorbidities; (3) the type of nonopioid medication; (4) dose of medication; (5) duration of treatment.

Evidence was too limited to evaluate how comparative effectiveness and harms of nonopioid therapy varied in subgroups, due to the small number of trials. One trial reported that results were similar in the patients with ureteric calculi on imaging compared with all patients presenting clinically with renal colic. ${ }^{233,234}$

\section{Nonpharmacologic Therapy}

KQs $7 \mathrm{~m}$ and $7 \mathrm{n}$ address the comparative effectiveness of nonopioid pharmacologic therapy versus: (1) inactive controls or (2) other nonpharmacologic therapies.

\section{Acupuncture Versus NSAID or Acetaminophen}

One fair quality trial $(\mathrm{n}=121)$ compared a single acupuncture session versus a single dose of intramuscular diclofenac (75 mg) or intravenous acetaminophen (1000 mg) for acute renal colic (Appendixes E and F, Tables E-10 and F-1; Table 38). ${ }^{228}$ Outcomes were assessed at 2 hours. Details of the acupuncture treatment were not provided. Acupuncture was associated with higher pain intensity than either medication at 2 hours (mean 4.52 vs. 2.75 and 2.10 on a 0 to 10 scale, $\mathrm{p}<0.005$ for acupuncture versus NSAID and for acupuncture versus acetaminophen). Few adverse events were reported.

Table 38. Acupuncture versus NSAID or acetaminophen for acute renal colic

\begin{tabular}{|l|l|l|l|}
\hline $\begin{array}{l}\text { Author, } \\
\text { Year } \\
\text { Quality }\end{array}$ & Interventions & $\begin{array}{l}\text { Followup Duration } \\
\text { Sample Size }\end{array}$ & Pain Intensity \\
\hline $\begin{array}{l}\text { Kaynar, } \\
2015^{228}\end{array}$ & A: Acupuncture, appears to be a single & 2 hours & Pain intensity (mean [SD \\
Fair & session & $\mathrm{n}=160$ & NR], 0 to 10 VAS): 4.52 vs. \\
& B: Diclofenac $75 \mathrm{mg} \mathrm{IM} \times 1$ & & 2.75 vs. 2.10, $\mathrm{p}<0.005$ for \\
& C: Acetaminophen $1 \mathrm{~g} / 100 \mathrm{~mL}$ IV $\times 1$ & & B and C \\
\hline
\end{tabular}

Abbreviations: NR = not reported; SD = standard deviation; VAS = visual analog scale 
KQs 7o and 7p address how the comparative effectiveness and harms of nonpharmacologic therapy vary depending on: (1) patient demographics (e.g., age, gender); (2) patient medical and psychiatric comorbidities; (3) the type of treatment used; (4) the frequency of therapy; (5) the duration of therapy.

No evidence was found for KQs 7o or 7p.

\section{KQ 8. Sickle Cell Crisis}

\section{Key Points}

- Opioid agonist or partial agonist vs. mixed agent

o Evidence insufficient from two poor quality trials (SOE: insufficient).

- Relaxation vs. attention control

o Insufficient evidence from one small trial (SOE: insufficient).

\section{Summary of Findings}

Evidence on the comparative effectiveness of interventions for acute sickle cell pain was limited to three small ( $\mathrm{n}=18,24$, and 68) trials (Appendix E, Table E-11). ${ }^{238-240}$ Two trials evaluated opioid therapy (KQ 8a and 8c), and one trial evaluated nonpharmacologic therapy (KQ $8 \mathrm{i}$ and $8 \mathrm{j}$ ). The mean age of patients ranged from 25 to 32 years, and the proportion of females was 28 percent to 70 percent. Baseline pain scores ranged from 6.2 to 7.6, and duration of pain was $<7$ days in one trial ${ }^{240}$ and not described in the other two trials. ${ }^{238,239}$ One trial of opioid therapy excluded patients with a history of substance use disorder; otherwise, the trials did not describe psychiatric or medical comorbidities. One trial ${ }^{238}$ was rated fair quality and two trials were rated poor quality (Appendix F, Table F-1). ${ }^{239,240}$ Methodological limitations in the trials included unclear blinding of patients and caregivers, failure to report adequate randomization and allocation concealment methods, and unclear use of intention to treat analysis. In one of the poor quality trials, some patients were randomized to interventions multiple times, and it was unclear how many patients received each intervention. ${ }^{239}$

\section{Detailed Synthesis}

\section{Opioid Therapy}

KQs 8a and 8c address the comparative effectiveness and harms of opioid therapy versus: (1) nonopioid pharmacologic therapy (e.g., acetaminophen, NSAIDs, antidepressants, anticonvulsants) or (2) nonpharmacologic therapy (e.g., exercise, cognitive behavioral therapy, acupuncture).

\section{Opioid Agonist or Partial Agonist Versus Mixed Agent}

Two poor quality trials compared different opioid types for acute sickle cell pain in ED settings (Table 39). ${ }^{239,240}$ One trial $(n=68)$ found a single intravenous infusion of the pure opioid agonist meperidine $1 \mathrm{mg} / \mathrm{kg}$ associated with decreased pain intensity versus the mixed agent tramadol $1.5 \mathrm{mg} / \mathrm{kg}$ at 120 minutes (mean difference -2.9 on a 0 to 10 scale; $95 \%$ CI, -4.3 to $-1.5) .{ }^{240}$ Adverse events were not reported. Parenteral tramadol is not available in the United States, though it is available in other parts of the world. The dose of meperidine mg in morphine 
equivalent dose was $0.4 \mathrm{mg} / \mathrm{kg}$. A published conversion ratio for parenteral tramadol was not available; based on the conversion ratio for oral tramadol the dose was $0.3 \mathrm{mg} / \mathrm{kg}$.

The other trial ( $\mathrm{n}=18$ with 45 acute pain episodes) compared intramuscular butorphanol $2 \mathrm{mg}$ (a partial agonist) versus morphine $6 \mathrm{mg}$ (a pure agonist). ${ }^{239}$ After initial administration, the dose could be repeated in 30 to 60 minutes if needed for pain relief and then every 2 to 4 hours until pain relief or discharge. Pain was assessed at 60 and 120 minutes following each dose and at discharge. There was no difference between butorphanol versus morphine in pain intensity (adjusted mean scores 3.8 vs. 4.2 on a 0 to 10 scale, $\mathrm{p}=0.70$ ) or pain relief (adjusted mean scores 4.9 vs. 4.2 on a 0 to 10 scale, $\mathrm{p}=0.80$ ). Results were similar when the analysis was restricted to the first acute pain episode for each patient. There was also no difference in the likelihood of ED discharge (68\% vs. 70\%, p=0.92) or in global assessments of treatment (likelihood of a "good" or "excellent" global assessment of treatment 52\% vs. 71\%; p value for difference across all categories=0.75). There was no difference in adverse events, but few events (primarily nausea and vomiting) were reported ( $23 \%$ vs. $13 \%, \mathrm{p}=0.46)$.

Table 39. Opioid agonist or partial agonist versus mixed agent for sickle cell pain

\begin{tabular}{|c|c|c|c|c|}
\hline $\begin{array}{l}\text { Author, } \\
\text { Year } \\
\text { Quality }\end{array}$ & Interventions & $\begin{array}{l}\text { Followup } \\
\text { Duration } \\
\text { Sample Size }\end{array}$ & Pain Intensity & Other Pain Results \\
\hline $\begin{array}{l}\text { Gonzalez, } \\
1988^{239} \\
\text { Poor }\end{array}$ & $\begin{array}{l}\text { A. Butorphonol, } 2 \mathrm{mg} \text { IM } \\
\text { B. Morphine, } 6 \mathrm{mg} \mathrm{IM}\end{array}$ & $\begin{array}{l}60 \text { and } 120 \\
\text { minutes and at } \\
\text { discharge } \\
\mathrm{n}=18 \text { with } 45 \\
\text { randomized } \\
\text { episodes }\end{array}$ & $\begin{array}{l}\text { Pain (adjusted mean } \\
\text { [SD NR], } 0 \text { to } 100 \text { VAS } \\
\text { converted to } 0 \text { to } 10 \\
\text { scale): } 3.8 \text { vs. } 4.2, \\
p=0.698\end{array}$ & $\begin{array}{l}\text { Pain relief (adjusted } \\
\text { mean [SD NR], } 0 \text { to } \\
100 \text { VAS converted to } \\
0 \text { to } 10 \text { scale) } \\
4.9 \text { vs. } 5.2, p=0.8005\end{array}$ \\
\hline $\begin{array}{l}\text { Uzun, } \\
2010^{240} \\
\text { Poor }\end{array}$ & $\begin{array}{l}\text { A. Meperidine, } 1 \mathrm{mg} / \mathrm{kg} \text { IV } \\
(\mathrm{n}=34) \\
\text { B. Tramadol, } 1.5 \mathrm{mg} / \mathrm{kg} \\
\text { slow infusion }(\mathrm{n}=34)\end{array}$ & $\begin{array}{l}120 \text { minutes } \\
n=68\end{array}$ & $\begin{array}{l}\text { Pain (mean [SD] o to } \\
10 \text { VAS score): } 3.5 \\
\text { (2.9) vs. } 6.4(3.0), \mathrm{MD} \text {, } \\
-2.9 \text { (95\% } \mathrm{Cl}-4.3 \text { to } \\
-1.5)\end{array}$ & NR \\
\hline
\end{tabular}

Abbreviations: $\mathrm{CI}=$ confidence interval; IM = intramuscular; IV = intravenous; $\mathrm{MD}=$ mean difference; $\mathrm{NR}=$ not reported; $\mathrm{SD}$ = standard deviation; VAS = visual analog scale

No evidence was found for other subquestions (KQs 8b and 8d to 8h) related to opioid therapy for acute sickle cell pain.

\section{Nonopioid Pharmacologic Therapy}

KQs $8 \mathrm{i}$ and 8k address the comparative effectiveness and harms of nonopioid pharmacologic therapy versus: (1) nonopioid pharmacologic therapy or (2) nonpharmacologic therapy.

No studies of nonopioid pharmacologic therapy for sickle cell pain met inclusion criteria.

\section{Nonpharmacologic Therapy}

KQs 8m and 8n address the comparative effectiveness of nonopioid pharmacologic therapy versus: (1) inactive controls or (2) other nonpharmacologic therapy.

\section{Relaxation Versus Attention Control}

One small ( $n=24)$, fair quality, open-label trial compared a guided audio-visual relaxation intervention versus an attention control in patients with sickle cell disease in a clinic or prior to discharge from an acute care center or hospital (Table 40). ${ }^{238}$ The relaxation intervention was administered via six 2 to 20 minute video clips; participants were instructed to practice guided relaxation at least once a day for two weeks. The attention control was a 12 minute computer- 
based discussion about sickle cell disease experiences and daily tracking of stress and pain. At two weeks, there was no statistically significant difference between relaxation versus attention control in pain intensity, though the estimate was imprecise and favored relaxation (mean difference -0.70 on a 0 to 10 scale; $95 \%$ CI, -1.72 to 0.32 ). Adverse events were not reported.

Table 40. Nonpharmacologic interventions for sickle cell pain

\begin{tabular}{|c|c|c|c|}
\hline $\begin{array}{l}\text { Author, Year } \\
\text { Quality }\end{array}$ & Interventions & $\begin{array}{l}\text { Followup } \\
\text { Duration } \\
\text { Sample } \\
\text { Size }\end{array}$ & Pain Results \\
\hline $\begin{array}{l}\text { Ezenwa, } \\
2016^{238} \\
\text { Fair }\end{array}$ & $\begin{array}{l}\text { A: Tablet-based guided audio-visual } \\
\text { relaxation--Video clips for guided } \\
\text { relaxation lasting } 2 \text { to } 20 \text { minutes; } \\
\text { patients told to practice guided relaxation } \\
\text { at least once per day for two weeks } \\
\text { B: Control }\end{array}$ & $\begin{array}{l}14 \text { days } \\
n=24\end{array}$ & $\begin{array}{l}\text { Pain intensity, (mean [SD], } 0 \text { to } \\
100 \text { Composite Pain Index } \\
\text { score converted to } 0 \text { to } 10 \\
\text { scale): } 3.5 \text { (1.2) vs. } 4.2(1.2) \\
\text { MD }-0.70(95 \% \mathrm{Cl}-1.72 \text { to } \\
0.32)\end{array}$ \\
\hline
\end{tabular}

Abbreviations: $\mathrm{CI}$ = confidence interval; $\mathrm{MD}$ = mean difference; $\mathrm{SD}$ = standard deviation

KQs 80 and 8p address how the comparative effectiveness and harms of nonpharmacologic therapy vary depending on: (1) patient demographics (e.g., age, gender); (2) patient medical and psychiatric comorbidities; (3) the type of treatment used; (4) the frequency of therapy; (5) the duration of therapy.

No evidence was found for KQs 80 and 8p. 


\section{Discussion}

\section{Key Findings and Strength of Evidence}

This review synthesized the evidence on the comparative effectiveness of opioid and nonopioid pharmacologic therapy and the effectiveness and comparative effectiveness of nonpharmacologic therapy for eight acute pain conditions. It also synthesized the evidence on the accuracy and effectiveness of risk assessment instruments in persons with acute pain being considered for opioid therapy, the association between opioid prescribing versus not prescribing for acute pain and subsequent (including long-term) opioid use, and factors associated with opioid prescribing, for each of these conditions. The key findings for effectiveness and comparative effectiveness are summarized in Table 41 (focusing on pain intensity, stratified by followup duration) and the strength of evidence table (Appendix $G$ ), which also includes findings for function and adverse events.

Evidence was available from 183 randomized controlled trials on the comparative effectiveness of interventions for acute pain or effectiveness of nonpharmacologic therapy. The conditions with the most robust evidence were low back pain, postoperative pain and dental pain; evidence was particularly sparse for neck pain, neuropathic pain, and sickle cell pain. Pain was the main outcome addressed in the trials, though methods for measuring pain varied (e.g., differences in mean pain intensity, the likelihood of clinically significant improvement in pain, summed or average measures of pain, or the likelihood of rescue or repeat medication use). For postoperative pain, dental pain, and kidney stone pain, most comparative effectiveness trials of pharmacologic therapy (opioid or nonopioid) evaluated effects of a single dose on pain at $<1$ day (usually 8 hours or less) followup. Across conditions, most trials of pharmacologic therapy focused on outcomes at $<1$ week followup. The emphasis on short-term outcomes in the trials may reflect the natural history of acute pain, which tends to rapidly improvement in the first days to week. Effects on function and quality of life were more commonly measured in trials that evaluated nonpharmacologic therapy and those that evaluated outcomes at longer followup. 
Table 41. Summary of evidence of treatments for acute pain: pain

\begin{tabular}{|c|c|c|c|c|c|c|}
\hline Key Question & $\begin{array}{l}\text { Intervention and } \\
\text { Comparison }\end{array}$ & $\begin{array}{l}\text { Pain } \\
\text { (Effect Size/SOE) }^{a} \\
<1 \text { Day }\end{array}$ & $\begin{array}{l}\text { Pain } \\
\text { (Effect Size/SOE)a }^{a} \\
1 \text { Day to }<1 \text { Week }\end{array}$ & $\begin{array}{l}\text { Pain } \\
\text { (Effect Size/SOE) }^{a} \\
1 \text { Week to }<2 \text { Weeks }\end{array}$ & $\begin{array}{l}\text { Pain } \\
\text { (Effect Size/SOE) }^{a} \\
2 \text { Weeks to } 4 \text { Weeks }\end{array}$ & $\begin{array}{l}\text { Pain } \\
\text { (Effect Size/SOE) }^{a} \\
\geq 4 \text { Weeks }\end{array}$ \\
\hline \multirow{12}{*}{ KQ1: Back pain } & Opioid vs. NSAID & $\begin{array}{c}\text { None } \\
+\end{array}$ & $\begin{array}{c}\text { None } \\
+\end{array}$ & No evidence & No evidence & No evidence \\
\hline & $\begin{array}{l}\text { Opioid vs. muscle } \\
\text { relaxant }\end{array}$ & No evidence & $\begin{array}{c}\text { None } \\
+ \\
\end{array}$ & No evidence & No evidence & $\begin{array}{c}\text { None } \\
+\end{array}$ \\
\hline & $\begin{array}{l}\text { Muscle relaxant vs. } \\
\text { benzodiazepine }\end{array}$ & No evidence & $\begin{array}{c}\text { Small to moderate } \\
+\end{array}$ & No evidence & No evidence & No evidence \\
\hline & $\begin{array}{l}\text { NSAID or muscle } \\
\text { relaxant vs. } \\
\text { manipulation }\end{array}$ & No evidence & No evidence & $\begin{array}{c}\text { None } \\
+\end{array}$ & $\begin{array}{c}\text { None } \\
+\end{array}$ & $\begin{array}{c}\text { None } \\
+\end{array}$ \\
\hline & $\begin{array}{l}\text { Acupuncture vs. } \\
\text { NSAID }\end{array}$ & No evidence & Insufficient & No evidence & $\begin{array}{c}\text { Moderate } \\
+\end{array}$ & $\begin{array}{c}\text { Moderate } \\
+\end{array}$ \\
\hline & $\begin{array}{l}\text { Exercise vs. usual } \\
\text { care }\end{array}$ & No evidence & No evidence & $\begin{array}{c}\text { None } \\
+\end{array}$ & $\begin{array}{c}\text { None } \\
+\end{array}$ & $\begin{array}{c}\text { None } \\
+\end{array}$ \\
\hline & Exercise vs. bed rest & No evidence & No evidence & $\begin{array}{c}\text { None } \\
++\end{array}$ & $\begin{array}{c}\text { None } \\
++\end{array}$ & $\begin{array}{c}\text { None } \\
++\end{array}$ \\
\hline & $\begin{array}{l}\text { Traditional Chinese } \\
\text { acupuncture vs. sham }\end{array}$ & No evidence & No evidence & No evidence & $\begin{array}{c}\text { Moderate vs. } \\
\text { nonpenetrating sham } \\
\text { or usual care; } \\
\text { None vs. needle } \\
\text { sham } \\
+\end{array}$ & $\begin{array}{c}\text { None } \\
+\end{array}$ \\
\hline & $\begin{array}{l}\text { Brace vs. no brace, } \\
\text { osteoporotic } \\
\text { compression fracture }\end{array}$ & No evidence & No evidence & No evidence & $\begin{array}{c}\text { None } \\
+\end{array}$ & $\begin{array}{c}\text { None } \\
+\end{array}$ \\
\hline & $\begin{array}{l}\text { Heat therapy vs. } \\
\text { usual care or placebo }\end{array}$ & No evidence & $\begin{array}{c}\text { Moderate } \\
++\end{array}$ & $\begin{array}{c}\text { Moderate } \\
+\end{array}$ & $\begin{array}{c}\text { Moderate } \\
+\end{array}$ & No evidence \\
\hline & $\begin{array}{l}\text { Manipulation vs. } \\
\text { inactive controls }\end{array}$ & No evidence & $\begin{array}{c}\text { None } \\
+\end{array}$ & $\begin{array}{c}\text { None } \\
+\end{array}$ & $\begin{array}{c}\text { None } \\
++\end{array}$ & $\begin{array}{c}\text { None } \\
+\end{array}$ \\
\hline & $\begin{array}{l}\text { Manipulation vs. } \\
\text { sham, radiculopathy }\end{array}$ & No evidence & No evidence & $\begin{array}{c}\text { Moderate } \\
+\end{array}$ & No evidence & $\begin{array}{c}\text { Moderate } \\
+\end{array}$ \\
\hline \multirow{4}{*}{ KQ2: Neck pain } & $\begin{array}{l}\text { Collar vs. usual } \\
\text { activity, radiculopathy }\end{array}$ & No evidence & No evidence & No evidence & $\begin{array}{c}\text { Moderate to large } \\
+\end{array}$ & $\begin{array}{c}\text { Moderate to large } \\
+\end{array}$ \\
\hline & $\begin{array}{l}\text { Collar vs. exercise, } \\
\text { radiculopathy }\end{array}$ & No evidence & No evidence & No evidence & $\begin{array}{c}\text { None } \\
+ \\
\end{array}$ & $\begin{array}{c}\text { None } \\
+ \\
\end{array}$ \\
\hline & $\begin{array}{l}\text { Exercise vs. usual } \\
\text { activity, radiculopathy }\end{array}$ & No evidence & No evidence & No evidence & $\begin{array}{c}\text { Moderate } \\
+\end{array}$ & $\begin{array}{c}\text { Moderate } \\
+\end{array}$ \\
\hline & $\begin{array}{l}\text { Collar vs. usual } \\
\text { activity, whiplash } \\
\text { neck sprain }\end{array}$ & No evidence & No evidence & No evidence & No evidence & $\begin{array}{c}\text { No difference } \\
+\end{array}$ \\
\hline
\end{tabular}




\begin{tabular}{|c|c|c|c|c|c|c|}
\hline Key Question & $\begin{array}{l}\text { Intervention and } \\
\text { Comparison }\end{array}$ & $\begin{array}{l}\text { Pain } \\
\text { (Effect Size/SOE) }^{a} \\
<1 \text { Day }\end{array}$ & $\begin{array}{l}\text { Pain } \\
\text { (Effect Size/SOE) }^{a} \\
1 \text { Day to <1 Week }\end{array}$ & $\begin{array}{l}\text { Pain } \\
\text { (Effect Size/SOE) }^{\mathrm{a}} \\
1 \text { Week to }<2 \text { Weeks }\end{array}$ & $\begin{array}{l}\text { Pain } \\
\text { (Effect Size/SOE) }^{\mathrm{a}} \\
2 \text { Weeks to } 4 \text { Weeks }\end{array}$ & $\begin{array}{l}\text { Pain } \\
\text { (Effect Size/SOE) }^{a} \\
\geq 4 \text { Weeks }\end{array}$ \\
\hline & $\begin{array}{l}\text { Collar vs. exercise, } \\
\text { whiplash neck sprain }\end{array}$ & No evidence & No evidence & No evidence & No evidence & $\begin{array}{c}\text { No difference } \\
+\end{array}$ \\
\hline & $\begin{array}{l}\text { Exercise vs. usual } \\
\text { activity, whiplash } \\
\text { neck sprain }\end{array}$ & No evidence & No evidence & No evidence & No evidence & $\begin{array}{c}\text { No difference } \\
+\end{array}$ \\
\hline & $\begin{array}{l}\text { Ultrasound vs. sham } \\
\text { whiplash neck sprain }\end{array}$ & No evidence & No evidence & $\begin{array}{c}\text { None } \\
+\end{array}$ & $\begin{array}{c}\text { Small increase } \\
+\end{array}$ & No evidence \\
\hline \multirow{3}{*}{$\begin{array}{c}\text { KQ 3: Other } \\
\text { musculoskeletal } \\
\text { pain }\end{array}$} & $\begin{array}{l}\text { NSAID vs. } \\
\text { acetaminophen }\end{array}$ & $\begin{array}{c}\text { None } \\
++\end{array}$ & $\begin{array}{c}\text { None } \\
++\end{array}$ & No evidence & No evidence & No evidence \\
\hline & $\begin{array}{l}\text { Ultrasound vs. sham } \\
\text { ultrasound }\end{array}$ & No evidence & $\begin{array}{c}\text { None } \\
+ \\
\end{array}$ & No evidence & No evidence & $\begin{array}{c}\text { None } \\
+ \\
\end{array}$ \\
\hline & $\begin{array}{l}\text { Acupressure vs. } \\
\text { sham acupressure or } \\
\text { usual care }\end{array}$ & No evidence & $\begin{array}{c}\text { Moderate } \\
+\end{array}$ & No evidence & No evidence & $\begin{array}{c}\text { Moderate } \\
+\end{array}$ \\
\hline $\begin{array}{c}\text { KQ4: Acute } \\
\text { neuropathic pain }\end{array}$ & Opioid vs. gabapentin & No evidence & No evidence & $\begin{array}{c}\text { Moderate } \\
+\end{array}$ & No evidence & $\begin{array}{c}\text { Moderate } \\
+\end{array}$ \\
\hline \multirow{8}{*}{$\begin{array}{c}\text { KQ 5: } \\
\text { Postoperative } \\
\text { pain }\end{array}$} & $\begin{array}{l}\text { Opioid vs. NSAID, } \\
\text { single dose, various } \\
\text { surgeries }\end{array}$ & $\begin{array}{c}\text { None } \\
+\end{array}$ & No evidence & No evidence & No evidence & No evidence \\
\hline & $\begin{array}{l}\text { Opioid vs. NSAID, } \\
\text { multidose course, } \\
\text { various surgeries }\end{array}$ & Insufficient ${ }^{b}$ & No evidence & No evidence & No evidence & No evidence \\
\hline & $\begin{array}{l}\text { Opioid vs. } \\
\text { acetaminophen, } \\
\text { single dose, cesarean } \\
\text { section }\end{array}$ & $\begin{array}{c}\text { None } \\
+\end{array}$ & No evidence & No evidence & No evidence & No evidence \\
\hline & $\begin{array}{l}\text { NSAID vS. } \\
\text { acetaminophen, } \\
\text { various surgeries }\end{array}$ & Insufficient & No evidence & No evidence & No evidence & No evidence \\
\hline & $\begin{array}{l}\text { Acupuncture vs. } \\
\text { sham, various } \\
\text { surgeries }\end{array}$ & No evidence & Insufficient & No evidence & No evidence & No evidence \\
\hline & $\begin{array}{l}\text { Acupressure vs. } \\
\text { sham, knee } \\
\text { surgeries }^{c}\end{array}$ & Insufficient & Insufficient & No evidence & No evidence & No evidence \\
\hline & $\begin{array}{l}\text { Cold therapy vs. } \\
\text { sham, knee surgeries }\end{array}$ & No evidence & $\begin{array}{c}\text { None } \\
+\end{array}$ & No evidence & $\begin{array}{c}\text { None } \\
+\end{array}$ & $\begin{array}{c}\text { None } \\
+\end{array}$ \\
\hline & $\begin{array}{l}\text { Massage vs. no } \\
\text { massage, various } \\
\text { surgeries }\end{array}$ & $\begin{array}{c}\text { Moderate to large } \\
+\end{array}$ & No evidence & No evidence & No evidence & No evidence \\
\hline
\end{tabular}




\begin{tabular}{|c|c|c|c|c|c|c|}
\hline Key Question & $\begin{array}{l}\text { Intervention and } \\
\text { Comparison }\end{array}$ & $\begin{array}{l}\text { Pain } \\
\text { (Effect Size/SOE) }^{a} \\
<1 \text { Day }\end{array}$ & $\begin{array}{l}\text { Pain } \\
\text { (Effect Size/SOE) }^{a} \\
1 \text { Day to }<1 \text { Week }\end{array}$ & $\begin{array}{l}\text { Pain } \\
\text { (Effect Size/SOE) }^{a} \\
1 \text { Week to }<2 \text { Weeks }\end{array}$ & $\begin{array}{l}\text { Pain } \\
\text { (Effect Size/SOE) }^{a} \\
2 \text { Weeks to } 4 \text { Weeks }\end{array}$ & $\begin{array}{l}\text { Pain } \\
\text { (Effect Size/SOE) }^{a} \\
\geq 4 \text { Weeks }\end{array}$ \\
\hline & $\begin{array}{l}\text { Music therapy vs. no } \\
\text { music therapy, } \\
\text { various surgeries }\end{array}$ & $\begin{array}{c}\text { Moderate } \\
+\end{array}$ & $\begin{array}{c}\text { Small to moderate } \\
+\end{array}$ & No evidence & No evidence & No evidence \\
\hline & $\begin{array}{l}\text { Exercise vs. no } \\
\text { exercise, thyroid } \\
\text { surgery }\end{array}$ & No evidence & No evidence & $\begin{array}{l}\text { Large } \\
++\end{array}$ & No evidence & $\begin{array}{c}\text { None } \\
+\end{array}$ \\
\hline & $\begin{array}{l}\text { TENS vs. sham } \\
\text { TENS, liposuction }\end{array}$ & $\begin{array}{l}\text { Moderate to large } \\
+\end{array}$ & $\begin{array}{c}\text { Moderate to large } \\
+\end{array}$ & No evidence & No evidence & No evidence \\
\hline \multirow{6}{*}{ KQ 6: Dental pain } & $\begin{array}{l}\text { Opioid plus } \\
\text { acetaminophen vs. } \\
\text { acetaminophen, } \\
\text { single dose-surgical } \\
\text { pain }\end{array}$ & $\begin{array}{c}\text { Small } \\
++\end{array}$ & No evidence & No evidence & No evidence & No evidence \\
\hline & $\begin{array}{l}\text { Opioid vs. } \\
\text { acetaminophen, } \\
\text { single dose-surgical } \\
\text { pain }\end{array}$ & $\begin{array}{c}\text { None } \\
+\end{array}$ & No evidence & No evidence & No evidence & No evidence \\
\hline & $\begin{array}{l}\text { Opioid plus } \\
\text { acetaminophen or } \\
\text { NSAID vs. NSAID, } \\
\text { single dose surgical } \\
\text { pain }\end{array}$ & $\begin{array}{c}\text { Small to moderate } \\
\text { increase } \\
+\end{array}$ & No evidence & No evidence & No evidence & No evidence \\
\hline & $\begin{array}{l}\text { Opioid plus } \\
\text { acetaminophen or } \\
\text { NSAID vs. NSAID, } \\
\text { multidose course- } \\
\text { surgical pain }\end{array}$ & $\begin{array}{c}\text { None } \\
+\end{array}$ & No evidence & No evidence & No evidence & No evidence \\
\hline & $\begin{array}{l}\text { Opioid vs. NSAID } \\
\text { (surgical and } \\
\text { nonsurgical pain) }\end{array}$ & Insufficient & No evidence & No evidence & No evidence & No evidence \\
\hline & $\begin{array}{l}\text { NSAID vs. } \\
\text { acetaminophen, } \\
\text { single dose }\end{array}$ & $\begin{array}{c}\text { Moderate to large } \\
++\end{array}$ & No evidence & No evidence & No evidence & No evidence \\
\hline \multirow{3}{*}{$\begin{array}{l}\text { KQ7: Kidney } \\
\text { stone pain }\end{array}$} & $\begin{array}{l}\text { Morphine vs. NSAID, } \\
\text { single dose }\end{array}$ & $\begin{array}{c}\text { Small increase } \\
++\end{array}$ & No evidence & No evidence & No evidence & No evidence \\
\hline & $\begin{array}{l}\text { Meperidine vs. } \\
\text { NSAID, single dose }\end{array}$ & $\begin{array}{l}\text { Moderate to large } \\
\text { increase } \\
++\end{array}$ & No evidence & No evidence & No evidence & No evidence \\
\hline & $\begin{array}{l}\text { Morphine vs. } \\
\text { acetaminophen }\end{array}$ & $\begin{array}{c}\text { Small increase } \\
++\end{array}$ & No evidence & No evidence & No evidence & No evidence \\
\hline
\end{tabular}




\begin{tabular}{|c|c|c|c|c|c|c|}
\hline Key Question & $\begin{array}{l}\text { Intervention and } \\
\text { Comparison }\end{array}$ & $\begin{array}{l}\text { Pain } \\
\text { (Effect Size/SOE) }^{a} \\
<1 \text { Day }\end{array}$ & $\begin{array}{l}\text { Pain } \\
\text { (Effect Size/SOE) }^{a} \\
1 \text { Day to }<1 \text { Week }\end{array}$ & $\begin{array}{l}\text { Pain } \\
\text { (Effect Size/SOE) }^{a} \\
1 \text { Week to }<2 \text { Weeks }\end{array}$ & $\begin{array}{l}\text { Pain } \\
\text { (Effect Size/SOE) }^{a} \\
2 \text { Weeks to } 4 \text { Weeks }\end{array}$ & $\begin{array}{l}\text { Pain } \\
\text { (Effect Size/SOE) }^{a} \\
\geq 4 \text { Weeks }\end{array}$ \\
\hline & $\begin{array}{l}\text { NSAID vs. } \\
\text { acetaminophen }\end{array}$ & Insufficient & No evidence & No evidence & No evidence & No evidence \\
\hline & $\begin{array}{l}\text { Acupuncture vs. } \\
\text { NSAID or } \\
\text { acetaminophen }\end{array}$ & $\begin{array}{c}\text { Moderate increase } \\
+\end{array}$ & No evidence & No evidence & No evidence & No evidence \\
\hline $\begin{array}{l}\text { KQ8: Sickle cell } \\
\text { pain }\end{array}$ & Insufficient evidence & No evidence & No evidence & No evidence & No evidence & No evidence \\
\hline
\end{tabular}

${ }^{a}$ Effect size: None or small, moderate, or large decrease in pain for intervention A vs. B, unless indicated as an increase in pain; SOE: + = low, ++ = moderate, +++ = high, or insufficient

${ }^{b}$ Opioid was associated with increased likelihood of repeat or rescue medication use (SOE: moderate)

c Acupressure associated with decreased pain medication use versus sham acupressure at $<1$ day and 1 day to $<1$ week (SOE: low)

Abbreviations: ACL = anterior cruciate ligament; KQ = Key Question; NSAID = nonsteroidal anti-inflammatory drug; SOE = strength of evidence; TENS = transcutaneous electrical nerve stimulation 
There were no high strength of evidence ratings, due to small numbers of trials, methodological limitations, imprecision, and inconsistency. Most strength of evidence ratings were low or insufficient, reflecting the limitations in the evidence and high uncertainty in findings. For surgical dental pain and kidney stone pain, moderate strength of evidence indicated that opioids were associated with greater pain or need for rescue medication use versus nonsteroidal anti-inflammatory drugs (NSAIDs). Findings for postoperative pain were somewhat inconsistent. Although there was moderate strength of evidence that opioids are associated with increased likelihood of repeat or rescue medication use at 1 day to 1 week, evidence on pain intensity was insufficient due to inconsistency. In addition, results were based on a small number of trials and pain after a limited set of surgical procedures, most commonly cesarean section, anterior cruciate ligament (ACL) reconstruction, knee arthroplasty, and cholecystectomy. Due to variability in the severity and duration of pain following surgical procedures, findings cannot be broadly generalized to all postoperative pain. There was moderate strength of evidence that opioids were associated with increased risk of adverse events such as nausea, dizziness, and sedation versus nonopioid pharmacologic therapies, though the trials were not designed to assess serious adverse events, and few such events were reported. Evidence comparing opioids versus acetaminophen was less robust than for opioids versus NSAIDs, and somewhat mixed: for dental pain, moderate evidence indicated that opioids were associated with better pain outcomes on some measures, but for kidney stone pain, opioids were associated with worse pain. Evidence on NSAIDs versus acetaminophen was also somewhat mixed: for dental pain, evidence indicated that NSAIDs were associated with better pain outcomes, but for kidney stone pain, results were inconsistent. Evidence on nonopioid pharmacologic therapies other than opioids, NSAIDs, or acetaminophen was very limited. Determinations regarding the effectiveness of nonpharmacologic therapies for acute pain were also constrained by the available evidence. For low back pain, heat therapy was associated with a moderate decrease in pain versus usual care or placebo at 1 day to $<1$ week and at 2 to $<4$ weeks. There was likely no difference between manipulation versus inactive controls for non-radicular low back pain, though one trial of patients with radiculopathy found manipulation associated with increased likelihood of improvement in pain at 2 to $<4$ weeks, and at $\geq 4$ weeks. ${ }^{76}$ There was low strength of evidence supporting acupuncture over an NSAID for low back pain, but findings were based on one trial that evaluated one session of acupuncture and a single dose of an NSAID. ${ }^{48}$ There was low strength of evidence supporting effectiveness of massage for postoperative pain, with likely no difference between cold therapy versus no cold therapy for this condition, with the possible exception of decreased pain medication use at $<1$ week. There was also low strength of evidence supporting effectiveness of acupressure for acute musculoskeletal pain. Reporting of harms for nonpharmacologic therapies was suboptimal, making it difficult to compare harms of pharmacologic and nonpharmacologic therapies. However, the noninvasive nonpharmacologic therapies evaluated in this report are generally not thought to be associated with serious harms, and harms were few when reported.

Trials of opioid therapy were not designed to evaluate effects on long-term use of opioids or associated outcomes such as misuse or substance use disorder. Adverse events associated with use of these and other medications for chronic pain were recently summarized in reports funded by the Agency for Healthcare Research and Quality on opioids and nonopioid pharmacologic therapy. ${ }^{43,44}$ Limited evidence from observational studies found being prescribed an opioid for acute low back pain or after minor or elective surgical procedures was associated with increased likelihood of opioid use at longer term (e.g., 6 months or 1 year) followup. Evidence on factors 
associated with opioid prescribing in patients with the acute pain conditions addressed in this report was very limited, but suggested that legislation mandating use of prescription drug monitoring program data prior to prescribing was not associated with decreases in opioid prescribing for low back pain or postoperative pain. No study evaluated the accuracy or effectiveness of risk assessment instruments to inform use of opioids for acute pain; risk assessment instruments have focused on patients with chronic pain. ${ }^{43}$

\section{Findings in Relationship to What Is Already Known}

Our review differs from most previously published reviews by focusing on head-to-head trials of pharmacologic therapy. Therefore, our findings complement the findings of reviews that evaluated the effectiveness of pharmacologic interventions based on placebo-controlled trials, but are generally consistent with them. For low back pain, such reviews found insufficient evidence to determine the effectiveness of opioid therapy for acute low back pain, ${ }^{241}$ moderate evidence of small short-term effects of NSAIDs versus placebo on pain, ${ }^{242}$ ineffectiveness of acetaminophen, ${ }^{243}$ and high-quality evidence of short-term benefits for pain relief with muscle relaxants. ${ }^{244}$ For dental pain, a review of systematic reviews of placebo-controlled and head-tohead trials of pharmacologic therapy found that a number of NSAIDs, alone or in combination with acetaminophen, were associated with similar or increased likelihood of achieving clinically significant pain relief versus various opioids, alone or in combination with an NSAID or acetaminophen. ${ }^{245}$ For acute pain due to kidney stones, our findings are consistent with a recent systematic review of placebo-controlled trials that found NSAIDs were more effective than opioids or acetaminophen for pain, though it focused on outcomes at 30 minutes after administration. ${ }^{29}$ Like our review, a recent guideline on management of sickle cell pain found little evidence on pharmacologic therapy. ${ }^{246}$ Recent reviews of pharmacologic therapy for neuropathic pain focused on effectiveness in patients with chronic symptoms. ${ }^{247-249}$ Reviews have also found limited evidence suggesting effectiveness of NSAIDs relative to placebo ${ }^{250}$ for acute neck pain and strong evidence suggesting effectiveness of NSAIDs for acute ankle sprain. $^{251}$

For nonpharmacologic therapy, our review differs from previously published reviews by focusing on management of pain in outpatients or in patients soon to be discharged, and restricting inclusion to patients with symptom duration of $<4$ weeks. For low back pain, one prior review that included patients with pain for up to 6 weeks also found low to very low quality evidence suggesting no difference in effects of manipulation versus inert interventions, sham manipulation, or when added to another intervention, and evidence that manipulation was no better than active therapies. ${ }^{252}$ Another review that included studies of patients with low back pain for up to 12 weeks found inconsistent evidence that acupuncture was more effective than NSAIDs for pain and limited evidence that acupuncture was more effective than sham. ${ }^{253}$ For postoperative pain, a prior review found evidence of effectiveness for transcutaneous electrical nerve stimulation (TENS) and cognitive behavioral therapy, but included trials of inpatient management following major surgical procedures and trials in which the intervention was initiated pre- or perioperatively. ${ }^{254}$ For acute ankle sprain, systematic reviews found evidence to support bracing and neuromuscular training to prevent recurrence of ankle sprain and early mobilization, exercise, and manual therapy for treatment of acute pain. ${ }^{251}$ However, the purpose of these treatments was primarily to manage the underlying condition rather than treatment of pain, the focus of our review. 


\section{Applicability}

A number of issues could impact the applicability of our findings. Most randomized trials were conducted in emergency department or postoperative care unit settings, which might reduce applicability to outpatient management of acute pain. Further, trials of pharmacologic therapy frequently evaluated a single dose and some trials of nonpharmacologic therapy evaluated a single treatment session, potentially limiting the applicability of findings to a multidose course of treatment. Trials excluded important patient subgroups, such as persons with a history of substance use disorder, prior opioid use, and psychological or medical comorbidities, or did not report information regarding these factors. In addition, trials were not designed to evaluate how benefits or harms varied in subgroups defined by these factors or others, such as age, sex, and race/ethnicity. Another limitation to applicability is that most trials-particularly trials of pharmacologic therapy - were designed to assess short-term ( $<1$ week, and often $<1$ day) effects on pain, with few trials evaluating effects on non-pain outcomes or at longer term followup. Finally, the applicability of findings for one pain condition (including pain due to specific surgical procedures) addressed in this review to another pain condition in this review, or to acute pain conditions not addressed in this review, is uncertain. For example, opioids were associated with decreased pain versus acetaminophen for dental pain, but increased pain versus acetaminophen for kidney stone pain. The applicability of findings from one acute pain condition to others may vary depending on the type and nature of the pain. For example, evidence on pharmacologic therapy for low back pain may have high applicability to neck pain, another musculoskeletal condition in the spine, but less applicable to sickle cell pain, neuropathic pain, or abdominal pain.

\section{Implications for Clinical and Policy Decision Making}

Our review has potential implications for clinical and policy decision making. A number of policies on management of acute pain have focused on limiting the amount or duration of opioids prescribed. Although such policies are associated with decreases in the rate and amount of opioid prescribing, ${ }^{255-258}$ effects of such policies on pain and other patient outcomes are not well studied. ${ }^{259}$ The findings of our review that opioids are not superior to NSAIDs for some commonly encountered acute pain conditions, and in some cases may be inferior, may provide indirect evidence that such policies may not adversely affect outcomes among patients with those conditions, provided that NSAIDs or other effective alternatives are utilized. Evidence of an increased risk of adverse events with opioids compared with other pharmacologic therapies and indicating an association between acute opioid prescribing for low back pain and postoperative pain and long-term use also may provide indirect support for policies aimed at reducing overprescribing of opioids for acute pain, though such findings are susceptible to residual confounding.

There was a lack of evidence to guide clinical and policy decision making around most nonpharmacologic therapies. In addition to considerations regarding effectiveness, use of nonpharmacologic therapies as an alternate or in addition to pharmacologic therapy should account for issues related to timely access, burdens (e.g., time off work to attend treatment sessions), costs, availability, and reimbursement. Heat therapy has been incorporated into low back pain guidelines as a potential self-care option that does not require a visit with a healthcare provider and relatively low costs. ${ }^{260}$ Although guidelines on low back pain and postoperative pain suggest use of nonpharmacologic therapies (massage, acupuncture, and spinal manipulation 
for low back pain and transcutaneous electrical nerve stimulation and cognitive-behavioral therapies for postoperative pain) these recommendations were based in part on evidence not eligible for this review (e.g., subacute pain, pre- or perioperative interventions, and inpatient management of major surgical procedures). ${ }^{254,260}$

The lack of evidence about how benefits and harms of therapies vary in subgroups (such as older adults, pregnant persons, or those with comorbidities, including history of substance use disorder or prior opioid use) is an important limitation on clinical and policy decision making. Evidence on the association between enactment of legislation requiring use of prescription drug management program data prior to prescribing an opioid for acute pain has shown little effect on opioid prescribing rates. Research is needed to identify risk mitigation, education, and other medication management factors associated with prescribing practices (ideally, overprescribing), in order to better inform policy decision.

\section{Limitations of the Review Process}

We excluded non-English language articles and did not search for studies published only as abstracts. We did not conduct statistical and graphical methods for assessing small sample effects (a potential marker for publication bias) due to small numbers of trials and heterogeneity in study design methods, patient populations, and outcomes. Searches on clinical trial registries and public solicitation did not identify unpublished studies suggesting publication bias. We did not have access to individual patient data, which limited our ability to evaluate subgroup effects. Meta-analyses could not be conducted for most questions due to small numbers of studies, methodological limitations, and heterogeneity across studies in interventions, study designs, and outcomes. There was also variability within the predefined acute pain categories (e.g., presence of absence of radiculopathy, type of musculoskeletal pain, surgical or nonsurgical dental pain, and specific surgical procedure), which could obscure potential differential effects by specific condition or procedure, though results were reported by specific condition when possible. Because of anticipated statistical heterogeneity, we utilized a random effects model when metaanalysis was performed. We defined acute pain as lasting $<4$ weeks, though other definitions have utilized longer thresholds (e.g., $<6$ or $<12$ weeks), which decreased the evidence included in this review. However, pain improves rapidly in the first few days and the trajectory for improvement slows and responsiveness to treatments tends to decrease the longer that pain is present. Therefore, the 4 week threshold potentially reduced heterogeneity due to variability among patients related to duration of pain. Within the prespecified 4 week threshold, variability in duration (e.g., 1 or 2 days versus 3 to 4 weeks) could also potentially impact effects of treatment, but evidence was insufficient to determine the impact of acute pain duration. We excluded trials that combined multiple therapies (e.g., manipulation, massage, and physical modalities) because it was not possible to disentangle the effects of the individual components. The exception was an opioid plus acetaminophen or an NSAID, because this combination is commonly used in clinical practice and excluding such studies would have substantially reduced the available evidence on opioids. When sufficient data were available, we analyzed effects of an opioid plus acetaminophen or an NSAID separately from an opioid alone. We analyzed NSAIDs as a class, which might obscure potential within-class differences. However, a previous systematic review found that analgesic effects of different NSAIDs were similar. ${ }^{261}$ We also excluded trials in which the outcomes were only assessed at $<1$ hour; therefore, our review does not address the immediate effects of interventions. The report did not address acute headache, 
which is being addressed in a separate AHRQ-funded review, and other types of acute pain (e.g., abdominal pain, chest pain, gynecological or urological pain, and others).

\section{Limitations of the Evidence Base}

The evidence base had important limitations. Importantly, there was very little evidence for sickle cell pain, acute neuropathic pain, and neck pain. Evidence was also limited for musculoskeletal pain other than low back pain and kidney stone pain. Evidence on the effectiveness of nonpharmacologic therapy for acute pain was generally more limited than evidence on the effectiveness of pharmacologic therapy, despite broader inclusion criteria (i.e., effectiveness and comparative effectiveness studies, rather than just comparative effectiveness studies) for nonpharmacologic therapy. Most trials had important methodological limitations, reducing certainty in findings. The trials primarily focused on outcomes related to pain, usually at $<1$ week and often at $<1$ day, with relatively limited measurement of function and quality of life. In addition, trials were not designed to evaluate the likelihood of long-term opioid use or harms related to the addiction and overdose potential of opioids. Most trials of pharmacologic therapy and some trials of nonpharmacologic therapy evaluated a single dose or session of treatment, which may not indicate benefits and harms of multiple doses or sessions. In addition, therapy was usually initiated in emergency department or postoperative care settings, with few trials focusing on outpatient management of acute pain. The trials were not designed to evaluate how benefits and harms varied in subgroups defined by demographics (including age, sex, and race/ethnicity) or clinical factors (including history of substance use disorder, prior opioid use, and history of cancer). Patients with a history of substance use disorder or prior opioid use represent an important clinical challenge, but were excluded or trials did not describe these characteristics. The trials were also not designed to evaluate how benefits and harms varied according to opioid dose or amount prescribed. Evidence on effects of interventions on long-term opioid use was primarily available from observational studies, based on claims data on dispensing, which may not accurately measure actual use of prescribed opioids and does not capture use of unprescribed opioids. Evidence on the accuracy and effectiveness of risk assessment instruments was unavailable and evidence on how risk mitigation strategies, patient education, and other factors impact prescribing of opioids was very limited.

\section{Research Gaps}

Many research gaps limit the full understanding of the effectiveness, comparative effectiveness, and harms of treatments for acute pain, as well as aspects of opioid prescribing (risk assessment instruments, long-term use, and factors impacting prescribing). Among the acute pain conditions addressed in this review, evidence is particularly lacking for sickle cell pain, neuropathic pain, and neck pain. Research on management of acute sickle cell pain is an important priority, given the disparities in care associated with this condition. ${ }^{262}$ Neuropathic pain is more commonly managed as a chronic condition and evidence from management of low back pain may have applicability to neck pain. Research is needed to identify effective nonpharmacologic therapies. Cannabis has become legal in many states for medical as well as recreational use, though evidence on acute pain is lacking. Research is needed to determine the effectiveness of cannabis for acute pain.

Patients at higher risk for or with a history of or current opioid use disorder or misuse or with mental health and medical comorbidities are commonly encountered in clinical practice, but evidence on management of acute pain in these populations is very limited. Studies that enroll 
such patients and evaluate how benefits and harms vary compared with patients without such factors would be very helpful for informing treatment and policy approaches. Research is also needed to better understand the degree to which acute pain treatments can be individualized based on demographic, clinical, or genetic factors. Studies should measure multiple important outcomes, including pain, function, and quality of life, such as sleep, and mental health outcomes. Studies are also needed to better understand the association between use of opioid and nonopioid therapies and risk of misuse and opioid use disorder using standardized methods. Research is also needed to better understand how patients value different outcomes (beneficial and harmful) associated with interventions for acute pain, and effects of strategies that consider such preferences in decision making. Studies should address how policies aimed at reducing the duration or dose of opioid prescribing impact patient outcomes such as pain and quality of life and the effectiveness of interventions to mitigate such effects (e.g., identification and followup of patients with persistent pain and timely refills or referrals for treatment), in addition to effects on prescribing. Research is also needed to develop and validate instruments for accurately predicting risk of opioid use disorder or misuse in persons with acute pain, and to determine how using risk prediction instruments impacts treatment decisions and, ultimately, patient outcomes.

It is important for future studies on opioids to evaluate longer-term outcomes, including long-term use and potentially associated harms (e.g., opioid use disorder, overdose, impaired social and emotional cognition, and workforce nonparticipation). Well-designed clinical registries that prospectively enroll patients with acute pain prescribed and not prescribed opioids could complement randomized trials evaluating long-term outcomes.

\section{Conclusions}

Opioid therapy was associated with decreased or similar effectiveness for pain versus an NSAID for surgical dental pain, kidney stone pain, and low back pain. Opioids and NSAIDs were more effective than acetaminophen for surgical dental pain, but opioids were less effective than acetaminophen for kidney stone pain. Opioids were associated with increased risk of shortterm adverse events versus NSAIDs or acetaminophen, including any adverse event, nausea, dizziness, and somnolence. Serious adverse events were uncommon for all interventions, but studies were not designed to assess risk of overdose, opioid use disorder, or long-term harms. Being prescribed an opioid for acute low back pain or postoperative pain was associated with increased likelihood of use of opioids at long-term followup versus not being prescribed, based on observational studies. Evidence on nonpharmacological therapies was limited, but heat therapy, spinal manipulation, massage, acupuncture, acupressure, a cervical collar, and exercise were effective for specific acute pain conditions. Research is needed to determine the comparative effectiveness of therapies for sickle cell pain, acute neuropathic pain, neck pain, and management of postoperative pain following discharge; effects of therapies for acute pain on non-pain outcomes; effects of therapies on long-term outcomes, including long-term opioid use; and how benefits and harms of therapies vary in subgroups. 


\section{References}

1. Henschke N, Kamper SJ, Maher CG. The epidemiology and economic consequences of pain. Mayo Clin Proc. 2015

Jan;90(1):139-47. doi: 10.1016/j.mayocp.2014.09.010. PMID: 25572198.

2. Institute of Medicine. Relieving Pain in America: A Blueprint for Transforming Prevention, Care, Education, and Research. Washington, D.C.: The National Academies Press; 2011.

3. Tighe P, Buckenmaier CC, 3rd, Boezaart AP, et al. Acute Pain Medicine in the United States: A Status Report. Pain Med. 2015 Sep;16(9):1806-26. doi: https://dx.doi.org/10.1111/pme.12760. PMID: 26535424.

4. Kent ML, Tighe PJ, Belfer I, et al. The ACTTION-APS-AAPM Pain Taxonomy (AAAPT) Multidimensional Approach to Classifying Acute Pain Conditions. Pain Med. 2017 May 1;18(5):947-58. doi: 10.1093/pm/pnx019. PMID: 28482098.

5. Buvanendran A, Fiala J, Patel KA, et al. The Incidence and Severity of Postoperative Pain following Inpatient Surgery. Pain Med. 2015 Dec;16(12):2277-83. doi: https://dx.doi.org/10.1111/pme.12751. PMID: 25917518.

6. Cordell WH, Keene KK, Giles BK, et al. The high prevalence of pain in emergency medical care. Am J Emerg Med. 2002 May;20(3):165-9. PMID: 11992334.

7. Dix P, Sandhar B, Murdoch J, et al. Pain on medical wards in a district general hospital. Br J Anaesth. 2004 Feb;92(2):235-7. doi: 10.1093/bja/aeh052. PMID: 14722175.

8. The Interagency Pain Research Coordinating Committee. National pain strategy: a comprehensive population health level strategy for pain. Bethesda, MD: National Institutes of Health; 2016.

https://iprcc.nih.gov/National_Pain_Strategy /NPS_Main.htm.
9. Glare P, Aubrey K, Myles P. Postoperative pain management and opioids 1 (series): Transition from acute to chronic pain after surgery. The Lancet. 2019(393):1537-46 (epub ahead of print https://www.thelancet.com/pdfs/journals/lan cet/PIIS0140-6736(19)30352-6.pdf).

10. McGreevy K, Bottros MM, Raja SN. Preventing Chronic Pain following Acute Pain: Risk Factors, Preventive Strategies, and their Efficacy. Eur J Pain Suppl. 2011;5(2):365-72. doi: 10.1016/j.eujps.2011.08.013. PMID: 22102847.

11. Apfelbaum JL, Chen C, Mehta SS, et al. Postoperative pain experience: results from a national survey suggest postoperative pain continues to be undermanaged. Anesth Analg. 2003 Aug;97(2):534-40, table of contents. PMID: 12873949.

12. Moore RA, Wiffen PJ, Derry S, et al. Nonprescription (OTC) oral analgesics for acute pain - an overview of Cochrane reviews. Cochrane Database Syst Rev. 2015 Nov 04(11):CD010794. doi: https://dx.doi.org/10.1002/14651858.CD010 794.pub2. PMID: 26544675.

13. Darnall BD, Stacey BR, Chou R. Medical and psychological risks and consequences of long-term opioid therapy in women. Pain Med. 2012 Sep;13(9):1181-211. doi: 10.1111/j.1526-4637.2012.01467.x. PMID: 22905834.

14. Ezenwa MO, Fleming MF. Racial Disparities in Pain Management in Primary Care. J Health Dispar Res Pract. 2012;5(3):12-26. PMID: 24244911.

15. Goyal MK, Kuppermann N, Cleary SD, et al. Racial Disparities in Pain Management of Children With Appendicitis in Emergency Departments. JAMA Pediatr. 2015 Nov;169(11):996-1002. doi: 10.1001/jamapediatrics.2015.1915. PMID: 26366984. 
16. Nafiu OO, Chimbira WT, Stewart M, et al. Racial differences in the pain management of children recovering from anesthesia. Paediatr Anaesth. 2017 Jul;27(7):760-7. doi: https://dx.doi.org/10.1111/pan.13163. PMID: 28504322.

17. Dowell D, Kunins HV, Farley TA. Opioid Analgesics—Risky Drugs, Not Risky PatientsOpioid Analgesics. JAMA. 2013;309(21):2219-20. doi: 10.1001/jama.2013.5794.

18. National Institutes of Health National Institute on Drug Abuse. Overdose Death Rates. 2019. https://www.drugabuse.gov/relatedtopics/trends-statistics/overdose-death-rates. Accessed May 29, 2019.

19. Mundkur ML, Franklin JM, Abdia Y, et al. Days' Supply of Initial Opioid Analgesic Prescriptions and Additional Fills for Acute Pain Conditions Treated in the Primary Care Setting - United States, 2014. MMWR Morb Mortal Wkly Rep. 2019 Feb 15;68(6):140-3. doi: 10.15585/mmwr.mm6806a3. PMID: 30763301.

20. National Conference of State Legislatures. Prescribing Policies: States Confront Opioid Overdose Epidemic. 2018.

http://www.ncsl.org/research/health/prescrib ing-policies-states-confront-opioidoverdose-epidemic.aspx. Accessed May 29, 2019.

21. Reznikoff C. How acute pain leads to chronic opioid use. Cleve Clin J Med. 2018 Nov;85(11):837-41. doi: 10.3949/ccjm.85a.18038. PMID: 30395519.

22. Shah A, Hayes CJ, Martin BC. Characteristics of Initial Prescription Episodes and Likelihood of Long-Term Opioid Use - United States, 2006-2015. MMWR Morb Mortal Wkly Rep. 2017 Mar 17;66(10):265-9. doi:

10.15585/mmwr.mm6610a1. PMID: 28301454.

23. Brummett CM, Waljee JF, Goesling J, et al. New Persistent Opioid Use After Minor and Major Surgical Procedures in US Adults. JAMA Surg. 2017 Jun 21;152(6):e170504. doi: 10.1001/jamasurg.2017.0504. PMID: 28403427.
24. Brat GA, Agniel D, Beam A, et al. Postsurgical prescriptions for opioid naive patients and association with overdose and misuse: retrospective cohort study. BMJ. 201801 17;360:j5790. doi: 10.1136/bmj.j5790. PMID: 29343479.

25. Chang AK, Bijur PE, Esses D, et al. Effect of a Single Dose of Oral Opioid and Nonopioid Analgesics on Acute Extremity Pain in the Emergency Department: A Randomized Clinical Trial. JAMA. 2017 Nov 7;318(17):1661-7. doi: 10.1001/jama.2017.16190. PMID: 29114833.

26. Friedman BW, Dym AA, Davitt M, et al. Naproxen With Cyclobenzaprine, Oxycodone/Acetaminophen, or Placebo for Treating Acute Low Back Pain: A Randomized Clinical Trial. JAMA. 2015 Oct 20;314(15):1572-80. doi: 10.1001/jama.2015.13043. PMID: 26501533.

27. Lewis RA, Williams NH, Sutton AJ, et al. Comparative clinical effectiveness of management strategies for sciatica: systematic review and network metaanalyses. Spine J. 2015 Jun 1;15(6):1461-77. doi: 10.1016/j.spinee.2013.08.049. PMID: 24412033.

28. Moore PA, Hersh EV. Combining ibuprofen and acetaminophen for acute pain management after third-molar extractions: translating clinical research to dental practice. J Am Dent Assoc. 2013 Aug;144(8):898-908. PMID: 23904576.

29. Pathan SA, Mitra B, Cameron PA. A Systematic Review and Meta-analysis Comparing the Efficacy of Nonsteroidal Anti-inflammatory Drugs, Opioids, and Paracetamol in the Treatment of Acute Renal Colic. Eur Urol. 2018 04;73(4):58395. doi: https://dx.doi.org/10.1016/j.eururo.2017.11. 001. PMID: 29174580.

30. Franklin GM, Stover BD, Turner JA, et al. Early opioid prescription and subsequent disability among workers with back injuries: the Disability Risk Identification Study Cohort. Spine. 2008 Jan 15;33(2):199-204. doi:

https://dx.doi.org/10.1097/BRS.0b013e3181 60455c. PMID: 18197107. 
31. Webster BS, Verma SK, Gatchel RJ. Relationship between early opioid prescribing for acute occupational low back pain and disability duration, medical costs, subsequent surgery and late opioid use. Spine. 2007 Sep 01;32(19):2127-32. PMID: 17762815.

32. Bartels K, Mayes LM, Dingmann C, et al. Opioid Use and Storage Patterns by Patients after Hospital Discharge following Surgery. PLoS ONE. 2016;11(1):e0147972. doi: 10.1371/journal.pone.0147972. PMID: 26824844.

33. Bicket MC, Long JJ, Pronovost PJ, et al. Prescription Opioid Analgesics Commonly Unused After Surgery: A Systematic Review. JAMA Surg. 2017 Nov 1;152(11):1066-71. PMID: Pmc5701659.

34. Neuman M, Bateman B, Wunsch H. Postoperative pain management and opioids 2 (series): Inappropriate opioid prescription after surgery. The Lancet. 2019;393:154757 (epub ahead of print: https://www.thelancet.com/pdfs/journals/lan cet/PIIS0140-6736(19)30428-3.pdf).

35. Dowell D, Haegerich TM, Chou R. CDC Guideline for Prescribing Opioids for Chronic Pain--United States, 2016. JAMA. 2016 Apr 19;315(15):1624-45. doi: 10.1001/jama.2016.1464. PMID: 26977696.

36. Davis CS, Lieberman AJ, HernandezDelgado $\mathrm{H}$, et al. Laws limiting the prescribing or dispensing of opioids for acute pain in the United States: A national systematic legal review. Drug Alcohol Depend. 2019 Jan 1;194:166-72. doi: 10.1016/j.drugalcdep.2018.09.022. PMID: 30445274.

37. Chua KP, Brummett CM, Waljee JF. Opioid Prescribing Limits for Acute Pain: Potential Problems With Design and Implementation. JAMA. 2019 Feb 19;321(7):643-4. doi: 10.1001/jama.2019.0010. PMID: 30703214.
38. US Department of Health and Human Services Office of the Assistant Secretary for Health. Draft Report on Pain Management Best Practices: Updates, Gaps, Inconsistencies, and Recommendations. 2019. https://www.hhs.gov/ash/advisorycommittees/pain/reports/2018-12-draftreport-on-updates-gaps-inconsistenciesrecommendations/index.html\#1introduction. Accessed May 29, 2019.

39. Agency for Healthcare Research and Quality: Effective Health Care Program. Treatment of Acute Pain: An Evidence Map. 2019.

https://effectivehealthcare.ahrq.gov/topics/ac ute-pain-treatment/protocol. Accessed May 29, 2019.

40. Methods Guide for Effectiveness and Comparative Effectiveness Reviews. Rockville, MD: Agency for Healthcare Research and Quality; 2017.

https://effectivehealthcare.ahrq.gov/topics/ce r-methods-guide/overview. Accessed June 1, 2019.

41. Morton SC, Murad MH, O'Connor E, et al. Quantitative Synthesis-An Update. Methods Guide for Comparative Effectiveness Reviews. (Prepared by the Scientific Resource Center under Contract No. 290-2012-0004-C). AHRQ Publication No. 18-EHC007-EF. Rockville, MD: Agency for Healthcare Research and Quality; February 2018. Posted final reports are located on the Effective Health Care Program search page (https://effectivehealthcare.ahrq.gov/). https://doi.org/10.23970/AHRQEPCMETH GUIDE3.

42. Skelly AC, Chou R, Dettori JR, et al. Noninvasive Nonpharmacological Treatment for Chronic Pain: A Systematic Review. Comparative Effectiveness Review No. 209. (Prepared by the Pacific Northwest Evidence-based Practice Center under Contract No. 290-2015-00009-I.) AHRQ Publication No 18-EHC013-EF. : . Rockville, MD: Agency for Healthcare Research and Quality; June 2018. https://effectivehealthcare.ahrq.gov/topics/n onpharma-treatment-pain/research-2018 PMID: 30179389. 
43. Chou R, Hartung D, Turner J, et al. Opioid Treatments for Chronic Pain. Comparative Effectiveness Review No. 229. Agency for Healthcare Research and Quality. 2020 Apr 16doi:

https://doi.org/10.23970/AHRQEPCCER22 9.

44. McDonagh M, Selph S, Buckle yD, et al. Nonopioid Pharmacologic Treatments for Chronic Pain. Comparative Effectiveness Review No. 228. Agency for Healthcare Research and Quality. 2020 Apr 16doi: https://doi.org/10.23970/AHRQEPCCER22 $\underline{8}$.

45. Chou R, Deyo R, Friedly J, et al. Noninvasive Treatments for Low Back Pain. Comparative Effectiveness Review No. 169. (Prepared by the Pacific Northwest Evidence-based Practice Center under Contract No. 290-2012-00014-I.) AHRQ Publication No. 16-EHC004-EF. Rockville, MD: Agency for Healthcare Research and Quality; February 2016.

46. Anders C, Hubner A. Influence of elastic lumbar support belts on trunk muscle function in patients with non-specific acute lumbar back pain. PLoS ONE. 2019;14(1):e0211042. doi: https://dx.doi.org/10.1371/journal.pone.0211 042. PMID: 30677095.

47. Kim HJ, Yi JM, Cho HG, et al. Comparative study of the treatment outcomes of osteoporotic compression fractures without neurologic injury using a rigid brace, a soft brace, and no brace: a prospective randomized controlled non-inferiority trial. J Bone Joint Surg Am. 2014 Dec 3;96(23):1959-66. doi: 10.2106/jbjs.N.00187. PMID: 25471910.

48. Shin JS, Ha IH, Lee J, et al. Effects of motion style acupuncture treatment in acute low back pain patients with severe disability: a multicenter, randomized, controlled, comparative effectiveness trial. Pain. 2013 Jul;154(7):1030-7. doi: 10.1016/j.pain.2013.03.013. PMID: 23639822.

49. Wilkinson MJ. Does 48 hours' bed rest influence the outcome of acute low back pain? Br J Gen Pract. 1995

Sep;45(398):481-4. PMID: 7546872.
50. Basmajian JV. Acute back pain and spasm. A controlled multicenter trial of combined analgesic and antispasm agents. Spine. 1989 Apr;14(4):438-9. PMID: 2524114.

51. Biondi D, Xiang J, Benson C, et al. Tapentadol immediate release versus oxycodone immediate release for treatment of acute low back pain. Pain Physician. 2013 May-Jun;16(3):E237-46. PMID: 23703422.

52. Boyles W. Management of acute musculoskeletal conditions : thoracolumbar strain or sprain. A double-blind evaluation comparing the efficacy and safety of carisoprodol with diazepam. Today's Ther Trends. 1983 1983;1:1-16.

53. Brown FL, Jr., Bodison S, Dixon J, et al. Comparison of diflunisal and acetaminophen with codeine in the treatment of initial or recurrent acute low back strain. Clin Ther. 1986;9 Suppl C:52-8. PMID: 2951012.

54. Dehghan M, Farahbod F. The efficacy of thermotherapy and cryotherapy on pain relief in patients with acute low back pain, a clinical trial study. J Clin Diagn Res. 2014;8(9):LC01-LC4. doi: 10.7860/JCDR/2014/7404.4818. PMID: 25386469.

55. Erhard RE, Delitto A, Cibulka MT. Relative effectiveness of an extension program and a combined program of manipulation and flexion and extension exercises in patients with acute low back syndrome. Phys Ther. 1994 Dec;74(12):1093-100. doi: 10.1093/ptj/74.12.1093. PMID: 7991650.

56. Evans DP, Burke MS, Newcombe RG. Medicines of choice in low back pain. Curr Med Res Opin. 1980;6(8):540-7. PMID: 6446445.

57. Gilbert JR, Taylor DW, Hildebrand A, et al. Clinical trial of common treatments for low back pain in family practice. Br Med J (Clin Res Ed). 1985 Sep 21;291(6498):791-4. PMID: 2931153.

58. Glover JR, Morris JG, Khosla T. Back pain: a randomized clinical trial of rotational manipulation of the trunk. Br J Ind Med. 1974 Jan;31(1):59-64. doi: 10.1136/oem.31.1.59. PMID: 4274488. 
59. Hackett GI, Seddon D, Kaminski D. Electroacupuncture compared with paracetamol for acute low back pain. Practitioner. 1988 Feb 22;232(1443):163-4. PMID: 2973008.

60. Hadler NM, Curtis P, Gillings DB, et al. A benefit of spinal manipulation as adjunctive therapy for acute low-back pain: a stratified controlled trial. Spine. 1987 Sep;12(7):7026. PMID: 2961085.

61. Hallegraeff JM, de Greef M, Winters JC, et al. Manipulative therapy and clinical prediction criteria in treatment of acute nonspecific low back pain. Percept Mot Skills. 2009 Feb;108(1):196-208. doi: 10.2466/pms.108.1.196-208. PMID: 19425461.

62. Hancock MJ, Maher CG, Latimer J, et al. Assessment of diclofenac or spinal manipulative therapy, or both, in addition to recommended first-line treatment for acute low back pain: a randomised controlled trial. Lancet. 2007 Nov 10;370(9599):1638-43. PMID: 17993364.

63. Hasegawa TM, Baptista AS, de Souza MC, et al. Acupuncture for acute non-specific low back pain: a randomised, controlled, double-blind, placebo trial. Acupunct Med. 2014 Apr;32(2):109-15. doi: 10.1136/acupmed-2013-010333. PMID: 24316509.

64. Hennies OL. A new skeletal muscle relaxant (DS 103-282) compared to diazepam in the treatment of muscle spasm of local origin. J Int Med Res. 1981;9(1):62-8. PMID: 6451461.

65. Hofstee DJ, Gijtenbeek JM, Hoogland PH, et al. Westeinde sciatica trial: randomized controlled study of bed rest and physiotherapy for acute sciatica. J Neurosurg. 2002 Jan;96(1 Suppl):45-9. PMID: 11797655.

66. Hoiriis KT, Pfleger B, McDuffie FC, et al. A randomized clinical trial comparing chiropractic adjustments to muscle relaxants for subacute low back pain. J Manipulative Physiol Ther. 2004 Jul-Aug;27(6):388-98. PMID: 15319761.

67. Hussain I, Hussain Shah SI, Amjad I. Efficacy of spinal manual therapy in nonspecific acute low back pain. Rawal Medical Journal. 2013;38(4):358-60.
68. Innes GD, Croskerry P, Worthington J, et al. Ketorolac versus acetaminophen-codeine in the emergency department treatment of acute low back pain. J Emerg Med. 1998 Jul-Aug;16(4):549-56. PMID: 9696169.

69. Juni P, Battaglia M, Nuesch E, et al. A randomised controlled trial of spinal manipulative therapy in acute low back pain. Ann Rheum Dis. 2009 Sep;68(9):1420-7. doi: 10.1136/ard.2008.093757. PMID: 18775942.

70. Kettenmann B, Wille C, Lurie-Luke E, et al. Impact of continuous low level heatwrap therapy in acute low back pain patients: subjective and objective measurements. Clin J Pain. 2007 Oct;23(8):663-8. doi: 10.1097/AJP.0b013e31813543ef. PMID: 17885344.

71. Liu J, Li N. Clinical observation of a combination of acupuncture and drug administration for nonspecific acute lumbar sprain. Journal of Acupuncture and Tuina Science. 2010 Feb;8(1):47-9. doi: 10.1007/s11726-010-0047-2.

72. Malmivaara A, Hakkinen U, Aro T, et al. The treatment of acute low back pain--bed rest, exercises, or ordinary activity? N Engl J Med. 1995 Feb 09;332(6):351-5. PMID: 7823996.

73. Nadler SF, Steiner DJ, Erasala GN, et al. Continuous low-level heatwrap therapy for treating acute nonspecific low back pain. Arch Phys Med Rehabil. 2003a Mar;84(3):329-34. doi: 10.1053/apmr.2003.50102. PMID: 12638099.

74. Nadler SF, Steiner DJ, Petty SR, et al. Overnight use of continuous low-level heatwrap therapy for relief of low back pain. Arch Phys Med Rehabil. 2003b Mar;84(3):335-42. PMID: 12638100.

75. Postacchini F, Facchini M, Palieri P. Efficacy of various forms of conservative treatment in low back pain. A comparative study. Neuro-orthopedics. 1988;6(1):28-35.

76. Santilli V, Beghi E, Finucci S. Chiropractic manipulation in the treatment of acute back pain and sciatica with disc protrusion: a randomized double-blind clinical trial of active and simulated spinal manipulations. Spine J. 2006 Mar-Apr;6(2):131-7. PMID: 16517383. 
77. Stark J, Petrofsky J, Berk L, et al. Continuous low-level heatwrap therapy relieves low back pain and reduces muscle stiffness. Phys Sportsmed. 2014 Nov;42(4):39-48. doi: 10.3810/psm.2014.11.2090. PMID: 25419887.

78. Tao XG, Bernacki EJ. A randomized clinical trial of continuous low-level heat therapy for acute muscular low back pain in the workplace. J Occup Environ Med. 2005 Dec;47(12):1298-306. doi: 10.1097/01.jom.0000184877.01691.a3. PMID: 16340712.

79. Underwood MR, Morgan J. The use of a back class teaching extension exercises in the treatment of acute low back pain in primary care. Fam Pract. 1998 Feb;15(1):915. PMID: 9527292.

80. Vas J, Aranda JM, Modesto M, et al. Acupuncture in patients with acute low back pain: a multicentre randomised controlled clinical trial. Pain. 2012 Sep;153(9):1883-9. doi: 10.1016/j.pain.2012.05.033. PMID: 22770838.

81. von Heymann WJ, Schloemer P, Timm J, et al. Spinal high-velocity low amplitude manipulation in acute nonspecific low back pain: a double-blinded randomized controlled trial in comparison with diclofenac and placebo. Spine. 2013 Apr 01;38(7):540-8. doi: 10.1097/BRS.0b013e318275d09c. PMID: 23026869.

82. Vorsanger GJ, Farrell J, Xiang J, et al. Tapentadol, oxycodone or placebo for acute pain of vertebral compression fractures: a randomized Phase IIIb study. Pain Manag. 2013;3(2):109-18. PMID: 24645994.

83. Lee SS, Choi Y, Pransky GS. Extent and Impact of Opioid Prescribing for Acute Occupational Low Back Pain in the Emergency Department. J Emerg Med. 2016 Mar;50(3):376-84.e1-2. doi: 10.1016/j.jemermed.2015.10.015. PMID: 26754559.
84. Webster BS, Cifuentes M, Verma S, et al. Geographic variation in opioid prescribing for acute, work-related, low back pain and associated factors: a multilevel analysis. Am J Ind Med. 2009 Feb;52(2):162-71. doi: https://dx.doi.org/10.1002/ajim.20655. PMID: 19016267.

85. Hoppe JA, McStay C, Sun BC, et al. Emergency Department Attending Physician Variation in Opioid Prescribing in Low Acuity Back Pain. West J Emerg Med. 2017 Oct;18(6):1135-42. doi: 10.5811/westjem.2017.7.33306. PMID: 29085548.

86. Khobrani M, Perona S, Patanwala AE. Effect of a legislative mandate on opioid prescribing for back pain in the emergency department. Am J Emerg Med. 2019 Nov;37(11):2035-8. doi: 10.1016/j.ajem.2019.02.031. PMID: 30824275.

87. Sun BC, Charlesworth CJ, Lupulescu-Mann $\mathrm{N}$, et al. Effect of Automated Prescription Drug Monitoring Program Queries on Emergency Department Opioid Prescribing. Ann Emerg Med. 2018 Mar;71(3):33747.e6. doi: 10.1016/j.annemergmed.2017.10.023. PMID: 29248333.

88. Kongsted A, Qerama E, Kasch H, et al. Neck collar, "act-as-usual" or active mobilization for whiplash injury? A randomized parallel-group trial. Spine (Phila Pa 1976). 2007 Mar 15;32(6):618-26. doi: 10.1097/01.brs.0000257535.77691.bd. PMID: 17413465.

89. Borchgrevink GE, Kaasa A, McDonagh D, et al. Acute treatment of whiplash neck sprain injuries. A randomized trial of treatment during the first 14 days after a car accident. Spine. 1998 Jan 01;23(1):25-31. doi: 10.1097/00007632-199801010-00006. PMID: 9460148.

90. Kuijper B, Tans JT, Beelen A, et al. Cervical collar or physiotherapy versus wait and see policy for recent onset cervical radiculopathy: randomised trial. BMJ. 2009 Oct 7;339:b3883. doi: 10.1136/bmj.b3883. PMID: 19812130. 
91. Ruiz-Molinero C, Jimenez-Rejano JJ, Chillon-Martinez R, et al. Efficacy of therapeutic ultrasound in pain and joint mobility in whiplash traumatic acute and subacute phases. Ultrasound Med Biol. 2014 Sep;40(9):2089-95. doi:

10.1016/j.ultrasmedbio.2014.04.016. PMID: 25023094.

92. Schnabel M, Ferrari R, Vassiliou T, et al. Randomised, controlled outcome study of active mobilisation compared with collar therapy for whiplash injury. Emerg Med J. 2004 May;21(3):306-10. PMID: 15107368.

93. AlRuthia Y, Alghadeer S, Balkhi B, et al. Efficacy of acetaminophen versus ibuprofen for the management of rotator cuff-related shoulder pain: Randomized open-label study. Saudi Pharm J. 2019;27(6):882-8. doi: 10.1016/j.jsps.2019.06.001. PMID: 31516331.

94. Andersen LA, Gøtzsche PC. Naproxen and aspirin in acute musculoskeletal disorders: a double-blind, parallel study in patients with sports injuries. Pharmatherapeutica. 1984;3(8):531-7. PMID: 6366809.

95. Dalton JD, Jr., Schweinle JE. Randomized controlled noninferiority trial to compare extended release acetaminophen and ibuprofen for the treatment of ankle sprains. Ann Emerg Med. 2006 Nov;48(5):615-23. doi: 10.1016/j.annemergmed.2006.05.015. PMID: 17052565.

96. Duncan JJ, Farr JE. Comparison of diclofenac sodium and aspirin in the treatment of acute sports injuries. Am J Sports Med. 1988 Nov-Dec;16(6):656-9. doi: 10.1177/036354658801600618. PMID: 3149152.

97. Indelicato PA. Comparison of diflunisal and acetaminophen with codeine in the treatment of mild to moderate pain due to strains and sprains. Clin Ther. 1986;8(3):269-74. PMID: 3521855.

98. Jalili M, Fathi M, Moradi-Lakeh M, et al. Sublingual buprenorphine in acute pain management: a double-blind randomized clinical trial. Ann Emerg Med. 2012 Apr;59(4):276-80. doi: 10.1016/j.annemergmed.2011.10.021. PMID: 22115823.
99. Kayali C, Agus H, Surer L, et al. The efficacy of paracetamol in the treatment of ankle sprains in comparison with diclofenac sodium. Saudi Med J. 2007 Dec;28(12):1836-9. PMID: 18060212.

100. Lazcano A, Dougherty JM, Kruger M. Use of rib belts in acute rib fractures. Am J Emerg Med. 1989 Jan;7(1):97-100. PMID: 2643965.

101. Lyrtzis C, Natsis K, Papadopoulos C, et al. Efficacy of paracetamol versus diclofenac for Grade II ankle sprains. Foot Ankle Int. 2011 Jun;32(6):571-5. doi: 10.3113/fai.2011.0571. PMID: 21733417.

102. Man SY, Woo WK, Lam PKW, et al. Feasibility Study Comparing Oral Paracetamol and Oral Non-Steroidal AntiInflammatory Drugs for Treating Pain after Musculoskeletal Injury: a Randomised, Double Blind, Controlled Trial. Hong Kong Journal of Emergency Medicine. 2004 2004/04/01;11(2):78-84. doi: 10.1177/102490790401100203.

103. Ridderikhof ML, Lirk P, Goddijn H, et al. Acetaminophen or Nonsteroidal AntiInflammatory Drugs in Acute Musculoskeletal Trauma: A Multicenter, Double-Blind, Randomized, Clinical Trial. Ann Emerg Med. 2018 Mar;71(3):35768.e8. doi: 10.1016/j.annemergmed.2017.08.041. PMID: 29033294.

104. Zhao M, Gao W, Zhang L, et al. Acupressure Therapy for Acute Ankle Sprains: A Randomized Clinical Trial. Pm R. 2018 Jan;10(1):36-44. doi: 10.1016/j.pmrj.2017.06.009. PMID: 28634002.

105. Aghababian RV. Comparison of diflunisal and acetaminophen with codeine in the management of grade 2 ankle sprain. Clin Ther. 1986;8(5):520-6. PMID: 3094957.

106. Basur RL, Shephard E, Mouzas GL. A cooling method in the treatment of ankle sprains. Practitioner. 1976 Jun;216(1296):708-11. PMID: 821050. 
107. Bondarsky EE, Domingo AT, Matuza NM, et al. Ibuprofen vs acetaminophen vs their combination in the relief of musculoskeletal pain in the ED: a randomized, controlled trial. Am J Emerg Med. 2013

Sep;31(9):1357-60. doi: 10.1016/j.ajem.2013.06.007. PMID: 23896011.

108. Heere LP. Piroxicam in acute musculoskeletal disorders and sports injuries. Am J Med. 1988 May 20;84(5A):50-5. PMID: 3287912.

109. Hewitt DJ, Todd KH, Xiang J, et al. Tramadol/acetaminophen or hydrocodone/acetaminophen for the treatment of ankle sprain: a randomized, placebo-controlled trial. Ann Emerg Med. 2007 Apr;49(4):468-80, 80.e1-2. PMID: 17113683.

110. Hocutt JE, Jr., Jaffe R, Rylander CR, et al. Cryotherapy in ankle sprains. Am J Sports Med. 1982 Sep-Oct;10(5):316-9. doi: 10.1177/036354658201000512. PMID: 6814272.

111. Muckle DS. A comparative study of flurbiprofen and aspirin in soft tissue trauma. Br J Sports Med. 1976 Mar;10(1):11-3. doi: 10.1136/bjsm.10.1.11. PMID: 786422.

112. Muncie HL, Jr., King DE, DeForge B. Treatment of mild to moderate pain of acute soft tissue injury: diflunisal vs acetaminophen with codeine. J Fam Pract. 1986 Aug;23(2):125-7. PMID: 2942630.

113. Nyanzi CS, Langridge J, Heyworth JR, et al. Randomized controlled study of ultrasound therapy in the management of acute lateral ligament sprains of the ankle joint. Clin Rehabil. 1999 Feb;13(1):16-22. doi: 10.1177/026921559901300103. PMID: 10327093.

114. Ordog GJ. Transcutaneous electrical nerve stimulation versus oral analgesic: a randomized double-blind controlled study in acute traumatic pain. Am J Emerg Med. 1987 Jan;5(1):6-10. doi: 10.1016/07356757(87)90281-6. PMID: 3545246.
115. Oswal P, Nagarathna R, Ebnezar J, et al. The effect of add-on yogic prana energization technique (YPET) on healing of fresh fractures: a randomized control study. J Altern Complement Med. 2011 Mar;17(3):253-8. doi: 10.1089/acm.2010.0001. PMID: 21417810.

116. Pagliara L, Tornago S, Metastasio J, et al. Tramadol compared with diclofenac in traumatic musculoskeletal pain. Current Therapeutic Research. 1997 Aug;58(8):47391. doi: 10.1016/S0011393X\%2897\%2980039-2.

117. Sloan JP, Hain R, Pownall R. Clinical benefits of early cold therapy in accident and emergency following ankle sprain. Arch Emerg Med. 1989 Mar;6(1):1-6. doi: 10.1136/emj.6.1.1. PMID: 2712981.

118. Turturro MA, Paris PM, Larkin GL. Tramadol versus hydrocodoneacetaminophen in acute musculoskeletal pain: a randomized, double-blind clinical trial. Ann Emerg Med. 1998 Aug;32(2):13943. PMID: 9701294.

119. Williamson JB, George TK, Simpson DC, et al. Ultrasound in the treatment of ankle sprains. Injury. 1986 May;17(3):176-8. doi: 10.1016/0020-1383(86)90327-x. PMID: 3102375.

120. Woo WW, Man SY, Lam PK, et al. Randomized double-blind trial comparing oral paracetamol and oral nonsteroidal antiinflammatory drugs for treating pain after musculoskeletal injury. Ann Emerg Med. 2005 Oct;46(4):352-61. PMID: 16187469.

121. Zammit E, Herrington L. Ultrasound therapy in the management of acute lateral ligament sprains of the ankle joint. Phys Ther Sport. 2005;6(3):116-21.

122. Whitefield M, O'Kane CJ, Anderson S. Comparative efficacy of a proprietary topical ibuprofen gel and oral ibuprofen in acute soft tissue injuries: a randomized, double-blind study. J Clin Pharm Ther. 2002 Dec;27(6):409-17. PMID: 12472980.

123. Balakrishnan S, Bhushan K, Bhargava VK, et al. A randomized parallel trial of topical aspirin-moisturizer solution vs. oral aspirin for acute herpetic neuralgia. Int J Dermatol. 2001 Aug;40(8):535-8. doi: 10.1046/j.13654362.2001.01265.x. PMID: 11703529. 
124. Dworkin RH, Barbano RL, Tyring SK, et al. A randomized, placebo-controlled trial of oxycodone and of gabapentin for acute pain in herpes zoster. Pain. 2009 Apr;142(3):20917. doi: 10.1016/j.pain.2008.12.022. PMID: 19195785.

125. Abbaspoor Z, Akbari M, Najar S. Effect of foot and hand massage in post-cesarean section pain control: a randomized control trial. Pain Manag Nurs. 2014

Mar;15(1):132-6. doi: https://dx.doi.org/10.1016/j.pmn.2012.07.00 8. PMID: 23352729.

126. Daniels SE, Upmalis D, Okamoto A, et al. A randomized, double-blind, phase III study comparing multiple doses of tapentadol IR, oxycodone IR, and placebo for postoperative (bunionectomy) pain. Curr Med Res Opin. 2009b Mar;25(3):765-76. doi: https://dx.doi.org/10.1185/03007990902728 183. PMID: 19203298.

127. Degirmen N, Ozerdogan N, Sayiner D, et al. Effectiveness of foot and hand massage in postcesarean pain control in a group of Turkish pregnant women. Appl Nurs Res. 2010 Aug;23(3):153-8. doi: 10.1016/j.apnr.2008.08.001. PMID: 20643325.

128. Ffrench-O'Carroll R, Steinhaeuser H, Duff $\mathrm{S}$, et al. A randomized controlled trial comparing tapentadol with oxycodone in non-breastfeeding women post elective cesarean section. Curr Med Res Opin. 2019 06;35(6):975-81. doi: https://dx.doi.org/10.1080/03007995.2018.1 550059. PMID: 30444145.

129. Holmstrom A, Hardin BC. Cryo/Cuff compared to epidural anesthesia after knee unicompartmental arthroplasty: a prospective, randomized and controlled study of 60 patients with a 6-week followup. J Arthroplasty. 2005 Apr;20(3):316-21. PMID: 15809948.

130. Nguyen KK, Liu YF, Chang C, et al. A Randomized Single-Blinded Trial of Ibuprofen- versus Opioid-Based Primary Analgesic Therapy in Outpatient Otolaryngology Surgery. Otolaryngol Head Neck Surg. 2019 May;160(5):839-46. doi: 10.1177/0194599819832528. PMID: 30832548.
131. Ripa M, Betts B, Dhaliwal S, et al. Survey of Postoperative Pain in Photorefractive Keratectomy Using Topical versus Oral Nonsteroidal Anti-Inflammatory Drugs. Clinical Ophthalmology. 2020;14:1459-66. doi:

https://dx.doi.org/10.2147/OPTH.S255441. PMID: 32546954.

132. Dambros C, Martimbianco AL, Polachini LO, et al. Effectiveness of cryotherapy after anterior cruciate ligament reconstruction. Acta Ortop Bras. 2012;20(5):285-90. doi: 10.1590/s1413-78522012000500008. PMID: 24453619.

133. Dervin GF, Taylor DE, Keene GC. Effects of cold and compression dressings on early postoperative outcomes for the arthroscopic anterior cruciate ligament reconstruction patient. J Orthop Sports Phys Ther. 1998 Jun;27(6):403-6. doi: 10.2519/jospt.1998.27.6.403. PMID: 9617725.

134. Edwards DJ, Rimmer M, Keene GC. The use of cold therapy in the postoperative management of patients undergoing arthroscopic anterior cruciate ligament reconstruction. Am J Sports Med. 1996 MarApr;24(2):193-5. doi: 10.1177/036354659602400213. PMID: 8775119.

135. Langenbach MR, Aydemir-Dogruyol K, Issel R, et al. Randomized sham-controlled trial of acupuncture for postoperative pain control after stapled haemorrhoidopexy. Colorectal Dis. 2012 Aug;14(8):e486-91. doi: 10.1111/j.1463-1318.2012.02984.x. PMID: 22330010.

136. Lessard LA, Scudds RA, Amendola A, et al. The efficacy of cryotherapy following arthroscopic knee surgery. J Orthop Sports Phys Ther. 1997 Jul;26(1):14-22. doi: 10.2519/jospt.1997.26.1.14. PMID: 9201637.

137. Ohkoshi Y, Ohkoshi M, Nagasaki S, et al. The effect of cryotherapy on intraarticular temperature and postoperative care after anterior cruciate ligament reconstruction. Am J Sports Med. 1999 May-Jun;27(3):35762. doi: 10.1177/03635465990270031601. PMID: 10352774. 
138. Ayhan H, Tastan S, Iyigun E, et al. The Effectiveness of Neck Stretching Exercises Following Total Thyroidectomy on Reducing Neck Pain and Disability: A Randomized Controlled Trial. Worldviews Evid Based Nurs. 2016 Jun;13(3):224-31. doi: 10.1111/wvn.12136. PMID: 26773539.

139. Barber FA, McGuire DA, Click S.

Continuous-flow cold therapy for outpatient anterior cruciate ligament reconstruction. Arthroscopy. 1998 Mar;14(2):130-5. doi: 10.1016/s0749-8063(98)70030-1. PMID: 9531122.

140. Berti M, Albertin A, Casati A, et al. A prospective, randomized comparison of dexketoprofen, ketoprofen or paracetamol for postoperative analgesia after outpatient knee arthroscopy. Minerva Anestesiol. 2000 Jul-Aug;66(7-8):549-54. PMID: 10965735.

141. Bugada D, Lavand'homme P, Ambrosoli AL, et al. Effect of postoperative analgesia on acute and persistent postherniotomy pain: a randomized study. J Clin Anesth. 2015 12;27(8):658-64. doi: 10.1016/j.jclinane.2015.06.008. PMID: 26329661.

142. Castellano JJ, Rojas AM, Karia R, et al. A Randomized, Double-Blind, PlaceboControlled Study of Neuromuscular Electrical Stimulation (NMES) use for Recovery after Elective Total Hip Replacement Surgery. Bull Hosp Jt Dis (2013). 2016 Nov;74(4):275-81. PMID: 27815950 .

143. da Silva MP, Liebano RE, Rodrigues VA, et al. Transcutaneous electrical nerve stimulation for pain relief after liposuction: a randomized controlled trial. Aesthetic Plast Surg. 2015 Apr;39(2):262-9. doi: https://dx.doi.org/10.1007/s00266-0150451-6. PMID: 25665520.

144. Daniel DM, Stone ML, Arendt DL. The effect of cold therapy on pain, swelling, and range of motion after anterior cruciate ligament reconstructive surgery. Arthroscopy. 1994 Oct;10(5):530-3. doi: 10.1016/s0749-8063(05)80008-8. PMID: 7999161.
145. Daniels S, Casson E, Stegmann JU, et al. A randomized, double-blind, placebocontrolled phase 3 study of the relative efficacy and tolerability of tapentadol IR and oxycodone IR for acute pain. Curr Med Res Opin. 2009a Jun;25(6):1551-61. doi: 10.1185/03007990902952825. PMID: 19445652.

146. Felhendler D, Lisander B. Pressure on acupoints decreases postoperative pain. Clin J Pain. 1996 Dec;12(4):326-9. doi: 10.1097/00002508-199612000-00012. PMID: 8969878.

147. Finlay KA, Wilson JA, Gaston P, et al. Postoperative pain management through audioanalgesia: Investigating musical constructs. Psychol Music. 2016;44(3):493-513.

148. Forchuk C, Baruth P, Prendergast M, et al. Postoperative arm massage: a support for women with lymph node dissection. Cancer Nurs. 2004 Jan-Feb;27(1):25-33. doi: 10.1097/00002820-200401000-00004. PMID: 15108949.

149. Gamermann PW, Martins ALC, Rosa L, et al. Acupuncture as a complement to the pharmacological management of pain, nausea and vomiting after cesarean section: a randomized clinical trial. Acupuncture and Related Therapies. 2014;3(1):11-4. doi: 10.1016/j.arthe.2014.12.002.

150. Gimbel JS, Brugger A, Zhao W, et al. Efficacy and tolerability of celecoxib versus hydrocodone/acetaminophen in the treatment of pain after ambulatory orthopedic surgery in adults. Clin Ther. 2001 Feb;23(2):228-41. PMID: 11293556.

151. He BJ, Tong PJ, Li J, et al. Auricular acupressure for analgesia in perioperative period of total knee arthroplasty. Pain Med. 2013 Oct;14(10):1608-13. doi: 10.1111/pme.12197. PMID: 23865512.

152. Hebertson RM, Storey N. The comparative efficacy of diclofenac potassium, aspirin, and placebo in the treatment of patients with pain following gynecologic surgery. Todays Therapeutic Trends. 1995;12:33-.

153. Helmerhorst GTT, Zwiers R, Ring D, et al. Pain Relief After Operative Treatment of an Extremity Fracture: A Noninferiority Randomized Controlled Trial. J Bone Joint Surg Am. 2017 Nov 15;99(22):1908-15. doi: 10.2106/JBJS.17.00149. PMID: 29135664. 
154. Hulme J, Waterman H, Hillier VF. The effect of foot massage on patients' perception of care following laparoscopic sterilization as day case patients. J Adv Nurs. 1999 Aug;30(2):460-8. doi: 10.1046/j.1365-2648.1999.01101.x. PMID: 10457249.

155. Koc M, Tez M, Yoldas O, et al. Cooling for the reduction of postoperative pain: prospective randomized study. Hernia. 2006 Apr;10(2):184-6. doi: 10.1007/s10029-0050062-2. PMID: 16432641.

156. Koras K, Karabulut N. The Effect of Foot Massage on Postoperative Pain and Anxiety Levels in Laparoscopic Cholecystectomy Surgery: A Randomized Controlled Experimental Study. J Perianesth Nurs. 2019 Jun;34(3):551-8. doi: https://dx.doi.org/10.1016/j.jopan.2018.07.0 06. PMID: 30470466.

157. Morrison NA, Repka MX. Ketorolac versus acetaminophen or ibuprofen in controlling postoperative pain in patients with strabismus. Ophthalmology. 1994 May;101(5):915-8. PMID: 8190480.

158. Nilsson U, Unosson M, Rawal N. Stress reduction and analgesia in patients exposed to calming music postoperatively: a randomized controlled trial. Eur J Anaesthesiol. 2005 Feb;22(2):96-102. doi: 10.1017/s0265021505000189. PMID: 15816586.

159. Oh JH, Seo HJ, Lee YH, et al. Do Selective COX-2 Inhibitors Affect Pain Control and Healing After Arthroscopic Rotator Cuff Repair? A Preliminary Study. Am J Sports Med. 2018 Mar;46(3):679-86. doi: 10.1177/0363546517744219. PMID: 29253346.

160. Rawal N, Allvin R, Amilon A, et al. Postoperative analgesia at home after ambulatory hand surgery: a controlled comparison of tramadol, metamizol, and paracetamol. Anesth Analg. 2001 Feb;92(2):347-51. PMID: 11159230.

161. Rosenberg M, Curtis L, Bourke DL. Transcutaneous electrical nerve stimulation for the relief of postoperative pain. Pain. 1978 Aug;5(2):129-33. doi: 10.1016/03043959\%2878\%2990034-9. PMID: 14716650.
162. Saatsaz S, Rezaei R, Alipour A, et al. Massage as adjuvant therapy in the management of post-cesarean pain and anxiety: A randomized clinical trial. Complement Ther Clin Pract. 2016 Aug;24:92-8. doi: 10.1016/j.ctcp.2016.05.014. PMID: 27502807.

163. Schachtel BP, Thoden WR, Baybutt RI. Ibuprofen and acetaminophen in the relief of postpartum episiotomy pain. J Clin Pharmacol. 1989 Jun;29(6):550-3. PMID: 2666455.

164. Sniezek PJ, Brodland DG, Zitelli JA. A randomized controlled trial comparing acetaminophen, acetaminophen and ibuprofen, and acetaminophen and codeine for postoperative pain relief after Mohs surgery and cutaneous reconstruction. Dermatol Surg. 2011;37(7):1007-13. PMID: 21561527.

165. Sozen KK, Karabulut N. Efficacy of Hand and Foot Massage in Anxiety and Pain Management Following Laparoscopic Cholecystectomy: A Controlled Randomized Study. Surg Laparosc Endosc Percutan Tech. 2019 Dec 04;04:04. doi: https://dx.doi.org/10.1097/SLE.0000000000 000738. PMID: 31855924.

166. Speer KP, Warren RF, Horowitz L. The efficacy of cryotherapy in the postoperative shoulder. J Shoulder Elbow Surg. 1996 JanFeb;5(1):62-8. doi: 10.1016/s10582746(96)80032-2. PMID: 8919444.

167. Sunshine A, Olson NZ, Zighelboim I, et al. Ketoprofen, acetaminophen plus oxycodone, and acetaminophen in the relief of postoperative pain. Clin Pharmacol Ther. 1993 Nov;54(5):546-55. doi: 10.1038/clpt.1993.187. PMID: 8222498.

168. Thijs E, Schotanus MGM, Bemelmans YFL, et al. Reduced opiate use after total knee arthroplasty using computer-assisted cryotherapy. Knee Surg Sports Traumatol Arthrosc. 2019 Apr;27(4):1204-12. doi: 10.1007/s00167-018-4962-y. PMID: 29725749.

169. Viscusi ER, Allard R, Sohns M, et al. Tapentadol immediate release for moderate to severe acute post-surgery pain. J Opioid Manag. 2019 Jan/Feb;15(1):51-67. doi: 10.5055/jom.2019.0486. PMID: 30855723. 
170. Vorsanger GJ, Klopfer AM, Xiang J, et al. Immediate-release tapentadol or oxycodone for treatment of acute postoperative pain after elective arthroscopic shoulder surgery: a randomized, phase IIIb study. J Opioid Manag. 2013 Jul-Aug;9(4):281-90. doi: 10.5055/jom.2013.0170. PMID: 24353022.

171. Wirz S, Wartenberg H-C, Wittmann M, et al. Post-operative pain therapy with controlled release oxycodone or controlled release tramadol following orthopedic surgery: A prospective, randomized, doubleblind investigation. The Pain Clinic. 2005;17(4):367-76. doi: 10.1163/156856905774482733. PMID: 15259296

172. Alam A, Gomes T, Zheng H, et al. Longterm analgesic use after low-risk surgery: a retrospective cohort study. Arch Intern Med. 2012 Mar 12;172(5):425-30. doi: https://dx.doi.org/10.1001/archinternmed.20 11.1827. PMID: 22412106.

173. Thiels CA, Habermann EB, Hooten WM, et al. Chronic use of tramadol after acute pain episode: cohort study. BMJ. 2019 May 14;365:11849. doi: https://dx.doi.org/10.1136/bmj.l1849. PMID: 31088782.

174. Stucke RS, Kelly JL, Mathis KA, et al. Association of the Use of a Mandatory Prescription Drug Monitoring Program With Prescribing Practices for Patients Undergoing Elective Surgery. JAMA Surg. 2018 Dec 1;153(12):1105-10. doi: 10.1001/jamasurg.2018.2666. PMID: 30140927.

175. Akinbade AO, Ndukwe KC, Owotade FJ. Comparative analgesic efficacy and tolerability of celecoxib and tramadol on postoperative pain after mandibular third molar extraction: A double blind randomized controlled trial. Niger J Clin Pract. 2019 Jun;22(6):796-800. doi: https://dx.doi.org/10.4103/njcp.njcp_544_18 . PMID: 31187764.
176. Akural EI, Jarvimaki V, Lansineva A, et al. Effects of combination treatment with ketoprofen $100 \mathrm{mg}+$ acetaminophen 1000 mg on postoperative dental pain: a singledose, 10-hour, randomized, double-blind, active- and placebo-controlled clinical trial. Clin Ther. 2009 Mar;31(3):560-8. doi: 10.1016/j.clinthera.2009.03.017. PMID: 19393845.

177. Cattry E, Troullos E, Paredes-Diaz A. Efficacy and safety of naproxen sodium 440 $\mathrm{mg}$ versus acetaminophen $600 \mathrm{mg} /$ codeine phosphate $60 \mathrm{mg}$ in the treatment of postoperative dental pain. Am J Dent. 2020 Apr;33(2):106-12. PMID: 32259416.

178. Daniels S, Reader S, Berry P, et al. Onset of analgesia with sodium ibuprofen, ibuprofen acid incorporating poloxamer and acetaminophen-a single-dose, doubleblind, placebo-controlled study in patients with post-operative dental pain. Eur J Clin Pharmacol. 2009c;65(4):343-53. PMID: 19252905.

179. Doroschak AM, Bowles WR, Hargreaves KM. Evaluation of the combination of flurbiprofen and tramadol for management of endodontic pain. J Endod. 1999 Oct;25(10):660-3. doi: 10.1016/s00992399(99)80350-1. PMID: 10687523.

180. Kiersch TA, Halladay SC, Hormel PC. A single-dose, double-blind comparison of naproxen sodium, acetaminophen, and placebo in postoperative dental pain. Clin Ther. 1994;16(3):394-404. PMID: 7923306.

181. Moore RA, Gay-Escoda C, Figueiredo R, et al. Dexketoprofen/tramadol: randomised double-blind trial and confirmation of empirical theory of combination analgesics in acute pain. J Headache Pain.

2015;16:541. doi: https://dx.doi.org/10.1186/s10194-0150541-5. PMID: 26123824.

182. Searle S, Muse D, Paluch E, et al. Efficacy and Safety of Single and Multiple Doses of a Fixed-dose Combination of Ibuprofen and Acetaminophen in the Treatment of Postsurgical Dental Pain: Results from Two Phase 3, Randomized, Parallel-Group, Double-Blind, Placebo-Controlled Studies. Clin J Pain. 2020 Apr 07;07:07. doi: https://dx.doi.org/10.1097/AJP.0000000000 000828. PMID: 32271183. 
183. Altiparmak N, Bayram B, Diker N, et al. Efficacy of Ice Pack Therapy After Impacted Third Molar Surgery: A Randomized Controlled Clinical Trial. Turkiye Klinikleri. Dishekimligi Bilimleri Dergisi. 2018;24(1).

184. Bentley KC, Head TW. The additive analgesic efficacy of acetaminophen, 1000 $\mathrm{mg}$, and codeine, $60 \mathrm{mg}$, in dental pain. Clin Pharmacol Ther. 1987 Dec;42(6):634-40. doi: 10.1038/clpt.1987.211. PMID: 3690941.

185. Breivik E, Haanaes H, Barkvoll P. Upside assay sensitivity in a dental pain model. Eur J Pain. 1998;2(2):179-86. doi: 10.1016/S1090-3801\%2898\%2990010-6. PMID: 10700313.

186. Breivik EK, Barkvoll P, Skovlund E. Combining diclofenac with acetaminophen or acetaminophen-codeine after oral surgery: a randomized, double-blind single-dose study. Clin Pharmacol Ther. 1999 Dec;66(6):625-35. doi: 10.1053/cp.1999.v66.103629001. PMID: 10613619.

187. Brown JD, Daniels SE, Bandy DP, et al. Evaluation of multiday analgesia with etoricoxib in a double-blind, randomized controlled trial using the postoperative thirdmolar extraction dental pain model. Clin J Pain. 2013 Jun;29(6):492-8. doi: 10.1097/AJP.0b013e318260c144. PMID: 23247002.

188. Cooper SA, Breen JF, Giuliani RL. The relative efficacy of indoprofen compared with opioid-analgesic combinations. J Oral Surg. 1981 Jan;39(1):21-5. PMID: 6935400 .

189. Cooper SA, Engel J, Ladov M, et al. Analgesic efficacy of an ibuprofen-codeine combination. Pharmacotherapy. 1982 MayJun;2(3):162-7. doi: 10.1002/j.18759114.1982.tb04528.x. PMID: 6763202.

190. Cooper SA, Firestein A, Cohn P. Doubleblind comparison of meclofenamate sodium with acetaminophen, acetaminophen with codeine and placebo for relief of postsurgical dental pain. J Clin Dent. 1988 Fall;1(2):31-4. PMID: 3254707.
191. Cooper SA, Kupperman A. The analgesic efficacy of flurbiprofen compared to acetaminophen with codeine. J Clin Dent. 1991;2(3):70-4. PMID: 1930699.

192. Cooper SA, Precheur H, Rauch D, et al. Evaluation of oxycodone and acetaminophen in treatment of postoperative dental pain. Oral Surg Oral Med Oral Pathol. 1980 Dec;50(6):496-501. doi: 10.1016/0030-4220(80)90430-2. PMID: 7005804.

193. Cooper SA, Schachtel BP, Goldman E, et al. Ibuprofen and acetaminophen in the relief of acute pain: a randomized, double-blind, placebo-controlled study. J Clin Pharmacol. 1989 Nov;29(11):1026-30. PMID: 2689471.

194. Dionne RA, Snyder J, Hargreaves KM. Analgesic efficacy of flurbiprofen in comparison with acetaminophen, acetaminophen plus codeine, and placebo after impacted third molar removal. J Oral Maxillofac Surg. 1994 Sep;52(9):919-24; discussion 25-6. doi: 10.1016/s02782391(10)80068-0. PMID: 8064454.

195. Forbes JA, Kehm CJ, Grodin CD, et al. Evaluation of ketorolac, ibuprofen, acetaminophen, and an acetaminophencodeine combination in postoperative oral surgery pain. Pharmacotherapy. 1990;10(6 ( Pt 2)):94s-105s. PMID: 2082318.

196. Forouzanfar T, Sabelis A, Ausems S, et al. Effect of ice compression on pain after mandibular third molar surgery: a singleblind, randomized controlled trial. Int J Oral Maxillofac Surg. 2008 Sep;37(9):824-30. doi: 10.1016/j.ijom.2008.05.011. PMID: 18603410.

197. Hersh EV, Levin LM, Cooper SA, et al. Ibuprofen liquigel for oral surgery pain. Clin Ther. 2000;22(11):1306-18. PMID: 11117655.

198. Hill CM, Balkenohl M, Thomas DW, et al. Pregabalin in patients with postoperative dental pain. Eur J Pain. 2001;5(2):119-24. doi: 10.1053/eujp.2001.0235. PMID: 11465977. 
199. Ibikunle AA, Adeyemo WL. Oral healthrelated quality of life following third molar surgery with or without application of ice pack therapy. Oral Maxillofac Surg. 2016 Sep;20(3):239-47. doi: 10.1007/s10006-0160558-1. PMID: 27139019.

200. Lao L, Bergman S, Langenberg P, et al. Efficacy of Chinese acupuncture on postoperative oral surgery pain. Oral Surg Oral Med Oral Pathol Oral Radiol Endod. 1995 Apr;79(4):423-8. doi: 10.1016/s10792104(05)80121-0. PMID: 7614199.

201. Malmstrom K, Kotey P, Coughlin H, et al. A randomized, double-blind, parallel-group study comparing the analgesic effect of etoricoxib to placebo, naproxen sodium, and acetaminophen with codeine using the dental impaction pain model. Clin J Pain. 2004;20(3):147-55. PMID: 15100590.

202. Mehlisch DR, Aspley S, Daniels SE, et al. Comparison of the analgesic efficacy of concurrent ibuprofen and paracetamol with ibuprofen or paracetamol alone in the management of moderate to severe acute postoperative dental pain in adolescents and adults: a randomized, double-blind, placebocontrolled, parallel-group, single-dose, twocenter, modified factorial study. Clin Ther. 2010 May;32(5):882-95. doi: 10.1016/j.clinthera.2010.04.022. PMID: 20685496.

203. Mehlisch DR, Brown P. Single-dose therapy with diclofenac potassium, aspirin, or placebo following dental impaction surgery. Today's Ther Trends. 1995a;12:15-31.

204. Mehlisch DR, Sollecito WA, Helfrick JF, et al. Multicenter clinical trial of ibuprofen and acetaminophen in the treatment of postoperative dental pain. J Am Dent Assoc. 1990 Aug;121(2):257-63. PMID: 2205641.

205. Mehrvarzfar P, Abbott PV, Saghiri MA, et al. Effects of three oral analgesics on postoperative pain following root canal preparation: a controlled clinical trial. Int Endod J. 2012;45(1):76-82. PMID: 21902704.

206. Mishra A, Amalakara J, Avula H, et al. Effect of diclofenac mouthwash on postoperative pain after periodontal surgery. J Clin Diagn Res. 2017;11(4):ZC24-ZC6. doi: 10.7860/JCDR/2017/22165.965. PMID: 28571255.
207. Moore PA, Crout RJ, Jackson DL, et al. Tramadol hydrochloride: analgesic efficacy compared with codeine, aspirin with codeine, and placebo after dental extraction. J Clin Pharmacol. 1998 Jun;38(6):554-60. doi: 10.1002/j.1552-4604.1998.tb05794.x. PMID: 9650546.

208. Nelson S, Brahmin J. An evaluation of the analgesic efficacy of diclofenac potassium, aspirin, and placebo in postoperative dental pain. Today's Ther Trends. 1994;12:3-14.

209. Olson MNZ, Otero AM, Marrero I, et al. Onset of analgesia for liquigel ibuprofen 400 mg, acetaminophen $1000 \mathrm{mg}$, ketoprofen 25 $\mathrm{mg}$, and placebo in the treatment of postoperative dental pain. J Clin Pharmacol. 2001;41(11):1238-47. PMID: 11697757.

210. Sadeghein A, Shahidi N, Dehpour AR. A comparison of ketorolac tromethamine and acetaminophen codeine in the management of acute apical periodontitis. J Endod. 1999 Apr;25(4):257-9. PMID: 10425951.

211. Santini MF, Rosa RAD, Ferreira MBC, et al. Comparison of two combinations of opioid and non-opioid analgesics for acute periradicular abscess: a randomized clinical trial. Journal of Applied Oral Science. 2017 Sep-Oct;25(5):551-8. doi: 10.1590/16787757-2016-0407. PMID: 29069153.

212. Skoglund LA, Skjelbred P, Fyllingen G. Analgesic efficacy of acetaminophen 1000 $\mathrm{mg}$, acetaminophen $2000 \mathrm{mg}$, and the combination of acetaminophen $1000 \mathrm{mg}$ and codeine phosphate $60 \mathrm{mg}$ versus placebo in acute postoperative pain. Pharmacotherapy. 1991;11(5):364-9. PMID: 1745622.

213. Sunshine A, Marrero I, Olson N, et al. Comparative study of flurbiprofen, zomepirac sodium, acetaminophen plus codeine, and acetaminophen for the relief of postsurgical dental pain. Am J Med. 1986 Mar 24;80(3a):50-4. doi: 10.1016/00029343(86)90111-7. PMID: 3515924.

214. van der Westhuijzen AJ, Becker PJ, Morkel $\mathrm{J}$, et al. A randomized observer blind comparison of bilateral facial ice pack therapy with no ice therapy following third molar surgery. Int J Oral Maxillofac Surg. 2005 2005/05/01/;34(3):281-6. doi: https://doi.org/10.1016/j.ijom.2004.05.006. PMID: 15741037. 
215. Van Dyke T, Litkowski LJ, Kiersch TA, et al. Combination oxycodone $5 \mathrm{mg}$ /ibuprofen $400 \mathrm{mg}$ for the treatment of postoperative pain: a double-blind, placebo- and activecontrolled parallel-group study. Clin Ther. 2004 Dec;26(12):2003-14. doi: 10.1016/j.clinthera.2004.12.002. PMID: 15823764.

216. Voelker M, Schachtel BP, Cooper SA, et al. Efficacy of disintegrating aspirin in two different models for acute mild-to-moderate pain: sore throat pain and dental pain. Inflammopharmacology. 2016 Feb;24(1):4351. doi: 10.1007/s10787-015-0253-0. PMID: 26603742.

217. Zandi M, Amini P, Keshavarz A. Effectiveness of cold therapy in reducing pain, trismus, and oedema after impacted mandibular third molar surgery: a randomized, self-controlled, observer-blind, split-mouth clinical trial. Int J Oral Maxillofac Surg. 2016 2016/01/01/;45(1):118-23. doi: https://doi.org/10.1016/j.ijom.2015.10.021. PMID: 26597577.

218. Zupelari-Goncalves P, Weckwerth GM, Calvo AM, et al. Efficacy of oral diclofenac with or without codeine for pain control after invasive bilateral third molar extractions. Int J Oral Maxillofac Surg. 2017 May;46(5):621-7. doi: 10.1016/j.ijom.2017.01.008. PMID: 28161136.

219. Mehlisch DR, Jasper RD, Brown P, et al. Comparative study of ibuprofen lysine and acetaminophen in patients with postoperative dental pain. Clin Ther. 1995b;17(5):852-60. PMID: 8595637.

220. Daniels SE, Bandy DP, Christensen SE, et al. Evaluation of the dose range of etoricoxib in an acute pain setting using the postoperative dental pain model. Clin J Pain. 2011 Jan;27(1):1-8. doi: 10.1097/ajp.0b013e3181ed0639. PMID: 21188849.

221. Harbaugh CM, Nalliah RP, Hu HM, et al. Persistent Opioid Use After Wisdom Tooth Extraction. JAMA. 2018;320(5):504-6. doi: 10.1001/jama.2018.9023. PMID: 30088000.
222. Rasubala L, Pernapati L, Velasquez X, et al. Impact of a Mandatory Prescription Drug Monitoring Program on Prescription of Opioid Analgesics by Dentists. PLoS ONE. 2015;10(8):e0135957. doi: https://dx.doi.org/10.1371/journal.pone.0135 957. PMID: 26274819.

223. al-Sahlawi KS, Tawfik OM. Comparative study of the efficacy of lysine acetylsalicylate, indomethacin and pethidine in acute renal colic. Eur J Emerg Med. 1996 Sep;3(3):183-6. doi: 10.1097/00063110199609000-00008. PMID: 9023498.

224. Cordell WH, Wright SW, Wolfson AB, et al. Comparison of intravenous ketorolac, meperidine, and both (balanced analgesia) for renal colic. Ann Emerg Med. 1996 Aug;28(2):151-8. doi: 10.1016/s01960644(96)70055-0. PMID: 8759578.

225. Finlay IG, Scott R, McArdle CS. Prospective double-blind comparison of buprenorphine and pethidine in ureteric colic. Br Med J (Clin Res Ed). 1982 Jun 19;284(6332):1830-1. doi: 10.1136/bmj.284.6332.1830. PMID: 6805715.

226. Garcća-Alonso F. Comparative study of the efficacy of dipyrone, diclofenac sodium and pethidine in acute renal colic. Eur J Clin Pharmacol. 1991;40(6):543-6. PMID: 1884733.

227. Grissa MH, Claessens YE, Bouida W, et al. Paracetamol vs piroxicam to relieve pain in renal colic. Results of a randomized controlled trial. Am J Emerg Med. 2011 Feb;29(2):203-6. doi: 10.1016/j.ajem.2009.09.019. PMID: 20934829.

228. Kaynar M, Koyuncu F, Buldu I, et al. Comparison of the efficacy of diclofenac, acupuncture, and acetaminophen in the treatment of renal colic. Am J Emerg Med. 2015 Jun;33(6):749-53. doi: 10.1016/j.ajem.2015.02.033. PMID: 25827597.

229. Larkin GL, Peacock WFt, Pearl SM, et al. Efficacy of ketorolac tromethamine versus meperidine in the ED treatment of acute renal colic. Am J Emerg Med. 1999 Jan;17(1):6-10. doi: 10.1016/s07356757(99)90003-7. PMID: 9928687. 
230. Marthak KV, Gokarn AM, Rao AV, et al. A multi-centre comparative study of diclofenac sodium and a dipyrone/spasmolytic combination, and a single-centre comparative study of diclofenac sodium and pethidine in renal colic patients in India. Curr Med Res Opin. 1991;12(6):366-73. doi: 10.1185/03007999109111506. PMID: 2044396.

231. Narci H, Ugur M, Uzun H, et al. Combining $1000 \mathrm{mg}$ oral acetaminophen with $75 \mathrm{mg}$ intramuscular diclofenac of analgesic efficacy for acute renal colic treatment. Sci Res Essays. 2012 Jun 14;7(22):2017-21.

232. Oosterlinck W, Philp NH, Charig C, et al. A double-blind single dose comparison of intramuscular ketorolac tromethamine and pethidine in the treatment of renal colic. $\mathrm{J}$ Clin Pharmacol. 1990 Apr;30(4):336-41. doi: 10.1002/j.1552-4604.1990.tb03603.x. PMID: 2341581.

233. Pathan SA, Mitra B, Straney LD, et al. Delivering safe and effective analgesia for management of renal colic in the emergency department: a double-blind, multigroup, randomised controlled trial. Lancet. 2016 May 14;387(10032):1999-2007. doi: 10.1016/s0140-6736(16)00652-8. PMID: 26993881.

234. Sandhu DP, Iacovou JW, Fletcher MS, et al. A comparison of intramuscular ketorolac and pethidine in the alleviation of renal colic. Br J Urol. 1994 Dec;74(6):690-3. doi: 10.1111/j.1464-410x.1994.tb07107.x. PMID: 7827834.

235. Latta KS, Ginsberg B, Barkin RL. Meperidine: a critical review. Am J Ther. 2002 Jan-Feb;9(1):53-68. doi: 10.1097/00045391-200201000-00010. PMID: 11782820.

236. Pais VM, Jr., Sites BD. The association of nephrolithiasis with prescription opioid use. Clin Nephrol. 2019 Apr;91(4):231-6. doi: 10.5414/cn109717. PMID: 30862351.

237. Shoag JE, Patel N, Posada L, et al. Kidney Stones and Risk of Narcotic Use. J Urol. 2019 Jul;202(1):114-8. doi: 10.1097/ju.0000000000000197. PMID: 30829133.
238. Ezenwa MO, Yao Y, Engeland CG, et al. A randomized controlled pilot study feasibility of a tablet-based guided audio-visual relaxation intervention for reducing stress and pain in adults with sickle cell disease. $\mathrm{J}$ Adv Nurs. 2016 Jun;72(6):1452-63. doi: 10.1111/jan.12895. PMID: 26768753.

239. Gonzalez ER, Ornato JP, Ware D, et al. Comparison of intramuscular analgesic activity of butorphanol and morphine in patients with sickle cell disease. Ann Emerg Med. 1988 Aug;17(8):788-91. PMID: 3394980.

240. Uzun B, Kekec Z, Gurkan E. Efficacy of tramadol vs meperidine in vasoocclusive sickle cell crisis. Am J Emerg Med. 2010 May;28(4):445-9. doi: 10.1016/j.ajem.2009.01.016. PMID: 20466223.

241. Abdel Shaheed C, Maher CG, Williams KA, et al. Efficacy, Tolerability, and DoseDependent Effects of Opioid Analgesics for Low Back Pain: A Systematic Review and Meta-analysis. JAMA Intern Med. 2016 Jul 01;176(7):958-68. doi: https://dx.doi.org/10.1001/jamainternmed.20 16.1251. PMID: 27213267.

242. Machado GC, Maher CG, Ferreira PH, et al. Non-steroidal anti-inflammatory drugs for spinal pain: a systematic review and metaanalysis. Ann Rheum Dis. 2017 Jul;76(7):1269-78. doi: 10.1136/annrheumdis-2016-210597. PMID: 28153830.

243. Machado GC, Maher CG, Ferreira PH, et al. Efficacy and safety of paracetamol for spinal pain and osteoarthritis: systematic review and meta-analysis of randomised placebo controlled trials. BMJ : British Medical Journal. 2015;350:h1225. doi: 10.1136/bmj.h1225.

244. Abdel Shaheed C, Maher CG, Williams KA, et al. Efficacy and tolerability of muscle relaxants for low back pain: Systematic review and meta-analysis. Eur J Pain. 2017 02;21(2):228-37. doi: 10.1002/ejp.907. PMID: 27329976. 
245. Moore PA, Ziegler KM, Lipman RD, et al. Benefits and harms associated with analgesic medications used in the management of acute dental pain: An overview of systematic reviews. J Am Dent Assoc. 2018 2018/04/01/;149(4):256-65.e3. doi:

https://doi.org/10.1016/j.adaj.2018.02.012.

246. Evidence-Based Management of Sickle Cell Disease: Expert Panel Report, 2014.

Pediatrics. 2014;134(6):e1775. doi: 10.1542/peds.2014-2986.

247. Finnerup NB, Attal N, Haroutounian S, et al. Pharmacotherapy for neuropathic pain in adults: a systematic review and metaanalysis. Lancet neurol. 2015

Feb;14(2):162-73. doi: https://dx.doi.org/10.1016/S14744422(14)70251-0. PMID: 25575710.

248. Sommer C, Klose P, Welsch P, et al. Opioids for chronic non-cancer neuropathic pain. An updated systematic review and meta-analysis of efficacy, tolerability and safety in randomized placebo-controlled studies of at least 4 weeks duration. Eur $\mathrm{J}$ Pain. 2020 Jan;24(1):3-18. doi: 10.1002/ejp.1494. PMID: 31705717.

249. Meng H, Johnston B, Englesakis M, et al. Selective Cannabinoids for Chronic Neuropathic Pain: A Systematic Review and Meta-analysis. Anesth Analg. 2017 Nov;125(5):1638-52. doi: 10.1213/ane.0000000000002110. PMID: 28537982.

250. Wong JJ, Côté P, Ameis A, et al. Are nonsteroidal anti-inflammatory drugs effective for the management of neck pain and associated disorders, whiplash-associated disorders, or non-specific low back pain? A systematic review of systematic reviews by the Ontario Protocol for Traffic Injury Management (OPTIMa) Collaboration. Eur Spine J. 2016 Jan;25(1):34-61. doi: 10.1007/s00586-015-3891-4. PMID: 25827308.

251. Doherty C, Bleakley C, Delahunt E, et al. Treatment and prevention of acute and recurrent ankle sprain: an overview of systematic reviews with meta-analysis. Br J Sports Med. 2017 Jan;51(2):113-25. doi: 10.1136/bjsports-2016-096178. PMID: 28053200.
252. Rubinstein SM, Terwee CB, Assendelft WJ, et al. Spinal manipulative therapy for acute low-back pain. Cochrane Database Syst Rev. 2012 Sep 12;2012(9):Cd008880. doi: 10.1002/14651858.CD008880.pub2. PMID: 22972127.

253. Lee JH, Choi TY, Lee MS, et al. Acupuncture for acute low back pain: a systematic review. Clin J Pain. 2013 Feb;29(2):172-85. doi: 10.1097/AJP.0b013e31824909f9. PMID: 23269281.

254. Chou R, Gordon DB, de Leon-Casasola OA, et al. Management of Postoperative Pain: A Clinical Practice Guideline From the American Pain Society, the American Society of Regional Anesthesia and Pain Medicine, and the American Society of Anesthesiologists' Committee on Regional Anesthesia, Executive Committee, and Administrative Council. J Pain. 2016 Feb;17(2):131-57. doi: https://dx.doi.org/10.1016/j.jpain.2015.12.00 8. PMID: 26827847.

255. Frazee R, Garmon E, Isbell C, et al. Postoperative Opioid Prescription Reduction Strategy in a Regional Healthcare System. J Am Coll Surg. 2020;230(4):631-5. doi: 10.1016/j.jamcollsurg.2019.12.023.

256. Howard R, Waljee J, Brummett C, et al. Reduction in Opioid Prescribing Through Evidence-Based Prescribing Guidelines. JAMA Surg. 2018;153(3):285-7. doi: 10.1001/jamasurg.2017.4436.

257. Lee JS, Howard RA, Klueh MP, et al. The Impact of Education and Prescribing Guidelines on Opioid Prescribing for Breast and Melanoma Procedures. Ann Surg Oncol. 2019 Jan;26(1):17-24. doi: 10.1245/s10434018-6772-3. PMID: 30238243.

258. Louie CE, Kelly JL, Barth RJ, Jr. Association of Decreased Postsurgical Opioid Prescribing With Patients' Satisfaction With Surgeons. JAMA Surg. 2019;154(11):1049-54. doi: 10.1001/jamasurg.2019.2875.

259. National Academies of Sciences E, and Medicine,. Framing Opioid Prescribing Guidelines for Acute Pain: Developing the Evidence. Washington (DC): National Academies Press (US); 2020. 
260. Qaseem A, Wilt TJ, McLean RM, et al. Noninvasive Treatments for Acute, Subacute, and Chronic Low Back Pain: A Clinical Practice Guideline From the American College of Physicians. Ann Intern Med. 2017 Apr 04;166(7):514-30. doi: https://dx.doi.org/10.7326/M16-2367. PMID: 28192789.
261. Chou R, McDonagh MS, Nakamoto E, et al. AHRQ Comparative Effectiveness Reviews. Analgesics for Osteoarthritis: An Update of the 2006 Comparative Effectiveness Review. Rockville (MD): Agency for Healthcare Research and Quality (US); 2011.

262. Lee L, Smith-Whitley K, Banks S, et al. Reducing Health Care Disparities in Sickle Cell Disease: A Review. Public Health Rep. 2019 Nov/Dec;134(6):599-607. doi: 10.1177/0033354919881438. PMID: 31600481. 


\section{Abbreviations and Acronyms}

ACL

AHRQ

ARD

CDC

CI

DASH

ED

EPC

HVLA

IM

IV

IQR

$\mathrm{KQ}$

MD

MPQ

NMES

NR

NRS

NSAID

NS

ODI

PCA

PICOTS

PID

RCT

RMDQ

RR

SD

SEADS

SF-36

SMD

SOE

SPADI

SPID

SOE

TENS anterior cruciate ligament

Agency for Healthcare Research and Quality

absolute risk difference

Centers for Disease Control and Prevention

confidence interval

Disabilities of the Arm, Shoulder, and Hand

emergency department

Evidence-based Practice Center

high-velocity low amplitude

intramuscular

intravenous

interquartile range

Key Question

mean difference

McGill Pain Questionnaire

neuromuscular electrical stimulation

not reported

numerical rating scale

nonsteroidal anti-inflammatory drug

not significant

Oswestry Disability Index

patient-controlled analgesia

population, intervention, comparator, outcome, timing, setting

pain intensity difference

randomized controlled trial

Roland-Morris Disability Questionnaire

relative risk

standard deviation

Supplemental Evidence and Data for Systematic Review

36-Item Short-Form Survey

standardized mean difference

strength of evidence

Shoulder Pain and Disability Index

sum of pain intensity difference

strength of evidence

transcutaneous electrical nerve stimulation 
VAS

VRS

WOMAC visual analog scale verbal rating scale

Western Ontario and McMaster Universitie 


\section{Appendix A. Literature Search Strategies}

\section{Treatment Effectiveness and Harms}

\section{Ovid MEDLINE(R) 1946 to July Week 42020}

1. Pain/

2. Acute Pain/

3. Pain Management/

4. (acute adj3 pain).ti,ab,kf.

5. exp back pain/ or exp musculoskeletal pain/ or neck pain/ or exp neuralgia/ or exp Facial Pain/ or exp Nephrolithiasis/ or exp Anemia, Sickle Cell/ or Pain, Postoperative/

6. (back or spine or spinal or radicular or neck or musculoskeletal or fracture* or neuropathic or neuralgia or neuropathy or sciatica or "dental pain" or "ondotogenic pain" or "kidney stone*" or urolithiasis or nephrolithiasis or "sickle cell" or "postoperative pain").ti,ab,kf.

7. treatment outcome/

8. exp Therapeutics/

9. (dh or dt or pc or rh or th).fs.

10. (treatment or therap* or intervention*).ti,ab,kf.

11. (or/1-4) and (5 or 6) and (or/7-10)

12. exp cohort studies/

13. cohort\$.tw.

14. controlled clinical trial.pt.

15. epidemiologic methods/

16. limit 15 to $\mathrm{yr}=1966-1989$

17. exp case-control studies/

18. (case\$ and control\$).tw.

19. or $/ 12-14,16-18$

20. randomized controlled trial.pt.

21. (random* or placebo* or control* or trial or blind*).ti,ab.

22. (animals not humans).sh.

23. (comment or editorial or meta-analysis or practice-guideline or review or letter).pt.

24. (20 or 21$)$ not (22 or 23)

25. review.pt.

26. (medline or medlars or embase or pubmed or cochrane).tw,sh.

27. (scisearch or psychinfo or psycinfo).tw,sh.

28. (psychlit or psyclit).tw,sh.

29. cinahl.tw,sh.

30. ((hand adj2 search\$) or (manual\$ adj2 search\$)).tw,sh.

31. (electronic database\$ or bibliographic database\$ or computeri?ed database\$ or online database\$).tw,sh.

32. (pooling or pooled or mantel haenszel).tw,sh.

33. (peto or dersimonian or der simonian or fixed effect).tw,sh.

34. or/26-33

35. 25 and 34

36. meta-analysis.pt.

37. meta-analysis.sh.

38. (meta-analys\$ or meta analys\$ or metaanalys\$).tw,sh. 
39. (systematic\$ adj5 review\$).tw,sh.

40. (systematic\$ adj5 overview\$).tw,sh.

41. (quantitativ\$ adj5 review\$).tw,sh.

42. (quantitativ\$ adj5 overview\$).tw,sh.

43. (quantitativ\$ adj5 synthesis\$).tw,sh.

44. (methodologic\$ adj5 review\$).tw,sh.

45. (methodologic\$ adj5 overview\$).tw,sh.

46. (integrative research review $\$$ or research integration).tw.

47. or/36-46

48. 35 or 47

49. 19 or 24 or 48

50. 11 and 49

Ovid MEDLINE(R) In-Process \& Other Non-Indexed Citations 1946 to August 5, 2020, Ovid MEDLINE(R) Epub Ahead of Print August 5, 2020

1. (acute adj3 pain).ti,kf.

2. (back or spine or spinal or radicular or neck or musculoskeletal or fracture* or neuropathic or neuralgia or neuropathy or sciatica or "dental pain" or "ondotogenic pain" or "kidney stone*" or urolithiasis or nephrolithiasis or "sickle cell" or "postoperative pain").ti,kf.

3. (treatment or therap* or intervention*).ti,kf.

4. (1 or 2$)$ and 3

5. (random* or control* or placebo or sham or trial).ti,kw.

6. (systematic or "meta analysis" or metanalysis or medline).ti,kf.

7. 4 and (5 or 6$)$

8. 7 not (animal or mouse or mice or rat* or dog* or canine).ti.

9. 8 not chronic.ti.

10. "PubMed-not-MEDLINE".st.

11. 9 not 10

Ovid MEDLINE(R) ALL 1946 to August 5, 2020

Postoperative pain supplemental search

1. treatment outcome/

2. exp Therapeutics/

3. (dh or dt or pc or rh or th).fs.

4. (treatment or therap* or intervention*).ti,ab,kf.

5. Pain, Postoperative/

6. "postoperative pain".ti,ab,kf.

7. 5 or 6

8. or $/ 1-4$

9. 7 and 8

10. (opioid* or hydrocodone or oxycodone or hydromorphone or fentanyl or buprenorphine or naltrexone or naloxone or tramadol or tapentadol).tw.

11. (acetaminophen or "nonsteroidal anti-inflammatory" or NSAID* or "skeletal muscle relaxant*" or SMR* or benzodiazepine* or antidepressant* or anticonvulsant* or cannabis or cannabinoid*).tw. 
12. (exercise or "cognitive behavioral therapy" or CBT or meditation or relaxation or music or "virtual reality" or acupuncture or acupressure or electroacupuncture or massage or manipulation or mobilization or mobilisation or "physical modalit*" or "transcutaneous electrical nerve stimulation" or TENS or ultrasound or brace* or traction or heat or cold or cryo*).tw.

13. or $/ 10-12$

14. 9 and 13

15. 14 and (random* or control* or placebo or sham or trial).ti,ab,kf.

16. randomized controlled trial.pt.

17. (random* or placebo* or control* or trial or blind*).ti,ab.

18. (animals not humans).sh.

19. (comment or editorial or meta-analysis or practice-guideline or review or letter).pt.

20. (16 or 17$)$ not (18 or 19)

21. 14 and 20

22. 15 or 21

23. (pediatric* or preschool* or toddler* or infan* or child*).ti,ab.

24. 22 not 23

25. limit 24 to english language

Ovid MEDLINE(R) ALL 1946 to August 5, 2020

Musculoskeletal supplemental search

1 Pain/

2 Acute Pain/

3 Pain Management/

4 (acute adj3 pain).ti,ab,kf.

5 exp back pain/ or exp musculoskeletal pain/ or neck pain/ or exp neuralgia/ or exp Facial Pain/ or exp Nephrolithiasis/ or exp Anemia, Sickle Cell/ or Pain, Postoperative/

6 (back or spine or spinal or radicular or neck or musculoskeletal or fracture* or neuropathic or neuralgia or neuropathy or sciatica or "dental pain" or "ondotogenic pain" or "kidney stone*" or urolithiasis or nephrolithiasis or "sickle cell" or "postoperative pain").ti,ab,kf.

7 treatment outcome/

8 exp Therapeutics/

9 (dh or dt or pc or rh or th).fs.

10 (treatment or therap* or intervention*).ti,ab,kf.

11 (or/1-4) and (5 or 6) and (or/7-10)

12 exp cohort studies/

13 cohort\$.tw.

14 controlled clinical trial.pt.

15 epidemiologic methods/

16 limit 15 to $\mathrm{yr}=1966-1989$

17 exp case-control studies/

18 (case\$ and control\$).tw.

19 or/12-14,16-18

20 randomized controlled trial.pt.

21 (random* or placebo* or control* or trial or blind*).ti,ab.

22 (animals not humans).sh.

23 (comment or editorial or meta-analysis or practice-guideline or review or letter).pt. 
24 (20 or 21$)$ not (22 or 23$)$

25 review.pt.

26 (medline or medlars or embase or pubmed or cochrane).tw,sh.

27 (scisearch or psychinfo or psycinfo).tw,sh.

28 (psychlit or psyclit).tw,sh.

29 cinahl.tw,sh.

30 ((hand adj2 search\$) or (manual\$ adj2 search\$)).tw,sh.

31 (electronic database $\$$ or bibliographic database $\$$ or computeri?ed database $\$$ or online database\$).tw,sh.

32 (pooling or pooled or mantel haenszel).tw,sh.

33 (peto or dersimonian or der simonian or fixed effect).tw,sh.

34 or/26-33

$35 \quad 25$ and 34

36 meta-analysis.pt.

37 meta-analysis.sh.

38 (meta-analys\$ or meta analys\$ or metaanalys\$).tw,sh.

39 (systematic\$ adj5 review\$).tw,sh.

40 (systematic\$ adj5 overview\$).tw,sh.

41 (quantitativ\$ adj5 review\$).tw,sh.

42 (quantitativ\$ adj5 overview\$).tw,sh.

43 (quantitativ\$ adj5 synthesis\$).tw,sh.

44 (methodologic\$ adj5 review\$).tw,sh.

45 (methodologic\$ adj5 overview\$).tw,sh.

46 (integrative research review\$ or research integration).tw.

47 or/36-46

$48 \quad 35$ or 47

4919 or 24 or 48

$50 \quad 11$ and 49

51 or $/ 7-10$

52 or $/ 1-4$

$53 \quad 51$ and 52

54 (ultrasound or TENS or cold or cryotherapy).tw.

$55 \quad 53$ and 54

56 ankle.tw.

$57 \quad 55$ and 56

$58 \quad 57$ not 50

59 limit 58 to english language

$60 \quad 49$ and 59

61 musculoskeletal.tw.

6255 and 61

63 limit 62 to english language

$64 \quad 63$ not 50

6560 or 64

66 (animal* or mouse or mice or rat* or dog* or canine or cow* or bovine or horse* or mare* or pig* or porcine or rabbit* or llama* or sheep or ewe*).ti.

$67 \quad 65$ not 66 
Cochrane Central Register of Controlled Trials July 2020

1. Pain/

2. Acute Pain/

3. Pain Management/

4. (acute adj3 pain).ti,ab

5. exp back pain/ or exp musculoskeletal pain/ or neck pain/ or exp neuralgia/ or exp Facial Pain/ or exp Nephrolithiasis/ or exp Anemia, Sickle Cell/ or Pain, Postoperative/

6. (back or spine or spinal or radicular or neck or musculoskeletal or fracture* or neuropathic or neuralgia or neuropathy or sciatica or "dental pain" or "ondotogenic pain" or "kidney stone*" or urolithiasis or nephrolithiasis or "sickle cell" or "postoperative pain").ti,ab

7. treatment outcome/

8. exp Therapeutics/

9. (dh or dt or pc or rh or th).fs.

10. (treatment or therap* or intervention*).ti,ab

11. (or/1-4) and (5 or 6) and (or/7-10)

12. limit 11 to medline records

13. 11 not 12

14. conference abstract.pt.

15. "journal: conference abstract".pt.

16. "journal: conference review".pt.

17. or/14-16

18. 13 not 17

PsycINFO 1806 to August Week 12020

1. exp Pain/

2. chronic pain/

3. 1 not 2

4. sickle cell disease/

5. exp Back Pain/

6. exp neuralgia/ or exp peripheral neuropathy/

7. Pain Management/

8. pain.ti,ab.

9. (back or spine or spinal or radicular or neck or musculoskeletal or fracture* or neuropathic or neuralgia or neuropathy or sciatica or dental or ondotogenic or kidney or urolithiasis or nephrolithiasis or "sickle cell" or postoperative).ti,ab.

10. (7 or 8$)$ and 9

11. (acute adj3 pain).ti,ab.

12. 3 or 4 or 5 or 6 or 10 or 11

13. exp treatment outcomes/

14. treatment effectiveness evaluation/

15. 12 and (13 or 14)

16. exp clinical trials/

17. (random* or control* or placebo or sham or trial or blind*).ti,ab.

18. 15 and (16 or 17$)$

19. limit 18 to english language 
20. limit 19 to human

21. limit 20 to (childhood or adolescence $<13$ to 17 years $>$ )

22. 20 not 21

23. 22 not chronic.ti.

Cochrane Database of Systematic Reviews 2005 to August 5, 2020

1. (back or spine or spinal or radicular or neck or musculoskeletal or fracture* or neuropathic or neuralgia or neuropathy or sciatica or "dental pain" or "ondotogenic pain" or "kidney stone*" or urolithiasis or nephrolithiasis or "sickle cell" or "postoperative pain").ti.

2. (treatment or therap* or intervention*).ti,ab.

3. 1 and 2

4. limit 3 to full systematic reviews

5. 4 not chronic.ti.

6. 5 not children.ti.

7. 5 not 6

8. 7 and adult*.ti.

9. 6 or 8

Elsevier Embase August 5, 2020

('backache'/exp OR 'musculoskeletal pain'/exp OR 'neuropathic pain'/exp OR 'neuralgia'/exp OR 'tooth pain'/exp OR 'postoperative pain'/exp OR (('sickle cell anemia'/exp OR 'sickle cell crisis'/exp) AND ('pain'/exp OR pain:ti,ab,kw))) AND 'drug therapy'/exp AND ('article'/it OR 'review'/it) AND 'human'/de AND ('cohort analysis'/de OR 'comparative study'/de OR 'controlled study'/de OR 'meta analysis'/de OR 'randomized controlled trial'/de OR 'randomized controlled trial (topic)'/de OR 'systematic review'/de) AND [english]/lim AND [embase]/lim NOT ([embase]/lim AND [medline]/lim)

\section{Opioid Use Risk Assessment and Mitigation}

\section{Ovid MEDLINE(R) 1946 to July Week 42020}

1. Pain/

2. Acute Pain/

3. Pain Management/

4. (acute adj3 pain).ti,ab,kf.

5. exp back pain/ or exp musculoskeletal pain/ or neck pain/ or exp neuralgia/ or exp Facial Pain/ or exp Nephrolithiasis/ or exp Anemia, Sickle Cell/ or Pain, Postoperative/

6. (back or spine or spinal or radicular or neck or musculoskeletal or fracture* or neuropathic or neuralgia or neuropathy or sciatica or "dental pain" or "ondotogenic pain" or "kidney stone*" or urolithiasis or nephrolithiasis or "sickle cell" or "postoperative pain").ti,ab,kf.

7. (or/1-4) and (5 or 6)

8. exp Analgesics, Opioid/

9. opioid*.ti,ab,kw.

10. (buprenorphine or codeine or fentanyl or hydrocodone or hydromorphone or methadone or morphine or oxycodone or oxymorphone or tapentadol or tramadol).ti,ab,kw,sh,hw.

11. or/8-10

12. exp Opioid-Related Disorders/

13. (opioid adj2 (abuse or addict* or misuse or diversion)).ti,ab,kf. 
14. 12 or 13

15. 7 and (11 or 14$)$

16. Decision Support Techniques/

17. "Predictive Value of Tests"/

18. Prognosis/

19. Risk Assessment/

20. Risk Factors/

21. Proportional Hazards Models/

22. "Reproducibility of Results"/

23. "Sensitivity and Specificity"/

24. (sensitivity or specificity or accuracy).ti,ab,kf.

25. (risk and (predict\$ or assess\$)).ti,ab,kf.

26. or/16-25

27. Patient Compliance/

28. Health Services Misuse/

29. Substance Abuse Detection/

30. Drug Monitoring/

31. (urine adj7 (screen\$ or test\$ or detect\$)).ti,ab,kf.

32. Contracts/

33. Patient Education as Topic/

34. Drug Overdose/

35. or/27-34

36. risk\$.ti,ab,kf.

37. ("risk evaluation and mitigation" or "rems").ti,ab,kf.

38. Risk Reduction Behavior/ or Risk/

39. or/36-38

40. 26 or 35 or 39

41. 15 and 40

42. limit 41 to english language

Cochrane Central Register of Controlled Trials July 2020

1. Pain/

2. Acute Pain/

3. Pain Management/

4. (acute adj3 pain).ti,ab

5. exp back pain/ or exp musculoskeletal pain/ or neck pain/ or exp neuralgia/ or exp Facial Pain/ or exp Nephrolithiasis/ or exp Anemia, Sickle Cell/ or Pain, Postoperative/

6. (back or spine or spinal or radicular or neck or musculoskeletal or fracture* or neuropathic or neuralgia or neuropathy or sciatica or "dental pain" or "ondotogenic pain" or "kidney stone*" or urolithiasis or nephrolithiasis or "sickle cell" or "postoperative pain").ti,ab

7. (or/1-4) and (5 or 6)

8. exp Analgesics, Opioid/

9. opioid*.ti,ab,kw.

10. (buprenorphine or codeine or fentanyl or hydrocodone or hydromorphone or methadone or morphine or oxycodone or oxymorphone or tapentadol or tramadol).ti,ab,kw,sh,hw.

11. or/8-10 
12. exp Opioid-Related Disorders/

13. (opioid adj2 (abuse or addict* or misuse or diversion)).ti,ab

14. 12 or 13

15. 7 and (11 or 14$)$

16. Decision Support Techniques/

17. "Predictive Value of Tests"/

18. Prognosis/

19. Risk Assessment/

20. Risk Factors/

21. Proportional Hazards Models/

22. "Reproducibility of Results"/

23. "Sensitivity and Specificity"/

24. (sensitivity or specificity or accuracy).ti,ab

25. (risk and (predict\$ or assess\$)).ti,ab

26. or $/ 16-25$

27. Patient Compliance/

28. Health Services Misuse/

29. Substance Abuse Detection/

30. Drug Monitoring/

31. (urine adj7 (screen\$ or test\$ or detect\$)).ti,ab

32. Contracts/

33. Patient Education as Topic/

34. Drug Overdose/

35. or/27-34

36. risk\$.ti,ab

37. ("risk evaluation and mitigation" or "rems").ti,ab

38. Risk Reduction Behavior/ or Risk/

39. or/36-38

40. 26 or 35 or 39

41. 15 and 40

42. limit 41 to english language

43. limit 42 to medline records

44. 42 not 43 


\section{Appendix B. Methods}

\section{Details of Study Selection}

\section{Inclusion and Exclusion Criteria}

Studies were selected for inclusion using pre-established criteria based on the Key Questions and PICOTs (Table B-1). The focus of the report was on outpatient management of acute pain. Therefore, it excluded studies on the inpatient management of acute pain, including inpatient management of pain following major surgical procedures. However, acute pain is often initially managed in emergency department and acute care settings. Therefore, studies in which therapy was initiated in such studies were included, even if therapy was not continued following discharge (e.g. single dose studies of pharmacologic therapy or single session of pharmacologic therapy). Studies of therapies initiated in postoperative care settings were also included, if they were conducted in patients who had undergone outpatient surgical procedures, procedures in which the expected postoperative stay was no more than 1 day, or major surgical procedures shortly before discharge. Because few studies on outpatient management of sickle cell pain were expected, studies were not restricted by inpatient versus outpatient setting.

The population was restricted to patients with acute ( $<4$ weeks pain). Studies with mixed populations of acute and nonacute pain were included if the duration of pain was 4 weeks or longer in $<10 \%$ of patients or if the average duration of pain was $<2$ weeks.

The interventions evaluated in this report were opioid therapy, nonopioid pharmacologic therapy (NSAIDs, acetaminophen, skeletal muscle relaxants, benzodiazepines, antidepressants, anticonvulsants, or cannabis), and noninvasive nonpharmacologic therapy used for pain (exercise [and related therapies], cognitive behavioral therapy, meditation, relaxation, music therapy, virtual reality, acupuncture, massage, manipulation/mobilization, physical modalities [transcutaneous electrical nerve stimulation, ultrasound, braces, traction, heat, cold]). Interventions that involved combinations of different therapy were excluded, with the exception of an opioid plus acetaminophen or NSAID, since these medications are commonly administered together; this combination was classified as an opioid and analyzed separately from an opioid alone if sufficient data were available. Studies in which all patients received background therapy (i.e., not involved in the analyzed comparison) were included, as long as the background therapy could reasonably be considered usual care (e.g., non-opioid analgesics, education, or home exercises).

The comparisons addressed in this report varied depending on the intervention type. For pharmacologic therapy, (opioid or nonopioid), this report focused on studies of comparative effectiveness, to address decisional dilemmas regarding the choice of pharmacologic therapy. The efficacy of pharmacologic therapy versus placebo has been addressed in other reviews. For nonpharmacologic therapy, there is more uncertainty about efficacy for acute pain. Therefore, studies comparing nonpharmacologic therapy versus inactive therapies (placebo, sham therapy, no therapy, attention control, or minimal intervention) were included, as well as head-to-head studies of comparative effectiveness. Questions also addressed the accuracy and effectiveness of risk prediction instruments and factors associated with opioid prescribing.

Outcomes were pain (including satisfaction with pain relief), function, quality of life (including mood and sleep), and harms. One question addressed the accuracy of risk assessment instruments and one question addressed effects of prescribing versus no prescribing on long-term 
use. Outcomes were assessed at prespecified time periods: $<1$ day, 1 day to $<1$ week, 1 week to $<2$ weeks, 2 weeks to $<4$ weeks, and $\geq 4$ weeks.

For Key Questions related to effectiveness and comparative effectiveness, inclusion was restricted to RCTs. Controlled observational studies (cohort, case-control, and before-after studies) were eligible to assess effects of opioid prescribing versus no prescribing on long-term use, accuracy and effectiveness of risk prediction instruments, and factors influencing prescribing.

Table B-1. Inclusion criteria by PICOTS element

\begin{tabular}{|c|c|}
\hline $\begin{array}{l}\text { PICOTS } \\
\text { Element }\end{array}$ & Inclusion Criteria \\
\hline Population & $\begin{array}{l}\text { Adults with acute pain related to the following conditions: } \\
\text { 1. Acute back pain (including back pain with radiculopathy) } \\
\text { 2. Acute neck pain (including neck pain with radiculopathy) } \\
\text { 3. Other musculoskeletal pain (not requiring surgery) } \\
\text { 4. Peripheral neuropathic pain (related to herpes zoster and, trigeminal neuralgia) } \\
\text { 5. Postoperative pain (excluding inpatient management of pain following major surgical } \\
\text { procedures) } \\
\text { 6. Dental pain } \\
\text { 7. Kidney stones } \\
\text { 8. Sickle cell crisis (episodic pain) } \\
\text { *Special populations: } \\
\text { General adult } \\
\text { Older populations >65 years } \\
\text { Patients with history of substance use disorder } \\
\text { Patients currently under treatment for opioid use disorder with opioid agonist therapy or } \\
\text { naltrexone } \\
\text { Patients with a history of psychiatric illness } \\
\text { Patients with history of overdose } \\
\text { Pregnant/breastfeeding women } \\
\text { Patients with comorbidities (e.g., kidney disease, sleep disordered breathing) }\end{array}$ \\
\hline Interventions & $\begin{array}{l}\text { Opioid therapy: } \\
\text { a-e. Any systemic opioid, including agonists, partial agonists, and mixed mechanism opioids } \\
\text { (tapentadol or tramadol). } \\
\text { f. Instruments, genetic/metabolic tests for predicting risk of misuse, opioid use disorder, and } \\
\text { overdose } \\
\text { g. Use of risk prediction instruments, genetic/metabolic tests } \\
\text { h. The following factors: (1) existing opioid management plans; (2) patient education; (3) clinician } \\
\text { and patient values and preferences related to opioids; (4) urine drug screening; (5) use of } \\
\text { prescription drug monitoring program data; (6) availability of close followup } \\
\text { Nonopioid therapy: Oral, parenteral, or topical nonopioid pharmacological therapy used for acute } \\
\text { pain (acetaminophen, nonsteroidal anti-inflammatory drugs, skeletal muscle relaxants, } \\
\text { benzodiazepines, antidepressants, anticonvulsants, cannabis). } \\
\text { Noninvasive nonpharmacological therapy: Noninvasive nonpharmacological therapies used for } \\
\text { acute pain (exercise [and related therapies], cognitive behavioral therapy, meditation, relaxation, } \\
\text { music therapy, virtual reality, acupuncture, massage, manipulation/mobilization, physical } \\
\text { modalities [transcutaneous electrical nerve stimulation, ultrasound, braces, traction, heat, cold]) }\end{array}$ \\
\hline
\end{tabular}




\begin{tabular}{|c|c|}
\hline $\begin{array}{l}\text { PICOTS } \\
\text { Element }\end{array}$ & Inclusion Criteria \\
\hline Comparators & $\begin{array}{l}\text { Opioid therapy: } \\
\text { a-d. Usual care, another opioid, nonopioid drug, or noninvasive, nonpharmacological therapy } \\
\text { e. Usual care, another opioid, nonopioid drug, or noninvasive, nonpharmacological therapy, no } \\
\text { opioid/nothing prescribed } \\
\text { f. Reference standard for misuse, opioid use disorder, or overdose; or other benchmarks } \\
\text { g. Usual care } \\
\text { h. Not utilizing the factors specified in interventions (h) above } \\
\text { Nonopioid pharmacological therapy: } \\
\text { Other nonopioid pharmacological therapy or noninvasive nonpharmacological therapy } \\
\text { Noninvasive nonpharmacological therapy: } \\
\text { Sham treatment, waitlist, usual care, attention control, and no treatment; or other noninvasive } \\
\text { nonpharmacological therapy }\end{array}$ \\
\hline Outcomes & $\begin{array}{l}\text { Opioid therapy: } \\
\text { a-d, g, i. Pain, function, pain relief satisfaction, and quality of life, harms, adverse events } \\
\text { (including withdrawal, risk of misuse, opioid, opioid use disorder, overdose). } \\
\text { e. Persistent opioid use } \\
\text { f. Measures of diagnostic accuracy } \\
\text { h. Opioid prescribing rates } \\
\text { Nonopioid therapy: pain, function, pain relief satisfaction, quality of life and quality of life, harms, } \\
\text { adverse events, opioid use } \\
\text { Noninvasive nonpharmacological therapy: pain, function, pain relief satisfaction, quality of life and } \\
\text { quality of life, harms, adverse events, opioid use }\end{array}$ \\
\hline $\begin{array}{l}\text { Time of } \\
\text { followup }\end{array}$ & $<1$ day; 1 day to $<1$ week; 1 week to $<2$ weeks; 2 weeks to $<4$ weeks; $\geq 4$ weeks \\
\hline Setting & $\begin{array}{l}\text { Emergency department (initiation of therapy and following discharge), physician's office, } \\
\text { outpatient or inpatient surgical center, dental clinic or oral surgery center, inpatient (sickle cell } \\
\text { only) }\end{array}$ \\
\hline Study design & $\begin{array}{l}\text { All KQs: RCTs; in addition: } \\
\text { e. cohort studies (for long-term opioid use) } \\
\text { f. studies assessing diagnostic accuracy } \\
\text { h. cohort studies and before-after studies assessing effects on prescribing rates }\end{array}$ \\
\hline
\end{tabular}

Abbreviations: RCT = randomized controlled trial

Study Design. Controlled observational studies (cohort, case-control, and before-after studies) were eligible to assess effects of opioid prescribing versus no prescribing on long-term use, accuracy and effectiveness of risk prediction instruments, and factors influencing prescribing.

For all Key Questions, we included randomized controlled trials (RCTs). Controlled observational studies Controlled observational studies (cohort, case-control, and before-after studies) were eligible to assess effects of opioid prescribing versus no prescribing on long-term use, accuracy and effectiveness of risk prediction instruments, and factors influencing prescribing. For all KQs, we excluded uncontrolled observational studies, case series, and case reports. Systematic reviews were not included but references were reviewed for potentially relevant studies.

Non-English Language Studies: Inclusion was restricted to English-language articles, but did review English-language abstracts of non-English language articles to identify studies that would otherwise meet inclusion criteria, in order to inform assessments regarding the potential for language bias.

\section{Data Extraction}

Data were extracted from included studies into standardized Excel spreadsheets. Data abstracted were: study design, year, setting, country, sample size, eligibility criteria, population 
and clinical characteristics, intervention characteristics, and results. All study data was verified for accuracy and completeness by a second team member. A record of studies excluded at the full-text level with reasons for exclusion was maintained (Appendix H).

Many studies reported summary data based on multiple pain measurements over a specified time period (e.g., average pain or sum of pain intensity differences). When available, we reported outcomes reported at a specific time point; when necessary, data were estimated from figures provided in the studies. We extracted continuous as well as dichotomous results. We prioritized results for pain intensity in the following order: (1) mean difference in pain intensity at followup; (2) mean difference in pain intensity change from baseline; (3) mean difference in pain relief at followup; (4) other outcome for pain intensity. Summary data were used to supplement outcomes measured at specific time points. Effects on pain intensity were converted when necessary to a 0 to 10 scale to facilitate interpretation across studies using different scales. Opioid doses were calculated in milligram morphine equivalents using published conversions. ${ }^{1}$ Unadjusted relative risks and mean differences with 95 percent confidence intervals were calculated if necessary, using online calculators (MedCalc).

\section{Risk of Bias Assessment of Individual Studies}

Predefined criteria were used to assess the quality of individual controlled trials, systematic reviews, and observational studies. Randomized and nonrandomized trials were evaluated using criteria and methods developed by the Cochrane Back Review Group, ${ }^{2}$ cohort studies were evaluated using criteria developed by the U.S. Preventive Services Task Force, ${ }^{3}$ and studies of diagnostic accuracy were assessed using QUADAS-2. ${ }^{4}$ These criteria were used in conjunction with the approach recommended in the chapter, Assessing the Risk of Bias of Individual Studies When Comparing Medical Interventions in the Methods Guide for Effectiveness and Comparative Effectiveness Reviews developed by the Agency for Healthcare Research and Quality. ${ }^{5}$ Studies were assigned an overall rating of "good," "fair,” or "poor."

Studies rated "good" are considered to have the least risk of bias, and their results are generally considered valid. Good-quality intervention studies include clear descriptions of the population, setting, interventions, and comparison groups; a valid method for allocating patients to treatment; low dropout rates and clear reporting of dropouts; appropriate means for preventing bias; and appropriate measurement of outcomes.

Studies rated "fair" are susceptible to some bias, though not enough to invalidate the results. These studies may not meet all the criteria for a rating of good quality, but no flaw or combination of flaws is likely to cause major bias. The study may be missing information, making it difficult to assess limitations and potential problems. The fair-quality category is broad, and studies with this rating vary in their strengths and weaknesses. The results of some fair-quality studies are likely to be valid, while others may be only possibly valid.

Studies rated "poor" have significant flaws that imply biases of various types that may invalidate the results. They have a serious or "fatal" flaw (or combination of flaws) in design, analysis, or reporting; large amounts of missing information; discrepancies in reporting; or serious problems in the delivery of the intervention. The results of these studies are at least as likely to reflect flaws in the study design as to show true difference between the compared interventions. We did not exclude studies rated poor quality a priori, but poor-quality studies were considered less reliable and given less weight than higher-quality studies when synthesizing the evidence, particularly when discrepancies between studies were present. 
Two team members independently assessed quality. Disagreements were resolved by consensus.

\section{Data Synthesis and Analysis}

We constructed evidence tables showing study characteristics, results, and quality ratings for all included studies, and summary tables to highlight the main findings, organized by Key Question (acute pain condition).

Meta-analyses were limited by the small number of studies for each comparison and outcome, methodological limitations in the studies, and variability in the studies, including methods for measuring outcomes. ${ }^{6}$ Comparisons and outcomes for which there were sufficient studies to perform meta-analyses were limited to opioids versus NSAIDs or acetaminophen for dental pain and kidney stone pain, for the outcomes rescue or repeat medication use and selected harms. Meta-analyses were conducted using Review Manager 5.3 (The Nordic Cochrane Centre, the Cochrane Collaboration, 2014). Otherwise, evidence synthesis was qualitative.

Key Questions addressed how the comparative effectiveness and harms varied according to patient demographics, medical and psychiatric comorbidities, pain types, treatment features and dosing strategies. Although planned techniques to assess these factors included sensitivity and stratified analyses, evidence was too limited to apply these techniques.

The magnitude of effects for pain and function was classified using the same system used in other recent AHRQ reviews on pain conducted at our Evidence-based Practice Center (EPC). ${ }^{7-11}$ Using the same classifications provides a consistent benchmark for comparing results of pain interventions across acute and chronic pain conditions. Based on these criteria, a small/slight effect is defined for pain as a mean between-group difference following treatment of 5 to 10 points on a 0 - to 100 -point visual analog scale (VAS), 0.5 to 1.0 points on a 0 - to 10 -point numeric rating scale, or equivalent; for function as a mean difference of 5 to 10 points on the 0to 100-point Oswestry Disability Index (ODI) or 1 to 2 points on the 0 - to 24-point RolandMorris Disability Questionnaire (RDQ), or equivalent; and for any outcome as a standardized mean difference (SMD) of 0.2 to 0.5 . A moderate effect was defined for pain as a mean difference of 10 to 20 points on a 0 - to 100-point VAS, for function as a mean difference of 10 to 20 points on the ODI or 2 to 5 points on the RDQ, and for any outcome as an SMD of 0.5 to 0.8. Large/substantial effects were defined as greater than moderate. We applied similar thresholds to other outcomes measures. Small effects using this system may be below published thresholds for clinically meaningful effects. However, there is variability across individual patients regarding what constitutes a clinically meaningful effect, which is influenced by a number of factors such as preferences, duration and type of chronic pain, baseline symptom severity, harms, and costs. For some patients a small improvement in pain or function using a treatment with low cost or no serious harms may be important.

\section{Grading the Strength of the Body of Evidence}

Regardless of whether evidence was synthesized quantitatively or qualitatively, the strength of evidence for each Key Question/body of evidence was initially assessed by one researcher for each clinical outcome (see PICOTS) by using the approach described in the AHRQ Methods Guide. ${ }^{5}$ To ensure consistency and validity of the assessment, the strength of evidence grade was reviewed by the entire team of investigators prior to assigning a final grade. The strength of evidence grades were based on the following factors:

- Study limitations (low, medium, or high level of study limitations) 
- Consistency (consistent, inconsistent, or unknown/not applicable)

- Directness (direct or indirect)

- Precision (precise or imprecise)

- Reporting bias (suspected or undetected)

Each body of evidence was assigned an overall strength of evidence grade of high, moderate, low, or insufficient based on the assessment of the above domains:

- High-We are very confident that the estimate of effect lies close to the true effect for this outcome. The body of evidence has few or no deficiencies. We believe that the findings are stable, i.e., another study would not change the conclusions.

- Moderate-We are moderately confident that the estimate of effect lies close to the true effect for this outcome. The body of evidence has some deficiencies. We believe that the findings are likely to be stable, but some doubt remains.

- Low-We have limited confidence that the estimate of effect lies close to the true effect for this outcome. The body of evidence has major or numerous deficiencies (or both). We believe that additional evidence is needed before concluding either that the findings are stable or that the estimate of effect is close to the true effect.

- Insufficient-We have no evidence, we are unable to estimate an effect, or we have no confidence in the estimate of effect for this outcome. No evidence is available or the body of evidence has unacceptable deficiencies, precluding reaching a conclusion.

Plain-language statements are used in the Main Points and the Evidence Summary to convey the strength of evidence (SOE). High SOE is described as "is associated with" or simply "reduces/increases;" moderate SOE is described as "probably;" and low SOE is described as "might be."

\section{Peer Review and Public Commentary}

Peer reviewers were invited to provide written comments on the draft report based on their clinical, content, or methodological expertise. The EPC will consider all peer review comments prior to finalization of the report. Peer reviewers do not participate in writing or editing of the final report or other products. The final report does not necessarily represent the views of individual reviewers. The EPC will complete a disposition of all peer review comments. The disposition of comments for systematic reviews and technical briefs will be published 3 months after the publication of the evidence report.

Potential Peer Reviewers must disclose any financial conflicts of interest greater than $\$ 5,000$ and any other relevant business or professional conflicts of interest. Invited Peer Reviewers may not have any financial conflict of interest greater than $\$ 5,000$. Peer reviewers who disclose potential business or professional conflicts of interest may submit comments on draft reports through the public comment mechanism.

\section{Assessing Applicability}

Applicability was assessed in accordance with the AHRQ Methods Guide, ${ }^{12}$ which is based on the PICOTS framework. Applicability addresses the extent to which outcomes associated with an intervention are likely to be similar across different patients and settings in clinical practice based on the populations, interventions, comparisons, and outcomes evaluated in the 
studies. For example, exclusion of acute pain patients with psychiatric comorbidities reduces applicability to clinical practice since many patients with acute pain have such comorbidities, and may respond more poorly to treatment. Factors that may affect applicability which we have identified a priori include eligibility criteria and patient factors (e.g., demographic characteristics, duration or severity of pain, presence of medical and psychiatric comorbidities, event rates and symptom severity in treatment and control groups), intervention factors (e.g., dose and duration of therapy, intensity and frequency of monitoring, level of adherence support, use of co-interventions), comparisons (e.g., type of comparator, effectiveness and feasibility of active comparators), outcomes (e.g., use of unvalidated or nonstandardized outcomes, measurement of short-term or surrogate outcomes), settings (e.g., clinical setting, country), and study design features (e.g., use of run-in periods). We will use information regarding these factors to assess the situations in which the evidence is most relevant and to evaluate applicability to real-world clinical practice in typical U.S. settings, summarizing applicability assessments qualitatively.

\section{Appendix B References}

1. $\quad$ Nielsen S, Degenhardt L, Hoban B, et al. A synthesis of oral morphine equivalents (OME) for opioid utilisation studies. Pharmacoepidemiol Drug Saf. 2016 Jun;25(6):733-7. doi: 10.1002/pds.3945. PMID: 26693665.

2. Furlan AD, Pennick V, Bombardier C, et al. 2009 updated method guidelines for systematic reviews in the Cochrane Back Review Group. Spine (Phila Pa 1976). 2009 Aug 15;34(18):1929-41. doi: 10.1097/BRS.0b013e3181b1c99f. PMID: 19680101.

3. U.S. Preventive Services Task Force. Methods and processes. 2018. https://www.uspreventiveservicestaskforce.o rg/Page/Name/methods-and-processes. Accessed December 17, 2018.

4. Whiting PF, Rutjes AW, Westwood ME, et al. QUADAS-2: a revised tool for the quality assessment of diagnostic accuracy studies. Ann Intern Med. 2011 Oct 18;155(8):529-36. doi: 10.7326/0003-4819155-8-201110180-00009. PMID: 22007046.

5. Methods Guide for Effectiveness and Comparative Effectiveness Reviews. Rockville, MD: Agency for Healthcare Research and Quality; 2017. https://effectivehealthcare.ahrq.gov/topics/ce r-methods-guide/overview. Accessed June 1, 2019.
6. Morton SC, Murad MH, O'Connor E, et al. Quantitative Synthesis-An Update.

Methods Guide for Comparative Effectiveness Reviews. (Prepared by the Scientific Resource Center under Contract No. 290-2012-0004-C). AHRQ Publication No. 18-EHC007-EF. Rockville, MD: Agency for Healthcare Research and Quality; February 2018. Posted final reports are located on the Effective Health Care Program search page (https://effectivehealthcare.ahrq.gov/). https://doi.org/10.23970/AHRQEPCMETH GUIDE3.

7. Systematic Review Update: Noninvasive Nonpharmacologic Treatments for Chronic Pain. Agency for Healthcare Research and Quality. https://effectivehealthcare.ahrq.gov/topics/n oninvasive-nonpharm-pain-update/protocol. Accessed August 8, 2019.

8. Nonopioid Pharmacologic Treatments for Chronic Pain. Agency for Healthcare Research and Quality. https://effectivehealthcare.ahrq.gov/topics/n onopioid-chronic-pain/protocol. Accessed August 8, 2019. 
9. Chou R, Deyo R, Friedly J, et al.

Noninvasive Treatments for Low Back Pain. Comparative Effectiveness Review No. 169. (Prepared by the Pacific Northwest Evidence-based Practice Center under Contract No. 290-2012-00014-I.) AHRQ Publication No. 16-EHC004-EF. Rockville, MD: Agency for Healthcare Research and Quality; February 2016.

10. Skelly AC, Chou R, Dettori JR, et al. Noninvasive Nonpharmacological Treatment for Chronic Pain: A Systematic Review. Comparative Effectiveness Review No. 209. (Prepared by the Pacific Northwest Evidence-based Practice Center under Contract No. 290-2015-00009-I.) AHRQ Publication No 18-EHC013-EF. : . Rockville, MD: Agency for Healthcare Research and Quality; June 2018. https://effectivehealthcare.ahrq.gov/topics/n onpharma-treatment-pain/research-2018 PMID: 30179389.
11. Opioid Treatments for Chronic Pain. Agency for Healthcare Research and Quality.

https://effectivehealthcare.ahrq.gov/topics/o pioids-chronic-pain/protocol. Accessed August 8, 2019.

12. Atkins D, Chang SM, Gartlehner G, et al. Assessing applicability when comparing medical interventions: AHRQ and the Effective Health Care Program. J Clin Epidemiol. 2011 Nov;64(11):1198-207. doi: 10.1016/j.jclinepi.2010.11.021. PMID: 21463926 


\section{Appendix C. Included Studies List}

1. Abbaspoor Z, Akbari M, Najar S. Effect of foot and hand massage in post-cesarean section pain control: a randomized control trial. Pain Manag Nurs. 2014

Mar;15(1):132-6. doi: https://dx.doi.org/10.1016/j.pmn.2012.07.00 8. PMID: 23352729.

2. Aghababian RV. Comparison of diflunisal and acetaminophen with codeine in the management of grade 2 ankle sprain. Clin Ther. 1986;8(5):520-6. PMID: 3094957.

3. Akinbade AO, Ndukwe KC, Owotade FJ. Comparative analgesic efficacy and tolerability of celecoxib and tramadol on postoperative pain after mandibular third molar extraction: A double blind randomized controlled trial. Niger J Clin Pract. 2019 Jun;22(6):796-800. doi: https://dx.doi.org/10.4103/njcp.njcp_544_18 . PMID: 31187764.

4. Akural EI, Jarvimaki V, Lansineva A, et al. Effects of combination treatment with ketoprofen $100 \mathrm{mg}+$ acetaminophen 1000 mg on postoperative dental pain: a singledose, 10-hour, randomized, double-blind, active- and placebo-controlled clinical trial. Clin Ther. 2009 Mar;31(3):560-8. doi: 10.1016/j.clinthera.2009.03.017. PMID: 19393845.

5. Alam A, Gomes T, Zheng H, et al. Longterm analgesic use after low-risk surgery: a retrospective cohort study. Arch Intern Med. 2012 Mar 12;172(5):425-30. doi: https://dx.doi.org/10.1001/archinternmed.20 11.1827. PMID: 22412106.

6. AlRuthia Y, Alghadeer S, Balkhi B, et al. Efficacy of acetaminophen versus ibuprofen for the management of rotator cuff-related shoulder pain: Randomized open-label study. Saudi Pharm J. 2019;27(6):882-8. doi: 10.1016/j.jsps.2019.06.001. PMID: 31516331.

7. al-Sahlawi KS, Tawfik OM. Comparative study of the efficacy of lysine acetylsalicylate, indomethacin and pethidine in acute renal colic. Eur J Emerg Med. 1996 Sep;3(3):183-6. doi: 10.1097/00063110199609000-00008. PMID: 9023498.
8. Altiparmak N, Bayram B, Diker N, et al. Efficacy of Ice Pack Therapy After Impacted Third Molar Surgery: A Randomized Controlled Clinical Trial. Turkiye Klinikleri. Dishekimligi Bilimleri Dergisi. 2018;24(1).

9. Anders C, Hubner A. Influence of elastic lumbar support belts on trunk muscle function in patients with non-specific acute lumbar back pain. PLoS ONE.

2019;14(1):e0211042. doi: https://dx.doi.org/10.1371/journal.pone.0211 042. PMID: 30677095.

10. Andersen LA, Gøtzsche PC. Naproxen and aspirin in acute musculoskeletal disorders: a double-blind, parallel study in patients with sports injuries. Pharmatherapeutica. 1984;3(8):531-7. PMID: 6366809.

11. Ayhan $\mathrm{H}$, Tastan S, Iyigun E, et al. The Effectiveness of Neck Stretching Exercises Following Total Thyroidectomy on Reducing Neck Pain and Disability: A Randomized Controlled Trial. Worldviews Evid Based Nurs. 2016 Jun;13(3):224-31. doi: 10.1111/wvn.12136. PMID: 26773539.

12. Balakrishnan S, Bhushan K, Bhargava VK, et al. A randomized parallel trial of topical aspirin-moisturizer solution vs. oral aspirin for acute herpetic neuralgia. Int J Dermatol. 2001 Aug;40(8):535-8. doi: 10.1046/j.13654362.2001.01265.x. PMID: 11703529.

13. Barber FA, McGuire DA, Click S. Continuous-flow cold therapy for outpatient anterior cruciate ligament reconstruction. Arthroscopy. 1998 Mar;14(2):130-5. doi: 10.1016/s0749-8063(98)70030-1. PMID: 9531122.

14. Basmajian JV. Acute back pain and spasm. A controlled multicenter trial of combined analgesic and antispasm agents. Spine. 1989 Apr;14(4):438-9. PMID: 2524114.

15. Basur RL, Shephard E, Mouzas GL. A cooling method in the treatment of ankle sprains. Practitioner. 1976 Jun;216(1296):708-11. PMID: 821050. 
16. Bentley KC, Head TW. The additive analgesic efficacy of acetaminophen, 1000 $\mathrm{mg}$, and codeine, $60 \mathrm{mg}$, in dental pain. Clin Pharmacol Ther. 1987 Dec;42(6):634-40. doi: 10.1038/clpt.1987.211. PMID: 3690941.

17. Berti M, Albertin A, Casati A, et al. A prospective, randomized comparison of dexketoprofen, ketoprofen or paracetamol for postoperative analgesia after outpatient knee arthroscopy. Minerva Anestesiol. 2000 Jul-Aug;66(7-8):549-54. PMID: 10965735.

18. Biondi D, Xiang J, Benson C, et al. Tapentadol immediate release versus oxycodone immediate release for treatment of acute low back pain. Pain Physician. 2013 May-Jun;16(3):E237-46. PMID: 23703422.

19. Bondarsky EE, Domingo AT, Matuza NM, et al. Ibuprofen vs acetaminophen vs their combination in the relief of musculoskeletal pain in the ED: a randomized, controlled trial. Am J Emerg Med. 2013

Sep;31(9):1357-60. doi: 10.1016/j.ajem.2013.06.007. PMID: 23896011.

20. Borchgrevink GE, Kaasa A, McDonagh D, et al. Acute treatment of whiplash neck sprain injuries. A randomized trial of treatment during the first 14 days after a car accident. Spine. 1998 Jan 01;23(1):25-31. doi: 10.1097/00007632-199801010-00006. PMID: 9460148.

21. Boyles W. Management of acute musculoskeletal conditions : thoracolumbar strain or sprain. A double-blind evaluation comparing the efficacy and safety of carisoprodol with diazepam. Today's Ther Trends. 1983 1983;1:1-16.

22. Breivik E, Haanaes H, Barkvoll P. Upside assay sensitivity in a dental pain model. Eur J Pain. 1998;2(2):179-86. doi: 10.1016/S1090-3801\%2898\%2990010-6. PMID: 10700313.

23. Breivik EK, Barkvoll P, Skovlund E. Combining diclofenac with acetaminophen or acetaminophen-codeine after oral surgery: a randomized, double-blind single-dose study. Clin Pharmacol Ther. 1999 Dec;66(6):625-35. doi: 10.1053/cp.1999.v66.103629001. PMID: 10613619.
24. Brown FL, Jr., Bodison S, Dixon J, et al. Comparison of diflunisal and acetaminophen with codeine in the treatment of initial or recurrent acute low back strain. Clin Ther. 1986;9 Suppl C:52-8. PMID: 2951012.

25. Brown JD, Daniels SE, Bandy DP, et al. Evaluation of multiday analgesia with etoricoxib in a double-blind, randomized controlled trial using the postoperative thirdmolar extraction dental pain model. Clin J Pain. 2013 Jun;29(6):492-8. doi: 10.1097/AJP.0b013e318260c144. PMID: 23247002.

26. Bugada D, Lavand'homme P, Ambrosoli AL, et al. Effect of postoperative analgesia on acute and persistent postherniotomy pain: a randomized study. J Clin Anesth. 2015 12;27(8):658-64. doi: 10.1016/j.jclinane.2015.06.008. PMID: 26329661.

27. Castellano JJ, Rojas AM, Karia R, et al. A Randomized, Double-Blind, PlaceboControlled Study of Neuromuscular Electrical Stimulation (NMES) use for Recovery after Elective Total Hip Replacement Surgery. Bull Hosp Jt Dis (2013). 2016 Nov;74(4):275-81. PMID: 27815950.

28. Cattry E, Troullos E, Paredes-Diaz A. Efficacy and safety of naproxen sodium 440 $\mathrm{mg}$ versus acetaminophen $600 \mathrm{mg} /$ codeine phosphate $60 \mathrm{mg}$ in the treatment of postoperative dental pain. Am J Dent. 2020 Apr;33(2):106-12. PMID: 32259416.

29. Cooper SA, Breen JF, Giuliani RL. The relative efficacy of indoprofen compared with opioid-analgesic combinations. J Oral Surg. 1981 Jan;39(1):21-5. PMID: 6935400.

30. Cooper SA, Engel J, Ladov M, et al. Analgesic efficacy of an ibuprofen-codeine combination. Pharmacotherapy. 1982 MayJun;2(3):162-7. doi: 10.1002/j.18759114.1982.tb04528.x. PMID: 6763202.

31. Cooper SA, Firestein A, Cohn P. Doubleblind comparison of meclofenamate sodium with acetaminophen, acetaminophen with codeine and placebo for relief of postsurgical dental pain. J Clin Dent. 1988 Fall;1(2):31-4. PMID: 3254707. 
32. Cooper SA, Kupperman A. The analgesic efficacy of flurbiprofen compared to acetaminophen with codeine. J Clin Dent. 1991;2(3):70-4. PMID: 1930699.

33. Cooper SA, Precheur H, Rauch D, et al. Evaluation of oxycodone and acetaminophen in treatment of postoperative dental pain. Oral Surg Oral Med Oral Pathol. 1980 Dec;50(6):496-501. doi: 10.1016/0030-4220(80)90430-2. PMID: 7005804 .

34. Cooper SA, Schachtel BP, Goldman E, et al. Ibuprofen and acetaminophen in the relief of acute pain: a randomized, double-blind, placebo-controlled study. J Clin Pharmacol. 1989 Nov;29(11):1026-30. PMID: 2689471.

35. Cordell WH, Wright SW, Wolfson AB, et al. Comparison of intravenous ketorolac, meperidine, and both (balanced analgesia) for renal colic. Ann Emerg Med. 1996 Aug;28(2):151-8. doi: 10.1016/s01960644(96)70055-0. PMID: 8759578.

36. da Silva MP, Liebano RE, Rodrigues VA, et al. Transcutaneous electrical nerve stimulation for pain relief after liposuction: a randomized controlled trial. Aesthetic Plast Surg. 2015 Apr;39(2):262-9. doi: https://dx.doi.org/10.1007/s00266-0150451-6. PMID: 25665520.

37. Dalton JD, Jr., Schweinle JE. Randomized controlled noninferiority trial to compare extended release acetaminophen and ibuprofen for the treatment of ankle sprains. Ann Emerg Med. 2006 Nov;48(5):615-23. doi: 10.1016/j.annemergmed.2006.05.015. PMID: 17052565.

38. Dambros C, Martimbianco AL, Polachini LO, et al. Effectiveness of cryotherapy after anterior cruciate ligament reconstruction. Acta Ortop Bras. 2012;20(5):285-90. doi: 10.1590/s1413-78522012000500008. PMID: 24453619.

39. Daniel DM, Stone ML, Arendt DL. The effect of cold therapy on pain, swelling, and range of motion after anterior cruciate ligament reconstructive surgery. Arthroscopy. 1994 Oct;10(5):530-3. doi: 10.1016/s0749-8063(05)80008-8. PMID: 7999161.
40. Daniels S, Casson E, Stegmann JU, et al. A randomized, double-blind, placebocontrolled phase 3 study of the relative efficacy and tolerability of tapentadol IR and oxycodone IR for acute pain. Curr Med Res Opin. 2009a Jun;25(6):1551-61. doi: 10.1185/03007990902952825. PMID: 19445652.

41. Daniels S, Reader S, Berry P, et al. Onset of analgesia with sodium ibuprofen, ibuprofen acid incorporating poloxamer and acetaminophen - a single-dose, doubleblind, placebo-controlled study in patients with post-operative dental pain. Eur J Clin Pharmacol. 2009c;65(4):343-53. PMID: 19252905.

42. Daniels SE, Bandy DP, Christensen SE, et al. Evaluation of the dose range of etoricoxib in an acute pain setting using the postoperative dental pain model. Clin J Pain. 2011 Jan;27(1):1-8. doi: 10.1097/ajp.0b013e3181ed0639. PMID: 21188849.

43. Daniels SE, Upmalis D, Okamoto A, et al. A randomized, double-blind, phase III study comparing multiple doses of tapentadol IR, oxycodone IR, and placebo for postoperative (bunionectomy) pain. Curr Med Res Opin. 2009b Mar;25(3):765-76. doi: https://dx.doi.org/10.1185/03007990902728 183. PMID: 19203298.

44. Degirmen N, Ozerdogan N, Sayiner D, et al. Effectiveness of foot and hand massage in postcesarean pain control in a group of Turkish pregnant women. Appl Nurs Res. 2010 Aug;23(3):153-8. doi: 10.1016/j.apnr.2008.08.001. PMID: 20643325.

45. Dehghan M, Farahbod F. The efficacy of thermotherapy and cryotherapy on pain relief in patients with acute low back pain, a clinical trial study. J Clin Diagn Res.

2014;8(9):LC01-LC4. doi: 10.7860/JCDR/2014/7404.4818. PMID: 25386469.

46. Dervin GF, Taylor DE, Keene GC. Effects of cold and compression dressings on early postoperative outcomes for the arthroscopic anterior cruciate ligament reconstruction patient. J Orthop Sports Phys Ther. 1998 Jun;27(6):403-6. doi:

10.2519/jospt.1998.27.6.403. PMID: 9617725. 
47. Dionne RA, Snyder J, Hargreaves KM. Analgesic efficacy of flurbiprofen in comparison with acetaminophen, acetaminophen plus codeine, and placebo after impacted third molar removal. J Oral Maxillofac Surg. 1994 Sep;52(9):919-24; discussion 25-6. doi: 10.1016/s02782391(10)80068-0. PMID: 8064454.

48. Doroschak AM, Bowles WR, Hargreaves KM. Evaluation of the combination of flurbiprofen and tramadol for management of endodontic pain. J Endod. 1999 Oct;25(10):660-3. doi: 10.1016/s00992399(99)80350-1. PMID: 10687523.

49. Duncan JJ, Farr JE. Comparison of diclofenac sodium and aspirin in the treatment of acute sports injuries. Am J Sports Med. 1988 Nov-Dec;16(6):656-9. doi: 10.1177/036354658801600618. PMID: 3149152.

50. Dworkin RH, Barbano RL, Tyring SK, et al. A randomized, placebo-controlled trial of oxycodone and of gabapentin for acute pain in herpes zoster. Pain. 2009 Apr;142(3):20917. doi: 10.1016/j.pain.2008.12.022. PMID: 19195785.

51. Edwards DJ, Rimmer M, Keene GC. The use of cold therapy in the postoperative management of patients undergoing arthroscopic anterior cruciate ligament reconstruction. Am J Sports Med. 1996 MarApr;24(2):193-5. doi:

10.1177/036354659602400213. PMID: 8775119.

52. Erhard RE, Delitto A, Cibulka MT. Relative effectiveness of an extension program and a combined program of manipulation and flexion and extension exercises in patients with acute low back syndrome. Phys Ther. 1994 Dec;74(12):1093-100. doi: 10.1093/ptj/74.12.1093. PMID: 7991650.

53. Evans DP, Burke MS, Newcombe RG. Medicines of choice in low back pain. Curr Med Res Opin. 1980;6(8):540-7. PMID: 6446445.

54. Ezenwa MO, Yao Y, Engeland CG, et al. A randomized controlled pilot study feasibility of a tablet-based guided audio-visual relaxation intervention for reducing stress and pain in adults with sickle cell disease. $\mathrm{J}$ Adv Nurs. 2016 Jun;72(6):1452-63. doi: 10.1111/jan.12895. PMID: 26768753.
55. Felhendler D, Lisander B. Pressure on acupoints decreases postoperative pain. Clin J Pain. 1996 Dec;12(4):326-9. doi: 10.1097/00002508-199612000-00012. PMID: 8969878.

56. Ffrench-O'Carroll R, Steinhaeuser H, Duff $S$, et al. A randomized controlled trial comparing tapentadol with oxycodone in non-breastfeeding women post elective cesarean section. Curr Med Res Opin. 2019 06;35(6):975-81. doi: https://dx.doi.org/10.1080/03007995.2018.1 550059. PMID: 30444145.

57. Finlay IG, Scott R, McArdle CS. Prospective double-blind comparison of buprenorphine and pethidine in ureteric colic. Br Med J (Clin Res Ed). 1982 Jun 19;284(6332):1830-1. doi: 10.1136/bmj.284.6332.1830. PMID: 6805715.

58. Finlay KA, Wilson JA, Gaston P, et al. Postoperative pain management through audioanalgesia: Investigating musical constructs. Psychol Music. 2016;44(3):493-513.

59. Forbes JA, Kehm CJ, Grodin CD, et al. Evaluation of ketorolac, ibuprofen, acetaminophen, and an acetaminophencodeine combination in postoperative oral surgery pain. Pharmacotherapy. 1990;10(6 ( Pt 2)):94s-105s. PMID: 2082318.

60. Forchuk C, Baruth P, Prendergast M, et al. Postoperative arm massage: a support for women with lymph node dissection. Cancer Nurs. 2004 Jan-Feb;27(1):25-33. doi: 10.1097/00002820-200401000-00004. PMID: 15108949.

61. Forouzanfar T, Sabelis A, Ausems S, et al. Effect of ice compression on pain after mandibular third molar surgery: a singleblind, randomized controlled trial. Int J Oral Maxillofac Surg. 2008 Sep;37(9):824-30. doi: 10.1016/j.ijom.2008.05.011. PMID: 18603410.

62. Friedman BW, Dym AA, Davitt M, et al. Naproxen With Cyclobenzaprine, Oxycodone/Acetaminophen, or Placebo for Treating Acute Low Back Pain: A Randomized Clinical Trial. JAMA. 2015 Oct 20;314(15):1572-80. doi: 10.1001/jama.2015.13043. PMID: 26501533. 
63. Gamermann PW, Martins ALC, Rosa L, et al. Acupuncture as a complement to the pharmacological management of pain, nausea and vomiting after cesarean section: a randomized clinical trial. Acupuncture and Related Therapies. 2014;3(1):11-4. doi: 10.1016/j.arthe.2014.12.002.

64. Garcća-Alonso F. Comparative study of the efficacy of dipyrone, diclofenac sodium and pethidine in acute renal colic. Eur J Clin Pharmacol. 1991;40(6):543-6. PMID: 1884733.

65. Gilbert JR, Taylor DW, Hildebrand A, et al. Clinical trial of common treatments for low back pain in family practice. Br Med J (Clin Res Ed). 1985 Sep 21;291(6498):791-4. PMID: 2931153.

66. Gimbel JS, Brugger A, Zhao W, et al. Efficacy and tolerability of celecoxib versus hydrocodone/acetaminophen in the treatment of pain after ambulatory orthopedic surgery in adults. Clin Ther. 2001 Feb;23(2):228-41. PMID: 11293556.

67. Glover JR, Morris JG, Khosla T. Back pain: a randomized clinical trial of rotational manipulation of the trunk. Br J Ind Med. 1974 Jan;31(1):59-64. doi: 10.1136/oem.31.1.59. PMID: 4274488.

68. Gonzalez ER, Ornato JP, Ware D, et al. Comparison of intramuscular analgesic activity of butorphanol and morphine in patients with sickle cell disease. Ann Emerg Med. 1988 Aug;17(8):788-91. PMID: 3394980 .

69. Grissa MH, Claessens YE, Bouida W, et al. Paracetamol vs piroxicam to relieve pain in renal colic. Results of a randomized controlled trial. Am J Emerg Med. 2011 Feb;29(2):203-6. doi: 10.1016/j.ajem.2009.09.019. PMID: 20934829.

70. Hackett GI, Seddon D, Kaminski D. Electroacupuncture compared with paracetamol for acute low back pain. Practitioner. 1988 Feb 22;232(1443):163-4. PMID: 2973008.

71. Hadler NM, Curtis P, Gillings DB, et al. A benefit of spinal manipulation as adjunctive therapy for acute low-back pain: a stratified controlled trial. Spine. 1987 Sep;12(7):7026. PMID: 2961085.
72. Hallegraeff JM, de Greef M, Winters JC, et al. Manipulative therapy and clinical prediction criteria in treatment of acute nonspecific low back pain. Percept Mot Skills. 2009 Feb;108(1):196-208. doi: 10.2466/pms.108.1.196-208. PMID: 19425461.

73. Hancock MJ, Maher CG, Latimer J, et al. Assessment of diclofenac or spinal manipulative therapy, or both, in addition to recommended first-line treatment for acute low back pain: a randomised controlled trial. Lancet. 2007 Nov 10;370(9599):1638-43. PMID: 17993364.

74. Harbaugh CM, Nalliah RP, Hu HM, et al. Persistent Opioid Use After Wisdom Tooth Extraction. JAMA. 2018;320(5):504-6. doi: 10.1001/jama.2018.9023. PMID: 30088000.

75. Hasegawa TM, Baptista AS, de Souza MC, et al. Acupuncture for acute non-specific low back pain: a randomised, controlled, double-blind, placebo trial. Acupunct Med. 2014 Apr;32(2):109-15. doi: 10.1136/acupmed-2013-010333. PMID: 24316509.

76. He BJ, Tong PJ, Li J, et al. Auricular acupressure for analgesia in perioperative period of total knee arthroplasty. Pain Med. 2013 Oct;14(10):1608-13. doi: 10.1111/pme.12197. PMID: 23865512.

77. Hebertson RM, Storey N. The comparative efficacy of diclofenac potassium, aspirin, and placebo in the treatment of patients with pain following gynecologic surgery. Todays Therapeutic Trends. 1995;12:33-.

78. Heere LP. Piroxicam in acute musculoskeletal disorders and sports injuries. Am J Med. 1988 May 20;84(5A):50-5. PMID: 3287912.

79. Helmerhorst GTT, Zwiers R, Ring D, et al. Pain Relief After Operative Treatment of an Extremity Fracture: A Noninferiority Randomized Controlled Trial. J Bone Joint Surg Am. 2017 Nov 15;99(22):1908-15. doi: 10.2106/JBJS.17.00149. PMID: 29135664.

80. Hennies OL. A new skeletal muscle relaxant (DS 103-282) compared to diazepam in the treatment of muscle spasm of local origin. J Int Med Res. 1981;9(1):62-8. PMID: 6451461. 
81. Hersh EV, Levin LM, Cooper SA, et al. Ibuprofen liquigel for oral surgery pain. Clin Ther. 2000;22(11):1306-18. PMID: 11117655.

82. Hewitt DJ, Todd KH, Xiang J, et al. Tramadol/acetaminophen or hydrocodone/acetaminophen for the treatment of ankle sprain: a randomized, placebo-controlled trial. Ann Emerg Med. 2007 Apr;49(4):468-80, 80.e1-2. PMID: 17113683.

83. Hill CM, Balkenohl M, Thomas DW, et al. Pregabalin in patients with postoperative dental pain. Eur J Pain. 2001;5(2):119-24. doi: 10.1053/eujp.2001.0235. PMID: 11465977.

84. Hocutt JE, Jr., Jaffe R, Rylander CR, et al. Cryotherapy in ankle sprains. Am J Sports Med. 1982 Sep-Oct;10(5):316-9. doi: 10.1177/036354658201000512. PMID: 6814272.

85. Hofstee DJ, Gijtenbeek JM, Hoogland PH, et al. Westeinde sciatica trial: randomized controlled study of bed rest and physiotherapy for acute sciatica. J Neurosurg. 2002 Jan;96(1 Suppl):45-9. PMID: 11797655.

86. Hoiriis KT, Pfleger B, McDuffie FC, et al. A randomized clinical trial comparing chiropractic adjustments to muscle relaxants for subacute low back pain. J Manipulative Physiol Ther. 2004 Jul-Aug;27(6):388-98. PMID: 15319761.

87. Holmstrom A, Hardin BC. Cryo/Cuff compared to epidural anesthesia after knee unicompartmental arthroplasty: a prospective, randomized and controlled study of 60 patients with a 6-week followup. J Arthroplasty. 2005 Apr;20(3):316-21. PMID: 15809948.

88. Hulme J, Waterman H, Hillier VF. The effect of foot massage on patients' perception of care following laparoscopic sterilization as day case patients. J Adv Nurs. 1999 Aug;30(2):460-8. doi: 10.1046/j.1365-2648.1999.01101.x. PMID: 10457249.

89. Hussain I, Hussain Shah SI, Amjad I. Efficacy of spinal manual therapy in nonspecific acute low back pain. Rawal Medical Journal. 2013;38(4):358-60.
90. Ibikunle AA, Adeyemo WL. Oral healthrelated quality of life following third molar surgery with or without application of ice pack therapy. Oral Maxillofac Surg. 2016 Sep;20(3):239-47. doi: 10.1007/s10006-0160558-1. PMID: 27139019.

91. Indelicato PA. Comparison of diflunisal and acetaminophen with codeine in the treatment of mild to moderate pain due to strains and sprains. Clin Ther. 1986;8(3):269-74. PMID: 3521855.

92. Innes GD, Croskerry P, Worthington J, et al. Ketorolac versus acetaminophen-codeine in the emergency department treatment of acute low back pain. J Emerg Med. 1998 Jul-Aug;16(4):549-56. PMID: 9696169.

93. Jalili M, Fathi M, Moradi-Lakeh M, et al. Sublingual buprenorphine in acute pain management: a double-blind randomized clinical trial. Ann Emerg Med. 2012 Apr;59(4):276-80. doi: 10.1016/j.annemergmed.2011.10.021. PMID: 22115823.

94. Juni P, Battaglia M, Nuesch E, et al. A randomised controlled trial of spinal manipulative therapy in acute low back pain. Ann Rheum Dis. 2009 Sep;68(9):1420-7. doi: 10.1136/ard.2008.093757. PMID: 18775942.

95. Kayali C, Agus H, Surer L, et al. The efficacy of paracetamol in the treatment of ankle sprains in comparison with diclofenac sodium. Saudi Med J. 2007 Dec;28(12):1836-9. PMID: 18060212.

96. Kaynar M, Koyuncu F, Buldu I, et al. Comparison of the efficacy of diclofenac, acupuncture, and acetaminophen in the treatment of renal colic. Am J Emerg Med. 2015 Jun;33(6):749-53. doi: 10.1016/j.ajem.2015.02.033. PMID: 25827597.

97. Kettenmann B, Wille C, Lurie-Luke E, et al. Impact of continuous low level heatwrap therapy in acute low back pain patients: subjective and objective measurements. Clin J Pain. 2007 Oct;23(8):663-8. doi: 10.1097/AJP.0b013e31813543ef. PMID: 17885344. 
98. Kiersch TA, Halladay SC, Hormel PC. A single-dose, double-blind comparison of naproxen sodium, acetaminophen, and placebo in postoperative dental pain. Clin Ther. 1994;16(3):394-404. PMID: 7923306.

99. Kim HJ, Yi JM, Cho HG, et al. Comparative study of the treatment outcomes of osteoporotic compression fractures without neurologic injury using a rigid brace, a soft brace, and no brace: a prospective randomized controlled non-inferiority trial. J Bone Joint Surg Am. 2014 Dec 3;96(23):1959-66. doi: 10.2106/jbjs.N.00187. PMID: 25471910.

100. Koc M, Tez M, Yoldas O, et al. Cooling for the reduction of postoperative pain: prospective randomized study. Hernia. 2006 Apr;10(2):184-6. doi: 10.1007/s10029-0050062-2. PMID: 16432641.

101. Kongsted A, Qerama E, Kasch H, et al. Neck collar, "act-as-usual" or active mobilization for whiplash injury? A randomized parallel-group trial. Spine (Phila Pa 1976). 2007 Mar 15;32(6):618-26. doi: 10.1097/01.brs.0000257535.77691.bd. PMID: 17413465.

102. Koras K, Karabulut N. The Effect of Foot Massage on Postoperative Pain and Anxiety Levels in Laparoscopic Cholecystectomy Surgery: A Randomized Controlled Experimental Study. J Perianesth Nurs. 2019 Jun;34(3):551-8. doi: https://dx.doi.org/10.1016/j.jopan.2018.07.0 06. PMID: 30470466.

103. Kuijper B, Tans JT, Beelen A, et al. Cervical collar or physiotherapy versus wait and see policy for recent onset cervical radiculopathy: randomised trial. BMJ. 2009 Oct 7;339:b3883. doi: 10.1136/bmj.b3883. PMID: 19812130.

104. Langenbach MR, Aydemir-Dogruyol K, Issel R, et al. Randomized sham-controlled trial of acupuncture for postoperative pain control after stapled haemorrhoidopexy. Colorectal Dis. 2012 Aug;14(8):e486-91. doi: 10.1111/j.1463-1318.2012.02984.x. PMID: 22330010.
105. Lao L, Bergman S, Langenberg P, et al. Efficacy of Chinese acupuncture on postoperative oral surgery pain. Oral Surg Oral Med Oral Pathol Oral Radiol Endod. 1995 Apr;79(4):423-8. doi: 10.1016/s10792104(05)80121-0. PMID: 7614199.

106. Larkin GL, Peacock WFt, Pearl SM, et al. Efficacy of ketorolac tromethamine versus meperidine in the ED treatment of acute renal colic. Am J Emerg Med. 1999 Jan;17(1):6-10. doi: 10.1016/s07356757(99)90003-7. PMID: 9928687.

107. Lazcano A, Dougherty JM, Kruger M. Use of rib belts in acute rib fractures. Am J Emerg Med. 1989 Jan;7(1):97-100. PMID: 2643965.

108. Lee SS, Choi Y, Pransky GS. Extent and Impact of Opioid Prescribing for Acute Occupational Low Back Pain in the Emergency Department. J Emerg Med. 2016 Mar;50(3):376-84.e1-2. doi: 10.1016/j.jemermed.2015.10.015. PMID: 26754559.

109. Lessard LA, Scudds RA, Amendola A, et al. The efficacy of cryotherapy following arthroscopic knee surgery. J Orthop Sports Phys Ther. 1997 Jul;26(1):14-22. doi: 10.2519/jospt.1997.26.1.14. PMID: 9201637.

110. Liu J, Li N. Clinical observation of a combination of acupuncture and drug administration for nonspecific acute lumbar sprain. Journal of Acupuncture and Tuina Science. 2010 Feb;8(1):47-9. doi: 10.1007/s11726-010-0047-2.

111. Lyrtzis C, Natsis K, Papadopoulos C, et al. Efficacy of paracetamol versus diclofenac for Grade II ankle sprains. Foot Ankle Int. 2011 Jun;32(6):571-5. doi: 10.3113/fai.2011.0571. PMID: 21733417.

112. Malmivaara A, Hakkinen U, Aro T, et al. The treatment of acute low back pain--bed rest, exercises, or ordinary activity? N Engl J Med. 1995 Feb 09;332(6):351-5. PMID: 7823996.

113. Malmstrom K, Kotey P, Coughlin H, et al. A randomized, double-blind, parallel-group study comparing the analgesic effect of etoricoxib to placebo, naproxen sodium, and acetaminophen with codeine using the dental impaction pain model. Clin J Pain. 2004;20(3):147-55. PMID: 15100590. 
114. Man SY, Woo WK, Lam PKW, et al. Feasibility Study Comparing Oral Paracetamol and Oral Non-Steroidal AntiInflammatory Drugs for Treating Pain after Musculoskeletal Injury: a Randomised, Double Blind, Controlled Trial. Hong Kong Journal of Emergency Medicine. 2004 2004/04/01;11(2):78-84. doi: 10.1177/102490790401100203.

115. Marthak KV, Gokarn AM, Rao AV, et al. A multi-centre comparative study of diclofenac sodium and a dipyrone/spasmolytic combination, and a single-centre comparative study of diclofenac sodium and pethidine in renal colic patients in India. Curr Med Res Opin. 1991;12(6):366-73. doi: 10.1185/03007999109111506. PMID: 2044396.

116. Mehlisch DR, Aspley S, Daniels SE, et al. Comparison of the analgesic efficacy of concurrent ibuprofen and paracetamol with ibuprofen or paracetamol alone in the management of moderate to severe acute postoperative dental pain in adolescents and adults: a randomized, double-blind, placebocontrolled, parallel-group, single-dose, twocenter, modified factorial study. Clin Ther. 2010 May;32(5):882-95. doi: 10.1016/j.clinthera.2010.04.022. PMID: 20685496.

117. Mehlisch DR, Brown P. Single-dose therapy with diclofenac potassium, aspirin, or placebo following dental impaction surgery. Today's Ther Trends. 1995a;12:15-31.

118. Mehlisch DR, Jasper RD, Brown P, et al. Comparative study of ibuprofen lysine and acetaminophen in patients with postoperative dental pain. Clin Ther. 1995b;17(5):852-60. PMID: 8595637.

119. Mehlisch DR, Sollecito WA, Helfrick JF, et al. Multicenter clinical trial of ibuprofen and acetaminophen in the treatment of postoperative dental pain. J Am Dent Assoc. 1990 Aug;121(2):257-63. PMID: 2205641.

120. Mehrvarzfar P, Abbott PV, Saghiri MA, et al. Effects of three oral analgesics on postoperative pain following root canal preparation: a controlled clinical trial. Int Endod J. 2012;45(1):76-82. PMID: 21902704.
121. Mishra A, Amalakara J, Avula H, et al. Effect of diclofenac mouthwash on postoperative pain after periodontal surgery. J Clin Diagn Res. 2017;11(4):ZC24-ZC6. doi: 10.7860/JCDR/2017/22165.965. PMID: 28571255.

122. Moore PA, Crout RJ, Jackson DL, et al. Tramadol hydrochloride: analgesic efficacy compared with codeine, aspirin with codeine, and placebo after dental extraction. J Clin Pharmacol. 1998 Jun;38(6):554-60. doi: 10.1002/j.1552-4604.1998.tb05794.x. PMID: 9650546.

123. Moore RA, Gay-Escoda C, Figueiredo R, et al. Dexketoprofen/tramadol: randomised double-blind trial and confirmation of empirical theory of combination analgesics in acute pain. J Headache Pain.

2015;16:541. doi: https://dx.doi.org/10.1186/s10194-0150541-5. PMID: 26123824.

124. Morrison NA, Repka MX. Ketorolac versus acetaminophen or ibuprofen in controlling postoperative pain in patients with strabismus. Ophthalmology. 1994 May;101(5):915-8. PMID: 8190480.

125. Muckle DS. A comparative study of flurbiprofen and aspirin in soft tissue trauma. Br J Sports Med. 1976 Mar;10(1):11-3. doi: 10.1136/bjsm.10.1.11. PMID: 786422.

126. Muncie HL, Jr., King DE, DeForge B. Treatment of mild to moderate pain of acute soft tissue injury: diflunisal vs acetaminophen with codeine. J Fam Pract. 1986 Aug;23(2):125-7. PMID: 2942630.

127. Nadler SF, Steiner DJ, Erasala GN, et al. Continuous low-level heatwrap therapy for treating acute nonspecific low back pain. Arch Phys Med Rehabil. 2003a Mar;84(3):329-34. doi: 10.1053/apmr.2003.50102. PMID: 12638099.

128. Nadler SF, Steiner DJ, Petty SR, et al. Overnight use of continuous low-level heatwrap therapy for relief of low back pain. Arch Phys Med Rehabil. 2003b Mar;84(3):335-42. PMID: 12638100. 
129. Narci H, Ugur M, Uzun H, et al. Combining $1000 \mathrm{mg}$ oral acetaminophen with $75 \mathrm{mg}$ intramuscular diclofenac of analgesic efficacy for acute renal colic treatment. Sci Res Essays. 2012 Jun 14;7(22):2017-21.

130. Nelson S, Brahmin J. An evaluation of the analgesic efficacy of diclofenac potassium, aspirin, and placebo in postoperative dental pain. Today's Ther Trends. 1994;12:3-14.

131. Nguyen KK, Liu YF, Chang C, et al. A Randomized Single-Blinded Trial of Ibuprofen- versus Opioid-Based Primary Analgesic Therapy in Outpatient Otolaryngology Surgery. Otolaryngol Head Neck Surg. 2019 May;160(5):839-46. doi: 10.1177/0194599819832528. PMID: 30832548.

132. Nilsson U, Unosson M, Rawal N. Stress reduction and analgesia in patients exposed to calming music postoperatively: a randomized controlled trial. Eur J Anaesthesiol. 2005 Feb;22(2):96-102. doi: 10.1017/s0265021505000189. PMID: 15816586.

133. Nyanzi CS, Langridge J, Heyworth JR, et al. Randomized controlled study of ultrasound therapy in the management of acute lateral ligament sprains of the ankle joint. Clin Rehabil. 1999 Feb;13(1):16-22. doi: 10.1177/026921559901300103. PMID: 10327093.

134. Oh JH, Seo HJ, Lee YH, et al. Do Selective COX-2 Inhibitors Affect Pain Control and Healing After Arthroscopic Rotator Cuff Repair? A Preliminary Study. Am J Sports Med. 2018 Mar;46(3):679-86. doi: 10.1177/0363546517744219. PMID: 29253346.

135. Ohkoshi Y, Ohkoshi M, Nagasaki S, et al. The effect of cryotherapy on intraarticular temperature and postoperative care after anterior cruciate ligament reconstruction. Am J Sports Med. 1999 May-Jun;27(3):35762. doi: $10.1177 / 03635465990270031601$. PMID: 10352774.

136. Olson MNZ, Otero AM, Marrero I, et al. Onset of analgesia for liquigel ibuprofen 400 mg, acetaminophen $1000 \mathrm{mg}$, ketoprofen 25 $\mathrm{mg}$, and placebo in the treatment of postoperative dental pain. J Clin Pharmacol. 2001;41(11):1238-47. PMID: 11697757.
137. Oosterlinck W, Philp NH, Charig C, et al. A double-blind single dose comparison of intramuscular ketorolac tromethamine and pethidine in the treatment of renal colic. $\mathrm{J}$ Clin Pharmacol. 1990 Apr;30(4):336-41. doi: 10.1002/j.1552-4604.1990.tb03603.x. PMID: 2341581.

138. Ordog GJ. Transcutaneous electrical nerve stimulation versus oral analgesic: a randomized double-blind controlled study in acute traumatic pain. Am J Emerg Med. 1987 Jan;5(1):6-10. doi: 10.1016/07356757(87)90281-6. PMID: 3545246.

139. Oswal P, Nagarathna R, Ebnezar J, et al. The effect of add-on yogic prana energization technique (YPET) on healing of fresh fractures: a randomized control study. J Altern Complement Med. 2011 Mar;17(3):253-8. doi: 10.1089/acm.2010.0001. PMID: 21417810.

140. Pagliara L, Tornago S, Metastasio J, et al. Tramadol compared with diclofenac in traumatic musculoskeletal pain. Curr Ther Res. 1997 Aug;58(8):473-91. doi: 10.1016/S0011-393X\%2897\%2980039-2.

141. Pathan SA, Mitra B, Straney LD, et al. Delivering safe and effective analgesia for management of renal colic in the emergency department: a double-blind, multigroup, randomised controlled trial. Lancet. 2016 May 14;387(10032):1999-2007. doi: 10.1016/s0140-6736(16)00652-8. PMID: 26993881.

142. Postacchini F, Facchini M, Palieri P. Efficacy of various forms of conservative treatment in low back pain. A comparative study. Neuro-orthopedics. 1988;6(1):28-35.

143. Rasubala L, Pernapati L, Velasquez X, et al. Impact of a Mandatory Prescription Drug Monitoring Program on Prescription of Opioid Analgesics by Dentists. PLoS ONE. 2015;10(8):e0135957. doi: https://dx.doi.org/10.1371/journal.pone.0135 957. PMID: 26274819.

144. Rawal N, Allvin R, Amilon A, et al. Postoperative analgesia at home after ambulatory hand surgery: a controlled comparison of tramadol, metamizol, and paracetamol. Anesth Analg. 2001 Feb;92(2):347-51. PMID: 11159230. 
145. Ridderikhof ML, Lirk P, Goddijn H, et al. Acetaminophen or Nonsteroidal AntiInflammatory Drugs in Acute Musculoskeletal Trauma: A Multicenter, Double-Blind, Randomized, Clinical Trial. Ann Emerg Med. 2018 Mar;71(3):35768.e8. doi: 10.1016/j.annemergmed.2017.08.041. PMID: 29033294.

146. Ripa M, Betts B, Dhaliwal S, et al. Survey of Postoperative Pain in Photorefractive Keratectomy Using Topical versus Oral Nonsteroidal Anti-Inflammatory Drugs. Clin Ophthalmol. 2020;14:1459-66. doi: https://dx.doi.org/10.2147/OPTH.S255441. PMID: 32546954.

147. Rosenberg M, Curtis L, Bourke DL. Transcutaneous electrical nerve stimulation for the relief of postoperative pain. Pain. 1978 Aug;5(2):129-33. doi: 10.1016/03043959\%2878\%2990034-9. PMID: 14716650.

148. Ruiz-Molinero C, Jimenez-Rejano JJ, Chillon-Martinez R, et al. Efficacy of therapeutic ultrasound in pain and joint mobility in whiplash traumatic acute and subacute phases. Ultrasound Med Biol. 2014 Sep;40(9):2089-95. doi:

10.1016/j.ultrasmedbio.2014.04.016. PMID: 25023094.

149. Saatsaz S, Rezaei R, Alipour A, et al. Massage as adjuvant therapy in the management of post-cesarean pain and anxiety: A randomized clinical trial. Complement Ther Clin Pract. 2016

Aug;24:92-8. doi: 10.1016/j.ctcp.2016.05.014. PMID: 27502807.

150. Sadeghein A, Shahidi N, Dehpour AR. A comparison of ketorolac tromethamine and acetaminophen codeine in the management of acute apical periodontitis. J Endod. 1999 Apr;25(4):257-9. PMID: 10425951.

151. Sandhu DP, Iacovou JW, Fletcher MS, et al. A comparison of intramuscular ketorolac and pethidine in the alleviation of renal colic. Br J Urol. 1994 Dec;74(6):690-3. doi: 10.1111/j.1464-410x.1994.tb07107.x. PMID: 7827834.
152. Santilli V, Beghi E, Finucci S. Chiropractic manipulation in the treatment of acute back pain and sciatica with disc protrusion: a randomized double-blind clinical trial of active and simulated spinal manipulations. Spine J. 2006 Mar-Apr;6(2):131-7. PMID: 16517383.

153. Santini MF, Rosa RAD, Ferreira MBC, et al. Comparison of two combinations of opioid and non-opioid analgesics for acute periradicular abscess: a randomized clinical trial. J Appl Oral Sci. 2017 SepOct;25(5):551-8. doi: 10.1590/1678-77572016-0407. PMID: 29069153.

154. Schachtel BP, Thoden WR, Baybutt RI. Ibuprofen and acetaminophen in the relief of postpartum episiotomy pain. J Clin Pharmacol. 1989 Jun;29(6):550-3. PMID: 2666455.

155. Schnabel M, Ferrari R, Vassiliou T, et al. Randomised, controlled outcome study of active mobilisation compared with collar therapy for whiplash injury. Emerg Med J. 2004 May;21(3):306-10. PMID: 15107368.

156. Searle S, Muse D, Paluch E, et al. Efficacy and Safety of Single and Multiple Doses of a Fixed-dose Combination of Ibuprofen and Acetaminophen in the Treatment of Postsurgical Dental Pain: Results from Two Phase 3, Randomized, Parallel-Group, Double-Blind, Placebo-Controlled Studies. Clin J Pain. 2020 Apr 07;07:07. doi: https://dx.doi.org/10.1097/AJP.0000000000 000828. PMID: 32271183.

157. Shin JS, Ha IH, Lee J, et al. Effects of motion style acupuncture treatment in acute low back pain patients with severe disability: a multicenter, randomized, controlled, comparative effectiveness trial. Pain. 2013 Jul;154(7):1030-7. doi: 10.1016/j.pain.2013.03.013. PMID: 23639822.

158. Skoglund LA, Skjelbred P, Fyllingen G. Analgesic efficacy of acetaminophen 1000 $\mathrm{mg}$, acetaminophen $2000 \mathrm{mg}$, and the combination of acetaminophen $1000 \mathrm{mg}$ and codeine phosphate $60 \mathrm{mg}$ versus placebo in acute postoperative pain. Pharmacotherapy. 1991;11(5):364-9. PMID: 1745622. 
159. Sloan JP, Hain R, Pownall R. Clinical benefits of early cold therapy in accident and emergency following ankle sprain. Arch Emerg Med. 1989 Mar;6(1):1-6. doi: 10.1136/emj.6.1.1. PMID: 2712981.

160. Sniezek PJ, Brodland DG, Zitelli JA. A randomized controlled trial comparing acetaminophen, acetaminophen and ibuprofen, and acetaminophen and codeine for postoperative pain relief after Mohs surgery and cutaneous reconstruction. Dermatol Surg. 2011;37(7):1007-13. PMID: 21561527.

161. Sozen KK, Karabulut N. Efficacy of Hand and Foot Massage in Anxiety and Pain Management Following Laparoscopic Cholecystectomy: A Controlled Randomized Study. Surg Laparosc Endosc Percutan Tech. 2019 Dec 04;04:04. doi: https://dx.doi.org/10.1097/SLE.0000000000 000738. PMID: 31855924.

162. Speer KP, Warren RF, Horowitz L. The efficacy of cryotherapy in the postoperative shoulder. J Shoulder Elbow Surg. 1996 JanFeb;5(1):62-8. doi: 10.1016/s10582746(96)80032-2. PMID: 8919444.

163. Stark J, Petrofsky J, Berk L, et al. Continuous low-level heatwrap therapy relieves low back pain and reduces muscle stiffness. Phys Sportsmed. 2014 Nov;42(4):39-48. doi: 10.3810/psm.2014.11.2090. PMID: 25419887.

164. Stucke RS, Kelly JL, Mathis KA, et al. Association of the Use of a Mandatory Prescription Drug Monitoring Program With Prescribing Practices for Patients Undergoing Elective Surgery. JAMA Surg. 2018 Dec 1;153(12):1105-10. doi: 10.1001/jamasurg.2018.2666. PMID: 30140927.

165. Sunshine A, Marrero I, Olson N, et al. Comparative study of flurbiprofen, zomepirac sodium, acetaminophen plus codeine, and acetaminophen for the relief of postsurgical dental pain. Am J Med. 1986 Mar 24;80(3a):50-4. doi: 10.1016/00029343(86)90111-7. PMID: 3515924.
166. Sunshine A, Olson NZ, Zighelboim I, et al. Ketoprofen, acetaminophen plus oxycodone, and acetaminophen in the relief of postoperative pain. Clin Pharmacol Ther. 1993 Nov;54(5):546-55. doi: 10.1038/clpt.1993.187. PMID: 8222498.

167. Tao XG, Bernacki EJ. A randomized clinical trial of continuous low-level heat therapy for acute muscular low back pain in the workplace. J Occup Environ Med. 2005 Dec;47(12):1298-306. doi: 10.1097/01.jom.0000184877.01691.a3. PMID: 16340712.

168. Thiels CA, Habermann EB, Hooten WM, et al. Chronic use of tramadol after acute pain episode: cohort study. BMJ. 2019 May 14;365:11849. doi: https://dx.doi.org/10.1136/bmj.l1849. PMID: 31088782.

169. Thijs E, Schotanus MGM, Bemelmans YFL, et al. Reduced opiate use after total knee arthroplasty using computer-assisted cryotherapy. Knee Surg Sports Traumatol Arthrosc. 2019 Apr;27(4):1204-12. doi: 10.1007/s00167-018-4962-y. PMID: 29725749.

170. Turturro MA, Paris PM, Larkin GL. Tramadol versus hydrocodoneacetaminophen in acute musculoskeletal pain: a randomized, double-blind clinical trial. Ann Emerg Med. 1998 Aug;32(2):13943. PMID: 9701294.

171. Underwood MR, Morgan J. The use of a back class teaching extension exercises in the treatment of acute low back pain in primary care. Fam Pract. 1998 Feb;15(1):915. PMID: 9527292.

172. Uzun B, Kekec Z, Gurkan E. Efficacy of tramadol vs meperidine in vasoocclusive sickle cell crisis. Am J Emerg Med. 2010 May;28(4):445-9. doi: 10.1016/j.ajem.2009.01.016. PMID: 20466223.

173. van der Westhuijzen AJ, Becker PJ, Morkel $\mathrm{J}$, et al. A randomized observer blind comparison of bilateral facial ice pack therapy with no ice therapy following third molar surgery. Int J Oral Maxillofac Surg. 2005 2005/05/01/;34(3):281-6. doi: https://doi.org/10.1016/j.ijom.2004.05.006. PMID: 15741037. 
174. Van Dyke T, Litkowski LJ, Kiersch TA, et al. Combination oxycodone $5 \mathrm{mg} /$ ibuprofen $400 \mathrm{mg}$ for the treatment of postoperative pain: a double-blind, placebo- and activecontrolled parallel-group study. Clin Ther. 2004 Dec;26(12):2003-14. doi: 10.1016/j.clinthera.2004.12.002. PMID: 15823764.

175. Vas J, Aranda JM, Modesto M, et al. Acupuncture in patients with acute low back pain: a multicentre randomised controlled clinical trial. Pain. 2012 Sep;153(9):1883-9. doi: 10.1016/j.pain.2012.05.033. PMID: 22770838.

176. Viscusi ER, Allard R, Sohns M, et al. Tapentadol immediate release for moderate to severe acute post-surgery pain. J Opioid Manag. 2019 Jan/Feb;15(1):51-67. doi: 10.5055/jom.2019.0486. PMID: 30855723.

177. Voelker M, Schachtel BP, Cooper SA, et al. Efficacy of disintegrating aspirin in two different models for acute mild-to-moderate pain: sore throat pain and dental pain. Inflammopharmacology. 2016 Feb;24(1):4351. doi: 10.1007/s10787-015-0253-0. PMID: 26603742.

178. von Heymann WJ, Schloemer P, Timm J, et al. Spinal high-velocity low amplitude manipulation in acute nonspecific low back pain: a double-blinded randomized controlled trial in comparison with diclofenac and placebo. Spine. 2013 Apr 01;38(7):540-8. doi:

10.1097/BRS.0b013e318275d09c. PMID: 23026869.

179. Vorsanger GJ, Farrell J, Xiang J, et al. Tapentadol, oxycodone or placebo for acute pain of vertebral compression fractures: a randomized Phase IIIb study. Pain Manag. 2013;3(2):109-18. PMID: 24645994.

180. Vorsanger GJ, Klopfer AM, Xiang J, et al. Immediate-release tapentadol or oxycodone for treatment of acute postoperative pain after elective arthroscopic shoulder surgery: a randomized, phase IIIb study. J Opioid Manag. 2013 Jul-Aug;9(4):281-90. doi: 10.5055/jom.2013.0170. PMID: 24353022.
181. Webster BS, Verma SK, Gatchel RJ. Relationship between early opioid prescribing for acute occupational low back pain and disability duration, medical costs, subsequent surgery and late opioid use. Spine. 2007 Sep 01;32(19):2127-32. PMID: 17762815.

182. Whitefield M, O'Kane CJ, Anderson S. Comparative efficacy of a proprietary topical ibuprofen gel and oral ibuprofen in acute soft tissue injuries: a randomized, double-blind study. J Clin Pharm Ther. 2002 Dec;27(6):409-17. PMID: 12472980.

183. Wilkinson MJ. Does 48 hours' bed rest influence the outcome of acute low back pain? Br J Gen Pract. 1995 Sep;45(398):481-4. PMID: 7546872.

184. Williamson JB, George TK, Simpson DC, et al. Ultrasound in the treatment of ankle sprains. Injury. 1986 May;17(3):176-8. doi: 10.1016/0020-1383(86)90327-x. PMID: 3102375.

185. Wirz S, Wartenberg H-C, Wittmann M, et al. Post-operative pain therapy with controlled release oxycodone or controlled release tramadol following orthopedic surgery: A prospective, randomized, doubleblind investigation. The Pain Clinic. 2005;17(4):367-76. doi: 10.1163/156856905774482733. PMID: 15259296

186. Woo WW, Man SY, Lam PK, et al. Randomized double-blind trial comparing oral paracetamol and oral nonsteroidal antiinflammatory drugs for treating pain after musculoskeletal injury. Ann Emerg Med. 2005 Oct;46(4):352-61. PMID: 16187469.

187. Zammit E, Herrington L. Ultrasound therapy in the management of acute lateral ligament sprains of the ankle joint. Phys Ther Sport. 2005;6(3):116-21.

188. Zandi M, Amini P, Keshavarz A. Effectiveness of cold therapy in reducing pain, trismus, and oedema after impacted mandibular third molar surgery: a randomized, self-controlled, observer-blind, split-mouth clinical trial. Int J Oral Maxillofac Surg. 2016 2016/01/01/;45(1):118-23. doi: https://doi.org/10.1016/j.ijom.2015.10.021. PMID: 26597577. 
189. Zhao M, Gao W, Zhang L, et al. Acupressure Therapy for Acute Ankle Sprains: A Randomized Clinical Trial. PM R. 2018 Jan;10(1):36-44. doi: 10.1016/j.pmrj.2017.06.009. PMID: 28634002.
190. Zupelari-Goncalves P, Weckwerth GM, Calvo AM, et al. Efficacy of oral diclofenac with or without codeine for pain control after invasive bilateral third molar extractions. Int J Oral Maxillofac Surg. 2017 May;46(5):621-7. doi: 10.1016/j.ijom.2017.01.008. PMID: 28161136. 


\section{Appendix D. Forest Plots}

\section{Key Question 6. Dental Pain}

Figure D-1. Rescue or repeat medication use for opioid versus NSAID for dental pain

\begin{tabular}{|c|c|c|c|c|c|c|c|c|c|c|}
\hline \multirow[b]{2}{*}{ Study or Subgroup } & \multicolumn{2}{|c|}{ Opioid } & \multicolumn{2}{|c|}{ NSAID } & \multirow[b]{2}{*}{ Weight } & Risk Ratio & \multirow{2}{*}{\multicolumn{4}{|c|}{$\begin{array}{c}\text { Risk Ratio } \\
\text { M-H, Random, } 95 \% \mathrm{Cl}\end{array}$}} \\
\hline & Events & Total & Events & Total & & M-H, Random, $95 \% \mathrm{Cl}$ & & & & \\
\hline \multicolumn{11}{|l|}{ 2.1.1 Single dose } \\
\hline Breivik 1999 & 8 & 23 & 8 & 22 & $1.4 \%$ & $0.96[0.44,2.10]$ & & & 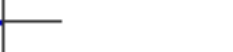 & \\
\hline Cattry 2020 Study 1 & 77 & 91 & 56 & 90 & $26.1 \%$ & $1.36[1.13,1.63]$ & & & - & \\
\hline Cattry 2020 Study 2 & 70 & 91 & 51 & 92 & $19.0 \%$ & $1.39[1.12,1.72]$ & & & - & \\
\hline Cooper 1988 & 18 & 31 & 19 & 36 & $4.7 \%$ & $1.10[0.72,1.69]$ & & & - & \\
\hline Cooper 1991 & 31 & 39 & 53 & 83 & $17.0 \%$ & $1.24[0.99,1.56]$ & & & - & \\
\hline Forbes 1990 & 32 & 38 & 56 & 98 & $18.2 \%$ & $1.47[1.18,1.84]$ & & & $\rightarrow$ & \\
\hline Malmstrom 2004 & 38 & 50 & 27 & 51 & $9.6 \%$ & $1.44[1.06,1.94]$ & & & - & \\
\hline Sunshine 1986 & 9 & 31 & 10 & 60 & $1.4 \%$ & $1.74[0.79,3.84]$ & & & & \\
\hline Subtotal $(95 \% \mathrm{Cl})$ & & 394 & & 532 & $97.5 \%$ & $1.36[1.23,1.49]$ & & & 1 & \\
\hline Total events & 283 & & 280 & & & & & & & \\
\hline \multirow{2}{*}{\multicolumn{11}{|c|}{$\begin{array}{l}\text { Heterogeneity: } \mathrm{Tau}^{2}=0.00 ; \mathrm{Chi}^{2}=3.33, \mathrm{df}=7(\mathrm{P}=0.85) ; \mathrm{I}^{2}=0 \% \\
\text { Test for overall effect: } Z=6.29(\mathrm{P}=0.00001)\end{array}$}} \\
\hline & & & & & & & & & & \\
\hline \multicolumn{11}{|l|}{ 2.1.2 Multidose } \\
\hline $\begin{array}{l}\text { Brown } 2013 \\
\text { Subtotal }(95 \% \mathrm{Cl})\end{array}$ & 12 & $\begin{array}{l}62 \\
62\end{array}$ & 34 & $\begin{array}{l}192 \\
192\end{array}$ & $\begin{array}{l}2.5 \% \\
2.5 \%\end{array}$ & $\begin{array}{l}1.09[0.60,1.98] \\
1.09[0.60,1.98]\end{array}$ & & & & \\
\hline Total events & 12 & & 34 & & & & & & & \\
\hline \multicolumn{11}{|c|}{ Heterogeneity: Not applicable } \\
\hline \multicolumn{11}{|c|}{ Test for overall effect: $Z=0.29(P=0.77)$} \\
\hline Total $(95 \% \mathrm{Cl})$ & & 456 & & 724 & $100.0 \%$ & $1.35[1.23,1.48]$ & & & 1 & \\
\hline Total events & 295 & & 314 & & & & & & & \\
\hline \multicolumn{7}{|c|}{ Heterogeneity: $\mathrm{Tau}^{2}=0.00 ; \mathrm{Chi}^{2}=3.88, \mathrm{df}=8(\mathrm{P}=0.87) ; \mathrm{I}^{2}=0 \%$} & 0.01 & $\begin{array}{l}0.1 \\
\text { Favors opioid }\end{array}$ & $\begin{array}{c}10 \\
\text { Favors NSAID }\end{array}$ & 100 \\
\hline
\end{tabular}

Abbreviations: $\mathrm{CI}$ = confidence interval; $\mathrm{df}$ = degrees of freedom; $\mathrm{M}-\mathrm{H}=$ Mantel-Haenszel; NSAID = nonsteroidal anti-inflammatory drug 
Figure D-2. Medication rated very good or excellent for opioid versus NSAID for dental pain

\begin{tabular}{|c|c|c|c|c|c|c|c|c|c|c|}
\hline \multirow{2}{*}{$\frac{\text { Study or Subgroup }}{2.2 .1 \text { Sinqle dose }}$} & \multicolumn{2}{|c|}{ Opioid } & \multicolumn{2}{|c|}{ NSAID } & \multicolumn{2}{|r|}{ Risk Ratio } & \multirow{2}{*}{\multicolumn{4}{|c|}{$\begin{array}{c}\text { Risk Ratio } \\
\text { M-H, Random, } 95 \% \mathrm{Cl} \\
\end{array}$}} \\
\hline & Events & Iotal & & Iotal & Weight & $\mathrm{IM}-\mathrm{H}$, Kandom, 95\% Cl & & & & \\
\hline Cattry 2020 Study 1 & 21 & 91 & 37 & 90 & $13.1 \%$ & $0.56[0.36,0.88]$ & & $\rightarrow$ & & \\
\hline Cattry 2020 Study 2 & 32 & 91 & 39 & 92 & $14.7 \%$ & $0.83[0.57,1.20]$ & & $\rightarrow$ & & \\
\hline Cooper 1988 & 15 & 31 & 17 & 19 & $14.2 \%$ & $0.54[0.36,0.80]$ & & $\rightarrow$ & & \\
\hline Cooper 1991 & 14 & 39 & 39 & 83 & $12.5 \%$ & $0.76[0.47,1.23]$ & & $\rightarrow$ & & \\
\hline $\begin{array}{l}\text { Malmstrom } 2004 \\
\text { Subtotal }(95 \% \mathrm{Cl})\end{array}$ & 24 & $\begin{array}{r}50 \\
302\end{array}$ & 43 & $\begin{array}{r}51 \\
335\end{array}$ & $\begin{array}{l}15.8 \% \\
70.3 \%\end{array}$ & $\begin{array}{l}0.57[0.42,0.78] \\
0.64[0.53,0.76]\end{array}$ & & $\rightarrow-$ & & \\
\hline $\begin{array}{l}\text { Total events } \\
\text { Heterogeneity: } \text { Tau }^{2}=0.00 \\
\text { Test for overall effect: } Z=6\end{array}$ & $\begin{aligned} & 106 \\
\mathrm{Chi}^{2} & =4.1 \\
05(\mathrm{P} & <0 .\end{aligned}$ & $\begin{array}{l}2, d f= \\
00001)\end{array}$ & $\begin{array}{r}175 \\
4(P=0\end{array}$ & $39) ; 1^{2}=$ & $=3 \%$ & & & & & \\
\hline 2.2.2 Multidose & & & & & & & & & & \\
\hline Brown 2013 & 29 & 62 & 115 & 192 & $16.3 \%$ & $0.78[0.58,1.04]$ & & $\rightarrow$ & & \\
\hline $\begin{array}{l}\text { Zupelari-Goncalves } 2017 \\
\text { Subtotal }(95 \% \mathrm{Cl})\end{array}$ & 30 & $\begin{array}{r}46 \\
108\end{array}$ & 17 & $\begin{array}{r}46 \\
238\end{array}$ & $\begin{array}{l}13.4 \% \\
29.7 \%\end{array}$ & $\begin{array}{l}1.76[1.15,2.72] \\
1.15[0.52,2.57]\end{array}$ & & & & \\
\hline $\begin{array}{l}\text { Total events } \\
\text { Heterogeneity: } \operatorname{Tau}^{2}=0.30 \\
\text { Test for overall effect: } Z=0\end{array}$ & $\begin{aligned} & 59 \\
\mathrm{Chi}^{2} & =9.4 \\
35 & (\mathrm{P}=0 .\end{aligned}$ & $\begin{array}{l}\text { 13, df }= \\
72)\end{array}$ & $\begin{array}{r}132 \\
1(\mathrm{P}=0 .\end{array}$ & $002) ; 1^{2}$ & $=89 \%$ & & & & & \\
\hline Total $(95 \% \mathrm{Cl})$ & & 410 & & 573 & $100.0 \%$ & $0.76[0.57,1.00]$ & & & & \\
\hline Total events & 165 & & 307 & & & & & & & \\
\hline $\begin{array}{l}\text { Heterogeneity: } \operatorname{Tau}^{2}=0.10 \\
\text { Test for overall effect: } Z=1\end{array}$ & $\begin{array}{l}\mathrm{Chi}^{2}=22 \\
95(\mathrm{P}=0 . \\
\mathrm{Chi}^{2}=\end{array}$ & $\begin{array}{l}78, \mathrm{df} \\
05) \\
205\end{array}$ & $=6(P=0$ & 000 & $i_{1}^{2}=74 \%$ & & 0.01 & $\begin{array}{c}0.1 \\
\text { Favors NSAID }\end{array}$ & $\begin{array}{c}10 \\
\text { Favors opioid }\end{array}$ & 100 \\
\hline
\end{tabular}

Abbreviations: $\mathrm{CI}$ = confidence interval; $\mathrm{df}$ = degrees of freedom; $\mathrm{M}-\mathrm{H}$ = Mantel-Haenszel; NSAID = nonsteroidal anti-inflammatory drug 
Figure D-3. Any adverse event for opioid versus NSAID for dental pain

\begin{tabular}{|c|c|c|c|c|c|c|c|c|c|}
\hline \multirow{2}{*}{$\frac{\text { Study or Subgroup }}{2.3 .1 \text { Single dose }}$} & \multicolumn{2}{|c|}{ Opioid } & \multicolumn{2}{|c|}{ NSAID } & \multicolumn{2}{|r|}{ Risk Ratio } & \multicolumn{3}{|c|}{$\begin{array}{c}\text { Risk Ratio } \\
\text { M-H, Random, } 95 \% \mathrm{Cl} \\
\end{array}$} \\
\hline & & & & & & & & & \\
\hline Cattry 2020 Study 1 & 23 & 92 & 6 & 91 & $7.5 \%$ & $3.79[1.62,8.88]$ & & & $\longrightarrow$ \\
\hline Cattry 2020 Study 2 & 37 & 91 & 28 & 92 & $15.5 \%$ & $1.34[0.90,1.99]$ & & & $=$ \\
\hline Cooper 1982 & 41 & 127 & 20 & 76 & $14.2 \%$ & $1.23[0.78,1.93]$ & & & - \\
\hline Cooper 1991 & 8 & 39 & 7 & 83 & $6.5 \%$ & $2.43[0.95,6.23]$ & & & \\
\hline Dionne 1994 & 9 & 24 & 8 & 48 & $7.9 \%$ & $2.25[0.99,5.09]$ & & & \\
\hline Forbes 1990 & 8 & 40 & 21 & 125 & $9.0 \%$ & $1.19[0.57,2.48]$ & & & \\
\hline Malmstrom 2004 & 25 & 50 & 19 & 51 & $14.3 \%$ & $1.34[0.85,2.11]$ & & & $=$ \\
\hline Sunshine 1986 & 3 & 31 & 0 & 60 & $0.9 \%$ & $13.34[0.71,250.43]$ & & & \\
\hline $\begin{array}{l}\text { Van Dyke } 2004 \\
\text { Subtotal }(95 \% \mathrm{Cl})\end{array}$ & 46 & $\begin{array}{l}250 \\
744\end{array}$ & 20 & $\begin{array}{l}186 \\
812\end{array}$ & $\begin{array}{l}13.4 \% \\
89.1 \%\end{array}$ & $\begin{array}{l}1.71[1.05,2.79] \\
1.59[1.25,2.02]\end{array}$ & & & $\bar{\bullet}$ \\
\hline \multicolumn{10}{|c|}{$\begin{array}{l}\text { Heterogeneity: } \text { Tau }^{2}=0.03 ; \mathrm{Chi}^{2}=10.86, \mathrm{df}=8(\mathrm{P}=0.21) ; \mathrm{I}^{2}=26 \% \\
\text { Test for overall effect: } \mathrm{Z}=3.77(\mathrm{P}=0.0002)\end{array}$} \\
\hline \multicolumn{10}{|l|}{ 2.3.2 Multidose } \\
\hline Akinbade 2019 & 25 & 45 & 0 & 45 & $1.0 \%$ & $51.00[3.20,812.96]$ & & & \\
\hline $\begin{array}{l}\text { Brown } 2013 \\
\text { Subtotal }(95 \% \mathrm{Cl})\end{array}$ & 11 & $\begin{array}{r}62 \\
107\end{array}$ & 20 & $\begin{array}{l}192 \\
237\end{array}$ & $\begin{array}{r}9.8 \% \\
10.9 \%\end{array}$ & $\begin{array}{r}1.70[0.86,3.35] \\
7.97[0.10,655.44]\end{array}$ & & & \\
\hline \multicolumn{9}{|c|}{$\begin{array}{l}\text { Total events } \quad 36 \quad 20 \\
\text { Heterogeneity: } \text { Tau }^{2}=9.15 ; \mathrm{Chi}^{2}=9.65, \mathrm{df}=1(\mathrm{P}=0.002) ; \mathrm{I}^{2}=90 \% \\
\text { Test for overall effect: } Z=0.92(\mathrm{P}=0.36)\end{array}$} & \\
\hline Total $(95 \% \mathrm{Cl})$ & & 851 & & 1049 & $100.0 \%$ & $1.72[1.29,2.28]$ & & & 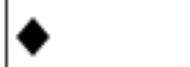 \\
\hline Total events & 236 & & 149 & & & & & & \\
\hline $\begin{array}{l}\text { Heterogeneity: } \mathrm{Tau}^{2}= \\
\text { Test for overall effect } \\
\text { Test for subgroup dif }\end{array}$ & $\begin{array}{l}0.10 ; \text { Chi } \\
Z=3.71 \\
\text { erences: }\end{array}$ & $\begin{array}{l}\mathrm{P}=18.9 \\
\mathrm{P}=0.0 \\
\mathrm{Chi}^{2}=0\end{array}$ & $\begin{array}{l}97, \mathrm{df}=1 \\
002) \\
0.51, \mathrm{df}=\end{array}$ & $0(P=0$ & $0.04) ; 1^{2}$ & $47 \%$ & 0.002 & $\begin{array}{c}0.1 \\
\text { Favors opioid }\end{array}$ & $\begin{array}{c}10 \\
\text { Favors NS }\end{array}$ \\
\hline
\end{tabular}

Abbreviations: $\mathrm{CI}$ = confidence interval; $\mathrm{df}$ = degrees of freedom; $\mathrm{M}-\mathrm{H}$ = Mantel-Haenszel; NSAID = nonsteroidal anti-inflammatory drug 
Figure D-4. Nausea for opioid versus NSAID for dental pain

\begin{tabular}{|c|c|c|c|c|c|c|c|c|c|c|}
\hline \multirow{2}{*}{$\frac{\text { Study or Subgroup }}{2.4 .1 \text { Single dose }}$} & \multicolumn{2}{|c|}{ Opioid } & \multicolumn{2}{|c|}{ NSAID } & Weight & $\begin{array}{l}\quad \text { Risk Ratio } \\
\text { M.H. Random } 95 \% \mathrm{Cl}\end{array}$ & \multicolumn{3}{|c|}{$\begin{array}{c}\text { Risk Ratio } \\
\text { M-H, Random, } 95 \% \mathrm{Cl}\end{array}$} & \\
\hline & Events & Total & Events & Total & vveigint & M-H, Random, $95 \% \mathrm{Cl}$ & & \\
\hline Breivik 1999 & 0 & 23 & 1 & 24 & $1.5 \%$ & $0.35[0.01,8.11]$ & & & & \\
\hline Cattry 2020 Study 1 & 8 & 92 & 1 & 91 & $3.6 \%$ & $7.91[1.01,62.00]$ & & & & \\
\hline Cattry 2020 Study 2 & 21 & 91 & 11 & 92 & $34.0 \%$ & $1.93[0.99,3.77]$ & & & $\longrightarrow$ & \\
\hline Cooper 1982 & 6 & 127 & 2 & 76 & $6.1 \%$ & $1.80[0.37,8.67]$ & & & & \\
\hline Cooper 1991 & 1 & 39 & 0 & 83 & $1.5 \%$ & $6.30[0.26,151.25]$ & & & & \\
\hline Dionne 1994 & 2 & 24 & 1 & 48 & $2.8 \%$ & $4.00[0.38,41.94]$ & & & & \\
\hline Forbes 1990 & 1 & 40 & 1 & 125 & $2.0 \%$ & $3.13[0.20,48.83]$ & & & & \\
\hline Malmstrom 2004 & 8 & 50 & 2 & 51 & $6.8 \%$ & $4.08[0.91,18.28]$ & & & & \\
\hline Moore 2015 & 12 & 367 & 2 & 182 & $6.9 \%$ & $2.98[0.67,13.15]$ & & & & \\
\hline Van Dyke 2004 & 25 & 250 & 7 & 186 & $22.9 \%$ & $2.66[1.17,6.01]$ & & & & \\
\hline Subtotal $(95 \% \mathrm{Cl})$ & & 1103 & & 958 & $88.1 \%$ & $2.48[1.64,3.76]$ & & & & \\
\hline Total events & 84 & & 28 & & & & & & & \\
\hline \multicolumn{11}{|c|}{$\begin{array}{l}\text { Heterogeneity: } \operatorname{Tau}^{2}=0.00 ; \mathrm{Chi}^{2}=4.48, \mathrm{df}=9(\mathrm{P}=0.88) ; \mathrm{I}^{2}=0 \% \\
\text { Test for overall effect: } Z=4.28(P=0.0001)\end{array}$} \\
\hline \multicolumn{11}{|l|}{ 2.4.2 Multidose } \\
\hline Akinbade 2019 & 5 & 45 & 0 & 45 & $1.9 \%$ & $11.00[0.63,193.25]$ & & & & \\
\hline $\begin{array}{l}\text { Brown } 2013 \\
\text { Subtotal }(95 \% \mathrm{Cl})\end{array}$ & 6 & $\begin{array}{r}62 \\
107\end{array}$ & 4 & $\begin{array}{l}192 \\
237\end{array}$ & $\begin{array}{l}10.0 \% \\
11.9 \%\end{array}$ & $\begin{array}{l}4.65[1.35,15.93] \\
5.31[1.71,16.49]\end{array}$ & & & & \\
\hline Total events & 11 & & 4 & & & & & & & \\
\hline \multicolumn{11}{|c|}{$\begin{array}{l}\text { Heterogeneity: } \operatorname{Tau}^{2}=0.00 ; \mathrm{Chi}^{2}=0.33, \mathrm{df}=1(\mathrm{P}=0.57) ; \mathrm{I}^{2}=0 \% \\
\text { Test for overall effect: } Z=2.89(P=0.004)\end{array}$} \\
\hline Total $(95 \% \mathrm{Cl})$ & & 1210 & & 1195 & $100.0 \%$ & $2.72[1.84,4.01]$ & & & & \\
\hline Total events & 95 & & 32 & & & & & & & \\
\hline $\begin{array}{l}\text { Heterogeneity: } \operatorname{Tau}^{2}= \\
\text { Test for overall effect }\end{array}$ & $\begin{array}{l}0.00 ; \mathrm{Chi} \\
\mathrm{Z}=5.02\end{array}$ & $\begin{array}{l}=6.31 \\
P<0.0\end{array}$ & $\begin{array}{l}1, d f=11 \\
0001)\end{array}$ & $(P=0.8$ & $85) ;\left.\right|^{2}=09$ & & 0.01 & $\begin{array}{l}0.1 \\
\text { Favors opioid }\end{array}$ & $\begin{array}{c}10 \\
\text { Favors NSAID }\end{array}$ & 100 \\
\hline
\end{tabular}

Abbreviations: $\mathrm{CI}$ = confidence interval; $\mathrm{df}$ = degrees of freedom; $\mathrm{M}-\mathrm{H}=$ Mantel-Haenszel; NSAID = nonsteroidal anti-inflammatory drug 
Figure D-5. Dizziness for opioid versus NSAID for dental pain

\begin{tabular}{|c|c|c|c|c|c|c|c|c|c|c|}
\hline \multirow{2}{*}{$\frac{\text { Study or Subgroup }}{2.6 .1 \text { Single dose }}$} & \multicolumn{2}{|c|}{ Opioid } & \multicolumn{2}{|c|}{ NSAID } & \multirow[t]{2}{*}{ Weight } & $\begin{array}{c}\text { Risk Ratio } \\
\text { M-H. Random. } 95 \% \mathrm{Cl}\end{array}$ & \multicolumn{4}{|c|}{$\begin{array}{c}\text { Risk Ratio } \\
\text { M-H, Random, } 95 \% \mathrm{Cl}\end{array}$} \\
\hline & & & & & & & & & & \\
\hline Cattry 2020 Study 1 & 7 & 92 & 0 & 91 & $4.8 \%$ & $14.84[0.86,256.06]$ & & & & \\
\hline Cattry 2020 Study 2 & 11 & 91 & 2 & 92 & $17.8 \%$ & $5.56[1.27,24.39]$ & & & & \\
\hline Cooper 1982 & 4 & 127 & 0 & 76 & $4.6 \%$ & $5.41[0.30,99.19]$ & & & & \\
\hline Cooper 1991 & 2 & 39 & 0 & 83 & $4.3 \%$ & $10.50[0.52,213.64]$ & & & & \\
\hline Forbes 1990 & 2 & 40 & 9 & 125 & $17.5 \%$ & $0.69[0.16,3.08]$ & & & & \\
\hline Malmstrom 2004 & 5 & 50 & 1 & 51 & $8.7 \%$ & $5.10[0.62,42.12]$ & & & & \\
\hline Moore 2015 & 9 & 367 & 2 & 182 & $16.8 \%$ & $2.23[0.49,10.22]$ & & & & \\
\hline $\begin{array}{l}\text { Van Dyke } 2004 \\
\text { Subtotal }(95 \% \mathrm{Cl})\end{array}$ & 8 & $\begin{array}{r}250 \\
1056\end{array}$ & 2 & $\begin{array}{l}186 \\
886\end{array}$ & $\begin{array}{l}16.5 \% \\
91.1 \%\end{array}$ & $\begin{array}{r}2.98[0.64,13.85] \\
3.02[1.57,5.81]\end{array}$ & & & & \\
\hline \multicolumn{11}{|c|}{$\begin{array}{l}\text { Heterogeneity: } \operatorname{Tau}^{2}=0.00 ; \mathrm{Chi}^{2}=6.89, \mathrm{df}=7(\mathrm{P}=0.44) ; \mathrm{I}^{2}=0 \% \\
\text { Test for overall effect: } Z=3.31(\mathrm{P}=0.0009)\end{array}$} \\
\hline \multicolumn{11}{|l|}{ 2.6.2 Multidose } \\
\hline Akinbade 2019 & 4 & 45 & 0 & 45 & $4.7 \%$ & $9.00[0.50,162.43]$ & & & & \\
\hline $\begin{array}{l}\text { Brown } 2013 \\
\text { Subtotal }(95 \% \mathrm{Cl})\end{array}$ & 0 & $\begin{array}{r}62 \\
107\end{array}$ & 2 & $\begin{array}{l}192 \\
237\end{array}$ & $\begin{array}{l}4.3 \% \\
8.9 \%\end{array}$ & $\begin{array}{l}0.61[0.03,12.59] \\
2.44[0.17,34.85]\end{array}$ & & & & \\
\hline \multicolumn{11}{|c|}{$\begin{array}{l}\text { Heterogeneity: } \operatorname{Tau}^{2}=1.41 ; \mathrm{Chi}^{2}=1.62, \mathrm{df}=1(\mathrm{P}=0.20) ; \mathrm{I}^{2}=38 \% \\
\text { Test for overall effect: } Z=0.66(P=0.51)\end{array}$} \\
\hline Total $(95 \% \mathrm{Cl})$ & & 1163 & & 1123 & $100.0 \%$ & $2.97[1.59,5.54]$ & & & & \\
\hline Total events & 52 & & 18 & & & & & & & \\
\hline $\begin{array}{l}\text { Heterogeneity: } \mathrm{Tau}^{2}= \\
\text { Test for overall effect: }\end{array}$ & $\begin{array}{l}0.00 ; \mathrm{Chi}^{2} \\
\mathrm{Z}=3.42(\end{array}$ & $\begin{array}{l}\mathrm{P}=8.5 \\
\mathrm{P}=0.0 \\
\mathrm{Chi}^{2}=1\end{array}$ & $\begin{array}{l}3 \mathrm{df}=9 \\
006)\end{array}$ & $P=0$. & $8) ; 1^{2}=0 \%$ & & 0.01 & $\begin{array}{l}0.1 \\
\text { Favors opioid }\end{array}$ & Favors NSAID & 100 \\
\hline
\end{tabular}

Abbreviations: $\mathrm{CI}$ = confidence interval; $\mathrm{df}$ = degrees of freedom; $\mathrm{M}-\mathrm{H}$ = Mantel-Haenszel; NSAID = nonsteroidal anti-inflammatory drug 
Figure D-6. Drowsiness for opioid versus NSAID for dental pain

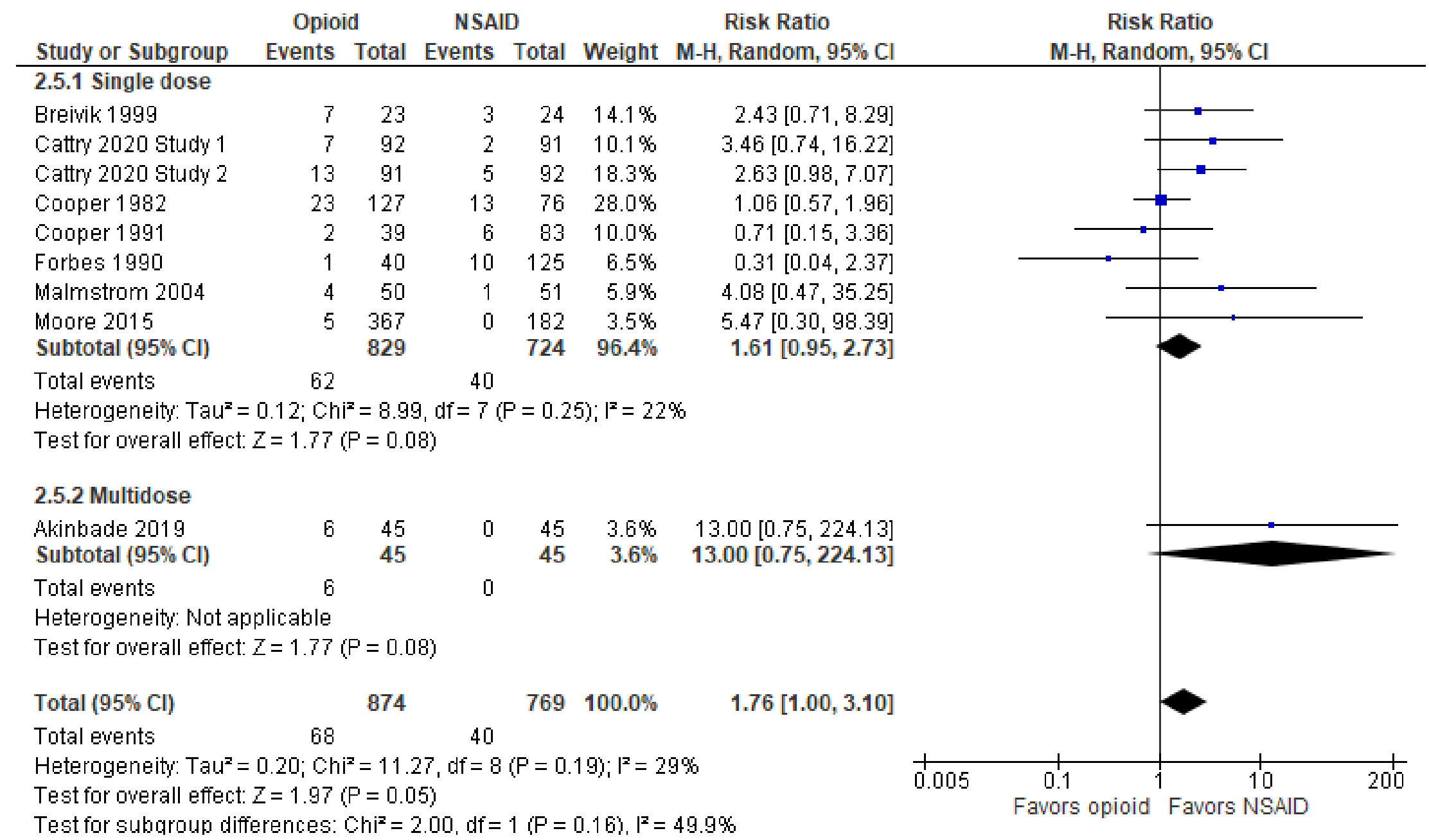

Abbreviations: $\mathrm{CI}$ = confidence interval; $\mathrm{df}$ = degrees of freedom; $\mathrm{M}-\mathrm{H}$ = Mantel-Haenszel; NSAID = nonsteroidal anti-inflammatory drug 
Figure D-7. Headache for opioid versus NSAID for dental pain

\begin{tabular}{|c|c|c|c|c|c|c|c|c|c|c|}
\hline \multirow{2}{*}{$\begin{array}{l}\text { Study or Subgroup } \\
2.7 .1 \text { Single dose }\end{array}$} & \multicolumn{2}{|c|}{ Opioid } & \multicolumn{2}{|c|}{ NSAID } & Weight & \multicolumn{2}{|l|}{ Risk Ratio } & \multicolumn{2}{|c|}{$\begin{array}{c}\text { Risk Ratio } \\
\text { M-H, Random, } 95 \% \mathrm{Cl}\end{array}$} & \\
\hline & & & & & & & & & & \\
\hline Cattry 2020 Study 1 & 1 & 92 & 2 & 91 & $8.5 \%$ & $0.49[0.05,5.36]$ & & & & \\
\hline Cattry 2020 Study 2 & 5 & 91 & 7 & 92 & $24.4 \%$ & $0.72[0.24,2.19]$ & & & & \\
\hline Cooper 1982 & 0 & 127 & 1 & 76 & $5.1 \%$ & $0.20[0.01,4.86]$ & & & & \\
\hline Cooper 1991 & 2 & 39 & 0 & 83 & $5.7 \%$ & $10.50[0.52,213.64]$ & & & & \\
\hline Dionne 1994 & 0 & 24 & 3 & 48 & $6.0 \%$ & $0.28[0.02,5.21]$ & & & & \\
\hline Forbes 1990 & 0 & 40 & 3 & 125 & $5.9 \%$ & $0.44[0.02,8.32]$ & & & & \\
\hline Malmstrom 2004 & 7 & 50 & 9 & 51 & $29.5 \%$ & $0.79[0.32,1.97]$ & & & & \\
\hline $\begin{array}{l}\text { Moore } 2015 \\
\text { Subtotal }(95 \% \mathrm{Cl})\end{array}$ & 1 & $\begin{array}{l}367 \\
830\end{array}$ & 0 & $\begin{array}{l}182 \\
748\end{array}$ & $\begin{array}{r}5.1 \% \\
90.1 \%\end{array}$ & $\begin{array}{r}1.49[0.06,36.44] \\
0.75[0.41,1.38]\end{array}$ & & & & \\
\hline $\begin{array}{l}\text { Total events } \\
\text { Heterogeneity: Tau }= \\
\text { Test for overall effect }\end{array}$ & $\begin{array}{c}16 \\
0.00 ; \mathrm{Chi}^{\prime} \\
z=0.92(\end{array}$ & $\begin{array}{l}i^{2}=4.49 \\
(P=0.3\end{array}$ & $\begin{array}{l}25 \\
9, d f=7(t \\
6)\end{array}$ & $=0.7$ & $2) ; I^{2}=0 \%$ & & & & & \\
\hline 2.7.2 Multidose & & & & & & & & & & \\
\hline $\begin{array}{l}\text { Brown } 2013 \\
\text { Subtotal }(95 \% \mathrm{Cl})\end{array}$ & 4 & $\begin{array}{l}62 \\
62\end{array}$ & 1 & $\begin{array}{l}192 \\
192\end{array}$ & $\begin{array}{l}9.9 \% \\
9.9 \%\end{array}$ & $\begin{array}{l}12.39[1.41,108.76] \\
12.39[1.41,108.76]\end{array}$ & & & & $\longrightarrow$ \\
\hline $\begin{array}{l}\text { Total events } \\
\text { Heterogeneity: Not a } \\
\text { Test for overall effect }\end{array}$ & $\begin{array}{l}4 \\
\text { olicable } \\
z=2.270\end{array}$ & $P=0.0$ & 1 & & & & & & & \\
\hline Total $(95 \% \mathrm{Cl})$ & & 892 & & 940 & $100.0 \%$ & $0.99[0.46,2.11]$ & & & & \\
\hline Total events & 20 & & 26 & & & & & & & \\
\hline $\begin{array}{l}\text { Heterogeneity: Tau } \\
\text { Test for overall effect } \\
\text { Test for subqroup dif }\end{array}$ & $\begin{array}{l}0.29 ; \mathrm{Chi}^{2} \\
\mathrm{Z}=0.03( \\
\text { rences: }\end{array}$ & $\begin{array}{l}\mathrm{j}^{2}=10.4 \\
\mathrm{P}=0.9 \\
\mathrm{Chi}^{2}=5\end{array}$ & $\begin{array}{l}\text { 47, } \mathrm{df}=8 \\
\text { 7) } \\
5.92, \mathrm{df}=\end{array}$ & $(P=0$. & $23) ;\left.\right|^{2}=2$ & $4 \%$ & 0.01 & $\begin{array}{l}0.1 \\
\text { Favors opioid }\end{array}$ & Favors NSAID & 100 \\
\hline
\end{tabular}

Abbreviations: $\mathrm{CI}$ = confidence interval; $\mathrm{df}$ = degrees of freedom; $\mathrm{M}-\mathrm{H}$ = Mantel-Haenszel; NSAID = nonsteroidal anti-inflammatory drug 
Figure D-8. Rescue or repeat medication use for opioid versus acetaminophen for dental pain

\begin{tabular}{|c|c|c|c|c|c|c|c|c|}
\hline \multirow[b]{2}{*}{ Study or Subgroup } & \multicolumn{2}{|c|}{ Opioid } & \multicolumn{2}{|c|}{ Acetaminophen } & \multicolumn{2}{|r|}{ Risk Ratio } & \multirow{2}{*}{\multicolumn{2}{|c|}{$\begin{array}{c}\text { Risk Ratio } \\
\text { M-H, Random, } 95 \% \mathrm{Cl}\end{array}$}} \\
\hline & Events & Total & Events & Total & Weight & M-H, Random, $95 \% \mathrm{Cl}$ & & \\
\hline \multicolumn{9}{|l|}{ 1.1.1 Single dose } \\
\hline Bentley 1987 & 16 & 41 & 27 & 41 & $11.9 \%$ & $0.59[0.38,0.92]$ & $\rightarrow$ & \\
\hline Breivik 1999 & 8 & 23 & 10 & 22 & $5.4 \%$ & $0.77[0.37,1.58]$ & $\rightarrow$ & - \\
\hline Cooper 1981 & 2 & 37 & 5 & 42 & $1.2 \%$ & $0.45[0.09,2.20]$ & & \\
\hline Cooper 1988 & 18 & 31 & 28 & 36 & $16.6 \%$ & $0.75[0.53,1.06]$ & $\rightarrow-$ & \\
\hline Cooper 1991 & 31 & 39 & 35 & 37 & $30.6 \%$ & $0.84[0.70,1.00]$ & $=$ & \\
\hline Forbes 1990 & 32 & 38 & 30 & 36 & $28.2 \%$ & $1.01[0.83,1.24]$ & 1 & 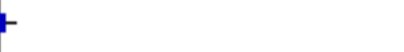 \\
\hline Sunshine 1986 & 9 & 31 & 14 & 30 & $6.1 \%$ & $0.62[0.32,1.22]$ & 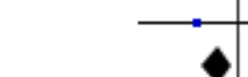 & \\
\hline $\begin{array}{l}\text { Subtotal }(95 \% \mathrm{Cl}) \\
\text { Total events }\end{array}$ & 116 & 240 & 149 & 244 & $100.0 \%$ & $0.81[0.67,0.97]$ & $\nabla$ & \\
\hline \multicolumn{9}{|c|}{$\begin{array}{l}\text { Heterogeneity: } \text { Tau }^{2}=0.02 ; \mathrm{Chi}^{2}=9.76, \mathrm{df}=6(\mathrm{P}=0.14) ; \mathrm{I}^{2}=39 \% \\
\text { Test for overall effect: } Z=2.35(\mathrm{P}=0.02)\end{array}$} \\
\hline Total $(95 \% \mathrm{Cl})$ & & 240 & & 244 & $100.0 \%$ & $0.81[0.67,0.97]$ & $\boldsymbol{\nabla}$ & \\
\hline Total events & 116 & & 149 & & & & & \\
\hline $\begin{array}{l}\text { Heterogeneity: } \operatorname{Tau}^{2} \\
\text { Test for overall effec } \\
\text { Test for subqroup di }\end{array}$ & $\begin{array}{l}0.02 ; \mathrm{Ch} \\
Z=2.35 \\
\text { erences: }\end{array}$ & $\begin{array}{l}P=9.76 \\
P=0.0 \\
\text { Vot app }\end{array}$ & $\begin{array}{l}\text { idf }=6(P \\
\text { 2) }\end{array}$ & $4 ; 1^{2}$ & $=39 \%$ & 0.01 & $\begin{array}{l}0.1 \\
\text { Favors opioid }\end{array}$ & $\begin{array}{cc}10 & 100 \\
\text { Favors acetaminophen }\end{array}$ \\
\hline
\end{tabular}

Abbreviations: $\mathrm{CI}$ = confidence interval; $\mathrm{df}=$ degrees of freedom; $\mathrm{M}-\mathrm{H}=$ Mantel-Haenszel 
Figure D-9. Any adverse event for opioid + acetaminophen versus acetaminophen for dental pain

\begin{tabular}{|c|c|c|c|c|c|c|c|c|c|c|}
\hline \multirow[b]{2}{*}{ Study or Subgroup } & \multicolumn{2}{|c|}{ Opioid + acetaminophen } & \multicolumn{2}{|c|}{ Acetaminophen } & \multicolumn{2}{|r|}{ Risk Ratio } & \multirow{2}{*}{\multicolumn{3}{|c|}{$\begin{array}{l}\text { Risk Ratio } \\
\text { M-H, Random, } 95 \% \mathrm{Cl}\end{array}$}} & \\
\hline & Events & Total & Events & Total & Weight & M-H, Random, $95 \% \mathrm{Cl}$ & & & & \\
\hline \multicolumn{11}{|l|}{ 1.2.1 Single dose } \\
\hline Bentley 1987 & 23 & 64 & 21 & 42 & $18.3 \%$ & $0.72[0.46,1.12]$ & & & & \\
\hline Cooper 1980 & 77 & 172 & 3 & 37 & $10.5 \%$ & $5.52[1.84,16.54]$ & & & & \\
\hline Cooper 1981 & 10 & 42 & 12 & 37 & $14.9 \%$ & $0.73[0.36,1.50]$ & & & & \\
\hline Cooper 1991 & 8 & 39 & 6 & 37 & $12.0 \%$ & $1.26[0.49,3.30]$ & & & & \\
\hline Dionne 1994 & 9 & 24 & 7 & 27 & $13.6 \%$ & $1.45[0.64,3.29]$ & & & & \\
\hline Forbes 1990 & 8 & 40 & 5 & 41 & $11.2 \%$ & $1.64[0.59,4.59]$ & & & & \\
\hline Skoglund 1991 & 13 & 37 & 12 & 69 & $15.4 \%$ & $2.02[1.03,3.97]$ & & & & \\
\hline Sunshine 1986 & 3 & 31 & 1 & 30 & $4.2 \%$ & $2.90[0.32,26.38]$ & & & & \\
\hline Subtotal $(95 \% \mathrm{Cl})$ & & 449 & & 320 & $100.0 \%$ & $1.43[0.87,2.37]$ & & & & \\
\hline Total events & 151 & & 67 & & & & & & & \\
\hline \multirow{2}{*}{\multicolumn{11}{|c|}{$\begin{array}{l}\text { Heterogeneity: } \mathrm{Tau}^{2}=0.31 ; \mathrm{Ch}^{2}=19.84, \mathrm{df}=7(\mathrm{P}=0.006) ; \mathrm{I}^{2}=65 \% \\
\text { Test for overall effect: } Z=1.41(\mathrm{P}=0.16)\end{array}$}} \\
\hline & & & & & & & & & & \\
\hline Total $(95 \% \mathrm{Cl})$ & & 449 & & 320 & $100.0 \%$ & $1.43[0.87,2.37]$ & & & & \\
\hline Total events & 151 & & 67 & & & & & & & \\
\hline \multicolumn{7}{|c|}{$\begin{array}{l}\text { Heterogeneity: } \operatorname{Tau}^{2}=0.31 ; \mathrm{Ch}^{2}=19.84, \mathrm{df}=7(\mathrm{P}=0.006) ;\left.\right|^{2}=65 \% \\
\text { Test for overall effect: } Z=1.41(\mathrm{P}=0.16)\end{array}$} & 0.01 & $\begin{array}{l}0.1 \\
\text { Favors opioid }\end{array}$ & Favors ace & $\begin{array}{cc}10 & 100 \\
\text { etaminophen }\end{array}$ \\
\hline
\end{tabular}

Abbreviations: $\mathrm{CI}$ = confidence interval; $\mathrm{df}$ = degrees of freedom; $\mathrm{M}-\mathrm{H}$ = Mantel-Haenszel 
Figure D-10. Nausea for opioid versus acetaminophen for dental pain

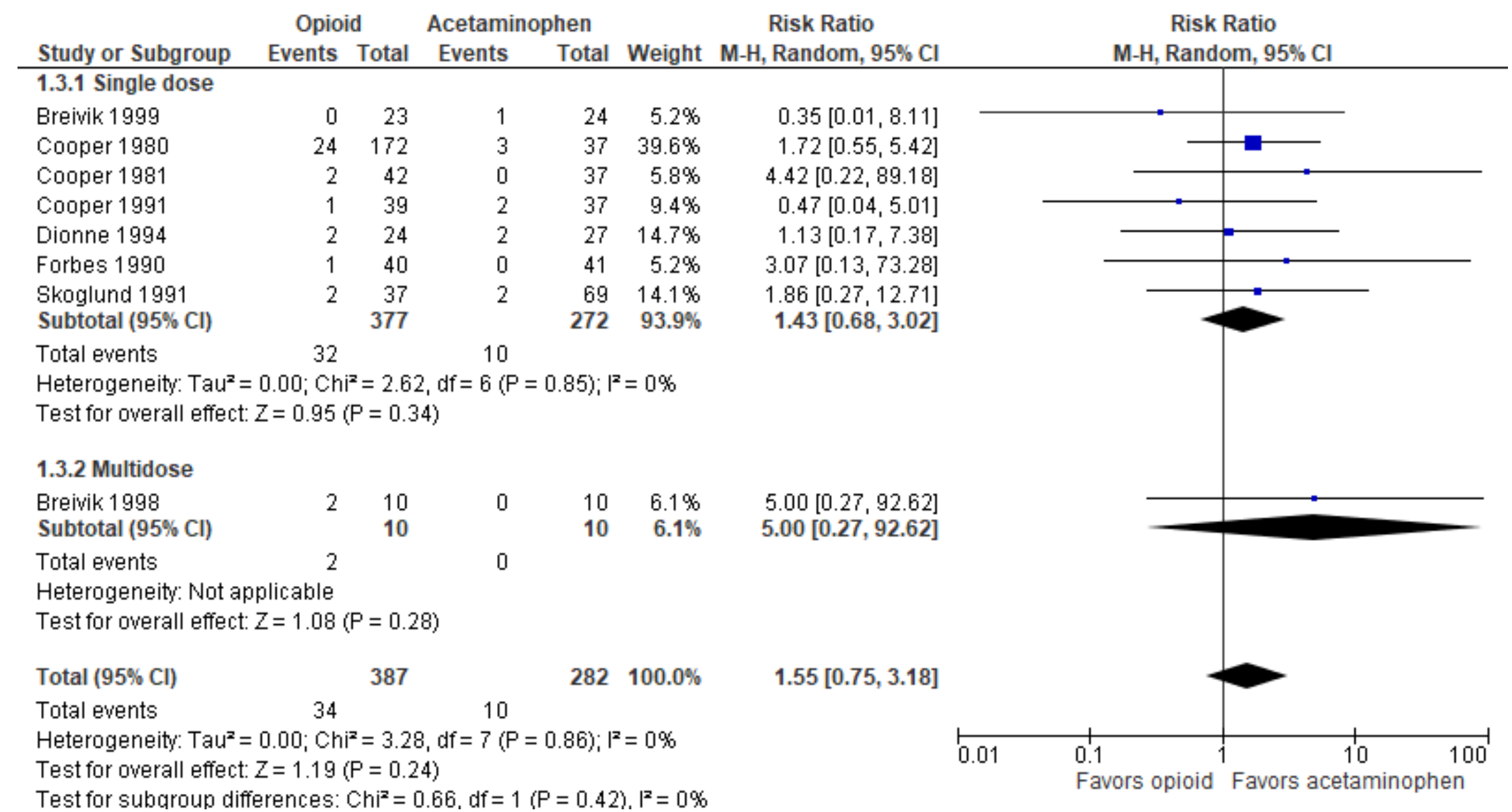

Abbreviations: $\mathrm{CI}=$ confidence interval; $\mathrm{df}=$ degrees of freedom; $\mathrm{M}-\mathrm{H}=$ Mantel - Haenszel 
Figure D-11. Drowsiness for opioid versus acetaminophen for dental pain

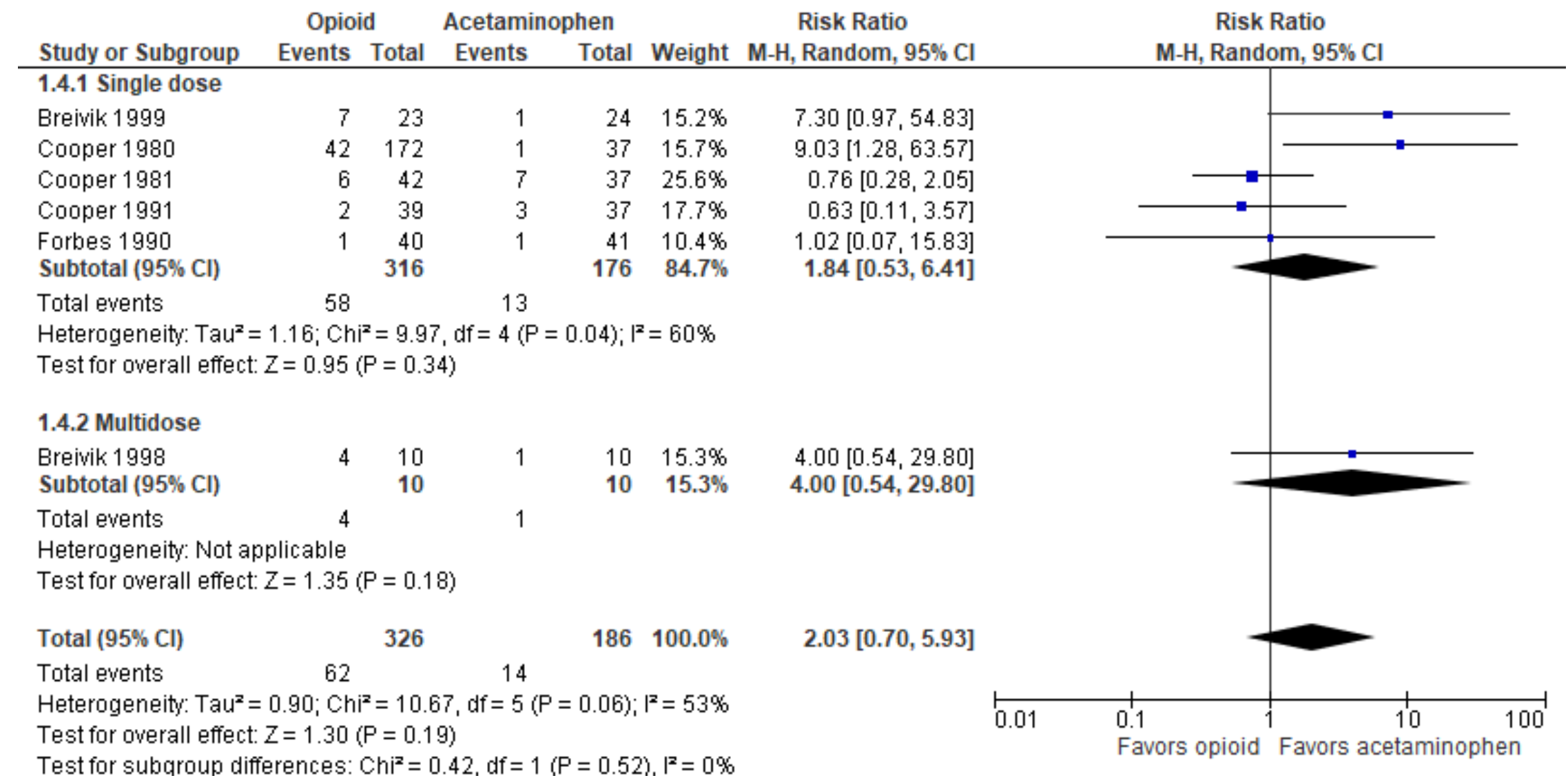

Abbreviations: $\mathrm{CI}=$ confidence interval; $\mathrm{df}=$ degrees of freedom; $\mathrm{M}-\mathrm{H}=$ Mantel-Haenszel 
Figure D-12. Dizziness for opioid versus acetaminophen for dental pain

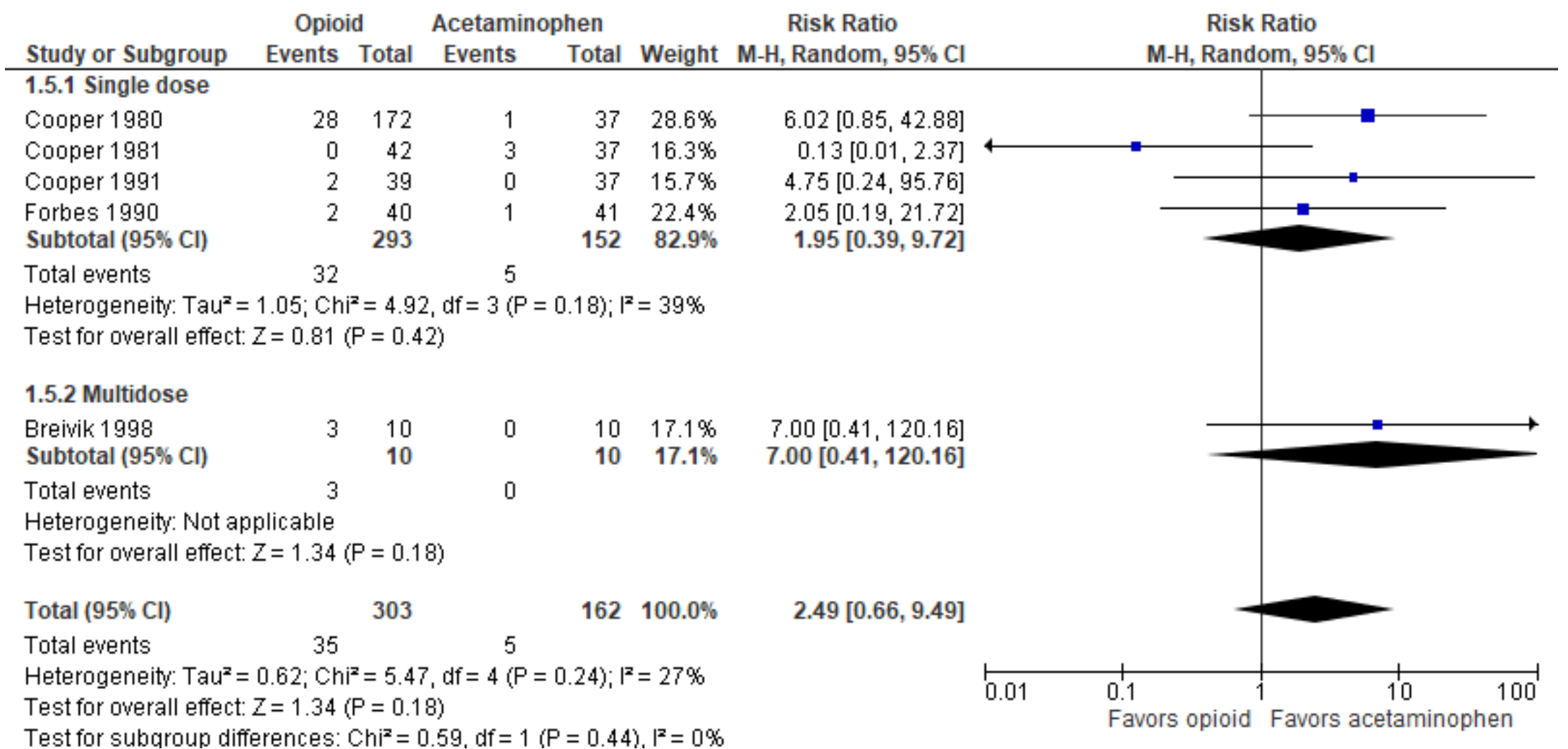

Abbreviations: $\mathrm{CI}$ = confidence interval; $\mathrm{df}$ = degrees of freedom; $\mathrm{M}-\mathrm{H}=$ Mantel-Haenszel 
Figure D-13. Headache for opioid versus acetaminophen for dental pain

\begin{tabular}{|c|c|c|c|c|c|c|c|c|c|c|}
\hline \multirow[b]{2}{*}{ Study or Subgroup } & \multicolumn{2}{|c|}{ Opioid } & \multicolumn{2}{|c|}{ Acetaminophen } & \multirow[b]{2}{*}{ Weight } & \multicolumn{2}{|l|}{ Risk Ratio } & \multirow{2}{*}{\multicolumn{2}{|c|}{$\begin{array}{c}\text { Risk Ratio } \\
\text { M-H, Random, } 95 \% \mathrm{Cl}\end{array}$}} & \\
\hline & Events & Total & Events & Total & & M-H, Random, $95 \% \mathrm{Cl}$ & & & & \\
\hline \multicolumn{11}{|l|}{ 1.6.1 Single dose } \\
\hline Cooper 1980 & 5 & 172 & 1 & 37 & $31.5 \%$ & $1.08[0.13,8.94]$ & & & & \\
\hline Cooper 1981 & 0 & 42 & 2 & 37 & $15.6 \%$ & $0.18[0.01,3.57]$ & $\longleftarrow$ & & & \\
\hline Cooper 1991 & 2 & 39 & 2 & 37 & $38.8 \%$ & $0.95[0.14,6.39]$ & & & & \\
\hline $\begin{array}{l}\text { Dionne } 1994 \\
\text { Subtotal }(95 \% \mathrm{Cl})\end{array}$ & 0 & $\begin{array}{r}24 \\
277\end{array}$ & 1 & $\begin{array}{r}27 \\
138\end{array}$ & $\begin{array}{r}14.2 \% \\
100.0 \%\end{array}$ & $\begin{array}{l}0.37[0.02,8.75] \\
0.67[0.20,2.18]\end{array}$ & & & & \\
\hline Total events & 7 & & 6 & & & & & & & \\
\hline \multicolumn{11}{|c|}{$\begin{array}{l}\text { Heterogeneity: } \mathrm{Tau}^{2}=0.00 ; \mathrm{Chi}^{2}=1.23, \mathrm{df}=3(\mathrm{P}=0.75) ; \mathrm{I}^{2}=0 \% \\
\text { Test for overall effect: } Z=0.67(\mathrm{P}=0.50)\end{array}$} \\
\hline Total $(95 \% \mathrm{Cl})$ & & 277 & & 138 & $100.0 \%$ & $0.67[0.20,2.18]$ & & & & \\
\hline Total events & 7 & & 6 & & & & & & & \\
\hline \multicolumn{7}{|c|}{$\begin{array}{l}\text { Heterogeneity: } \operatorname{Tau}^{2}=0.00 ; \mathrm{Chi}^{2}=1.23, \mathrm{df}=3(\mathrm{P}=0.75) ; \mathrm{I}^{2}=0 \% \\
\text { Test for overall effect: } Z=0.67(\mathrm{P}=0.50) \\
\text { Test for subqroup differences: Not applicable }\end{array}$} & 0.01 & $\begin{array}{l}0.1 \\
\text { Favors opioid }\end{array}$ & Favors acet & $10 \quad 100$ \\
\hline
\end{tabular}

Abbreviations: $\mathrm{CI}$ = confidence interval; $\mathrm{df}=$ degrees of freedom; $\mathrm{M}-\mathrm{H}=$ Mantel-Haenszel 
Figure D-14. Rescue or repeat medication use for NSAID versus acetaminophen for dental pain

\begin{tabular}{|c|c|c|c|c|c|c|c|c|c|}
\hline \multirow[b]{2}{*}{ Study or Subgroup } & \multicolumn{2}{|c|}{ NSAID } & \multicolumn{2}{|c|}{ Acetaminophen } & \multirow[b]{2}{*}{ Weight } & \multicolumn{2}{|l|}{ Risk Ratio } & \multirow{2}{*}{\multicolumn{2}{|c|}{$\begin{array}{c}\text { Risk Ratio } \\
\text { M-H, Random, } 95 \% \mathrm{Cl}\end{array}$}} \\
\hline & Events & Total & Events & Total & & M-H, Random, $95 \% \mathrm{Cl}$ & & & om, $95 \% \mathrm{Cl}$ \\
\hline \multicolumn{10}{|l|}{ 3.1.1 Single dose } \\
\hline Breivik 1999 & 8 & 22 & 10 & 22 & $1.9 \%$ & $0.80[0.39,1.64]$ & & 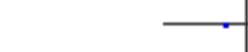 & 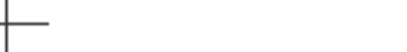 \\
\hline Cooper 1988 & 19 & 36 & 28 & 36 & $7.2 \%$ & $0.68[0.48,0.97]$ & & $\rightarrow$ & \\
\hline Cooper 1989 & 18 & 61 & 30 & 59 & $4.4 \%$ & $0.58[0.37,0.92]$ & & $\rightarrow-$ & \\
\hline Cooper 1991 & 53 & 83 & 35 & 37 & $20.9 \%$ & $0.68[0.56,0.81]$ & & 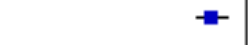 & \\
\hline Daniels 2009c & 44 & 160 & 35 & 80 & $7.2 \%$ & $0.63[0.44,0.90]$ & & $\rightarrow$ & \\
\hline Forbes 1990 & 56 & 98 & 30 & 36 & $15.1 \%$ & $0.69[0.55,0.86]$ & & $=$ & \\
\hline Hersh 2000 & 33 & 120 & 32 & 63 & $6.4 \%$ & $0.54[0.37,0.79]$ & & $\because$ & \\
\hline Mehlisch 1990 & 125 & 306 & 174 & 306 & $23.0 \%$ & $0.72[0.61,0.85]$ & & 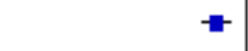 & \\
\hline Mehlisch 1995b & 25 & 98 & 61 & 101 & $6.5 \%$ & $0.42[0.29,0.61]$ & & $\rightarrow$ & \\
\hline Olson 2001 & 34 & 134 & 25 & 66 & $5.2 \%$ & $0.67[0.44,1.02]$ & & $\rightarrow$ & \\
\hline $\begin{array}{l}\text { Sunshine } 1986 \\
\text { Subtotal }(95 \% \mathrm{Cl})\end{array}$ & 10 & $\begin{array}{r}60 \\
1178\end{array}$ & 14 & $\begin{array}{r}30 \\
836\end{array}$ & $\begin{array}{r}2.1 \% \\
100.0 \%\end{array}$ & $\begin{array}{l}0.36[0.18,0.71] \\
0.64[0.58,0.71]\end{array}$ & & - & \\
\hline \multicolumn{10}{|c|}{$\begin{array}{l}\text { Heterogeneity: } \text { Tau }^{2}=0.00 ; \mathrm{Chi}^{2}=11.83, \mathrm{df}=10(\mathrm{P}=0.30) ; \mathrm{I}^{2}=15 \% \\
\text { Test for overall effect: } Z=8.59(\mathrm{P}=0.00001)\end{array}$} \\
\hline Total $(95 \% \mathrm{Cl})$ & & 1178 & & 836 & $100.0 \%$ & $0.64[0.58,0.71]$ & & $\bullet$ & \\
\hline Total events & 425 & & 474 & & & & & & \\
\hline $\begin{array}{l}\text { Heterogeneity: Tau } \\
\text { Test for overall effeo } \\
\text { Test for subqroup d }\end{array}$ & $\begin{array}{l}0.00 ; \mathrm{Ch} \\
Z=8.59 \\
\text { erences: }\end{array}$ & $\begin{array}{l}P^{2}=11 \\
P \leq 0.0 \\
\text { Not app }\end{array}$ & $\begin{array}{l}33, \mathrm{df}=10 \\
0001) \\
\text { olicable }\end{array}$ & $=0.30$ & $i^{2}=15 \%$ & & 0.01 & $\begin{array}{l}0.1 \\
\text { Favors NSAID }\end{array}$ & \begin{tabular}{|c|}
10 \\
100 \\
Favors acetaminophen
\end{tabular} \\
\hline
\end{tabular}

Abbreviations: C I= confidence interval; $\mathrm{df}$ = degrees of freedom; M-H = Mantel-Haenszel; NSAID = nonsteroidal anti-inflammatory drug 
Figure D-15. Medication rated very good or excellent for NSAID versus acetaminophen for dental pain

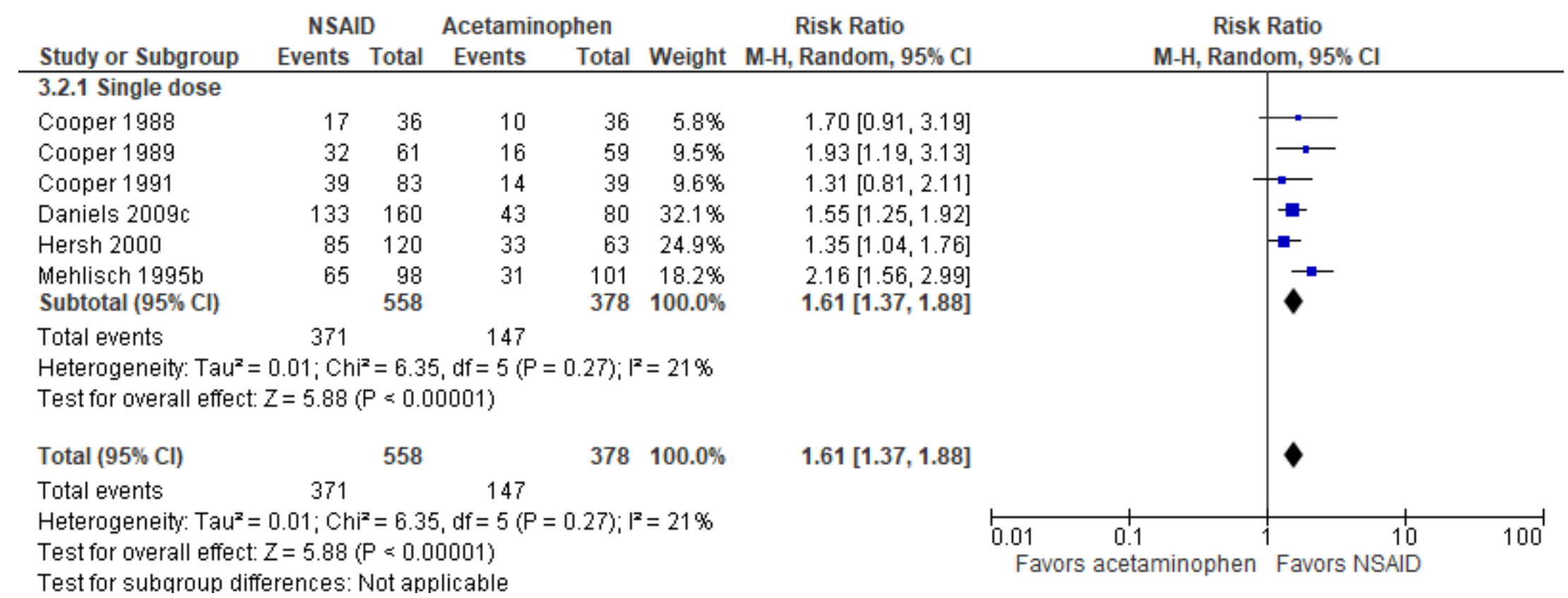

Abbreviations: $\mathrm{CI}$ = confidence interval; $\mathrm{df}$ = degrees of freedom; $\mathrm{M}-\mathrm{H}=$ Mantel-Haenszel; NSAID = nonsteroidal anti-inflammatory drug 
Figure D-16. Any adverse event for NSAID versus acetaminophen for dental pain

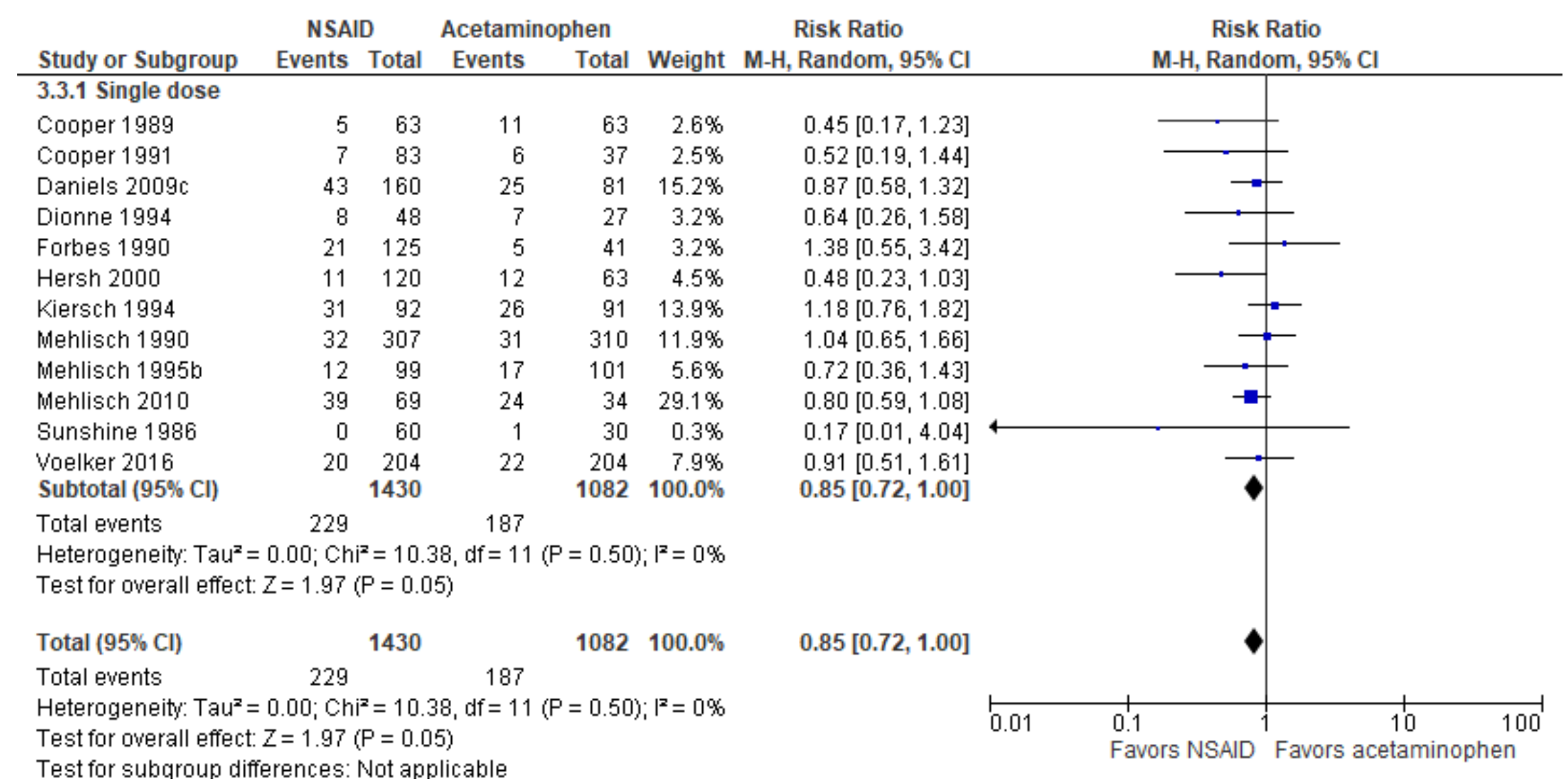

Abbreviations: $\mathrm{CI}$ = confidence interval; $\mathrm{df}$ = degrees of freedom; $\mathrm{M}-\mathrm{H}$ = Mantel-Haenszel; NSAID = nonsteroidal anti-inflammatory drug 
Figure D-17. Nausea for NSAID versus acetaminophen for dental pain

\begin{tabular}{|c|c|c|c|c|c|c|c|c|c|}
\hline \multirow[b]{2}{*}{ Study or Subgroup } & \multicolumn{2}{|c|}{ NSAID } & \multicolumn{2}{|c|}{ Acetaminophen } & \multirow[b]{2}{*}{ Weight } & Risk Ratio & \multirow{2}{*}{\multicolumn{3}{|c|}{$\begin{array}{l}\text { Risk Ratio } \\
\text { M-H, Random, } 95 \% \mathrm{Cl}\end{array}$}} \\
\hline & Events & Total & Events & Total & & M-H, Random, $95 \% \mathrm{Cl}$ & & & \\
\hline \multicolumn{10}{|l|}{ 3.4.1 Single dose } \\
\hline Akural 2009 & 4 & 20 & 4 & 18 & $4.9 \%$ & $0.90[0.26,3.08]$ & & & \\
\hline Breivik 1999 & 0 & 23 & 1 & 24 & $0.7 \%$ & $0.35[0.01,8.11]$ & & & \\
\hline Cooper 1989 & 0 & 63 & 2 & 63 & $0.8 \%$ & $0.20[0.01,4.08]$ & & & \\
\hline Cooper 1991 & 0 & 83 & 2 & 37 & $0.8 \%$ & $0.09[0.00,1.84]$ & & & \\
\hline Daniels 2009c & 14 & 160 & 8 & 81 & $10.8 \%$ & $0.89[0.39,2.02]$ & & & \\
\hline Dionne 1994 & 1 & 48 & 2 & 27 & $1.3 \%$ & $0.28[0.03,2.96]$ & & & \\
\hline Forbes 1990 & 1 & 125 & 0 & 41 & $0.7 \%$ & $1.00[0.04,24.08]$ & & & \\
\hline Hersh 2000 & 1 & 120 & 3 & 63 & $1.5 \%$ & $0.17[0.02,1.65]$ & & & - \\
\hline Kiersch 1994 & 13 & 92 & 12 & 91 & $13.8 \%$ & $1.07[0.52,2.22]$ & & & \\
\hline Mehlisch 2010 & 18 & 69 & 10 & 34 & $17.2 \%$ & $0.89[0.46,1.71]$ & & & - \\
\hline Olson 2001 & 3 & 134 & 2 & 66 & $2.4 \%$ & $0.74[0.13,4.31]$ & & & \\
\hline Searle 2020 & 24 & 175 & 33 & 165 & $31.8 \%$ & $0.69[0.42,1.11]$ & & $\rightarrow$ & \\
\hline Voelker 2016 & 11 & 204 & 16 & 204 & $13.3 \%$ & $0.69[0.33,1.44]$ & & $\rightarrow$ & \\
\hline Subtotal $(95 \% \mathrm{Cl})$ & & 1316 & & 914 & $100.0 \%$ & $0.75[0.57,0.98]$ & & & \\
\hline Total events & 90 & & 95 & & & & & & \\
\hline \multicolumn{10}{|c|}{$\begin{array}{l}\text { Heterogeneity: } \operatorname{Tau}^{2}=0.00 ; \mathrm{Chi}^{2}=6.84, \mathrm{df}=12(\mathrm{P}=0.87) ; \mathrm{I}^{2}=0 \% \\
\text { Test for overall effect: } Z=2.09(\mathrm{P}=0.04)\end{array}$} \\
\hline Total $(95 \% \mathrm{Cl})$ & & 1316 & & 914 & $100.0 \%$ & $0.75[0.57,0.98]$ & & & \\
\hline Total events & 90 & & 95 & & & & & & \\
\hline $\begin{array}{l}\text { Heterogeneity: Tau } \\
\text { Test for overall effec }\end{array}$ & $\begin{array}{l}0.00 ; \mathrm{Chi} \\
\mathrm{Z}=2.09\end{array}$ & $\begin{array}{l}P^{2}=6.8 \\
P=0.0\end{array}$ & $\begin{array}{l}4, d f=12 \\
4)\end{array}$ & $1.87) ;$ & $F^{2}=0 \%$ & & 0.005 & $\begin{array}{c}0.1 \\
\text { Favors NSAID }\end{array}$ & Favors acetaminophen \\
\hline
\end{tabular}

Abbreviations: $\mathrm{CI}$ = confidence interval; $\mathrm{df}$ = degrees of freedom; $\mathrm{M}-\mathrm{H}$ = Mantel-Haenszel; NSAID = nonsteroidal anti-inflammatory drug 
Figure D-18. Drowsiness for NSAID versus acetaminophen for dental pain

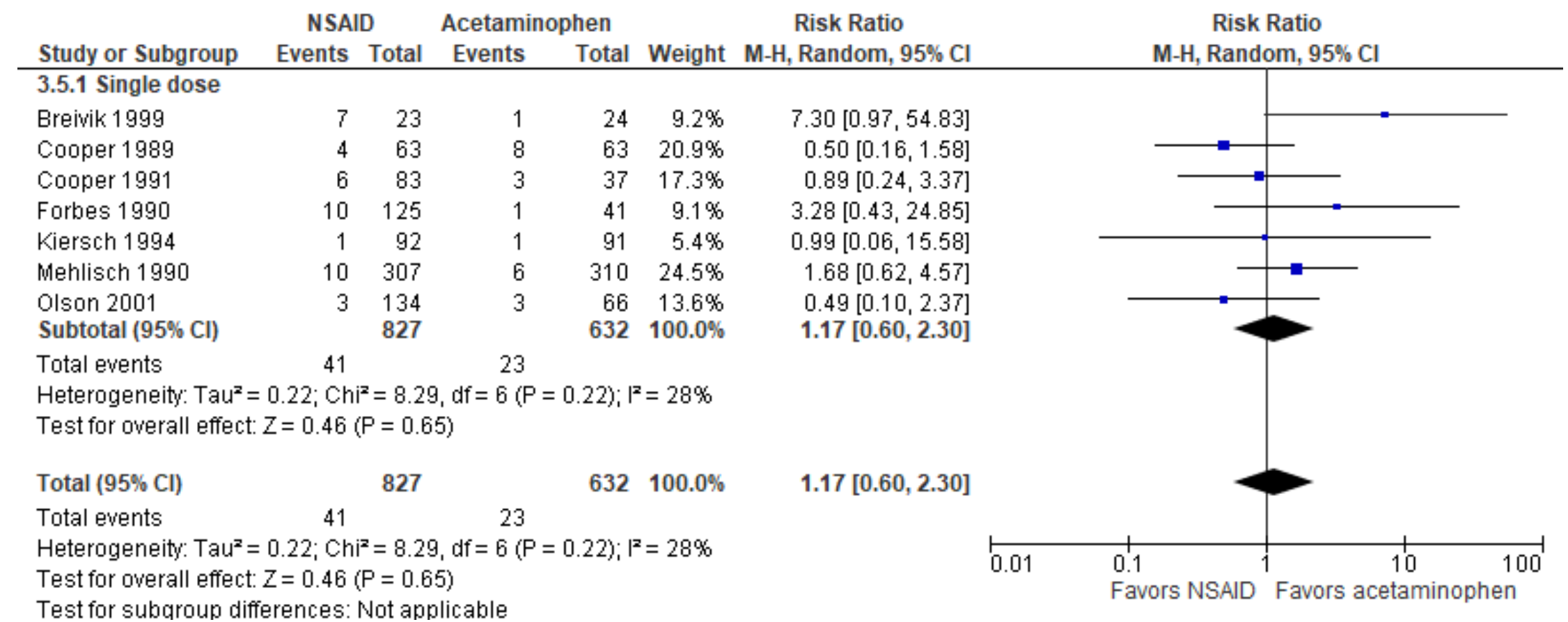

Abbreviations: $\mathrm{CI}$ = confidence interval; $\mathrm{df}$ = degrees of freedom; $\mathrm{M}-\mathrm{H}$ = Mantel-Haenszel; NSAID = nonsteroidal anti-inflammatory drug 
Figure D-19. Dizziness for NSAID versus acetaminophen for dental pain

\begin{tabular}{|c|c|c|c|c|c|c|c|c|c|}
\hline \multirow[b]{2}{*}{ Study or Subgroup } & \multicolumn{2}{|c|}{ NSAID } & \multicolumn{2}{|c|}{ Acetaminophen } & \multirow[b]{2}{*}{ Weight } & \multicolumn{2}{|l|}{ Risk Ratio } & \multirow{2}{*}{$\begin{array}{l}\text { Risk Ratio } \\
\text { M-H, Random, } 95 \% \mathrm{Cl}\end{array}$} & Ratio \\
\hline & Events & Total & Events & Total & & M-H, Random, $95 \% \mathrm{Cl}$ & & & $\mathrm{om}, 95 \% \mathrm{Cl}$ \\
\hline \multicolumn{10}{|l|}{ 3.6.1 Single dose } \\
\hline Cooper 1989 & 0 & 63 & 2 & 63 & $3.5 \%$ & $0.20[0.01,4.08]$ & & & \\
\hline Daniels $2009 \mathrm{c}$ & 4 & 160 & 5 & 81 & $18.0 \%$ & $0.41[0.11,1.47]$ & & & - \\
\hline Forbes 1990 & 9 & 125 & 1 & 41 & $7.6 \%$ & $2.95[0.39,22.60]$ & & & \\
\hline Hersh 2000 & 1 & 120 & 0 & 63 & $3.2 \%$ & $1.59[0.07,38.40]$ & & & \\
\hline Kiersch 1994 & 4 & 92 & 1 & 91 & $6.7 \%$ & $3.96[0.45,34.72]$ & & & \\
\hline Mehlisch 2010 & 6 & 69 & 7 & 34 & $27.6 \%$ & $0.42[0.15,1.16]$ & & & \\
\hline Searle 2020 & 6 & 175 & 12 & 165 & $30.2 \%$ & $0.47[0.18,1.23]$ & & & \\
\hline $\begin{array}{l}\text { Voelker } 2016 \\
\text { Subtotal }(95 \% \mathrm{Cl})\end{array}$ & 0 & $\begin{array}{r}204 \\
1008\end{array}$ & 1 & $\begin{array}{l}204 \\
742\end{array}$ & $\begin{array}{r}3.2 \% \\
100.0 \%\end{array}$ & $\begin{array}{l}0.33[0.01,8.13] \\
0.59[0.33,1.05]\end{array}$ & & & \\
\hline Total events & 30 & & 29 & & & & & & \\
\hline \multicolumn{10}{|c|}{$\begin{array}{l}\text { Heterogeneity: } \operatorname{Tau}^{2}=0.05 ; \mathrm{Chi}^{2}=7.46, \mathrm{df}=7(\mathrm{P}=0.38) ; \mathrm{I}^{2}=6 \% \\
\text { Test for overall effect: } Z=1.81(\mathrm{P}=0.07)\end{array}$} \\
\hline Total $(95 \% \mathrm{Cl})$ & & 1008 & & 742 & $100.0 \%$ & $0.59[0.33,1.05]$ & & & \\
\hline Total events & 30 & & 29 & & & & & & \\
\hline $\begin{array}{l}\text { Heterogeneity: } \operatorname{Tau}^{2}= \\
\text { Test for overall effect }\end{array}$ & $\begin{array}{l}0.05 ; \mathrm{Chi} \\
Z=1.81 \\
\end{array}$ & $\begin{array}{l}P^{2}=7.4 \\
P=0.0\end{array}$ & $6, d f=7(P$ & 38); $1^{2}$ & $=6 \%$ & & 0.01 & $\begin{array}{l}0.1 \\
\text { Favors NSAID }\end{array}$ & $\begin{array}{cc}10 & 100 \\
\text { Favors acetaminophen }\end{array}$ \\
\hline
\end{tabular}

Abbreviations: $\mathrm{CI}$ = confidence interval; $\mathrm{df}$ = degrees of freedom; $\mathrm{M}-\mathrm{H}$ = Mantel-Haenszel; NSAID = nonsteroidal anti-inflammatory drug 
Figure D-20. Headache for NSAID versus acetaminophen for dental pain

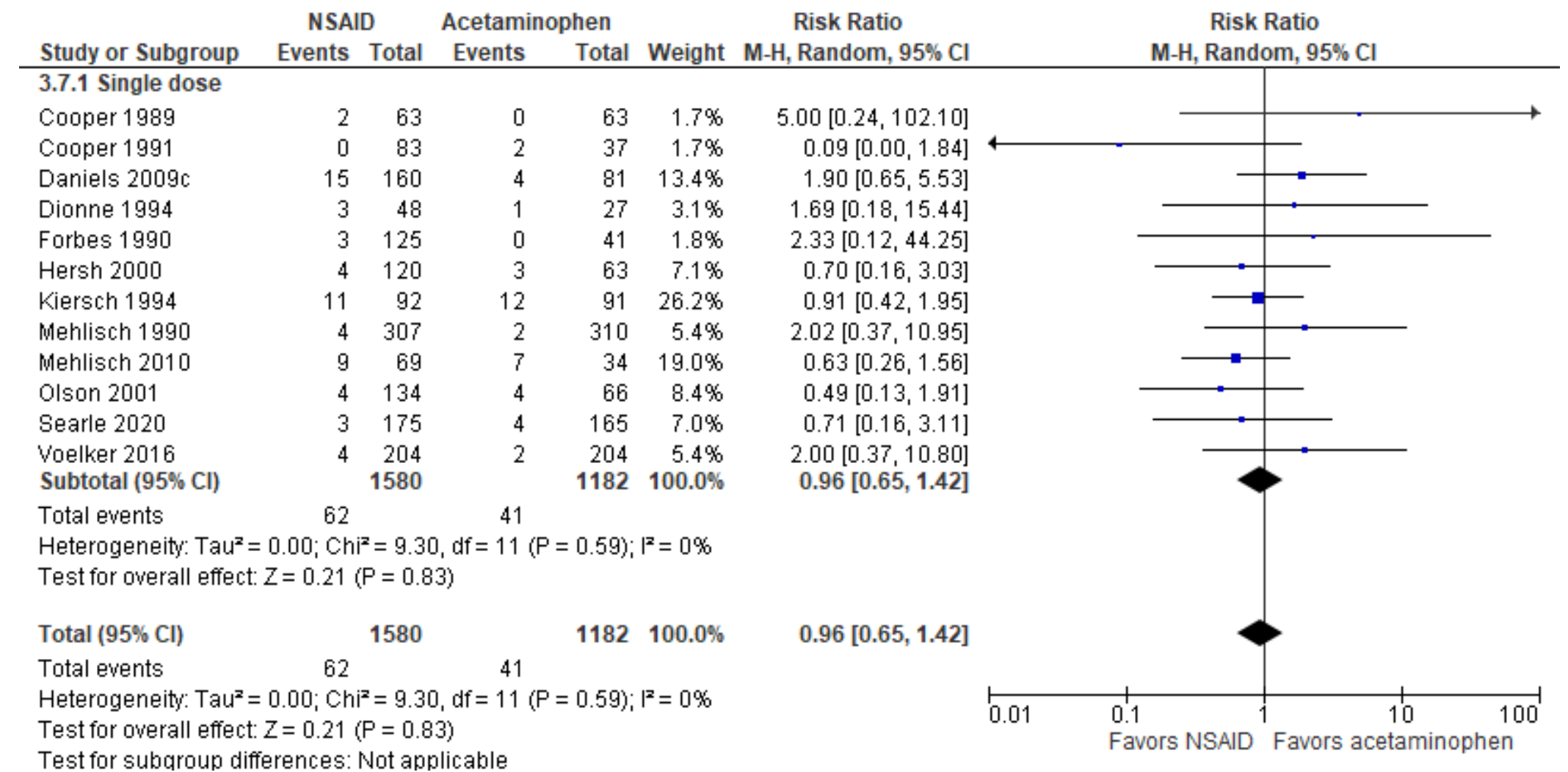

Test for subqroup differences: Not applicable

Abbreviations: $\mathrm{CI}$ = confidence interval; $\mathrm{df}$ = degrees of freedom; $\mathrm{M}-\mathrm{H}$ = Mantel-Haenszel; NSAID = nonsteroidal anti-inflammatory drug 


\section{Key Question 7. Kidney Stone Pain}

Figure D-21. Rescue or repeat medication use for opioid versus NSAID for acute renal colic

Opioid NSAID Risk Ratio

Study or Subgroup

Events Total Events Total Weight $\mathrm{M}-\mathrm{H}$, Random, $95 \% \mathrm{Cl}$

Risk Ratio

4.1.1 Morphine

Pathan 2016

Subtotal $(95 \% \mathrm{Cl})$

$126 \quad 549$

549

$63 \quad 547 \quad 19.8 \%$

$547 \quad 19.8 \%$

$1.99[1.51,2.63]$

Total events

$126 \quad 63$

Heterogeneity: Not applicable

Test for overall effect: $Z=4.86(P \leq 0.00001)$

\subsubsection{Meperidine}

al-Sahlawi 1996

Cordell 1996

Garcca-Alonso 1991

Larkin 1999

Oosterlinck 1990

Sandhu 1994

Subtotal $(95 \% \mathrm{Cl})$

Total events

$\begin{array}{rrrrr}0 & 50 & 2 & 50 & 1.2 \% \\ 31 & 35 & 23 & 36 & 19.9 \% \\ 23 & 118 & 19 & 116 & 14.2 \% \\ 16 & 37 & 11 & 33 & 13.2 \% \\ 18 & 20 & 6 & 36 & 10.9 \% \\ 53 & 72 & 44 & 68 & 20.8 \% \\ & 332 & & 339 & 80.2 \% \\ 141 & & 105 & & \end{array}$

$0.20[0.01,4.06]$

$1.39[1.06,1.82]$

$1.19[0.69,2.06]$

$1.30[0.71,2.38]$

$5.40[2.56,11.37]$

$1.14[0.91,1.42]$

$1.48[1.02,2.14]$

Heterogeneity: Tau $^{2}=0.12 ; \mathrm{Chi}^{2}=17.53, \mathrm{df}=5(\mathrm{P}=0.004) ; \mathrm{I}^{2}=71 \%$

Test for overall effect: $Z=2.07(P=0.04)$

Total $(95 \% \mathrm{Cl})$

881

$886 \quad 100.0 \%$

$1.57[1.12,2.20]$

Total events

267

168

Heterogeneity: $\operatorname{Tau}^{2}=0.13 ; \mathrm{Chi}^{2}=26.11, \mathrm{df}=6(\mathrm{P}=0.0002) ; \mathrm{I}^{2}=77 \%$

Test for overall effect: $Z=2.61(P=0.009)$

Test for subqroup differences: $\mathrm{Chi}^{2}=1.61, \mathrm{df}=1(\mathrm{P}=0.20), \mathrm{I}^{2}=38.0 \%$

$\mathrm{M}-\mathrm{H}$, Random, $95 \% \mathrm{Cl}$

Abbreviations: $\mathrm{CI}$ = confidence interval; $\mathrm{df}$ = degrees of freedom; $\mathrm{M}-\mathrm{H}$ = Mantel-Haenszel; NSAID = nonsteroidal anti-inflammatory drug 
Figure D-22. Any adverse event for opioid versus NSAID for acute renal colic

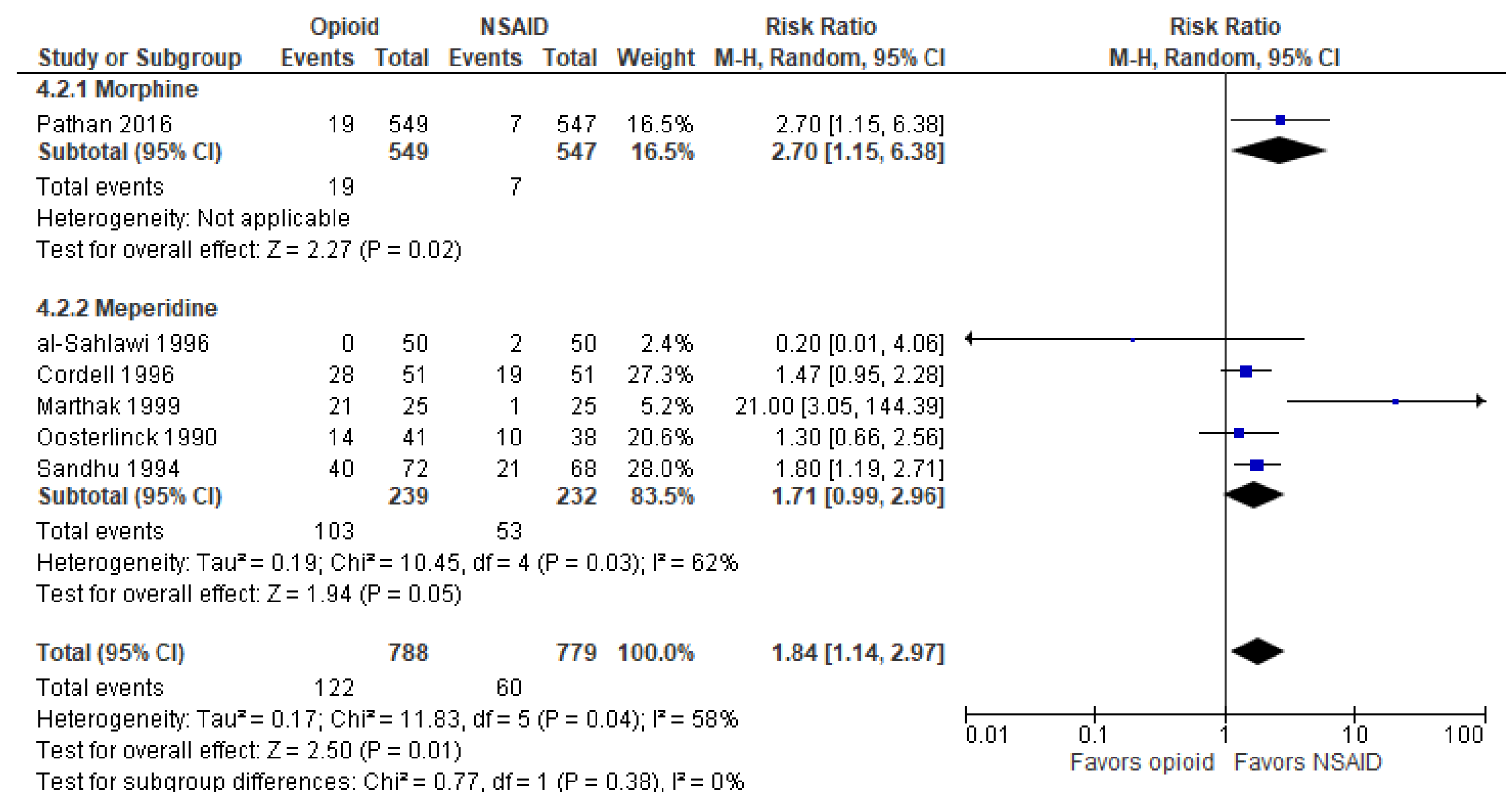

Abbreviations: $\mathrm{CI}$ = confidence interval; $\mathrm{df}$ = degrees of freedom; $\mathrm{M}-\mathrm{H}$ = Mantel-Haenszel; NSAID = nonsteroidal anti-inflammatory drug 
Figure D-23. Somnolence for opioid versus NSAID for acute renal colic

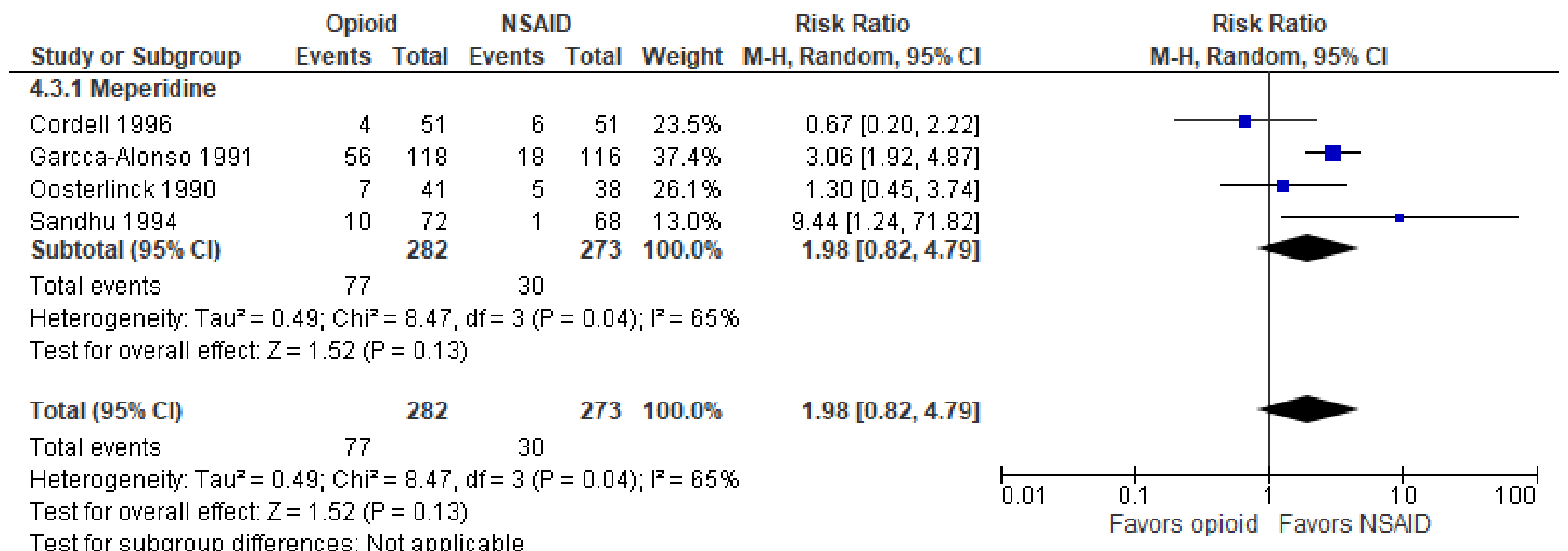

Abbreviations: $\mathrm{CI}$ = confidence interval; $\mathrm{df}$ = degrees of freedom; $\mathrm{M}-\mathrm{H}=$ Mantel-Haenszel; NSAID = nonsteroidal anti-inflammatory drug 
Figure D-24. Nausea for opioid versus NSAID for acute renal colic

\begin{tabular}{|c|c|c|c|c|c|c|c|c|c|c|}
\hline \multirow{2}{*}{$\begin{array}{l}\text { Study or Subgroup } \\
\text { 4.4.1 Meperidine }\end{array}$} & \multicolumn{2}{|c|}{ Opioid } & \multicolumn{2}{|c|}{ NSAID } & \multirow[t]{2}{*}{ Weight } & $\begin{array}{c}\text { Risk Ratio } \\
\text { M-H, Random, } 95 \% \mathrm{Cl}\end{array}$ & \multicolumn{4}{|c|}{$\begin{array}{c}\text { Risk Ratio } \\
\text { M-H, Random, } 95 \% \mathrm{Cl}\end{array}$} \\
\hline & & & & & & & & & & \\
\hline Garcca-Alonso 1991 & 46 & 118 & 15 & 116 & $38.7 \%$ & $3.01[1.79,5.09]$ & & & - & \\
\hline Larkin 1999 & 4 & 37 & 5 & 33 & $16.2 \%$ & $0.71[0.21,2.44]$ & & & & \\
\hline Marthak 1999 & 2 & 25 & 0 & 25 & $3.6 \%$ & $5.00[0.25,99.16]$ & & & & \\
\hline Oosterlinck 1990 & 0 & 41 & 1 & 38 & $3.2 \%$ & $0.31[0.01,7.37]$ & & & & \\
\hline $\begin{array}{l}\text { Sandhu } 1994 \\
\text { Subtotal }(95 \% \mathrm{Cl})\end{array}$ & 28 & $\begin{array}{r}72 \\
293\end{array}$ & 15 & $\begin{array}{r}68 \\
280\end{array}$ & $\begin{array}{r}38.3 \% \\
100.0 \%\end{array}$ & $\begin{array}{r}1.76[1.04,3.00] \\
1.84[1.02,3.31]\end{array}$ & & & & \\
\hline Total events & 80 & & 36 & & & & & & & \\
\hline \multicolumn{11}{|c|}{$\begin{array}{l}\text { Heterogeneity: } \operatorname{Tau}^{2}=0.16 ; \mathrm{Ch}^{2}=6.94, \mathrm{df}=4(\mathrm{P}=0.14) ; \mathrm{I}^{2}=42 \% \\
\text { Test for overall effect: } Z=2.04(\mathrm{P}=0.04)\end{array}$} \\
\hline Total $(95 \% \mathrm{Cl})$ & & 293 & & 280 & $100.0 \%$ & $1.84[1.02,3.31]$ & & & & \\
\hline Total events & 80 & & 36 & & & & & & & \\
\hline $\begin{array}{l}\text { Heterogeneity: } \operatorname{Tau}^{2}= \\
\text { Test for overall effect: } \\
\text { Test for subaroun diffe }\end{array}$ & $\begin{array}{l}16 ; \mathrm{Chi}^{2} \\
=2.04(\mathrm{P}\end{array}$ & $\begin{array}{l}=6.94 \\
=0.04 \\
\text { ot appli }\end{array}$ & $\mathrm{df}=4(\mathrm{P}$ & $=0.14)$ & $F^{2}=42 \%$ & & 0.01 & $\begin{array}{l}0.1 \\
\text { Favors opioid }\end{array}$ & $\begin{array}{c}10 \\
\text { Favors NSAID }\end{array}$ & 100 \\
\hline
\end{tabular}

Abbreviations: $\mathrm{CI}$ = confidence interval; $\mathrm{df}$ = degrees of freedom; $\mathrm{M}-\mathrm{H}$ = Mantel-Haenszel; NSAID = nonsteroidal anti-inflammatory drug 


\section{Appendix E. Evidence Tables}

Shown in associated Excel files. 


\section{Appendix F. Risk of Bias Assessment}

Shown in associated Excel files. 


\section{Appendix G. Details on Strength of Evidence}

Table G-1. Treatments for acute pain: findings and strength of evidence for interventions

\begin{tabular}{|c|c|c|c|c|c|c|c|c|c|c|}
\hline Intervention & Outcomes & $\begin{array}{l}\text { Timing of } \\
\text { Outcomes }\end{array}$ & $\begin{array}{l}\text { Number } \\
\text { of } \\
\text { Studies }\end{array}$ & $\begin{array}{l}\text { Number } \\
\text { of } \\
\text { Subjects }\end{array}$ & Directness & Precision & Quality & Consistency & Findings & SOE \\
\hline \multirow[t]{2}{*}{$\begin{array}{l}\text { KQ 1: Acute low } \\
\text { back pain }\end{array}$} & Pain & $\begin{array}{l}<1 \mathrm{~d} ; 1 \mathrm{~d} \\
\text { to }<1 \mathrm{w}\end{array}$ & 1 & 113 & Direct & Precise & Fair & $\begin{array}{l}\text { Unable to } \\
\text { assess }\end{array}$ & No differences & Low \\
\hline & $\begin{array}{l}\text { Serious } \\
\text { AEs, study } \\
\text { withdrawal } \\
\text { due to AEs, } \\
\text { any AE }\end{array}$ & $\begin{array}{l}<1 \mathrm{~d} ; 1 \mathrm{~d} \\
\text { to } 1 \mathrm{w}\end{array}$ & 1 & 113 & Direct & Precise & Fair & $\begin{array}{l}\text { Unable to } \\
\text { assess }\end{array}$ & $\begin{array}{l}\text { Higher risk } \\
\text { with opioid }\end{array}$ & Low \\
\hline \multirow{2}{*}{$\begin{array}{l}\text { KQ 1: Acute low } \\
\text { back pain } \\
\text { Opioid vs. } \\
\text { muscle relaxant }\end{array}$} & $\begin{array}{l}\text { Pain, } \\
\text { function }\end{array}$ & $\begin{array}{l}1 \text { to }<2 \mathrm{w} ; \\
\geq 4 \mathrm{w}\end{array}$ & 1 & 216 & Direct & Imprecise & Good & $\begin{array}{l}\text { Unable to } \\
\text { assess }\end{array}$ & No differences & Low \\
\hline & $\begin{array}{l}\text { Dizziness, } \\
\text { nausea or } \\
\text { vomiting }\end{array}$ & $\begin{array}{l}1 \text { to }<2 \mathrm{w} ; \\
\geq 4 \mathrm{w}\end{array}$ & 1 & 216 & Direct & Precise & Good & $\begin{array}{l}\text { Unable to } \\
\text { assess }\end{array}$ & $\begin{array}{l}\text { Higher risk } \\
\text { with opioid }\end{array}$ & Moderate \\
\hline \multirow[t]{2}{*}{$\begin{array}{l}\text { KQ 1: Acute low } \\
\text { back pain } \\
\text { Muscle relaxant } \\
\text { vs. } \\
\text { benzodiazepine }\end{array}$} & Pain & $\begin{array}{l}1 \mathrm{~d} \text { to }<1 \\
W\end{array}$ & 2 & 110 & Direct & Imprecise & Fair & Consistent & $\begin{array}{l}\text { Small to } \\
\text { moderate } \\
\text { decrease in } \\
\text { pain with } \\
\text { muscle } \\
\text { relaxant }\end{array}$ & Low \\
\hline & AEs & $\begin{array}{l}1 \mathrm{~d} \text { to }<1 \\
W\end{array}$ & 2 & 110 & Direct & Imprecise & Fair & Inconsistent & $\begin{array}{l}\text { Unable to } \\
\text { determine }\end{array}$ & Insufficient \\
\hline \multirow{2}{*}{$\begin{array}{l}\text { KQ 1: Acute low } \\
\text { back pain } \\
\text { NSAID or muscle } \\
\text { relaxant vs. } \\
\text { manipulation }\end{array}$} & $\begin{array}{l}\text { Pain, } \\
\text { function }\end{array}$ & $\begin{array}{l}1 \text { to }<2,2 \\
\text { to }<4, \text { and } \\
\geq 4 \mathrm{w}\end{array}$ & 3 & 320 & Direct & Precise & Fair & Inconsistent & $\begin{array}{l}\text { Likely no } \\
\text { differences }\end{array}$ & Low \\
\hline & AEs & $\begin{array}{l}1 \text { to }<2,2 \\
\text { to }<4, \text { and } \\
\geq 4 \mathrm{w}\end{array}$ & 3 & 320 & Direct & Imprecise & Fair & Inconsistent & $\begin{array}{l}\text { Unable to } \\
\text { determine }\end{array}$ & Insufficient \\
\hline $\begin{array}{l}\text { KQ 1: Acute low } \\
\text { back pain } \\
\text { Acupuncture vs. } \\
\text { NSAID }\end{array}$ & $\begin{array}{l}\text { Pain, } \\
\text { function }\end{array}$ & $\begin{array}{l}2 \text { to }<4 \mathrm{w} \\
\text { and } \geq 4 \mathrm{w}\end{array}$ & 1 & 58 & Direct & Imprecise & Fair & $\begin{array}{l}\text { Unable to } \\
\text { assess }\end{array}$ & $\begin{array}{l}\text { Moderate } \\
\text { improvement } \\
\text { in pain and } \\
\text { function with } \\
\text { acupuncture }\end{array}$ & Low \\
\hline $\begin{array}{l}\text { KQ 1: Acute low } \\
\text { back pain }\end{array}$ & $\begin{array}{l}\text { Pain, } \\
\text { function }\end{array}$ & 1 to $52 \mathrm{w}$ & 2 & 194 & Direct & Imprecise & Fair & Consistent & No differences & Low \\
\hline $\begin{array}{l}\text { Exercise vs. } \\
\text { usual care }\end{array}$ & AEs & -- & -- & -- & -- & -- & -- & -- & No evidence & Insufficient \\
\hline
\end{tabular}




\begin{tabular}{|c|c|c|c|c|c|c|c|c|c|c|}
\hline Intervention & Outcomes & $\begin{array}{l}\text { Timing of } \\
\text { Outcomes }\end{array}$ & $\begin{array}{l}\text { Number } \\
\text { of } \\
\text { Studies }\end{array}$ & $\begin{array}{l}\text { Number } \\
\text { of } \\
\text { Subjects }\end{array}$ & Directness & Precision & Quality & Consistency & Findings & SOE \\
\hline \multirow{3}{*}{$\begin{array}{l}\text { KQ 1: Acute low } \\
\text { back pain } \\
\text { Exercise vs. bed } \\
\text { rest }\end{array}$} & $\begin{array}{l}\text { Pain, } \\
\text { function }\end{array}$ & 1 to $52 \mathrm{w}$ & 3 & 384 & Direct & Precise & Fair & Consistent & No differences & Moderate \\
\hline & Sick days & $\begin{array}{l}2 \text { to } 4 w \\
\text { and } \geq 4 w\end{array}$ & 1 & 100 & Direct & Precise & Fair & $\begin{array}{l}\text { Unable to } \\
\text { assess }\end{array}$ & $\begin{array}{l}\text { Fewer sick } \\
\text { days with } \\
\text { opioid }\end{array}$ & Low \\
\hline & AEs & 1 to $52 \mathrm{w}$ & 3 & 384 & Direct & Imprecise & Fair & $\begin{array}{l}\text { Unable to } \\
\text { assess }\end{array}$ & Sparse data & Insufficient \\
\hline \multirow[t]{3}{*}{$\begin{array}{l}\text { KQ 1: Acute low } \\
\text { back pain } \\
\text { Traditional } \\
\text { Chinese } \\
\text { acupuncture vs. } \\
\text { sham or usual } \\
\text { care }\end{array}$} & $\begin{array}{l}\text { Pain, } \\
\text { function }\end{array}$ & 2 to $<4 \mathrm{w}$ & 1 & 261 & Direct & Imprecise & Fair & $\begin{array}{l}\text { Inconsistency } \\
\text { based on } \\
\text { type of sham }\end{array}$ & $\begin{array}{l}\text { Acupuncture } \\
\text { decreased } \\
\text { persistent pain } \\
\text { vs. non- } \\
\text { penetrating } \\
\text { sham or usual } \\
\text { care, but not } \\
\text { needle sham }\end{array}$ & Low \\
\hline & $\begin{array}{l}\text { Pain, } \\
\text { function }\end{array}$ & $\geq 4 \mathrm{w}$ & 1 & 261 & Direct & Imprecise & Fair & $\begin{array}{l}\text { Unable to } \\
\text { assess }\end{array}$ & No differences & Low \\
\hline & $\begin{array}{l}\text { Serious } \\
\text { AEs, study } \\
\text { withdrawal } \\
\text { due to AEs }\end{array}$ & $\begin{array}{l}2 \text { to }<4 \mathrm{w}, \\
\geq 4 \mathrm{w}\end{array}$ & 1 & 261 & Direct & Imprecise & Fair & $\begin{array}{l}\text { Unable to } \\
\text { assess }\end{array}$ & $\begin{array}{l}\text { No events } \\
\text { reported }\end{array}$ & Low \\
\hline $\begin{array}{l}\text { KQ 1: Acute low } \\
\text { back pain } \\
\text { Brace vs. no } \\
\text { brace, } \\
\text { osteoporotic } \\
\text { compression } \\
\text { fracture }\end{array}$ & $\begin{array}{l}\text { Pain, } \\
\text { function, } \\
\text { opioid use }\end{array}$ & $\begin{array}{l}2 \text { to }<4 \mathrm{w}, \\
\geq 4 \mathrm{w}\end{array}$ & 1 & 60 & Direct & Precise & Fair & $\begin{array}{l}\text { Unable to } \\
\text { assess }\end{array}$ & No differences & Low \\
\hline \multirow[t]{2}{*}{$\begin{array}{l}\text { KQ 1: Acute low } \\
\text { back pain } \\
\text { Heat therapy vs. } \\
\text { usual care or } \\
\text { placebo }\end{array}$} & $\begin{array}{l}\text { Pain, } \\
\text { function }\end{array}$ & $\begin{array}{l}1 \mathrm{~d} \text { to }<1 \\
\mathrm{w}, 1 \text { to }<2 \\
\mathrm{w}, 2 \text { to }<4 \\
\mathrm{w}\end{array}$ & 6 & 425 & Direct & $\begin{array}{l}\text { Imprecise } \\
\text { to precise }\end{array}$ & Fair & Consistent & $\begin{array}{l}\text { Moderate } \\
\text { improvement } \\
\text { in pain and } \\
\text { function with } \\
\text { heat therapy }\end{array}$ & $\begin{array}{l}\text { Low to } \\
\text { moderate }\end{array}$ \\
\hline & $\begin{array}{l}\text { Adverse } \\
\text { events }\end{array}$ & $\begin{array}{l}1 \mathrm{~d} \text { to }<1 \\
\mathrm{w}, 1 \text { to }<2 \\
\mathrm{w}, 2 \text { to }<4 \\
\mathrm{w}\end{array}$ & 6 & 425 & Direct & Imprecise & Fair & Consistent & $\begin{array}{l}\text { No serious } \\
\text { AEs and few } \\
\text { non-serious } \\
\text { AEs }\end{array}$ & Low \\
\hline
\end{tabular}




\begin{tabular}{|c|c|c|c|c|c|c|c|c|c|c|}
\hline Intervention & Outcomes & $\begin{array}{l}\text { Timing of } \\
\text { Outcomes }\end{array}$ & $\begin{array}{l}\text { Number } \\
\text { of } \\
\text { Studies }\end{array}$ & $\begin{array}{l}\text { Number } \\
\text { of } \\
\text { Subjects }\end{array}$ & Directness & Precision & Quality & Consistency & Findings & SOE \\
\hline \multirow{2}{*}{$\begin{array}{l}\text { KQ 1: Acute low } \\
\text { back pain } \\
\text { Manipulation vs. } \\
\text { inactive } \\
\text { controls, no } \\
\text { radiculopathy }\end{array}$} & $\begin{array}{l}\text { Pain, } \\
\text { function }\end{array}$ & $\begin{array}{l}1 \mathrm{~d} \text { to }<1 \\
\mathrm{w}, 1 \text { to }<2 \\
\mathrm{w}, 2 \text { to }<4 \\
\mathrm{w}, \text { or } \geq 4 \mathrm{w}\end{array}$ & 6 & 555 & Direct & Imprecise & Fair & Consistent & No differences & $\begin{array}{l}\text { Low to } \\
\text { moderate }\end{array}$ \\
\hline & $\begin{array}{l}\text { Adverse } \\
\text { events }\end{array}$ & $\begin{array}{l}1 \mathrm{~d} \text { to }<1 \\
\mathrm{w}, 1 \text { to }<2 \\
\mathrm{w}, 2 \text { to }<4 \\
\mathrm{w}, \text { or } \geq 4 \mathrm{w}\end{array}$ & 6 & 555 & Direct & Imprecise & Fair & Consistent & $\begin{array}{l}\text { Limited } \\
\text { reporting, few } \\
\text { or no serious } \\
\text { AEs }\end{array}$ & Low \\
\hline \multirow{2}{*}{$\begin{array}{l}\text { KQ 1: Acute low } \\
\text { back pain } \\
\text { Manipulation vs. } \\
\text { sham, } \\
\text { radiculopathy }\end{array}$} & Pain & $\begin{array}{l}2 \text { to }<4 \mathrm{w}, \\
\geq 4 \mathrm{w}\end{array}$ & 1 & 102 & Direct & Precise & Good & $\begin{array}{l}\text { Unable to } \\
\text { assess }\end{array}$ & $\begin{array}{l}\text { Decreased } \\
\text { likelihood of } \\
\text { pain with } \\
\text { manipulation }\end{array}$ & Low \\
\hline & AEs & $\begin{array}{l}2 \text { to }<4 \mathrm{w}, \\
\geq 4 \mathrm{w}\end{array}$ & 1 & 102 & Direct & Imprecise & Good & $\begin{array}{l}\text { Unable to } \\
\text { assess }\end{array}$ & $\begin{array}{l}\text { No AEs } \\
\text { reported in } \\
\text { either group }\end{array}$ & Low \\
\hline $\begin{array}{l}\text { KQ 2: Acute } \\
\text { neck pain } \\
\text { Collar vs. usual } \\
\text { activity, neck } \\
\text { pain with } \\
\text { radiculopathy }\end{array}$ & $\begin{array}{l}\text { Pain, } \\
\text { function }\end{array}$ & $\begin{array}{l}2 \text { to }<4 \mathrm{w}, \\
\geq 4 \mathrm{w}\end{array}$ & 1 & 135 & Direct & Imprecise & Fair & $\begin{array}{l}\text { Unable to } \\
\text { assess }\end{array}$ & $\begin{array}{l}\text { Moderate to } \\
\text { large decrease } \\
\text { in pain with } \\
\text { collar, no } \\
\text { difference in } \\
\text { function }\end{array}$ & Low \\
\hline $\begin{array}{l}\text { KQ 2: Acute } \\
\text { neck pain } \\
\text { Brace vs. } \\
\text { exercise, neck } \\
\text { pain with } \\
\text { radiculopathy }\end{array}$ & $\begin{array}{l}\text { Pain, } \\
\text { function }\end{array}$ & $\begin{array}{l}2 \text { to }<4 \mathrm{w}, \\
\geq 4 \mathrm{w}\end{array}$ & 1 & 139 & Direct & Imprecise & Fair & $\begin{array}{l}\text { Unable to } \\
\text { assess }\end{array}$ & No differences & Low \\
\hline $\begin{array}{l}\text { KQ 2: Acute } \\
\text { neck pain } \\
\text { Exercise vs. } \\
\text { usual activity, } \\
\text { neck pain with } \\
\text { radiculopathy }\end{array}$ & $\begin{array}{l}\text { Pain, } \\
\text { function }\end{array}$ & $\begin{array}{l}2 \text { to }<4 \mathrm{w}, \\
\geq 4 \mathrm{w}\end{array}$ & 1 & 136 & Direct & Imprecise & Fair & $\begin{array}{l}\text { Unable to } \\
\text { assess }\end{array}$ & $\begin{array}{l}\text { Moderate to } \\
\text { large decrease } \\
\text { in pain with } \\
\text { exercise, no } \\
\text { difference in } \\
\text { function }\end{array}$ & Low \\
\hline
\end{tabular}




\begin{tabular}{|c|c|c|c|c|c|c|c|c|c|c|}
\hline Intervention & Outcomes & $\begin{array}{l}\text { Timing of } \\
\text { Outcomes }\end{array}$ & $\begin{array}{l}\text { Number } \\
\text { of } \\
\text { Studies }\end{array}$ & $\begin{array}{l}\text { Number } \\
\text { of } \\
\text { Subjects }\end{array}$ & Directness & Precision & Quality & Consistency & Findings & SOE \\
\hline $\begin{array}{l}\text { KQ 2: Acute } \\
\text { neck pain } \\
\text { Ultrasound vs. } \\
\text { sham, neck pain } \\
\text { with } \\
\text { radiculopathy }\end{array}$ & Pain & $\begin{array}{l}1 \text { to }<2 w \\
2 \text { to }<4 w\end{array}$ & 1 & 54 & Direct & Imprecise & Fair & $\begin{array}{l}\text { Unable to } \\
\text { assess }\end{array}$ & $\begin{array}{l}\text { No difference } \\
\text { at } 1 \text { to }<2 \mathrm{w} \text {, } \\
\text { small } \\
\text { decrease with } \\
\text { ultrasound at } 2 \\
\text { to }<4 \mathrm{w}\end{array}$ & Low \\
\hline $\begin{array}{l}\text { KQ 2: Acute } \\
\text { neck pain } \\
\text { Collar vs. usual } \\
\text { activity, } \\
\text { whiplash neck } \\
\text { strain }\end{array}$ & $\begin{array}{l}\text { Pain, health } \\
\text { status }\end{array}$ & $\geq 4$ weeks & 1 & 303 & Direct & Precise & Fair & $\begin{array}{l}\text { Unable to } \\
\text { assess }\end{array}$ & $\begin{array}{l}\text { No difference } \\
\text { at } \geq 4 \text { weeks }\end{array}$ & Low \\
\hline $\begin{array}{l}\text { KQ 2: Acute } \\
\text { neck pain } \\
\text { Collar vs. } \\
\text { exercise, } \\
\text { whiplash neck } \\
\text { strain }\end{array}$ & $\begin{array}{l}\text { Pain, health } \\
\text { status }\end{array}$ & $\geq 4$ weeks & 1 & 297 & Direct & Precise & Fair & $\begin{array}{l}\text { Unable to } \\
\text { assess }\end{array}$ & $\begin{array}{l}\text { No difference } \\
\text { at } \geq 4 \text { weeks }\end{array}$ & Low \\
\hline $\begin{array}{l}\text { KQ 2: Acute } \\
\text { neck pain } \\
\text { Exercise vs. } \\
\text { usual activity, } \\
\text { whiplash neck } \\
\text { strain }\end{array}$ & $\begin{array}{l}\text { Pain, health } \\
\text { status }\end{array}$ & $\geq 4$ weeks & 1 & 296 & Direct & Precise & Fair & $\begin{array}{l}\text { Unable to } \\
\text { assess }\end{array}$ & $\begin{array}{l}\text { No difference } \\
\text { at } \geq 4 \text { weeks }\end{array}$ & Low \\
\hline $\begin{array}{l}\text { KQ 3: Other } \\
\text { musculoskeletal } \\
\text { pain } \\
\text { NSAID vs. } \\
\text { acetaminophen }\end{array}$ & Pain & $\begin{array}{l}<1 \mathrm{~d}, 1 \mathrm{~d} \\
\text { to }<1 \mathrm{w}, 1 \\
\text { to }<2 \mathrm{w}, \\
\geq 4 \mathrm{w}\end{array}$ & 8 & 1,100 & Direct & Imprecise & Good & Consistent & No differences & Moderate \\
\hline $\begin{array}{l}\text { KQ 3: Other } \\
\text { musculoskeletal } \\
\text { pain } \\
\text { Ultrasound vs. } \\
\text { sham }\end{array}$ & Pain & $\begin{array}{l}1 \mathrm{~d} \text { to }<1 \\
w, \geq 4 \mathrm{w}\end{array}$ & 3 & 190 & Direct & Imprecise & Fair & Consistent & No differences & Low \\
\hline
\end{tabular}




\begin{tabular}{|c|c|c|c|c|c|c|c|c|c|c|}
\hline Intervention & Outcomes & $\begin{array}{l}\text { Timing of } \\
\text { Outcomes }\end{array}$ & $\begin{array}{l}\text { Number } \\
\text { of } \\
\text { Studies }\end{array}$ & $\begin{array}{l}\text { Number } \\
\text { of } \\
\text { Subjects }\end{array}$ & Directness & Precision & Quality & Consistency & Findings & SOE \\
\hline $\begin{array}{l}\text { KQ 3: Other } \\
\text { musculoskeletal } \\
\text { pain } \\
\text { Acupressure vs. } \\
\text { sham } \\
\text { acupressure or } \\
\text { usual care }\end{array}$ & $\begin{array}{l}\text { Pain, health } \\
\text { status }\end{array}$ & $\begin{array}{l}1 \mathrm{~d} \text { to }<1 \\
w, \geq 4 \mathrm{w}\end{array}$ & 1 & 62 & Direct & Precise & Fair & $\begin{array}{l}\text { Unable to } \\
\text { assess }\end{array}$ & $\begin{array}{l}\text { Moderate } \\
\text { decrease in } \\
\text { pain and small } \\
\text { improvement } \\
\text { in health } \\
\text { status with } \\
\text { acupressure }\end{array}$ & Low \\
\hline \multirow{2}{*}{$\begin{array}{l}\text { KQ 4: Acute } \\
\text { neuropathic pain } \\
\text { Opioid vs. } \\
\text { gabapentin, } \\
\text { herpes zoster }\end{array}$} & Pain & $\begin{array}{l}1 \text { to }<2 w, \\
\geq 4 \mathrm{w}\end{array}$ & 1 & 45 & Direct & Imprecise & Fair & $\begin{array}{l}\text { Unable to } \\
\text { assess }\end{array}$ & $\begin{array}{l}\text { Increased } \\
\text { likelihood of } \\
\text { improvement } \\
\text { in pain }\end{array}$ & Low \\
\hline & Constipation & $\begin{array}{l}1 \text { to }<2 w, \\
\geq 4 \mathrm{w}\end{array}$ & 1 & 45 & Direct & Imprecise & Fair & $\begin{array}{l}\text { Unable to } \\
\text { assess }\end{array}$ & $\begin{array}{l}\text { Increased risk } \\
\text { of constipation } \\
\text { with opioid }\end{array}$ & Low \\
\hline $\begin{array}{l}\text { KQ 5: } \\
\text { Postoperative } \\
\text { pain }\end{array}$ & $\begin{array}{l}\text { Pain, rescue } \\
\text { medication } \\
\text { use }\end{array}$ & $<1 d$ & 2 & 421 & Direct & Imprecise & Fair & Consistent & No differences & Low \\
\hline \multirow{2}{*}{$\begin{array}{l}\text { KQ 5: } \\
\text { Postoperative } \\
\text { pain } \\
\text { Opioid vs. } \\
\text { NSAID, } \\
\text { multidose } \\
\text { course, various } \\
\text { surgeries }\end{array}$} & Pain & $\begin{array}{l}1 \mathrm{~d} \text { to }<1 \\
W\end{array}$ & 4 & 830 & Direct & Imprecise & Fair & Inconsistent & $\begin{array}{l}\text { Unable to } \\
\text { determine }\end{array}$ & Insufficient \\
\hline & $\begin{array}{l}\text { Rescue } \\
\text { medication } \\
\text { use }\end{array}$ & $\begin{array}{l}1 \mathrm{~d} \text { to }<1 \\
w\end{array}$ & 4 & 860 & Direct & Imprecise & Fair & Consistent & $\begin{array}{l}\text { RR } 1.22 \text { to } \\
2.04\end{array}$ & Moderate \\
\hline $\begin{array}{l}\text { KQ 5: } \\
\text { Postoperative } \\
\text { pain } \\
\text { Opioid vs. } \\
\text { acetaminophen, } \\
\text { single dose, } \\
\text { cesarean section }\end{array}$ & $\begin{array}{l}\text { Pain, re- } \\
\text { medication }\end{array}$ & $<1 \mathrm{~d}$ & 1 & 96 & Direct & Imprecise & Fair & $\begin{array}{l}\text { Unable to } \\
\text { assess }\end{array}$ & No difference & Low \\
\hline
\end{tabular}




\begin{tabular}{|c|c|c|c|c|c|c|c|c|c|c|}
\hline Intervention & Outcomes & $\begin{array}{l}\text { Timing of } \\
\text { Outcomes }\end{array}$ & $\begin{array}{l}\text { Number } \\
\text { of } \\
\text { Studies }\end{array}$ & $\begin{array}{l}\text { Number } \\
\text { of } \\
\text { Subjects }\end{array}$ & Directness & Precision & Quality & Consistency & Findings & SOE \\
\hline $\begin{array}{l}\text { KQ 5: } \\
\text { Postoperative } \\
\text { pain } \\
\text { Opioid vs. } \\
\text { acetaminophen, } \\
\text { multidose } \\
\text { course, various } \\
\text { surgeries }\end{array}$ & $\begin{array}{l}\text { Study } \\
\text { withdrawal } \\
\text { due to AEs }\end{array}$ & $\begin{array}{l}<1 \mathrm{~d}, 1 \mathrm{~d} \\
\text { to }<1 \mathrm{w}\end{array}$ & 3 & 252 & Direct & Imprecise & Fair & Consistent & $\begin{array}{l}\text { Increased risk } \\
\text { with opioid }\end{array}$ & Low \\
\hline $\begin{array}{l}\text { KQ 5: } \\
\text { Postoperative } \\
\text { pain } \\
\text { NSAID vs. } \\
\text { acetaminophen, } \\
\text { single dose, } \\
\text { various } \\
\text { surgeries }\end{array}$ & $\begin{array}{l}\text { Pain, rescue } \\
\text { medication } \\
\text { use }\end{array}$ & $<1 d$ & 2 & 113 & Direct & Imprecise & Fair & Inconsistent & $\begin{array}{l}\text { Unable to } \\
\text { determine }\end{array}$ & Insufficient \\
\hline $\begin{array}{l}\text { KQ 5: } \\
\text { Postoperative } \\
\text { pain } \\
\text { Acupuncture vs. } \\
\text { sham, various } \\
\text { surgeries }\end{array}$ & Pain & $\begin{array}{l}1 \mathrm{~d} \text { to }<1 \\
w\end{array}$ & 2 & 106 & Direct & Imprecise & Fair & Inconsistent & $\begin{array}{l}\text { Unable to } \\
\text { determine }\end{array}$ & Insufficient \\
\hline \multirow{3}{*}{$\begin{array}{l}\text { KQ 5: } \\
\text { Postoperative } \\
\text { pain } \\
\text { Acupressure vs. } \\
\text { sham, knee } \\
\text { surgeries }\end{array}$} & Pain & $\begin{array}{l}<1 \mathrm{~d}, 1 \mathrm{~d} \\
\text { to }<1 \mathrm{w}\end{array}$ & 2 & 130 & Direct & Imprecise & Fair & Consistent & $\begin{array}{l}\text { Unable to } \\
\text { determine }\end{array}$ & Insufficient \\
\hline & $\begin{array}{l}\text { Pain } \\
\text { medication } \\
\text { use }\end{array}$ & $\begin{array}{l}<1 \mathrm{~d}, 1 \mathrm{~d} \\
\text { to }<1 \mathrm{w}\end{array}$ & 2 & 130 & Direct & Imprecise & Fair & Consistent & $\begin{array}{l}\text { Decreased } \\
\text { with } \\
\text { acupuncture }\end{array}$ & Low \\
\hline & $\begin{array}{l}\text { Pain } \\
\text { intensity }\end{array}$ & $<1 w$ & 3 & 168 & Direct & Imprecise & Fair & Consistent & No differences & Low \\
\hline
\end{tabular}




\begin{tabular}{|c|c|c|c|c|c|c|c|c|c|c|}
\hline Intervention & Outcomes & $\begin{array}{l}\text { Timing of } \\
\text { Outcomes }\end{array}$ & $\begin{array}{l}\text { Number } \\
\text { of } \\
\text { Studies }\end{array}$ & $\begin{array}{l}\text { Number } \\
\text { of } \\
\text { Subjects }\end{array}$ & Directness & Precision & Quality & Consistency & Findings & SOE \\
\hline $\begin{array}{l}\text { KQ 5: } \\
\text { Postoperative } \\
\text { pain }\end{array}$ & $\begin{array}{l}\text { Pain } \\
\text { intensity; } \\
\text { function, } \\
\text { QoL }\end{array}$ & $\begin{array}{l}2 \text { to }<4 \mathrm{w} \\
\geq 4 \mathrm{w}\end{array}$ & 1 & 60 & Direct & Imprecise & Good & $\begin{array}{l}\text { Unable to } \\
\text { assess }\end{array}$ & No differences & Low \\
\hline $\begin{array}{l}\text { Cold therapy vs. } \\
\text { sham or usual } \\
\text { care, knee } \\
\text { surgeries }\end{array}$ & $\begin{array}{l}\text { Pain } \\
\text { medication } \\
\text { use }\end{array}$ & $<1 \mathrm{w}$ & 1 & 60 & Direct & Imprecise & Good & $\begin{array}{l}\text { Unable to } \\
\text { assess }\end{array}$ & $\begin{array}{l}\text { Decreased } \\
\text { with cold } \\
\text { therapy }\end{array}$ & Low \\
\hline $\begin{array}{l}\text { KQ 5: } \\
\text { Postoperative } \\
\text { pain } \\
\text { Massage vs. no } \\
\text { massage, } \\
\text { various } \\
\text { surgeries }\end{array}$ & $\begin{array}{l}\text { Pain } \\
\text { intensity, } \\
\text { decreased } \\
\text { pain } \\
\text { medication } \\
\text { use, anxiety }\end{array}$ & $<1 \mathrm{~d}$ & 2 to 5 & 733 & Direct & Precise & Poor & Consistent & $\begin{array}{l}\text { Moderate to } \\
\text { large decrease } \\
\text { with massage } \\
\text { at }<1 \text { day, } \\
\text { decreased } \\
\text { pain } \\
\text { medication } \\
\text { use, and } \\
\text { decreased } \\
\text { anxiety }\end{array}$ & Low \\
\hline $\begin{array}{l}\text { KQ 5: } \\
\text { Postoperative } \\
\text { pain } \\
\text { Music therapy } \\
\text { vs. no music } \\
\text { therapy, various } \\
\text { surgeries }\end{array}$ & Pain & $\begin{array}{l}<1 \mathrm{~d}, 1 \mathrm{~d} \\
\text { to }<1 \mathrm{w}\end{array}$ & 2 & 148 & Direct & Imprecise & Fair & Consistent & $\begin{array}{l}\text { Small to } \\
\text { moderate } \\
\text { decrease in } \\
\text { pain intensity }\end{array}$ & Low \\
\hline $\begin{array}{l}\text { KQ 5: } \\
\text { Postoperative } \\
\text { pain } \\
\text { Exercise vs. no } \\
\text { exercise, thyroid } \\
\text { surgery }\end{array}$ & Function & $\begin{array}{l}1 \text { to }<2 w, \\
\geq 4 w\end{array}$ & 1 & 80 & Direct & Imprecise & Fair & $\begin{array}{l}\text { Unable to } \\
\text { assess }\end{array}$ & $\begin{array}{l}\text { Large } \\
\text { decrease with } \\
\text { exercise at } 1 \\
\text { week, no } \\
\text { difference at } 1 \\
\text { month }\end{array}$ & Low \\
\hline $\begin{array}{l}\text { KQ 5: } \\
\text { Postoperative } \\
\text { pain } \\
\text { TENS vs. sham } \\
\text { TENS, } \\
\text { liposuction }\end{array}$ & $\begin{array}{l}\text { Pain } \\
\text { intensity, } \\
\text { analgesic } \\
\text { use }\end{array}$ & $\begin{array}{l}<1 \mathrm{~d}, 1 \mathrm{~d} \\
\text { to }<1 \mathrm{w}\end{array}$ & 1 & 42 & Direct & Imprecise & Fair & $\begin{array}{l}\text { Unable to } \\
\text { assess }\end{array}$ & $\begin{array}{l}\text { Moderate to } \\
\text { large decrease } \\
\text { in pain } \\
\text { intensity and } \\
\text { decreased } \\
\text { analgesic use } \\
\text { with TENS }\end{array}$ & Low \\
\hline
\end{tabular}




\begin{tabular}{|c|c|c|c|c|c|c|c|c|c|c|}
\hline Intervention & Outcomes & $\begin{array}{l}\text { Timing of } \\
\text { Outcomes }\end{array}$ & $\begin{array}{l}\text { Number } \\
\text { of } \\
\text { Studies }\end{array}$ & $\begin{array}{l}\text { Number } \\
\text { of } \\
\text { Subjects }\end{array}$ & Directness & Precision & Quality & Consistency & Findings & SOE \\
\hline \multirow[t]{2}{*}{$\begin{array}{l}\text { KQ 6: Dental } \\
\text { pain } \\
\text { Opioid + } \\
\text { acetaminophen } \\
\text { vs. } \\
\text { acetaminophen, } \\
\text { single dose }\end{array}$} & Pain & $<1 \mathrm{~d}$ & 11 & 828 & Direct & Precise & Fair & Inconsistent & $\begin{array}{l}\text { Inconsistent } \\
\text { effects on pain } \\
\text { intensity, but } \\
\text { larger sum of } \\
\text { pain intensity } \\
\text { differences } \\
\text { with opioid }\end{array}$ & $\begin{array}{l}\text { Moderate } \\
\text { (for sum of } \\
\text { pain intensity } \\
\text { differences) }\end{array}$ \\
\hline & $\begin{array}{l}\text { Rescue or } \\
\text { repeat } \\
\text { medication } \\
\text { use }\end{array}$ & $<1 \mathrm{~d}$ & 7 & 484 & Direct & Precise & Fair & Consistent & $\begin{array}{l}\text { RR } 0.81,95 \% \\
\text { Cl } 0.56 \text { to } 0.97\end{array}$ & Moderate \\
\hline $\begin{array}{l}\text { KQ 6: Dental } \\
\text { pain } \\
\text { Opioid vs. } \\
\text { acetaminophen, } \\
\text { single dose }\end{array}$ & $\begin{array}{l}\text { Pain, rescue } \\
\text { medication } \\
\text { use }\end{array}$ & $<1 \mathrm{~d}$ & 2 & 149 & Direct & Imprecise & Fair & Consistent & No differences & Low \\
\hline $\begin{array}{l}\text { KQ 6: Dental } \\
\text { pain } \\
\text { Opioid (with or } \\
\text { without } \\
\text { acetaminophen) } \\
\text { vs. } \\
\text { acetaminophen, } \\
\text { single dose }\end{array}$ & $\begin{array}{l}\text { Any AE, } \\
\text { nausea, } \\
\text { drowsiness, } \\
\text { dizziness }\end{array}$ & $<1 d$ & 4 to 8 & $\begin{array}{l}445 \text { to } \\
769\end{array}$ & Direct & Imprecise & Fair & Consistent & $\begin{array}{l}\text { Increased risk } \\
\text { with opioid }\end{array}$ & Low \\
\hline $\begin{array}{l}\text { KQ 6: Dental } \\
\text { pain } \\
\text { Opioid plus } \\
\text { acetaminophen } \\
\text { or NSAID vs. } \\
\text { NSAID, single } \\
\text { dose }\end{array}$ & $\begin{array}{l}\text { Pain, rescue } \\
\text { or repeat } \\
\text { medication } \\
\text { use }\end{array}$ & $<1 d$ & 8 to 12 & $\begin{array}{l}926 \text { to } \\
2,021\end{array}$ & Direct & Precise & Fair & $\begin{array}{l}\text { Inconsistent } \\
\text { (pain } \\
\text { intensity); } \\
\text { consistent } \\
\text { (rescue or } \\
\text { repeat } \\
\text { medication } \\
\text { use) }\end{array}$ & $\begin{array}{l}\text { Small to } \\
\text { moderate } \\
\text { increase in } \\
\text { pain intensity } \\
\text { with opioids, } \\
\text { increased } \\
\text { likelihood of } \\
\text { rescue or } \\
\text { repeat } \\
\text { medication } \\
\text { use (RR } 1.35 \text {, } \\
95 \% \mathrm{Cl} 1.23 \text { to } \\
1.48)\end{array}$ & $\begin{array}{l}\text { Low for pain; } \\
\text { moderate for } \\
\text { rescue or } \\
\text { repeat } \\
\text { medication } \\
\text { use }\end{array}$ \\
\hline
\end{tabular}




\begin{tabular}{|c|c|c|c|c|c|c|c|c|c|c|}
\hline Intervention & Outcomes & $\begin{array}{l}\text { Timing of } \\
\text { Outcomes }\end{array}$ & $\begin{array}{l}\text { Number } \\
\text { of } \\
\text { Studies }\end{array}$ & $\begin{array}{l}\text { Number } \\
\text { of } \\
\text { Subjects }\end{array}$ & Directness & Precision & Quality & Consistency & Findings & SOE \\
\hline $\begin{array}{l}\text { KQ 6: Dental } \\
\text { pain } \\
\text { Opioid vs. } \\
\text { acetaminophen, } \\
\text { multidose } \\
\text { course }\end{array}$ & $\begin{array}{l}\text { Pain } \\
\text { intensity }\end{array}$ & $\begin{array}{l}1 \mathrm{~d} \text { to }<1 \\
W\end{array}$ & 1 & 20 & Direct & Imprecise & Fair & Consistent & No difference & Low \\
\hline $\begin{array}{l}\text { KQ 6: Dental } \\
\text { pain } \\
\text { Opioid (with or } \\
\text { without } \\
\text { acetaminophen) } \\
\text { vs. NSAID }\end{array}$ & $\begin{array}{l}\text { Any } \mathrm{AE}, \\
\text { nausea, } \\
\text { dizziness, } \\
\text { drowsiness }\end{array}$ & $\begin{array}{l}<1 \mathrm{~d}, 1 \mathrm{~d} \\
\text { to }<1 \mathrm{w}\end{array}$ & 9 to 12 & $\begin{array}{l}1,959 \text { to } \\
2,784\end{array}$ & Direct & Precise & Fair & Consistent & $\begin{array}{l}\text { RR } 1.72(95 \% \\
\mathrm{Cl} 1.29 \text { to } \\
2.28) \text { for any } \\
\mathrm{AE}, 2.72 \text { (95\% } \\
\mathrm{Cl} 1.84 \text { to } \\
4.01) \text { for } \\
\text { nausea, } 2.97 \\
(95 \% \mathrm{Cl} 1.59 \\
\text { to } 5.54) \text { for } \\
\text { dizziness, and } \\
1.76 \text { ( } 95 \% \mathrm{Cl} \\
1.00 \text { to } 3.10) \\
\text { for drowsiness }\end{array}$ & Moderate \\
\hline \multirow[t]{2}{*}{$\begin{array}{l}\text { KQ 6: Dental } \\
\text { pain } \\
\text { NSAID vs. } \\
\text { acetaminophen, } \\
\text { single dose }\end{array}$} & $\begin{array}{l}\text { Pain } \\
\text { intensity, } \\
\text { rescue or } \\
\text { repeat } \\
\text { medication } \\
\text { use }\end{array}$ & $<1 d$ & 11 to 15 & $\begin{array}{l}2,014 \text { to } \\
2,506\end{array}$ & Direct & Precise & Fair & Consistent & $\begin{array}{l}\text { Moderate to } \\
\text { large decrease } \\
\text { in pain with } \\
\text { NSAID, } \\
\text { decreased } \\
\text { likelihood of } \\
\text { rescue or } \\
\text { repeat } \\
\text { medication } \\
\text { use (RR } 0.64 \text {, } \\
95 \% \mathrm{Cl} 0.58 \text { to } \\
0.71 \text { ) }\end{array}$ & Moderate \\
\hline & Any AE & $<1 d$ & 12 & 2,512 & Direct & Precise & Fair & Consistent & $\begin{array}{l}\text { RR } 0.85(95 \% \\
\mathrm{Cl} 0.72 \text { to } \\
1.00)\end{array}$ & Moderate \\
\hline
\end{tabular}




\begin{tabular}{|c|c|c|c|c|c|c|c|c|c|c|}
\hline Intervention & Outcomes & $\begin{array}{l}\text { Timing of } \\
\text { Outcomes }\end{array}$ & $\begin{array}{l}\text { Number } \\
\text { of } \\
\text { Studies }\end{array}$ & $\begin{array}{l}\text { Number } \\
\text { of } \\
\text { Subjects }\end{array}$ & Directness & Precision & Quality & Consistency & Findings & SOE \\
\hline \multirow[t]{2}{*}{$\begin{array}{l}\text { KQ 7: Kidney } \\
\text { stone pain } \\
\text { Morphine vs. } \\
\text { NSAID, single } \\
\text { dose }\end{array}$} & $\begin{array}{l}\text { Pain, rescue } \\
\text { medication } \\
\text { use }\end{array}$ & $<1 d$ & 1 & 1,097 & Direct & Precise & Good & $\begin{array}{l}\text { Unable to } \\
\text { assess }\end{array}$ & $\begin{array}{l}\text { Increased } \\
\text { likelihood of } \\
\text { pain, and } \\
\text { rescue } \\
\text { medication } \\
\text { use with } \\
\text { morphine }\end{array}$ & Moderate \\
\hline & Any $A E$ & $<1 d$ & 1 & 1,097 & Direct & Precise & Good & $\begin{array}{l}\text { Unable to } \\
\text { assess }\end{array}$ & $\begin{array}{l}3 \% \text { vs. } 1 \%, \\
\text { RR } 2.70(95 \% \\
\text { Cl } 1.15 \text { to } \\
6.38)\end{array}$ & Moderate \\
\hline \multirow[t]{2}{*}{$\begin{array}{l}\text { KQ 7: Kidney } \\
\text { stone pain } \\
\text { Meperidine vs. } \\
\text { NSAID, single } \\
\text { dose }\end{array}$} & $\begin{array}{l}\text { Pain, rescue } \\
\text { medication } \\
\text { use }\end{array}$ & $<1 \mathrm{~d}$ & 4 to 6 & $\begin{array}{l}475 \text { to } \\
671\end{array}$ & Direct & Precise & Fair & Inconsistent & $\begin{array}{l}\text { Moderate to } \\
\text { large increase } \\
\text { in pain } \\
\text { intensity with } \\
\text { meperidine, } \\
\text { increased } \\
\text { likelihood of } \\
\text { rescue } \\
\text { medication } \\
\text { use }\end{array}$ & Moderate \\
\hline & $\begin{array}{l}\text { Any } A E, \\
\text { somnolence, } \\
\text { nausea }\end{array}$ & $<1 d$ & 4 to 5 & $\begin{array}{l}471 \text { to } \\
573\end{array}$ & Direct & Imprecise & Fair & Inconsistent & $\begin{array}{l}\text { RR } 1.71(95 \% \\
\mathrm{Cl} 0.99 \text { to } \\
2.96) \text { for any } \\
\mathrm{AE}, \mathrm{RR} 1.98 \\
(95 \% \mathrm{Cl} 0.82 \\
\text { to } 4.79) \text { for } \\
\text { somnolence, } \\
\text { and RR } 1.84 \\
(95 \% \mathrm{Cl} 1.02 \\
\text { to } 3.31) \text { for } \\
\text { nausea }\end{array}$ & Low \\
\hline $\begin{array}{l}\text { KQ 7: Kidney } \\
\text { stone pain } \\
\text { Morphine vs. } \\
\text { acetaminophen, } \\
\text { single dose }\end{array}$ & $\begin{array}{l}\text { Pain, rescue } \\
\text { medication } \\
\text { use }\end{array}$ & $<1 d$ & 1 & 1,096 & Direct & Precise & Good & $\begin{array}{l}\text { Unable to } \\
\text { assess }\end{array}$ & $\begin{array}{l}\text { Increased } \\
\text { likelihood of } \\
\text { pain with } \\
\text { morphine, } \\
\text { similar rescue } \\
\text { medication } \\
\text { use }\end{array}$ & Moderate \\
\hline
\end{tabular}




\begin{tabular}{|c|c|c|c|c|c|c|c|c|c|c|}
\hline Intervention & Outcomes & $\begin{array}{l}\text { Timing of } \\
\text { Outcomes }\end{array}$ & $\begin{array}{l}\text { Number } \\
\text { of } \\
\text { Studies }\end{array}$ & $\begin{array}{l}\text { Number } \\
\text { of } \\
\text { Subjects }\end{array}$ & Directness & Precision & Quality & Consistency & Findings & SOE \\
\hline & Any $A E$ & $<1 \mathrm{~d}$ & 1 & 1,096 & Direct & Precise & Good & $\begin{array}{l}\text { Unable to } \\
\text { assess }\end{array}$ & $\begin{array}{l}3 \% \text { vs. } 1 \%, \\
\text { RR } 2.71(95 \% \\
\mathrm{Cl} 1.15 \text { to } \\
6.39)\end{array}$ & Moderate \\
\hline $\begin{array}{l}\text { KQ 7: Kidney } \\
\text { stone pain } \\
\text { NSAID vs. } \\
\text { acetaminophen, } \\
\text { single dose }\end{array}$ & $\begin{array}{l}\text { Pain, rescue } \\
\text { medication } \\
\text { use }\end{array}$ & $<1 \mathrm{~d}$ & 2 to 3 & $\begin{array}{l}1,145 \text { to } \\
1,225\end{array}$ & Direct & $\begin{array}{l}\text { Imprecise } \\
\text { (for pain) }\end{array}$ & Fair & $\begin{array}{l}\text { Inconsistent } \\
\text { (pain) }\end{array}$ & $\begin{array}{l}\text { Inconsistent } \\
\text { effects on } \\
\text { pain; } \\
\text { decreased } \\
\text { likelihood of } \\
\text { rescue } \\
\text { medication } \\
\text { use with } \\
\text { NSAID }\end{array}$ & $\begin{array}{l}\text { Low for } \\
\text { rescue } \\
\text { medication } \\
\text { use, } \\
\text { insufficient } \\
\text { for pain }\end{array}$ \\
\hline $\begin{array}{l}\text { KQ 7: Kidney } \\
\text { stone pain } \\
\text { Acupuncture vs. } \\
\text { NSAID or } \\
\text { acetaminophen }\end{array}$ & Pain & $<1 \mathrm{~d}$ & 1 & 160 & Direct & Imprecise & Fair & $\begin{array}{l}\text { Unable to } \\
\text { assess }\end{array}$ & $\begin{array}{l}\text { Moderate } \\
\text { increase in } \\
\text { pain with } \\
\text { acupuncture }\end{array}$ & Low \\
\hline $\begin{array}{l}\text { KQ8: Sickle cell } \\
\text { pain } \\
\text { Insufficient } \\
\text { evidence }\end{array}$ & -- & -- & -- & -- & -- & -- & -- & -- & -- & -- \\
\hline
\end{tabular}

Abbreviations: $\mathrm{AE}=$ adverse event; $\mathrm{CI}=$ confidence interval; $\mathrm{D}=$ day; $\mathrm{KQ}=$ Key Question NSAID = nonsteroidal anti-inflammatory drug; $\mathrm{QoL}=$ quality of life; $\mathrm{RR}=$ relative risk; SOE = strength of evidence; TENS = transcutaneous electrical nerve stimulation; $\mathrm{W}=$ week 


\section{Appendix H. Excluded Studies List}

1. Fingolimod (Gilenya): An oral sphingosine 1-phosphate receptor modulator. Formulary. 2010;45(12). Exclusion reason: Ineligible publication type

2. The effects of gabapentin and ibuprofen on pain and reduction of morphine consumption in patients suffering from orthopedic fractures admitted in hospital emergency room. International Journal of Pharmaceutical Research. 2019;11(4):191-6. Exclusion reason: Not in English

3. Abdallah FW, Halpern SH, Aoyama K, et al. Will the Real Benefits of Single-Shot Interscalene Block Please Stand Up? A Systematic Review and Meta-Analysis. Anesth Analg. 2015 May;120(5):1114-29. doi:

https://dx.doi.org/10.1213/ANE.0000000000 000688. PMID: 25822923. Exclusion

reason: Publication used as source document

4. Abdel Shaheed C, Maher CG, Williams KA, et al. Efficacy, Tolerability, and DoseDependent Effects of Opioid Analgesics for Low Back Pain: A Systematic Review and Meta-analysis. JAMA Intern Med. 2016 Jul 01;176(7):958-68. doi: https://dx.doi.org/10.1001/jamainternmed.20 16.1251. PMID: 27213267. Exclusion reason: Publication used as source document

5. Abdel Shaheed C, Maher CG, Williams KA, et al. Interventions available over the counter and advice for acute low back pain: systematic review and meta-analysis. J Pain. 2014 Jan;15(1):2-15. doi: 10.1016/j.jpain.2013.09.016. PMID: 24373568. Exclusion reason: Publication used as source document

6. Abdel Shaheed C, Maher CG, Williams KA, et al. Efficacy and tolerability of muscle relaxants for low back pain: Systematic review and meta-analysis. Eur J Pain. 2017 02;21(2):228-37. doi: 10.1002/ejp.907. PMID: 27329976. Exclusion reason: Publication used as source document
7.

Abou-Karam M, Dube S, Kvann HS, et al. Parental Report of Morphine Use at Home after Pediatric Surgery. J Pediatr. 2015 Sep;167(3):599-604.e1-2. doi: https://dx.doi.org/10.1016/j.jpeds.2015.06.0 35. PMID: 26205183. Exclusion reason: Background information only

8. Aboursheid T, Albaroudi O, Alahdab F. Inhaled nitric oxide for treating pain crises in people with sickle cell disease. Cochrane Database Syst Rev. 2019(10) PMID: 31603241. Exclusion reason: Ineligible intervention

9. Abou-Setta AM, Beaupre LA, Rashiq S, et al. Comparative effectiveness of pain management interventions for hip fracture: a systematic review. Ann Intern Med. 2011 Aug 16;155(4):234-45. doi: 10.7326/00034819-155-4-201108160-00346. PMID: 21844549. Exclusion reason: Publication used as source document

10. Acevedo JI, Beskin JL. Complications of plantar fascia rupture associated with corticosteroid injection. Foot Ankle Int. 1998 Feb;19(2):91-7. doi: 10.1177/107110079801900207. PMID: 9498581. Exclusion reason: Ineligible population

11. Achterberg J, Kenner C, Casey D. Behavioral strategies for the reduction of pain and anxiety associated with orthopedic trauma. Biofeedback Self Regul. 1989 Jun;14(2):101-14. PMID: 2675983.

Exclusion reason: Ineligible study design

12. Acosta-Olivo C, Siller-Adame A, TamezMata Y, et al. Laser Treatment on Acupuncture Points Improves Pain and Wrist Functionality in Patients Undergoing Rehabilitation Therapy after Wrist Bone Fracture. A Randomized, Controlled, Blinded Study. Acupunct Electrother Res. 2017 Jan;42(1):11-25. doi: 10.3727/036012917x14908026365007. PMID: 29772132. Exclusion reason: Ineligible intervention 
13. Adachi N, Munesada M, Yamada N, et al. Effects of aromatherapy massage on facedown posture-related pain after vitrectomy: a randomized controlled trial. Pain Manag Nurs. 2014 Jun;15(2):482-9. doi: 10.1016/j.pmn.2012.12.004. PMID: 23466193. Exclusion reason: Ineligible setting

14. Adamek S, Matouskova O, Polanecky O, et al. The effect of postoperative pain treatment on the incidence of anastomotic insufficiency after rectal and rectosigmoideal surgery. Prague Med Rep. 2013;114(4):214-21. doi: 10.14712/23362936.2014.10. PMID: 24485338. Exclusion reason: Ineligible study design

15. Adhikari S, Koirala P, Kafle D. Comparison of Scapular Manipulation With External Rotation Method of Reduction of Acute Anterior Shoulder Dislocation for Sedation Requirements and Success Rates. J Spec Oper Med. 2018 2018;18(3):34-7. PMID: 30222834. Exclusion reason: Ineligible intervention

16. Adib-Hajbaghery M, Etri M. Effect of acupressure of Ex-Le7 point on pain, nausea and vomiting after appendectomy: A randomized trial. J Res Med Sci. 2013;18(6):482. PMID: 24250696.

Exclusion reason: Ineligible population

17. Afshar K, Jafari S, Marks AJ, et al. Nonsteroidal anti-inflammatory drugs (NSAIDs) and non-opioids for acute renal colic. Cochrane Database Syst Rev. 2015 Jun 29(6):CD006027. doi: 10.1002/14651858.CD006027.pub2. PMID: 26120804. Exclusion reason: Publication used as source document

18. Aghababian RV, Volturo GA, Heifetz IN. Comparison of diflunisal and naproxen in the management of acute low back strain. Clin Ther. 1986;9 Suppl C:47-51. PMID: 2951011. Exclusion reason: Ineligible comparator
19. Ahiskalioglu EO, Ahiskalioglu A, Aydin P, et al. Effects of a single-dose preemptive pregabalin on acute and chronic pain after inguinal hernia repair with mesh under spinal anaesthesia: A randomised controlled trial. Eur J Anaesthesiol. 2016

Aug;33(8):605-7. doi: 10.1097/EJA.0000000000000395. PMID: 27120252. Exclusion reason: Ineligible intervention

20. Ahmed HE, Craig WF, White PF, et al. Percutaneous electrical nerve stimulation: an alternative to antiviral drugs for acute herpes zoster. Anesth Analg. 1998 Oct;87(4):911-4. doi: 10.1097/00000539-199810000-00031. PMID: 9768793. Exclusion reason: Ineligible comparator

21. Ahn NY, Park HJ. Effects of Korean hand acupressure on opioid-related nausea and vomiting, and pain after caesarean delivery using spinal anaesthesia. Complement Ther Clin Pract. 2017 Aug;28:101-7. doi: 10.1016/j.ctcp.2017.05.014. PMID: 28779916. Exclusion reason: Ineligible study design

22. Aida N, Shibuya M, Yoshino K, et al. Respiratory muscle stretch gymnastics in patients with post coronary artery bypass grafting pain: impact on respiratory muscle function, activity, mood and exercise capacity. J Med Dent Sci. 2002 Dec;49(4):157-70. PMID: 12641387. Exclusion reason: Ineligible outcome

23. Airaksinen OV, Kyrklund N, Latvala K, et al. Efficacy of cold gel for soft tissue injuries: a prospective randomized doubleblinded trial. Am J Sports Med. 2003 SepOct;31(5):680-4. doi: 10.1177/03635465030310050801. PMID: 12975186. Exclusion reason: Ineligible comparator

24. Akarsu S, Sahin S, Kara C, et al. A comparison between parenteral paracetamol and diclofenac for acute postoperetive pain treatment in patients after caeserean section. Turk jinekoloji ve obstetrik dernegi dergisi. 2010;7(4):262-6. Exclusion reason: Not in English 
25. Akbari GA, Entezariasl M, Isazadehfar K. Comparison of the effects of indomethacin, diclofenac and acetaminophen suppositories on pain score and pethidine usage after cesarean section. Journal of Isfahan Medical School. 2012;30(179). Exclusion reason: Not in English

26. Akhter N, Zahid Siddiq M. Comparative efficacy of diclofenac sodium alone and in combination with thiocolchicoside in patients with low back pain. Medical Forum Monthly. 2017;28(11):93-6. Exclusion reason: Ineligible comparator

27. Akmese ZB, Oran NT. Effects of Progressive Muscle Relaxation Exercises Accompanied by Music on Low Back Pain and Quality of Life During Pregnancy. J Midwifery Womens Health. 2014 SepOct;59(5):503-9. doi: 10.1111/jmwh.12176. PMID: 24965313. Exclusion reason: Ineligible population

28. Aksoy C, Karan A, Diraçoğlu D. Low back pain: Results of an open clinical trial comparing the standard treatment alone to the combination of standard treatment and thiocolchicoside. J Orthop Traumatol. 2002;3(2):103-8. doi: 10.1007/s101950200036. Exclusion reason: Ineligible comparator

29. Aksoy MK, Altan L, Guner A. The effectiveness of soft and semi-rigid cervical collars on acute cervical radiculopathy. European Research Journal. 2018;4(1):1625. Exclusion reason: Ineligible population

30. Al Hajeri A, Fedorowicz Z. Piracetam for reducing the incidence of painful sickle cell disease crises. Cochrane Database Syst Rev. 2016 Feb 12;2:CD006111. doi: https://dx.doi.org/10.1002/14651858.CD006 111.pub3. PMID: 26869149. Exclusion reason: Publication used as source document

31. Albin SR, Koppenhaver SL, Marcus R, et al. Short-term Effects of Manual Therapy in Patients After Surgical Fixation of Ankle and/or Hindfoot Fracture: A Randomized Clinical Trial. J Orthop Sports Phys Ther. 2019 May;49(5):310-9. doi: 10.2519/jospt.2019.8864. PMID: 30759357.

Exclusion reason: Ineligible population
32. Alexander L, Hall E, Eriksson L, et al. The combination of non-selective NSAID 400 $\mathrm{mg}$ and paracetamol $1000 \mathrm{mg}$ is more effective than each drug alone for treatment of acute pain. A systematic review. Swed Dent J. 2014;38(1):1-14. PMID: 26995806. Exclusion reason: Publication used as source document

33. Ali AA. Management of the first episode of acute low back pain: a comparison between two treatment protocols $(\mathrm{PhD})$. Texas Woman's University. 2002. Exclusion reason: Ineligible publication type

34. Ali AA. Management of the first episode of acute low back pain: a comparison between two treatment protocols. Texas Woman's University. 2002. Exclusion reason: Ineligible publication type

35. Alijanipour P, Tan TL, Matthews CN, et al. Periarticular Injection of Liposomal Bupivacaine Offers No Benefit Over Standard Bupivacaine in Total Knee Arthroplasty: A Prospective, Randomized, Controlled Trial. J Arthroplasty. 2017 02;32(2):628-34. doi: 10.1016/j.arth.2016.07.023. PMID: 27667533. Exclusion reason: Ineligible intervention

36. Allred KD, Byers JF, Sole ML. The effect of music on postoperative pain and anxiety. Pain Manag Nurs. 2010 Mar;11(1):15-25. doi: 10.1016/j.pmn.2008.12.002. PMID: 20207324. Exclusion reason: Ineligible intervention

37. Almeida CC, Silva V, Junior GC, et al. Transcutaneous electrical nerve stimulation and interferential current demonstrate similar effects in relieving acute and chronic pain: a systematic review with metaanalysis. Braz J Phys Ther. 2018 Sep Oct;22(5):347-54. doi: https://dx.doi.org/10.1016/j.bjpt.2017.12.00 5. PMID: 29426587. Exclusion reason: Publication used as source document

38. Alpaslan C, Kahraman S, Guner B, et al. Does the use of soft or hard splints affect the short-term outcome of temporomandibular joint arthrocentesis? Int J Oral Maxillofac Surg. 2008 May;37(5):424-7. doi: 10.1016/j.ijom.2008.01.022. PMID: 18356022. Exclusion reason: Ineligible study design 
39. Alshahrani MS, Alsulaibikh AH, ElTahan MM, et al. 226 Ketamine Use for Acute Painful Crisis of Sickle Cell Disease: a Randomized Controlled Trial. Ann Emerg Med. 2019;74(4):S89-S90. Exclusion reason: Ineligible publication type

40. Al-Sukhun J, Al-Sukhun S, Penttila H, et al. Preemptive analgesic effect of low doses of celecoxib is superior to low doses of traditional nonsteroidal anti-inflammatory drugs. J Craniofac Surg. 2012

Mar;23(2):526-9. doi: 10.1097/SCS.0b013e31824cd4fb. PMID: 22421863. Exclusion reason: Ineligible intervention

41. Altan L, Kasapoglu Aksoy M, Kosegil Ozturk E. Efficacy of diclofenac \& thiocolchioside gel phonophoresis comparison with ultrasound therapy on acute low back pain; a prospective, doubleblind, randomized clinical study. Ultrasonics. 2019 Jan;91:201-5. doi: 10.1016/j.ultras.2018.08.008. PMID: 30139568. Exclusion reason: Ineligible comparator

42. Altman R, Daniels S, Young CL. Indomethacin submicron particle capsules provide effective pain relief in patients with acute pain: a phase 3 study. Phys Sportsmed. 2013 Nov;41(4):7-15. doi:

10.3810/psm.2013.11.2031. PMID: 24231592. Exclusion reason: Ineligible comparator

43. Aluko A, DeSouza L, Peacock J. The effect of core stability exercises on variations in acceleration of trunk movement, pain, and disability during an episode of acute nonspecific low back pain: a pilot clinical trial. J Manipulative Physiol Ther. 2013 Oct;36(8):497-504.e1-3. doi: 10.1016/j.jmpt.2012.12.012. PMID: 23948426. Exclusion reason: Ineligible comparator

44. Alvarenga MB, de Oliveira SM, Francisco AA, et al. Effect of low-level laser therapy on pain and perineal healing after episiotomy: A triple-blind randomized controlled trial. Lasers Surg Med. 2017 02;49(2):181-8. doi: 10.1002/lsm.22559. PMID: 27426042. Exclusion reason: Ineligible intervention
45. Amato F, Morrone EG, Lacquaniti G. Efficacy, safety and tolerance of subcutaneous injection of high dosages of diclofenac in patients with neuropathic noncancer pain and neuropathic cancer pain: Data from a clinical setting. Eur J Inflamm. 2015;13(1):32-9. doi: 10.1177/1721727X15576850. Exclusion reason: Ineligible population

46. Ameis A, Randhawa K, Yu H, et al. The Global Spine Care Initiative: a review of reviews and recommendations for the noninvasive management of acute osteoporotic vertebral compression fracture pain in lowand middle-income communities. Eur Spine J. 2018 09;27(Suppl 6):861-9. doi: https://dx.doi.org/10.1007/s00586-0175273-6. PMID: 29038868. Exclusion reason: Publication used as source document

47. Amin-Hanjani S, Corcoran J, Chatwani A. Cold therapy in the management of postoperative cesarean section pain. Am J Obstet Gynecol. 1992 Jul;167(1):108-9. doi: 10.1016/s0002-9378(11)91638-x. PMID: 1442907. Exclusion reason: Ineligible outcome

48. Andersen LL, Jay K, Andersen CH, et al. Acute effects of massage or active exercise in relieving muscle soreness: randomized controlled trial. J Strength Cond Res. 2013 Dec;27(12):3352-9. doi: 10.1519/JSC.0b013e3182908610. PMID: 23524365. Exclusion reason: Ineligible population

49. Andersen LL, Kjaer M, Sogaard K, et al. Effect of two contrasting types of physical exercise on chronic neck muscle pain. Arthritis Rheum. 2008 Jan 15;59(1):84-91. doi: 10.1002/art.23256. PMID: 18163419. Exclusion reason: Ineligible population

50. Andersen T, Christensen FB, Ernst C, et al. The effect of electrical stimulation on lumbar spinal fusion in older patients: a randomized, controlled, multi-center trial: part 1: functional outcome. Spine. 2009 Oct 01;34(21):2241-7. doi: 10.1097/BRS.0b013e3181b02988. PMID: 19934802. Exclusion reason: Ineligible intervention 
51. Andersson S, Fredin H, Lindberg H, et al. Ibuprofen and compression bandage in the treatment of ankle sprains. Acta Orthop Scand. 1983 Apr;54(2):322-5. doi: 10.3109/17453678308996578. PMID: 6342332. Exclusion reason: Ineligible comparator

52. Angelo JL, Wu J, Sirody J, et al. Reduction in Prescribed Opioids after General Surgery Procedures at a Public Hospital. Am Surg. 2019 Oct 01;85(10):1198-203. PMID: 31657324. Exclusion reason: Ineligible intervention

53. Anie KA, Green J. Psychological therapies for sickle cell disease and pain [Systematic Review]. Cochrane Database Syst Rev. 2015;5:5. PMID: 25966336 Exclusion reason: Publication used as source document

54. Anonymous. Pain Management in the Opioid-Dependent Pregnant Woman. J Perinat Neonatal Nurs. 2017

Apr/Jun;31(2):E1. doi: 10.1097/JPN.0000000000000261. PMID: 28437303. Exclusion reason: Ineligible publication type

55. Antall GF, Kresevic D. The use of guided imagery to manage pain in an elderly orthopaedic population. Orthop Nurs. 2004 Sep-Oct;23(5):335-40. doi: 10.1097/00006416-200409000-00012. PMID: 15554471. Exclusion reason: Ineligible setting

56. Anthony CA, Volkmar A, Shah AS, et al. Communication with Orthopedic Trauma Patients via an Automated Mobile Phone Messaging Robot. Telemed J E Health. 2018 07;24(7):504-9. doi: https://dx.doi.org/10.1089/tmj.2017.0188. PMID: 29261036. Exclusion reason: Background information only

57. Aoki T, Numajiri M, Yamamoto M. A well controlled comparative study of piroxicam gel, indomethacin gel and placebo gel in the treatment of trauma. Japanese Pharmacology and Therapeutics. 1984;12(12):101-17.

Exclusion reason: Ineligible comparator
58. Aparna P, Geetha P, Shanmugasundaram P. Comparison of aceclofenac and combination (Aceclofenac + thiocolchicoside) therapy in acute low back pain patients. Res J Pharm Technol. 2017;9(11):1927-9. Exclusion reason: Ineligible study design

59. Aqua K, Gimbel JS, Singla N, et al. Efficacy and tolerability of oxymorphone immediate release for acute postoperative pain after abdominal surgery: a randomized, doubleblind, active- and placebo-controlled, parallel-group trial. Clin Ther. 2007 Jun;29(6):1000-12. doi: 10.1016/j.clinthera.2007.06.001. PMID: 17692717. Exclusion reason: Ineligible setting

60. Araki S, Kawamura O, Mataka T. Randomized controlled trial comparing the effect of manual acupuncture with sham acupuncture for acute low back pain. Journal of Japanese Society for Acupuncture. 2001;51(382). Exclusion reason: Not in English

61. Araujo FX, Scholl Schell M, Ribeiro DC. Effectiveness of Physiotherapy interventions plus Extrinsic Feedback for neck disorders: A systematic review with meta-analysis. Musculoskelet Sci Pract. 2017 06;29:13243. doi: 10.1016/j.msksp.2017.04.005. PMID: 28412631. Exclusion reason: Publication used as source document

62. Archer KR, Coronado RA, Haug CM, et al. A comparative effectiveness trial of postoperative management for lumbar spine surgery: changing behavior through physical therapy (CBPT) study protocol. BMC Musculoskelet Disord. 2014 Oct 01;15:325. doi: https://dx.doi.org/10.1186/1471-247415-325. PMID: 25273991. Exclusion reason: Background information only

63. Argoff C, McCarberg B, Gudin J, et al. SoluMatrix Diclofenac: Sustained OpioidSparing Effects in a Phase 3 Study in Patients with Postoperative Pain. Pain Med. 2016 10;17(10):1933-41. doi: 10.1093/pm/pnw012. PMID: 26995799. Exclusion reason: Ineligible comparator 
64. Arias-Buria JL, Valero-Alcaide R, Cleland $\mathrm{JA}$, et al. Inclusion of trigger point dry needling in a multimodal physical therapy program for postoperative shoulder pain: a randomized clinical trial. J Manipulative Physiol Ther. 2015 Mar-Apr;38(3):179-87. doi: 10.1016/j.jmpt.2014.11.007. PMID: 25666690. Exclusion reason: Ineligible comparator

65. Arici E, Tastan S, Can MF. The effect of using an abdominal binder on postoperative gastrointestinal function, mobilization, pulmonary function, and pain in patients undergoing major abdominal surgery: A randomized controlled trial. Int J Nurs Stud. 2016 Oct;62:108-17. doi:

10.1016/j.ijnurstu.2016.07.017. PMID: 27474943. Exclusion reason: Ineligible setting

66. Armond ACV, Gloria JCR, Dos Santos CRR, et al. Acupuncture on anxiety and inflammatory events following surgery of mandibular third molars: a split-mouth, randomized, triple-blind clinical trial. Int J Oral Maxillofac Surg. 2019 Feb;48(2):27481. doi: 10.1016/j.ijom.2018.07.016. PMID: 30139712. Exclusion reason: Ineligible intervention

67. Arriaga-Pizano L, Gomez-Jimenez DC, Flores-Mejia LA, et al. Low back pain in athletes can be controlled with acupuncture by a catecholaminergic pathway: clinical trial. Acupunct Med. 2020 May 20:964528420912251. doi: https://dx.doi.org/10.1177/09645284209122 51. PMID: 32429680. Exclusion reason: Ineligible population

68. Arsh A, Darain H, Iqbal M, et al. Effectiveness of manual therapy to the cervical spine with and without manual therapy to the upper thoracic spine in the management of non-specific neck pain; a randomized controlled trial. J Pak Med Assoc. 2020 Mar;70(3):399-403. doi: https://dx.doi.org/10.5455/JPMA.300523. PMID: 32207414. Exclusion reason: Ineligible population
69. Arslan H, Ahmed HMA, Yildiz ED, et al. Acupuncture reduces the postoperative pain in teeth with symptomatic apical periodontitis: a preliminary randomized placebo-controlled prospective clinical trial. Quintessence Int. 2019;50(4):270-7. doi: https://dx.doi.org/10.3290/j.qi.a42153. PMID: 30887960. Exclusion reason: Ineligible intervention

70. Arslan S, Gokce Kutsal Y. Comparison of the efficacy of etofenamate and tenoxicam in the treatment of low back pain. Journal of Rheumatology and Medical Rehabilitation. 1999;10(1):14-8. Exclusion reason: Not in English

71. Asenlof P, Denison E, Lindberg P. Individually tailored treatment targeting activity, motor behavior, and cognition reduces pain-related disability: a randomized controlled trial in patients with musculoskeletal pain. J Pain. 2005 Sep;6(9):588-603. doi: 10.1016/j.jpain.2005.03.008. PMID: 16139778. Exclusion reason: Ineligible population

72. Aslan Telci E, Karaduman A. Effects of three different conservative treatments on pain, disability, quality of life, and mood in patients with cervical spondylosis.

Rheumatol Int. 2012 Apr;32(4):1033-40. doi: 10.1007/s00296-010-1751-4. PMID: 21246365. Exclusion reason: Ineligible population

73. Asnani MR, Francis DK, Brandow AM, et al. Interventions for treating neuropathic pain in people with sickle cell disease. Cochrane Database Syst Rev. 2019(7)doi: 10.1002/14651858.CD012943.pub2. PMID: 31273755. Exclusion reason: Publication used as source document

74. Aspinall SL, Jacques A, Leboeuf-Yde C, et al. No difference in pressure pain threshold and temporal summation after lumbar spinal manipulation compared to sham: A randomised controlled trial in adults with low back pain. Musculoskelet Sci Pract. 2019 Oct;43:18-25. doi: 10.1016/j.msksp.2019.05.011. PMID: 31176287. Exclusion reason: Ineligible population 
75. As-Sanie S, Till SR, Mowers EL, et al. Opioid Prescribing Patterns, Patient Use, and Postoperative Pain After Hysterectomy for Benign Indications. Obstet Gynecol. 2017 12;130(6):1261-8. doi: 10.1097/AOG.0000000000002344. PMID: 29112660. Exclusion reason: Ineligible intervention

76. Attal N, Ayache SS, Ciampi De Andrade D, et al. Repetitive transcranial magnetic stimulation and transcranial direct-current stimulation in neuropathic pain due to radiculopathy: a randomized shamcontrolled comparative study. Pain. 2016 Jun;157(6):1224-31. doi: 10.1097/j.pain.0000000000000510. PMID: 26845524. Exclusion reason: Ineligible population

77. Au AH, Choi SW, Cheung CW, et al. The Efficacy and Clinical Safety of Various Analgesic Combinations for Post-Operative Pain after Third Molar Surgery: A Systematic Review and Meta-Analysis. PLoS ONE [Electronic Resource]. 2015;10(6):e0127611. doi: 10.1371/journal.pone.0127611. PMID: 26053953. Exclusion reason: Publication used as source document

78. Aufwerber S, Heijne A, Edman G, et al. Early mobilization does not reduce the risk of deep venous thrombosis after Achilles tendon rupture: a randomized controlled trial. Knee Surg Sports Traumatol Arthrosc. 2019 Nov 02;02:02. doi: https://dx.doi.org/10.1007/s00167-01905767-x. PMID: 31679069. Exclusion reason: Ineligible outcome

79. Awadalla R, Gnjidic D, Patanwala A, et al. The Effectiveness of Stewardship Interventions to Reduce the Prescribing of Extended-Release Opioids for Acute Pain: A Systematic Review. Pain Med. 2020 Jun 03;03:03. doi: https://dx.doi.org/10.1093/pm/pnaa139. PMID: 32488237. Exclusion reason: Ineligible population
80. Awotidebe AW, Inglis-Jassiem G, Young T. Does Low-level Laser Therapy Provide Additional Benefits to Exercise in Patients with Shoulder Musculoskeletal Disorders? A Meta-analysis of Randomised Controlled Trials. Ortop Traumatol Rehabil. 2019 Dec 31;21(6):407-16. doi: https://dx.doi.org/10.5604/01.3001.0013.739 8. PMID: 32100715. Exclusion reason: Ineligible intervention

81. Ay S, Dogan SK, Evcik D. Is low-level laser therapy effective in acute or chronic low back pain? Clin Rheumatol. 2010 Aug;29(8):905-10. doi: 10.1007/s10067010-1460-0. PMID: 20414695. Exclusion reason: Ineligible population

82. Aynehchi BB, Cerrati EW, Rosenberg DB. The efficacy of oral celecoxib for acute postoperative pain in face-lift surgery. JAMA Facial Plast Surg. 2014 SepOct;16(5):306-9. doi: 10.1001/jamafacial.2014.351. PMID: 25010711. Exclusion reason: Ineligible study design

83. Ayo D, Blumberg SN, Rockman CR, et al. Compression versus No Compression after Endovenous Ablation of the Great Saphenous Vein: A Randomized Controlled Trial. Ann Vasc Surg. 2017 Jan;38:72-7. doi: https://dx.doi.org/10.1016/j.avsg.2016.08.00 8. PMID: 27554689. Exclusion reason: Ineligible intervention

84. Ayub A, Osama M, Ahmad S. Effects of active versus passive upper extremity neural mobilization combined with mechanical traction and joint mobilization in females with cervical radiculopathy: A randomized controlled trial. J Back Musculoskelet Rehabil. 2019 Jan 11;11:11. doi: 10.3233/BMR-170887. PMID: 30664500. Exclusion reason: Ineligible population

85. Azimova YE, Tabeeva GR. Tenoxicam (texamen) in the treatment of acute cervicalgia: Results of an open comparative trial. Zh Nevrol Psikhiatr Im S S Korsakova. 2014;2014(4):47-51. PMID: 24874317. Exclusion reason: Not in English 
86. Azizkhani R, Pourafzali SM, Baloochestani $E$, et al. Comparing the analgesic effect of intravenous acetaminophen and morphine on patients with renal colic pain referring to the emergency department: A randomized controlled trial. J Res Med Sci. 2013 Sep;18(9):772-6. PMID: 24381620. Exclusion reason: Ineligible duration

87. Babbar S, Marier JF, Mouksassi MS, et al. Pharmacokinetic analysis of capsaicin after topical administration of a highconcentration capsaicin patch to patients with peripheral neuropathic pain. Ther Drug Monit. 2009 Aug;31(4):502-10. doi: 10.1097/FTD.0b013e3181a8b200. PMID: 19494795. Exclusion reason: Ineligible population

88. Backonja M, Wallace MS, Blonsky ER, et al. NGX-4010, a high-concentration capsaicin patch, for the treatment of postherpetic neuralgia: a randomised, double-blind study. Lancet Neurol. 2008 Dec;7(12):1106-12. doi: 10.1016/S14744422(08)70228-X. PMID: 18977178. Exclusion reason: Ineligible population

89. Backonja MM. High-concentration capsaicin for the treatment of post-herpetic neuralgia and other types of peripheral neuropathic pain. Eur J Pain Supplements. 2010 Aug;4(S2):170-4. doi: 10.1016/S17543207\%2810\%2970529-0. Exclusion reason: Ineligible study design

90. Baek SH, Oh JW, Shin JS, et al. Long term follow-up of cervical intervertebral disc herniation inpatients treated with integrated complementary and alternative medicine: a prospective case series observational study. BMC Complement Altern Med. 2016 Feb 04;16:52. doi: 10.1186/s12906-016-1034-z. PMID: 26850111. Exclusion reason: Ineligible study design

91. Bai Y, Miller T, Tan M, et al. Lidocaine patch for acute pain management: a metaanalysis of prospective controlled trials. Curr Med Res Opin. 2015 Mar;31(3):57581. doi: 10.1185/03007995.2014.973484. PMID: 25290665. Exclusion reason: Publication used as source document
92. Bakhsha F, Niaki AS, Jafari SY, et al. The Effects of Diclofenac Suppository and Intravenous Acetaminophen and their Combination on the Severity of Postoperative Pain in Patients Undergoing Spinal Anaesthesia During Cesarean Section. J Clin Diagn Res. 2016 Jul;10(7):UC09-12. doi: https://dx.doi.org/10.7860/JCDR/2016/1509 3.8120. PMID: 27630929. Exclusion reason: Ineligible setting

93. Bakker CJ, Wise KL, Williams BR, et al. Complementary and Alternative Medicine for Postoperative Pain: A Systematic Review. J Bone Joint Surg. 2020 May 20;102 Suppl 1:S36-S46. doi: https://dx.doi.org/10.2106/JBJS.19.01439. PMID: 32251133. Exclusion reason: Publication used as source document

94. Bakshi R, Thumb N, Broll H. Treatment of acute lumbosacral back pain with diclofenac resinate: results of a double-blind comparative trial versus piroxicam. Drug Investig 1994;8:288-93. Exclusion reason: Ineligible comparator

95. Bakshi R, Thumb N, Broll H, et al. Treatment of acute lumbosacral back pain with diclofenac resinate. Results of a double-blind comparative trial versus pixoxicam. Drug Investig 1994;8(5):288-93. PMID: 12740678. Exclusion reason: Ineligible comparator

96. Balani M, Gawade P, Maheshgauri S, et al. Results of two multicentric, comparative, randomized, parallel group clinical trials to evaluate the efficacy and safety of dexketoprofen trometamol in the treatment of dental pain and dysmenorrhoea in Indian patients. J Clin Diagn Res. 2008;2(5):108691. Exclusion reason: Ineligible intervention

97. Balazcs E, Sieper J, Bickham K, et al. A randomized, clinical trial to assess the relative efficacy and tolerability of two doses of etoricoxib versus naproxen in patients with ankylosing spondylitis. BMC Musculoskelet Disord. 201610 13;17(1):426. doi: 10.1186/s12891-0161275-5. PMID: 27737664. Exclusion reason: Ineligible population 
98. Bali C, Ergenoglu P, Ozmete O, et al. Comparison of the postoperative analgesic effects of naproxen sodium and naproxen sodium-codeine phosphate for arthroscopic meniscus surgery. Braz J Anesthesiol. 2016 Mar-Apr;66(2):151-6. doi: https://dx.doi.org/10.1016/j.bjane.2014.08.0 06. PMID: 26952223. Exclusion reason: Ineligible intervention

99. Balko R, Hurley R, Jatoi A. Poly (ADPribose) polymerase inhibition for chemotherapy-induced peripheral neuropathy: A meta-analysis of placebocontrolled trials. J Palliat Med. 2019 Aug;22(8):977-80. doi: http://dx.doi.org/10.1089/jpm.2018.0572. PMID: 30839241. Exclusion reason: Ineligible intervention

100. Ballas SK. Update on pain management in sickle cell disease. Hemoglobin. 2011;35(56):520-9. doi:

10.3109/03630269.2011.610478. PMID: 21910604. Exclusion reason: Ineligible intervention

101. Bamgbose BO, Akinwande JA, Adeyemo WL, et al. Prospective, randomized, openlabel, pilot clinical trial comparing the effects of dexamethasone coadministered with diclofenac potassium or acetaminophen and diclofenac potassium monotherapy after third-molar extraction in adults. Curr Ther Res Clin Exp. 2006;67(4):229-40. doi: 10.1016/j.curtheres.2006.07.001. PMID: 24678099. Exclusion reason: Ineligible intervention

102. Bandpei MAM, Ahmadshirvani M, Fakhri $M$, et al. The effect of an exercise program and ergonomic advices on treatment of pregnancy-related low back pain: a randomized controlled clinical trial. Journal of Mazandaran University of Medical Sciences. 2010;20(77):10-9. Exclusion reason: Not in English

103. Banerjee P, Rogers BA. Systematic review of high-volume multimodal wound infiltration in total knee arthroplasty. Orthopedics. 2014 Jun;37(6):403-12. doi: 10.3928/01477447-20140528-07. PMID: 24972430. Exclusion reason: Publication used as source document
104. Banting J, Meriano T. Journal Club: Ketamine in the Emergency Department. J Spec Oper Med. 2015;15(3):94-7. PMID: 26360362. Exclusion reason: Ineligible study design

105. Barasch A, Safford MM, McNeal SF, et al. Patterns of postoperative pain medication prescribing after invasive dental procedures. Spec Care Dentist. 2011 Mar-Apr;31(2):537. doi: https://dx.doi.org/10.1111/j.17544505.2011.00181.x. PMID: 21371065.

Exclusion reason: Background information only

106. Barber FA. A comparison of crushed ice and continuous flow cold therapy. Am J Knee Surg. 2000;13(2):97-101; discussion 2. PMID: 11281337. Exclusion reason: Ineligible intervention

107. Barker R, Kober A, Hoerauf K, et al. Outof-hospital auricular acupressure in elder patients with hip fracture: a randomized double-blinded trial. Acad Emerg Med. 2006 Jan;13(1):19-23. doi: 10.1197/j.aem.2005.07.014. PMID: 16365322. Exclusion reason: Ineligible duration

108. Barnaby DP, Chertoff AE, Restivo AJ, et al. Randomized Controlled Trial of Intravenous Acetaminophen Versus Intravenous Hydromorphone for the Treatment of Acute Pain in the Emergency Department. Ann Emerg Med. 2019 Feb;73(2):133-40. doi: https://dx.doi.org/10.1016/j.annemergmed.2 018.06.019. PMID: 30119941. Exclusion reason: Background information only

109. Baron R, Mayoral V, Leijon G, et al. 5\% lidocaine medicated plaster versus pregabalin in post-herpetic neuralgia and diabetic polyneuropathy: an open-label, noninferiority two-stage RCT study. Curr Med Res Opin. 2009 Jul;25(7):1663-76. doi: 10.1185/03007990903047880. PMID: 19485723. Exclusion reason: Ineligible population

110. Barra M, Remák E, Liu DD, et al. A costconsequence analysis of parecoxib and opioids vs opioids alone for postoperative pain: Chinese perspective. Clinicoecon Outcomes Res. 2019;11:169-77. doi: 10.2147/CEOR.S183404. PMID: 30863130.

Exclusion reason: Ineligible setting 
111. Barrett T, Kostenbader K, Nalamachu S, et al. Safety and Tolerability of Biphasic Immediate-Release/Extended-Release Oxycodone/Acetaminophen Tablets: Analysis of 11 Clinical Trials. Pain Pract. 2016 09;16(7):856-68. doi: https://dx.doi.org/10.1111/papr.12324. PMID: 26296448. Exclusion reason: Ineligible comparator

112. Barrington JW, Hansen RN, Lovelace B, et al. Impact of Intravenous Acetaminophen on Lengths of Stay and Discharge Status after Total Knee Arthroplasty. J Knee Surg. 2019 Jan;32(1):111-6. doi: 10.1055/s-00381636908. PMID: 29514362. Exclusion reason: Ineligible duration

113. Barrington R. Single-blind study of diflunisal versus mefenamic acid in the treatment of pain after Colles' fracture. Curr Med Res Opin. 1980;6(9):630-3. PMID: 7408529. Exclusion reason: Ineligible intervention

114. Barron J. The control of postoperative pain by the use of local anesthetic pumps. Henry Ford Hosp Med Bull. 1959 Sep;7:219-20. PMID: 13797102. Exclusion reason: Ineligible publication type

115. Bartels K, Mayes LM, Dingmann C, et al. Opioid Use and Storage Patterns by Patients after Hospital Discharge following Surgery. PLoS ONE [Electronic Resource]. 2016;11(1):e0147972. doi: 10.1371/journal.pone.0147972. PMID: 26824844. Exclusion reason: Ineligible study design

116. Basmajian JV. Reflex cervical muscle spasm: treatment by diazepam, phenobarbital or placebo. Arch Phys Med Rehabil. 1983 Mar;64(3):121-4. PMID: 6338860. Exclusion reason: Ineligible population

117. Bates AS, Knepil GJ. Systematic review and meta-analysis of the efficacy of hilotherapy following oral and maxillofacial surgery. Int J Oral Maxillofac Surg. 2016 Jan;45(1):1107. doi: 10.1016/j.ijom.2015.08.983. PMID: 26362489. Exclusion reason: Publication used as source document
118. Batista JA, Errigo MM. Preoperative gabapentin as adjuvant in the management of acute postoperative pain in abdominal hysterectomy. Revista de la sociedad espanola del dolor. 2015;22(5):200-4. Exclusion reason: Not in English

119. Batistella CE, Bidin F, Giacomelli I, et al. Effects of the Russian current in the treatment of low back pain in women: A randomized clinical trial. J Bodyw Mov Ther. 2020 Apr;24(2):118-22. doi: https://dx.doi.org/10.1016/j.jbmt.2019.10.00 9. PMID: 32507136. Exclusion reason: Ineligible population

120. Bauer BA, Cutshall SM, Wentworth LJ, et al. Effect of massage therapy on pain, anxiety, and tension after cardiac surgery: a randomized study. Complement Ther Clin Pract. 2010 May;16(2):70-5. doi: 10.1016/j.ctcp.2009.06.012. PMID: 20347836. Exclusion reason: Ineligible setting

121. Baverel L, Cucurulo T, Lutz C, et al. Anesthesia and analgesia methods for outpatient anterior cruciate ligament reconstruction. Orthop Traumatol Surg Res. 2016 12;102(8S):S251-S5. doi: 10.1016/j.otsr.2016.08.007. PMID: 27687059. Exclusion reason: Ineligible intervention

122. Baxendale BR, Vater M, Lavery KM. Dexamethasone reduces pain and swelling following extraction of third molar teeth. Anaesthesia. 1993 Nov;48(11):961-4. doi: 10.1111/j.1365-2044.1993.tb07474.x. PMID: 8250191. Exclusion reason: Ineligible intervention

123. Baxi V. Management of Acute Pain. J Assoc Physicians India. 2015 Feb;63(2 Suppl):1420. PMID: 26529854. Exclusion reason: Ineligible study design

124. Bayoumi AB, Ikizgul O, Karaali CN, et al. Antidepressants in Spine Surgery: A Systematic Review to Determine Benefits and Risks. Asian Spine J. 2019

Dec;13(6):1036-46. doi: https://dx.doi.org/10.31616/asj.2018.0237. PMID: 31422644. Exclusion reason: Ineligible outcome 
125. Bean B, Aeppli D, Balfour HH, Jr. Acyclovir in shingles. J Antimicrob Chemother. 1983 Sep;12 Suppl B:123-7. PMID: 6355047. Exclusion reason: Ineligible intervention

126. Beauchamp GA, Winstanley EL, Ryan SA, et al. Moving beyond misuse and diversion: the urgent need to consider the role of iatrogenic addiction in the current opioid epidemic. Am J Public Health. 2014 Nov;104(11):2023-9. doi: https://dx.doi.org/10.2105/AJPH.2014.3021 47. PMID: 25211712. Exclusion reason: Background information only

127. Beaudoin FL, Gutman R, Merchant RC, et al. Persistent pain after motor vehicle collision: comparative effectiveness of opioids vs nonsteroidal antiinflammatory drugs prescribed from the emergency department-a propensity matched analysis. Pain. 2017 02;158(2):289-95. doi: 10.1097/j.pain.0000000000000756. PMID: 28092325. Exclusion reason: Ineligible study design

128. Beaudoin FL, Janicki A, Zhai W, et al. Trends in opioid prescribing before and after implementation of an emergency department opioid prescribing policy. Am J Emerg Med. 2018 Feb;36(2):329-31. doi: 10.1016/j.ajem.2017.07.068. PMID: 28760380. Exclusion reason: Ineligible outcome

129. Beaulieu P. Effects of nabilone, a synthetic cannabinoid, on postoperative pain. Can J Anaesth. 2006 Aug;53(8):769-75. PMID: 16873343. Exclusion reason: Background information only

130. Becker A, Held H, Redaelli M, et al. Implementation of a guideline for low back pain management in primary care: a costeffectiveness analysis. Spine. 2012 Apr 15;37(8):701-10. doi: 10.1097/BRS.0b013e31822b01bd. PMID: 21738095. Exclusion reason: Ineligible outcome

131. Becker WC, Gordon K, Edelman EJ, et al. Trends in Any and High-Dose Opioid Analgesic Receipt Among Aging Patients With and Without HIV. AIDS Behav. 2016 03;20(3):679-86. doi: 10.1007/s10461-0151197-5. PMID: 26384973. Exclusion reason: Ineligible population
132. Beckmann M, Bruun-Olsen V, Pripp AH, et al. Effect of exercise interventions in the early phase to improve physical function after hip fracture - A systematic review and meta-analysis. Physiotherapy. 2020 May 20;108:90-7. doi: https://dx.doi.org/10.1016/j.physio.2020.04. 009. PMID: 32726713. Exclusion reason: Ineligible outcome

133. Beckwee D, Bautmans I, Lefeber N, et al. Effect of Transcutaneous Electric Nerve Stimulation on Pain after Total Knee Arthroplasty: A Blind Randomized Controlled Trial. J Knee Surg. 2018 Feb;31(2):189-96. doi: https://dx.doi.org/10.1055/s-0037-1602134. PMID: 28460406. Exclusion reason: Ineligible duration

134. Beebe MJ, Allen R, Anderson MB, et al. Continuous femoral nerve block using $0.125 \%$ bupivacaine does not prevent early ambulation after total knee arthroplasty. Clin Orthop Relat Res. 2014 May;472(5):1394-9. doi: 10.1007/s11999-013-3164-7. PMID: 23857316. Exclusion reason: Ineligible intervention

135. Befeler D, Giattini JF. Pentazocine intramuscularly for control of postoperative pain in orthopedic patients. J Am Geriatr Soc. 1970 Dec;18(12):962-7. PMID: 5508242. Exclusion reason: Ineligible study design

136. Behrbalk E, Halpern P, Boszczyk BM, et al. Anxiolytic medication as an adjunct to morphine analgesia for acute low back pain management in the emergency department: a prospective randomized trial. Spine. 2014 Jan 01;39(1):17-22. doi: 10.1097/BRS.0000000000000038. PMID: 24270933. Exclusion reason: Ineligible intervention

137. Beinert K, Keller M, Taube W. Neck muscle vibration can improve sensorimotor function in patients with neck pain. Spine J. 2015 Mar 01;15(3):514-21. doi: 10.1016/j.spinee.2014.10.013. PMID: 25452010. Exclusion reason: Ineligible study design 
138. Bekawi MS, El Wakeel LM, Al Taher WM, et al. Clinical study evaluating pregabalin efficacy and tolerability for pain management in patients undergoing laparoscopic cholecystectomy. Clin J Pain. 2014 Nov;30(11):944-52. doi: 10.1097/AJP.0000000000000060. PMID: 24662499. Exclusion reason: Ineligible comparator

139. Beloeil H, Albaladejo P, Sion A, et al. Multicentre, prospective, double-blind, randomised controlled clinical trial comparing different non-opioid analgesic combinations with morphine for postoperative analgesia: the OCTOPUS study. Br J Anaesth. 2019 Jun;122(6):e98e106. doi: https://dx.doi.org/10.1016/j.bja.2018.10.058. PMID: 30915987. Exclusion reason: Ineligible setting

140. Benedetti F, Amanzio M, Casadio C, et al. Control of postoperative pain by transcutaneous electrical nerve stimulation after thoracic operations. Ann Thorac Surg. 1997 Mar;63(3):773-6. doi: 10.1016/s00034975(96)01249-0. PMID: 9066400.

Exclusion reason: Ineligible setting

141. Benson RC. Double Blind Evaluation of Analgesic Agents in the Postpartum Patient. West J Surg Obstet Gynecol. 1963 JulAug;71:167-9. PMID: 14057471. Exclusion reason: Ineligible comparator

142. Bergeria CL, Huhn AS, Dunn KE. Randomized comparison of two web-based interventions on immediate and 30-day opioid overdose knowledge in three unique risk groups. Prev Med. 2019 May 10;10:10. doi: 10.1016/j.ypmed.2019.05.006. PMID: 31078564. Exclusion reason: Ineligible population

143. Bergquist-Ullman M, Larsson U. Acute low back pain in industry. A controlled prospective study with special reference to therapy and confounding factors Acta Orthop Scand.. 1977(170):1-117. doi: 10.3109/ort.1977.48.suppl-170.01. PMID: 146394. Exclusion reason: Ineligible population
144. Bernstein DN, St John M, Rubery PT, et al. PROMIS Pain Interference Is Superior to the Likert Pain Scale for Pain Assessment in Spine Patients. Spine. 2019 Jul 15;44(14):E852-E6. doi: https://dx.doi.org/10.1097/BRS.0000000000 002979. PMID: 30633118. Exclusion reason: Background information only

145. Bert JM, Stark JG, Maschka K, et al. The effect of cold therapy on morbidity subsequent to arthroscopic lateral retinacular release. Orthop Rev. 1991 Sep;20(9):755-8. PMID: 1945511. Exclusion reason: Ineligible setting

146. Bertalanffy A, Kober A, Bertalanffy P, et al. Transcutaneous electrical nerve stimulation reduces acute low back pain during emergency transport. Acad Emerg Med. 2005 Jul;12(7):607-11. doi: 10.1197/j.aem.2005.01.013. PMID: 15995091. Exclusion reason: Ineligible duration

147. Berthelot JM, Darrieutort-Lafitte C, Le Goff $\mathrm{B}$, et al. Strong opioids for noncancer pain due to musculoskeletal diseases: Not more effective than acetaminophen or NSAIDs. Joint, Bone, Spine: Rev Rhum Engl Ed. 2015 Dec;82(6):397-401. doi: https://dx.doi.org/10.1016/j.jbspin.2015.08.0 03. PMID: 26453108. Exclusion reason: Background information only

148. Bertozzi L, Gardenghi I, Turoni F, et al. Effect of therapeutic exercise on pain and disability in the management of chronic nonspecific neck pain: systematic review and meta-analysis of randomized trials. Phys Ther 2013 Aug;93(8):1026-36. doi: https://dx.doi.org/10.2522/ptj.20120412. PMID: 23559524. Exclusion reason: Publication used as source document

149. Best AD, De Silva RK, Thomson WM, et al. Efficacy of Codeine When Added to Paracetamol (Acetaminophen) and Ibuprofen for Relief of Postoperative Pain After Surgical Removal of Impacted Third Molars: A Double-Blinded Randomized Control Trial. J Oral Maxillofac Surg. 2017 Oct;75(10):2063-9. doi: 10.1016/j.joms.2017.04.045. PMID: 28586638. Exclusion reason: Ineligible intervention 
150. Beutner KR, Friedman DJ, Forszpaniak C, et al. Valaciclovir compared with acyclovir for improved therapy for herpes zoster in immunocompetent adults. Antimicrobial Agents \& Chemotherapy. 1995 Jul;39(7):1546-53. doi: 10.1128/aac.39.7.1546. PMID: 7492102.

Exclusion reason: Ineligible intervention

151. Bianchini C, Malago M, Crema L, et al. Post-operative pain management in head and neck cancer patients: predictive factors and efficacy of therapy. Acta Otorhinolaryngol Ital. 2016 Apr;36(2):91-6. doi: 10.14639/0392-100X-499. PMID: 27196072. Exclusion reason: Ineligible study design

152. Bijur PE, Esses D, Chang AK, et al. Dosing and titration of intravenous opioid analgesics administered to ED patients in acute severe pain. Am J Emerg Med. 2012 Sep;30(7):1241-4. doi:

10.1016/j.ajem.2011.06.015. PMID: 21908134. Exclusion reason: Ineligible intervention

153. Bijur PE, Mills AM, Chang AK, et al. Comparative Effectiveness of PatientControlled Analgesia for Treating Acute Pain in the Emergency Department. Ann Emerg Med. 2017 Dec;70(6):809-18.e2. doi: 10.1016/j.annemergmed.2017.03.064. PMID: 28601270. Exclusion reason: Ineligible intervention

154. Binder A, Baron R. Utility of transcutaneous electrical nerve stimulation in neurologic pain disorders. Neurology. 2010 Jan 12;74(2):104-5. doi: 10.1212/WNL.0b013e3181cbb8af. PMID: 20042703. Exclusion reason: Ineligible publication type

155. Binhas M, Egbeola-Martial J, Kluger MD, et al. Opioids and nonopioids for postoperative pain control in patients with chronic kidney disease. J Opioid Manag. 2017 Jan/Feb;13(1):17-25. doi: https://dx.doi.org/10.5055/jom.2017.0364. PMID: 28345743. Exclusion reason: Background information only
156. BinKharfi M, AlSagre A. BET 2: Safety and efficacy of low-dose ketamine versus opioids for acute pain management in the ED. Emerg Med J. 2019 Feb;36(2):128-9. doi: 10.1136/emermed-2019-208441.2. PMID: 30696780. Exclusion reason: Publication used as source document

157. Binning AR, Przesmycki K, Sowinski P, et al. A randomised controlled trial on the efficacy and side-effect profile (nausea/vomiting/sedation) of morphine-6glucuronide versus morphine for postoperative pain relief after major abdominal surgery. Eur J Pain. 2011 Apr;15(4):402-8. doi: 10.1016/j.ejpain.2010.09.007. PMID: 21041105. Exclusion reason: Ineligible intervention

158. Binns-Turner PG, Wilson LL, Pryor ER, et al. Perioperative music and its effects on anxiety, hemodynamics, and pain in women undergoing mastectomy. AANA J. 2011 Aug;79(4 Suppl):S21-7. PMID: 22403963. Exclusion reason: Ineligible setting

159. Binny J, Joshua Wong NL, Garga S, et al. Transcutaneous electric nerve stimulation (TENS) for acute low back pain: systematic review. Scandinavian J Pain. 201904 24;19(2):225-33. doi: https://dx.doi.org/10.1515/sjpain-2018-0124. PMID: 30849052. Exclusion reason: Publication used as source document

160. Bishop MD, Beneciuk JM, George SZ. Immediate reduction in temporal sensory summation after thoracic spinal manipulation. Spine J. 2011 May;11(5):4406. doi: 10.1016/j.spinee.2011.03.001. PMID: 21463970. Exclusion reason: Ineligible population

161. Bishop PB, Quon JA, Fisher CG, et al. The Chiropractic Hospital-based Interventions Research Outcomes (CHIRO) study: a randomized controlled trial on the effectiveness of clinical practice guidelines in the medical and chiropractic management of patients with acute mechanical low back pain. Spine J. 2010 Dec;10(12):1055-64. doi: 10.1016/j.spinee.2010.08.019. PMID: 20889389. Exclusion reason: Ineligible comparator 
162. Bjersa K, Andersson T. High frequency TENS as a complement for pain relief in postoperative transition from epidural to general analgesia after pancreatic resection. Complement Ther Clin Pract. 2014

Feb;20(1):5-10. doi:

10.1016/j.ctcp.2013.11.004. PMID: 24439637. Exclusion reason: Ineligible setting

163. Bjune K, Stubhaug A, Dodgson MS, et al. Additive analgesic effect of codeine and paracetamol can be detected in strong, but not moderate, pain after Caesarean section. Baseline pain-intensity is a determinant of assay-sensitivity in a postoperative analgesic trial. Acta Anaesthesiol Scand. 1996 Apr;40(4):399-407. doi: 10.1111/j.13996576.1996.tb04460.x. PMID: 8738682.

Exclusion reason: Ineligible setting

164. Bjurstrom MF, Irwin MR. Perioperative Pharmacological Sleep-Promotion and Pain Control: A Systematic Review. Pain Pract. 2019 06;19(5):552-69. doi: https://dx.doi.org/10.1111/papr.12776. PMID: 30762974. Exclusion reason: Ineligible intervention

165. Black P, Max MB, Desjardins P, et al. A randomized, double-blind, placebocontrolled comparison of the analgesic efficacy, onset of action, and tolerability of ibuprofen arginate and ibuprofen in postoperative dental pain. Clin Ther. 2002 Jul;24(7):1072-89. doi: 10.1016/s01492918(02)80020-0. PMID: 12182253.

Exclusion reason: Ineligible comparator

166. Blay N, Donoghue J. The effect of preadmission education on domiciliary recovery following laparoscopic cholecystectomy. Aust J Adv Nurs. 2005 Jun-Aug;22(4):14-9. PMID: 16496831.

Exclusion reason: Ineligible intervention

167. Bleakley CM, McDonough SM, MacAuley DC, et al. Cryotherapy for acute ankle sprains: a randomised controlled study of two different icing protocols. Br J Sports Med. 2006 Aug;40(8):700-5; discussion 5. PMID: 16611722. Exclusion reason: Ineligible comparator
168. Bleakley CM, O'Connor SR, Tully MA, et al. Effect of accelerated rehabilitation on function after ankle sprain: randomised controlled trial. BMJ. 2010 May 10;340:c1964. doi: 10.1136/BMJ.c1964. PMID: 20457737. Exclusion reason: Ineligible outcome

169. Blomberg S, Hallin G, Grann K, et al. Manual therapy with steroid injections--a new approach to treatment of low back pain. A controlled multicenter trial with an evaluation by orthopedic surgeons. Spine. 1994 Mar 01;19(5):569-77. doi: 10.1097/00007632-199403000-00013. PMID: 8184352. Exclusion reason: Ineligible intervention

170. Blomberg S, Svardsudd K, Tibblin G. Manual therapy with steroid injections in low-back pain. Improvement of quality of life in a controlled trial with four months' follow-up. Scand J Prim Health Care. 1993 Jun;11(2):83-90. doi: 10.3109/02813439308994908. PMID: 8356370. Exclusion reason: Ineligible intervention

171. Blondell RD, Azadfard M, Wisniewski AM. Pharmacologic therapy for acute pain. Am Fam Physician. 2013 Jun 01;87(11):766-72. PMID: 23939498. Exclusion reason: Ineligible publication type

172. Bloomfield SS, Barden TP, Mitchell J. Asprin and codeine in two postpartum pain models. Clin Pharmacol Ther. 1976 Oct;20(4):499-503. doi: 10.1002/cpt1976204499. PMID: 788993. Exclusion reason: Ineligible population

173. Bloomquist DS. Pain control in endodontics. Dental Clinics of North America. 1979 Oct;23(4):543-53. PMID: 294388.

Exclusion reason: Ineligible study design

174. Bocanegra M, Seijas A, Gonzalez Yibirin $\mathrm{M}$. Effectiveness and tolerability of oncedaily nimesulide versus ibuprofen in pain management after surgical extraction of an impacted third molar: a 24-hour, doubleblind, randomized, double-dummy, parallelgroup study. Curr Ther Res Clin Exp. 2005;66(3):172-80. doi: 10.1016/j.curtheres.2005.06.007. PMID: 24672121. Exclusion reason: Ineligible comparator 
175. Bocanegra M, Seijas A, Yibirín MG. Efficacy and tolerability of conventional nimesulide versus beta-cyclodextrin nimesulide in patients with pain after surgical dental extraction: A multicenter, prospective, randomized, double-blind, double-dummy study. Curr Ther Res Clin Exp. 2003;64(5):279-89. doi: 10.1016/S0011-393X(03)00087-0. PMID: 24944376 Exclusion reason: Ineligible intervention

176. Bodhise PB, Dejoie M, Brandon Z, et al. Non-pharmacologic management of sickle cell pain. Hematology. 2004 Jun;9(3):235-7. doi: $10.1080 / 10245330410001701495$. PMID: 15204105. Exclusion reason: Ineligible study design

177. Boersma K, Linton SJ. Expectancy, fear and pain in the prediction of chronic pain and disability: a prospective analysis. Eur J Pain. 2006 Aug;10(6):551-7. doi: 10.1016/j.ejpain.2005.08.004. PMID: 16199189. Exclusion reason: Ineligible intervention

178. Boghdady W, Lotfy M, William E. Diclofenac potassium in the management of dental pain: a multicenter double-blind comparison with glafenine. Egypt Dent J. 1993 Jul;39(3):461-6. PMID: 9590971.

Exclusion reason: Ineligible intervention

179. Bohl DD, Louie PK, Shah N, et al. Multimodal Versus Patient-Controlled Analgesia After an Anterior Cervical Decompression and Fusion. Spine. 2016 Jun;41(12):994-8. doi: 10.1097/BRS.0000000000001380. PMID: 26679869. Exclusion reason: Ineligible study design

180. Bood SA, Sundequist U, Kjellgren A, et al. Eliciting the relaxation response with the help of flotation-rest (restricted environmental stimulation technique) in patients with stress-related ailments. Int $\mathrm{J}$ Stress Manag. 2006 May;13(2):154-75. doi: 10.1037/1072-5245.13.2.154. Exclusion reason: Ineligible population

181. Borenstein DG, Lacks S, Wiesel SW. Cyclobenzaprine and naproxen versus naproxen alone in the treatment of acute low back pain and muscle spasm. Clin Ther. 1990 Mar-Apr;12(2):125-31. PMID: 2141299. Exclusion reason: Ineligible comparator
182. Borges MR, de Oliveira NML, Antonelli IBS, et al. Transcutaneous electrical nerve stimulation is superior than placebo and control for postoperative pain relief. Pain Manag 2020 Jul;10(4):235-46. doi: https://dx.doi.org/10.2217/pmt-2019-0063. PMID: 32564661. Exclusion reason: Ineligible duration

183. Borms T. Comparison of injectable formulations of tiaprofenic acid and ketoprofen in acute lumbar sciatica. Singleblind randomised trial. Drugs. 1988;35 Suppl 1:85-7. PMID: 3282869. Exclusion reason: Ineligible comparator

184. Bosch HC, Sigmund R, Hettich M. Efficacy and tolerability of intramuscular and oral meloxicam in patients with acute lumbago: a comparison with intramuscular and oral piroxicam. Curr Med Res Opin. 1997;14(1):29-38. doi: 10.1185/03007999709113340. PMID: 9524791. Exclusion reason: Ineligible comparator

185. Bot AG, Bekkers S, Arnstein PM, et al. Opioid use after fracture surgery correlates with pain intensity and satisfaction with pain relief. Clin Orthop Relat Res. 2014 Aug;472(8):2542-9. doi: 10.1007/s11999014-3660-4. PMID: 24777731. Exclusion reason: Ineligible study design

186. Boulet SL, Okoroh EM, Azonobi I, et al. Sickle cell disease in pregnancy: maternal complications in a Medicaid-enrolled population. Matern Child Health J. 2013 Feb;17(2):200-7. doi: 10.1007/s10995-0121216-3. PMID: 23315242. Exclusion reason: Ineligible population

187. Bouzia A, Tassoudis V, Karanikolas M, et al. Pregabalin Effect on Acute and Chronic Pain after Cardiac Surgery. Anesthesiol Res Pract. 2017;2017doi: 10.1155/2017/2753962. PMID: 28539936. Exclusion reason: Ineligible intervention

188. Bowers KJ, McAllister KB, Ray M, et al. Ketamine as an Adjunct to Opioids for Acute Pain in the Emergency Department: A Randomized Controlled Trial. Acad Emerg Med. 2017 06;24(6):676-85. doi: 10.1111/acem.13172. PMID: 28177167. Exclusion reason: Ineligible population 
189. Bowsher D. Postherpetic neuralgia and its treatment: a retrospective survey of 191 patients. J Pain Symptom Manage. 1996 Nov;12(5):290-9. doi: 10.1016/s08853924(96)00180-7. PMID: 8942124.

Exclusion reason: Ineligible study design

190. Boyd C, Crawford C, Paat CF, et al. The Impact of Massage Therapy on Function in Pain Populations-A Systematic Review and Meta-Analysis of Randomized Controlled Trials: Part III, Surgical Pain Populations. Pain Med. 2016 Sep;17(9):1757-72. doi: 10.1093/pm/pnw101. PMID: 27165970.

Exclusion reason: Publication used as source document

191. Braden JB, Fan MY, Edlund MJ, et al. Trends in use of opioids by noncancer pain type 2000-2005 among Arkansas Medicaid and HealthCore enrollees: results from the TROUP study. J Pain. 2008 Nov;9(11):1026-35. doi: https://dx.doi.org/10.1016/j.jpain.2008.06.00 2. PMID: 18676205. Exclusion reason: Background information only

192. Bradley C, Perchet C, Lelekov-Boissard T, et al. Not an Aspirin: No Evidence for Acute Anti-Nociception to Laser-Evoked Pain After Motor Cortex rTMS in Healthy Humans. Brain Stimul. 2016 JanFeb;9(1):48-57. doi: 10.1016/j.brs.2015.08.015. PMID: 26433607. Exclusion reason: Ineligible population

193. Bradley LA, Young LD, Anderson KO, et al. Psychological approaches to the management of arthritis pain. Soc Sci Med. 1984;19(12):1353-60. doi: http://dx.doi.org/10.1016/02779536\%2884\%2990024-8. PMID: 6531714.

Exclusion reason: Background information only

194. Brain P, Leyva R, Doyle G, et al. Onset of analgesia and efficacy of ibuprofen sodium in postsurgical dental pain: a randomized, placebo-controlled study versus standard ibuprofen. Clin J Pain. 2015 05;31(5):44450. doi: 10.1097/AJP.0000000000000142. PMID: 25119511. Exclusion reason: Ineligible comparator
195. Bramness JG, Skurtveit S, Morland J, et al. The risk of traffic accidents after prescriptions of carisoprodol. Accid Anal Prev. 2007 Sep;39(5):1050-5. doi: 10.1016/j.aap.2007.02.002. PMID: 17854578. Exclusion reason: Ineligible population

196. Brandal D, Keller MS, Lee C, et al. Impact of Enhanced Recovery After Surgery and Opioid-Free Anesthesia on Opioid Prescriptions at Discharge From the Hospital: A Historical-Prospective Study. Anesth Analg. 2017 11;125(5):1784-92. doi: 10.1213/ANE.0000000000002510. PMID: 29049123. Exclusion reason: Ineligible study design

197. Brandow AM, DeBaun MR. Key Components of Pain Management for Children and Adults with Sickle Cell Disease. Hematol Oncol Clin North Am. 2018 06;32(3):535-50. doi: https://dx.doi.org/10.1016/j.hoc.2018.01.014 . PMID: 29729787. Exclusion reason: Background information only

198. Brandsson S, Rydgren B, Hedner T, et al. Postoperative analgesic effects of an external cooling system and intra-articular bupivacaine/morphine after arthroscopic cruciate ligament surgery. Knee Surg Sports Traumatol Arthrosc. 1996;4(4):200-5. PMID: 9046503. Exclusion reason: Ineligible intervention

199. Brannan SK, Mallinckrodt CH, Brown EB, et al. Duloxetine $60 \mathrm{mg}$ once-daily in the treatment of painful physical symptoms in patients with major depressive disorder. J Psychiatr Res. 2005 Jan;39(1):43-53. doi: 10.1016/j.jpsychires.2004.04.011. PMID: 15504423. Exclusion reason: Ineligible population

200. Braschi E, Garrison S, Allan GM. Cyclobenzaprine for acute back pain. Can Fam Physician. 2015 Dec;61(12):1074. PMID: 26668287. Exclusion reason: Ineligible publication type

201. Brat GA, Agniel D, Beam A, et al. Postsurgical prescriptions for opioid naive patients and association with overdose and misuse: retrospective cohort study. BMJ. 201801 17;360:j5790. doi: 10.1136/BMJ.j5790. PMID: 29343479. Exclusion reason: Ineligible comparator 
202. Bratton RL, Montero DP, Adams KS, et al. Effect of "ionized" wrist bracelets on musculoskeletal pain: a randomized, doubleblind, placebo-controlled trial. Mayo Clin Proc. 2002 Nov;77(11):1164-8. PMID: 12440551. Exclusion reason: Ineligible intervention

203. Brattwall M, Turan I, Jakobsson J. Pain management after elective hallux valgus surgery: a prospective randomized doubleblind study comparing etoricoxib and tramadol. Anesth Analg. 2010

Aug;111(2):544-9. doi: 10.1213/ANE.0b013e3181e3d87c. PMID: 20584877. Exclusion reason: Ineligible comparator

204. Bremner S, Webster F, Katz J, et al. Older adults' postoperative pain medication usage after total knee arthroplasty: a qualitative descriptive study. J Opioid Manag. 2012 May-Jun;8(3):145-52. doi: 10.5055/jom.2012.0110. PMID: 22798174. Exclusion reason: Ineligible study design

205. Brennan GP, Fritz JM, Hunter SJ, et al. Identifying subgroups of patients with acute/subacute "nonspecific" low back pain: results of a randomized clinical trial. Spine. 2006 Mar 15;31(6):623-31. doi: 10.1097/01.brs.0000202807.72292.a8. PMID: 16540864. Exclusion reason: Ineligible study design

206. Bresnahan BW, Rundell SD, Dagadakis $\mathrm{MC}$, et al. A systematic review to assess comparative effectiveness studies in epidural steroid injections for lumbar spinal stenosis and to estimate reimbursement amounts. PM R. 2013 Aug;5(8):705-14. doi: https://dx.doi.org/10.1016/j.pmrj.2013.05.01 2. PMID: 23953016. Exclusion reason: Publication used as source document

207. Brignardello-Petersen R. Patients with idiopathic trigeminal neuralgia seem to benefit from acupuncture and sham acupuncture, but we are uncertain about whether these benefits are beyond those attained by pharmacologic treatment alone. $\mathrm{J}$ Am Dent Assoc. 2018 Mar;149(3):e57. doi: 10.1016/j.adaj.2017.11.022. PMID: 29287739. Exclusion reason: Ineligible publication type
208. Broadsword AM, Sedgley CM, Kohli R, et al. The impact of the Federal Schedule II prescribing mandate on opioid prescribing practices within a dental school setting. J Endod. 2019;45(e26). Exclusion reason: Ineligible outcome

209. Brockmann R, Klein HM. Pain-diminishing effects of Kinesio taping after median sternotomy. Physiother Theory Pract. 2018 Jun;34(6):433-41. doi: 10.1080/09593985.2017.1422205. PMID: 29308962. Exclusion reason: Ineligible intervention

210. Brockow T, Dillner A, Franke A, et al. Analgesic effectiveness of subcutaneous carbon-dioxide insufflations as an adjunct treatment in patients with non-specific neck or low back pain. Complement Ther Med. 2001 Jun;9(2):68-76. doi:

10.1054/ctim.2001.0434. PMID: 11444885. Exclusion reason: Ineligible comparator

211. Brockow T, Heissner T, Franke A, et al. Evaluation of the efficacy of subcutaneous carbon dioxide insufflations for treating acute non specific neck pain in general practice: A sham controlled randomized trial. Eur J Pain. 2008 Jan;12(1):9-16. doi: 10.1016/j.ejpain.2007.01.002. PMID: 17845862. Exclusion reason: Ineligible comparator

212. Bronfort G. Chiropractic treatment of low back pain: a prospective survey. J Manipulative Physiol Ther. 1986 Jun;9(2):99-113. PMID: 2942620. Exclusion reason: Ineligible study design

213. Bronfort G. Spinal manipulation: current state of research and its indications. Neurol Clin. 1999 Feb;17(1):91-111. PMID: 9855673. Exclusion reason: Background information only

214. Bronfort G, Evans R, Anderson A, et al. A randomized controlled trial of spinal manipulation, medication or home exercise for acute and subacute neck pain. BMC Complement Altern Med. 2012;Conference(Suppl 1):International. Exclusion reason: Ineligible population 
215. Bronfort G, Evans R, Anderson AV, et al. Spinal manipulation, medication, or home exercise with advice for acute and subacute neck pain: a randomized trial. Ann Intern Med. 2012 Jan 03;156(1 Pt 1):1-10. doi: 10.7326/0003-4819-156-1-20120103000002. PMID: 22213489. Exclusion reason: Ineligible population

216. Brookoff D, Polomano R. Treating sickle cell pain like cancer pain. Ann Intern Med. 1992 Mar 01;116(5):364-8. PMID: 1736768. Exclusion reason: Ineligible outcome

217. Brown C, Schwartz K, Wild V, et al. Comparison of anirolac with morphine and placebo for postoperative pain. Curr Ther Res. 1991 Sep;50(3):379-85. Exclusion reason: Ineligible comparator

218. Brown JH. DMSO--its efficacy in acute musculoskeletal problems as evaluated by a "double blind" study. Ind Med Surg. 1966 Sep;35(9):777-81. PMID: 5223142.

Exclusion reason: Ineligible comparator

219. Brown MR, Farquhar-Smith P, Williams JE, et al. The use of high-intensity focused ultrasound as a novel treatment for painful conditions-a description and narrative review of the literature. Br J Anaesth. 2015 Oct;115(4):520-30. doi: https://dx.doi.org/10.1093/bja/aev302. PMID: 26385662. Exclusion reason: Publication used as source document

220. Brummett CM, Waljee JF, Goesling J, et al. New Persistent Opioid Use After Minor and Major Surgical Procedures in US Adults. JAMA Surg. 2017 Jun 21;152(6):e170504. doi: 10.1001/jamasurg.2017.0504. PMID: 28403427. Exclusion reason: Ineligible outcome

221. Bryans R, Decina P, Descarreaux M, et al. Evidence-based guidelines for the chiropractic treatment of adults with neck pain. J Manipulative Physiol Ther. 2014 Jan;37(1):42-63. doi: https://dx.doi.org/10.1016/j.jmpt.2013.08.01 0. PMID: 24262386. Exclusion reason: Background information only
222. Buchbinder R, Johnston RV, Rischin KJ, et al. Percutaneous vertebroplasty for osteoporotic vertebral compression fracture. Cochrane Database Syst Rev. 201811 06;11:CD006349. doi: https://dx.doi.org/10.1002/14651858.CD006 349.pub4. PMID: 30399208. Exclusion

reason: Publication used as source document

223. Buck M, Paice JA. Pharmacologic management of acute pain in the orthopaedic patient. Orthop Nurs. 1994 Nov-

Dec;13(6):14-23; quiz 4. PMID: 7870475. Exclusion reason: Ineligible study design

224. Budiansky AS, Margarson MP, Eipe N. Acute pain management in morbid obesity an evidence based clinical update. Surg Obes Relat Dis. 2017 Mar;13(3):523-32. doi:

https://dx.doi.org/10.1016/j.soard.2016.09.0 13. PMID: 27771314. Exclusion reason: Ineligible publication type

225. Bundsen P, Ericson K, Peterson LE, et al. Pain relief in labor by transcutaneous electrical nerve stimulation. Testing of a modified stimulation technique and evaluation of the neurological and biochemical condition of the newborn infant. Acta Obstet Gynecol Scand. 1982;61(2):129-36. doi: 10.3109/00016348209156543. PMID: 7051741. Exclusion reason: Ineligible population

226. Burgess JA, Sommers EE, Truelove EL, et al. Short-term effect of two therapeutic methods on myofascial pain and dysfunction of the masticatory system. J Prosthet Dent. 1988 Nov;60(5):606-10. doi: 10.1016/00223913(88)90223-5. PMID: 3199321.

Exclusion reason: Ineligible population

227. Burton AK, Tillotson KM, Cleary J. Singleblind randomised controlled trial of chemonucleolysis and manipulation in the treatment of symptomatic lumbar disc herniation. Eur Spine J. 2000 Jun;9(3):2027. doi: 10.1007/s005869900113. PMID: 10905437. Exclusion reason: Ineligible population 
228. Burton AK, Waddell G, Tillotson KM, et al. Information and advice to patients with back pain can have a positive effect. A randomized controlled trial of a novel educational booklet in primary care. Spine. 1999 Dec 01;24(23):2484-91. doi: 10.1097/00007632-199912010-00010. PMID: 10626311. Exclusion reason: Ineligible population

229. Busse JW, Sadeghirad B, Oparin Y, et al. Management of Acute Pain From Non-Low Back, Musculoskeletal Injuries : A Systematic Review and Network Metaanalysis of Randomized Trials. Ann Intern Med. 2020 Nov 3;173(9):730-8. doi: 10.7326/m19-3601. PMID: 32805127. Exclusion reason: Publication used as source document

230. Butera KA, George SZ, Borsa PA, et al. Prolonged Reduction in Shoulder Strength after Transcutaneous Electrical Nerve Stimulation Treatment of Exercise-Induced Acute Muscle Pain. Pain Pract. 2018 11;18(8):954-68. doi: 10.1111/papr.12690. PMID: 29505689. Exclusion reason: Ineligible outcome

231. Buttagat V, Narktro T, Onsrira K, et al. Short-term effects of traditional Thai massage on electromyogram, muscle tension and pain among patients with upper back pain associated with myofascial trigger points. Complement Ther Med. 2016 Oct;28:8-12. doi: 10.1016/j.ctim.2016.07.004. PMID: 27670864. Exclusion reason: Ineligible population

232. Buvanendran A, Kroin JS, Rajagopal A, et al. Oral Ketamine for Acute Pain Management After Amputation Surgery. Pain Med. 201806 01;19(6):1265-70. doi: 10.1093/pm/pnx229. PMID: 29025089.

Exclusion reason: Ineligible study design

233. Buyukyilmaz F, Asti T. The effect of relaxation techniques and back massage on pain and anxiety in Turkish total hip or knee arthroplasty patients. Pain Manag Nurs. 2013 Sep;14(3):143-54. doi: https://dx.doi.org/10.1016/j.pmn.2010.11.00 1. PMID: 23972865. Exclusion reason: Ineligible intervention
234. Byrne K, Nolan A, Barnard J, et al. Managing Postoperative Analgesic Failure: Tramadol Versus Morphine for Refractory Pain in the Post-Operative Recovery Unit. Pain Med. 201702 01;18(2):348-55. doi: 10.1093/pm/pnw084. PMID: 28204722.

Exclusion reason: Ineligible setting

235. Cabitza P, Randelli P. Efficacy and safety of eperisone in patients with low back pain: a double blind randomized study. Eur Rev Med Pharmacol Sci. 2008 JulAug;12(4):229-35. PMID: 18727454. Exclusion reason: Ineligible intervention

236. Cameron ID, Wang E, Sindhusake D. A randomized trial comparing acupuncture and simulated acupuncture for subacute and chronic whiplash. Spine. 2011 Dec 15;36(26):E1659-65. doi: 10.1097/BRS.0b013e31821bf674. PMID: 21494196. Exclusion reason: Ineligible population

237. Campos J, Lobo C, Queiroz AM, et al. Treatment of the acute sickle cell vasoocclusive crisis in the Emergency Department: a Brazilian method of switching from intravenous to oral morphine. Eur J Haematol. 2014 Jul;93(1):34-40. doi: 10.1111/ejh.12293. PMID: 24571671. Exclusion reason: Ineligible study design

238. Cankaya A, Saritas S. Effect of Classic Foot Massage on Vital Signs, Pain, and Nausea/Vomiting Symptoms After Laparoscopic Cholecystectomy. Surg Laparosc Endosc Percutan Tech. 2018 Dec;28(6):359-65. doi: https://dx.doi.org/10.1097/SLE.0000000000 000586. PMID: 30312194. Exclusion

reason: Ineligible duration

239. Cao S, Karmouta R, Li DG, et al. Opioid Prescribing Patterns and Complications in the Dermatology Medicare Population. JAMA Dermatol. 201803 01;154(3):31722. doi:

https://dx.doi.org/10.1001/jamadermatol.201 7.5835. PMID: 29417134. Exclusion reason: Background information only

240. Carey TS, Garrett JM. The relation of race to outcomes and the use of health care services for acute low back pain. Spine. 2003 Feb 15;28(4):390-4. PMID: 12590217. Exclusion reason: Background information only 
241. Carne X, Rios J, Torres F. Postmarketing cohort study to assess the safety profile of oral dexketoprofen trometamol for mild to moderate acute pain treatment in primary care. Methods Find Exp Clin. 2009

Oct;31(8):533-40. doi:

10.1358/mf.2009.31.8.1419070. PMID: 19967102. Exclusion reason: Ineligible study design

242. Carter MR, Green BR. Renal calculi: emergency department diagnosis and treatment. Emerg Med Pract. 2011 Jul;13(7):1-17; quiz 8. PMID: 22164398. Exclusion reason: Background information only

243. Casale R. Acute low back pain. Symptomatic treatment with a muscle relaxant drug. Clin J Pain. 1988;4(2):81-8.

Exclusion reason: Ineligible comparator

244. Cashin AG, Lee H, Bagg MK, et al. A systematic review highlights the need to improve the quality and applicability of trials of physical therapy interventions for low back pain. J Clin Epidemiol. 2020 Jun 27;126:106-15. doi: https://dx.doi.org/10.1016/j.jclinepi.2020.06. 025. PMID: 32603685. Exclusion reason: Ineligible outcome

245. Casserley-Feeney SN, Daly L, Hurley DA. The access randomized clinical trial of public versus private physiotherapy for low back pain. Spine. 2012 Jan 15;37(2):85-96. doi: 10.1097/BRS.0b013e3182127457. PMID: 21289590. Exclusion reason: Ineligible intervention

246. Cassidy JD, Lopes AA, Yong-Hing K. The immediate effect of manipulation versus mobilization on pain and range of motion in the cervical spine: a randomized controlled trial. J Manipulative Physiol Ther. 1992 Nov-Dec;15(9):570-5. PMID: 1469341. Exclusion reason: Ineligible population

247. Castaldo M, Catena A, Chiarotto A, et al. Do Subjects with Whiplash-Associated Disorders Respond Differently in the ShortTerm to Manual Therapy and Exercise than Those with Mechanical Neck Pain? Pain Med. 201704 01;18(4):791-803. doi: 10.1093/pm/pnw266. PMID: 28034987.

Exclusion reason: Ineligible study design
248. Castillo-Saavedra L, Gebodh N, Bikson M, et al. Clinically effective treatment of fibromyalgia pain with high-definition transcranial direct current stimulation: Phase ii open-label dose optimization. J Pain. 2016 Jan;17(1):14-26. doi: 10.1016/j.jpain.2015.09.009. PMID: 26456677. Exclusion reason: Ineligible study design

249. Castro Rivera LA. Estudio abierto comparativo entre piroxicam y sulindac en el tratamiento de la lumbalgia aguda= open comparative study between piroxicam and sulindac in the treatment of acute low back pain. 1992. Exclusion reason: Not in English

250. Castro-Sanchez AM, Mataran-Penarrocha GA, Sanchez-Labraca N, et al. A randomized controlled trial investigating the effects of craniosacral therapy on pain and heart rate variability in fibromyalgia patients. Clin Rehabil. 2011 Jan;25(1):2535. doi: $10.1177 / 0269215510375909$. PMID: 20702514. Exclusion reason: Ineligible population

251. Cauley CE, Anderson G, Haynes AB, et al. Predictors of In-hospital Postoperative Opioid Overdose After Major Elective Operations: A Nationally Representative Cohort Study. Ann Surg. 2017 04;265(4):702-8. doi: https://dx.doi.org/10.1097/SLA.000000000 001945. PMID: 28267693. Exclusion reason: Ineligible outcome

252. Ceccio CM. Postoperative pain relief through relaxation in elderly patients with fractured hips. Orthop Nurs. 1984 MayJun;3(3):11-9. doi: 10.1097/00006416198405000-00003. PMID: 6374586. Exclusion reason: Ineligible setting

253. Cepeda M, Alvarez H, Morales O, et al. Addition of ultralow dose naloxone to postoperative morphine PCA: unchanged analgesia and opioid requirement but decreased incidence of opioid side effects. Pain. 2004 Jan;107(1-2):41-6. doi: 10.1016/j.pain.2003.09.011. PMID: 14715387. Exclusion reason: Ineligible intervention 
254. Cepeda MS, Delgado M, Ponce M, et al. Equivalent outcomes during postoperative patient-controlled intravenous analgesia with lidocaine plus morphine versus morphine alone. Anesth Analg. 1996 Jul;83(1):102-6. doi: 10.1097/00000539199607000-00018. PMID: 8659717. Exclusion reason: Ineligible population

255. Chai PR, Carreiro S, Innes BJ, et al. Digital Pills to Measure Opioid Ingestion Patterns in Emergency Department Patients With Acute Fracture Pain: A Pilot Study. JMIR. 201701 13;19(1):e19. doi: 10.2196/jmir.7050. PMID: 28087496. Exclusion reason: Ineligible intervention

256. Chakote K, Guggenheimer J. Implications of use of opioid-containing analgesics for palliation of acute dental pain. J Opioid Manag. 2019 Jan/Feb;15(1):35-41. doi: 10.5055/jom.2019.0484. PMID: 30855721. Exclusion reason: Ineligible study design

257. Chakrabarty S, Biswas S, Maiti T, et al. Pregabalin and amitriptyline as monotherapy or as low-dose combination in patients of neuropathic pain: A randomized, controlled trial to evaluate efficacy and safety in an Eastern India teaching hospital. Ann Indian Acad Neurol. 2019;22(4):437-41. doi: 10.4103/aian.AIAN_144_18. PMID: 31736565. Exclusion reason: Ineligible population

258. Chan VW, Clark AJ, Davis JC, et al. The post-operative analgesic efficacy and tolerability of lumiracoxib compared with placebo and naproxen after total knee or hip arthroplasty. Acta Anaesthesiol Scand. 2005 Nov;49(10):1491-500. PMID: 16223396. Exclusion reason: Ineligible comparator

259. Chandanwale AS, Sundar S, Latchoumibady $\mathrm{K}$, et al. Efficacy and safety profile of combination of tramadol-diclofenac versus tramadol-paracetamol in patients with acute musculoskeletal conditions, postoperative pain, and acute flare of osteoarthritis and rheumatoid arthritis: a Phase III, 5-day open-label study. J Pain Res. 2014;7:455-63. doi: 10.2147/JPR.S67817. PMID: 25152629. Exclusion reason: Ineligible comparator
260. Chandrasekar T, Glick L, Wong D, et al. Simple frameshifts in minimally invasive surgery postoperative pain management significantly reduce opiate prescriptions. Can J Urol. 2020 06;27(3):10250-6. PMID: 32544049. Exclusion reason: Ineligible intervention

261. Chang AK, Bijur PE, Holden L, et al. Comparative Analgesic Efficacy of Oxycodone/Acetaminophen Versus Hydrocodone/Acetaminophen for Shortterm Pain Management in Adults Following ED Discharge. Acad Emerg Med. 2015 Nov;22(11):1254-60. doi: 10.1111/acem.12813. PMID: 26479162. Exclusion reason: Ineligible comparator

262. Chang AK, Bijur PE, Lupow JB, et al. Randomized clinical trial of the $2 \mathrm{mg}$ hydromorphone bolus protocol versus the "1+1" hydromorphone titration protocol in treatment of acute, severe pain in the first hour of emergency department presentation. Ann Emerg Med. 2013 Oct;62(4):304-10. doi: 10.1016/j.annemergmed.2013.02.023. PMID: 23694801. Exclusion reason: Ineligible population

263. Chang AK, Bijur PE, Lupow JB, et al. Randomized clinical trial of efficacy and safety of a single 2-mg intravenous dose of hydromorphone versus usual care in the management of acute pain. Acad Emerg Med. 2013 Feb;20(2):185-92. doi: 10.1111/acem.12071. PMID: 23406078. Exclusion reason: Ineligible population

264. Chang DJ, Bird SR, Bohidar NR, et al. Analgesic efficacy of rofecoxib compared with codeine/acetaminophen using a model of acute dental pain. Oral Surg Oral Med Oral Pathol Oral Radiol Endod. 2005 Oct;100(4):e74-80. doi: 10.1016/j.tripleo.2005.04.026. PMID: 16182156. Exclusion reason: Ineligible intervention

265. Chang DJ, Desjardins PJ, Chen E, et al. Comparison of the analgesic efficacy of rofecoxib and enteric-coated diclofenac sodium in the treatment of postoperative dental pain: a randomized, placebocontrolled clinical trial. Clin Ther. 2002 Apr;24(4):490-503. doi: 10.1016/s01492918(02)85126-8. PMID: 12017395.

Exclusion reason: Ineligible comparator 
266. Chang DJ, Desjardins PJ, King TR, et al. The analgesic efficacy of etoricoxib compared with oxycodone/acetaminophen in an acute postoperative pain model: a randomized, double-blind clinical trial. Anesth Analg. 2004 Sep;99(3):807-15, table of contents. doi:

10.1213/01.ANE.0000133141.75831.57. PMID: 15333415. Exclusion reason: Ineligible comparator

267. Chang DJ, Fricke JR, Bird SR, et al. Rofecoxib versus codeine/acetaminophen in postoperative dental pain: a double-blind, randomized, placebo- and active comparator-controlled clinical trial. Clin Ther. 2001 Sep;23(9):1446-55. doi: 10.1016/s0149-2918(01)80119-3. PMID: 11589259. Exclusion reason: Ineligible comparator

268. Chapa HO, Venegas G, VanDuyne CP, et al. In-office thermachoice III ablation: a comparison of two anesthetic techniques. Gynecol Obstet Invest. 2010;69(2):140-4. doi: 10.1159/000267323. PMID: 20029223.

Exclusion reason: Ineligible intervention

269. Chaparro LE, Clarke H, Valdes PA, et al. Adding pregabalin to a multimodal analgesic regimen does not reduce pain scores following cosmetic surgery: a randomized trial. J Anesth. 2012 Dec;26(6):829-35. doi: 10.1007/s00540012-1447-x. PMID: 22797880. Exclusion reason: Ineligible comparator

270. Chapman CR, Vierck CJ. The Transition of Acute Postoperative Pain to Chronic Pain: An Integrative Overview of Research on Mechanisms. J Pain. 2017 04;18(4):359.e1.e38. doi:

https://dx.doi.org/10.1016/j.jpain.2016.11.00 4. PMID: 27908839. Exclusion reason: Background information only

271. Charney RL, Yan Y, Schootman M, et al. Oxycodone versus codeine for triage pain in children with suspected forearm fracture: a randomized controlled trial. Pediatr Emerg Care. 2008 Sep;24(9):595-600. doi: 10.1097/PEC.0b013e3181850ca3. PMID: 18772726. Exclusion reason: Ineligible population
272. Chase T, Jha A, Brooks CA, et al. A pilot feasibility study of massage to reduce pain in people with spinal cord injury during acute rehabilitation. Spinal Cord. 2013 Nov;51(11):847-51. doi: https://dx.doi.org/10.1038/sc.2013.104. PMID: 24042991. Exclusion reason: Ineligible population

273. Chau SW, Soo LY, Lu DV, et al. Clinical experience of pain treatment for postherpetic neuralgia in elderly patients. Acta Anaesthesiol Taiwan. 2007 Jun;45(2):95101. PMID: 17694685. Exclusion reason: Ineligible comparator

274. Chauny JM, Paquet J, Lavigne G, et al. Percentage of pain intensity difference on an 11-point numerical rating scale underestimates acute pain resolution. Eur J Pain. 2014 Sep;18(8):1103-11. doi: https://dx.doi.org/10.1002/j.15322149.2014.00452.x. PMID: 24449521. Exclusion reason: Background information only

275. Chauny JM, Paquet J, Lavigne G, et al. Evaluating acute pain intensity relief: challenges when using an 11-point numerical rating scale. Pain. 2016 Feb;157(2):355-60. doi: https://dx.doi.org/10.1097/j.pain.000000000 0000382. PMID: 26447700. Exclusion reason: Background information only

276. Cheing GL, Wan JW, Kai Lo S. Ice and pulsed electromagnetic field to reduce pain and swelling after distal radius fractures. $\mathrm{J}$ Rehabil Med. 2005 Nov;37(6):372-7. doi: 10.1080/16501970510041055. PMID: 16287669. Exclusion reason: Ineligible comparator

277. Chen CC, Yang CC, Hu CC, et al. Acupuncture for pain relief after total knee arthroplasty: a randomized controlled trial. Reg Anesth Pain Med. 2015 JanFeb;40(1):31-6. doi: 10.1097/AAP.0000000000000138. PMID: 25158837. Exclusion reason: Ineligible intervention 
278. Chen KB, Huang Y, Jin XL, et al.

Electroacupuncture or transcutaneous electroacupuncture for postoperative ileus after abdominal surgery: A systematic review and meta-analysis. Int J Surg. 2019 Oct;70:93-101. doi: https://dx.doi.org/10.1016/j.ijsu.2019.08.034 . PMID: 31494334. Exclusion reason: Publication used as source document

279. Chen L, Arayssi T. Is there a role for topical nonsteroidal anti-inflammatory drugs in the treatment of tendonitis? Aging Health. 2006;2(1):59-60. doi: 10.2217/1745509X.2.1.59. Exclusion reason: Ineligible comparator

280. Chen T, Wang K, Xu J, et al. Electroacupuncture Reduces Postoperative Pain and Analgesic Consumption in Patients Undergoing Thoracic Surgery: A

Randomized Study. Evid Based Complement Alternat Med. 2016;2016:2126416. doi: https://dx.doi.org/10.1155/2016/2126416. PMID: 27073400. Exclusion reason: Ineligible setting

281. Chen Y. Clinical observation of electroacupuncture at S13 in addition to drug therapy in acute lumbar sprain. $\mathrm{J}$ Community Med. 2010;8(39). Exclusion reason: Not in English

282. Cheng YH, Hsu CY, Lin YN. The effect of mechanical traction on low back pain in patients with herniated intervertebral disks: a systemic review and meta-analysis. Clin Rehabil. 2019 Aug 28:269215519872528. doi: https://dx.doi.org/10.1177/02692155198725 28. PMID: 31456418. Exclusion reason: Publication used as source document

283. Cheng YJ. Lidocaine Skin Patch (Lidopat 5\%) Is Effective in the Treatment of Traumatic Rib Fractures: A Prospective Double-Blinded and Vehicle-Controlled Study. Med Princ Pract. 2016;25(1):36-9. doi: https://dx.doi.org/10.1159/000441002. PMID: 26539836. Exclusion reason: Ineligible comparator
284. Cherasse A, Muller G, Ornetti P, et al. Tolerability of opioids in patients with acute pain due to nonmalignant musculoskeletal disease. A hospital-based observational study. Joint, Bone, Spine: Rev Rhum Engl Ed. 2004 Nov;71(6):572-6. doi: 10.1016/j.jbspin.2003.10.014. PMID: 15589442. Exclusion reason: Ineligible setting

285. Cherkin DC, Deyo RA, Battie M, et al. A comparison of physical therapy, chiropractic manipulation, and provision of an educational booklet for the treatment of patients with low back pain. N Engl J Med. 1998 Oct 8;339(15):1021-9. doi: 10.1056/nejm199810083391502. PMID: 9761803. Exclusion reason: Ineligible population

286. Cherkin DC, Deyo RA, Street JH, et al. Pitfalls of patient education. Limited success of a program for back pain in primary care. Spine. 1996 Feb 01;21(3):345-55. doi: 10.1097/00007632-199602010-00019. PMID: 8742212. Exclusion reason: Ineligible population

287. Chesterton LS, van der Windt DA, Sim J, et al. Transcutaneous electrical nerve stimulation for the management of tennis elbow: a pragmatic randomized controlled trial: the TATE trial (ISRCTN 87141084). BMC Musculoskelet Disord. 2009 Dec 11;10:156. doi: 10.1186/1471-2474-10-156. PMID: 20003341. Exclusion reason: Ineligible study design

288. Cheung CW, Ching Wong SS, Qiu Q, et al. Oral Oxycodone for Acute Postoperative Pain: A Review of Clinical Trials. Pain Physician. 2017 02;20(2S):SE33-SE52. PMID: 28226340. Exclusion reason: Publication used as source document

289. Cheung R, Krishnaswami S, Kowalski K. Analgesic efficacy of celecoxib in postoperative oral surgery pain: a singledose, two-center, randomized, double-blind, active- and placebo-controlled study. Clin Ther. 2007;29 Suppl:2498-510. doi: 10.1016/j.clinthera.2007.12.008. PMID: 18164917. Exclusion reason: Ineligible comparator 
290. Chia KL, Lam RPK, Lam CK, et al. Acupuncture in the emergency department: a systematic review of randomised controlled trials. Acupunct Med. 2018 Jun;36(3):183-92. doi: 10.1136/acupmed2017-011547. PMID: 29581138. Exclusion reason: Publication used as source document

291. Childers MK, Borenstein D, Brown RL, et al. Low-dose cyclobenzaprine versus combination therapy with ibuprofen for acute neck or back pain with muscle spasm: a randomized trial. Curr Med Res Opin. 2005 Sep;21(9):1485-93. doi: 10.1185/030079905X61938. PMID: 16197668. Exclusion reason: Ineligible comparator

292. Childs JD, Flynn TW, Fritz JM. A perspective for considering the risks and benefits of spinal manipulation in patients with low back pain. Man Ther. 2006 Nov;11(4):316-20. doi: 10.1016/j.math.2005.09.002. PMID: 16839800. Exclusion reason: Ineligible comparator

293. Childs JD, Fritz JM, Flynn TW, et al. A clinical prediction rule to identify patients with low back pain most likely to benefit from spinal manipulation: a validation study. Ann Intern Med. 2004 Dec 21;141(12):920-

8. doi: 10.7326/0003-4819-141-12200412210-00008. PMID: 15611489.

Exclusion reason: Ineligible comparator

294. Chiodo CP, Keyser C, Palms D, et al. The Impact of Music Therapy in the Postoperative Setting on Overall Patient Satisfaction Using Validated Outcome Scores. J Perianesth Nurs. 2019 Apr;34(2):354-8. doi: https://dx.doi.org/10.1016/j.jopan.2018.04.0 06. PMID: 30025665. Exclusion reason: Ineligible outcome

295. Chiu JH, Chen WS, Chen $\mathrm{CH}$, et al. Effect of transcutaneous electrical nerve stimulation for pain relief on patients undergoing hemorrhoidectomy: prospective, randomized, controlled trial. Dis Colon Rectum. 1999 Feb;42(2):180-5. doi: 10.1007/bf02237124. PMID: 10211493.

Exclusion reason: Ineligible setting
296. Cho HW, Hwang EH, Lim B, et al. How current Clinical Practice Guidelines for low back pain reflect Traditional Medicine in East Asian Countries: a systematic review of Clinical Practice Guidelines and systematic reviews. PLoS ONE [Electronic Resource]. 2014;9(2):e88027. doi: https://dx.doi.org/10.1371/journal.pone.0088 027. PMID: 24505363. Exclusion reason: Publication used as source document

297. Cho YH, Kim CK, Heo KH, et al. Acupuncture for acute postoperative pain after back surgery: a systematic review and meta-analysis of randomized controlled trials. Pain Pract. 2015 Mar;15(3):279-91. doi: 10.1111/papr.12208. PMID: 24766648. Exclusion reason: Publication used as source document

298. Choi WJ, Kim WY, Sohn CH, et al. Emergency Department-Based Treatment of Acute Ureteral Colic with Hydromorphone and Pethidine: Prospective, Randomized Control Trial. Journal of The Korean Society of Emergency Medicine. 2011 4;22(2):151-5. Exclusion reason: Not in English

299. Choiniere M, Rittenhouse BE, Perreault S, et al. Efficacy and costs of patient-controlled analgesia versus regularly administered intramuscular opioid therapy.

Anesthesiology. 1998 Dec;89(6):1377-88. doi: 10.1097/00000542-199812000-00015. PMID: 9856712. Exclusion reason: Ineligible comparator

300. Chok B, Lee R, Latimer J, et al. Endurance training of the trunk extensor muscles in people with subacute low back pain. Phys Ther 1999 Nov;79(11):1032-42. PMID: 10534796. Exclusion reason: Ineligible comparator

301. Choo KJ, Grace TR, Khanna K, et al. A Goal-directed Quality Improvement Initiative to Reduce Opioid Prescriptions After Orthopaedic Procedures. J Am Acad Orthop Surg Glob Res Rev. 2019 Sep;3(9):e109. doi: https://dx.doi.org/10.5435/JAAOSGlobal-D19-00109. PMID: 31773081. Exclusion reason: Ineligible population 
302. Chou R. Nonpharmacologic Therapies for Low Back Pain. Ann Intern Med. 2017 Oct 17;167(8):606-7. doi: 10.7326/117-0395. PMID: 29049772. Exclusion reason: Publication used as source document

303. Chou R, Deyo R, Friedly J, et al. Nonpharmacologic Therapies for Low Back Pain: A Systematic Review for an American College of Physicians Clinical Practice Guideline. Ann Intern Med. 2017 Apr 04;166(7):493-505. doi: https://dx.doi.org/10.7326/M16-2459. PMID: 28192793. Exclusion reason: Publication used as source document

304. Chou R, Deyo R, Friedly J, et al. Systemic Pharmacologic Therapies for Low Back Pain: A Systematic Review for an American College of Physicians Clinical Practice Guideline. Ann Intern Med. 2017 Apr 04;166(7):480-92. doi:

https://dx.doi.org/10.7326/M16-2458. PMID: 28192790. Exclusion reason: Publication used as source document

305. Chou R, Gordon DB, de Leon-Casasola OA, et al. Management of Postoperative Pain: A Clinical Practice Guideline From the American Pain Society, the American Society of Regional Anesthesia and Pain Medicine, and the American Society of Anesthesiologists' Committee on Regional Anesthesia, Executive Committee, and Administrative Council. J Pain. 2016 Feb;17(2):131-57. doi: https://dx.doi.org/10.1016/j.jpain.2015.12.00 8. PMID: 26827847. Exclusion reason: Publication used as source document

306. Chou R, Gordon DB, de Leon-Casasola OA, et al. Management of Postoperative Pain: A Clinical Practice Guideline From the American Pain Society, the American Society of Regional Anesthesia and Pain Medicine, and the American Society of Anesthesiologists' Committee on Regional Anesthesia, Executive Committee, and Administrative Council. J Pain. 2016 Feb;17(2):131-57. doi: 10.1016/j.jpain.2015.12.008. PMID: 26827847. Exclusion reason: Ineligible publication type
307. Chou R, Huffman LH, American Pain S, et al. Nonpharmacologic therapies for acute and chronic low back pain: a review of the evidence for an American Pain Society/American College of Physicians clinical practice guideline. Ann Intern Med. 2007 Oct 02;147(7):492-504. PMID: 17909210. Exclusion reason: Publication used as source document

308. Chou R, Qaseem A, Snow V, et al. Diagnosis and treatment of low back pain: a joint clinical practice guideline from the American College of Physicians and the American Pain Society. Ann Intern Med. 2007 Oct 2;147(7):478-91. PMID: 17909209. Exclusion reason: Ineligible publication type

309. Christensen K, Rogers E, Green GA, et al. Safety and efficacy of intranasal ketamine for acute postoperative pain. Acute Pain. 2007;9(4):183-92. doi: 10.1016/j.acpain.2007.09.001. Exclusion reason: Ineligible comparator

310. Christensen KS, Cawkwell GD. Valdecoxib versus rofecoxib in acute postsurgical pain: results of a randomized controlled trial. J Pain Symptom Manage. 2004 May;27(5):460-70. doi:

10.1016/j.jpainsymman.2004.02.001. PMID: 15120774. Exclusion reason: Ineligible intervention

311. Chung F, Tong D, Miceli PC, et al. Controlled-release codeine is equivalent to acetaminophen plus codeine for postcholecystectomy analgesia. Can J Anaesth. 2004 Mar;51(3):216-21. doi: 10.1007/BF03019098. PMID: 15010401. Exclusion reason: Ineligible comparator

312. Chung JY, Lee JJ, Seo HY, et al. Effect of Tramadol/Acetaminophen Combination Drug in Acute Pain After Spinal Surgery. Journal of Korean Society of Spine Surgery. 2007;14(3):137-43. Exclusion reason: Background information only

313. Chung YC, Chien HC, Chen HH, et al. Acupoint stimulation to improve analgesia quality for lumbar spine surgical patients. Pain Manag Nurs. 2014 Dec;15(4):738-47. doi:

https://dx.doi.org/10.1016/j.pmn.2013.07.01 0. PMID: 24144572. Exclusion reason: Ineligible population 
314. Chung YC, Tsou MY, Chen HH, et al. Integrative acupoint stimulation to alleviate postoperative pain and morphine-related side effects: a sham-controlled study. Int J Nurs Stud. 2014 Mar;51(3):370-8. doi: https://dx.doi.org/10.1016/j.ijnurstu.2013.06 .007. PMID: 23866092. Exclusion reason: Ineligible population

315. Cibrian K. Nondrug Interventions Reduce Pain and Opioid Use After Total Knee Arthroplasty. AJN. 2017 Nov;117(11):62. doi: 10.1097/01.NAJ.0000526753.99420.45. PMID: 29076861. Exclusion reason: Ineligible study design

316. Cibulka MT, Delitto A. A comparison of two different methods to treat hip pain in runners. J Orthop Sports Phys Ther. 1993 Apr;17(4):172-6. doi: 10.2519/jospt.1993.17.4.172. PMID: 8467341. Exclusion reason: Ineligible population

317. Cifuentes M, Powell R, Webster B. Shorter time between opioid prescriptions associated with reduced work disability among acute low back pain opioid users. Journal of Occupational \& Environmental Medicine. 2012 Apr;54(4):491-6. doi:

10.1097/JOM.0b013e3182479fae. PMID: 22441492. Exclusion reason: Ineligible outcome

318. Cifuentes M, Webster B, Genevay S, et al. The course of opioid prescribing for a new episode of disabling low back pain: opioid features and dose escalation. Pain. 2010 Oct;151(1):22-9. doi: 10.1016/j.pain.2010.04.012. PMID: 20705393. Exclusion reason: Ineligible comparator

319. Cipriano G, Jr., de Camargo Carvalho AC, Bernardelli GF, et al. Short-term transcutaneous electrical nerve stimulation after cardiac surgery: effect on pain, pulmonary function and electrical muscle activity. Interact Cardiovasc Thorac Surg. 2008 Aug;7(4):539-43. doi: 10.1510/icvts.2007.168542. PMID: 18417519. Exclusion reason: Ineligible setting
320. Cizmic Z, Edusei E, Anoushiravani AA, et al. The Effect of Psychosensory Therapy on Short-term Outcomes of Total Joint Arthroplasty: A Randomized Controlled Trial. Orthopedics. 2018 Nov 01;41(6):e848-e53. doi: 10.3928/0147744720181010-04. PMID: 30321440. Exclusion reason: Ineligible intervention

321. Clarke CL, Ryan CG, Martin DJ. Pain neurophysiology education for the management of individuals with chronic low back pain: systematic review and metaanalysis. Man Ther. 2011 Dec;16(6):544-9. doi:

https://dx.doi.org/10.1016/j.math.2011.05.00 3. PMID: 21705261. Exclusion reason: Publication used as source document

322. Clarke H, Soneji N, Ko DT, et al. Rates and risk factors for prolonged opioid use after major surgery: population based cohort study. BMJ. 2014 Feb 11;348:g1251. doi: 10.1136/BMJ.g1251. PMID: 24519537. Exclusion reason: Ineligible population

323. Clarke R, Derry S, Moore AR. Single dose oral etoricoxib for acute postoperative pain in adults. Cochrane Database Syst Rev. 2019(5) PMID: 24809657. Exclusion reason: Publication used as source document

324. Cleland JA, Childs JD, McRae M, et al. Immediate effects of thoracic manipulation in patients with neck pain: a randomized clinical trial. Man Ther. 2005

May;10(2):127-35. doi: 10.1016/j.math.2004.08.005. PMID: 15922233. Exclusion reason: Ineligible population

325. Cleland JA, Childs JD, Palmer JA, et al. Slump stretching in the management of nonradicular low back pain: a pilot clinical trial. Man Ther. 2006 Nov;11(4):279-86. doi: 10.1016/j.math.2005.07.002. PMID: 16380286. Exclusion reason: Ineligible population 
326. Cleland JA, Fritz JM, Kulig K, et al. Comparison of the effectiveness of three manual physical therapy techniques in a subgroup of patients with low back pain who satisfy a clinical prediction rule: a randomized clinical trial. Spine (Phila Pa 1976). 2009 Dec 1;34(25):2720-9. doi: 10.1097/BRS.0b013e3181b48809. PMID: 19940729. Exclusion reason: Ineligible comparator

327. Cleland JA, Glynn P, Whitman JM, et al. Short-term effects of thrust versus nonthrust mobilization/manipulation directed at the thoracic spine in patients with neck pain: a randomized clinical trial. Phys Ther 2007 Apr;87(4):431-40. doi: 10.2522/ptj.20060217. PMID: 17341509. Exclusion reason: Ineligible population

328. Co LL, Schmitz TH, Havdala H, et al. Acupuncture: an evaluation in the painful crises of sickle cell anaemia. Pain. 1979 Oct;7(2):181-5. doi: 10.1016/03043959(79)90009-5. PMID: 523174.

Exclusion reason: Ineligible study design

329. Coan RM, Wong G, Ku SL, et al. The acupuncture treatment of low back pain: a randomized controlled study. Am J Chin Med. 1980 Spring-Summer;8(1-2):181-9. PMID: 6446852. Exclusion reason: Ineligible population

330. Cohen E, Hafner R, Rotenberg Z, et al. Comparison of ketorolac and diclofenac in the treatment of renal colic. Eur J Clin Pharmacol. 1998 Aug;54(6):455-8. doi: 10.1007/s002280050492. PMID: 9776434.

Exclusion reason: Ineligible comparator

331. Colberg K, Hettich M, Sigmund R, et al. The efficacy and tolerability of an 8-day administration of intravenous and oral meloxicam: a comparison with intramuscular and oral diclofenac in patients with acute lumbago. German Meloxicam Ampoule Study Group. Curr Med Res Opin. 1996;13(7):363-77. PMID: 8862936.

Exclusion reason: Ineligible comparator

332. Comeaux T, Comeaux T. The effect of complementary music therapy on the patient's postoperative state anxiety, pain control, and environmental noise satisfaction. MEDSURG Nurs. 2013 SepOct;22(5):313-8. PMID: 24358573.

Exclusion reason: Ineligible duration
333. Comelon M, Wisloeff-Aase K, Raeder J, et al. A comparison of oxycodone prolongedrelease vs. oxycodone + naloxone prolonged-release after laparoscopic hysterectomy. Acta Anaesthesiol Scand. 2013 Apr;57(4):509-17. doi: https://dx.doi.org/10.1111/aas.12051. PMID: 23301686. Exclusion reason: Ineligible comparator

334. Conn IG. Transcutaneous electrical nerve stimulation following appendicectomy: the placebo effect. Ann R Coll Surg Engl. 1987 May;69(3):145-6. PMID: 19311137. Exclusion reason: Ineligible setting

335. Conti PC, de Alencar EN, da Mota Correa AS, et al. Behavioural changes and occlusal splints are effective in the management of masticatory myofascial pain: a short-term evaluation. J Oral Rehabil. 2012 Oct;39(10):754-60. doi: 10.1111/j.13652842.2012.02327.x. PMID: 22672361.

Exclusion reason: Ineligible population

336. Cook C, Learman K, Houghton S, et al. The addition of cervical unilateral posterioranterior mobilisation in the treatment of patients with shoulder impingement syndrome: a randomised clinical trial. Man Ther. 2014 Feb;19(1):18-24. doi: 10.1016/j.math.2013.05.007. PMID: 23791561. Exclusion reason: Ineligible population

337. Cook CE, Rhon DI, Lewis BD, et al. Postoperative opioid pain management patterns for patients who receive hip surgery. Substance Abuse Treatment, Prevention, \& Policy. 201703 16;12(1):14. doi: 10.1186/s13011-017-0094-5. PMID: 28298221. Exclusion reason: Ineligible study design

338. Cooper SA, Desjardins P, Brain P, et al. Longer analgesic effect with naproxen sodium than ibuprofen in post-surgical dental pain: a randomized, double-blind, placebo-controlled, single-dose trial. Curr Med Res Opin. 2019 12;35(12):2149-58. doi:

https://dx.doi.org/10.1080/03007995.2019.1 655257. PMID: 31402718. Exclusion

reason: Ineligible comparator 
339. Cooper TE, Hambleton IR, Ballas SK, et al. Pharmacological interventions for painful sickle cell vaso-occlusive crises in adults. Cochrane Database Syst Rev. 201911 14;11(11):14. doi: https://dx.doi.org/10.1002/14651858.CD012 187.pub2. PMID: 31742673. Exclusion reason: Publication used as source document

340. Cope AG, Wetzstein MM, Mara KC, et al. Abdominal Ice after Laparoscopic Hysterectomy: A Randomized Controlled Trial. J Minim Invasive Gynecol. 2020 Jul 03;03:03. doi:

https://dx.doi.org/10.1016/j.jmig.2020.06.02 7. PMID: 32622918. Exclusion reason: Ineligible setting

341. Corbett M, South E, Harden M, et al. Brain and spinal stimulation therapies for phantom limb pain: a systematic review. Health Technol Assess. 2018 11;22(62):1-94. doi: https://dx.doi.org/10.3310/hta22620. PMID: 30407905. Exclusion reason: Publication used as source document

342. Cordell WH, Larson TA, Lingeman JE, et al. Indomethacin suppositories versus intravenously titrated morphine for the treatment of ureteral colic. Ann Emerg Med. 1994 Feb;23(2):262-9. doi: 10.1016/s01960644(94)70038-9. PMID: 8304606.

Exclusion reason: Ineligible duration

343. Cornelius R, Herr KA, Gordon DB, et al. Evidence-Based Practice Guideline : Acute Pain Management in Older Adults. J Gerontol Nurs. 2017 Feb 01;43(2):18-27. doi: https://dx.doi.org/10.3928/0098913420170111-08. PMID: 28128395. Exclusion reason: Background information only

344. Cotta BH, Nguyen V, Sur RL, et al. Opiates prescribed for acute renal colic are associated with prolonged use. World J Urol. 2020 Aug 01;01:01. doi: https://dx.doi.org/10.1007/s00345-02003386-7. PMID: 32740804. Exclusion reason: Ineligible study design

345. Coudeyre E, Tubach F, Rannou F, et al. Effect of a simple information booklet on pain persistence after an acute episode of low back pain: a non-randomized trial in a primary care setting. PLoS ONE [Electronic Resource]. 2007 Aug 08;2(8):e706. PMID: 17684553. Exclusion reason: Ineligible intervention
346. Coxhead CE. A clinical trial of the management of sciatica with or without low back pain. Physiotherapy. 1974

Mar;60(3):72-4. PMID: 4445302.

Exclusion reason: Ineligible population

347. Coxhead CE, Inskip H, Meade TW, et al. Multicentre trial of physiotherapy in the management of sciatic symptoms. Lancet. 1981 May 16;1(8229):1065-8. doi: 10.1016/s0140-6736(81)92238-8. PMID: 6112444. Exclusion reason: Ineligible population

348. Cramer GD, Humphreys CR, Hondras MA, et al. The Hmax/Mmax ratio as an outcome measure for acute low back pain. J Manipulative Physiol Ther. 1993 Jan;16(1):7-13. PMID: 8423429. Exclusion reason: Ineligible comparator

349. Crawford C, Boyd C, Paat CF, et al. The Impact of Massage Therapy on Function in Pain Populations-A Systematic Review and Meta-Analysis of Randomized Controlled Trials: Part I, Patients Experiencing Pain in the General Population. Pain Med. 2016 Jul 1;17(7):1353-75. doi: 10.1093/pm/pnw099. PMID: 27165971. Exclusion reason:

Publication used as source document

350. Crespin DJ, Griffin KH, Johnson JR, et al. Acupuncture provides short-term pain relief for patients in a total joint replacement program. Pain Med. 2015 Jun;16(6):1195203. doi: 10.1111/pme.12685. PMID: 25586769. Exclusion reason: Ineligible study design

351. Crocker CG. Acute postoperative pain: cause and control (continuing education credit). Orthop Nurs. 1986 Mar-Apr;5(2):116. doi: 10.1097/00006416-19860300000003. PMID: 3635022. Exclusion reason: Ineligible study design

352. Cruser A, Maurer D, Hensel K, et al. A randomized, controlled trial of osteopathic manipulative treatment for acute low back pain in active duty military personnel. J Man Manip Ther. 2012;20(1):5-15. doi: 10.1179/2042618611Y.0000000016. PMID: 23372389. Exclusion reason: Ineligible comparator 
353. Curatolo M. Pharmacological and Interventional Management of Pain After Whiplash Injury. J Orthop Sports Phys Ther. 2016 Oct;46(10):845-50. PMID: 27594660. Exclusion reason: Ineligible publication type

354. Curkovic B, Bernik N. Three-week open multi-centre study of sustained-release tramadol in various pain conditions. Arzneimittelforschung. 2003;53(7):503-6. PMID: 12918216. Exclusion reason: Ineligible study design

355. Curry C, Kelly AM. Intravenous tenoxicam for the treatment of renal colic. N Z Med J. 1995 Jun 14;108(1001):229-30. PMID: 7603654. Exclusion reason: Ineligible intervention

356. Cuschieri RJ, Morran CG, McArdle CS. Transcutaneous electrical stimulation for postoperative pain. Ann R Coll Surg Engl. 1985 Mar;67(2):127-9. PMID: 3883879.

Exclusion reason: Ineligible setting

357. Dabu-Bondoc S, Vadivelu N, Benson J, et al. Hemispheric synchronized sounds and perioperative analgesic requirements. Anesth Analg. 2010 Jan 01;110(1):208-10. doi: 10.1213/ANE.0b013e3181bea424. PMID: 19861358. Exclusion reason: Ineligible setting

358. Daljord OA, Barstad S, Norenberg P. [Ambulatory treatment of an acute attack in urinary calculi. A randomized study of the effects of Petidin, Fortralin, Temgesic and Confortid]. Tidsskr Nor Laegeforen. 1983 Apr 30;103(12):1006-8. PMID: 6192515. Exclusion reason: Not in English

359. Daniels S, Melson T, Hamilton DA, et al. Analgesic efficacy and safety of a novel injectable formulation of diclofenac compared with intravenous ketorolac and placebo after orthopedic surgery: A multicenter, randomized, double-blinded, multiple-dose trial. Clin J Pain. 2013 Aug;29(8):655-63. doi: 10.1097/AJP.0b013e318270f957. PMID: 23328337. Exclusion reason: Ineligible intervention
360. Daniels SE, Atkinson HC, Stanescu I, et al. Analgesic Efficacy of an Acetaminophen/Ibuprofen Fixed-dose Combination in Moderate to Severe Postoperative Dental Pain: A Randomized, Double-blind, Parallel-group, Placebocontrolled Trial. Clin Ther. 2018 10;40(10):1765-76.e5. doi: 10.1016/j.clinthera.2018.08.019. PMID: 30245281. Exclusion reason: Ineligible comparator

361. Daniels SE, Desjardins PJ, Bird SR, et al. Rofecoxib $50 \mathrm{mg}$ and valdecoxib 20 or 40 mg in adults and adolescents with postoperative pain after third molar extraction: results of two randomized, double-blind, placebo-controlled, singledose studies. Clin Ther. 2006

Jul;28(7):1022-34. doi: 10.1016/j.clinthera.2006.07.005. PMID: 16990080. Exclusion reason: Ineligible comparator

362. Daniels SE, Goulder MA, Aspley S, et al. A randomised, five-parallel-group, placebocontrolled trial comparing the efficacy and tolerability of analgesic combinations including a novel single-tablet combination of ibuprofen/paracetamol for postoperative dental pain. Pain. 2011;152(3):632-42. PMID: 21257263. Exclusion reason: Ineligible population

363. Daniels SE, Grossman EH, Kuss ME, et al. A double-blind, randomized comparison of intramuscularly and intravenously administered parecoxib sodium versus ketorolac and placebo in a post-oral surgery pain model. Clin Ther. 2001 Jul;23(7):101831. doi: 10.1016/s0149-2918(01)80088-6. PMID: 11519767. Exclusion reason: Ineligible intervention

364. Daniels SE, Playne R, Stanescu I, et al. Efficacy and Safety of an Intravenous Acetaminophen/Ibuprofen Fixed-dose Combination After Bunionectomy: a Randomized, Double-Blind, Factorial, Placebo-controlled Trial. Clin Ther. 2019

Aug 22;22:22. doi:

10.1016/j.clinthera.2019.07.008. PMID: 31447129. Exclusion reason: Ineligible intervention 
365. Danielsen JM, Johnsen R, Kibsgaard SK, et al. Early aggressive exercise for postoperative rehabilitation after discectomy. Spine. 2000 Apr 15;25(8):101520. doi: 10.1097/00007632-20000415000017. PMID: 10767815. Exclusion reason: Ineligible population

366. Danoff JR, Goel R, Sutton R, et al. How Much Pain Is Significant? Defining the Minimal Clinically Important Difference for the Visual Analog Scale for Pain After Total Joint Arthroplasty. J Arthroplasty. 2018 07;33(7S):S71-S5.e2. doi: https://dx.doi.org/10.1016/j.arth.2018.02.02 9. PMID: 29567002. Exclusion reason: Background information only

367. Daoust R, Emond M, Bergeron E, et al. Risk factors of significant pain syndrome 90 days after minor thoracic injury: trajectory analysis. Acad Emerg Med. 2013 Nov;20(11):1139-45. doi: https://dx.doi.org/10.1111/acem.12248. PMID: 24238316. Exclusion reason: Background information only

368. Daoust R, Paquet J, Cournoyer A, et al. Quantity of opioids consumed following an emergency department visit for acute pain: a Canadian prospective cohort study. BMJ Open. 201809 17;8(9):e022649. doi: https://dx.doi.org/10.1136/BMJopen-2018022649. PMID: 30224393. Exclusion reason: Ineligible outcome

369. Dario AB, Moreti Cabral A, Almeida L, et al. Effectiveness of telehealth-based interventions in the management of nonspecific low back pain: a systematic review with meta-analysis. Spine J. 2017 09;17(9):1342-51. doi: https://dx.doi.org/10.1016/j.spinee.2017.04. 008. PMID: 28412562. Exclusion reason: Publication used as source document

370. Das SK, Banerjee M, Mondal S, et al. A comparative study of efficacy and safety of lornoxicam versus tramadol as analgesics after surgery on head and neck. Indian J Otolaryngol Head Neck Surg. 2013 Jul;65(Suppl 1):126-30. doi: https://dx.doi.org/10.1007/s12070-0130617-y. PMID: 24427628. Exclusion reason: Ineligible publication type
371. Dash A, Maiti R, Akantappa Bandakkanavar $\mathrm{TK}$, et al. Intramuscular drotaverine and diclofenac in acute renal colic: A comparative study of analgesic efficacy and safety. Pain Med. 2012 Mar;13(3):466-71. doi: 10.1111/j.1526-4637.2011.01314.x. PMID: 22295884. Exclusion reason: Ineligible comparator

372. Datta R, Upadhyay KK. A randomized clinical trial of three different steroid agents for treatment of low backache through the caudal route. Med J Armed Forces India. 2011;67(1):25-33. doi: 10.1016/S03771237(11)80007-9. PMID: 27365757. Exclusion reason: Ineligible intervention

373. Datta S, Lee M, Falco FJ, et al. Systematic assessment of diagnostic accuracy and therapeutic utility of lumbar facet joint interventions. Pain Physician. 2009 MarApr;12(2):437-60. PMID: 19305489. Exclusion reason: Publication used as source document

374. Davies JR. Ineffective transcutaneous nerve stimulation following epidural anaesthesia. Anaesthesia. 1982 Apr;37(4):453-4. doi: 10.1111/j.1365-2044.1982.tb01159.x. PMID: 6979269. Exclusion reason: Ineligible setting

375. Davis KM, Esposito MA, Meyer BA. Oral analgesia compared with intravenous patient-controlled analgesia for pain after cesarean delivery: a randomized controlled trial. Am J Obstet Gynecol. 2006

Apr;194(4):967-71. doi: 10.1016/j.ajog.2006.02.025. PMID: 16580284. Exclusion reason: Ineligible setting

376. Davis L, Evans S, Fishman B, et al. Predictors of attrition in HIV-positive subjects with peripheral neuropathic pain. AIDS Care. 2004 Apr;16(3):395-402. doi: 10.1080/09540120410001665394. PMID: 15203432. Exclusion reason: Ineligible population

377. De Benedittis G, Besana F, Lorenzetti A. A new topical treatment for acute herpetic neuralgia and post-herpetic neuralgia: the aspirin/diethyl ether mixture. An open-label study plus a double-blind controlled clinical trial. Pain. 1992 Mar;48(3):383-90. PMID: 1594261. Exclusion reason: Ineligible comparator 
378. de Franceschi L, Finco G, Vassanelli A, et al. A pilot study on the efficacy of ketorolac plus tramadol infusion combined with erythrocytapheresis in the management of acute severe vaso-occlusive crises and sickle cell pain. Haematologica. 2004

Nov;89(11):1389-91. PMID: 15531461. Exclusion reason: Ineligible study design

379. De Franceschi L, Mura P, Schweiger V, et al. Fentanyl Buccal Tablet: A New Breakthrough Pain Medication in Early Management of Severe Vaso-Occlusive Crisis in Sickle Cell Disease. Pain Pract. 2016 07;16(6):680-7. doi:

10.1111/papr.12313. PMID: 26009799.

Exclusion reason: Ineligible comparator

380. De Gregori M, Diatchenko L, Ingelmo PM, et al. Human Genetic Variability Contributes to Postoperative Morphine Consumption. J Pain. 2016 05;17(5):628-36. doi: https://dx.doi.org/10.1016/j.jpain.2016.02.00 3. PMID: 26902643. Exclusion reason: Background information only

381. de Jong JR, Vlaeyen JW, van Eijsden M, et al. Reduction of pain-related fear and increased function and participation in work-related upper extremity pain (WRUEP): effects of exposure in vivo. Pain. 2012 Oct;153(10):2109-18. doi: 10.1016/j.pain.2012.07.001. PMID: 22902198. Exclusion reason: Ineligible population

382. de Klerk JE, du Plessis WF, Steyn HS, et al. Hypnotherapeutic ego strengthening with male South African coronary artery bypass patients. Am J Clin Hypn. 2004

Oct;47(2):79-92. doi: 10.1080/00029157.2004.10403627. PMID: 15554461. Exclusion reason: Ineligible population

383. De Meulemeester KE, Castelein B, Coppieters I, et al. Comparing Trigger Point Dry Needling and Manual Pressure Technique for the Management of Myofascial Neck/Shoulder Pain: A Randomized Clinical Trial. J Manipulative Physiol Ther. 2017 01;40(1):11-20. doi: https://dx.doi.org/10.1016/j.jmpt.2016.10.00 8. PMID: 28017188. Exclusion reason: Ineligible population
384. DeAndrade JR, Maslanka M, Maneatis T, et al. The use of ketorolac in the management of postoperative pain. Orthopedics. 1994 Feb;17(2):157-66. PMID: 8190679.

Exclusion reason: Ineligible publication type

385. Dehghan M, Farahbod F. Evaluation of the therapeutic effect of oral gabapentin on the severity of acute low back pain. Scientific Journal of Kurdistan University of Medical Sciences. 2015;20(1):97-104. Exclusion reason: Not in English

386. Dehner C, Hartwig E, Strobel P, et al. Comparison of the relative benefits of 2 versus 10 days of soft collar cervical immobilization after acute whiplash injury. Arch Phys Med Rehabil. 2006

Nov;87(11):1423-7. doi: 10.1016/j.apmr.2006.07.268. PMID: 17084114. Exclusion reason: Ineligible comparator

387. del Pozo-Cruz B, Gusi N, Adsuar JC, et al. Musculoskeletal fitness and health-related quality of life characteristics among sedentary office workers affected by subacute, non-specific low back pain: a crosssectional study. Physiotherapy. 2013 Sep;99(3):194-200. doi: https://dx.doi.org/10.1016/j.physio.2012.06. 006. PMID: 23219627. Exclusion reason: Background information only

388. Dellemijn PL, van Duijn H, Vanneste JA. Prolonged treatment with transdermal fentanyl in neuropathic pain. J Pain Symptom Manage. 1998 Oct;16(4):220-9. doi: 10.1016/S0885-3924\%2898\%29000700. PMID: 9803049. Exclusion reason: Ineligible population

389. Demir B, Saritas S. Effect of hand massage on pain and anxiety in patients after liver transplantation: A randomised controlled trial. Complement Ther Clin Pract. 2020 May;39:101152. doi: https://dx.doi.org/10.1016/j.ctcp.2020.10115 2. PMID: 32379634. Exclusion reason: Ineligible intervention

390. Deogaonkar M, Slavin KV. Peripheral nerve/field stimulation for neuropathic pain. Neurosurg Clin N Am. 2014 Jan;25(1):1-10. doi: 10.1016/j.nec.2013.10.001. PMID: 24262894. Exclusion reason: Ineligible publication type 
391. Derleth BM, Dexter DD, Arndt R, et al. Effect of a Statewide Controlled-Substance Monitoring Requirement on the Opioid Prescribing Practice for Treatment of Acute Pain. WMJ. 2020 Mar;119(1):33-6. PMID: 32348069. Exclusion reason: Ineligible population

392. Derry S, Best J, Moore AR. Single dose oral dexibuprofen. Cochrane Database Syst Rev. 2019(5) PMID: 24151035. Exclusion

reason: Ineligible comparator

393. Derry S, Best J, Moore AR. Single dose oral dexibuprofen [S(+)-ibuprofen] for acute postoperative pain in adults [Systematic Review]. Cochrane Database Syst Rev. 2019a;5:5. PMID: 24151035. Exclusion reason: Publication used as source document

394. Derry S, Derry CJ, Moore RA. Single dose oral ibuprofen plus oxycodone for acute postoperative pain in adults. Cochrane Database Syst Rev. 2013 Jun 26(6):CD010289. doi: https://dx.doi.org/10.1002/14651858.CD010 289.pub2. PMID: 23801549. Exclusion reason: Publication used as source document

395. Derry S, Karlin SM, Moore RA. Single dose oral ibuprofen plus codeine for acute postoperative pain in adults. Cochrane Database Syst Rev. 2015 Feb 05(2):CD010107. doi: https://dx.doi.org/10.1002/14651858.CD010 107.pub3. PMID: 25927097. Exclusion reason: Ineligible comparator

396. Derry S, Matthews PR, Wiffen PJ, et al. Salicylate-containing rubefacients for acute and chronic musculoskeletal pain in adults. Cochrane Database Syst Rev. 2014 Nov 26(11):CD007403. doi: https://dx.doi.org/10.1002/14651858.CD007 403.pub3. PMID: 25425092. Exclusion reason: Publication used as source document

397. Derry S, Moore AR, Gaskell H, et al. Topical NSAIDs for acute musculoskeletal pain in adults. Cochrane Database Syst Rev. 2019(5) PMID: 27386729. Exclusion reason: Publication used as source document
398. Derry S, Moore RA, Gaskell H, et al. Topical NSAIDs for acute musculoskeletal pain in adults. Cochrane Database Syst Rev. 2015 Jun 11(6):CD007402. doi: https://dx.doi.org/10.1002/14651858.CD007 402.pub3. PMID: 26068955. Exclusion reason: Publication used as source document

399. Derry S, Wiffen PJ, Kalso EA, et al. Topical analgesics for acute and chronic pain in adults - an overview of Cochrane Reviews. Cochrane Database Syst Rev. 201705 12;5:CD008609. doi: https://dx.doi.org/10.1002/14651858.CD008 609.pub2. PMID: 28497473. Exclusion reason: Publication used as source document

400. Derry S, Wiffen PJ, Moore AR, et al. Topical lidocaine for neuropathic pain in adults. Cochrane Database Syst Rev. 2019(5) PMID: 25058164. Exclusion reason: Publication used as source document

401. Derry S, Wiffen PJ, Moore RA. Single dose oral ibuprofen plus caffeine for acute postoperative pain in adults. Cochrane Database Syst Rev. 2015 Jul 14(7):CD011509. doi: https://dx.doi.org/10.1002/14651858.CD011 509.pub2. PMID: 26171993. Exclusion reason: Ineligible comparator

402. Dersh J, Mayer TG, Gatchel RJ, et al. Prescription opioid dependence is associated with poorer outcomes in disabling spinal disorders. Spine. 2008 Sep 15;33(20):221927. doi: 10.1097/BRS.0b013e31818096d1. PMID: 18725868. Exclusion reason: Ineligible population

403. Desai PC, Brittain JE, Jones SK, et al. A pilot study of eptifibatide for treatment of acute pain episodes in sickle cell disease. Blood. 2102;120(2102)doi: 10.1016/j.thromres.2013.08.002. PMID: 23973010. Exclusion reason: Ineligible intervention 
404. Desai SN, Badiger SV, Tokur SB, et al. Safety and efficacy of transdermal buprenorphine versus oral tramadol for the treatment of post-operative pain following surgery for fracture neck of femur: a prospective, randomised clinical study. Indian J Anaesth. 2017;61(3):225-9. doi: 10.4103/ija.IJA_208_16. PMID: 28405035. Exclusion reason: Ineligible intervention

405. DeSantana JM, Walsh DM, Vance C, et al. Effectiveness of transcutaneous electrical nerve stimulation for treatment of hyperalgesia and pain. Curr Rheumatol Rep. 2008 Dec;10(6):492-9. PMID: 19007541.

Exclusion reason: Background information only

406. Desjardins PJ, Black PM, Daniels S, et al. A randomized controlled study comparing rofecoxib, diclofenac sodium, and placebo in post-bunionectomy pain. Curr Med Res Opin. 2004 Oct;20(10):1523-37. doi: 10.1185/030079904X3069. PMID: 15462686. Exclusion reason: Ineligible comparator

407. Desmoulin GT, Yasin NI, Chen DW. Initial results using Khan Kinetic Treatment ${ }^{\mathrm{TM}}$ as a low back pain treatment option. J Musculoskelet Pain. 2007;15(3):91-102. doi: 10.1300/J094v15n03_12. Exclusion reason: Ineligible population

408. Dettori JR, Bullock SH, Sutlive TG, et al. The effects of spinal flexion and extension exercises and their associated postures in patients with acute low back pain. Spine. 1995 Nov 01;20(21):2303-12. doi: 10.1097/00007632-199511000-00008. PMID: 8553118. Exclusion reason: Ineligible comparator

409. Dever C. Treating Acute Pain in the OpiateDependent Patient. J Trauma Nurs. 2017 Sep/Oct;24(5):292-9. doi: https://dx.doi.org/10.1097/JTN.0000000000 000309. PMID: 28885516. Exclusion reason: Background information only

410. Deyo RA, Diehl AK, Rosenthal M. How many days of bed rest for acute low back pain? A randomized clinical trial. N Engl J Med. 1986 Oct 23;315(17):1064-70. doi: 10.1056/NEJM198610233151705. PMID: 2945109. Exclusion reason: Ineligible comparator
411. Diaz C, Gasso FC, Panades JN. Doubleblind study of the analgesic activity of fosfosal in patients with musculoskeletal and articular pain: comparison with lysine acetylsalicylate and placebo. Clin Ther. 1981;4(2):121-6. PMID: 6794911. Exclusion reason: Ineligible intervention

412. Dingemann J, Plewig B, Baumann I, et al. Acupuncture in posttonsillectomy pain : a prospective, double-blinded, randomized, controlled trial. HNO. 2017 Jan;65(Suppl 1):73-9. doi: 10.1007/s00106-016-0289-5. PMID: 28070602. Exclusion reason: Ineligible population

413. Dinges HC, Otto S, Stay DK, et al. Side Effect Rates of Opioids in Equianalgesic Doses via Intravenous Patient-Controlled Analgesia: A Systematic Review and Network Meta-analysis. Anesth Analg. 2019 10;129(4):1153-62. doi:

https://dx.doi.org/10.1213/ANE.0000000000 003887. PMID: 30418234. Exclusion reason: Ineligible population

414. Dinis J, Soto E, Pedroza C, et al. Nonopioid versus opioid analgesia after hospital discharge following cesarean delivery: a randomized equivalence trial. Am J Obstet Gynecol. 2020 05;222(5):488.e1-.e8. doi: https://dx.doi.org/10.1016/j.ajog.2019.12.00 1. PMID: 31816306. Exclusion reason: Ineligible comparator

415. do Nascimento-Junior EM, Dos Santos GMS, Tavares Mendes ML, et al. Cryotherapy in reducing pain, trismus, and facial swelling after third-molar surgery: Systematic review and meta-analysis of randomized clinical trials. J Am Dent Assoc. 2019 04;150(4):269-77.e1. doi: https://dx.doi.org/10.1016/j.adaj.2018.11.00 8. PMID: 30798949. Exclusion reason: Publication used as source document

416. Doleman B, Read D, Lund J, et al. Preemptive paracetamol reduces postoperative opioid consumption and pain scores following surgery: systematic review and meta-analysis. Anaesthesia. 2015;70(11). Exclusion reason: Publication used as source document 
417. Donec V, Krisciunas A. The effectiveness of Kinesio Taping after total knee replacement in early postoperative rehabilitation period. A randomized controlled trial. Eur J Phys Rehabil Med. 2014 Aug;50(4):363-71. PMID: 24819349. Exclusion reason: Ineligible setting

418. Dong CS, Zhang J, Lu Q, et al. Effect of Dexmedetomidine combined with sufentanil for post- thoracotomy intravenous analgesia:a randomized, controlled clinical study. BMC Anesthesiol. $2017 \mathrm{Mar}$ 1;17(1):33. doi: 10.1186/s12871-017-03244. PMID: 28249614. Exclusion reason: Ineligible population

419. Dong DS, Yu X, Wan CF, et al. Efficacy of Short-Term Spinal Cord Stimulation in Acute/Subacute Zoster-Related Pain: A Retrospective Study. Pain Physician. 2017 07;20(5):E633-E45. PMID: 28727708. Exclusion reason: Ineligible study design

420. Doran DM, Newell DJ. Manipulation in treatment of low back pain: a multicentre study. Br Med J. 1975 Apr 26;2(5964):1614. doi: 10.1136/BMJ.2.5964.161. PMID: 123815. Exclusion reason: Ineligible population

421. Dougados M, Le Henanff A, Logeart I, et al. Short-term efficacy of rofecoxib and diclofenac in acute shoulder pain: A placebo-controlled randomized trial. PLoS Clin Trials. 2007;2(3)doi: 10.1371/journal.pctr.0020009. PMID: 17347681. Exclusion reason: Ineligible comparator

422. Dowell D, Haegerich TM, Chou R. CDC Guideline for Prescribing Opioids for Chronic Pain--United States, 2016. JAMA. 2016 Apr 19;315(15):1624-45. doi: 10.1001/jama.2016.1464. PMID: 26977696. Exclusion reason: Publication used as source document

423. Downie AS, Hancock MJ, Rzewuska M, et al. Trajectories of acute low back pain: a latent class growth analysis. Pain. 2016 Jan;157(1):225-34. doi: 10.1097/j.pain.0000000000000351. PMID: 26397929. Exclusion reason: Ineligible study design
424. Doyle G, Jayawardena S, Ashraf E, et al. Efficacy and tolerability of nonprescription ibuprofen versus celecoxib for dental pain. $\mathrm{J}$ Clin Pharmacol. 2002 Aug;42(8):912-9. doi: 10.1177/009127002401102830. PMID: 12162474. Exclusion reason: Ineligible comparator

425. Drake G, Williams AC de C. Nursing Education Interventions for Managing Acute Pain in Hospital Settings: A Systematic Review of Clinical Outcomes and Teaching Methods. Pain Manag Nurs. 2017 02;18(1):3-15. doi: https://dx.doi.org/10.1016/j.pmn.2016.11.00 1. PMID: 28038974. Exclusion reason: Publication used as source document

426. Draper DO, Klyve D, Ortiz R, et al. Effect of low-intensity long-duration ultrasound on the symptomatic relief of knee osteoarthritis: a randomized, placebo-controlled doubleblind study. J Orthop Surg. 2018 Oct 16;13(1):257. doi: 10.1186/s13018-0180965-0. PMID: 30326947. Exclusion reason: Ineligible population

427. Dreiser RL, Le Parc JM, Velicitat P, et al. Oral meloxicam is effective in acute sciatica: two randomised, double-blind trials versus placebo or diclofenac. Inflamm Res. 2001 Mar;50 Suppl 1:S17-23. PMID: 11339516. Exclusion reason: Ineligible comparator

428. Dreiser RL, Marty M, Ionescu E, et al. Relief of acute low back pain with diclofenac-K 12.5 mg tablets: a flexible dose, ibuprofen $200 \mathrm{mg}$ and placebocontrolled clinical trial. Int J Clin Pharmacol Ther. 2003 Sep;41(9):375-85. doi: 10.5414/cpp41375. PMID: 14518597. Exclusion reason: Ineligible comparator

429. Drez D, Jr., Ritter M, Rosenberg TD. Pain relief after arthroscopy: naproxen sodium compared to propoxyphene napsylate with acetaminophen. South Med J. 1987 Apr;80(4):440-3. doi: 10.1097/00007611198704000-00008. PMID: 2882607.

Exclusion reason: Ineligible comparator

430. Du E, Farzal Z, Stephenson E, et al. Multimodal Analgesia Protocol after Head and Neck Surgery: Effect on Opioid Use and Pain Control. Otolaryngol Head Neck Surg. 2019 09;161(3):424-30. doi: https://dx.doi.org/10.1177/01945998198418 85. PMID: 30961428. Exclusion reason: Ineligible population 
431. Du S, Hu L, Dong J, et al. Self-management program for chronic low back pain: A systematic review and meta-analysis. Patient Educ Couns. 2017 01;100(1):37-49. doi: https://dx.doi.org/10.1016/j.pec.2016.07.029 . PMID: 27554077. Exclusion reason: Publication used as source document

432. Duncan C, Riley B. BET 2: Low-dose ketamine for acute pain in the ED. Emerg Med J. 2016 Dec;33(12):892-3. doi: 10.1136/emermed-2016-206440.2. PMID: 27864394. Exclusion reason: Publication used as source document

433. Dunlop RJ, Bennett KC. Pain management for sickle cell disease. Cochrane Database Syst Rev. 2006 Apr 19(2):CD003350. PMID: 16625580. Exclusion reason: Ineligible publication type

434. Dunning JR, Cleland JA, Waldrop MA, et al. Upper cervical and upper thoracic thrust manipulation versus nonthrust mobilization in patients with mechanical neck pain: a multicenter randomized clinical trial. J Orthop Sports Phys Ther. 2012 Jan;42(1):518. doi: 10.2519/jospt.2012.3894. PMID: 21979312. Exclusion reason: Ineligible population

435. Dupuis F, Barrett E, Dubé MO, et al. Cryotherapy or gradual reloading exercises in acute presentations of rotator cuff tendinopathy: a randomised controlled trial. BMJ Open Sport Exerc Med. 2018;4(1):e000477. doi: 10.1136/BMJsem2018-000477. PMID: 30622733. Exclusion reason: Ineligible population

436. Durand Z, Nechuta S, Krishnaswami S, et al. Prevalence and Risk Factors Associated With Long-term Opioid Use After Injury Among Previously Opioid-Free Workers. JAMA Netw Open. 2019 Jul 3;2(7):e197222. doi: 10.1001/jamanetworkopen.2019.7222. PMID: 31314119. Exclusion reason: Ineligible outcome

437. Durkin B, Page C, Glass P. Pregabalin for the treatment of postsurgical pain. Expert Opin Pharmacother. 2010 Nov;11(16):27518. doi: 10.1517/14656566.2010.526106. PMID: 20977407. Exclusion reason: Ineligible study design
438. Dworkin RH, Turk DC, Basch E, et al. Considerations for extrapolating evidence of acute and chronic pain analgesic efficacy. Pain. 2011 Aug;152(8):1705-8. doi: 10.1016/j.pain.2011.02.026. PMID: 21396781. Exclusion reason: Background information only

439. Dworkin RH, White R, O'Connor AB, et al. Healthcare costs of acute and chronic pain associated with a diagnosis of herpes zoster. J Am Geriatr Soc. 2007 Aug;55(8):1168-75. PMID: 17661954. Exclusion reason: Background information only

440. Eardley W, Toth C. An open-label, nonrandomized comparison of venlafaxine and gabapentin as monotherapy or adjuvant therapy in the management of neuropathic pain in patients with peripheral neuropathy. J Pain Res. 2010;3:33-49. doi: 10.2147/jpr.s8675. PMID: 21197308.

Exclusion reason: Ineligible population

441. Ebneshahidi A, Mohseni M. The effect of patient-selected music on early postoperative pain, anxiety, and hemodynamic profile in cesarean section surgery. J Altern Complement Med. 2008 Sep;14(7):827-31. doi: 10.1089/acm.2007.0752. PMID: 18803492. Exclusion reason: Ineligible setting

442. Ebrahimzadeh MH, Mousavi SK, Ashraf H, et al. Transdermal fentanyl patches versus patient-controlled intravenous morphine analgesia for postoperative pain management. Iranian Red Crescent Medical Journal. 2014;16(5) PMID: 25031847. Exclusion reason: Ineligible population

443. Ee CC, Manheimer E, Pirotta MV, et al. Acupuncture for pelvic and back pain in pregnancy: a systematic review. Am J Obstet Gynecol. 2008 Mar;198(3):254-9. doi: 10.1016/j.ajog.2007.11.008. PMID: 18313444. Exclusion reason: Publication used as source document

444. Effiong A, Kumari P. Integrating palliative care and emergency medicine for optimal management of sickle cell pain in the wake of the United States opioid epidemic. Ann Palliat Med. 2018 Apr;7(2):274-6. doi: https://dx.doi.org/10.21037/apm.2017.12.01. PMID: 29307211. Exclusion reason: Background information only 
445. Eid AI, DePesa C, Nordestgaard AT, et al. Variation of Opioid Prescribing Patterns among Patients undergoing Similar Surgery on the Same Acute Care Surgery Service of the Same Institution: Time for

Standardization? Surgery. 2018

Nov;164(5):926-30. doi:

10.1016/j.surg.2018.05.047. PMID:

30049481. Exclusion reason: Ineligible study design

446. Eiff MP, Smith AT, Smith GE. Early mobilization versus immobilization in the treatment of lateral ankle sprains. Am J Sports Med. 1994 Jan-Feb;22(1):83-8. PMID: 8129116. Exclusion reason: Ineligible intervention

447. Eipe N, Penning J, Yazdi F, et al. Perioperative use of pregabalin for acute pain-a systematic review and meta-analysis. Pain. 2015 Jul;156(7):1284-300. doi: https://dx.doi.org/10.1097/j.pain.000000000 0000173. PMID: 25830925. Exclusion

reason: Publication used as source document

448. Eisenberg DM, Post DE, Davis RB, et al. Addition of choice of complementary therapies to usual care for acute low back pain: a randomized controlled trial. Spine. 2007 Jan 15;32(2):151-8. doi: 10.1097/01.brs.0000252697.07214.65. PMID: 17224808. Exclusion reason: Ineligible comparator

449. Eisenhart AW, Gaeta TJ, Yens DP. Osteopathic manipulative treatment in the emergency department for patients with acute ankle injuries. J Am Osteopath Assoc. 2003 Sep;103(9):417-21. PMID: 14527076. Exclusion reason: Ineligible intervention

450. Eke FU, Obamyonyi A, Eke NN, et al. An open comparative study of dispersible piroxicam versus soluble acetylsalicylic acid for the treatment of osteoarticular painful attack during sickle cell crisis. Trop Med Int Health. 2000 Feb;5(2):81-4. doi: 10.1046/j.1365-3156.2000.00507.x. PMID: 10747265. Exclusion reason: Ineligible population
451. Elander J, Lusher J, Bevan D, et al. Pain management and symptoms of substance dependence among patients with sickle cell disease. Soc Sci Med. 2003

Nov;57(9):1683-96. doi: 10.1016/s02779536(02)00553-1. PMID: 12948577. Exclusion reason: Ineligible study design

452. Elgebaly AS. Does the Use of Nitroglycerin Patch Improve Local Anaesthetic Effects in Bier's Block? A Double-Blind Placebo Controlled Study. Anesthesiol Res Pract. 2018;2018doi: 10.1155/2018/9674731. PMID: 29706996 Exclusion reason: Ineligible intervention

453. Elmallah RK, Ramkumar PN, Khlopas A, et al. Postoperative Pain and Analgesia: Is There a Genetic Basis to the Opioid Crisis? Surg Technol Int. 201806 01;32:306-14. PMID: 29791710. Exclusion reason: Background information only

454. Eloy JD, Anthony C, Amin S, et al. Gabapentin does not appear to improve postoperative pain and sleep patterns in patients who concomitantly receive regional anesthesia for lower extremity orthopedic surgery: A randomized control trial. Pain Res Manag. 2017;2017doi: 10.1155/2017/2310382. PMID: 28348503.

Exclusion reason: Ineligible setting

455. Elzaki WM, Abubakr NH, Ziada HM, et al. Double-blind Randomized Placebocontrolled Clinical Trial of Efficiency of Nonsteroidal Anti-inflammatory Drugs in the Control of Post-endodontic Pain. J Endod. 2016 Jun;42(6):835-42. doi: 10.1016/j.joen.2016.02.014. PMID: 27080115. Exclusion reason: Ineligible comparator

456. Emmiler M, Solak O, Kocogullari C, et al. Control of acute postoperative pain by transcutaneous electrical nerve stimulation after open cardiac operations: a randomized placebo-controlled prospective study. Heart Surg Forum. 2008;11(5):E300-3. doi: 10.1532/HSF98.20081083. PMID: 18948245. Exclusion reason: Ineligible setting 
457. Engen DJ, Carns PE, Allen MS, et al. Evaluating efficacy and feasibility of transcutaneous electrical nerve stimulation for postoperative pain after video-assisted thoracoscopic surgery: A randomized pilot trial. Complement Ther Clin Pract. 2016 May;23:141-8. doi: 10.1016/j.ctcp.2015.04.002. PMID: 25935320. Exclusion reason: Ineligible setting

458. Eppstein AC, Sakamoto B. The novel use of different bupivacaine preparations with combined regional techniques for postoperative pain management in nonopioid-based laparoscopic inguinal herniorrhaphy. J Clin Anesth. 2016 Nov;34:403-6. doi: 10.1016/j.jclinane.2016.05.011. PMID: 27687421. Exclusion reason: Ineligible intervention

459. Erden S, Demir SG, Kanatli U, et al. The effect of standard pain assessment on pain and analgesic consumption amount in patients undergoing arthroscopic shoulder surgery. Appl Nurs Res. 2017 02;33:121-6. doi: 10.1016/j.apnr.2016.11.009. PMID: 28096004. Exclusion reason: Ineligible intervention

460. Erden S, Senol Celik S. The effect of transcutaneous electrical nerve stimulation on post-thoracotomy pain. Contemp Nurse. 2015 Oct-Dec;51(2-3):163-70. doi: https://dx.doi.org/10.1080/10376178.2016.1 166971. PMID: 26986188. Exclusion reason: Ineligible setting

461. Erdogan M, Erdogan A, Erbil N, et al. Prospective, Randomized, Placebocontrolled Study of the Effect of TENS on postthoracotomy pain and pulmonary function. World J Surg. 2005 Dec;29(12):1563-70. doi: 10.1007/s00268005-7934-6. PMID: 16331341. Exclusion reason: Ineligible setting

462. Ergün H, Polat O, Demirkan NA, et al. The efficacy, safety, and pharmacokinetics of intramuscular and oral phenyramidol in patients with low back pain in an emergency department. Turk J Med Sci. 2010;40(1):716. doi: 10.3906/sag-0811-15. Exclusion reason: Ineligible comparator
463. Ericson L, Ambring A, Björholt I, et al. Opioid rotation in patients initiated on oxycodone or morphine: A register study. J Pain Res. 2013;6:379-86. doi: 10.2147/JPR.S44571. PMID: 23717049. Exclusion reason: Background information only

464. Ernst E. Complementary treatments in rheumatic diseases. Rheum Dis Clin North Am. 2008 May;34(2):455-67. doi: 10.1016/j.rdc.2008.03.007. PMID: 18638686. Exclusion reason: Ineligible publication type

465. Ernst E, Fialka V. Ice freezes pain? A review of the clinical effectiveness of analgesic cold therapy. J Pain Symptom Manage. 1994 Jan;9(1):56-9. PMID: 8169463. Exclusion reason: Publication used as source document

466. Eryildirim B, Kuyumcuoglu U, Tarhan F, et al. Comparison of three analgesic treatment protocols for pain management during extracorporeal shock wave lithotripsy. Urol Int. 2009;82(3):276-9. doi:

10.1159/000209357. PMID: 19440013. Exclusion reason: Ineligible intervention

467. Esmat IM, Farag HM. Comparative study between paracetamol and two different doses of pregabalin on postoperative pain in laparoscopic cholecystectomy. Saudi Journal of Anaesthesia. 2015;9(4):376-80. doi: 10.4103/1658-354X.159459. PMID: 26543452. Exclusion reason: Ineligible intervention

468. Essex MN, Xu H, Parsons B, et al. Parecoxib relieves pain and has an opioidsparing effect following major gastrointestinal surgery. Int J Gen Med. 2017;10:319-27. doi: 10.2147/IJGM.S143837. PMID: 29026330. Exclusion reason: Ineligible setting

469. Esteban Gonzalez P, Novoa NM, Varela G. Transcutaneous Electrical Nerve Stimulation Reduces Post-Thoractomy Ipsilateral Shoulder Pain. A Prospective Randomized Study. Arch Bronconeumol. 2015 Dec;51(12):621-6. doi: 10.1016/j.arbres.2014.11.002. PMID: 25555523. Exclusion reason: Ineligible setting 
470. Evans R, Bronfort G, Bittell S, et al. A pilot study for a randomized clinical trial assessing chiropractic care, medical care, and self-care education for acute and subacute neck pain patients. J Manipulative Physiol Ther. 2003 Sep;26(7):403-11. doi: 10.1016/S0161-4754(03)00093-9. PMID: 12975626. Exclusion reason: Ineligible population

471. Evron S, Schenker JG, Olshwang D, et al. Postoperative analgesia by percutaneous electrical stimulation in gynecology and obstetrics. Eur J Obstet Gynecol Reprod Biol. 1981 Nov;12(5):305-13. doi: 10.1016/0028-2243(81)90053-8. PMID: 6977467. Exclusion reason: Ineligible outcome

472. Eyler EC. Chronic and acute pain and pain management for patients in methadone maintenance treatment. Am J Addict. 2013 Jan;22(1):75-83. doi: 10.1111/j.15210391.2013.00308.x. PMID: 23398230.

Exclusion reason: Ineligible publication type

473. Ezenwa MO, Molokie RE, Wang ZJ, et al. Outpatient pain predicts subsequent oneyear acute health care utilization among adults with sickle cell disease. J Pain Symptom Manage. 2014 Jul;48(1):65-74. doi:

https://dx.doi.org/10.1016/j.jpainsymman.20 13.08.020. PMID: 24636960. Exclusion reason: Background information only

474. Faas A, Chavannes AW, van Eijk JT, et al. A randomized, placebo-controlled trial of exercise therapy in patients with acute low back pain. Spine. 1993 Sep 01;18(11):138895. PMID: 8235809. Exclusion reason: Ineligible outcome

475. Faas A, van Eijk JT, Chavannes AW, et al. A randomized trial of exercise therapy in patients with acute low back pain. Efficacy on sickness absence. Spine. 1995 Apr 15;20(8):941-7. doi: 10.1097/00007632199504150-00012. PMID: 7644960.

Exclusion reason: Ineligible outcome
476. Fabritius ML, Strom C, Koyuncu S, et al. Benefit and harm of pregabalin in acute pain treatment: a systematic review with metaanalyses and trial sequential analyses. $\mathrm{Br} \mathrm{J}$ Anaesth. 2017 Oct 01;119(4):775-91. doi: https://dx.doi.org/10.1093/bja/aex227. PMID: 29121288. Exclusion reason: Publication used as source document

477. Falco FJ, Erhart S, Wargo BW, et al. Systematic review of diagnostic utility and therapeutic effectiveness of cervical facet joint interventions. Pain Physician. 2009 Mar-Apr;12(2):323-44. PMID: 19305483. Exclusion reason: Publication used as source document

478. Falco FJ, Manchikanti L, Datta S, et al. Systematic review of the therapeutic effectiveness of cervical facet joint interventions: an update. Pain Physician. 2012 Nov-Dec;15(6):E839-68. PMID: 23159978. Exclusion reason: Publication used as source document

479. Fanelli A, Sorella MC, Chelly JE. Iontophoretic transdermal fentanyl for the management of acute perioperative pain in hospitalized patients. Expert Opin Pharmacother. 2016;17(4):571-7. doi: 10.1517/14656566.2016.1146684. PMID: 26809270. Exclusion reason: Ineligible intervention

480. Farrell JP, Twomey LT. Acute low back pain. Comparison of two conservative treatment approaches. Med J Aust. $1982 \mathrm{Feb}$ 20;1(4):160-4. PMID: 6210835. Exclusion reason: Ineligible comparator

481. Fashner J, Bell AL. Herpes zoster and postherpetic neuralgia: prevention and management. Am Fam Physician. 2011 Jun 15;83(12):1432-7. PMID: 21671543.

Exclusion reason: Background information only

482. Fassoulaki A, Patris K, Sarantopoulos C, et al. The analgesic effect of gabapentin and mexiletine after breast surgery for cancer. Anesth Analg. 2002 Oct;95(4):985-91, table of contents. doi: 10.1097/00000539200210000-00036. PMID: 12351281. Exclusion reason: Ineligible population 
483. Fazio AN. Control of postoperative pain: a comparison of the efficacy and safety of pentazocine, methotrimeprazine, meperidine, and a placebo. Curr Ther Res Clin Exp. 1970 Feb;12(2):73-7. PMID: 4984322. Exclusion reason: Ineligible intervention

484. Feinberg AE, Chesney TR, Srikandarajah S, et al. Opioid Use After Discharge in Postoperative Patients: A Systematic Review. Ann Surg. 2018 06;267(6):1056-62. doi:

https://dx.doi.org/10.1097/SLA.0000000000 002591. PMID: 29215370. Exclusion

reason: Background information only

485. Feng X, Ye T, Wang Z, et al. Transcutaneous acupoint electrical stimulation pain management after surgical abortion: A cohort study. Int J Surg. 2016 Jun;30:104-8. doi: 10.1016/j.ijsu.2016.04.042. PMID: 27142864. Exclusion reason: Ineligible study design

486. Fernandes IA, Armond ACV, Falci SGM. The Effectiveness of the Cold Therapy (cryotherapy) in the Management of Inflammatory Parameters after Removal of Mandibular Third Molars: A Meta-Analysis. Int Arch Otorhinolaryngol. 2019

Apr;23(2):221-8. doi: https://dx.doi.org/10.1055/s-0039-1677755. PMID: 30956709. Exclusion reason: Publication used as source document

487. Fernandes R, Mazzarello S, Majeed H, et al. Treatment of taxane acute pain syndrome (TAPS) in cancer patients receiving taxanebased chemotherapy-a systematic review. Support Care Cancer. 2016 Apr;24(4):158394. doi: https://dx.doi.org/10.1007/s00520015-2941-0. PMID: 26386706. Exclusion reason: Publication used as source document

488. Fernandez M, Ferreira PH. Acupuncture for sciatica and a comparison with Western Medicine (PEDro synthesis). Br J Sports Med. 2017 Mar;51(6):539-40. doi: 10.1136/bjsports-2016-096203. PMID: 27034130. Exclusion reason: Ineligible publication type
489. Fernandez-De-Las-Penas C, Cleland JA, Huijbregts P, et al. Repeated applications of thoracic spine thrust manipulation do not lead to tolerance in patients presenting with acute mechanical neck pain: a secondary analysis. J Man Manip Ther.

2009;17(3):154-62. doi:

10.1179/jmt.2009.17.3.154. PMID: 20046622. Exclusion reason: Ineligible comparator

490. Fernández-de-las-Peñas C, FernándezCarnero J, Fernández AP, et al. Dorsal Manipulation in Whiplash Injury Treatment. Journal of Whiplash \& Related Disorders. 2004 2004/01/01;3(2):55-72. doi: 10.3109/J180v03n02_05. Exclusion reason: Ineligible population

491. Fernandez-Sabate A, Portabella F. Comparative multiple-dose study of ketorolac tromethamine and diflunisal for pain following orthopaedic surgery. J Int Med Res. 1991 May-Jun;19(3):210-8. doi: 10.1177/030006059101900306. PMID: 1936510. Exclusion reason: Ineligible comparator

492. Ferreira AP, Costa DR, Oliveira AI, et al. Short-term transcutaneous electrical nerve stimulation reduces pain and improves the masticatory muscle activity in temporomandibular disorder patients: a randomized controlled trial. J Appl Oral Sci. 2017 Mar-Apr;25(2):112-20. doi: 10.1590/1678-77572016-0173. PMID: 28403351. Exclusion reason: Ineligible population

493. Fetherston CM, Ward S. Relationships between post operative pain management and short term functional mobility in total knee arthroplasty patients with a femoral nerve catheter: a preliminary study. J Orthop Surg. 2011 Feb 07;6:7. doi: 10.1186/1749799X-6-7. PMID: 21294923. Exclusion reason: Ineligible intervention

494. Fewster KM, Gallagher KM, Callaghan JP. The effect of standing interventions on acute low-back postures and muscle activation patterns. Appl Ergon. 2017 Jan;58:281-6. doi: 10.1016/j.apergo.2016.07.002. PMID: 27633223. Exclusion reason: Ineligible population 
495. Ffrench-O'Carroll R, Steinhaeuser H, Duff $\mathrm{S}$, et al. A randomized controlled trial comparing tapentadol with oxycodone in non-breastfeeding women post elective cesarean section: tapentadol vs. oxycodone post elective cesarean section. Curr Med Res Opin. 2018. Exclusion reason: Ineligible population

496. Fiddian J, Marx J. Comparison of passive mobilization and McKenzie protocol in treatment of low back pain. Aust J Physiother. 1986;32(3):205. Exclusion reason: Ineligible publication type

497. Field T, Diego M, Gonzalez G, et al. Neck arthritis pain is reduced and range of motion is increased by massage therapy.

Complement Ther Clin Pract. 2014

Nov;20(4):219-23. doi:

10.1016/j.ctcp.2014.09.001. PMID:

25444416. Exclusion reason: Ineligible population

498. Field T, Diego M, Solien-Wolfe L. Massage therapy plus topical analgesic is more effective than massage alone for hand arthritis pain. J Bodyw Mov Ther. 2014 Jul;18(3):322-5. doi: 10.1016/j.jbmt.2013.12.002. PMID: 25042302. Exclusion reason: Ineligible population

499. Finan MA, Roberts WS, Hoffman MS, et al. The effects of cold therapy on postoperative pain in gynecologic patients: a prospective, randomized study. Am J Obstet Gynecol. 1993 Feb;168(2):542-4. doi: 10.1016/00029378(93)90489-6. PMID: 7679885.

Exclusion reason: Ineligible setting

500. Fioravanti A, Bellisai B, Iacoponi F, et al. Phytothermotherapy in osteoarthritis: a randomized controlled clinical trial. Journal of Alternative \& Complementary Medicine. 2011 May;17(5):407-12. doi:

10.1089/acm.2010.0294. PMID: 21563963.

Exclusion reason: Ineligible population

501. Fiore JF, Jr., Olleik G, El-Kefraoui C, et al. Preventing opioid prescription after major surgery: a scoping review of opioid-free analgesia. Br J Anaesth. 2019 11;123(5):627-36. doi: https://dx.doi.org/10.1016/j.bja.2019.08.014. PMID: 31563269. Exclusion reason:

Background information only
502. Firanescu C, Lohle PN, de Vries J, et al. A randomised sham controlled trial of vertebroplasty for painful acute osteoporotic vertebral fractures (VERTOS IV). Trials [Electronic Resource]. 2011 Apr 05;12:93. doi: 10.1186/1745-6215-12-93. PMID: 21466679. Exclusion reason: Ineligible intervention

503. Fischer CA, Neubauer E, Adams HS, et al. Effects of multidisciplinary pain treatment can be predicted without elaborate questionnaires. Int Orthop. 2014 Mar;38(3):617-26. doi: 10.1007/s00264013-2156-2. PMID: 24292284. Exclusion reason: Ineligible study design

504. Fisher LR, Alvar BA, Maher SF, et al. Short-term Effects of Thoracic Spine Thrust Manipulation, Exercise, and Education in Individuals With Low Back Pain: A Randomized Controlled Trial. J Orthop Sports Phys Ther. 2020 Jan;50(1):24-32. doi:

https://dx.doi.org/10.2519/jospt.2020.8928. PMID: 31810405. Exclusion reason: Ineligible population

505. Fitzcharles MA, Ste-Marie PA, Hauser W, et al. Efficacy, Tolerability, and Safety of Cannabinoid Treatments in the Rheumatic Diseases: A Systematic Review of Randomized Controlled Trials. Arthritis Care Res (Hoboken). 2016 May;68(5):6818. doi: 10.1002/acr.22727. PMID: 26548380. Exclusion reason: Publication used as source document

506. Flaherty GG, Fitzpatrick JJ. Relaxation technique to increase comfort level of postoperative patients: a preliminary study. Nurs Res. 1978 Nov-Dec;27(6):352-5. PMID: 362379. Exclusion reason: Ineligible setting

507. Fleckenstein J, Kramer S, Hoffrogge P, et al. Acupuncture in acute herpes zoster pain therapy (ACUZoster)-design and protocol of a randomised controlled trial. BMC Complement Altern Med. 2009 Aug 12;9:31. doi: 10.1186/1472-6882-9-31. PMID: 19674449. Exclusion reason: Ineligible study design 
508. Flor H. Psychological pain interventions and neurophysiology: Implications for a mechanism-based approach. Am Psychol. 2014 Feb-Mar;69(2):188-96. doi: http://dx.doi.org/10.1037/a0035254. PMID: 24547804. Exclusion reason: Background information only

509. Foley-Nolan D, Barry C, Coughlan RJ, et al. Pulsed high frequency (27MHz) electromagnetic therapy for persistent neck pain. A double blind, placebo-controlled study of 20 patients. Orthopedics. 1990 Apr;13(4):445-51. PMID: 2185460. Exclusion reason: Ineligible population

510. Forbes JA, Beaver WT, White EH, et al. Diflunisal. A new oral analgesic with an unusually long duration of action. JAMA. 1982 Nov 5;248(17):2139-42. doi: 10.1001/jama.248.17.2139. PMID: 6750171. Exclusion reason: Ineligible study design

511. Forbes JA, Keller CK, Smith JW, et al. Analgesic effect of naproxen sodium, codeine, a naproxen-codeine combination and aspirin on the postoperative pain of oral surgery. Pharmacotherapy. 1986 SepOct;6(5):211-8. doi: 10.1002/j.18759114.1986.tb03479.x. PMID: 3540871. Exclusion reason: Ineligible population

512. Forbes JA, Kolodny AL, Beaver WT, et al. A 12-hour evaluation of the analgesic efficacy of diflunisal, acetaminophen, and acetaminophen-codeine combination, and placebo in postoperative pain. Pharmacotherapy. 1983 Mar-Apr;3(2 Pt 2):47s-54s. PMID: 6344039. Exclusion reason: Ineligible setting

513. Forgach L, Ong BY. Failure of meperidine wound infiltration to reduce pain after laparoscopic tubal ligation. Can J Anaesth. 1995 Dec;42(12):1085-9. doi:

10.1007/BF03015093. PMID: 8595682.

Exclusion reason: Ineligible intervention
514. Forogh B, Aslanpour H, Fallah E, et al. Adding high-frequency transcutaneous electrical nerve stimulation to the first phase of post anterior cruciate ligament reconstruction rehabilitation does not improve pain and function in young male athletes more than exercise alone: a randomized single-blind clinical trial. Disabil Rehabil. 2019 03;41(5):514-22. doi: https://dx.doi.org/10.1080/09638288.2017.1 399294. PMID: 29117738. Exclusion

reason: Ineligible comparator

515. Forouzanfar MH. Comparison of intravenous ibuprofen with intravenous ketorolac in renal colic pain management; A clinical trial. Anesth Pain Med. 2019;9(1) PMID: 30881914. Exclusion reason: Ineligible intervention

516. Forozeshfard M, Bakhtiary AH, Aminianfar A, et al. Short term effects of kinesio taping on pain and functional disability in young females with menstrual low back pain: A randomised control trial study. J Back Musculoskelet Rehabil. 2016 Nov 21;29(4):709-15. doi: 10.3233/BMR160673. PMID: 26966819. Exclusion reason: Ineligible intervention

517. Forrest WH, Jr. Orally administered zomepirac and parenterally administered morphine. Comparison for the treatment of postoperative pain. JAMA. $1980 \mathrm{Nov}$ 21;244(20):2298-302. PMID: 6776300. Exclusion reason: Ineligible comparator

518. Forsbrand MH, Turkiewicz A, Petersson IF, et al. Long-term effects on function, healthrelated quality of life and work ability after structured physiotherapy including a workplace intervention. A secondary analysis of a randomised controlled trial (WorkUp) in primary care for patients with neck and/or back pain. Scand J Prim Health Care. 2020 Mar;38(1):92-100. doi: https://dx.doi.org/10.1080/02813432.2020.1 717081. PMID: 32000558. Exclusion reason: Ineligible population

519. Forsgren H, Heimdahl A, Johansson B, et al. Effect of application of cold dressings on the postoperative course in oral surgery. Int $\mathrm{J}$ Oral Surg. 1985;14(3):223-8. PMID: 3926665. Exclusion reason: Ineligible outcome 
520. Forster EL, Kramer JF, Lucy SD, et al. Effect of TENS on pain, medications, and pulmonary function following coronary artery bypass graft surgery. Chest. 1994 Nov;106(5):1343-8. doi: 10.1378/chest.106.5.1343. PMID: 7956382. Exclusion reason: Ineligible setting

521. Fountas KN, Kapsalaki EZ, Johnston KW, et al. Postoperative lumbar microdiscectomy pain. Minimalization by irrigation and cooling. Spine (Phila Pa 1976). 1999 Sep 15;24(18):1958-60. doi: 10.1097/00007632199909150-00016. PMID: 10515023. Exclusion reason: Ineligible intervention

522. Fox LM, Murakami M, Danesh H, et al. Battlefield acupuncture to treat low back pain in the emergency department. Am J Emerg Med. 2018 Jun;36(6):1045-8. doi: 10.1016/j.ajem.2018.02.038. PMID: 29550099. Exclusion reason: Ineligible duration

523. Fraga A, de Almeida M, Moreira-da-Silva $\mathrm{V}$, et al. Intramuscular Etofenamate versus Diclofenac in the Relief of Renal Colic : A Randomised, Single-Blind, Comparative Study. Clin Drug Investig. 2003;23(11):7016. doi: 10.2165/00044011-20032311000002. PMID: 17536883. Exclusion reason: Ineligible comparator

524. Franke H, Franke JD, Fryer G. Osteopathic manipulative treatment for nonspecific low back pain: a systematic review and metaanalysis. BMC Musculoskelet Disord. 2014 Aug 30;15:286. doi: 10.1186/1471-2474-15286. PMID: 25175885. Exclusion reason: Publication used as source document

525. Franke H, Fryer G, Ostelo RW, et al. Muscle energy technique for non-specific low-back pain. Cochrane Database Syst Rev. 2015 Feb 27(2):CD009852. doi: 10.1002/14651858.CD009852.pub2. PMID: 25723574. Exclusion reason: Publication used as source document

526. Fraser JJ, Saliba SA, Hart JM, et al. Effects of midfoot joint mobilization on ankle-foot morphology and function following acute ankle sprain. A crossover clinical trial. Musculoskelet Sci Pract. 2020 Apr;46:102130. doi: https://dx.doi.org/10.1016/j.msksp.2020.102 130. PMID: 32217275. Exclusion reason: Ineligible outcome
527. Fredenburg HA, Silverman MJ. Effects of music therapy on positive and negative affect and pain with hospitalized patients recovering from a blood and marrow transplant: A randomized effectiveness study. The Arts in Psychotherapy. 2014 Apr;41(2):174-80. doi: 10.1016/j.aip.2014.01.007. Exclusion reason: Ineligible population

528. Freedman BM, Balakrishnan TP, O'Hara EL. Celecoxib reduces narcotic use and pain following augmentation mammaplasty. Aesthet Surg J. 2006;26(1):24-8. doi: 10.1016/j.asj.2005.11.001. PMID: 19338879. Exclusion reason: Ineligible comparator

529. Frey ME, Manchikanti L, Benyamin RM, et al. Spinal cord stimulation for patients with failed back surgery syndrome: a systematic review. Pain Physician. 2009 MarApr;12(2):379-97. PMID: 19305486. Exclusion reason: Publication used as source document

530. Fricke JR, Jr., Hewitt DJ, Jordan DM, et al. A double-blind placebo-controlled comparison of tramadol/acetaminophen and tramadol in patients with postoperative dental pain. Pain. 2004 Jun;109(3):250-7. PMID: 15157685. Exclusion reason: Ineligible comparator

531. Friedman BW, Cisewski D, Irizarry E, et al. A Randomized, Double-Blind, PlaceboControlled Trial of Naproxen With or Without Orphenadrine or Methocarbamol for Acute Low Back Pain. Ann Emerg Med. 2018 03;71(3):348-56.e5. doi: 10.1016/j.annemergmed.2017.09.031. PMID: 29089169. Exclusion reason: Ineligible comparator

532. Friedman BW, Irizarry E, Chertoff A, et al. Ibuprofen + acetaminophen versus ibuprofen alone for acute low back pain. An ED-based randomized study. Acad Emerg Med. 2019 PMID: 31811673. Exclusion reason: Ineligible comparator 
533. Friedman BW, Irizarry E, Chertoff A, et al. Ibuprofen Plus Acetaminophen Versus Ibuprofen Alone for Acute Low Back Pain: An Emergency Department-based Randomized Study. Acad Emerg Med. 2020 03;27(3):229-35. doi:

https://dx.doi.org/10.1111/acem.13898. PMID: 31811673. Exclusion reason: Ineligible comparator

534. Friedman BW, Irizarry E, Solorzano C, et al. Diazepam Is No Better Than Placebo When Added to Naproxen for Acute Low Back Pain. Ann Emerg Med. 2017 Aug;70(2):169-76.e1. doi: https://dx.doi.org/10.1016/j.annemergmed.2 016.10.002. PMID: 28187918. Exclusion reason: Background information only

535. Friedman BW, Irizarry E, Solorzano C, et al. A Randomized, Placebo-Controlled Trial of Ibuprofen Plus Metaxalone, Tizanidine, or Baclofen for Acute Low Back Pain. Ann Emerg Med. 2019 10;74(4):512-20. doi: https://dx.doi.org/10.1016/j.annemergmed.2 019.02.017. PMID: 30955985. Exclusion reason: Ineligible intervention

536. Friedman Z, Arzola C, Postonogova T, et al. Physician and Patient Survey of Taper Schedule and Family Physician Letters Following Discharged from the Acute Pain Service. Pain Pract. 2017 03;17(3):366-70. doi: 10.1111/papr.12457. PMID: 27160386. Exclusion reason: Ineligible outcome

537. Fritz JM, Childs JD, Flynn TW. Pragmatic application of a clinical prediction rule in primary care to identify patients with low back pain with a good prognosis following a brief spinal manipulation intervention. BMC Fam Pract. 2005 Jul 14;6(1):29. doi: 10.1186/1471-2296-6-29. PMID: 16018809. Exclusion reason: Ineligible comparator

538. Fritz JM, Delitto A, Erhard RE. Comparison of classification-based physical therapy with therapy based on clinical practice guidelines for patients with acute low back pain: a randomized clinical trial. Spine. $2003 \mathrm{Jul}$ 01;28(13):1363-71; discussion 72. doi: 10.1097/01.BRS.0000067115.61673.FF. PMID: 12838091. Exclusion reason: Ineligible comparator
539. Fritz JM, Kim M, Magel JS, et al. CostEffectiveness of Primary Care Management With or Without Early Physical Therapy for Acute Low Back Pain: Economic Evaluation of a Randomized Clinical Trial. Spine. 2017 Mar;42(5):285-90. doi: https://dx.doi.org/10.1097/BRS.0000000000 001729. PMID: 27270641. Exclusion reason: Background information only

540. Froehner Junior I, Kotze PG, Rocha JG, et al. Postoperative topical analgesia of hemorrhoidectomy with policresulen and cinchocaine: a prospective and controlled study. Rev Col Bras Cir. 2014 MarApr;41(2):92-8. doi: 10.1590/s010069912014000200004. PMID: 24918721.

Exclusion reason: Ineligible intervention

541. Fryda-Kaurimsky Z, Muller-Fassbender H. Tizanidine (DS 103-282) in the treatment of acute paravertebral muscle spasm: a controlled trial comparing tizanidine and diazepam. J Int Med Res. 1981;9(6):501-5. PMID: 6459256. Exclusion reason: Ineligible population

542. Fujii MH, Hodges AC, Russell RL, et al. Post-Discharge Opioid Prescribing and Use after Common Surgical Procedure. J Am Coll Surg. 2018 06;226(6):1004-12. doi: https://dx.doi.org/10.1016/j.jamcollsurg.201 8.01.058. PMID: 29499361. Exclusion reason: Ineligible outcome

543. Fujii-Abe K, Umino M, Kawahara H, et al. New method for postoperative pain relief using a combination of noxious and nonnoxious stimuli after impacted wisdom tooth extraction. J Oral Sci. 2019;61(2):364-9. doi: https://dx.doi.org/10.2334/josnusd.180187. PMID: 31217388. Exclusion reason: Ineligible duration

544. Fujiki EN, Netto NA, Kraychete DC, et al. Efficacy and safety of loxoprofen sodium topical patch for the treatment of pain in patients with minor acute traumatic limb injuries in Brazil: A randomized, doubleblind, noninferiority trial. Pain. 2019;160(7):1606-13. doi: 10.1097/j.pain.0000000000001549. PMID: 30839430. Exclusion reason: Ineligible intervention 
545. Fujumaki E, et al. Clinical evaluation of piroxicam gel versus indomethacin gel and placebo in the treatment of muscle pain: a double-blind, multicenter study. Japanese Pharmacology and Therapeutics. 1985;12(12):119-37. Exclusion reason: Ineligible comparator

546. Furlan AD, Giraldo M, Baskwill A, et al. Massage for low-back pain. Cochrane Database Syst Rev. 2015 Sep

01(9):CD001929. doi: 10.1002/14651858.CD001929.pub3. PMID: 26329399. Exclusion reason: Publication used as source document

547. Gallagher LM, Gardner V, Bates D, et al. Impact of Music Therapy on Hospitalized Patients Post-Elective Orthopaedic Surgery: A Randomized Controlled Trial. Orthop Nurs. 2018 Mar/Apr;37(2):124-33. doi: 10.1097/nor.0000000000000432. PMID: 29570546. Exclusion reason: Ineligible duration

548. Galli TT, Chiavegato LD, Liebano RE. Effects of TENS in living kidney donors submitted to open nephrectomy: a randomized placebo-controlled trial. Eur J Pain. 2015 Jan;19(1):67-76. doi: https://dx.doi.org/10.1002/ejp.521. PMID: 24831862. Exclusion reason: Ineligible setting

549. Galloway DJ, Boyle P, Burns HJ, et al. A clinical assessment of electroanalgesia following abdominal operations. Surg Gynecol Obstet. 1984 Nov;159(5):453-6. PMID: 6387977. Exclusion reason: Ineligible setting

550. Gammaitoni AR, Galer BS, Bulloch S, et al. Randomized, double-blind, placebocontrolled comparison of the analgesic efficacy of oxycodone 10 mg/acetaminophen $325 \mathrm{mg}$ versus controlled-release oxycodone $20 \mathrm{mg}$ in postsurgical pain. J Clin Pharmacol. 2003 Mar;43(3):296-304. PMID: 12638399. Exclusion reason: Ineligible population

551. Gan EY, Tian EA, Tey HL. Management of herpes zoster and post-herpetic neuralgia. Am J Clin Dermatol. 2013 Apr;14(2):77-85. doi: https://dx.doi.org/10.1007/s40257-0130011-2. PMID: 23456596. Exclusion reason: Background information only
552. Gan TJ, Joshi GP, Zhao SZ, et al. Presurgical intravenous parecoxib sodium and follow-up oral valdecoxib for pain management after laparoscopic cholecystectomy surgery reduces opioid requirements and opioid-related adverse effects. Acta Anaesthesiol Scand. 2004 Oct;48(9):1194-207. PMID: 15352969. Exclusion reason: Ineligible intervention

553. Gan TJ, Lubarsky DA, Flood EM, et al. Patient preferences for acute pain treatment. Br J Anaesth. 2004 May;92(5):681-8. PMID: 15003986. Exclusion reason: Ineligible intervention

554. Gan TJ, Singla N, Daniels SE, et al. Cardiovascular safety of hydroxypropylbeta-cyclodextrin-diclofenac in the management of acute postsurgical pain: a pooled analysis of 2 randomized, doubleblind, placebo- and active comparatorcontrolled phase III clinical trials. J Clin Anesth. 2016 Jun;31:249-58. doi: https://dx.doi.org/10.1016/j.jclinane.2016.01 .020. PMID: 27185721. Exclusion reason: Ineligible setting

555. Ganesh GS, Sahu PK, Das SP, et al. A subgroup analysis to compare patients with acute low back pain classified as per treatment-based classification. Physiother Res Int. 2019 Jan;24(1):e1747. doi: 10.1002/pri.1747. PMID: 30226651. Exclusion reason: Ineligible study design

556. Gao H, Wei C. Extrapoint acupuncture treatement of 36 cases of acute lumbar sprain. Journal of Gansu College of Traditional Chinese Medicine. 2006;2006:49-50. Exclusion reason: Not in English

557. Garcia Filho RJ, Korukian M, dos Santos FPE, et al. A randomized, double-blind clinical trial, comparing the combination of caffeine, carisoprodol, sodium diclofenac and paracetamol versus cyclobenzaprine, to evaluate efficacy and safety in the treatment of patients with acute low back pain and lumboischialgia. Acta ortopedica brasileira. 2006;14:11-6. Exclusion reason: Ineligible comparator 
558. Garcia-Perdomo HA, Echeverria-Garcia F, Lopez $\mathrm{H}$, et al. Pharmacologic interventions to treat renal colic pain in acute stone episodes: Systematic review and metaanalysis. Prog Urol. 2017 Oct;27(12):65465. doi: 10.1016/j.purol.2017.05.011. PMID: 28651994. Exclusion reason: Publication used as source document

559. Garra G, Singer AJ, Leno R, et al. Heat or cold packs for neck and back strain: a randomized controlled trial of efficacy. Acad Emerg Med. 2010 May;17(5):484-9. doi: 10.1111/j.1553-2712.2010.00735.x. PMID: 20536800. Exclusion reason: Ineligible duration

560. Garshasbi A, Faghih Zadeh S. The effect of exercise on the intensity of low back pain in pregnant women. Int J Gynaecol Obstet. 2005 Mar;88(3):271-5. doi: 10.1016/j.ijgo.2004.12.001. PMID: 15733880. Exclusion reason: Ineligible population

561. Garza AF, Monroy-Maya R, Soto-Rios M, et al. A pilot study of the effect of diclofenac with $B$ vitamins for the treatment of acute pain following lower-limb fracture and surgery. Proceedings of the Western Pharmacology Society. 2008;51:70-2. PMID: 19544682. Exclusion reason: Ineligible comparator

562. Gasanova I, Alexander J, Ogunnaike B, et al. Transversus Abdominis Plane Block Versus Surgical Site Infiltration for Pain Management After Open Total Abdominal Hysterectomy. Anesth Analg. 2015 Nov;121(5):1383-8. doi: 10.1213/ANE.0000000000000909. PMID: 26252171. Exclusion reason: Ineligible intervention

563. Gaskell H, Derry S, Moore AR, et al. Single dose oral oxycodone and oxycodone plus paracetamol (acetaminophen) for acute postoperative pain in adults. Cochrane Database Syst Rev. 2019(5) PMID: 19588335. Exclusion reason: Ineligible comparator

564. Gaskell H, Derry S, Wiffen PJ, et al. Single dose oral ketoprofen or dexketoprofen for acute postoperative pain in adults. Cochrane Database Syst Rev. 2019(5) PMID: 28540716. Exclusion reason: Ineligible comparator
565. Gaskell H, Derry S, Wiffen PJ, et al. Single dose oral ketoprofen or dexketoprofen for acute postoperative pain in adults. Cochrane Database Syst Rev. 201705

25;5:CD007355. doi: https://dx.doi.org/10.1002/14651858.CD007 355.pub3. PMID: 28540716. Exclusion reason: Ineligible comparator

566. Gastaldi R, Durand M, Roustit M, et al. Short-term Efficiency and Tolerance of Ketoprofen and Methylprednisolone in Acute Sciatica: a Randomized Trial. Pain Med. 2018doi: 10.1093/pm/pny252. PMID: 30576555. Exclusion reason: Ineligible setting

567. Gatchel RJ, Polatin PB, Noe C, et al. Treatment- and cost-effectiveness of early intervention for acute low-back pain patients: a one-year prospective study. J Occup Rehabil. 2003 Mar;13(1):1-9. PMID: 12611026. Exclusion reason: Ineligible intervention

568. Gatewood CT, Tran AA, Dragoo JL. The efficacy of post-operative devices following knee arthroscopic surgery: a systematic review. Knee Surg Sports Traumatol Arthrosc. 2017 Feb;25(2):501-16. doi: 10.1007/s00167-016-4326-4. PMID: 27695905. Exclusion reason: Publication used as source document

569. Gattie E, Cleland JA, Snodgrass S. The Effectiveness of Trigger Point Dry Needling for Musculoskeletal Conditions by Physical Therapists: A Systematic Review and Metaanalysis. J Orthop Sports Phys Ther. 2017 Mar;47(3):133-49. doi: 10.2519/jospt.2017.7096. PMID: 28158962. Exclusion reason: Publication used as source document

570. Gaubitz M, Schiffer T, Holm C, et al. Efficacy and safety of nicoboxil/nonivamide ointment for the treatment of acute pain in the low back - A randomized, controlled trial. Eur J Pain. 2016 Feb;20(2):263-73. doi: 10.1002/ejp.719. PMID: 25929250. Exclusion reason: Ineligible intervention

571. Gaul C, Eschalier A. Dose can help to achieve effective pain relief for acute mild to moderate pain with over-the-counter paracetamol. Open Pain Journal. 2018;11(1):12-20. doi: 10.2174/1876386301811010012. Exclusion reason: Ineligible publication type 
572. Gavin M, Litt M, Khan A, et al. A prospective, randomized trial of cognitive intervention for postoperative pain. Am Surg. 2006 May;72(5):414-8. PMID: 16719196. Exclusion reason: Ineligible setting

573. Gay C, Planas E, Donado M, et al. Analgesic efficacy of low doses of dexketoprofen in the dental pain model. A randomised, double-blind, placebocontrolled study. Clinical Drug Investig. 1996;11(6):320-30. Exclusion reason: Ineligible intervention

574. Gay-Escoda C, Hanna M, Montero A, et al. Tramadol/dexketoprofen (TRAM/DKP) compared with tramadol/paracetamol in moderate to severe acute pain: results of a randomised, double-blind, placebo and active-controlled, parallel group trial in the impacted third molar extraction pain model (DAVID study). BMJ Open. 201902 19;9(2):e023715. doi: https://dx.doi.org/10.1136/BMJopen-2018023715. PMID: 30782886. Exclusion reason: Ineligible comparator

575. Gemmell HA, Jacobson BH. The immediate effect of activator vs. meric adjustment on acute low back pain: a randomized controlled trial. J Manipulative Physiol Ther. 1995 Sep;18(7):453-6. PMID: 8568427. Exclusion reason: Ineligible comparator

576. Genord C, Frost T, Eid D. Opioid exit plan: A pharmacist's role in managing acute postoperative pain. J Am Pharm Assoc. 2017 Mar - Apr;57(2S):S92-S8. doi: 10.1016/j.japh.2017.01.016. PMID: 28292507. Exclusion reason: Ineligible outcome

577. George Steven Z. The effect of a fearavoidance based intervention for patients with acute low back pain. A randomized clinical trial. Proquest Dissertations and Theses. 2002. Exclusion reason: Ineligible publication type

578. George SZ, Fritz JM, Bialosky JE, et al. The effect of a fear-avoidance-based physical therapy intervention for patients with acute low back pain: results of a randomized clinical trial. Spine. 2003 Dec 01;28(23):2551-60. PMID: 14652471.

Exclusion reason: Ineligible comparator
579. George SZ, Fritz JM, Childs JD, et al. Sex differences in predictors of outcome in selected physical therapy interventions for acute low back pain. J Orthop Sports Phys Ther. 2006 Jun;36(6):354-63. PMID: 16776485. Exclusion reason: Ineligible study design

580. George SZ, Zeppieri G, Jr., Cere AL, et al. A randomized trial of behavioral physical therapy interventions for acute and subacute low back pain (NCT00373867). Pain. 2008 Nov 15;140(1):145-57. doi: 10.1016/j.pain.2008.07.029. PMID: 18786762. Exclusion reason: Ineligible population

581. Gerrish AW, Fogel S, Lockhart ER, et al. Opioid prescribing practices during implementation of an enhanced recovery program at a tertiary care hospital. Surgery. 2018 10;164(4):674-9. doi:

https://dx.doi.org/10.1016/j.surg.2018.06.03 4. PMID: 30098812. Exclusion reason: Ineligible population

582. Gertzbein SD, Tile M, McMurty RY, et al. Analysis of the analgesic efficacy of acetaminophen $1000 \mathrm{mg}$, codeine phosphate $60 \mathrm{mg}$, and the combination of acetaminophen $1000 \mathrm{mg}$ and codeine phosphate $60 \mathrm{mg}$ in the relief of postoperative pain. Pharmacotherapy. 1986 May-Jun;6(3):104-7. doi: 10.1002/j.18759114.1986.tb03462.x. PMID: 3737440.

Exclusion reason: Ineligible setting

583. Gerwig WH, Jr., Alpert S, Coakley CS, et al. Control of pain following

hemorrhoidectomy. Surgery. 1953

Nov;34(5):880-1. PMID: 13113510.

Exclusion reason: Ineligible study design

584. Gevirtz C, Vadivelu N, Kaye AD.

Postoperative pain control in drug abusing patients. Substance abuse: Inpatient and outpatient management for every clinician. New York, NY: Springer Science + Business Media; US; 2015:379-87.

Exclusion reason: Background information only 
585. Gharibo CG, Argoff C, Markenson JA, et al. Opioid-sparing Effects of SoluMatrix Indomethacin in a Phase 3 Study in Patients With Acute Postoperative Pain. Clin J Pain. 2018 02;34(2):138-44. doi: 10.1097/AJP.0000000000000525. PMID: 28591082. Exclusion reason: Ineligible comparator

586. Ghetti CM. Active music engagement with emotional-approach coping to improve wellbeing in liver and kidney transplant recipients. J Music Ther. 2011;48(4):463-85. PMID: 22506300. Exclusion reason: Ineligible population

587. Ghoseiri K, Allami M, Soroush MR, et al. Assistive technologies for pain management in people with amputation: a literature review. Mil Med Res. 201801 23;5(1):1. doi: https://dx.doi.org/10.1186/s40779-0180151-z. PMID: 29502531. Exclusion reason: Background information only

588. Giaccari LG, Coppolino F, Aurilio C, et al. Sufentanil Sublingual for Acute PostOperative Pain: A Systematic Literature Review Focused on Pain Intensity, Adverse Events, and Patient Satisfaction. Pain Ther. 2020 Jun;9(1):217-30. doi: https://dx.doi.org/10.1007/s40122-02000166-4. PMID: 32303979. Exclusion reason: Publication used as source document

589. Giannakopoulos NN, Katsikogianni EN, Hellmann D, et al. Comparison of three different options for immediate treatment of painful temporomandibular disorders: a randomized, controlled pilot trial. Acta Odontol Scand. 2016 Aug;74(6):480-6. doi: 10.1080/00016357.2016.1204558. PMID: 27410169. Exclusion reason: Ineligible population

590. Gibbons CE, Solan MC, Ricketts DM, et al. Cryotherapy compared with Robert Jones bandage after total knee replacement: a prospective randomized trial. Int Orthop. 2001;25(4):250-2. doi:

10.1007/s002640100227. PMID: 11561502. Exclusion reason: Ineligible setting
591. Gibbs JC, MacIntyre NJ, Ponzano M, et al. Exercise for improving outcomes after osteoporotic vertebral fracture. Cochrane Database Syst Rev. 2019(7)doi: 10.1002/14651858.CD008618.pub3. PMID: 31273764. Exclusion reason: Publication used as source document

592. Gibofsky A, Silberstein S, Argoff C, et al. Lower-dose diclofenac submicron particle capsules provide early and sustained acute patient pain relief in a phase 3 study. Postgrad Med. 2013 Sep;125(5):130-8. doi: 10.3810/pgm.2013.09.2693. PMID: 24113671. Exclusion reason: Ineligible comparator

593. Gibson T, Grahame R, Harkness J, et al. Controlled comparison of short-wave diathermy treatment with osteopathic treatment in non-specific low back pain. Lancet. 1985 Jun 1;1(8440):1258-61. doi: 10.1016/s0140-6736(85)92323-2. PMID: 2860453. Exclusion reason: Ineligible population

594. Giglio JA, Laskin DM. Double-blind comparison of meclofenamate sodium plus codeine, meclofenamate sodium, codeine, and placebo for relief of pain following surgical removal of third molars. J Oral Maxillofac Surg. 1990 Aug;48(8):785-90. PMID: 2197381. Exclusion reason: Ineligible comparator

595. Gil JA, Gunaseelan V, DeFroda SF, et al. Risk of Prolonged Opioid Use Among Opioid-Naive Patients After Common Shoulder Arthroscopy Procedures. Am J Sports Med. 2019 04;47(5):1043-50. doi: https://dx.doi.org/10.1177/03635465188197 80. PMID: 30735622. Exclusion reason: Ineligible intervention

596. Gil KM, Wilson JJ, Edens JL, et al. Effects of cognitive coping skills training on coping strategies and experimental pain sensitivity in African American adults with sickle cell disease. Health Psychol. 1996 Jan;15(1):310. PMID: 8788535. Exclusion reason: Ineligible study design

597. Gilbert JM, Gledhill T, Law N, et al. Controlled trial of transcutaneous electrical nerve stimulation (TENS) for postoperative pain relief following inguinal herniorrhaphy. Br J Surg. 1986 Sep;73(9):749-51. doi: 10.1002/bjs.1800730923. PMID: 3530368. Exclusion reason: Ineligible setting 
598. Gilbert MM, Koepke HH. Relief of musculoskeletal and associated psychopathological symptoms with meprobamate and aspirin: a controlled study. Curr Ther Res Clin Exp. 1973 Nov;15(11):820-32. PMID: 4201595. Exclusion reason: Ineligible intervention

599. Gilbert MM, Sanzari NP, Losada M, et al. Double-blind oral analgesic study of butorphanol in musculoskeletal pain: a comparison with codeine and placebo. J Int Med Res. 1978;6(1):14-23. doi: 10.1177/030006057800600104. PMID: 342300. Exclusion reason: Ineligible population

600. Gilmore SJ, McClelland JA, Davidson M. Physiotherapeutic interventions before and after surgery for degenerative lumbar conditions: a systematic review. Physiotherapy. 2015 Jun;101(2):111-8. doi: 10.1016/j.physio.2014.06.007. PMID: 25287631. Exclusion reason: Publication used as source document

601. Gilron I, Orr E, Tu D, et al. A randomized, double-blind, controlled trial of perioperative administration of gabapentin, meloxicam and their combination for spontaneous and movement-evoked pain after ambulatory laparoscopic cholecystectomy. Anesth Analg. 2009 Feb;108(2):623-30. doi: https://dx.doi.org/10.1213/ane.0b013e31819 3cd1b. PMID: 19151299. Exclusion reason: Ineligible intervention

602. Gimbel J, Jacobs D, Pixton G, et al. Effectiveness and safety of diclofenac epolamine topical patch $1.3 \%$ for the treatment of acute pain due to back strain: an open-label, uncontrolled study. Phys Sportsmed. 2011 Feb;39(1):11-8. doi: 10.3810/psm.2011.02.1857. PMID: 21378482. Exclusion reason: Ineligible study design

603. Gimbel J, Linn R, Hale M, et al. Lidocaine patch treatment in patients with low back pain: results of an open-label, nonrandomized pilot study. Am J Ther. 2005 Jul-Aug;12(4):311-9. PMID: 16041194.

Exclusion reason: Ineligible population
604. Ginnerup-Nielsen E, Christensen R, Thorborg K, et al. Physiotherapy for pain: a meta-epidemiological study of randomised trials. Br J Sports Med. 2016

Aug;50(16):965-71. doi: https://dx.doi.org/10.1136/bjsports-2015095741. PMID: 27015855. Exclusion reason: Publication used as source document

605. Giombini A, Casciello G, Di Cesare MC, et al. A controlled study on the effects of hyperthermia at $434 \mathrm{MHz}$ and conventional ultrasound upon muscle injuries in sport. J Sports Med Phys Fitness. 2001 Dec;41(4):521-7. PMID: 11687773.

Exclusion reason: Ineligible duration

606. Givens M, Rutherford C, Joshi G, et al. Impact of an emergency department pain management protocol on the pattern of visits by patients with sickle cell disease. J Emerg Med. 2007 Apr;32(3):239-43. PMID: 17394984. Exclusion reason: Ineligible study design

607. Gkegkes ID, Minis EE, Iavazzo C. Oxycodone/naloxone in postoperative pain management of surgical patients. J Opioid Manag. 2018 Jan/Feb;14(1):52-60. doi: https://dx.doi.org/10.5055/jom.2018.0429. PMID: 29508896. Exclusion reason: Publication used as source document

608. Gladwin MT, Kato GJ, Weiner D, et al. Nitric oxide for inhalation in the acute treatment of sickle cell pain crisis: a randomized controlled trial. JAMA. 2011 Mar 02;305(9):893-902. doi: 10.1001/jama.2011.235. PMID: 21364138. Exclusion reason: Ineligible intervention

609. Glassberg J. Evidence-based management of sickle cell disease in the emergency department. Emerg Med Pract. 2011 Aug;13(8):1-20; quiz PMID: 22164362. Exclusion reason: Ineligible publication type

610. Glassberg JA. Improving Emergency Department-Based Care of Sickle Cell Pain. Hematology. 201712 08;2017(1):412-7. doi:

https://dx.doi.org/10.1182/asheducation2017.1.412. PMID: 29222286. Exclusion reason: Background information only 
611. Godfrey CM, Morgan PP, Schatzker J. A randomized trial of manipulation for lowback pain in a medical setting. Spine (Phila Pa 1976). 1984 Apr;9(3):301-4. doi: 10.1097/00007632-198404000-00015. PMID: 6233718. Exclusion reason: Ineligible comparator

612. Goebel A, Moore A, Weatherall R, et al. Intravenous immunoglobulin in the treatment of primary trigeminal neuralgia refractory to carbamazepine: A study protocol[isrctn33042138]. BMC Neurology Vol 3 2003, ArtID 1. 2003 Jan;3doi: 10.1186/1471-2377-3-1. PMID: 12590652. Exclusion reason: Ineligible publication type

613. Goertz CM, Long CR, Hondras MA, et al. Adding chiropractic manipulative therapy to standard medical care for patients with acute low back pain: results of a pragmatic randomized comparative effectiveness study. Spine. 2013 Apr 15;38(8):627-34. doi: 10.1097/BRS.0b013e31827733e7. PMID: 23060056. Exclusion reason: Ineligible comparator

614. Goertz CM, Niemtzow R, Burns SM, et al. Auricular acupuncture in the treatment of acute pain syndromes: A pilot study. Mil Med. 2006 Oct;171(10):1010-4. doi: 10.7205/milmed.171.10.1010. PMID: 17076456. Exclusion reason: Ineligible population

615. Gogoularadja A, Bakshi SS. A Randomized Study on the Efficacy of Music Therapy on Pain and Anxiety in Nasal Septal Surgery. Int Arch Otorhinolaryngol. 2020

Apr;24(2):e232-e6. doi: https://dx.doi.org/10.1055/s-0039-3402438. PMID: 32296470. Exclusion reason: Ineligible intervention

616. Gomes C, Dibai-Filho AV, Moreira WA, et al. Effect of Adding Interferential Current in an Exercise and Manual Therapy Program for Patients With Unilateral Shoulder Impingement Syndrome: A Randomized Clinical Trial. J Manipulative Physiol Ther. 2018 Mar - Apr;41(3):218-26. doi: 10.1016/j.jmpt.2017.09.009. PMID: 29459121. Exclusion reason: Ineligible population
617. Gomes-Neto M, Lopes JM, Conceicao CS, et al. Stabilization exercise compared to general exercises or manual therapy for the management of low back pain: A systematic review and meta-analysis. Phys Ther Sport. 2017 Jan;23:136-42. doi: https://dx.doi.org/10.1016/j.ptsp.2016.08.00 4. PMID: 27707631. Exclusion reason: Publication used as source document

618. Gonzalez Ramallo VJ, Muino Miguez A, Rodriguez de Castro E, et al. [Intramuscular buprenorphine in the symptomatic treatment of renal colic]. Rev Clin Esp. 1990 May;186(8):414. PMID: 2236778. Exclusion reason: Not in English

619. Gonzalez-Barnadas A, Camps-Font O, Martin-Fatas P, et al. Efficacy and safety of selective COX-2 inhibitors for pain management after third molar removal: a meta-analysis of randomized clinical trials. Clin Oral Investig. 2020 Jan;24(1):79-96. doi: https://dx.doi.org/10.1007/s00784-01902910-3. PMID: 31016540. Exclusion

reason: Publication used as source document

620. Gonzalez-Iglesias J, Fernandez-de-las-Penas C, Cleland JA, et al. Inclusion of thoracic spine thrust manipulation into an electrotherapy/thermal program for the management of patients with acute mechanical neck pain: a randomized clinical trial. Man Ther. 2009 Jun;14(3):306-13. doi: 10.1016/j.math.2008.04.006. PMID: 18692428. Exclusion reason: Ineligible intervention

621. Gonzalez-Iglesias J, Fernandez-de-las-Penas C, Cleland JA, et al. Thoracic spine manipulation for the management of patients with neck pain: a randomized clinical trial. J Orthop Sports Phys Ther. 2009 Jan;39(1):20-7. PMID: 19209478.

Exclusion reason: Ineligible intervention

622. Gonzalez-Iglesias J, Fernandez-de-LasPenas C, Cleland JA, et al. Short-term effects of cervical kinesio taping on pain and cervical range of motion in patients with acute whiplash injury: a randomized clinical trial. J Orthop Sports Phys Ther. 2009 Jul;39(7):515-21. doi: 10.2519/jospt.2009.3072. PMID: 19574662. Exclusion reason: Ineligible intervention 
623. Gonzalez-Urzelai V, Palacio-Elua L, Lopezde-Munain J. Routine primary care management of acute low back pain: adherence to clinical guidelines. Eur Spine J. 2003 Dec;12(6):589-94. PMID: 14605973.

Exclusion reason: Background information only

624. Good M. A comparison of the effects of jaw relaxation and music on postoperative pain. Nurs Res. 1995 Jan-Feb;44(1):52-7. PMID: 7862546. Exclusion reason: Ineligible population

625. Good M, Ahn S. Korean and American music reduces pain in Korean women after gynecologic surgery. Pain Manag Nurs. 2008 Sep;9(3):96-103. doi:

10.1016/j.pmn.2008.02.002. PMID:

18706380. Exclusion reason: Ineligible study design

626. Good M, Albert JM, Anderson GC, et al. Supplementing relaxation and music for pain after surgery. Nurs Res. 2010 JulAug;59(4):259-69. doi: 10.1097/NNR.0b013e3181dbb2b3. PMID: 20585222. Exclusion reason: Ineligible setting

627. Good M, Anderson GC, Ahn S, et al. Relaxation and music reduce pain following intestinal surgery. Res Nurs Health. 2005 Jun;28(3):240-51. PMID: 15884029. Exclusion reason: Ineligible study design

628. Good M, Anderson GC, Stanton-Hicks M, et al. Relaxation and music reduce pain after gynecologic surgery. Pain Manag Nurs. 2002 Jun;3(2):61-70. PMID: 12050837. Exclusion reason: Ineligible setting

629. Good M, Chin CC. The effects of Western music on postoperative pain in Taiwan. Kaohsiung J Med Sci. 1998 Feb;14(2):94103. PMID: 9542366. Exclusion reason: Ineligible setting

630. Good M, Stanton-Hicks M, Grass JA, et al. Relaxation and music to reduce postsurgical pain. J Adv Nurs. 2001 Jan;33(2):208-15. PMID: 11168704. Exclusion reason: Ineligible setting
631. Goodwin VA, Hall AJ, Rogers E, et al. Orthotics and taping in the management of vertebral fractures in people with osteoporosis: a systematic review. BMJ Open. 201605 04;6(5):e010657. doi: 10.1136/BMJopen-2015-010657. PMID: 27147384. Exclusion reason: Publication used as source document

632. Gopal MG, Shannoma, Sharath Kumar BC, et al. A Comparative study to evaluate the efficacy and safety of acyclovir and famciclovir in the management of herpes zoster. J Clin Diagn Res. 2013;7(12):2904-7. doi: 10.7860/JCDR/2013/7884.3670. PMID: 24551671 Exclusion reason: Ineligible intervention

633. Gopikrishna V, Parameswaran A. Effectiveness of prophylactic use of rofecoxib in comparison with ibuprofen on postendodontic pain. J Endod. 2003 Jan;29(1):62-4. PMID: 12540224.

Exclusion reason: Ineligible intervention

634. Gordon DB, de Leon-Casasola OA, Wu CL, et al. Research Gaps in Practice Guidelines for Acute Postoperative Pain Management in Adults: Findings From a Review of the Evidence for an American Pain Society Clinical Practice Guideline. J Pain. 2016 Feb;17(2):158-66. doi: https://dx.doi.org/10.1016/j.jpain.2015.10.02 3. PMID: 26719073. Exclusion reason: Background information only

635. Gorevski E, Wead S, Tevar A, et al. Retrospective evaluation of donor pain and pain management after laprascopic nephrectomy. Transplant Proc. 2011 Sep;43(7):2487-91. doi: 10.1016/j.transproceed.2011.06.038. PMID: 21911110. Exclusion reason: Ineligible study design

636. Gorodetskyi IG, Gorodnichenko AI, Tursin PS, et al. Non-invasive interactive neurostimulation in the post-operative recovery of patients with a trochanteric fracture of the femur. A randomised, controlled trial. J Bone Joint Surg Br. 2007 Nov;89(11):1488-94. PMID: 17998187. Exclusion reason: Ineligible setting 
637. Gorrell LM, Beath K, Engel RM. Manual and Instrument Applied Cervical Manipulation for Mechanical Neck Pain: A Randomized Controlled Trial. J

Manipulative Physiol Ther. 2016

06;39(5):319-29. doi:

10.1016/j.jmpt.2016.03.003. PMID:

27180949. Exclusion reason: Ineligible population

638. Goswick Jr CB. Ibuprofen versus propoxyphene hydrochloride and placebo in acute musculoskeletal trauma. Curr Ther Res Clin Exp. 1983;34(4 II):685-92.

Exclusion reason: Ineligible comparator

639. Gottlieb M, Njie A. Comparison of naproxen with cyclobenzaprine, oxycodoneacetaminophen, and placebo for the treatment of acute low back pain. CJEM. 2016 Nov;18(6):491-4. PMID: 27640607. Exclusion reason: Ineligible publication type

640. Gourlay GK, Willis RJ, Lamberty J. A double-blind comparison of the efficacy of methadone and morphine in postoperative pain control. Anesthesiology. 1986 Mar;64(3):322-7. PMID: 3954126.

Exclusion reason: Ineligible setting

641. Grabow L. Controlled study of the analgetic effectivity of acupuncture.

Arzneimittelforschung. 1994 Apr;44(4):5548. PMID: 8011011. Exclusion reason: Ineligible setting

642. Graff-Radford SB, Shaw LR, Naliboff BN. Amitriptyline and fluphenazine in the treatment of postherpetic neuralgia. Clin J

Pain. 2000 Sep;16(3):188-92. doi: 10.1097/00002508-200009000-00002. PMID: 11014390. Exclusion reason: Ineligible intervention

643. Grape S, Schug SA, Lauer S, et al. Formulations of fentanyl for the management of pain. Drugs. 2010;70(1):5772. doi: https://dx.doi.org/10.2165/11531740000000000-00000. PMID: 20030425.

Exclusion reason: Background information only
644. Gray SL, Eggen AE, Blough D, et al. Benzodiazepine use in older adults enrolled in a health maintenance organization. Am J Geriatr Psychiatry. 2003 Sep-Oct;11(5):56876. PMID: 14506091. Exclusion reason: Background information only

645. Green T, Refshauge K, Crosbie J, et al. A randomized controlled trial of a passive accessory joint mobilization on acute ankle inversion sprains. Phys Ther. 2001 Apr;81(4):984-94. PMID: 11276181. Exclusion reason: Ineligible outcome

646. Greenstein G. Therapeutic efficacy of cold therapy after intraoral surgical procedures: a literature review. J Periodontol. 2007 May;78(5):790-800. doi: 10.1902/jop.2007.060319. PMID: 17470011. Exclusion reason: Ineligible publication type

647. Gregory J, McGowan L. An examination of the prevalence of acute pain for hospitalised adult patients: a systematic review. J Clin Nurs. 2016 Mar;25(5-6):583-98. doi: https://dx.doi.org/10.1111/jocn.13094. PMID: 26778249. Exclusion reason: Publication used as source document

648. Greig AM, Bennell KL, Briggs AM, et al. Postural taping decreases thoracic kyphosis but does not influence trunk muscle electromyographic activity or balance in women with osteoporosis. Man Ther. 2008 Jun;13(3):249-57. doi: 10.1016/j.math.2007.01.011. PMID: 17433756. Exclusion reason: Ineligible population

649. Griebeler ML, Morey-Vargas OL, Brito JP, et al. Pharmacologic interventions for painful diabetic neuropathy: An umbrella systematic review and comparative effectiveness network meta-analysis. Ann Intern Med. 2014 Nov 04;161(9):639-49. doi: 10.7326/M14-0511. PMID: 25364885. Exclusion reason: Publication used as source document

650. Griffin JR, Davis MD.

Amitriptyline/Ketamine as therapy for neuropathic pruritus and pain secondary to herpes zoster. J Drugs Dermatol. 2015 Feb;14(2):115-8. PMID: 25689805. Exclusion reason: Ineligible publication type 
651. Griffin XL, Parsons N, Costa ML, et al. Ultrasound and shockwave therapy for acute fractures in adults. Cochrane Database Syst Rev. 2015(1)doi: 10.1097/NOR.0000000000000118. PMID: 25607624. Exclusion reason: Publication used as source document

652. Grillo CM, Wada RS, da Luz Rosario de Sousa M. Acupuncture in the management of acute dental pain. J Acupunct Meridian Stud. 2014 Apr;7(2):65-70. doi: 10.1016/j.jams.2013.03.005. PMID: 24745864. Exclusion reason: Ineligible study design

653. Grisham JE, Vichinsky EP. Ketorolac versus meperidine in vasooclusive crisis: a study of safety and efficacy. COLLOQUESINSTITUT NATIONAL DE LA SANTE ET DE LA RECHERCHE MEDICALE COLLOQUES ET SEMINAIRES. 1995:431-2. Exclusion reason: Ineligible population

654. Grissa MH, Baccouche H, Boubaker H, et al. Acupuncture vs intravenous morphine in the management of acute pain in the ED. Am J Emerg Med. 2016 Nov;34(11):2112-6. doi: 10.1016/j.ajem.2016.07.028. PMID: 27475042. Exclusion reason: Ineligible population

655. Groeneweg R, Kropman H, Leopold H, et al. The effectiveness and cost-evaluation of manual therapy and physical therapy in patients with sub-acute and chronic non specific neck pain. Rationale and design of a Randomized Controlled Trial (RCT). BMC Musculoskelet Disord. 2010 Jan 24;11:14. doi: 10.1186/1471-2474-11-14. PMID: 20096136. Exclusion reason: Ineligible population

656. Grondin F, Bourgault P, Bolduc N. Intervention focused on the patient and family for better postoperative pain relief. Pain Manag Nurs. 2014 Mar;15(1):76-86. doi: 10.1016/j.pmn.2012.06.006. PMID: 24602427. Exclusion reason: Ineligible intervention

657. Gross A, Kay TM, Paquin JP, et al. Exercises for mechanical neck disorders. Cochrane Database Syst Rev. 2015 Jan 28;1:CD004250. doi: 10.1002/14651858.CD004250.pub5. PMID: 25629215. Exclusion reason: Ineligible outcome
658. Gross A, Langevin P, Burnie SJ, et al. Manipulation and mobilisation for neck pain contrasted against an inactive control or another active treatment. Cochrane Database Syst Rev. 2015 Sep 23(9):CD004249. doi: 10.1002/14651858.CD004249.pub4. PMID: 26397370. Exclusion reason: Publication used as source document

659. Gross AR, Aker PD, Goldsmith CH, et al. Conservative management of mechanical neck disorders. A systematic overview and meta-analysis. Online J Curr Clin Trials. 1996 Jul 30;Doc No 200-201:[34457 words; 185 paragraphs]. PMID: 9110943.

Exclusion reason: Publication used as source document

660. Grunnesjo MI, Bogefeldt JP, Blomberg SI, et al. A randomized controlled trial of the effects of muscle stretching, manual therapy and steroid injections in addition to 'stay active' care on health-related quality of life in acute or subacute low back pain. Clin Rehabil. 2011 Nov;25(11):999-1010. doi: 10.1177/0269215511403512. PMID: 21831926. Exclusion reason: Ineligible population

661. Grunnesjo MI, Bogefeldt JP, Svardsudd KF, et al. A randomized controlled clinical trial of stay-active care versus manual therapy in addition to stay-active care: functional variables and pain. J Manipulative Physiol Ther. 2004 Sep;27(7):431-41. PMID: 15389174. Exclusion reason: Ineligible population

662. Guan Dw, Li Y, Wang C, et al. Analgesic effect and security of tramadol/acetaminophen during perioperative total knee arthroplasty. Chinese Journal of Tissue Engineering Research. 2012;16(26):4781-5. doi: 10.3969/j.issn.1673-8225.2012.26.007. Exclusion reason: Not in English

663. Guevara Lopez U, Mendoza Feria J, Jimenez Martinez E, et al. Efficacy and effectively of butorphanol NS versus buprenorphine SL in the treatment of acute postoperative pain. Revista mexicana de anestesiologia. 1997;20(3):116-21. Exclusion reason: Not in English 
664. Guldiken S, Guldiken B, Arikan E, et al. Complete relief of pain in acute painful diabetic neuropathy of rapid glycaemic control (insulin neuritis) with venlafaxine HCL. Diabetes, Nutrition \& Metabolism Clinical \& Experimental. 2004

Aug;17(4):247-9. PMID: 15575347.

Exclusion reason: Ineligible publication type

665. Gulenc B, Kuyucu E, Bicer H, et al. Kinesiotaping Reduces Knee Diameter but Has No Effect on Differences Pain and Edema Following Knee Artroscopy. Acta Chirurgiae Orthopaedicae et Traumatologiae Cechoslovaca. 2018;85(4):285-90. PMID: 30257761. Exclusion reason: Ineligible intervention

666. Gundogdu EC, Arslan H. Effects of Various Cryotherapy Applications on Postoperative Pain in Molar Teeth with Symptomatic Apical Periodontitis: A Preliminary Randomized Prospective Clinical Trial. J Endod. 2018 Mar;44(3):349-54. doi: https://dx.doi.org/10.1016/j.joen.2017.11.00 2. PMID: 29398090. Exclusion reason: Ineligible intervention

667. Gunes Y, Ozbek TH, Gunduz HM, et al. Patient-controlled analgesia comparison of morphine to dexmedetomidine plus morphine in patients undergoing laminectomy. Neurosurg Q. 2008;18(3):17881. doi: 10.1097/WNQ.0b013e318182099d. Exclusion reason: Ineligible intervention

668. Gupta A, Fant F, Axelsson K, et al. Postoperative analgesia after radical retropubic prostatectomy: a double-blind comparison between low thoracic epidural and patient-controlled intravenous analgesia. Anesthesiology. 2006 Oct;105(4):784-93. PMID: 17006078. Exclusion reason: Ineligible comparator

669. Gurnani A, Sharma PK, Rautela RS, et al. Analgesia for acute musculoskeletal trauma: low-dose subcutaneous infusion of ketamine. Anaesth Intensive Care. 1996 Feb;24(1):32-6. PMID: 8669651.

Exclusion reason: Ineligible intervention
670. Gurusamy KS, Vaughan J, Toon CD, et al. Pharmacological interventions for prevention or treatment of postoperative pain in people undergoing laparoscopic cholecystectomy. Cochrane Database Syst Rev. 2014 Mar 28(3):CD008261. doi: https://dx.doi.org/10.1002/14651858.CD008 261.pub2. PMID: 24683057. Exclusion reason: Ineligible comparator

671. Gustin SM, Schwarz A, Birbaumer N, et al. NMDA-receptor antagonist and morphine decrease CRPS-pain and cerebral pain representation. Pain. 2010 Oct;151(1):69-76. doi: 10.1016/j.pain.2010.06.022. PMID: 20630656. Exclusion reason: Ineligible population

672. Gwak MS, Yang M, Hahm TS, et al. Effect of cryoanalgesia combined with intravenous continuous analgesia in thoracotomy patients. J Korean Med Sci. 2004 Feb;19(1):74-8. doi: 10.3346/jkms.2004.19.1.74. PMID: 14966345. Exclusion reason: Ineligible population

673. Gwirtz KH, Young JV, Byers RS, et al. The safety and efficacy of intrathecal opioid analgesia for acute postoperative pain: seven years' experience with 5969 surgical patients at Indiana University Hospital. Anesth Analg. 1999 Mar;88(3):599-604. doi: 10.1097/00000539-199903000-00026. PMID: 10072014. Exclusion reason: Ineligible intervention

674. Haanpaa M, Cruccu G, Nurmikko TJ, et al. Capsaicin 8\% patch versus oral pregabalin in patients with peripheral neuropathic pain. Eur J Pain. 2016 Feb;20(2):316-28. doi: 10.1002/ejp.731. PMID: 26581442.

Exclusion reason: Ineligible population

675. Haas M, Sharma R, Stano M. Costeffectiveness of medical and chiropractic care for acute and chronic low back pain. J Manipulative Physiol Ther. 2005 Oct;28(8):555-63. PMID: 16226622. Exclusion reason: Ineligible intervention

676. Haase O, Schwenk W, Hermann C, et al. Guided imagery and relaxation in conventional colorectal resections: a randomized, controlled, partially blinded trial. Dis Colon Rectum. 2005 Oct;48(10):1955-63. doi: 10.1007/s10350005-0114-9. PMID: 15991068. Exclusion reason: Ineligible intervention 
677. Habibi V, Kiabi FH, Sharifi H. The Effect of Dexmedetomidine on the Acute Pain After Cardiothoracic Surgeries: A Systematic Review. Braz J Cardiovasc Surg. 2018 JulAug;33(4):404-17. doi: 10.21470/16789741-2017-0253. PMID: 30184039.

Exclusion reason: Publication used as source document

678. Hagen EM, Odelien KH, Lie SA, et al. Adding a physical exercise programme to brief intervention for low back pain patients did not increase return to work. Scand J Public Health. 2010 Nov;38(7):731-8. doi: 10.1177/1403494810382472. PMID: 20817653. Exclusion reason: Ineligible population

679. Hah J, Mackey SC, Schmidt P, et al. Effect of Perioperative Gabapentin on Postoperative Pain Resolution and Opioid Cessation in a Mixed Surgical Cohort: A Randomized Clinical Trial. JAMA Surg. 201804 01;153(4):303-11. doi: https://dx.doi.org/10.1001/jamasurg.2017.49 15. PMID: 29238824. Exclusion reason: Ineligible comparator

680. Hahne AJ, Ford JJ, Richards MC, et al. Who Benefits Most From Individualized Physiotherapy or Advice for Low Back Disorders? A Preplanned Effect Modifier Analysis of a Randomized Controlled Trial. Spine. 2017 Nov 01;42(21):E1215-E24. doi: 10.1097/BRS.0000000000002148. PMID: 28263227. Exclusion reason: Ineligible population

681. Haider R, Bashir MS, Adeel M, et al. Comparison of conservative exercise therapy with and without Maitland Thoracic Manipulative therapy in patients with subacromial pain: Clinical trial. J Pak Med Assoc. 2018 Mar;68(3):381-7. PMID: 29540872. Exclusion reason: Ineligible population

682. Haldorsen EM, Kronholm K, Skouen JS, et al. Multimodal cognitive behavioral treatment of patients sicklisted for musculoskeletal pain: a randomized controlled study. Scand J Rheumatol. 1998;27(1):16-25. PMID: 9506873.

Exclusion reason: Ineligible population
683. Hale M, Upmalis D, Okamoto A, et al. Tolerability of tapentadol immediate release in patients with lower back pain or osteoarthritis of the hip or knee over 90 days: a randomized, double-blind study. Curr Med Res Opin. 2009 May;25(5):1095104. doi: 10.1185/03007990902816970. PMID: 19301989. Exclusion reason: Ineligible population

684. Haleem R, Wright R. A Scoping Review on Clinical Trials of Pain Reduction With Cannabis Administration in Adults. Journal of Clinical Medicine Research. 2020 Jun;12(6):344-51. doi: https://dx.doi.org/10.14740/jocmr4210. PMID: 32587650. Exclusion reason: Ineligible comparator

685. Hall PE, Derry S, Moore AR, et al. Single dose oral lornoxicam for acute postoperative pain in adults. Cochrane Database Syst Rev. 2019(5) PMID: 19821419. Exclusion reason: Publication used as source document

686. Halvorsen M, Falla D, Gizzi L, et al. Shortand long-term effects of exercise on neck muscle function in cervical radiculopathy: a randomized clinical trial. Man Ther. Conference: IFOMPT. 2016;25(pp e72)doi: 10.2340/16501977-2120. PMID: 27494094. Exclusion reason: Ineligible population

687. Hamina A, Taipale H, Tanskanen A, et al. Differences in analgesic use in communitydwelling persons with and without Alzheimer's disease. Eur J Pain. 2017 04;21(4):658-67. doi: https://dx.doi.org/10.1002/ejp.969. PMID: 27862681. Exclusion reason: Background information only

688. Hammack JE, Michalak JC, Loprinzi CL, et al. Phase III evaluation of nortriptyline for alleviation of symptoms of cis-platinuminduced peripheral neuropathy. Pain. 2002 Jul;98(1-2):195-203. doi: 10.1016/S03043959\%2802\%2900047-7. PMID: 12098632. Exclusion reason: Ineligible population

689. Hamner JB, Fleming MD. Lymphedema therapy reduces the volume of edema and pain in patients with breast cancer. Ann Surg Oncol. 2007 Jun;14(6):1904-8. PMID: 17342565. Exclusion reason: Background information only 
690. Hamza MA, White PF, Ahmed HE, et al. Effect of the frequency of transcutaneous electrical nerve stimulation on the postoperative opioid analgesic requirement and recovery profile. Anesthesiology. 1999 Nov;91(5):1232-8. doi: 10.1097/00000542199911000-00012. PMID: 10551571.

Exclusion reason: Ineligible setting

691. Han C, Li XD, Jiang HQ, et al. The use of gabapentin in the management of postoperative pain after total hip arthroplasty: a meta-analysis of randomised controlled trials. J Orthop Surg. 2016 Jul 12;11(1):79. doi: https://dx.doi.org/10.1186/s13018-0160412-z. PMID: 27405805. Exclusion reason: Ineligible comparator

692. Han SS, Lee YH, Oh JH, et al. Randomized, controlled trial of multimodal shoulder injection or intravenous patient-controlled analgesia after arthroscopic rotator cuff repair. Knee Surg Sports Traumatol Arthrosc. 2013 Dec;21(12):2877-83. doi: 10.1007/s00167-012-2202-4. PMID: 22990950. Exclusion reason: Ineligible comparator

693. Hancock MJ, Maher CG, Latimer J, et al. Manipulative therapy and/or NSAIDs for acute low back pain: design of a randomized controlled trial [ACTRN012605000036617]. BMC Musculoskelet Disord. 2005 Nov 10;6:57. PMID: 16280089. Exclusion reason: Ineligible publication type

694. Hancock MJ, Maher CG, Latimer J, et al. Can predictors of response to NSAIDs be identified in patients with acute low back pain? Clin J Pain. 2009 Oct;25(8):659-65. doi:

https://dx.doi.org/10.1097/AJP.0b013e3181a 7ee3a. PMID: 19920714. Exclusion reason: Background information only

695. Handoll HGH, Elliott J. Rehabilitation for distal radial fractures in adults. Cochrane Database Syst Rev. 2015(9) PMID: 26403335. Exclusion reason: Publication used as source document

696. Hanks GW, Rose NM, Aherne GW, et al. Controlled-release morphine tablets. A double-blind trial in dental surgery patients. Br J Anaesth. 1981 Dec;53(12):1259-64. PMID: 7032558. Exclusion reason: Ineligible comparator
697. Hansen BB, Kirkeskov L, Begtrup LM, et al. Early occupational intervention for people with low back pain in physically demanding jobs: A randomized clinical trial. PLoS Medicine / Public Library of Science. 2019 Aug;16(8):e1002898. doi: 10.1371/journal.pmed.1002898. PMID: 31419219. Exclusion reason: Ineligible population

698. Hansen H, Manchikanti L, Simopoulos TT, et al. A systematic evaluation of the therapeutic effectiveness of sacroiliac joint interventions. Pain Physician. 2012 MayJun;15(3):E247-78. PMID: 22622913. Exclusion reason: Publication used as source document

699. Hansen LE, Stone GL, Matson CA, et al. Total Joint Arthroplasty in Patients Taking Methadone or Buprenorphine/Naloxone Preoperatively for Prior Heroin Addiction: A Prospective Matched Cohort Study. J Arthroplasty. 2016 08;31(8):1698-701. doi: 10.1016/j.arth.2016.01.032. PMID: 26899477. Exclusion reason: Ineligible study design

700. Hanson KT, Thiels CA, Polites SF, et al. The opioid epidemic in acute care surgeryCharacteristics of overprescribing following laparoscopic cholecystectomy. J Trauma Acute Care Surg. 2018 07;85(1):62-70. doi: https://dx.doi.org/10.1097/TA.00000000000 01834. PMID: 29462081. Exclusion reason: Background information only

701. Hansson P, Ekblom A. Influence of stimulus frequency and probe size on vibrationinduced alleviation of acute orofacial pain. Appl Neurophysiol. 1986;49(3):155-65. PMID: 3548587. Exclusion reason: Ineligible intervention

702. Harkapaa K, Jarvikoski A, Mellin G, et al. A controlled study on the outcome of inpatient and outpatient treatment of low back pain. Part I. Pain, disability, compliance, and reported treatment benefits three months after treatment. Scandinavian J Rehabil Med. 1989;21(2):81-9. PMID: 2526364. Exclusion reason: Ineligible population 
703. Harkapaa K, Jarvikoski A, Mellin G, et al. Health locus of control beliefs and psychological distress as predictors for treatment outcome in low-back pain patients: results of a 3-month follow-up of a controlled intervention study. Pain. 1991 Jul;46(1):35-41. PMID: 1832753.

Exclusion reason: Ineligible intervention

704. Harkapaa K, Mellin G, Jarvikoski A, et al. A controlled study on the outcome of inpatient and outpatient treatment of low back pain. Part III. Long-term follow-up of pain, disability, and compliance. Scandinavian J Rehabil Med. 1990;22(4):181-8. PMID: 2148221. Exclusion reason: Ineligible population

705. Harkin C, Parker R. A prospective, randomised control trial of acupuncture for select common conditions within the emergency department. J Chin Med. 2007(85):45-53. Exclusion reason: Ineligible publication type

706. Harkless LB, DeLellis S, Carnegie DH, et al. Improved foot sensitivity and pain reduction in patients with peripheral neuropathy after treatment with monochromatic infrared photo energy-MIRE. J Diabetes Complications. 2006 MarApr;20(2):81-7. doi:

10.1016/j.jdiacomp.2005.06.002. PMID: 16504836. Exclusion reason: Ineligible intervention

707. Harrison RK, DiMeo T, Klinefelter RD, et al. Multi-Modal Pain Control in Ambulatory Hand Surgery. Am J Orthop. 2018 Jun;47(6)doi: 10.12788/ajo.2018.0042. PMID: 29979803. Exclusion reason: Ineligible intervention

708. Harte AA, Baxter GD, Gracey JH. The effectiveness of motorised lumbar traction in the management of LBP with lumbo sacral nerve root involvement: a feasibility study. BMC Musculoskelet Disord. 2007 Nov 29;8:118. PMID: 18047650. Exclusion reason: Ineligible population
709. Hartford LB, Murphy PB, Gray DK, et al. The Standardization of Outpatient Procedure (STOP) Narcotics after anorectal surgery: a prospective non-inferiority study to reduce opioid use. Tech Coloproctol. 2020 Jun;24(6):563-71. doi: https://dx.doi.org/10.1007/s10151-02002190-0. PMID: 32232594. Exclusion reason: Ineligible study design

710. Hartung DM, Middleton L, Haxby DG, et al. Rates of adverse events of long-acting opioids in a state Medicaid program. Ann Pharmacother. 2007 Jun;41(6):921-8. doi: 10.1345/aph.1K066. PMID: 17504834. Exclusion reason: Ineligible outcome

711. Harvey JN. Update on treatments for neuropathic pain. J Pain Palliat Care Pharmacother. 2008;22(1):54-7. PMID: 19042825. Exclusion reason: Background information only

712. Hasenbring M, Ulrich HW, Hartmann M, et al. The efficacy of a risk factor-based cognitive behavioral intervention and electromyographic biofeedback in patients with acute sciatic pain. An attempt to prevent chronicity. Spine. 1999 Dec 01;24(23):2525-35. PMID: 10626316. Exclusion reason: Ineligible population

713. Hashemirad F, Karimi N, Keshavarz R. The effect of Kinesio taping technique on trigger points of the piriformis muscle. J Bodyw Mov Ther. 2016 Oct;20(4):807-14. doi: 10.1016/j.jbmt.2016.02.002. PMID: 27814861. Exclusion reason: Ineligible population

714. Hassan Hashisha RK, Ali SM, Awad TE. Impact of tramadol abuse on clinical outcome of lumbar discectomy patients'. Neurotoxicology. 2019 12;75:9-13. doi: https://dx.doi.org/10.1016/j.neuro.2019.06.0 09. PMID: 31326535. Exclusion reason: Ineligible study design

715. Hastings V, McCallister AM, Curtis SA, et al. Efficacy of Osteopathic Manipulative Treatment for Management of Postpartum Pain. J Am Osteopath Assoc. 2016 Aug 01;116(8):502-9. doi: 10.7556/jaoa.2016.103. PMID: 27455099. Exclusion reason: Ineligible comparator 
716. Hasue M, Fujiwara M. Epidemiologic and clinical studies of long-term prognosis of low-back pain and sciatica. Spine. 1979 Mar-Apr;4(2):150-5. PMID: 162551. Exclusion reason: Background information only

717. Hatfield LA, Murphy N, Karp K, et al. A Systematic Review of Behavioral and Environmental Interventions for Procedural Pain Management in Preterm Infants. J Pediatr Nurs. 2019 Jan - Feb;44:22-30. doi: https://dx.doi.org/10.1016/j.pedn.2018.10.00 4. PMID: 30683278. Exclusion reason: Publication used as source document

718. Hauser W, Fitzcharles MA, Radbruch L, et al. Cannabinoids in Pain Management and Palliative Medicine. Dtsch Arztebl Int. 2017 Sep 22;114(38):627-34. doi: https://dx.doi.org/10.3238/arztebl.2017.0627 . PMID: 29017688. Exclusion reason: Publication used as source document

719. Hay E, Barlas P, Foster N, et al. Is acupuncture a useful adjunct to physiotherapy for older adults with knee pain?: the "acupuncture, physiotherapy and exercise" (APEX) study [ISRCTN88597683]. BMC Musculoskelet Disord. 2004 Sep 02;5:31. PMID: 15345098. Exclusion reason: Ineligible study design

720. He BS, Li Y, Gui T. Preliminary Clinical Evaluation of Acupuncture Therapy in Patients With Postpartum Sciatica. J Midwifery Womens Health. 2018 03;63(2):214-20. doi: 10.1111/jmwh.12681. PMID: 29569351. Exclusion reason: Ineligible population

721. He J, Zhao T, Zhang W, et al. A new analgesic method, two-minute sciatic nerve press, for immediate pain relief: A randomized trial. BMC Anesthesiol. 2008;8doi: 10.1186/1471-2253-8-1. PMID: 18221518. Exclusion reason: Ineligible population

722. He L, Zhang XM. Clinical effect of sphenopalatine ganglion needling in treating primary trigeminal neuralgia of Liver-yang upsurge syndrome type. Chin J Integr Med. 2012 Mar;18(3):214-8. doi: 10.1007/s11655-012-1021-2. PMID: 22466947. Exclusion reason: Ineligible comparator
723. Healy WL, Seidman J, Pfeifer BA, et al. Cold compressive dressing after total knee arthroplasty. Clin Orthop Relat Res. 1994 Feb(299):143-6. PMID: 7907012.

Exclusion reason: Ineligible setting

724. Hebertson RM, Storey N, Turner JL. Analgesic efficacy of meclofenamate sodium in episiotomy pain.

Pharmacotherapy:The Journal of Human Pharmacology \& Drug Therapy. 1986 SepOct;6(5):205-10. doi: 10.1002/j.18759114.1986.tb03478.x. PMID: 3540870. Exclusion reason: Ineligible comparator

725. Hedderson WC, Dover GC, George SZ, et al. Expectancy Reduces Symptoms but not Functional Impairment Following Exerciseinduced Musculoskeletal Injury. Clin J Pain. 2018 Jan;34(1):1-7. doi: https://dx.doi.org/10.1097/AJP.0000000000 000484. PMID: 28157138. Exclusion reason: Background information only

726. Heggannavar A, Naik P. Comparison of muscle energy technique and positional release therapy in acute low back pain-RCT. Physiotherapy (United Kingdom). 2011;Conference: World Physical Therapy 2011 Amsterdam Netherlands. Conference Start: 20110620 Conference End: 20110623. Conference

Publication:(var.pagings):eS470. Exclusion reason: Ineligible publication type

727. Heikkila H, Heikkila E, Eisemann M. Predictive factors for the outcome of a multidisciplinary pain rehabilitation programme on sick-leave and life satisfaction in patients with whiplash trauma and other myofascial pain: a follow-up study. Clin Rehabil. 1998 Dec;12(6):487-96. PMID: 9869252. Exclusion reason: Ineligible population

728. Heiser RM, Chiles K, Fudge M, et al. The use of music during the immediate postoperative recovery period. AORN J. 1997 Apr;65(4):777-8, 81-5. PMID: 9093740. Exclusion reason: Ineligible study design

729. Helander EM, Menard BL, Harmon CM, et al. Multimodal Analgesia, Current Concepts, and Acute Pain Considerations. Curr Pain Headache Rep. 2017 Jan;21(1):3. doi: https://dx.doi.org/10.1007/s11916-0170607-y. PMID: 28132136. Exclusion reason: Background information only 
730. Helm S, 2nd, Racz GB, Gerdesmeyer L, et al. Percutaneous and Endoscopic Adhesiolysis in Managing Low Back and Lower Extremity Pain: A Systematic Review and Meta-analysis. Pain Physician. 2016 Feb;19(2):E245-82. PMID: 26815254.

Exclusion reason: Publication used as source document

731. Helminen EE, Sinikallio SH, Valjakka AL, et al. Effectiveness of a cognitive-behavioral group intervention for knee osteoarthritis pain: protocol of a randomized controlled trial. BMC Musculoskelet Disord. 2013 Jan 29;14:46. doi: 10.1186/1471-2474-14-46. PMID: 23356455. Exclusion reason: Ineligible publication type

732. Henriksen MG, Jensen MB, Hansen HV, et al. Enforced mobilization, early oral feeding, and balanced analgesia improve convalescence after colorectal surgery. Nutrition. 2002 Feb;18(2):147-52. PMID: 11844646. Exclusion reason: Ineligible intervention

733. Hensel KL, Buchanan S, Brown SK, et al. Pregnancy Research on Osteopathic Manipulation Optimizing Treatment Effects: the PROMOTE study. Am J Obstet Gynecol. 2015 Jan;212(1):108.e1-9. doi: 10.1016/j.ajog.2014.07.043. PMID: 25068560. Exclusion reason: Ineligible population

734. Herman E, Williams R, Stratford P, et al. A randomized controlled trial of transcutaneous electrical nerve stimulation (CODETRON) to determine its benefits in a rehabilitation program for acute occupational low back pain. Spine. 1994 Mar 01;19(5):561-8. PMID: 8184351. Exclusion reason: Ineligible population

735. Hermans L, Calders P, Van Oosterwijck J, et al. An Overview of Offset Analgesia and the Comparison with Conditioned Pain Modulation: A Systematic Literature Review. Pain Physician. 2016 07;19(6):30726. PMID: 27454261. Exclusion reason: Publication used as source document
736. Hernandez NM, Parry JA, Mabry TM, et al. Patients at Risk: Preoperative Opioid Use Affects Opioid Prescribing, Refills, and Outcomes After Total Knee Arthroplasty. J Arthroplasty. 2018 07;33(7S):S142-S6. doi: 10.1016/j.arth.2018.01.004. PMID: 29402712. Exclusion reason: Ineligible comparator

737. Hernandez NM, Parry JA, Taunton MJ. Patients at Risk: Large Opioid Prescriptions After Total Knee Arthroplasty. J Arthroplasty. 2017 08;32(8):2395-8. doi: https://dx.doi.org/10.1016/j.arth.2017.02.06 0. PMID: 28392133. Exclusion reason: Ineligible study design

738. Herrmann WA, Geertsen MS. Efficacy and safety of lornoxicam compared with placebo and diclofenac in acute sciatica/lumbosciatica: an analysis from a randomised, double-blind, multicentre, parallel-group study. Int J Clin Pract. 2009 Nov;63(11):1613-21. doi: 10.1111/j.17421241.2009.02187.x. PMID: 19832818.

Exclusion reason: Ineligible comparator

739. Hershman M, Cheadle W, Swift R, et al. Transcutaneous electrical nerve stimulation as adjunctive analgesia in patients undergoing abdominal procedures. Surg Res Commun. 1989;7:65-9. Exclusion reason: Ineligible population

740. Hetherington JW, Philp NH. Diclofenac sodium versus pethidine in acute renal colic. Br Med J (Clin Res Ed). 1986 Jan 25;292(6515):237-8. doi: 10.1136/BMJ.292.6515.237. PMID: 3081085. Exclusion reason: Ineligible duration

741. Hidalgo B, Hall T, Bossert J, et al. The efficacy of manual therapy and exercise for treating non-specific neck pain: A systematic review. J Back Musculoskelet Rehabil. 2017 Nov 06;30(6):1149-69. doi: https://dx.doi.org/10.3233/BMR-169615. PMID: 28826164. Exclusion reason: Publication used as source document 
742. Hidalgo B, Pitance L, Hall T, et al. Shortterm effects of Mulligan mobilization with movement on pain, disability, and kinematic spinal movements in patients with nonspecific low back pain: a randomized placebo-controlled trial. J Manipulative Physiol Ther. 2015 Jul-Aug;38(6):365-74. doi: 10.1016/j.jmpt.2015.06.013. PMID: 26215900. Exclusion reason: Ineligible population

743. Hides JA, Jull GA, Richardson CA. Longterm effects of specific stabilizing exercises for first-episode low back pain. Spine. 2001 Jun 01;26(11):E243-8. PMID: 11389408. Exclusion reason: Ineligible comparator

744. Hides JA, Richardson CA, Jull GA. Multifidus muscle recovery is not automatic after resolution of acute, first-episode low back pain. Spine. 1996 Dec 01;21(23):27639. PMID: 8979323. Exclusion reason: Ineligible comparator

745. Hildebrand KA, Buckley RE, Mohtadi NG, et al. Functional outcome measures after displaced intra-articular calcaneal fractures. J Bone Joint Surg Br. 1996 Jan;78(1):11923. PMID: 8898141. Exclusion reason: Ineligible intervention

746. Hindle TH, 3rd. Comparison of carisoprodol, butabarbital, and placebo in treatment of the low back syndrome. Calif Med. 1972 Aug;117(2):7-11. PMID: 4262210. Exclusion reason: Ineligible comparator

747. Hingorani K, Biswas AK. Double-blind controlled trial comparing oxyphenbutazone and indomethacin in the treatment of acute low back pain. Br J Clin Pract. 1970 Mar;24(3):120-3. PMID: 4245599. Exclusion reason: Ineligible comparator

748. Hingorani K, Templeton JS. A comparative trial of azapropazone and ketoprofen in the treatment of acute backache. Curr Med Res Opin. 1975;3(6):407-12. PMID: 126848. Exclusion reason: Ineligible comparator

749. Hirunwiwatkul P, Charakorn N, Teerapraipruk B, et al. The effects of a cold pack on postoperative turbinate and/or septal bleeding and pain. Am J Rhinol Allergy. 2016 May;30(3):222-5. doi: https://dx.doi.org/10.2500/ajra.2016.30.4306 . PMID: 27216354. Exclusion reason: Ineligible duration
750. Hlobil H, Staal JB, Twisk J, et al. The effects of a graded activity intervention for low back pain in occupational health on sick leave, functional status and pain: 12-month results of a randomized controlled trial. J Occup Rehabil. 2005 Dec;15(4):569-80. PMID: 16254756. Exclusion reason: Ineligible population

751. Ho KY, Huh BK, White WD, et al. Topical amitriptyline versus lidocaine in the treatment of neuropathic pain. Clin J Pain. 2008 Jan;24(1):51-5. doi: 10.1097/AJP.0b013e318156db26. PMID: 18180637. Exclusion reason: Ineligible population

752. Ho MK, Chung CH. A prospective, randomised clinical trial comparing oral diclofenac potassium and intramuscular diclofenac sodium in acute pain relief. Hong Kong J Emerg Med. 2004;11(2):69-77. doi: 10.1177/102490790401100202. Exclusion reason: Ineligible intervention

753. Hodgson SA, Mawson SJ, Stanley D. Rehabilitation after two-part fractures of the neck of the humerus. J Bone Joint Surg Br. 2003 Apr;85(3):419-22. PMID: 12729121. Exclusion reason: Ineligible comparator

754. Hodzic J, Golka K, Selinski S, et al. Akupunktur während der ESWL von Nierensteinen - erste Ergebnisse. Der Urologe. 2007 2007/07/01;46(7):740-7. doi: 10.1007/s00120-007-1362-1. Exclusion reason: Not in English

755. Hoehler FK, Tobis JS, Buerger AA. Spinal manipulation for low back pain. JAMA. 1981 May 8;245(18):1835-8. PMID: 6453240. Exclusion reason: Ineligible population

756. Hofstee DJ, Gijtenbeek JJM, Hoogland PH, et al. Bed rest and physiotherapy are of no added value in the management of acute lumbosacral radicular pain: a randomised clinical study. Nederlands tijdschrift voor geneeskunde. 2003;147(6):249-54.

Exclusion reason: Not in English 
757. Hogan TJ, Silverman MJ. Coping-Infused Dialogue through Patient-Preferred Live Music: A Medical Music Therapy Protocol and Randomized Pilot Study for Hospitalized Organ Transplant Patients. J Music Ther. 2015;52(3):420-36. doi: 10.1093/jmt/thv008. PMID: 26315143. Exclusion reason: Ineligible duration

758. Hoiriis KT, McDuffie FC, Pfleger B. Design and implementation of a randomized controlled clinical trial of chiropractic care versus drug therapy for sub-acute low back pain. Chiropractic Research Journal. 1997;4(2):50-63. Exclusion reason: Ineligible population

759. Holland WC, Hunold KM, Mangipudi SA, et al. A Prospective Evaluation of Shared Decision-making Regarding Analgesics Selection for Older Emergency Department Patients With Acute Musculoskeletal Pain. Acad Emerg Med. 2016 Mar;23(3):306-14. doi: https://dx.doi.org/10.1111/acem.12888. PMID: 26728174. Exclusion reason: Background information only

760. Hollman F, Wolterbeek N, Zijl JAC, et al. Abduction Brace Versus Antirotation Sling After Arthroscopic Cuff Repair: The Effects on Pain and Function. Arthroscopy. 2017 Sep;33(9):1618-26. doi: https://dx.doi.org/10.1016/j.arthro.2017.02.0 10. PMID: 28427872. Exclusion reason: Ineligible comparator

761. Homayouni K, Naseri M, Zaravar F, et al. Comparison of the effect of aquatic physical therapy and conventional physical therapy in patients with lumbar spinal stenosis (a randomized controlled trial). J Musculoskelet Res. 2015;18(1)doi: 10.1142/S0218957715500025. Exclusion reason: Ineligible population

762. Hommez G, Ongena B, Cauwels R, et al. Analgesia (mis)usage on a dental emergency service: a patient survey. Clin Oral Investig. 2018 Apr;22(3):1297-302. doi: https://dx.doi.org/10.1007/s00784-0172228-6. PMID: 28983670. Exclusion reason: Background information only

763. Honig S, Murray KA. An appraisal of codeine as an analgesic: single-dose analysis. J Clin Pharmacol. 1984 FebMar;24(2-3):96-102. doi: 10.1002/j.15524604.1984.tb02771.x. PMID: 6371063. Exclusion reason: Ineligible population
764. Honig WJ, Van Ochten J. A multiple-dose comparison of ketorolac tromethamine with diflunisal and placebo in postmeniscectomy pain. J Clin Pharmacol. 1986 Nov-

Dec;26(8):700-5. PMID: 3540032.

Exclusion reason: Ineligible intervention

765. Hopkins D, Shipton EA, Potgieter D, et al. Comparison of tramadol and morphine via subcutaneous PCA following major orthopaedic surgery. Can J Anaesth. 1998 May;45(5 Pt 1):435-42. PMID: 9598258. Exclusion reason: Ineligible comparator

766. Hopton A, MacPherson H. Acupuncture for chronic pain: is acupuncture more than an effective placebo? A systematic review of pooled data from meta-analyses. Pain Pract. 2010 Mar-Apr;10(2):94-102. doi: https://dx.doi.org/10.1111/j.15332500.2009.00337.x. PMID: 20070551.

Exclusion reason: Publication used as source document

767. Horgas AL, Snigurska U, Farland MZ, et al. Analyzing analgesic medications in community-dwelling older adults. Pain Med. 2019 Jan;20(1):58-67. doi: http://dx.doi.org/10.1093/pm/pnx340. PMID: 29408973. Exclusion reason: Background information only

768. Horst J, Frei-Jones M, Deych E, et al. Pharmacokinetics and analgesic effects of methadone in children and adults with sickle cell disease. Pediatr Blood Cancer. 2016 12;63(12):2123-30. doi: 10.1002/pbc.26207. PMID: 27572136. Exclusion reason: Ineligible comparator

769. Hota LS, Warda HA, Haviland MJ, et al. Opioid use following gynecologic and pelvic reconstructive surgery. Int Urogynecol J. 2018 Oct;29(10):1441-5. doi: 10.1007/s00192-017-3474-5. PMID: 28889218. Exclusion reason: Ineligible study design

770. Hotta K, Endo T, Taira K, et al. Comparison of the analgesic effects of continuous extrapleural block and continuous epidural block after video-assisted thoracoscopic surgery. J Cardiothorac Vasc Anesth. 2011 Dec;25(6):1009-13. doi: 10.1053/j.jvca.2011.07.026. PMID: 21955830. Exclusion reason: Ineligible intervention 
771. Howard J. Managing acute painful sickle cell episodes in hospital. Nursing Times. 2012 Sep 25-Oct 1;108(39):23. PMID: 23155904. Exclusion reason: Background information only

772. Howard R, Alameddine M, Klueh M, et al. Spillover Effect of Evidence-Based Postoperative Opioid Prescribing. J Am Coll Surg. 2018 09;227(3):374-81. doi: https://dx.doi.org/10.1016/j.jamcollsurg.201 8.06.007. PMID: 30056059. Exclusion reason: Ineligible intervention

773. Howard R, Vu J, Lee J, et al. A Pathway for Developing Postoperative Opioid Prescribing Best Practices. Ann Surg. 2020 01;271(1):86-93. doi: https://dx.doi.org/10.1097/SLA.0000000000 003434. PMID: 31478976. Exclusion reason: Ineligible publication type

774. Howe DH, Newcombe RG, Wade MT. Manipulation of the cervical spine--a pilot study. J R Coll Gen Pract. 1983 Sep;33(254):574-9. PMID: 6355460. Exclusion reason: Ineligible population

775. Hruschak V, Cochran G. Psychosocial predictors in the transition from acute to chronic pain: a systematic review. Psychology Health \& Medicine. 2018 12;23(10):1151-67. doi: https://dx.doi.org/10.1080/13548506.2018.1 446097. PMID: 29490476. Exclusion reason: Publication used as source document

776. Hsia HL, Takemoto S, van de Ven T, et al. Acute pain is associated with chronic opioid use after total knee arthroplasty. Reg Anesth Pain Med. 2018 Oct;43(7):705-11. doi: 10.1097/AAP.0000000000000831. PMID: 29975257. Exclusion reason: Ineligible comparator

777. Hsieh CY, Phillips RB, Adams AH, et al. Functional outcomes of low back pain: comparison of four treatment groups in a randomized controlled trial. J Manipulative Physiol Ther. 1992 Jan;15(1):4-9. PMID: 1531488. Exclusion reason: Ineligible population
778. Hsieh L-F, Hong C-Z, Chern S-H, et al. Efficacy and side effects of diclofenac patch in treatment of patients with myofascial pain syndrome of the upper trapezius. J Pain Symptom Manage. 2010 Jan;39(1):116-25. doi: 10.1016/j.jpainsymman.2009.05.016. PMID: 19822404 Exclusion reason: Ineligible comparator

779. Hsiung WT, Chang YC, Yeh ML, et al. Acupressure improves the postoperative comfort of gastric cancer patients: A randomised controlled trial. Complement Ther Med. 2015 Jun;23(3):339-46. doi: https://dx.doi.org/10.1016/j.ctim.2015.03.01 0. PMID: 26051568. Exclusion reason: Ineligible setting

780. Hu J, Huang D, Li M, et al. Effects of a single dose of preoperative pregabalin and gabapentin for acute postoperative pain: A network meta-analysis of randomized controlled trials. J Pain Res. 2018;11:263343. doi: 10.2147/JPR.S170810. PMID: 30519075. Exclusion reason: Ineligible setting

781. Huang A, Katz J, Clarke H. Ensuring safe prescribing of controlled substances for pain following surgery by developing a transitional pain service. Pain Manag 2015;5(2):97-105. doi: 10.2217/pmt.15.7. PMID: 25806904. Exclusion reason: Ineligible publication type

782. Hug K, Roosli M. Therapeutic effects of whole-body devices applying pulsed electromagnetic fields (PEMF): a systematic literature review. Bioelectromagnetics. 2012 Feb;33(2):95-105. doi: https://dx.doi.org/10.1002/bem.20703. PMID: 21938735. Exclusion reason: Publication used as source document

783. Hui F, Boyle E, Vayda E, et al. A randomized controlled trial of a multifaceted integrated complementary-alternative therapy for chronic herpes zoster-related pain. Altern Med Rev. 2012 Mar;17(1):5768. PMID: 22502623. Exclusion reason: Ineligible population 
784. Humble SR, Dalton AJ, Li L. A systematic review of therapeutic interventions to reduce acute and chronic post-surgical pain after amputation, thoracotomy or mastectomy. Eur J Pain. 2015 Apr;19(4):451-65. doi: 10.1002/ejp.567. PMID: 25088289.

Exclusion reason: Publication used as source document

785. Hung KKC, Graham CA, Lo RSL, et al. Oral paracetamol and/or ibuprofen for treating pain after soft tissue injuries: Single centre double-blind, randomised controlled clinical trial. PLoS ONE [Electronic Resource]. 2018;13(2):e0192043. doi: 10.1371/journal.pone.0192043. PMID: 29408866. Exclusion reason: Ineligible population

786. Huong QBT, Luu HT, Thanh TV. Local infiltration analgesia with bupivacaine reduces postoperative pain and opioid consumption after joint replacements in a Vietnamese Hospital. Pharmaceutical Sciences Asia. 2019;46(3):147-55. doi: 10.29090/PSA.2019.03.017.0014.

Exclusion reason: Ineligible setting

787. Hurley DA, McDonough SM, Dempster M, et al. A randomized clinical trial of manipulative therapy and interferential therapy for acute low back pain. Spine. 2004 Oct 15;29(20):2207-16. PMID: 15480130.

Exclusion reason: Ineligible population

788. Hurley DA, Minder PM, McDonough SM, et al. Interferential therapy electrode placement technique in acute low back pain: a preliminary investigation. Arch Phys Med Rehabil. 2001 Apr;82(4):485-93. PMID: 11295009. Exclusion reason: Ineligible population

789. Hurwitz EL, Morgenstern H, Harber P, et al. Second Prize: The effectiveness of physical modalities among patients with low back pain randomized to chiropractic care: findings from the UCLA low back pain study. J Manipulative Physiol Ther. 2002 Jan;25(1):10-20. PMID: 11898014.

Exclusion reason: Ineligible intervention
790. Hurwitz EL, Morgenstern H, Harber P, et al. A randomized trial of chiropractic manipulation and mobilization for patients with neck pain: clinical outcomes from the UCLA neck-pain study. Am J Public Health. 2002 Oct;92(10):1634-41. doi: 10.2105/ajph.92.10.1634. PMID: 12356613. Exclusion reason: Ineligible population

791. Hurwitz EL, Morgenstern H, Vassilaki M, et al. Adverse reactions to chiropractic treatment and their effects on satisfaction and clinical outcomes among patients enrolled in the UCLA Neck Pain Study. J Manipulative Physiol Ther. 2004 Jan;27(1):16-25. doi: 10.1016/j.jmpt.2003.11.002. PMID: 14739870. Exclusion reason: Ineligible population

792. Hurwitz EL, Morgenstern H, Yu F. Satisfaction as a predictor of clinical outcomes among chiropractic and medical patients enrolled in the UCLA low back pain study. Spine. 2005 Oct 01;30(19):2121-8. PMID: 16205336. Exclusion reason: Ineligible intervention

793. Husebo BS, Achterberg W, Flo E. Identifying and Managing Pain in People with Alzheimer's Disease and Other Types of Dementia: A Systematic Review. CNS Drugs. 2016 06;30(6):481-97. doi: https://dx.doi.org/10.1007/s40263-0160342-7. PMID: 27240869. Exclusion reason: Publication used as source document

794. Hwang BY, Kwon JY, Kim E, et al. Oxycodone vs. fentanyl patient-controlled analgesia after laparoscopic cholecystectomy. Int J Med Sci. 2014;11(7):658-62. doi: 10.7150/ijms.8331. PMID: 24843313. Exclusion reason: Ineligible setting

795. Hwang U, Belland LK, Handel DA, et al. Is all pain is treated equally? A multicenter evaluation of acute pain care by age. Pain. 2014 Dec;155(12):2568-74. doi: https://dx.doi.org/10.1016/j.pain.2014.09.01 7. PMID: 25244947. Exclusion reason: Background information only 
796. Iacobone M, Citton M, Zanella S, et al. The effects of acupuncture after thyroid surgery: A randomized, controlled trial. Surgery. 2014 Dec;156(6):1605-12; discussion 12-3. doi:

https://dx.doi.org/10.1016/j.surg.2014.08.06 2. PMID: 25456960. Exclusion reason: Ineligible intervention

797. Iero PT, Mulherin DR, Jensen O, et al. A Prospective, Randomized, Open-Label Study Comparing an Opioid-Sparing Postsurgical Pain Management Protocol With and Without Liposomal Bupivacaine for Full-Arch Implant Surgery. Int J Oral Maxillofac Implants. 2018

Sep/Oct;33(5):1155-64. doi:

10.11607/jomi.5938. PMID: 30231106.

Exclusion reason: Ineligible intervention

798. Ilfeld BM, Ball ST, Cohen SP, et al. Percutaneous Peripheral Nerve Stimulation to Control Postoperative Pain, Decrease Opioid Use, and Accelerate Functional Recovery Following Orthopedic Trauma. Mil Med. 201903 01;184(Suppl 1):557-64. doi: 10.1093/milmed/usy378. PMID: 30901395. Exclusion reason: Ineligible study design

799. Ilfeld BM, Mariano ER, Girard PJ, et al. A multicenter, randomized, triple-masked, placebo-controlled trial of the effect of ambulatory continuous femoral nerve blocks on discharge-readiness following total knee arthroplasty in patients on general orthopaedic wards. Pain. 2010

Sep;150(3):477-84. doi: 10.1016/j.pain.2010.05.028. PMID: 20573448. Exclusion reason: Ineligible intervention

800. Ilyas AM, Miller AJ, Graham JG, et al. A Prospective, Randomized, Double-Blinded Trial Comparing Acetaminophen, Ibuprofen, and Oxycodone for Pain Management After Hand Surgery. Orthopedics. 2019 Mar 01;42(2):110-5. doi: https://dx.doi.org/10.3928/0147744720190221-02. PMID: 30810754. Exclusion reason: Ineligible outcome
801. Imafuku S, Nakayama J, Higa K, et al. Oneyear follow-up of zoster-associated pain in 764 immunocompetent patients with acute herpes zoster treated with famciclovir (FAMILIAR study). J Eur Acad Dermatol Venereol. 2014 Dec;28(12):1716-22. PMID: 25564680. Exclusion reason: Ineligible intervention

802. Imai R, Osumi M, Morioka S. Influence of illusory kinesthesia by vibratory tendon stimulation on acute pain after surgery for distal radius fractures: a quasi-randomized controlled study. Clin Rehabil. 2016 Jun;30(6):594-603. doi: 10.1177/0269215515593610. PMID: 26198893. Exclusion reason: Ineligible intervention

803. Inacio MC, Hansen C, Pratt NL, et al. Risk factors for persistent and new chronic opioid use in patients undergoing total hip arthroplasty: a retrospective cohort study. BMJ Open. 2016 Apr 29;6(4):e010664. doi: https://dx.doi.org/10.1136/BMJopen-2015010664. PMID: 27130165. Exclusion reason: Ineligible comparator

804. Iqbal ZA, Rajan R, Khan SA, et al. Effect of deep cervical flexor muscles training using pressure biofeedback on pain and disability of school teachers with neck pain. J Phys Ther Sci. 2013 Jun;25(6):657-61. doi: 10.1589/jpts.25.657. PMID: 24259822. Exclusion reason: Ineligible intervention

805. Irvine AB, Russell $\mathrm{H}$, Manocchia $\mathrm{M}$, et al. Mobile-Web app to self-manage low back pain: randomized controlled trial. JMIR. 2015 Jan 02;17(1):e1. doi: 10.2196/jmir.3130. PMID: 25565416. Exclusion reason: Ineligible population

806. Irving $G$, Jensen M, Cramer $M$, et al. Efficacy and tolerability of gastric-retentive gabapentin for the treatment of postherpetic neuralgia: results of a double-blind, randomized, placebo-controlled clinical trial. Clin J Pain. 2009 Mar-Apr;25(3):18592. doi: 10.1097/AJP.0b013e3181934276. PMID: 19333167. Exclusion reason: Ineligible population 
807. Ishida K, Shibanuma N, Matsumoto T, et al. Periarticular multimodal drug injection improves post-operative pain and functional recovery after total knee arthroplasty. $\mathrm{J}$ Orthop Sci. 2016 Mar;21(2):178-83. doi: 10.1016/j.jos.2015.12.004. PMID: 26723225. Exclusion reason: Ineligible intervention

808. Isiordia-Espinoza MA, de Jesus PozosGuillen A, Aragon-Martinez OH. Analgesic efficacy and safety of single-dose tramadol and non-steroidal anti-inflammatory drugs in operations on the third molars: a systematic review and meta-analysis. British J Oral Maxillofac Surg. 2014 Nov;52(9):775-83. doi: https://dx.doi.org/10.1016/j.bjoms.2014.05.0 05. PMID: 24930627. Exclusion reason: Publication used as source document

809. Isiordia-Espinoza MA, Pozos-Guillen A, Martinez-Rider R, et al. Comparison of the analgesic efficacy of oral ketorolac versus intramuscular tramadol after third molar surgery: A parallel, double-blind, randomized, placebo-controlled clinical trial. Medicina Oral, Patologia Oral y Cirugia Bucal. 2016 Sep 01;21(5):e637-43. PMID: 27475688. Exclusion reason: Ineligible intervention

810. Isner-Horobeti ME, Dufour SP, Schaeffer $\mathrm{M}$, et al. High-Force Versus Low-Force Lumbar Traction in Acute Lumbar Sciatica Due to Disc Herniation: A Preliminary Randomized Trial. J Manipulative Physiol Ther. 2016 Nov - Dec;39(9):645-54. doi: 10.1016/j.jmpt.2016.09.006. PMID: 27838140. Exclusion reason: Ineligible comparator

811. Jacobson J, Bertilson SO. Analgesic efficacy of paracetamol/codeine and paracetamol/dextropropoxyphene in pain after episiotomy and ruptures in connection with childbirth. J Int Med Res. 1987 MarApr;15(2):89-95. PMID: 3556264.

Exclusion reason: Ineligible comparator
812. Jahangirifard A, Razavi M, Ahmadi ZH, et al. Effect of TENS on Postoperative Pain and Pulmonary Function in Patients Undergoing Coronary Artery Bypass Surgery. Pain Manag Nurs. 2018 08;19(4):408-14. doi: https://dx.doi.org/10.1016/j.pmn.2017.10.01 8. PMID: 29396016. Exclusion reason: Ineligible setting

813. Jakobsen MD, Sundstrup E, Brandt M, et al. Effect of workplace- versus home-based physical exercise on pain in healthcare workers: study protocol for a single blinded cluster randomized controlled trial. BMC Musculoskelet Disord. 2014 Apr 07;15:119. doi: 10.1186/1471-2474-15-119. PMID: 24708570. Exclusion reason: Ineligible publication type

814. Jamieson MD, Everhart JS, Lin JS, et al. Reduction of Opioid Use After UpperExtremity Surgery through a Predictive Pain Calculator and Comprehensive Pain Plan. J Hand Surg Am. 2019 Dec;44(12):1050-9.e4. doi:

https://dx.doi.org/10.1016/j.jhsa.2019.10.00 2. PMID: 31806120. Exclusion reason: Ineligible comparator

815. Jansen JP, Peters JH. Efficacy and tolerability of the non-steroidal antirheumatic agent nabumetone in daily practice. Journal fur Pharmakologie und Therapie. 2008;17(5):147-53. Exclusion reason: Not in English

816. Jawahar R, Oh U, Yang S, et al. A systematic review of pharmacological pain management in multiple sclerosis. Drugs. 2013 Oct;73(15):1711-22. doi: https://dx.doi.org/10.1007/s40265-0130125-0. PMID: 24085618. Exclusion reason: Publication used as source document

817. Jellema P, van der Windt DA, van der Horst HE, et al. Why is a treatment aimed at psychosocial factors not effective in patients with (sub)acute low back pain? Pain. 2005 Dec 05;118(3):350-9. PMID: 16289797. Exclusion reason: Ineligible intervention 
818. Jellish WS, Owen K, Fluder E, et al. Patientcontrolled analgesia combined with either ondansetron or ondansetron plus prochlorperazine for control of pain and nausea and vomiting in patients undergoing abdominal surgery. J Clin Anesth. 2008 Dec;20(8):594-600. doi: 10.1016/j.jclinane.2008.06.008. PMID: 19100932. Exclusion reason: Ineligible intervention

819. Jensen JE, Conn RR, Hazelrigg G, et al. The use of transcutaneous neural stimulation and isokinetic testing in arthroscopic knee surgery. Am J Sports Med. 1985 JanFeb;13(1):27-33. doi:

10.1177/036354658501300105. PMID: 3872082. Exclusion reason: Ineligible study design

820. Jeon HR, Chae WS, Lee SJ, et al. A comparison of sufentanil and fentanyl for patientcontrolled epidural analgesia in arthroplasty. Korean J Anesthesiol. 2011;60(1):41-6. doi: 10.4097/kjae.2011.60.1.41. PMID: 21359080. Exclusion reason: Ineligible intervention

821. Jesus RR, Leite AM, Leite SS, et al. Anesthetic therapy for acute pain relief after laparoscopic cholecystectomy: systematic review. Rev Col Bras Cir. 2018 Jul 30;45(4):e1885. doi: https://dx.doi.org/10.1590/0100-6991e20181885. PMID: 30066738. Exclusion reason: Publication used as source document

822. Jin M, Chen J. Acupuncture treatment for 40 cases of acute lumbar sprain. Journal of Gansu College of Traditional Chinese Medicine. 2008;2006:49-50. Exclusion reason: Not in English

823. Jin X, Mi W. Adenosine for postoperative analgesia: A systematic review and metaanalysis. PLoS ONE [Electronic Resource]. 2017;12(3):e0173518. doi: https://dx.doi.org/10.1371/journal.pone.0173 518. PMID: 28333936. Exclusion reason: Publication used as source document
824. Jivraj NK, Raghavji F, Bethell J, et al. Persistent Postoperative Opioid Use: A Systematic Literature Search of Definitions and Population-based Cohort Study. Anesthesiology. 2020 Jun;132(6):1528-39. doi: 10.1097/aln.0000000000003265. PMID: 32243330. Exclusion reason: Publication used as source document

825. Johannsen M, O'Connor M, O'Toole MS, et al. Efficacy of Mindfulness-Based Cognitive Therapy on Late Post-Treatment Pain in Women Treated for Primary Breast Cancer: A Randomized Controlled Trial. J Clin Oncol. 201610 01;34(28):3390-9. doi: 10.1200/JCO.2015.65.0770. PMID: 27325850. Exclusion reason: Ineligible population

826. Johnson GH, Van Wagoner JD, Brown J, et al. Bromfenac sodium, acetaminophen/oxycodone, ibuprofen, and placebo for relief of postoperative pain. Clin Ther. 1997 May-Jun;19(3):507-19. doi: 10.1016/s0149-2918(97)80135-x. PMID: 9220215. Exclusion reason: Ineligible setting

827. Johnson L. Sickle cell disease patients and patient-controlled analgesia. Br J Nurs. 2003 Feb 13-26;12(3):144-53. PMID: 12610369. Exclusion reason: Ineligible intervention

828. Johnstone R, Donaghy M, Martin D. A pilot study of a cognitive-behavioural therapy approach to physiotherapy, for acute low back pain patients, who show signs of developing chronic pain. Adv Physiother. 2002;4(4):182-8. Exclusion reason: Ineligible comparator

829. Jonassaint CR, Kang C, Prussien KV, et al. Feasibility of implementing mobile technology-delivered mental health treatment in routine adult sickle cell disease care. Transl Behav Med. 2018doi: 10.1093/tbm/iby107. PMID: 30508141. Exclusion reason: Ineligible population

830. Jonsson PE, Olsson AM, Petersson BA, et al. Intravenous indomethacin and oxyconepapaverine in the treatment of acute renal colic. A double-blind study. Br J Urol. 1987 May;59(5):396-400. doi: 10.1111/j.1464410x.1987.tb04833.x. PMID: 3297230.

Exclusion reason: Ineligible comparator 
831. Joshi A, Snowdon AT, Rood JP, et al. Pain control after routine dento-alveolar day surgery: a patient satisfaction survey. $\mathrm{Br}$ Dent J. 2000 Oct 28;189(8):439-42. PMID: 11093393. Exclusion reason: Ineligible study design

832. Juhl GI, Norholt SE, Tonnesen E, et al. Analgesic efficacy and safety of intravenous paracetamol (acetaminophen) administered as a $2 \mathrm{~g}$ starting dose following third molar surgery. Eur J Pain. 2006 May;10(4):371-7. doi: 10.1016/j.ejpain.2005.06.004. PMID: 16085437. Exclusion reason: Ineligible intervention

833. Jung JH, Jang HJ, Bang CS, et al. Efficacy of submucosal bupivacaine injection for pain relief after endoscopic submucosal dissection: A multicenter, prospective, randomized controlled, and double-blind trial. Medicine. 2019 Apr;98(17):e15360. doi: 10.1097/MD.0000000000015360. PMID: 31027120. Exclusion reason: Ineligible intervention

834. Justins DM, Richardson PH. Clinical management of acute pain. Br Med Bull. 1991 Jul;47(3):561-83. PMID: 1794072. Exclusion reason: Background information only

835. Kadhim-Saleh A, Maganti H, Ghert M, et al. Is low-level laser therapy in relieving neck pain effective? Systematic review and metaanalysis. Rheumatol Int. 2013

Oct;33(10):2493-501. doi: https://dx.doi.org/10.1007/s00296-0132742-z. PMID: 23579335. Exclusion reason: Publication used as source document

836. Kadi MR, Hepguler S, Atamaz FC, et al. Is interferential current effective in the management of pain, range of motion, and edema following total knee arthroplasty surgery? A randomized double-blind controlled trial. Clin Rehabil. 2019 Jun;33(6):1027-34. doi: http://dx.doi.org/10.1177/026921551982985 6. PMID: 30764635. Exclusion reason: Ineligible intervention
837. Kadiroglu AK, Sit D, Kayabasi H, et al. The effect of venlafaxine $\mathrm{HCl}$ on painful peripheral diabetic neuropathy in patients with type 2 diabetes mellitus. J Diabetes Complications. 2008 Jul-Aug;22(4):241-5. doi: 10.1016/j.jdiacomp.2007.03.010. PMID: 18413214. Exclusion reason: Ineligible population

838. Kaijser Alin C, Uzunel E, Grahn Kronhed AC, et al. Effect of treatment on back pain and back extensor strength with a spinal orthosis in older women with osteoporosis: a randomized controlled trial. Arch Osteoporos. 2019 Jan 09;14(1):5. doi: 10.1007/s11657-018-0555-0. PMID: 30623268. Exclusion reason: Ineligible population

839. Kainu JP, Sarvela J, Halonen P, et al. Continuous wound infusion with ropivacaine fails to provide adequate analgesia after caesarean section. Int $\mathrm{J}$ Obstet Anesth. 2012 Apr;21(2):119-24. doi: 10.1016/j.ijoa.2011.12.009. PMID: 22341892. Exclusion reason: Ineligible intervention

840. Kalron A, Bar-Sela S. A systematic review of the effectiveness of Kinesio Taping--fact or fashion? Eur J Phys Rehabil Med. 2013 Oct;49(5):699-709. PMID: 23558699.

Exclusion reason: Publication used as source document

841. Kalus SM, Kornman LH, Quinlivan JA. Managing back pain in pregnancy using a support garment: a randomised trial. BJOG. 2008 Jan;115(1):68-75. doi: 10.1111/j.14710528.2007.01538.x. PMID: 17999695.

Exclusion reason: Ineligible population

842. Kam E, Eslick G, Campbell I. An audit of the effectiveness of acupuncture on musculoskeletal pain in primary health care. Acupunct Med. 2002 Mar;20(1):35-8. PMID: 11926604. Exclusion reason: Background information only

843. Kampe S, Wolter K, Warm M, et al. Clinical equivalence of controlled-release oxycodone $20 \mathrm{mg}$ and controlled-release tramadol 200 mg after surgery for breast cancer. Pharmacology. 2009;84(5):276-81. doi: 10.1159/000242998. PMID: 19797937. Exclusion reason: Ineligible intervention 
844. Kankkunen P, Vaajoki A. The effectiveness of music on postoperative pain among adults and children: the unused resource. Pain Manag. 2019 Jul;9(4):339-41. doi: https://dx.doi.org/10.2217/pmt-2018-0074. PMID: 31215333. Exclusion reason: Ineligible publication type

845. Kanlayanaphotporn R, Chiradejnant A, Vachalathiti R. The immediate effects of mobilization technique on pain and range of motion in patients presenting with unilateral neck pain: a randomized controlled trial.

Arch Phys Med Rehabil. 2009

Feb;90(2):187-92. doi:

10.1016/j.apmr.2008.07.017. PMID:

19236972. Exclusion reason: Ineligible population

846. Kanlayanaphotporn R, Chiradejnant A, Vachalathiti R. Immediate effects of the central posteroanterior mobilization technique on pain and range of motion in patients with mechanical neck pain. Disabil Rehabil. 2010;32(8):622-8. doi: 10.3109/09638280903204716. PMID: 20205574. Exclusion reason: Ineligible population

847. Kantor T, Cavaliere MB, Hopper M, et al. A double-blind parallel comparison of ketoprofen, codeine, and placebo in patients with moderate to severe postpartum pain. J Clin Pharmacol. 1984 May-Jun;24(5-6):22834. PMID: 6747019. Exclusion reason: Ineligible setting

848. Kaplan S, Alpayci M, Karaman E, et al. Short-Term Effects of Kinesio Taping in Women with Pregnancy-Related Low Back Pain: A Randomized Controlled Clinical Trial. Med Sci Monit. 2016 Apr 18;22:1297301. PMID: 27088271. Exclusion reason: Ineligible population

849. Kara B, Baskurt F, Acar S, et al. The effect of TENS on pain, function, depression, and analgesic consumption in the early postoperative period with spinal surgery patients. Turk Neurosurg. 2011;21(4):61824. doi: 10.5137/1019-5149.JTN .4985-11.0. PMID: 22194125. Exclusion reason: Ineligible intervention
850. Karabayirli S, Demircioglu RI, Muslu B, et al. The comparative effects of transdermal and intramuscular diclofenac on postlaparoscopic surgery pain. Surg Laparosc Endosc Percutan Tech. 2012 Aug;22(4):374-8. doi: 10.1097/SLE.0b013e31825b2b76. PMID: 22874692. Exclusion reason: Ineligible comparator

851. Karanikolas M, Aretha D, Tsolakis I, et al. Optimized perioperative analgesia reduces chronic phantom limb pain intensity, prevalence, and frequency: a prospective, randomized, clinical trial. Anesthesiology. 2011 May;114(5):1144-54. doi: 10.1097/ALN.0b013e31820fc7d2. PMID: 21368651. Exclusion reason: Ineligible population

852. Karcioglu O, Topacoglu H, Dikme O, et al. A systematic review of the pain scales in adults: Which to use? Am J Emerg Med. 2018 Apr;36(4):707-14. doi:

https://dx.doi.org/10.1016/j.ajem.2018.01.00 8. PMID: 29321111. Exclusion reason: Publication used as source document

853. Karhade AV, Ogink PT, Thio Q, et al. Development of machine learning algorithms for prediction of prolonged opioid prescription after surgery for lumbar disc herniation. Spine J. 2019

11;19(11):1764-71. doi: https://dx.doi.org/10.1016/j.spinee.2019.06. 002. PMID: 31185292. Exclusion reason: Ineligible study design

854. Karmkar KK, Khambete DD, Gurav AN, et al. Comparision of efficacy of low dose (25 $\mathrm{mg}$ ) with high dose diclofenac (50 mg) in management of postoperative pain after periodontal flap surgery: A randomised clinical trial. J Clin Diagn Res.

2018;12(12):31-5. doi: 10.7860/JCDR/2018/31147.12394.

Exclusion reason: Ineligible comparator

855. Kashanian M, Akbari Z, Alizadeh MH. The effect of exercise on back pain and lordosis in pregnant women. Int J Gynaecol Obstet. 2009 Nov;107(2):160-1. doi: 10.1016/j.ijgo.2009.06.018. PMID: 19682681. Exclusion reason: Ineligible population 
856. Katz NP, Mou J, Paillard FC, et al. Predictors of response in patients with postherpetic neuralgia and HIV-associated neuropathy treated with the $8 \%$ capsaicin patch (Qutenza). Clin J Pain. 2015 Oct;31(10):859-66. doi: http://dx.doi.org/10.1097/AJP.00000000000 00186. PMID: 25503598. Exclusion reason: Publication used as source document

857. Kavanagh T, Hu P, Minogue S. Daycase laparoscopic cholecystectomy: a prospective study of post-discharge pain, analgesic and antiemetic requirements. Ir J Med Sci. 2008 Jun;177(2):111-5. doi: 10.1007/s11845-0080131-5. PMID: 18270763. Exclusion reason: Ineligible study design

858. Kawchuk GN, Edgecombe TL, Wong AY, et al. A non-randomized clinical trial to assess the impact of nonrigid, inelastic corsets on spine function in low back pain participants and asymptomatic controls. Spine J. 2015 Oct 01;15(10):2222-7. doi: 10.1016/j.spinee.2015.06.047. PMID: 26101179. Exclusion reason: Ineligible study design

859. Kay B. A study of strong oral analgesics: the relief of postoperative pain using dextromoramide, pentazocine and bezitramide. Br J Anaesth. 1973 Jun;45(6):623-8. PMID: 4577940. Exclusion reason: Ineligible intervention

860. Kay TM, Gross A, Goldsmith C, et al. Exercises for mechanical neck disorders. Cochrane Database Syst Rev. 2005 Jul 20(3):CD004250. PMID: 16034925.

Exclusion reason: Publication used as source document

861. Kaydok E, Levendoglu F, Ozerbil MO, et al. Comparison of the efficacy of gabapentin and pregabalin for neuropathic pain in patients with spinal cord injury: A crossover study. 2014;30(6):1343-8. Exclusion reason: Ineligible population

862. Keilani C, Simondet N, Maalouf R, et al. Effects of music intervention on anxiety and pain reduction in ambulatory maxillofacial and otorhinolaryngology surgery: a descriptive survey of 27 cases. Oral Maxillofac Surg 2017 Jun;21(2):227-32. doi: 10.1007/s10006-017-0616-3. PMID: 28365803. Exclusion reason: Ineligible study design
863. Keita H, Geachan N, Dahmani S, et al. Comparison between patient-controlled analgesia and subcutaneous morphine in elderly patients after total hip replacement. Br J Anaesth. 2003 Jan;90(1):53-7. PMID: 12488379. Exclusion reason: Ineligible duration

864. Keller A, Hayden J, Bombardier C, et al. Effect sizes of non-surgical treatments of non-specific low-back pain. Eur Spine J. 2007 Nov;16(11):1776-88. PMID: 17619914. Exclusion reason: Background information only

865. Kelley BP, Shauver MJ, Chung KC. Management of Acute Postoperative Pain in Hand Surgery: A Systematic Review. J Hand Surg Am. 2015 Aug;40(8):1610-9, 9.e1. doi: 10.1016/j.jhsa.2015.05.024. PMID: 26213198. Exclusion reason: Publication used as source document

866. Kellstein D, Leyva R. Evaluation of FixedDose Combinations of Ibuprofen and Acetaminophen in the Treatment of Postsurgical Dental Pain: A Pilot, DoseRanging, Randomized Study. Drugs in R \& D. 2020 Jun 06;06:06. doi: https://dx.doi.org/10.1007/s40268-02000310-7. PMID: 32506309. Exclusion reason: Ineligible comparator

867. Kempf KK, Konzelman JL, Schultz RE, et al. Comparison of meclofenamate sodium with buffered aspirin and placebo for the relief of postoperative dental pain. Clin Ther. 1987;9(6):594-601. PMID: 3326678. Exclusion reason: Ineligible comparator

868. Kennedy S, Baxter GD, Kerr DP, et al. Acupuncture for acute non-specific low back pain: a pilot randomised nonpenetrating sham controlled trial. Complement Ther Med. 2008 Jun;16(3):139-46. doi: 10.1016/j.ctim.2007.03.001. PMID: 18534326. Exclusion reason: Ineligible population

869. Kenney MP, Milling LS. The effectiveness of virtual reality distraction for reducing pain: A meta-analysis. Psychol Conscious. 2016 Sep;3(3):199-210. doi: http://dx.doi.org/10.1037/cns0000084. Exclusion reason: Publication used as source document 
870. Kerpsack JM, Fankhauser RA. The use of controlled-release versus scheduled oxycodone in the immediate postoperative period following total joint arthroplasty. Orthopedics. 2005 May;28(5):491-4. PMID: 15945606. Exclusion reason: Ineligible comparator

871. Kertesz SG, Gordon AJ. A crisis of opioids and the limits of prescription control: United States. Addiction. 2019 Jan;114(1):169-80. doi: http://dx.doi.org/10.1111/add.14394. PMID: 30039595. Exclusion reason: Background information only

872. Keskin C, Ozdemir O, Uzun I, et al. Effect of intracanal cryotherapy on pain after single-visit root canal treatment. Aust Endod J. 2017 Aug;43(2):83-8. doi: https://dx.doi.org/10.1111/aej.12175. PMID: 27699913. Exclusion reason: Ineligible intervention

873. Keskin EA, Onur O, Keskin HL, et al. Transcutaneous electrical nerve stimulation improves low back pain during pregnancy. Gynecol Obstet Invest. 2012;74(1):76-83. doi: 10.1159/000337720. PMID: 22722614.

Exclusion reason: Ineligible population

874. Khalifa MS, Sharkawi MA. Treatment of pain owing to acute ureteral obstruction with prostaglandin-synthetase inhibitor: a prospective randomized study. J Urol. 1986 Aug;136(2):393-5. doi: 10.1016/s00225347(17)44878-6. PMID: 3090274.

Exclusion reason: Ineligible comparator

875. Khaliq W, Alam S, Puri KN. Topical lidocaine for the treatment of postherpetic neuralgia. Cochrane Database Syst Rev. 2019(6) PMID: 17443559. Exclusion reason: Publication used as source document

876. Khanasuk Y, Ngarmukos S. Contemporary pain management in total knee arthroplasty. J Med Assoc Thai. 2012 Oct;95 Suppl 10:S238-44. PMID: 23451469. Exclusion reason: Background information only
877. Khedr EM, Sharkawy ESA, Attia AMA, et al. Role of transcranial direct current stimulation on reduction of postsurgical opioid consumption and pain in total knee arthroplasty: Double randomized clinical trial. Eur J Pain. 2017 09;21(8):1355-65. doi: https://dx.doi.org/10.1002/ejp.1034. PMID: 28440034. Exclusion reason: Ineligible intervention

878. Kho HG, Eijk RJ, Kapteijns WM, et al. Acupuncture and transcutaneous stimulation analgesia in comparison with moderate-dose fentanyl anaesthesia in major surgery. Clinical efficacy and influence on recovery and morbidity. Anaesthesia. 1991 Feb;46(2):129-35. doi: 10.1111/j.13652044.1991.tb09359.x. PMID: 1908190. Exclusion reason: Ineligible setting

879. Khobrani M, Perona S, Patanwala AE. Effect of a legislative mandate on opioid prescribing for back pain in the emergency department. Am J Emerg Med. 2019 Nov;37(11):2035-8. doi: 10.1016/j.ajem.2019.02.031. PMID: 30824275. Exclusion reason: Ineligible population

880. Khooshideh M, Latifi Rostami SS, Sheikh M, et al. Pulsed Electromagnetic Fields for Postsurgical Pain Management in Women Undergoing Cesarean Section: A Randomized, Double-Blind, Placebocontrolled Trial. Clin J Pain. 2017 02;33(2):142-7. doi: 10.1097/AJP.0000000000000376. PMID: 28060214. Exclusion reason: Ineligible intervention

881. Khoury M, Caspi S, Stalnikowics R, et al. Emergency Department Administration of Oxycodone by Nurses Treating Musculoskeletal Pain: An Observational Prospective. Isr Med Assoc J. 2018 05;5(20):281-5. PMID: 29761672. Exclusion reason: Ineligible intervention

882. Khurana G, Jindal P, Sharma JP, et al. Postoperative pain and long-term functional outcome after administration of gabapentin and pregabalin in patients undergoing spinal surgery. Spine (Phila Pa 1976). 2014 Mar 15;39(6):E363-8. doi: 10.1097/brs.0000000000000185. PMID: 24384657. Exclusion reason: Ineligible population 
883. Kieburtz K, Simpson D, Yiannoutsos C, et al. A randomized trial of amitriptyline and mexiletine for painful neuropathy in HIV infection. AIDS Clinical Trial Group 242 Protocol Team. Neurology. 1998 Dec;51(6):1682-8. PMID: 9855523. Exclusion reason: Ineligible population

884. Kiersch TA, Minic MR. The onset of action and the analgesic efficacy of Saridon (a propyphenazone/paracetamol/ caffeine combination) in comparison with paracetamol, ibuprofen, aspirin and placebo (pooled statistical analysis). Curr Med Res Opin. 2002;18(1):18-25. doi: 10.1185/030079902125000101. PMID: 11999141. Exclusion reason: Ineligible study design

885. Kihlstrand M, Stenman B, Nilsson S, et al. Water-gymnastics reduced the intensity of back/low back pain in pregnant women. Acta Obstet Gynecol Scand. 1999 Mar;78(3):180-5. PMID: 10078577. Exclusion reason: Ineligible population

886. Kim DG, Chung SH, Jung HB. The effects of neural mobilization on cervical radiculopathy patients' pain, disability, ROM, and deep flexor endurance. J Back Musculoskelet Rehabil. 2017 Sep 22;30(5):951-9. doi: 10.3233/BMR-140191. PMID: 28453446. Exclusion reason: Ineligible population

887. Kim K, Jo D, Kim E. Pulsed Radiofrequency to the Dorsal Root Ganglion in Acute Herpes Zoster and Postherpetic Neuralgia. Pain Physician. 2017 03;20(3):E411-E8. PMID: 28339440.

Exclusion reason: Ineligible study design

888. Kim S, Brathwaite R, Kim O. EvidenceBased Practice Standard Care for Acute Pain Management in Adults With Sickle Cell Disease in an Urgent Care Center. Qual Manag Health Care. 2017

Apr/Jun;26(2):108-15. doi: https://dx.doi.org/10.1097/QMH.000000000 0000135. PMID: 28375958. Exclusion

reason: Background information only
889. Kim SC, Choudhry N, Franklin JM, et al. Patterns and predictors of persistent opioid use following hip or knee arthroplasty. Osteoarthritis Cartilage. 2017 09;25(9):1399-406. doi: 10.1016/j.joca.2017.04.002. PMID: 28433815. Exclusion reason: Ineligible comparator

890. Kim SI, Ha KY, An HS, et al. Sex-specific and Age-specific Analgesia for Early Postoperative Pain Management after Lumbar Decompressive Surgery: a Randomized Clinical Trial. Clin Spine Surg. 2018doi: 10.1097/BSD.0000000000000761. PMID: 30585803 Exclusion reason: Ineligible setting

891. Kim SK, Min A, Jeon C, et al. Clinical outcomes and cost-effectiveness of massage chair therapy versus basic physiotherapy in lower back pain patients: A randomized controlled trial. Medicine. 2020 Mar;99(12):e19514. doi: https://dx.doi.org/10.1097/MD.0000000000 019514. PMID: 32195952. Exclusion reason: Ineligible population

892. King AA, Noetzel M, White DA, et al. Blood transfusion therapy is feasible in a clinical trial setting in children with sickle cell disease and silent cerebral infarcts. Pediatr Blood Cancer. 2008 Mar;50(3):599602. PMID: 17985350. Exclusion reason: Ineligible population

893. Kisaalita N, Staud R, Hurley R, et al. Placebo use in pain management: The role of medical context, treatment efficacy, and deception in determining placebo acceptability. Pain. 2014 Dec;155(12):263845. doi:

https://dx.doi.org/10.1016/j.pain.2014.09.02 9. PMID: 25267208. Exclusion reason: Background information only

894. Kishore-Kumar R, Schafer SC, Lawlor BA, et al. Single doses of the serotonin agonists buspirone and m-chlorophenylpiperazine do not relieve neuropathic pain. Pain. 1989 May;37(2):223-7. PMID: 2664664.

Exclusion reason: Ineligible comparator

895. Kittang G, Melvaer T, Baerheim A. Acupuncture versus antiflogistica by acute low back pain in general practice. Tidsskrift for den norske laegeforening. 2001;121(10):1207-10. Exclusion reason: Not in English 
896. Kizhakkeveettil A, Rose KA, Kadar GE, et al. Integrative Acupuncture and Spinal Manipulative Therapy Versus Either Alone for Low Back Pain: A Randomized Controlled Trial Feasibility Study. J Manipulative Physiol Ther. 2017 Mar Apr;40(3):201-13. doi: 10.1016/j.jmpt.2017.01.002. PMID: 28259496. Exclusion reason: Ineligible population

897. Kizhakkeveettil A, Rose KA, Kadar GE, et al. An Exploratory Analysis of Gender as a Potential Modifier of Treatment Effect Among Patients in a Randomized Controlled Trial of Integrative Acupuncture and Spinal Manipulation for Low Back Pain. J Manipulative Physiol Ther. 2019 Mar Apr;42(3):177-86. doi: 10.1016/j.jmpt.2018.11.005. PMID: 31253252. Exclusion reason: Ineligible population

898. Kjaer Petersen K, Lunn TH, Husted H, et al. The influence of pre- and perioperative administration of gabapentin on pain 3-4 years after total knee arthroplasty. Scandinavian J Pain. 201804 25;18(2):23745. doi: 10.1515/sjpain-2018-0027. PMID: 29794296. Exclusion reason: Ineligible intervention

899. Klaiman MD, Shrader JA, Danoff JV, et al. Phonophoresis versus ultrasound in the treatment of common musculoskeletal conditions. Medicine \& Science in Sports \& Exercise. 1998 Sep;30(9):1349-55. PMID: 9741602. Exclusion reason: Ineligible population

900. Klasser GD, Ahmed AS. How to manage acute herpes zoster affecting trigeminal nerves. Dent J. 2014;80:e42. PMID: 25437934. Exclusion reason: Background information only

901. Knox CR, Lall R, Hansen Z, et al. Treatment compliance and effectiveness of a cognitive behavioural intervention for low back pain: a complier average causal effect approach to the BeST data set. BMC Musculoskelet Disord. 2014 Jan 14;15:17. doi: 10.1186/1471-2474-15-17. PMID: 24423146. Exclusion reason: Ineligible population
902. Kober A, Dobrovits M, Djavan B, et al. Local active warming: an effective treatment for pain, anxiety and nausea caused by renal colic. J Urol. 2003 Sep;170(3):741-4. PMID: 12913687. Exclusion reason: Ineligible duration

903. Kober A, Scheck T, Greher M, et al. Prehospital analgesia with acupressure in victims of minor trauma: a prospective, randomized, double-blinded trial. Anesth Analg. 2002 Sep;95(3):723-7, table of contents. doi: 10.1097/00000539200209000-00035. PMID: 12198060. Exclusion reason: Ineligible duration

904. Koes BW, Bouter LM, van Mameren H, et al. Randomised clinical trial of manipulative therapy and physiotherapy for persistent back and neck complaints: results of one year follow up. BMJ. $1992 \mathrm{Mar}$ 07;304(6827):601-5. PMID: 1532760. Exclusion reason: Ineligible population

905. Koffler SP, Hampstead BM, Irani F, et al. The neurocognitive effects of 5 day anesthetic ketamine for the treatment of refractory complex regional pain syndrome. Arch Clin Neuropsychol. 2007 Aug;22(6):719-29. PMID: 17611073. Exclusion reason: Ineligible population

906. Kohama T, Inoue M. Pycnogenol alleviates pain associated with pregnancy. Phytother Res. 2006 Mar;20(3):232-4. PMID: 16521117. Exclusion reason: Ineligible intervention

907. Kohli M, Murali T, Gupta R, et al. Optimization of subarachanoid block by oral pregabalin for hysterectomy. J Anaesthesiol Clin Pharmacol. 2011;27(1):101-5. PMID: 21804717. Exclusion reason: Ineligible intervention

908. Kolliker K. Pentazocine, pethidine and placebo for postoperative pain. An assessment by means of ventilatory measurements and subjective evaluation. Acta Anaesthesiol Scand. 1972;16(1):11-6. PMID: 5068732. Exclusion reason: Ineligible intervention 
909. Kong J, Fufa DT, Gerber AJ, et al. Psychophysical Outcomes From a Randomized Pilot Study of Manual, Electro, and Sham Acupuncture Treatment on Experimentally Induced Thermal Pain. $\mathrm{J}$

Pain. 2005 Jan;6(1):55-64. doi: 10.1016/j.jpain.2004.10.005. PMID: 15629419 Exclusion reason: Ineligible population

910. Konkaev AK, Eltaeva AA, Zabolotskikh IB, et al. Evaluation of Efficacy and Safety

Postoperative Pain Management by Intramuscular Analgesia after Different Types of Anaesthesia: Pilot Clinical Prospective Study. Anesteziologiia i Reanimatologiia. 2016 Nov;61(6):407-11. PMID: 29894606. Exclusion reason: Not in English

911. Konrath GA, Lock T, Goitz HT, et al. The use of cold therapy after anterior cruciate ligament reconstruction. A prospective, randomized study and literature review. Am J Sports Med. 1996 Sep-Oct;24(5):629-33. doi: 10.1177/036354659602400511. PMID: 8883683. Exclusion reason: Ineligible outcome

912. Konstantinovic LM, Kanjuh ZM, Milovanovic AN, et al. Acute low back pain with radiculopathy: a double-blind, randomized, placebo-controlled study. Photomed Laser Surg. 2010 Aug;28(4):55360. doi: 10.1089/pho.2009.2576. PMID: 20001318. Exclusion reason: Ineligible intervention

913. Koo KI, Park DK, Youm YS, et al. Enhanced Reality Showing Long-Lasting Analgesia after Total Knee Arthroplasty: Prospective, Randomized Clinical Trial. Scientific Reports. 2018 02 05;8(1):2343. doi: https://dx.doi.org/10.1038/s41598-01820260-0. PMID: 29402908. Exclusion reason: Ineligible comparator

914. Koo PJ. Addressing stakeholders' needs: economics and patient satisfaction. Am J Health Syst Pharm. 2007 Mar 15;64(6 Suppl 4):S11-5. PMID: 17353555. Exclusion reason: Background information only
915. Koranyi S, Barth J, Trelle S, et al. Psychological interventions for acute pain after open heart surgery. Cochrane Database Syst Rev. 2014 May 26(5):CD009984. doi: 10.1002/14651858.CD009984.pub2. PMID: 24861376. Exclusion reason: Ineligible study design

916. Kordi R, Abolhasani M, Rostami M, et al. Comparison between the effect of lumbopelvic belt and home based pelvic stabilizing exercise on pregnant women with pelvic girdle pain; a randomized controlled trial. J Back Musculoskelet Rehabil. 2013;26(2):133-9. doi: 10.3233/BMR-201200357. PMID: 23640314. Exclusion reason: Ineligible population

917. Korthuis PT, McCarty D, Weimer M, et al. Primary Care-Based Models for the Treatment of Opioid Use Disorder: A Scoping Review. Ann Intern Med. 2017 Feb 21;166(4):268-78. doi: 10.7326/M16-2149. PMID: 27919103. Exclusion reason: Background information only

918. Kouzounias K, Lind G, Schechtmann G, et al. Comparison of percutaneous balloon compression and glycerol rhizotomy for the treatment of trigeminal neuralgia. $\mathrm{J}$ Neurosurg. 2010 Sep;113(3):486-92. doi: 10.3171/2010.1.JNS091106. PMID: 20187700. Exclusion reason: Ineligible intervention

919. Krammer A, Horton S, Tumilty S. Pulsed electromagnetic energy as an adjunct to physiotherapy for the treatment of acute low back pain: a randomised controlled trial. New Zealand Journal of Physiotherapy. 2015;43:16-22. doi: 10.7860/JCDR/2014/7404.4818. PMID: 25386469. Exclusion reason: Ineligible comparator

920. Krammer G, Stricker K, Jayawardene S, et al. Evaluation of the analgesic efficacy of two lumiracoxib regimens in the treatment of post-operative dental pain: Single-centre, double-blind, randomised placebocontrolled, parallel-group study. Journal of Clinical Research. 2008;11(25-39):25-39.

Exclusion reason: Not in English 
921. Krauss J, Creighton D, Ely JD, et al. The immediate effects of upper thoracic translatoric spinal manipulation on cervical pain and range of motion: a randomized clinical trial. J Man Manip Ther. 2008;16(2):93-9. doi: 10.1179/106698108790818530. PMID: 19119394. Exclusion reason: Ineligible population

922. Krishna R, Nataraj MS. Efficacy of a single dose of a transdermal diclofenac patch as pre-emptive postoperative analgesia: A comparison with intramuscular diclofenac. Southern African Journal of Anaesthesia and Analgesia. 2012;18(4):194-7. doi: 10.1080/22201173.2012.10872852.

Exclusion reason: Ineligible intervention

923. Kroslak M, Pirapakaran K, Murrell GAC. Counterforce bracing of lateral epicondylitis: a prospective, randomized, double-blinded, placebo-controlled clinical trial. J Shoulder Elbow Surg. 2019

Feb;28(2):288-95. doi:

10.1016/j.jse.2018.10.002. PMID: 30658774. Exclusion reason: Ineligible population

924. Kuhlmann AYR, de Rooij A, Kroese LF, et al. Meta-analysis evaluating music interventions for anxiety and pain in surgery. Br J Surg. 2018 06;105(7):773-83. doi: https://dx.doi.org/10.1002/bjs.10853. PMID: 29665028. Exclusion reason: Publication used as source document

925. Kuijpers T, van Middelkoop M, Rubinstein SM, et al. A systematic review on the effectiveness of pharmacological interventions for chronic non-specific lowback pain. Eur Spine J. 2011 Jan;20(1):4050. doi: https://dx.doi.org/10.1007/s00586010-1541-4. PMID: 20680369. Exclusion reason: Publication used as source document

926. Kukushkin ML, Brylev LV, Laskov VB, et al. [Results of a randomized double blind parallel study on the efficacy and safety of tolpersione in patients with acute nonspecific low back pain.] Zhurnal nevrologii i psihiatrii imeni s s korsakova. 2017;117(11):69-78. PMID: 29265090.

Exclusion reason: Not in English
927. Kuttila M, Le Bell Y, Savolainen-Niemi E, et al. Efficiency of occlusal appliance therapy in secondary otalgia and temporomandibular disorders. Acta Odontol Scand. 2002 Aug;60(4):248-54. PMID: 12222651. Exclusion reason: Ineligible population

928. Kuusniemi K, Zollner J, Sjovall S, et al. Prolonged-release oxycodone/naloxone in postoperative pain management: from a randomized clinical trial to usual clinical practice. J Int Med Res. 2012;40(5):177593. PMID: 23206459. Exclusion reason: Ineligible study design

929. Kvien TK, Viktil K. Pharmacotherapy for regional musculoskeletal pain. Best Pract Res Clin Rheumatol. 2003 Feb;17(1):13750. PMID: 12659825. Exclusion reason: Background information only

930. Kvorning N, Holmberg C, Grennert L, et al. Acupuncture relieves pelvic and low-back pain in late pregnancy. Acta Obstet Gynecol Scand. 2004 Mar;83(3):246-50. doi: 10.1111/j.0001-6349.2004.0215.x. PMID: 14995919. Exclusion reason: Ineligible population

931. Kwon IS, Kim J, Park KM. Effects of music therapy on pain, discomfort, and depression for patients with leg fractures. Daehan Ganho Haghoeji. 2006 Jun;36(4):630-6. PMID: 16825847. Exclusion reason: Ineligible population

932. Kwong WJ, Hammond G, Upmalis D, et al. Bowel function after tapentadol and oxycodone immediate release (IR) treatment in patients with low back or osteoarthritis pain. Clin J Pain. 2013 Aug;29(8):664-72. doi: 10.1097/AJP.0b013e318274b695. PMID: 23835764. Exclusion reason: Ineligible population

933. Laba E. Clinical evaluation of ice therapy for acute ankle sprain injuries. New Zealand Journal of Physiotherapy. 1989;17:7-9. Exclusion reason: Ineligible population 
934. Lai YT, Chan HL, Lin SH, et al. Farinfrared ray patches relieve pain and improve skin sensitivity in myofascial pain syndrome: A double-blind randomized controlled study. Complement Ther Med. 2017 Dec;35:127-32. doi: 10.1016/j.ctim.2017.10.007. PMID: 29154057. Exclusion reason: Ineligible population

935. Lakkakula B, Sahoo R, Verma H, et al. Pain Management Issues as Part of the Comprehensive Care of Patients with Sickle Cell Disease. Pain Manag Nurs. 2018 12;19(6):558-72. doi: https://dx.doi.org/10.1016/j.pmn.2018.06.00 4. PMID: 30076112. Exclusion reason: Background information only

936. Lall MP, Restrepo E. Predictors of Weeks to Opioid Cessation after Lumbar Fusion: A Prospective Cohort Study. Pain Manag Nurs. 2018 10;19(5):525-34. doi: 10.1016/j.pmn.2018.04.007. PMID: 29779792. Exclusion reason: Ineligible study design

937. Lam DM, Choi SW, Wong SS, et al. Efficacy of Pregabalin in Acute Postoperative Pain Under Different Surgical Categories: A Meta-Analysis. Medicine. 2015 Nov;94(46):e1944. doi: https://dx.doi.org/10.1097/MD.0000000000 001944. PMID: 26579802. Exclusion reason: Publication used as source document

938. Lam OT, Strenger DM, Chan-Fee M, et al. Effectiveness of the McKenzie Method of Mechanical Diagnosis and Therapy for Treating Low Back Pain: Literature Review With Meta-analysis. J Orthop Sports Phys Ther. 2018 06;48(6):476-90. doi: https://dx.doi.org/10.2519/jospt.2018.7562. PMID: 29602304. Exclusion reason: Publication used as source document

939. Lan F, Ma YH, Xue JX, et al. Transcutaneous electrical nerve stimulation on acupoints reduces fentanyl requirement for postoperative pain relief after total hip arthroplasty in elderly patients. Minerva Anestesiol. 2012;78(8):887-95. Exclusion reason: Ineligible setting
940. Landen BR. Heat or cold for the relief of low back pain? Phys Ther. 1967

Dec;47(12):1126-8. doi: 10.1093/ptj/47.12.1126. PMID: 4229712.

Exclusion reason: Ineligible setting

941. Lang T, Barker R, Steinlechner B, et al. TENS relieves acute posttraumatic hip pain during emergency transport. Journal of Trauma-Injury Infection \& Critical Care. 2007b Jan;62(1):184-8; discussion 8. PMID: 17215752. Exclusion reason: Ineligible duration

942. Lang T, Hager H, Funovits V, et al. Prehospital analgesia with acupressure at the Baihui and Hegu points in patients with radial fractures: a prospective, randomized, double-blind trial. Am J Emerg Med. 2007a Oct;25(8):887-93. PMID: 17920972. Exclusion reason: Ineligible duration

943. Lange B, Murray M, Chreiteh SS, et al. Postural control and shoulder steadiness in F-16 pilots: a randomized controlled study. Aviat Space Environ Med. 2014 Apr;85(4):420-5. PMID: 24754203. Exclusion reason: Ineligible population

944. Langenfeld A, Humphreys BK, de Bie RA, et al. Effect of manual versus mechanically assisted manipulations of the thoracic spine in neck pain patients: study protocol of a randomized controlled trial. Trials [Electronic Resource]. 2015 May 27;16:233. doi: 10.1186/s13063-015-0763-5. PMID: 26013142. Exclusion reason: Ineligible population

945. Langenfeld A, Humphreys BK, De Bie RA, et al. Comparing manual and mechanically assisted manipulations of the thoracic spine in neck pain patients: a pilot study. F1000Res. 2018;7. Exclusion reason: Ineligible population

946. Langevin P, Roy JS, Desmeules F. Cervical radiculopathy: study protocol of a randomised clinical trial evaluating the effect of mobilisations and exercises targeting the opening of intervertebral foramen [NCT01500044]. BMC Musculoskelet Disord. 2012 Jan 31;13:10. doi: 10.1186/1471-2474-13-10. PMID: 22293092. Exclusion reason: Ineligible study design 
947. Lao L, Bergman S, Hamilton GR, et al. Evaluation of acupuncture for pain control after oral surgery: a placebo-controlled trial. Arch Otolaryngol Head Neck Surg. 1999

May;125(5):567-72. doi:

10.1001/archotol.125.5.567. PMID:

10326816. Exclusion reason: Ineligible outcome

948. Lapane KL, Quilliam BJ, Benson C, et al. Impact of noncancer pain on health-related quality of life. Pain Pract. 2015

Apr;15(4):333-42. doi: https://dx.doi.org/10.1111/papr.12184. PMID: 24571122. Exclusion reason: Background information only

949. Larsen MK, Kofod T, Starch-Jensen T. Therapeutic efficacy of cryotherapy on facial swelling, pain, trismus and quality of life after surgical removal of mandibular third molars: A systematic review. J Oral Rehabil. 2019 Jun;46(6):563-73. doi: https://dx.doi.org/10.1111/joor.12789. PMID: 30869171. Exclusion reason: Publication used as source document

950. Lateef TA, Al-Anee AM, Agha MTF. Evaluation the Efficacy of Hilotherm Cooling System in Reducing Postoperative Pain and Edema in Maxillofacial Traumatized Patients and Orthognathic Surgeries. J Craniofac Surg. 2018 Oct;29(7):e697-e706. doi: https://dx.doi.org/10.1097/SCS.0000000000 004951. PMID: 30192290. Exclusion reason: Ineligible setting

951. Lau PM, Chow DH, Pope MH. Early physiotherapy intervention in an Accident and Emergency Department reduces pain and improves satisfaction for patients with acute low back pain: a randomised trial. Aust J Physiother. 2008;54(4):243-9. PMID: 19025504. Exclusion reason: Ineligible comparator

952. Laureano Filho JR, de Oliveira e Silva ED, Camargo IB, et al. The influence of cryotherapy on reduction of swelling, pain and trismus after third-molar extraction: A preliminary study. The J Am Dent Assoc. 2005 2005/06/01/;136(6):774-8. doi: https://doi.org/10.14219/jada.archive.2005.0 261. PMID: 16022042. Exclusion reason: Ineligible intervention
953. Lavin RA, Tao XG, Yuspeh L, et al. Relationship Between Opioid Prescribing Patterns and Claim Duration and Cost. Journal of Occupational \& Environmental Medicine. 2016 Mar;58(3):e90-3. doi: 10.1097/JOM.0000000000000625. PMID: 26949894. Exclusion reason: Ineligible outcome

954. Lazarus AA, Mayne TJ. Relaxation: Some limitations, side effects, and proposed solutions. Psychotherapy: Theory, Research, Practice, Training. 1990 Sum;27(2):261-6. doi: http://dx.doi.org/10.1037/00333204.27.2.261. Exclusion reason: Background information only

955. Le Parc JM, Van Ganse E, Moore N, et al. Comparative tolerability of paracetamol, aspirin and ibuprofen for short-term analgesia in patients with musculoskeletal conditions: results in 4291 patients. Clin Rheumatol. 2002 Feb;21(1):28-31. doi: 10.1007/s100670200007. PMID: 11954880. Exclusion reason: Ineligible population

956. Le Roux PD, Samudrala S. Postoperative pain after lumbar disc surgery: a comparison between parenteral ketorolac and narcotics. Acta Neurochirurgica. 1999;141(3):261-7. PMID: 10214482. Exclusion reason: Ineligible population

957. Leaver AM, Maher CG, Herbert RD, et al. A randomized controlled trial comparing manipulation with mobilization for recent onset neck pain. Arch Phys Med Rehabil. 2010 Sep;91(9):1313-8. doi: 10.1016/j.apmr.2010.06.006. PMID: 20801246. Exclusion reason: Ineligible comparator

958. Lee A, Chan SK, Chen PP, et al. The costs and benefits of extending the role of the acute pain service on clinical outcomes after major elective surgery. Anesth Analg. 2010 Oct;111(4):1042-50. doi: 10.1213/ANE.0b013e3181ed1317. PMID: 20705784. Exclusion reason: Ineligible comparator 
959. Lee AC, Driban JB, Price LL, et al. Responsiveness and minimally important differences for 4 Patient-Reported Outcomes Measurement Information System Short Forms: Physical Function, Pain Interference, Depression, and Anxiety in knee osteoarthritis. J Pain. 2017 Sep;18(9):1096110. doi: 10.1016/j.jpain.2017.05.001. PMID: 28501708. Exclusion reason: Ineligible study design

960. Lee CH, Lee TY, Her JS, et al. SingleBlinded, Randomized Preliminary Study Evaluating the Effect of Transcutaneous Electrical Nerve Stimulation on Postoperative Pain in Patients with Colles' Fracture. Journal of Alternative \& Complementary Medicine. 2015 Dec;21(12):754-8. doi: 10.1089/acm.2015.0119. PMID: 26496205. Exclusion reason: Ineligible setting

961. Lee EG, Lee HJ, Hyun DJ, et al. Efficacy of low dose gabapentin in acute herpes zoster for preventing postherpetic neuralgia: a prospective controlled study. Dermatologic Therapy. 2016 May;29(3):184-90. doi: 10.1111/dth.12331. PMID: 26799145. Exclusion reason: Ineligible comparator

962. Lee GY, Yamada J, Kyololo O, et al. Pediatric clinical practice guidelines for acute procedural pain: a systematic review. Pediatrics. 2014 Mar;133(3):500-15. doi: https://dx.doi.org/10.1542/peds.2013-2744. PMID: 24488733. Exclusion reason: Publication used as source document

963. Lee H, Moseley GL, Hubscher M, et al. Understanding how pain education causes changes in pain and disability: protocol for a causal mediation analysis of the PREVENT trial. J Physiother. 2015 Jul;61(3):156. doi: 10.1016/j.jphys.2015.03.004. PMID: 26070650. Exclusion reason: Ineligible study design

964. Lee J, Park HP, Jeong MH, et al. Efficacy of ketamine for postoperative pain following robotic thyroidectomy: A prospective randomised study. J Int Med Res. 2018 Mar;46(3):1109-20. doi: 10.1177/0300060517734679. PMID: 29124992. Exclusion reason: Ineligible intervention
965. Lee JH, Choi TY, Lee MS, et al. Acupuncture for acute low back pain: a systematic review. Clin J Pain. 2013 Feb;29(2):172-85. doi: 10.1097/AJP.0b013e31824909f9. PMID: 23269281. Exclusion reason: Publication used as source document

966. Lee JH, Kim DH, Kim DH, et al. Comparison of Clinical Efficacy of Epidural Injection With or Without Steroid in Lumbosacral Disc Herniation: A Systematic Review and Meta-analysis. Pain Physician. 2018 09;21(5):449-68. PMID: 30282390.

Exclusion reason: Publication used as source document

967. Lee JH, Shin KH, Bahk SJ, et al. Comparison of clinical efficacy of transforaminal and caudal epidural steroid injection in lumbar and lumbosacral disc herniation: A systematic review and metaanalysis. Spine J. 2018 12;18(12):2343-53. doi: https://dx.doi.org/10.1016/j.spinee.2018.06. 720. PMID: 30030083. Exclusion reason: Publication used as source document

968. Lee JH, Shin KH, Park SJ, et al. Comparison of Clinical Efficacy Between Transforaminal and Interlaminar Epidural Injections in Lumbosacral Disc Herniation: A Systematic Review and Meta-Analysis. Pain Physician. 2018 09;21(5):433-48. PMID: 30282389. Exclusion reason: Publication used as source document

969. Lee TG, Kang SB, Kim DW, et al. Comparison of early mobilization and diet rehabilitation program with conventional care after laparoscopic colon surgery: a prospective randomized controlled trial. Dis Colon Rectum. 2011 Jan;54(1):21-8. doi: 10.1007/DCR.0b013e3181fcdb3e. PMID: 21160309. Exclusion reason: Ineligible population

970. Lee TG, Shin JS, Ha IH, et al. Immediate effects of motion style acupuncture treatment (MSAT) in acute low back pain with severe disability: A multicenter, randomized, controlled trial. Osteoarthritis Cartilage. Conference. 2012;20(pp S269) PMID: 23639822. Exclusion reason: Ineligible publication type 
971. Lee YH, Lee WC, Chen MT, et al.

Acupuncture in the treatment of renal colic.

J Urol. 1992 Jan;147(1):16-8. doi:

10.1016/s0022-5347(17)37121-5. PMID:

1729516. Exclusion reason: Ineligible duration

972. Lehtola V, Luomajoki H, Leinonen V, et al. Efficacy of movement control exercises versus general exercises on recurrent subacute nonspecific low back pain in a subgroup of patients with movement control dysfunction. Protocol of a randomized controlled trial. BMC Musculoskelet Disord. 2012 Apr 11;13:55. doi: https://dx.doi.org/10.1186/1471-2474-13-55. PMID: 22494776. Exclusion reason: Background information only

973. Lehtola V, Luomajoki H, Leinonen V, et al. Sub-classification based specific movement control exercises are superior to general exercise in sub-acute low back pain when both are combined with manual therapy: A randomized controlled trial. BMC Musculoskelet Disord. 2016 Mar 22;17:135. doi: 10.1186/s12891-016-0986-y. PMID: 27005470. Exclusion reason: Ineligible population

974. Lehtonen T, Kellokumpu I, Permi J, et al. Intravenous indomethacin in the treatment of ureteric colic. A clinical multicentre study with pethidine and metamizol as the control preparations. Ann Clin Res. 1983;15(56):197-9. PMID: 6364951. Exclusion reason: Ineligible duration

975. Leininger B, Bronfort G, Evans R, et al. Spinal manipulation or mobilization for radiculopathy: a systematic review. Phys Med Rehabil Clin N Am. 2011

Feb;22(1):105-25. doi: 10.1016/j.pmr.2010.11.002. PMID: 21292148. Exclusion reason: Publication used as source document

976. Leininger BD, Evans R, Bronfort G. Exploring patient satisfaction: a secondary analysis of a randomized clinical trial of spinal manipulation, home exercise, and medication for acute and subacute neck pain. J Manipulative Physiol Ther. 2014 Oct;37(8):593-601. doi: https://dx.doi.org/10.1016/j.jmpt.2014.08.00 5. PMID: 25199824. Exclusion reason: Ineligible population
977. Lekic D, Cenic D. Pain and tooth pulp evoked potentials. Clinical

Electroencephalography. 1992 Jan;23(1):3746. PMID: 1733621. Exclusion reason: Ineligible population

978. Leman P, Kapadia Y, Herington J. Randomised controlled trial of the onset of analgesic efficacy of dexketoprofen and diclofenac in lower limb injury. Emerg Med J. 2003 Nov;20(6):511-3. PMID: 14623834. Exclusion reason: Ineligible comparator

979. Lemos L, Fontes R, Flores S, et al. Effectiveness of the association between carbamazepine and peripheral analgesic block with ropivacaine for the treatment of trigeminal neuralgia. J Pain Res. 2010;3:201-12. doi: 10.2147/JPR.S13154. PMID: 21197324. Exclusion reason: Ineligible comparator

980. Lenhardt R, Marker E, Goll V, et al. Mild intraoperative hypothermia prolongs postanesthetic recovery. Anesthesiology. 1997 Dec;87(6):1318-23. PMID: 9416715. Exclusion reason: Ineligible population

981. Leung PC. Treatment of low back pain with acupuncture. Am J Chin Med.

1979;7(4):372-8. PMID: 161691.

Exclusion reason: Ineligible study design

982. Levrini L, Carraro M, Rizzo S, et al. Prescriptions of NSAIDs to patients undergoing third molar surgery : an observational, prospective, multicentre survey. Clin Drug Investig. 2008;28(10):657-68. PMID: 18783304. Exclusion reason: Ineligible outcome

983. Levy AS, Marmar E. The role of cold compression dressings in the postoperative treatment of total knee arthroplasty. Clin Orthop Relat Res. 1993 Dec(297):174-8. PMID: 7902225. Exclusion reason: Ineligible setting

984. Lewis C, Souvlis T, Sterling M. StrainCounterstrain therapy combined with exercise is not more effective than exercise alone on pain and disability in people with acute low back pain: a randomised trial. J Physiother. 2011;57(2):91-8. doi: 10.1016/S1836-9553(11)70019-4. PMID: 21684490. Exclusion reason: Ineligible intervention 
985. Lewith GT, Turner GM. Retrospective analysis of the management of acute low back pain. Practitioner. 1982

Sep;226(1371):1614-8. PMID: 6216470.

Exclusion reason: Ineligible study design

986. Li F, Ma J, Kuang M, et al. The efficacy of pregabalin for the management of postoperative pain in primary total knee and hip arthroplasty: a meta-analysis. J Orthop

Surg. 2017 Mar 24;12(1):49. doi: https://dx.doi.org/10.1186/s13018-0170540-0. PMID: 28340617. Exclusion reason: Ineligible comparator

987. Li J, Li H, Xv ZK, et al. Enhanced recovery care versus traditional care following laminoplasty: A retrospective case-cohort study. Medicine. 2018 Nov;97(48):e13195. doi: 10.1097/MD.0000000000013195. PMID: 30508899. Exclusion reason: Ineligible study design

988. Li J, Song Y. Transcutaneous electrical nerve stimulation for postoperative pain control after total knee arthroplasty: A metaanalysis of randomized controlled trials. Medicine. 2017 Sep;96(37):e8036. doi: https://dx.doi.org/10.1097/MD.0000000000 008036. PMID: 28906393. Exclusion reason: Publication used as source document

989. Li L, Wang H, Shen L, et al. Early application of salvia miltiorrhiza at huatuojiaji points reduces incidence of postherpetic neuralgia. Int J Clin Exp Med. 2018;11(7):7216-23. Exclusion reason: Ineligible intervention

990. Li M, Law SW, Cheng J, et al. A comparison study on the efficacy of SpinoMed(R) and soft lumbar orthosis for osteoporotic vertebral fracture. Prosthet Orthot Int. 2015 Aug;39(4):270-6. doi: 10.1177/0309364614528204. PMID: 24711165. Exclusion reason: Ineligible population

991. Li Y, Chen B, Lin D. Effect of Abdominal Acupuncture Therapy on Postoperative Residual Symptoms in Patients with Lumbar Intervertebral Disc Herniation: An Observation of 45 Cases. Journal of New Chinese Medicine. 2008;5(049). Exclusion reason: Not in English
992. Li Z, Chen Y. Ketamine reduces pain and opioid consumption after total knee arthroplasty: A meta-analysis of randomized controlled studies. Int J Surg. 2019 Oct;70:70-83. doi: https://dx.doi.org/10.1016/j.ijsu.2019.08.026 . PMID: 31446006. Exclusion reason: Ineligible intervention

993. Li Z, Liu D, Dong J, et al. Effects of Cold Irrigation on Early Results after Total Knee Arthroplasty: A Randomized, Double-Blind, Controlled Study. Medicine. 2016 Jun;95(24):e3563. doi: 10.1097/MD.0000000000003563. PMID: 27310945. Exclusion reason: Ineligible intervention

994. Liaw MY, Chen CL, Chen JF, et al. Effects of Knight-Taylor brace on balance performance in osteoporotic patients with vertebral compression fracture. J Back Musculoskelet Rehabil. 2009;22(2):75-81. doi: 10.3233/bmr-2009-0218. PMID: 20023334. Exclusion reason: Ineligible outcome

995. Licciardone JC, Aryal S. Prevention of progressive back-specific dysfunction during pregnancy: an assessment of osteopathic manual treatment based on Cochrane Back Review Group criteria. J Am Osteopath Assoc. 2013 Oct;113(10):728-36. doi: 10.7556/jaoa.2013.043. PMID: 24084800. Exclusion reason: Ineligible population

996. Liddle SD, Pennick V. Interventions for preventing and treating low-back and pelvic pain during pregnancy. Cochrane Database Syst Rev. 2015(9)doi: 10.1002/14651858.CD001139.pub4. PMID: 26422811. Exclusion reason: Publication used as source document

997. Liebana-Hermoso S, Manzano-Moreno FJ, Vallecillo-Capilla MF, et al. Oral pregabalin for acute pain relief after cervicofacial surgery: a systematic review. Clin Oral Investig. 2018 Jan;22(1):119-29. doi: 10.1007/s00784-017-2272-2. PMID: 29101547. Exclusion reason: Publication used as source document 
998. Lim KT, Hwang EH, Cho JH, et al.

Comparative effectiveness of Chuna manual therapy versus conventional usual care for non-acute low back pain: a pilot randomized controlled trial. Trials [Electronic Resource]. 2019 Apr 15;20(1):216. doi:

10.1186/s13063-019-3302-y. PMID: 30987662. Exclusion reason: Ineligible population

999. Lim YH, Song JM, Choi EH, et al. Effects of Repetitive Peripheral Magnetic Stimulation on Patients With Acute Low Back Pain: a Pilot Study. Ann Rehabil Med. 2018;42(2):229-38. doi: 10.5535/arm.2018.42.2.229. PMID: 29765876 Exclusion reason: Ineligible intervention

1000. Lima AC, Fernandes GA, Gonzaga IC, et al. Low-Level Laser and Light-Emitting Diode Therapy for Pain Control in Hyperglycemic and Normoglycemic Patients Who Underwent Coronary Bypass Surgery with Internal Mammary Artery Grafts: A Randomized, Double-Blind Study with Follow-Up. Photomed Laser Surg. 2016 Jun;34(6):244-51. doi: 10.1089/pho.2015.4049. PMID: 27081873. Exclusion reason: Ineligible intervention

1001. Lin CL, Hwang SL, Jiang P, et al. Effect of Music Therapy on Pain After Orthopedic Surgery-A Systematic Review and MetaAnalysis. Pain Pract. 2019 Nov 30;30:30. doi: https://dx.doi.org/10.1111/papr.12864. PMID: 31785131. Exclusion reason: Publication used as source document

1002. Lin FS, Lin WY, Lai CH, et al. Analgesic efficacy of tramadol/acetaminophen and propoxyphene/acetaminophen for relief of postoperative wound pain. Acta Anaesthesiol Taiwan. 2012 Jun;50(2):49-53. doi: 10.1016/j.aat.2012.05.009. PMID: 22769857. Exclusion reason: Ineligible intervention

1003. Lin L, Bohnert AS, Price AM, et al. Pain acceptance and opiate use disorders in addiction treatment patients with comorbid pain. Drug and Alcohol Dependence. 2015 Dec;157:136-42. doi: http://dx.doi.org/10.1016/j.drugalcdep.2015. 10.017. PMID: 26530502. Exclusion reason: Background information only
1004. Lin PL, Fan SZ, Huang CH, et al. Analgesic effect of lidocaine patch $5 \%$ in the treatment of acute herpes zoster: a double-blind and vehicle-controlled study. Reg Anesth Pain Med. 2008 Jul-Aug;33(4):320-5. doi: 10.1016/j.rapm.2007.02.015. PMID: 18675742. Exclusion reason: Ineligible comparator

1005. Lindahl JB, Nydert P, Giesecke K, et al. Prepacked take-home analgesics in ambulatory surgery. Acute Pain. 2006;8(1):13-21. doi: 10.1016/j.acpain.2006.01.001. Exclusion reason: Ineligible comparator

1006. Lindback Y, Tropp H, Enthoven P, et al. PREPARE: Pre-surgery physiotherapy for patients with degenerative lumbar spine disorder: a randomized controlled trial protocol. BMC Musculoskelet Disord. 2016 07 11;17:270. doi: 10.1186/s12891-0161126-4. PMID: 27400960. Exclusion reason: Ineligible intervention

1007. Lindenhovius AL, Helmerhorst GT, Schnellen AC, et al. Differences in prescription of narcotic pain medication after operative treatment of hip and ankle fractures in the United States and The Netherlands. Journal of Trauma-Injury Infection \& Critical Care. 2009 Jul;67(1):160-4. doi: https://dx.doi.org/10.1097/TA.0b013e31818 c12ee. PMID: 19590328. Exclusion reason: Background information only

1008. Lingner H, Blase L, Grosshennig A, et al. Manual therapy applied by general practitioners for nonspecific low back pain: results of the ManRuck pilot-study. Chiropr Man Therap. 2018;26(1)doi: 10.1186/s12998-018-0202-2. PMID: 30186593. Exclusion reason: Ineligible study design

1009. Linton SJ, Andersson T. Can chronic disability be prevented? A randomized trial of a cognitive-behavioral intervention and two forms of information for patients with spinal pain. Spine. 2000;25(21):2825-31. doi: 10.1097/00007632-200011010-00017. PMID: 11064530. Exclusion reason: Ineligible population 
1010. Linton SJ, Andersson T. Can chronic disability be prevented? A randomized trial of a cognitive-behavior intervention and two forms of information for patients with spinal pain. Spine. 2000 Nov 01;25(21):2825-31; discussion 4. doi: 10.1097/00007632200011010-00017. PMID: 11064530. Exclusion reason: Ineligible study design

1011. Linton SJ, Hellsing AL, Andersson D. A controlled study of the effects of an early intervention on acute musculoskeletal pain problems. Pain. 1993 Sep;54(3):353-9. PMID: 8233552. Exclusion reason: Ineligible intervention

1012. Lisinski P, Huber J, Wilkosz P, et al. Supervised versus uncontrolled rehabilitation of patients after rotator cuff repair-clinical and neurophysiological comparative study. Int J Artif Organs. 2012 Jan;35(1):45-54. doi: 10.5301/ijao.5000037. PMID: 22287203. Exclusion reason: Ineligible intervention

1013. Lison JF, Amer-Cuenca JJ, Piquer-Marti S, et al. Transcutaneous Nerve Stimulation for Pain Relief During Office Hysteroscopy: A Randomized Controlled Trial. Obstet Gynecol. 2017 02;129(2):363-70. doi: 10.1097/AOG.0000000000001842. PMID: 28079781. Exclusion reason: Ineligible population

1014. Lithgow DM, Blecher J. A controlled trial of glifanan and omnopon in postoperative pain. South African Medical Journal. SuidAfrikaanse Tydskrif Vir Geneeskunde. 1971 Feb 20;45(8):203-5. PMID: 4928687.

Exclusion reason: Ineligible intervention

1015. Litkowski LJ, Christensen SE, Adamson DN, et al. Analgesic efficacy and tolerability of oxycodone $5 \mathrm{mg} /$ ibuprofen $400 \mathrm{mg}$ compared with those of oxycodone 5 mg/acetaminophen $325 \mathrm{mg}$ and hydrocodone $7.5 \mathrm{mg}$ /acetaminophen $500 \mathrm{mg}$ in patients with moderate to severe postoperative pain: a randomized, doubleblind, placebo-controlled, single-dose, parallel-group study in a dental pain model. Clin Ther. 2005 Apr;27(4):418-29. PMID: 15922815. Exclusion reason: Ineligible population
1016. Liu CC. Effect of otopoint-pellet pressure combined with Gabapentin Capsules in the treatment of acute pain of herpes zoster of stagnated heat of liver channel. Chinese Medicine Modern Distance Education of China [zhong guo zhong yi yao xian dai yuan cheng jiao yu]. 2015;13(23):82-3. Exclusion reason: Not in English

1017. Liu S, Carpenter RL, Mulroy MF, et al. Intravenous versus epidural administration of hydromorphone. Effects on analgesia and recovery after radical retropubic prostatectomy. Anesthesiology. 1995 Mar;82(3):682-8. PMID: 7533484.

Exclusion reason: Ineligible comparator

1018. Liu SY. Thirty cases of shingles at acute stage treated by the method of purging stasis and stopping pain with acupuncture. Henan Traditional Chinese Medicine [he nan zhong yi]. 2015;35(5):1144-5. Exclusion reason: Not in English

1019. Liu X, Hanney WJ, Masaracchio M, et al. Immediate Physical Therapy Initiation in Patients With Acute Low Back Pain Is Associated With a Reduction in Downstream Health Care Utilization and Costs. Phys Ther 201805 01;98(5):336-47. doi: https://dx.doi.org/10.1093/ptj/pzy023. PMID: 29669083. Exclusion reason: Background information only

1020. Liu XL, Tan JY, Molassiotis A, et al. Acupuncture-Point Stimulation for Postoperative Pain Control: A Systematic Review and Meta-Analysis of Randomized Controlled Trials. Evid Based Complement Alternat Med. 2015;2015:657809. doi: https://dx.doi.org/10.1155/2015/657809. PMID: 26568767. Exclusion reason: Publication used as source document

1021. Liu Y, Petrini MA. Effects of music therapy on pain, anxiety, and vital signs in patients after thoracic surgery. Complement Ther Med. 2015 Oct;23(5):714-8. doi: 10.1016/j.ctim.2015.08.002. PMID: 26365452. Exclusion reason: Ineligible population

1022. Liu Y, Zhang K, Liu Y, et al. OxyContin tablets for postoperative analgesia. Eur J Pain Supplements. 2007 Sep;1(S1):101-2. doi: 10.1016/S1754-3207\%2808\%29600346. Exclusion reason: Ineligible publication type 
1023. Liu YT, Chiu CW, Chang CF, et al. Efficacy and Safety of Acupuncture for Acute Low Back Pain in Emergency Department: a Pilot Cohort Study. Evid Based Complement Alternat Med. 2015doi: 10.1155/2015/179731. PMID: 26346626. Exclusion reason: Ineligible study design

1024. Lo A, Macpherson N, Spiwak R. Prospective randomized trial of patientcontrolled analgesia with ketamine and morphine or morphine alone after hysterectomy. Can J Hosp Pharm. 2008;61(5):334-9. Exclusion reason: Ineligible intervention

1025. Lo PH, Tsou MY, Chang KY. Modeling the trajectory of analgesic demand over time after total knee arthroplasty using the latent curve analysis. Clin J Pain. 2015;31(9):77681. doi: 10.1097/AJP.0000000000000172. PMID: 25370137. Exclusion reason: Ineligible intervention

1026. Long DM. Electrical stimulation for the control of pain. Arch Surg. 1977 Jul;112(7):884-8. PMID: 301734. Exclusion reason: Background information only

1027. Longo L, Tamburini A, Monti A, et al. Treatment with $904 \mathrm{~nm}$ and $10600 \mathrm{~nm}$ laser of acute lumbago: double blind control. Laser Clinical Research. 1988;3:16-20. Exclusion reason: Ineligible intervention

1028. Longo UG, Loppini M, Denaro L, et al. Osteoporotic vertebral fractures: current concepts of conservative care. Br Med Bull. 2012 Jun;102:171-89. doi: https://dx.doi.org/10.1093/bmb/ldr048. PMID: 22130906. Exclusion reason: Publication used as source document

1029. Lopez-Cedrun J, Videla S, Burgueno M, et al. Co-crystal of Tramadol-Celecoxib in Patients with Moderate to Severe Acute Post-surgical Oral Pain: A Dose-Finding, Randomised, Double-Blind, Placebo- and Active-Controlled, Multicentre, Phase II Trial. Drugs in R \& D. 2018 Jun;18(2):13748. doi: 10.1007/s40268-018-0235-y. PMID: 29799099. Exclusion reason: Ineligible intervention
1030. Lopez-de-Uralde-Villanueva I, MunozGarcia D, Gil-Martinez A, et al. A Systematic Review and Meta-Analysis on the Effectiveness of Graded Activity and Graded Exposure for Chronic Nonspecific Low Back Pain. Pain Med. 2016 Jan;17(1):172-88. PMID: 26235368. Exclusion reason: Publication used as source document

1031. Lourenzi VDGCM, Jones A, Lourenzi FM, et al. THU0638-HPR Effectiveness of the Transcutaneous Electrical Nerve Stimulation in Pain Control of Patients with Acute Low Back Pain: A Randomized Controlled Trial. Ann Rheum Dis. 2015;74(Suppl 2):1322. doi: 10.1136/annrheumdis-2015-eular.4989. Exclusion reason: Ineligible publication type

1032. Lovell SJ, Taira T, Rodriguez E, et al. Comparison of valdecoxib and an oxycodone-acetaminophen combination for acute musculoskeletal pain in the emergency department: a randomized controlled trial. Acad Emerg Med. 2004 Dec;11(12):127882. PMID: 15576517. Exclusion reason: Ineligible intervention

1033. Lowenstein M, Grande D, Delgado MK. Opioid Prescribing Limits for Acute Pain Striking the Right Balance. N Engl J Med. 2018 Aug 09;379(6):504-6. doi: https://dx.doi.org/10.1056/NEJMp1803661. PMID: 30089064. Exclusion reason: Ineligible publication type

1034. Lu K, Cheng MC, Ge X, et al. A retrospective review of acupuncture use for the treatment of pain in sickle cell disease patients: descriptive analysis from a single institution. Clin J Pain. 2014 Sep;30(9):82530. doi: 10.1097/AJP.0000000000000036. PMID: 24322996. Exclusion reason: Ineligible study design

1035. Lu VM, Goyal A, Graffeo CS, et al. Glossopharyngeal Neuralgia Treatment Outcomes After Nerve Section, Microvascular Decompression, or Stereotactic Radiosurgery: A Systematic Review and Meta-Analysis. World Neurosurg. 2018 Dec;120:572-82.e7. doi: https://dx.doi.org/10.1016/j.wneu.2018.09.0 42. PMID: 30240868. Exclusion reason: Publication used as source document 
1036. Lu YY, Su ML, Gau ML, et al. The efficacy of cold-gel packing for relieving episiotomy pain - a quasi-randomised control trial. Contemp Nurse. 2015;50(1):26-35. doi: 10.1080/10376178.2015.1010257. PMID: 26058405. Exclusion reason: Ineligible setting

1037. Luckett-Gatopoulos S, Thoma B, Milne K, et al. SGEM Hot Off the Press: Regional Nerve Blocks for Hip and Femoral Neck Fractures: A Systematic Review. CJEM. 2016 Jul;18(4):296-300. doi: https://dx.doi.org/10.1017/cem.2016.332. PMID: 27435938. Exclusion reason: Publication used as source document

1038. Lund PG, Jensen SK, Therkildsen MH, et al. [Treatment of acute pain due to ureteral calculi with intravenous indomethacin or pethidine]. Ugeskr Laeger. 1986 Jun 23;148(26):1601-4. PMID: 3529546. Exclusion reason: Not in English

1039. Lundstam SO, Leissner KH, Wahlander LA, et al. Prostaglandin-synthetase inhibition with diclofenac sodium in treatment of renal colic: comparison with use of a narcotic analgesic. Lancet. 1982 May 15;1(8281):1096-7. doi: 10.1016/s01406736(82)92278-4. PMID: 6122892.

Exclusion reason: Ineligible comparator

1040. Lunn TH, Husted H, Laursen MB, et al. Analgesic and sedative effects of perioperative gabapentin in total knee arthroplasty: a randomized, double-blind, placebo-controlled dose-finding study. Pain. 2015 Dec;156(12):2438-48. doi: 10.1097/j.pain.0000000000000309. PMID: 26230741. Exclusion reason: Ineligible comparator

1041. Luo P, Lou J, Yang S. Pain Management during Rehabilitation after Distal Radius Fracture Stabilized with Volar Locking Plate: A Prospective Cohort Study. Biomed Res Int. 2018;2018:5786089. doi: 10.1155/2018/5786089. PMID: 30519581. Exclusion reason: Ineligible study design

1042. Lurie-Luke E, Neubauer G, Lindl C, et al. An exploratory workplace study to investigate the perceived value of continuous low-level heatwrap therapy in manual workers. Occupational Medicine (Oxford, England). 2003;53(3):173-8. PMID: 12724551. Exclusion reason: Ineligible study design
1043. Lynch ME, Clark AJ, Sawynok J, et al. Topical 2\% amitriptyline and 1\% ketamine in neuropathic pain syndromes: a randomized, double-blind, placebocontrolled trial. Anesthesiology. 2005 Jul;103(1):140-6. PMID: 15983466. Exclusion reason: Ineligible population

1044. Lyon C, Piggott S, Langner S, et al. Clinical Inquiry: How do oral NSAIDs compare to other oral analgesics right after an acute musculoskeletal injury? J Fam Pract. 2018 02;67(2):110-1. PMID: 29400903.

Exclusion reason: Background information only

1045. MacAuley D. Back pain and physiotherapy. BMJ. 2004 Sep;329(7468):694-5. doi: 10.1136/BMJ.329.7468.694. PMID: 15388588. Exclusion reason: Ineligible publication type

1046. MacDonald RS, Bell CM. An open controlled assessment of osteopathic manipulation in nonspecific low-back pain. Spine (Phila Pa 1976). 1990 May;15(5):36470. doi: 10.1097/00007632-19900500000005. PMID: 2141951. Exclusion reason: Ineligible population

1047. Macedo LG, Saragiotto BT, Yamato TP, et al. Motor control exercise for acute nonspecific low back pain. Cochrane Database Syst Rev. 2016 Feb 10;2:CD012085. doi: 10.1002/14651858.CD012085. PMID: 26863390. Exclusion reason: Publication used as source document

1048. Machado LA, Maher CG, Herbert RD, et al. The effectiveness of the McKenzie method in addition to first-line care for acute low back pain: a randomized controlled trial. BMC Med. 2010 Jan 26;8:10. doi: 10.1186/1741-7015-8-10. PMID: 20102596. Exclusion reason: Ineligible intervention

1049. MacIntyre B, Hamilton J, Fricke T, et al. The efficacy of healing touch in coronary artery bypass surgery recovery: a randomized clinical trial. Altern Ther Health Med. 2008 Jul-Aug;14(4):24-32. PMID: 18616066. Exclusion reason: Ineligible setting 
1050. Macintyre PE, Huxtable CA, Flint SL, et al. Costs and consequences: a review of discharge opioid prescribing for ongoing management of acute pain. Anaesth Intensive Care. 2014 Sep;42(5):558-74. PMID: 25233168. Exclusion reason: Background information only

1051. MacLean CD, Fujii M, Ahern TP, et al. Impact of Policy Interventions on Postoperative Opioid Prescribing. Pain Med. 2019 Jun 1;20(6):1212-8. doi: 10.1093/pm/pny215. PMID: 30412235. Exclusion reason: Ineligible comparator

1052. Macznik AK, Schneiders AG, Athens J, et al. Does Acupressure Hit the Mark? A Three-Arm Randomized Placebo-Controlled Trial of Acupressure for Pain and Anxiety Relief in Athletes With Acute Musculoskeletal Sports Injuries. Clin J Sport Med. 2017 Jul;27(4):338-43. doi: 10.1097/JSM.0000000000000378. PMID: 28653963. Exclusion reason: Ineligible duration

1053. Madden K, George A, van der Hoek NJ, et al. Cannabis for pain in orthopedics: a systematic review focusing on study methodology. Can J Surg. 201912 01;62(6):369-80. doi: https://dx.doi.org/10.1503/cjs.001018. PMID: 31782292. Exclusion reason: Ineligible comparator

1054. Madhusudhan SK. Novel analgesic combination of tramadol, paracetamol, caffeine and taurine in the management of moderate to moderately severeacute low back pain. J Orthop. 2013;10(3):144-8. doi: 10.1016/j.jor.2013.07.001. PMID: 24396231. Exclusion reason: Ineligible comparator

1055. Magel J, Fritz JM, Greene T, et al. Outcomes of Patients With Acute Low Back Pain Stratified by the STarT Back Screening Tool: Secondary Analysis of a Randomized Trial. Phys Ther 201703 01;97(3):330-7. doi: 10.2522/ptj.20160298. PMID: 28204740. Exclusion reason: Ineligible intervention
1056. Maheshwari K, Cummings KC, 3rd, Farag E, et al. A temporal analysis of opioid use, patient satisfaction, and pain scores in colorectal surgery patients. J Clin Anesth. 2016 Nov;34:661-7. doi: https://dx.doi.org/10.1016/j.jclinane.2016.07 .005. PMID: 27687467. Exclusion reason: Background information only

1057. Mahler DL, Forrest Jr WH, Brown CR, et al. Assay of aspirin and naproxen analgesia. Clin Pharmacol Ther. 1976;19(1):18-23. PMID: 1106936. Exclusion reason: Ineligible population

1058. Mahmoud F, Tampi RR. Pharmacotherapy for neuropathic pain in the elderly: Focus on postherpetic neuralgia. Clin Geriatr. 2012;20(1):30-4. Exclusion reason: Ineligible study design

1059. Maiers MJ, Hartvigsen J, Schulz C, et al. Chiropractic and exercise for seniors with low back pain or neck pain: the design of two randomized clinical trials. BMC Musculoskelet Disord. 2007 Sep 18;8:94. PMID: 17877825. Exclusion reason: Background information only

1060. Majlesi J, Unalan H. High-power pain threshold ultrasound technique in the treatment of active myofascial trigger points: a randomized, double-blind, casecontrol study. Arch Phys Med Rehabil. 2004 May;85(5):833-6. PMID: 15129409. Exclusion reason: Ineligible comparator

1061. Makela K, Palomaki O, Pokkinen S, et al. Oral versus patient-controlled intravenous administration of oxycodone for pain relief after cesarean section. Arch Gynecol Obstet. 2019 10;300(4):903-9. doi: https://dx.doi.org/10.1007/s00404-01905260-3. PMID: 31422458. Exclusion reason: Ineligible comparator

1062. Makuloluwe RT, Mouzas GL. Ultrasound in the treatment of sprained ankles. Practitioner. 1977 Apr;218(1306):586-8. PMID: 323842. Exclusion reason: Ineligible outcome

1063. Malanga GA, Yan N, Stark J. Mechanisms and efficacy of heat and cold therapies for musculoskeletal injury. Postgrad Med. 2015 Jan;127(1):57-65. PMID: 25526231. Exclusion reason: Background information only 
1064. Maldonado-Avila M, Garduno-Arteaga LM, Vela-Mollinedo RA, et al. Comparison of three analgesic drug regimens with twelfth subcostal nerve block for pain control during extracorporeal shock wave lithotripsy. Int Urol Nephrol. 2018 Jan;50(1):49-53. doi: 10.1007/s11255-017-1746-0. PMID: 29151179. Exclusion reason: Ineligible intervention

1065. Malmivaara A, Aro T. The treatment of acute low back pain--bed rest, exercise therapy or ordinary activity? Duodecim; laaketieteellinen aikakauskirja. 1995;111(22):2101-2. PMID: 9841169. Exclusion reason: Not in English

1066. Malmstrom K, Ang J, Fricke JR, et al. The analgesic effect of etoricoxib relative to that of two opioid-acetaminophen analgesics: a randomized, controlled single-dose study in acute dental impaction pain. Curr Med Res Opin. 2005;21(1):141-9. Exclusion reason: Ineligible comparator

1067. Malmstrom K, Fricke JR, Kotey P, et al. A comparison of rofexocix versus celecoxib in treating pain after dental surgery: A singlecenter, randomized, double-blind, placeboand active-comparator-controlled, parallelgroup, single-dose study using the dental impaction pain model. Clin Ther: The International Peer-Reviewed Journal of Drug Therapy. 2002 Oct;24(10):1549-60. doi: 10.1016/S0149-2918\%2802\%29800595. PMID: 12462285. Exclusion reason: Ineligible comparator

1068. Manabe H, Dan K, Hirata K, et al. Optimum pain relief with continuous epidural infusion of local anesthetics shortens the duration of zoster-associated pain. Clin J Pain. 2004 Sep-Oct;20(5):302-8. PMID: 15322436. Exclusion reason: Ineligible intervention

1069. Manchikanti L, Kaye AD, Boswell MV, et al. A Systematic Review and Best Evidence Synthesis of the Effectiveness of Therapeutic Facet Joint Interventions in Managing Chronic Spinal Pain. Pain Physician. 2015 Jul-Aug;18(4):E535-82. PMID: 26218948. Exclusion reason: Publication used as source document
1070. Manvelian G, Daniels S, Gibofsky A. A phase 2 study evaluating the efficacy and safety of a novel, proprietary, nanoformulated, lower dose oral diclofenac. Pain Med. 2012 Nov;13(11):1491-8. doi: 10.1111/j.1526-4637.2012.01479.x. PMID: 23043637. Exclusion reason: Ineligible comparator

1071. Manvelian G, Hochberg MC, Daniels SE, et al. A phase 2 study of lower-dose, indomethacin submicron particle capsules demonstrates early onset of acute pain relief. Clin J Pain. 2014 Oct;30(10):846-51. doi: 10.1097/AJP.0000000000000053. PMID: 24300220. Exclusion reason: Ineligible comparator

1072. Manwaring P, Platt D. Acute pain management: the role of patient-controlled analgesia. Connecticut Medicine. 1988 Apr;52(4):223-5. PMID: 2897268. Exclusion reason: Ineligible publication type

1073. Marco CA, Plewa MC, Buderer N, et al. Comparison of oxycodone and hydrocodone for the treatment of acute pain associated with fractures: a double-blind, randomized, controlled trial. Acad Emerg Med. 2005 Apr;12(4):282-8. PMID: 15805317. Exclusion reason: Ineligible comparator

1074. Marcus DA. Duloxetine use in painful conditions. Expert Opin Pharmacother. 2011 Jun;12(8):1333-40. doi: 10.1517/14656566.2011.580739. PMID: 21548724. Exclusion reason: Ineligible publication type

1075. Marin TJ, Van Eerd D, Irvin E, et al. Multidisciplinary biopsychosocial rehabilitation for subacute low back pain. Cochrane Database Syst Rev. 201706 28;6:CD002193. doi: https://dx.doi.org/10.1002/14651858.CD002 193.pub2. PMID: 28656659. Exclusion reason: Publication used as source document

1076. Marinelli L, Mori L, Solaro C, et al. Effect of radial shock wave therapy on pain and muscle hypertonia: a double-blind study in patients with multiple sclerosis. Multiple Sclerosis. 2015 Apr;21(5):622-9. doi: 10.1177/1352458514549566. PMID: 25257616. Exclusion reason: Ineligible population 
1077. Markman JD, Frazer ME, Rast SA, et al. Double-blind, randomized, controlled, crossover trial of pregabalin for neurogenic claudication. Neurology. 2015 Jan 20;84(3):265-72. doi: 10.1212/WNL.0000000000001168. PMID: 25503625. Exclusion reason: Ineligible comparator

1078. Markman JD, Gewandter JS, Frazer ME, et al. A Randomized, Double-blind, PlaceboControlled Crossover Trial of Oxymorphone Hydrochloride and Propoxyphene/Acetaminophen Combination for the Treatment of Neurogenic Claudication Associated With Lumbar Spinal Stenosis. Spine. 2015 May 15;40(10):684-91. doi: 10.1097/BRS.0000000000000837. PMID: 25705958. Exclusion reason: Ineligible population

1079. Maroon JC, Bost JW. Omega-3 fatty acids (fish oil) as an anti-inflammatory: an alternative to nonsteroidal anti-inflammatory drugs for discogenic pain. Surgical Neurology. 2006 Apr;65(4):326-31. PMID: 16531187. Exclusion reason: Ineligible study design

1080. Marques EM, Blom AW, Lenguerrand E, et al. Local anaesthetic wound infiltration in addition to standard anaesthetic regimen in total hip and knee replacement: long-term cost-effectiveness analyses alongside the APEX randomised controlled trials. BMC Med. 2015 Jun 26;13:151. doi: 10.1186/s12916-015-0389-1. PMID: 26116078. Exclusion reason: Ineligible study design

1081. Marques EM, Jones HE, Elvers KT, et al. Local anaesthetic infiltration for perioperative pain control in total hip and knee replacement: systematic review and metaanalyses of short- and long-term effectiveness. BMC Musculoskelet Disord. 2014 Jul 05;15:220. doi: https://dx.doi.org/10.1186/1471-2474-15220. PMID: 24996539. Exclusion reason: Publication used as source document

1082. Marshall RC. Tramadol or hydrocodoneacetaminophen for acute musculoskeletal pain? J Fam Pract. 1998 Nov;47(5):330-1. PMID: 9834758. Exclusion reason: Ineligible publication type
1083. Martelete M, Fiori AM. Comparative study of the analgesic effect of transcutaneous nerve stimulation (TNS); electroacupuncture (EA) and meperidine in the treatment of postoperative pain. Acupunct Electrother Res. 1985;10(3):183-93. doi: 10.3727/036012985816714432. PMID: 2866672. Exclusion reason: Ineligible population

1084. Martell BA, O'Connor PG, Kerns RD, et al. Systematic review: opioid treatment for chronic back pain: prevalence, efficacy, and association with addiction. Ann Intern Med. 2007 Jan 16;146(2):116-27. PMID: 17227935. Exclusion reason: Publication used as source document

1085. Marti-Carvajal AJ, Conterno LO, KnightMadden JM. Antibiotics for treating acute chest syndrome in people with sickle cell disease. Cochrane Database Syst Rev. 2019(9) PMID: 25749695. Exclusion reason: Ineligible intervention

1086. Martin PR, Rose MJ, Nichols PJ, et al. Physiotherapy exercises for low back pain: process and clinical outcome. Int Rehabil Med. 1986;8(1):34-8. PMID: 2942511. Exclusion reason: Ineligible population

1087. Martin W, Forouzanfar T, Schulten E, et al. Effect of cold compression on pain after orthognathic surgery. 2014:61. Exclusion reason: Ineligible setting

1088. Martin WJ, Skorpil NE, Ashton-James CE, et al. Effect of vasoconstriction on pain after mandibular third molar surgery: A singleblind, randomized controlled trial. Quintessence Int. 2016;47(7):589-96. doi: 10.3290/j.qi.a36174. PMID: 27284581. Exclusion reason: Ineligible intervention

1089. Martinez V, Beloeil H, Marret E, et al. Nonopioid analgesics in adults after major surgery: systematic review with network meta-analysis of randomized trials. Br J Anaesth. 2017 Jan;118(1):22-31. doi: https://dx.doi.org/10.1093/bja/aew391. PMID: 28039239. Exclusion reason: Publication used as source document 
1090. Martinez-Segura R, Fernandez-de-las-Penas C, Ruiz-Saez M, et al. Immediate effects on neck pain and active range of motion after a single cervical high-velocity low-amplitude manipulation in subjects presenting with mechanical neck pain: a randomized controlled trial. J Manipulative Physiol Ther. 2006 Sep;29(7):511-7. doi: 10.1016/j.jmpt.2006.06.022. PMID: 16949939. Exclusion reason: Ineligible population

1091. Martins LD, Rezende M, Loguercio AD, et al. Analgesic efficacy of ketorolac associated with a tramadol/acetaminophen combination after third molar surgery - a randomized, triple-blind clinical trial. Medicina Oral, Patologia Oral y Cirugia Bucal. 2019 Jan 01;24(1):e96-e102. doi: https://dx.doi.org/10.4317/medoral.22744. PMID: 30573715. Exclusion reason: Ineligible comparator

1092. Martin-Saavedra JS, Vergara-Mendez LD, Talero-Gutierrez C. Music is an effective intervention for the management of pain: An umbrella review. Complement Ther Clin Pract. 2018 Aug;32:103-14. doi: 10.1016/j.ctcp.2018.06.003. PMID: 30057035. Exclusion reason: Publication used as source document

1093. Martorella G, Cote J, Racine M, et al. Webbased nursing intervention for selfmanagement of pain after cardiac surgery: pilot randomized controlled trial. JMIR. 2012 Dec 14;14(6):e177. doi: 10.2196/jmir.2070. PMID: 23241361.

Exclusion reason: Ineligible intervention

1094. Masaracchio M, Cleland JA, Hellman M, et al. Short-term combined effects of thoracic spine thrust manipulation and cervical spine nonthrust manipulation in individuals with mechanical neck pain: a randomized clinical trial. J Orthop Sports Phys Ther. 2013

Mar;43(3):118-27. doi: 10.2519/jospt.2013.4221. PMID: 23221367. Exclusion reason: Ineligible comparator
1095. Masiero S, Poli P, Bonaldo L, et al. Supervised training and home-based rehabilitation in patients with stabilized ankylosing spondylitis on TNF inhibitor treatment: a controlled clinical trial with a 12-month follow-up. Clin Rehabil. 2014 Jun;28(6):562-72. doi: 10.1177/0269215513512214. PMID: 24285801. Exclusion reason: Ineligible population

1096. Massey T, Derry S, Moore RA, et al. Topical NSAIDs for acute pain in adults. Cochrane Database Syst Rev. 2008(4)doi: 10.1002/14651858.CD007402. PMID: 20556778. Exclusion reason: Publication used as source document

1097. Masters CS, Yelland MJ. The use of therapeutic medications for soft-tissue injuries in sports medicine. Med J Aust. 2006 Feb 20;184(4):198; author reply -9. PMID: 16489911. Exclusion reason: Ineligible study design

1098. Matsutani N, Dejima H, Takahashi Y, et al. Pregabalin reduces post-surgical pain after thoracotomy: a prospective, randomized, controlled trial. Surg Today. 2015 Nov;45(11):1411-6. doi: https://dx.doi.org/10.1007/s00595-0141088-9. PMID: 25430812. Exclusion

reason: Ineligible setting

1099. Matthews AM, Fu R, Dana T, et al. Intranasal or transdermal nicotine for the treatment of postoperative pain. Cochrane Database Syst Rev. 2016 Jan 12(1):CD009634. doi: https://dx.doi.org/10.1002/14651858.CD009 634.pub2. PMID: 26756459. Exclusion reason: Ineligible comparator

1100. Maxwell L. Acute pain management: i.v. patient controlled analgesia places the patient in control. Can Oper Room Nurs J. 1992 Sep-Oct;10(3):12-5. PMID: 1451046. Exclusion reason: Ineligible publication type

1101. Mayer JM, Ralph L, Look M, et al. Treating acute low back pain with continuous lowlevel heat wrap therapy and/or exercise: a randomized controlled trial. Spine J. 2005 Jul-Aug;5(4):395-403. PMID: 15996609. Exclusion reason: Ineligible population 
1102. Mayer TG, Ruoff GE. Clinical evaluation of zomepirac in the treatment of acute orthopedic pain. J Clin Pharmacol. 1980;20(4 II):285-91. doi: 10.1002/j.15524604.1980.tb01710.x. PMID: 6991546.

Exclusion reason: Ineligible comparator

1103. McCaffrey R, Locsin R. The effect of music on pain and acute confusion in older adults undergoing hip and knee surgery. Holist Nurs Pract. 2006 Sep-Oct;20(5):218-24; quiz 25-6. doi: 10.1097/00004650200609000-00002. PMID: 16974175.

Exclusion reason: Ineligible setting

1104. McCormick Z, Chang-Chien G, Marshall B, et al. Phantom limb pain: a systematic neuroanatomical-based review of pharmacologic treatment. Pain Med. 2014 Feb;15(2):292-305. doi: https://dx.doi.org/10.1111/pme.12283. PMID: 24224475. Exclusion reason: Publication used as source document

1105. McDonald EL, Daniel JN, Rogero RG, et al. How Does Perioperative Ketorolac Affect Opioid Consumption and Pain Management After Ankle Fracture Surgery? Clin Orthop Relat Res. 2020 Jan;478(1):144-51. doi: https://dx.doi.org/10.1097/CORR.00000000 00000978. PMID: 31567579. Exclusion reason: Ineligible intervention

1106. McEvoy A, Livingstone JI, Cahill CJ. Comparison of diclofenac sodium and morphine sulphate for postoperative analgesia after day case inguinal hernia surgery. Ann R Coll Surg Engl. 1996 Jul;78(4):363-6. PMID: 8712652.

Exclusion reason: Ineligible comparator

1107. McFarland C. A comparison of clinical outcomes between early physical therapy intervention and usual care in individuals following anterior cervical fusion. Diss Abstr Int. 2014;74(7-B(E)):No Pagination Specified. Exclusion reason: Ineligible publication type

1108. McGrath B, Elgendy H, Chung F, et al. Thirty percent of patients have moderate to severe pain $24 \mathrm{hr}$ after ambulatory surgery: a survey of 5,703 patients. Can J Anaesth. 2004 Nov;51(9):886-91. PMID: 15525613. Exclusion reason: Background information only
1109. McGregor AH, Dore CJ, Morris TP, et al. ISSLS prize winner: Function After Spinal Treatment, Exercise, and Rehabilitation (FASTER): a factorial randomized trial to determine whether the functional outcome of spinal surgery can be improved. Spine. 2011 Oct 01;36(21):1711-20. doi: 10.1097/BRS.0b013e318214e3e6. PMID: 21378603. Exclusion reason: Ineligible population

1110. McGuinness BW. A double-blind comparison in general practice of a combination tablet containing orphenadrine citrate and paracetamol ('Norgesic') with paracetamol alone. J Int Med Res. 1983;11(1):42-5. doi: 10.1177/030006058301100109. PMID: 6219903. Exclusion reason: Ineligible comparator

1111. McGuirk B, Bogduk N. Evidence-based care for low back pain in workers eligible for compensation. Occup Med (Oxford). 2007 Jan;57(1):36-42. PMID: 17046988.

Exclusion reason: Background information only

1112. McGurk M, Robinson P, Rajayogeswaran V, et al. Clinical comparison of dexketoprofen trometamol, ketoprofen, and placebo in postoperative dental pain. J Clin Pharmacol. 1998 12;38(S1):46S-54S. PMID: 9882082. Exclusion reason: Ineligible intervention

1113. McMorland G, Suter E. Chiropractic management of mechanical neck and lowback pain: a retrospective, outcome-based analysis. J Manipulative Physiol Ther. 2000 Jun;23(5):307-11. PMID: 10863249.

Exclusion reason: Ineligible study design

1114. McNicol ED, Midbari A, Eisenberg E. Opioids for neuropathic pain. Cochrane Database Syst Rev. 2019(1). Exclusion reason: Publication used as source document

1115. McQuay HJ, Carroll D, Jadad AR, et al. Dextromethorphan for the treatment of neuropathic pain: A double-blind randomized controlled crossover trial with integral n-of-1 design. Pain. 1994 Oct;59(1):127-33. doi: 10.1016/03043959\%2894\%2990056-6. PMID: 7854793. Exclusion reason: Ineligible population 
1116. McQuay HJ, Moore RA, Berta A, et al. Randomized clinical trial of dexketoprofen/tramadol $25 \mathrm{mg} / 75 \mathrm{mg}$ in moderate-to-severe pain after total hip arthroplasty. Br J Anaesth. 2016

Feb;116(2):269-76. doi: 10.1093/bja/aev457. PMID: 26787797.

Exclusion reason: Ineligible intervention

1117. McQuay HJ, Moore RA, Eccleston C, et al. Systematic review of outpatient services for chronic pain control. Health Technol Assess. 1997;1(6):i-iv, 1-135. PMID: 9483161.

Exclusion reason: Publication used as source document

1118. McReynolds TM, Sheridan BJ. Intramuscular ketorolac versus osteopathic manipulative treatment in the management of acute neck pain in the emergency department: a randomized clinical trial. J Am Osteopath Assoc. 2005 Feb;105(2):5768. PMID: 15784928. Exclusion reason: Ineligible comparator

1119. Meglio M, Cioni B, Visocchi M, et al. Spinal cord stimulation in low back and leg pain. Stereotact Funct Neurosurg. 1994;62(1-4):263-6. PMID: 7631079.

Exclusion reason: Ineligible population

1120. Mehlisch DR, Aspley S, Daniels SE, et al. A single-tablet fixed-dose combination of racemic ibuprofen/paracetamol in the management of moderate to severe postoperative dental pain in adult and adolescent patients: a multicenter, two-stage, randomized, double-blind, parallel-group, placebo-controlled, factorial study. Clin Ther. 2010 Jun;32(6):1033-49. doi: 10.1016/j.clinthera.2010.06.002. PMID: 20637958. Exclusion reason: Ineligible intervention

1121. Meier T, Wasner G, Faust M, et al. Efficacy of lidocaine patch $5 \%$ in the treatment of focal peripheral neuropathic pain syndromes: a randomized, double-blind, placebo-controlled study. Pain. 2003 Nov;106(1-2):151-8. PMID: 14581122. Exclusion reason: Ineligible population
1122. Melcher C, Wegener B, Jansson V, et al. [Managment of acute low back pain without trauma - an algorithm]. Zeitschrift fur Orthopadie \& Unfallchirurgie. 2018 Oct;156(5):554-60. doi: https://dx.doi.org/10.1055/a-0586-4815. PMID: 29758581. Exclusion reason: Not in English

1123. Melzack R, Guite S, Gonshor A. Relief of dental pain by ice massage of the hand. Can Med Assoc J. 1980 Jan 26;122(2):189-91. PMID: 7363212. Exclusion reason: Ineligible comparator

1124. Melzack R, Jeans ME, Stratford JG, et al. Ice massage and transcutaneous electrical stimulation: comparison of treatment for low-back pain. Pain. 1980 Oct;9(2):209-17. doi: 10.1016/0304-3959(80)90008-1. PMID: 6450393. Exclusion reason: Ineligible population

1125. Melzack R, Vetere P, Finch L. Transcutaneous electrical nerve stimulation for low back pain. A comparison of TENS and massage for pain and range of motion. Phys Ther 1983 Apr;63(4):489-93. PMID: 6220415. Exclusion reason: Ineligible population

1126. Menke JM. Do manual therapies help low back pain? A comparative effectiveness meta-analysis. Spine. 2014 Apr 01;39(7):E463-72. doi: https://dx.doi.org/10.1097/BRS.0000000000 000230. PMID: 24480940. Exclusion

reason: Publication used as source document

1127. Menzel NJ, Martinson IM. Effects of electrical surface stimulation on control of acute postoperative pain and prevention of atelectasis and ileus in patients having abdominal surgery. Commun Nurs Res. 1977 Mar;8:273-83. PMID: 585603. Exclusion reason: Ineligible intervention

1128. Menzel NN, Robinson ME. Back Pain in Direct Patient Care Providers: Early Intervention with Cognitive Behavioral Therapy. Pain Manag Nurs. 2006 Jun;7(2):53-63. doi: 10.1016/j.pmn.2006.02.002. PMID: 16730318. Exclusion reason: Ineligible population 
1129. Mercadante S, Villari P, Ferrera P, et al. Optimization of opioid therapy for preventing incident pain associated with bone metastases. J Pain Symptom Manage. 2004 Nov;28(5):505-10. doi:

10.1016/j.jpainsymman.2004.02.024. PMID: 15504626. Exclusion reason: Ineligible population

1130. Merrill HM, Dean DM, Mottla JL, et al. Opioid Consumption Following Foot and Ankle Surgery. Foot Ankle Int. 2018 06;39(6):649-56. doi: https://dx.doi.org/10.1177/10711007187575 27. PMID: 29506395. Exclusion reason: Background information only

1131. Merry AF, Gibbs RD, Edwards J, et al. Combined acetaminophen and ibuprofen for pain relief after oral surgery in adults: a randomized controlled trial. Br J Anaesth. 2010 Jan;104(1):80-8. doi: 10.1093/bja/aep338. PMID: 20007794. Exclusion reason: Ineligible intervention

1132. Miao Q, Qiang JH, Jin YL. Effectiveness of percutaneous neuromuscular electrical stimulation for neck pain relief in patients with cervical spondylosis. Medicine. 2018 Jun;97(26):e11080. doi: 10.1097/MD.0000000000011080. PMID: 29952946. Exclusion reason: Ineligible population

1133. Mibielli MA, Nunes CP, Cohen JC, et al. Treatment of acute, non-traumatic pain using a combination of diclofenaccholestyramine, uridine triphosphate, cytidine monophosphate, and hydroxycobalamin. Proceedings of the Western Pharmacology Society. 2010;53:512. PMID: 22128442. Exclusion reason: Ineligible intervention

1134. Michalek-Sauberer A, Heinzl H, SatorKatzenschlager SM, et al. Perioperative auricular electroacupuncture has no effect on pain and analgesic consumption after third molar tooth extraction. Anesth Analg. 2007 Mar;104(3):542-7. doi: 10.1213/01.ane.0000253233.51490.dd. PMID: 17312205. Exclusion reason: Ineligible intervention
1135. Michell AW, Sesath HGR. Feasibility Trial of Treatment of Ulnar Neuropathy at the Elbow Using a Specifically Designed Splint. J Clin Rheumatol. 2018 Jun 15;15:15. doi: 10.1097/RHU.0000000000000828. PMID: 29912777. Exclusion reason: Ineligible study design

1136. Michelotti A, Iodice G, Vollaro S, et al. Evaluation of the short-term effectiveness of education versus an occlusal splint for the treatment of myofascial pain of the jaw muscles. J Am Dent Assoc. 2012 Jan;143(1):47-53. doi: 10.14219/jada.archive.2012.0018. PMID: 22207667. Exclusion reason: Ineligible population

1137. Middleton RS. A comparison of two analgesic muscle relaxant combinations in acute back pain. Br J Clin Pract. 1984 Mar;38(3):107-9. PMID: 6231940. Exclusion reason: Ineligible comparator

1138. Mikanagi K, et al. Therapeutic Efficacy of Diflunisal for Acute Low Back Pain: a Multicenter Double-Blind Comparison with Mepirizole. Rinsho to kenkyu (the Japanese Journal of Clinical and Experimental Medicine). 1980;57(3):898-906. Exclusion reason: Not in English

1139. Miki K, Ikemoto T, Hayashi K, et al. Randomized open-labbel non-inferiority trial of acetaminophen or loxoprofen for patients with acute low back pain. J Orthop Sci. 2018 May;23(3):483-7. doi: 10.1016/j.jos.2018.02.007. PMID: 29503036. Exclusion reason: Ineligible intervention

1140. Miladinia M, Pishgooie AH, Aliyari S, et al. The comparison of the effect of two complementary medicine methods (Music therapy and massage therapy) on postoperative acute pain after abdominal surgery: a randomized clinical trial study. Iranian Red Crescent Medical Journal. 2017;19(6). Exclusion reason: Ineligible setting 
1141. Miller RC, Leenstra J, Qun R, et al. N09C6 (Alliance) - A Phase 3, Randomized Double-Blind Study of Doxepin Rinse Versus Placebo in the Treatment of Acute Oral Mucositis Pain in Patients Receiving Head and Neck Radiation Therapy With or Without Chemotherapy. Int J Radiat Oncol Biol Phys. 2013;85(1):21. Exclusion reason: Ineligible publication type

1142. Miller RR. Evaluation of the analgesic efficacy of ibuprofen. Pharmacotherapy: The Journal of Human Pharmacology \& Drug Therapy. 1981 Jul-Aug;1(1):21-7. PMID: 6765484. Exclusion reason: Background information only

1143. Miller-Matero LR, Coleman JP, SmithMason CE, et al. A Brief Mindfulness Intervention for Medically Hospitalized Patients with Acute Pain: A Pilot Randomized Clinical Trial. Pain Med. 2019 Apr 24;24:24. doi: 10.1093/pm/pnz082. PMID: 31329961. Exclusion reason: Ineligible setting

1144. Miller-Shahabar I, Schreuer N, Katsevman $\mathrm{H}$, et al. Efficacy of Compression Gloves in the Rehabilitation of Distal Radius Fractures: Randomized Controlled Study. Am J Phys Med Rehabil. 2018 12;97(12):904-10. doi: https://dx.doi.org/10.1097/PHM.000000000 0000998. PMID: 29994792. Exclusion reason: Ineligible population

1145. Miner JR, Moore J, Gray RO, et al. Oral versus intravenous opioid dosing for the initial treatment of acute musculoskeletal pain in the emergency department. Acad Emerg Med. 2008 Dec;15(12):1234-40. doi: 10.1111/j.1553-2712.2008.00266.x. PMID: 18945240. Exclusion reason: Ineligible population

1146. Minkowitz HS, Yarmush J, Donnell MT, et al. Safety and tolerability of fentanyl iontophoretic transdermal system: findings from a pooled data analysis of four clinical trials. J Opioid Manag. 2010 MayJun;6(3):203-10. PMID: 20642249.

Exclusion reason: Ineligible outcome
1147. Mishra H, Khan FA. A double-blind, placebo controlled randomised comparison of pre \& post operative administration of tramadol for dental extraction pain. Journal International Medical Sciences Academy. 2013;26(2):97-9. PMID: 22557747. Exclusion reason: Ineligible comparator

1148. Mishriky BM, Waldron NH, Habib AS. Impact of pregabalin on acute and persistent postoperative pain: a systematic review and meta-analysis. Br J Anaesth. 2015 Jan;114(1):10-31. doi: 10.1093/bja/aeu293. PMID: 25209095. Exclusion reason: Publication used as source document

1149. Mistiaen P, van Osch M, van Vliet L, et al. The effect of patient-practitioner communication on pain: a systematic review. Eur J Pain. 2016 May;20(5):675-88. doi: https://dx.doi.org/10.1002/ejp.797. PMID: 26492629. Exclusion reason: Publication used as source document

1150. Mitchinson AR, Kim HM, Rosenberg JM, et al. Acute postoperative pain management using massage as an adjuvant therapy: a randomized trial. Arch Surg. 2007 Dec;142(12):1158-67; discussion 67. PMID: 18086982. Exclusion reason: Ineligible setting

1151. Miyamoto GC, Costa LO, Cabral CM. Efficacy of the Pilates method for pain and disability in patients with chronic nonspecific low back pain: a systematic review with meta-analysis. Braz J Phys Ther. 2013 Nov-Dec;17(6):517-32. doi: https://dx.doi.org/10.1590/S141335552012005000127. PMID: 24346291. Exclusion reason: Publication used as source document

1152. Miyazaki S, Hagihara A, Kanda R, et al. Applicability of press needles to a doubleblind trial: a randomized, double-blind, placebo-controlled trial. Clin J Pain. 2009 Jun;25(5):438-44. doi: 10.1097/AJP.0b013e318193a6e1. PMID: 19454879. Exclusion reason: Ineligible population 
1153. Moeller C, Pawlowski J, Pappas AL, et al. The safety and efficacy of intravenous ketorolac in patients undergoing primary endoscopic sinus surgery: a randomized, double-blinded clinical trial. International Forum of Allergy \& Rhinology. 2012 JulAug;2(4):342-7. doi: 10.1002/alr.21028. PMID: 22411639. Exclusion reason: Ineligible intervention

1154. Mohamed SA, Abdel-Ghaffar HS. Effect of the addition of clonidine to locally administered bupivacaine on acute and chronic postmastectomy pain. J Clin Anesth. 2013 Feb;25(1):20-7. doi: 10.1016/j.jclinane.2012.05.006. PMID: 23391342. Exclusion reason: Ineligible intervention

1155. Mojsa IM, Pokrowiecki R, Lipczynski K, et al. Effect of submucosal dexamethasone injection on postoperative pain, oedema, and trismus following mandibular third molar surgery: a prospective, randomized, doubleblind clinical trial. Int J Oral Maxillofac Surg. 2017 Apr;46(4):524-30. doi: 10.1016/j.ijom.2016.11.006. PMID: 28012633. Exclusion reason: Ineligible intervention

1156. Molins-Cubero S, Rodriguez-Blanco C, Oliva-Pascual-Vaca A, et al. Changes in pain perception after pelvis manipulation in women with primary dysmenorrhea: a randomized controlled trial. Pain Med. 2014 Sep;15(9):1455-63. doi: 10.1111/pme.12404. PMID: 24666560. Exclusion reason: Ineligible duration

1157. Mondanaro JF, Homel P, Lonner B, et al. Music Therapy Increases Comfort and Reduces Pain in Patients Recovering From Spine Surgery. Am J Orthop (Chatham, $\mathrm{Nj}$ ). 2017 Jan/Feb;46(1):E13-E22. PMID: 28235116. Exclusion reason: Ineligible duration

1158. Montero-Oleas N, Arevalo-Rodriguez I, Nunez-Gonzalez S, et al. Therapeutic use of cannabis and cannabinoids: an evidence mapping and appraisal of systematic reviews. BMC Complementary Medicine and Therapies. 2020 Jan 15;20(1):12. doi: https://dx.doi.org/10.1186/s12906-0192803-2. PMID: 32020875. Exclusion reason: Ineligible population
1159. Moon JY, Lee PB, Kim YC, et al. Efficacy and Safety of $0.625 \%$ and $1.25 \%$ Capsaicin Patch in Peripheral Neuropathic Pain: MultiCenter, Randomized, and Semi-Double Blind Controlled Study. Pain Physician. 2017 02;20(2):27-35. PMID: 28158151. Exclusion reason: Ineligible population

1160. Moon YE, Seok H, Kim SH, et al. Extracorporeal shock wave therapy for sacroiliac joint pain: A prospective, randomized, sham-controlled short-term trial. J Back Musculoskelet Rehabil. 2017;30(4):779-84. doi: 10.3233/BMR150405. PMID: 28372309. Exclusion reason: Ineligible population

1161. Moore A, McQuay H, Gavaghan D. Deriving dichotomous outcome measures from continuous data in randomised controlled trials of analgesics: verification from independent data. Pain. 1997 Jan;69(12):127-30. PMID: 9060022. Exclusion reason: Background information only

1162. Moore AR, Collins SL, Edwards J, et al. Single dose oral dextropropoxyphene, alone and with paracetamol (acetaminophen), for postoperative pain. Cochrane Database Syst Rev. 2019(5) PMID: 10796793. Exclusion reason: Publication used as source document

1163. Moore AR, Derry S, Aldington D, et al. Single dose oral analgesics for acute postoperative pain in adults - an overview of Cochrane reviews. Cochrane Database Syst Rev. 2019(5) PMID: 26414123. Exclusion reason: Ineligible comparator

1164. Moore AR, Derry S, Mason L, et al. Single dose oral indometacin for the treatment of acute postoperative pain. Cochrane Database Syst Rev. 2019(5) PMID: 15495100.

Exclusion reason: Ineligible comparator

1165. Moore AR, Edwards J, Derry S, et al. Single dose oral dihydrocodeine for acute postoperative pain. Cochrane Database Syst Rev. 2019(5) PMID: 11034754. Exclusion reason: Ineligible comparator

1166. Moore AR, Edwards J, Loke KY, et al. Single dose oral piroxicam for acute postoperative pain. Cochrane Database Syst Rev. 2019(5) PMID: 11034755. Exclusion reason: Ineligible comparator 
1167. Moore D, Chong MS, Shetty A, et al. A systematic review of rescue analgesic strategies in acute exacerbations of primary trigeminal neuralgia. Br J Anaesth. 2019 Aug;123(2):e385-e96. doi: https://dx.doi.org/10.1016/j.bja.2019.05.026. PMID: 31208761. Exclusion reason: Ineligible intervention

1168. Moore N. Diclofenac potassium 12.5mg tablets for mild to moderate pain and fever: a review of its pharmacology, clinical efficacy and safety. Clin Drug Investig 2007;27(3):163-95. PMID: 17305413. Exclusion reason: Background information only

1169. Moore N, Charlesworth A, Van Ganse E, et al. Risk factors for adverse events in analgesic drug users: results from the PAIN study. Pharmacoepidemiology \& Drug Safety. 2003 Oct-Nov;12(7):601-10. PMID: 14558184. Exclusion reason: Ineligible study design

1170. Moore PA. Pain management in dental practice: tramadol vs. codeine combinations. J Am Dent Assoc. 1999 Jul;130(7):1075-9. PMID: 10422401. Exclusion reason: Ineligible study design

1171. Moore PA, Hersh EV. Combining ibuprofen and acetaminophen for acute pain management after third-molar extractions: translating clinical research to dental practice. J Am Dent Assoc. 2013 Aug;144(8):898-908. PMID: 23904576. Exclusion reason: Background information only

1172. Moore R, Gavaghan D, Tramer M, et al. Size is everything-Large amounts of information are needed to overcome random effects in estimating direction and magnitude of treatment effects. Pain. 1998 Dec;78(3):209-16. doi: http://dx.doi.org/10.1016/S03043959\%2898\%2900140-7. PMID: 9870574. Exclusion reason: Background information only

1173. Moore RA, Derry S. Diclofenac Potassium in Acute Postoperative Pain and Dysmenorrhoea: Results from Comprehensive Clinical Trial Reports. Pain Res Manag. 2018;2018:9493413. doi: https://dx.doi.org/10.1155/2018/9493413. PMID: 29623148. Exclusion reason: Publication used as source document
1174. Moore RA, Derry S, Aldington D, et al. Adverse events associated with single dose oral analgesics for acute postoperative pain in adults - an overview of Cochrane reviews. Cochrane Database Syst Rev. 2015 Oct 13(10):CD011407. doi: https://dx.doi.org/10.1002/14651858.CD011 407.pub2. PMID: 26461263. Exclusion reason: Ineligible comparator

1175. Moore RA, Derry S, Aldington D, et al. Single dose oral analgesics for acute postoperative pain in adults - an overview of Cochrane reviews. Cochrane Database Syst Rev. 2015 Sep 28(9):CD008659. doi: https://dx.doi.org/10.1002/14651858.CD008 659.pub3. PMID: 26414123. Exclusion reason: Ineligible comparator

1176. Moore RA, Derry S, Straube S, et al. Faster, higher, stronger? Evidence for formulation and efficacy for ibuprofen in acute pain. Pain. 2014 Jan;155(1):14-21. doi: 10.1016/j.pain.2013.08.013. PMID: 23969325. Exclusion reason: Ineligible intervention

1177. Moore RA, Derry S, Wiffen PJ, et al. Estimating relative efficacy in acute postoperative pain: network meta-analysis is consistent with indirect comparison to placebo alone. Pain. 2018 Nov;159(11):2234-44. doi: https://dx.doi.org/10.1097/j.pain.000000000 0001322. PMID: 29965830. Exclusion

reason: Publication used as source document

1178. Moore RA, Derry S, Wiffen PJ, et al. Overview review: Comparative efficacy of oral ibuprofen and paracetamol (acetaminophen) across acute and chronic pain conditions. Eur J Pain. 2015 Oct;19(9):1213-23. doi: https://dx.doi.org/10.1002/ejp.649. PMID: 25530283. Exclusion reason: Publication used as source document

1179. Moore RA, McQuay HJ, Tomaszewski J, et al. Dexketoprofen/tramadol 25 mg/75 mg: randomised double-blind trial in moderateto-severe acute pain after abdominal hysterectomy. BMC Anesthesiol. 2016 Jan 22;16:9. doi: https://dx.doi.org/10.1186/s12871-0160174-5. PMID: 26801905. Exclusion reason: Ineligible population 
1180. Moore RA, Wiffen PJ, Derry S, et al. Nonprescription (OTC) oral analgesics for acute pain - an overview of Cochrane reviews. Cochrane Database Syst Rev. 2015 Nov 04(11):CD010794. doi: https://dx.doi.org/10.1002/14651858.CD010 794.pub2. PMID: 26544675. Exclusion reason: Ineligible comparator

1181. Mora B, Giorni E, Dobrovits M, et al. Transcutaneous electrical nerve stimulation: an effective treatment for pain caused by renal colic in emergency care. J Urol. 2006 May;175(5):1737-41; discussion 41. PMID: 16600745. Exclusion reason: Ineligible duration

1182. Morrison BW, Fricke J, Brown J, et al. The optimal analgesic dose of rofecoxib: Overview of six randomized controlled trials. J Am Dent Assoc. 2000 Dec;131(12):1729-37. PMID: 11143737. Exclusion reason: Ineligible intervention

1183. Morrison GE, Chase W, Young V, et al. Back pain: treatment and prevention in a community hospital. Arch Phys Med Rehabil. 1988 Aug;69(8):605-9. PMID: 2970250. Exclusion reason: Ineligible intervention

1184. Morrison JD, Loan WB, Dundee JW. Controlled comparison of the efficacy of fourteen preparations in the relief of postoperative pain. British Medical Journal. 1971 Jul 31;3(5769):287-90. PMID: 4934047. Exclusion reason: Ineligible setting

1185. Morton JE. Manipulation in the treatment of acute low back pain. J Man Manip Ther. 1999;7(4):182-9. Exclusion reason: Ineligible comparator

1186. Mostafavifar M, Wertz J, Borchers J. A systematic review of the effectiveness of kinesio taping for musculoskeletal injury. Phys Sportsmed. 2012 Nov;40(4):33-40. doi: 10.3810/psm.2012.11.1986. PMID: 23306413. Exclusion reason: Publication used as source document
1187. Moukarzel M, Di Rienzo F, Lahoud J-C, et al. The therapeutic role of motor imagery during the acute phase after total knee arthroplasty: A pilot study. Disability and Rehabilitation: An International, Multidisciplinary Journal. 2019 Apr;41(8):926-33. doi: http://dx.doi.org/10.1080/09638288.2017.14 19289. PMID: 29275638. Exclusion reason: Ineligible intervention

1188. Mouravska N, Zielinski L, Bhatt M, et al. Adverse outcomes associated with opioid prescription for acute low back pain: a systematic review protocol. Systematic Reviews. 201708 14;6(1):163. doi: https://dx.doi.org/10.1186/s13643-0170556-x. PMID: 28807047. Exclusion reason: Publication used as source document

1189. Mousa SA, Abd Elfatah Alsobky H. Efficacy and effect of TIVA with propofol or dexmedetomidine versus sevoflurane without muscle relaxant during repair of the brachial plexus. Egyptian Journal of Anaesthesia. 2013;29(1):31-40. doi: 10.1016/j.egja.2012.08.001. Exclusion reason: Ineligible intervention

1190. Moyano JR, Zambrano SC. The influence of information leaflets on morphine consumption in postoperative patients using patient-controlled analgesia. J Pain Palliat Care Pharmacother. 2011;25(4):335-9. doi: 10.3109/15360288.2011.621515. PMID: 22126164. Exclusion reason: Ineligible intervention

1191. Mozafari J, Maleki Verki M, Motamed H, et al. Comparing intranasal ketamine with intravenous fentanyl in reducing pain in patients with renal colic: A double-blind randomized clinical trial. Am J Emerg Med. 2020 03;38(3):549-53. doi: https://dx.doi.org/10.1016/j.ajem.2019.05.04 9. PMID: 31155169. Exclusion reason: Ineligible duration

1192. Muckle DS. Flurbiprofen for the treatment of soft tissue trauma. American Journal of Medicine. 1986 Mar 24;80(3A):76-80. PMID: 2938471. Exclusion reason: Ineligible publication type 
1193. Mulroy MF, Burgess FW, Emanuelsson BM. Ropivacaine $0.25 \%$ and $0.5 \%$, but not $0.125 \%$, provide effective wound infiltration analgesia after outpatient hernia repair, but with sustained plasma drug levels. Reg Anesth Pain Med. 1999 Mar-Apr;24(2):13641. PMID: 10204899. Exclusion reason: Ineligible population

1194. Mumith A, Pavlou P, Barrett M, et al. Enhancing Postoperative Rehabilitation Following Knee Arthroplasty Using a New Cryotherapy Product: A Prospective Study. Geriatr Orthop Surg Rehabil. 2015 Dec;6(4):316-21. doi: 10.1177/2151458515609722. PMID: 26623168. Exclusion reason: Ineligible comparator

1195. Murgier J, Cassard X. Cryotherapy with dynamic intermittent compression for analgesia after anterior cruciate ligament reconstruction. Preliminary study. Orthop Traumatol Surg Res. 2014 May;100(3):30912. doi: 10.1016/j.otsr.2013.12.019. PMID: 24679367. Exclusion reason: Ineligible duration

1196. Murphy GS, Avram MJ, Greenberg SB, et al. Postoperative Pain and Analgesic Requirements in the First Year after Intraoperative Methadone for Complex Spine and Cardiac Surgery. Anesthesiology. 2020 02;132(2):330-42. doi: https://dx.doi.org/10.1097/ALN.0000000000 003025. PMID: 31939849. Exclusion reason: Ineligible intervention

1197. Myers RA, Woolf CJ, Mitchell D. Management of acute traumatic pain by peripheral transcutaneous electrical stimulation. South African Medical Journal. Suid-Afrikaanse Tydskrif Vir Geneeskunde. 1977 Aug 13;52(8):309-12. PMID: 302494. Exclusion reason: Ineligible study design

1198. Myers SS, Phillips RS, Davis RB, et al. Patient expectations as predictors of outcome in patients with acute low back pain. Journal of General Internal Medicine. 2008 Feb;23(2):148-53. PMID: 18066631. Exclusion reason: Ineligible study design
1199. Mylonas KS, Reinhorn M, Ott LR, et al. Patient-reported opioid analgesic requirements after elective inguinal hernia repair: A call for procedure-specific opioidadministration strategies. Surgery. 2017 11;162(5):1095-100. doi: https://dx.doi.org/10.1016/j.surg.2017.06.01 7. PMID: 28778580. Exclusion reason: Background information only

1200. Mystakidou K, Parpa E, Tsilika E, et al. Long-term management of noncancer pain with transdermal therapeutic systemfentanyl. J Pain. 2003 Aug;4(6):298-306. PMID: 14622686. Exclusion reason: Ineligible population

1201. Nadler SF, Steiner DJ, Erasala GN, et al. Continuous low-level heat wrap therapy provides more efficacy than Ibuprofen and acetaminophen for acute low back pain. Spine. 2002 May 15;27(10):1012-7. PMID: 12004166. Exclusion reason: Ineligible population

1202. Naesh O, Niles LA, Gilbert JG, et al. A randomized, placebo-controlled study of rofecoxib with paracetamol in early posttonsillectomy pain in adults. Eur $\mathrm{J}$ Anaesthesiol. 2005 Oct;22(10):768-73. PMID: 16211736. Exclusion reason: Ineligible intervention

1203. Naik P, Heggannavar A, Khatri S. Comparison of Muscle Energy Technique and positional release therapy in acute low back pain a RCT. Indian J Physiother and Occupational Therapy. 2010;4(2):32-6.

Exclusion reason: Ineligible comparator

1204. Naja ZM, El-Rajab M, Ziade F, et al. Preoperative vs. postoperative bilateral paravertebral blocks for laparoscopic cholecystectomy: a prospective randomized clinical trial. Pain Pract. 2011 NovDec;11(6):509-15. doi: 10.1111/j.15332500.2011.00447.x. PMID: 21447078. Exclusion reason: Ineligible intervention

1205. Nakashima H, Kanemura T, Ando K, et al. Is Pregabalin Effective Against Acute Lumbar Radicular Pain ? Spine Surgery \& Related Research. 2019 Jan 25;3(1):61-6. doi: https://dx.doi.org/10.22603/ssrr.20180003. PMID: 31435553. Exclusion reason: Ineligible intervention 
1206. Namba RS, Singh A, Paxton EW, et al. Patient Factors Associated With Prolonged Postoperative Opioid Use After Total Knee Arthroplasty. J Arthroplasty. 2018 08;33(8):2449-54. doi: 10.1016/j.arth.2018.03.068. PMID: 29753617. Exclusion reason: Ineligible intervention

1207. Nash MS, van de Ven I, van Elk N, et al. Effects of circuit resistance training on fitness attributes and upper-extremity pain in middle-aged men with paraplegia. Arch Phys Med Rehabil. 2007 Jan;88(1):70-5. PMID: 17207678. Exclusion reason: Ineligible population

1208. National Academy of Medicine. Evidencebased Clinical Practice Guidelines for Prescribing Opioids for Acute Pain. 2019. http://nationalacademies.org/hmd/Activities/ MentalHealth/GuidelinesforPrescribingOpio ids.aspx. Accessed May 29, 2019.

Exclusion reason: Ineligible publication type

1209. National Academy of Medicine. Action Collaborative on Countering the U.S. Opioid Epidemic. 2019.

https://nam.edu/programs/actioncollaborative-on-countering-the-u-s-opioidepidemic/. Accessed May 29, 2019.

Exclusion reason: Background information only

1210. Natour J, Puertas EB, Radu AS, et al. Loxoprofen in the treatment of low back pain - Clinical efficacy and safety in comparison to diclofenac. Revista brasileira de medicina. 2002;59(3):161-70. Exclusion reason: Not in English

1211. Navarathnam RG, Wang IY, Thomas D, et al. Evaluation of the transcutaneous electrical nerve stimulator for postoperative analgesia following cardiac surgery.

Anaesth Intensive Care. 1984

Nov;12(4):345-50. doi: 10.1177/0310057x8401200411. PMID: 6335005. Exclusion reason: Ineligible setting
1212. Nee RJ, Vicenzino B, Jull GA, et al. Neural tissue management provides immediate clinically relevant benefits without harmful effects for patients with nerve-related neck and arm pain: a randomised trial. J Physiother. 2012;58(1):23-31. doi: 10.1016/S1836-9553(12)70069-3. PMID: 22341379. Exclusion reason: Ineligible population

1213. Nelemans PJ, de Bie RA, de Vet HC, et al. Injection therapy for subacute and chronic benign low back pain. Cochrane Database Syst Rev. 2000(2):CD001824. PMID: 10796449. Exclusion reason: Background information only

1214. Neri G, Baffa C, Vitullio F, et al. Singleblind evaluation of post-tonsillectomy pain treatment with an eutectic mixture of local anesthetics. Eur J Inflamm. 2006;4(3):181-9. doi: 10.1177/1721727X0600400307.

Exclusion reason: Ineligible intervention

1215. Neto JO, Machado MD, de Almeida Correa $\mathrm{M}$, et al. Methadone patient-controlled analgesia for postoperative pain: a randomized, controlled, double-blind study. J Anesth. 2014 Aug;28(4):505-10. doi: 10.1007/s00540-013-1785-3. PMID: 24445560. Exclusion reason: Ineligible setting

1216. Neuman MD, Hennessy S, Small DS, et al. Drug Enforcement Agency 2014 Hydrocodone Rescheduling Rule and Opioid Dispensing after Surgery. Anesthesiology. 2020 05;132(5):1151-64. doi: https://dx.doi.org/10.1097/ALN.0000000000 003188. PMID: 32101973. Exclusion reason: Ineligible intervention

1217. Newcomer KL, Vickers Douglas KS, Shelerud RA, et al. Is a videotape to change beliefs and behaviors superior to a standard videotape in acute low back pain? A randomized controlled trial. Spine J. 2008 Nov-Dec;8(6):940-7. PMID: 18037355. Exclusion reason: Ineligible comparator

1218. Newman M, Minns Lowe C, Barker K. Spinal Orthoses for Vertebral Osteoporosis and Osteoporotic Vertebral Fracture: A Systematic Review. Arch Phys Med Rehabil. 2016 06;97(6):1013-25. doi: 10.1016/j.apmr.2015.10.108. PMID: 26615791. Exclusion reason: Publication used as source document 
1219. Newsome RJ, May S, Chiverton N, et al. A prospective, randomised trial of immediate exercise following lumbar microdiscectomy: a preliminary study. Physiotherapy. 2009 Dec;95(4):273-9. doi: 10.1016/j.physio.2009.06.004. PMID: 19892091. Exclusion reason: Ineligible outcome

1220. Newton WP. Bed rest, exercises, or ordinary activity for acute low back pain? J Fam Pract. 1995 Jul;41(1):96-7. PMID: 7798072. Exclusion reason: Ineligible publication type

1221. Nguyen L, Bowlds S, Munford C, et al. Decreasing Postoperative Opioid Prescribing through Education. Journal of Surgical Education. 2020 May Jun;77(3):615-20. doi: https://dx.doi.org/10.1016/j.jsurg.2019.11.01 0. PMID: 31859229. Exclusion reason: Ineligible intervention

1222. Nguyen TC, Lombana NF, Zavlin D, et al. Transition to Nonopioid Analgesia Does Not Impair Pain Control After Major Aesthetic Plastic Surgery. Aesthet Surg J. 2018 Sep 14;38(10):1139-44. doi: 10.1093/asj/sjy050. PMID: 29608639. Exclusion reason: Ineligible study design

1223. Nielsen PR, Jorgensen LD, Dahl B, et al. Prehabilitation and early rehabilitation after spinal surgery: randomized clinical trial. Clin Rehabil. 2010 Feb;24(2):137-48. doi: 10.1177/0269215509347432. PMID: 20103575. Exclusion reason: Ineligible setting

1224. Nikanne E, Kokki H, Salo J, et al. Celecoxib and ketoprofen for pain management during tonsillectomy: a placebo-controlled clinical trial. Otolaryngol Head Neck Surg. 2005 Feb;132(2):287-94. PMID: 15692543.

Exclusion reason: Ineligible comparator

1225. Nilsson H, Limchaichana N, Nilner M, et al. Short-term treatment of a resilient appliance in TMD pain patients: a randomized controlled trial. J Oral Rehabil. 2009 Aug;36(8):547-55. doi: 10.1111/j.13652842.2009.01973.x. PMID: 19604318.

Exclusion reason: Ineligible population
1226. Nilsson U, Rawal N, Enqvist B, et al. Analgesia following music and therapeutic suggestions in the PACU in ambulatory surgery; a randomized controlled trial. Acta Anaesthesiol Scand. 2003 Mar;47(3):27883. doi: 10.1034/j.1399-6576.2003.00064.x. PMID: 12648193. Exclusion reason: Ineligible setting

1227. Nilsson U, Rawal N, Unosson M. A comparison of intra-operative or postoperative exposure to music--a controlled trial of the effects on postoperative pain. Anaesthesia. 2003 Jul;58(7):699-703. PMID: 12886915. Exclusion reason: Ineligible study design

1228. Nordemar R, Thorner C. Treatment of acute cervical pain--a comparative group study. Pain. 1981 Feb;10(1):93-101. PMID: 6972028. Exclusion reason: Ineligible comparator

1229. Nordin CA, Michaelson P, Gard G, et al. Effects of the Web Behavior Change Program for Activity and Multimodal Pain Rehabilitation: Randomized Controlled Trial. JMIR. 201610 05;18(10):e265. PMID: 27707686. Exclusion reason: Ineligible population

1230. North RB. Spinal cord stimulation for intractable pain: long-term follow-up. J Spinal Disord. 1990 Dec;3(4):356-61. PMID: 2151991. Exclusion reason: Ineligible publication type

1231. Nottage KA, Hankins JS, Faughnan LG, et al. Addressing challenges of clinical trials in acute pain: The Pain Management of Vasoocclusive Crisis in Children and Young Adults with Sickle Cell Disease Study. Clinical Trials. 2016 08;13(4):409-16. doi: https://dx.doi.org/10.1177/17407745166365 73. PMID: 27000103. Exclusion reason: Background information only

1232. Nuhr M, Hoerauf K, Bertalanffy A, et al. Active warming during emergency transport relieves acute low back pain. Spine. 2004 Jul 15;29(14):1499-503. doi: 10.1097/01.brs.0000131439.87553.99. PMID: 15247569. Exclusion reason: Ineligible duration 
1233. Nunes GS, Bender PU, de Menezes FS, et al. Massage therapy decreases pain and perceived fatigue after long-distance Ironman triathlon: a randomised trial. J Physiother. 2016 Apr;62(2):83-7. doi: 10.1016/j.jphys.2016.02.009. PMID: 27025688. Exclusion reason: Ineligible population

1234. Oakland C. A comparison of the efficacy of the topical NSAID felbinac and ultrasound in the treatment of acute ankle injuries. British Journal of Clinical Research. 1993;13:16-22. Exclusion reason: Ineligible publication type

1235. Oberhofer D, Šakić K, Nesek-Adam V, et al. Low dose spinal morphine and intravenous diclofenac for postoperative analgesia after total hip and knee arthroplasty. Periodicum Biologorum. 2011;113(2):191-6. Exclusion reason: Ineligible comparator

1236. O'Brien J, Keaveny J, Pollard V, et al. Advancing Nursing Practice: Management of Neuropathic Pain With Capsaicin 8\% Without Physician Supervision. Clinical Nurse Specialist. 2017 May/Jun;31(3):15762. doi: 10.1097/NUR.0000000000000296. PMID: 28383334. Exclusion reason: Ineligible comparator

1237. Okomo U, Meremikwu MM. Fluid replacement therapy for acute episodes of pain in people with sickle cell disease. Cochrane Database Syst Rev. 201707 31;7:CD005406. doi: https://dx.doi.org/10.1002/14651858.CD005 406.pub5. PMID: 28759112. Exclusion reason: Publication used as source document

1238. Okoro T, Ibrahim Y, Mansour N, et al. The Use of Cryotherapy in the Early Postoperative Period after Total Hip Arthroplasty. Ortop Traumatol Rehabil. 2019 Oct 31;21(5):339-48. PMID: 31774061. Exclusion reason: Ineligible study design

1239. Oktaviani I. Pilates workouts can reduce pain in pregnant women. Complement Ther Clin Pract. 2018 May;31:349-51. doi: 10.1016/j.ctcp.2017.11.007. PMID: 29173892. Exclusion reason: Ineligible comparator
1240. Olaya-Contreras P, Styf J, Arvidsson D, et al. The effect of the stay active advice on physical activity and on the course of acute severe low back pain. BMC sports science, medicine and rehabilitation. 2015;7(19)doi: 10.1186/s13102-015-0013-x. PMID: 26322232 Exclusion reason: Ineligible comparator

1241. Oleisky ER, Pennings JS, Hills J, et al. Comparing different chronic preoperative opioid use definitions on outcomes after spine surgery. Spine J. 2019 06;19(6):98494. doi: https://dx.doi.org/10.1016/j.spinee.2018.12. 014. PMID: 30611889. Exclusion reason: Ineligible intervention

1242. Oleske DM, Lavender SA, Andersson GB, et al. Are back supports plus education more effective than education alone in promoting recovery from low back pain?: Results from a randomized clinical trial. Spine (Phila $\mathrm{Pa}$ 1976). 2007 Sep 1;32(19):2050-7. doi: 10.1097/BRS.0b013e3181453fcc. PMID: 17762804. Exclusion reason: Ineligible comparator

1243. Olsen MF, Bjerre E, Hansen MD, et al. Pain relief that matters to patients: systematic review of empirical studies assessing the minimum clinically important difference in acute pain. BMC Med. 2017 Feb 20;15(1):35. doi: https://dx.doi.org/10.1186/s12916-0160775-3. PMID: 28215182. Exclusion reason: Publication used as source document

1244. Olson NZ, Sunshine A, Zighelboim I, et al. Onset and duration of analgesia of diclofenac potassium in the treatment of postepisiotomy pain. Am J Ther. 1997 JulAug;4(7-8):239-46. doi: 10.1097/00045391199707000-00004. PMID: 10423616.

Exclusion reason: Ineligible setting

1245. Oltean H, Robbins C, van Tulder MW, et al. Herbal medicine for low-back pain. Cochrane Database Syst Rev. 2014 Dec 23(12):CD004504. doi: 10.1002/14651858.CD004504.pub4. PMID: 25536022. Exclusion reason: Publication used as source document 
1246. Oncel M, Sencan S, Yildiz H, et al. Transcutaneous electrical nerve stimulation for pain management in patients with uncomplicated minor rib fractures. European Journal of Cardio-Thoracic Surgery. 2002 Jul;22(1):13-7. PMID: 12103366. Exclusion reason: Ineligible population

1247. Onda A, Ogoshi A, Itoh M, et al. Comparison of the effects of treatment with celecoxib, loxoprofen, and acetaminophen on postoperative acute pain after arthroscopic knee surgery: A randomized, parallel-group trial. J Orthop Sci. 2016 Mar;21(2):172-7. doi: 10.1016/j.jos.2015.11.005. PMID: 26888227. Exclusion reason: Ineligible setting

1248. O'Neil JT, Wang ML, Kim N, et al. Prospective Evaluation of Opioid Consumption After Distal Radius Fracture Repair Surgery. Am J Orthop (Chatham, Nj). 2017 Jan/Feb;46(1):E35-E40. PMID: 28235120. Exclusion reason: Ineligible intervention

1249. O'Neill O. The efficacy of oral analgesia for postoperative pain. British Journal of Theatre Nursing. 1998 Dec;8(9):5-6, 8. PMID: 10076230. Exclusion reason: Ineligible study design

1250. Ono F, Yasumoto S, Furumura M, et al. Comparison between famciclovir and valacyclovir for acute pain in adult Japanese immunocompetent patients with herpes zoster. Journal of Dermatology. 2012 Nov;39(11):902-8. doi: 10.1111/j.13468138.2012.01584.x. PMID: 22670895.

Exclusion reason: Ineligible intervention

1251. Orava S. Medical treatment of acute low back pain. Diflunisal compared with indomethacin in acute lumbago. International J Clin Pharmacol Research. 1986;6(1):45-51. PMID: 2937744.

Exclusion reason: Ineligible comparator

1252. Oro L. A comparison between meptazinol and dextropropoxyphene plus paracetamol in elderly patients with musculoskeletal pains. Curr Med Res Opin. 1984;9(4):240-5. PMID: 6391829. Exclusion reason: Ineligible intervention
1253. Orringer EP, Casella JF, Ataga KI, et al. Purified poloxamer 188 for treatment of acute vaso-occlusive crisis of sickle cell disease: A randomized controlled trial. JAMA. 2001 Nov 07;286(17):2099-106. PMID: 11694150. Exclusion reason: Ineligible study design

1254. Ortega A, Gauna F, Munoz D, et al. Music Therapy for Pain and Anxiety Management in Nasal Bone Fracture Reduction: Randomized Controlled Clinical Trial. Otolaryngol Head Neck Surg. 2019 Jun 11:194599819856604. doi: 10.1177/0194599819856604. PMID: 31184266. Exclusion reason: Ineligible intervention

1255. Osama M, Shakil Ur Rehman S. Effects of static stretching as compared to autogenic inhibition and reciprocal inhibition muscle energy techniques in the management of mechanical neck pain: a randomized controlled trial. J Pak Med Assoc. 2020 May;70(5):786-90. doi: https://dx.doi.org/10.5455/JPMA.9596. PMID: 32400728. Exclusion reason: Ineligible population

1256. Osmundson SS, Raymond BL, Kook BT, et al. Individualized Compared With Standard Postdischarge Oxycodone Prescribing After Cesarean Birth: A Randomized Controlled Trial. Obstet Gynecol. 2018 09;132(3):62430. doi:

https://dx.doi.org/10.1097/AOG.000000000 0002782. PMID: 30095773. Exclusion reason: Ineligible outcome

1257. Ostenfeld T, Price J, Albanese M, et al. A randomized, controlled study to investigate the analgesic efficacy of single doses of the cannabinoid receptor-2 agonist GW842166, ibuprofen or placebo in patients with acute pain following third molar tooth extraction. Clin J Pain. 2011 Oct;27(8):668-76. doi: 10.1097/AJP.0b013e318219799a. PMID: 21540741. Exclusion reason: Ineligible intervention

1258. Ostojic P, Radunovic G, Lazovic M, et al. Ibuprofen plus paracetamol versus ibuprofen in acute low back pain: a randomized open label multicenter clinical study. Acta Reumatologica Portuguesa. 2017 JanMar;42(1):18-25. PMID: 27978532.

Exclusion reason: Ineligible comparator 
1259. O'Sullivan LM, Ahmed N, Sidebottom AJ. Dental pain management - a cause of significant morbidity due to paracetamol overdose. Br Dent J. 201804 27;224(8):6264. doi: https://dx.doi.org/10.1038/sj.bdj.2018.264. PMID: 29674735. Exclusion reason: Background information only

1260. Ou M-C, Lee Y-F, Li C-C, et al. The effectiveness of essential oils for patients with neck pain: A randomized controlled study. The Journal of Alternative and Complementary Medicine. 2014 Oct;20(10):771-9. doi: 10.1089/acm.2013.0453. PMID: 25192562 Exclusion reason: Ineligible population

1261. Ouellette RD. Buprenorphine and morphine efficacy in postoperative pain: a doubleblind multiple-dose study. J Clin Pharmacol. 1982 Apr;22(4):165-72. PMID: 7047580. Exclusion reason: Ineligible population

1262. Oyler D, Bernard AC, VanHoose JD, et al. Minimizing opioid use after acute major trauma. Am J Health Syst Pharm. 201802 01;75(3):105-10. doi: 10.2146/ajhp161021. PMID: 29371190. Exclusion reason: Ineligible setting

1263. Oyler DR, Parli SE, Bernard AC, et al. Nonopioid management of acute pain associated with trauma: Focus on pharmacologic options. J Trauma Acute Care Surg. 2015 Sep;79(3):475-83. doi: 10.1097/TA.0000000000000755. PMID: 26307883. Exclusion reason: Ineligible study design

1264. Ozgencil E, Yalcin S, Tuna H, et al. Perioperative administration of gabapentin $1,200 \mathrm{mg}$ day-1 and pregabalin $300 \mathrm{mg}$ day1 for pain following lumbar laminectomy and discectomy: a randomised, doubleblinded, placebo-controlled study. Singapore Med J. 2011 Dec;52(12):883-9. PMID: 22159931. Exclusion reason: Ineligible comparator

1265. Paanalahti K, Holm LW, Nordin M, et al. Adverse events after manual therapy among patients seeking care for neck and/or back pain: a randomized controlled trial. BMC Musculoskelet Disord. 2014 Mar 12;15:77. doi: 10.1186/1471-2474-15-77. PMID: 24618345. Exclusion reason: Ineligible population
1266. Paatelma M, Kilpikoski S, Simonen R, et al. Orthopaedic manual therapy, McKenzie method or advice only for low back pain in working adults: a randomized controlled trial with one year follow-up. J Rehabil Med. 2008 Nov;40(10):858-63. doi: 10.2340/16501977-0262. PMID: 19242624. Exclusion reason: Ineligible population

1267. Pabst H, Schaefer A, Staiger C, et al. Combination of comfrey root extract plus methyl nicotinate in patients with conditions of acute upper or low back pain: a multicentre randomised controlled trial. Phytother Res. 2013 Jun;27(6):811-7. doi: 10.1002/ptr.4790. PMID: 22887778.

Exclusion reason: Ineligible intervention

1268. Paige NM, Miake-Lye IM, Booth MS, et al. Association of Spinal Manipulative Therapy With Clinical Benefit and Harm for Acute Low Back Pain: Systematic Review and Meta-analysis. JAMA. 2017 Apr

11;317(14):1451-60. doi: 10.1001/jama.2017.3086. PMID: 28399251. Exclusion reason: Publication used as source document

1269. Pais VM, Jr., Sites BD. The association of nephrolithiasis with prescription opioid use. Clin Nephrol. 2019 Apr;91(4):231-6. doi: 10.5414/cn109717. PMID: 30862351. Exclusion reason: Ineligible outcome

1270. Paisley P, Serpell M. Diagnosis and management of postherpetic neuralgia. Practitioner. 2015 Jan;259(1778):21-4, 2-3. PMID: 25726617. Exclusion reason: Background information only

1271. Pakravan M, Roshani M, Yazdani S, et al. Pregabalin and gabapentin for postphotorefractive keratectomy pain: a randomized controlled trial. Eur J Ophthalmol. 2012;22 Suppl 7:S106-13. doi: 10.5301/ejo.5000143. PMID: 22577038.

Exclusion reason: Ineligible comparator

1272. Pal S, Dixit R, Moe S, et al. Transcutaneous electrical nerve stimulation (TENS) for pain management in sickle cell disease. Cochrane Database Syst Rev. 2020(3) PMID: PMC6483494. Exclusion reason: Ineligible population 
1273. Palangio M, Morris E, Doyle RT, Jr., et al. Combination hydrocodone and ibuprofen versus combination oxycodone and acetaminophen in the treatment of moderate or severe acute low back pain. Clin Ther. 2002 Jan;24(1):87-99. PMID: 11833838. Exclusion reason: Ineligible comparator

1274. Palangio M, Wideman GL, Keffer M, et al. Combination hydrocodone and ibuprofen versus combination oxycodone and acetaminophen in the treatment of postoperative obstetric or gynecologic pain. Clin Ther. 2000;22(5):600-12. PMID: 10868557. Exclusion reason: Ineligible comparator

1275. Palmer M. Mobilization following lumbar discectomy: a comparison of two methods of bed transfer. Physiotherapy Canada. 1989;41:146-53. Exclusion reason: Ineligible population

1276. Panagopoulos J, Hancock MJ, Ferreira P, et al. Does the addition of visceral manipulation alter outcomes for patients with low back pain? A randomized placebo controlled trial. Eur J Pain. 2015 Aug;19(7):899-907. doi: 10.1002/ejp.614. PMID: 25378096. Exclusion reason: Ineligible population

1277. Panella L, Caserta AV, Ballarati R, et al. Control of post-operative pain and rehabilitation compliance of patients undergoing knee replacement. Clinical Practice. 2016;13(2):39-45. doi: 10.4172/clinical-practice.100092. Exclusion reason: Ineligible study design

1278. Pantalone AL, Fisher AJ, Thomas GJ. Control of post-anorectal surgery pain with repository tubocurarine (tubadil). Current Researches in Anesth Analg. 1955 MayJun;34(3):160-7. PMID: 14365207.

Exclusion reason: Ineligible intervention

1279. Pape E, Collin C, Camelot F, et al. Paracetamol Misuse and Dental Pain: Results from the French Observational DAntaLor Study. J Oral Facial Pain Headache. 2019 Winter;33(1):123-9. doi: https://dx.doi.org/10.11607/ofph.1861. PMID: 30703177. Exclusion reason: Ineligible study design
1280. Papoian V, Handy KG, Villano AM, et al. Randomized control trial of opioid- versus nonopioid-based analgesia after thyroidectomy. Surgery. 2020 Jun;167(6):957-61. doi: https://dx.doi.org/10.1016/j.surg.2020.01.01 1. PMID: 32127178. Exclusion reason: Ineligible comparator

1281. Pareek A, Chandurkar N, Chandanwale AS, et al. Aceclofenac-tizanidine in the treatment of acute low back pain: a double-blind, double-dummy, randomized, multicentric, comparative study against aceclofenac alone. Eur Spine J. 2009 Dec;18(12):183642. doi: 10.1007/s00586-009-1019-4. PMID: 19421791. Exclusion reason: Ineligible intervention

1282. Park CH, Yong A, Lee SH. Involvement of selective alpha-2 adrenoreceptor in sympathetically maintained pain. Journal of Korean Neurosurgical Society.

2010;47(6):420-3. doi:

10.3340/jkns.2010.47.6.420. PMID:

20617085. Exclusion reason: Ineligible population

1283. Park SJ, Yoon KB, Yoon DM, et al. Botulinum Toxin Treatment for Nocturnal Calf Cramps in Patients With Lumbar Spinal Stenosis: A Randomized Clinical Trial. Arch Phys Med Rehabil. 2017 05;98(5):957-63. doi: 10.1016/j.apmr.2017.01.017. PMID: 28209505. Exclusion reason: Ineligible population

1284. Park SS, Kim DH, Nam IC, et al. The effectiveness of pregabalin for posttonsillectomy pain control: a randomized controlled trial. PLoS One. 2015;10(2):e0117161. doi: 10.1371/journal.pone.0117161. PMID: 25706948. Exclusion reason: Ineligible intervention

1285. Park YB, Ha CW, Cho SD, et al. A randomized study to compare the efficacy and safety of extended-release and immediate-release tramadol $\mathrm{HCl} /$ acetaminophen in patients with acute pain following total knee replacement. Curr Med Res Opin. 2015 Jan;31(1):75-84. doi: 10.1185/03007995.2014.975338. PMID: 25299350. Exclusion reason: Ineligible comparator 
1286. Park YH, Song JH, Kim TJ, et al. Comparison of the use of evaporative coolants and ice packs for the management of preoperative edema and pain in ankle fractures: a prospective randomized controlled trial. Arch Orthop Trauma Surg. 2019 Oct;139(10):1399-405. doi: https://dx.doi.org/10.1007/s00402-01903222-7. PMID: 31203381. Exclusion reason: Ineligible comparator

1287. Parkin-Smith GF, Norman IJ, Briggs E, et al. A structured protocol of evidence-based conservative care compared with usual care for acute nonspecific low back pain: a randomized clinical trial. Arch Phys Med Rehabil. 2012 Jan;93(1):11-20. doi: 10.1016/j.apmr.2011.08.022. PMID: 22200382. Exclusion reason: Ineligible population

1288. Parkin-Smith GF, Penter CS. A clinical trial investigating the effect of two manipulative approaches in the treatment of mechanical neck pain: a pilot study. Journal of the Neuromusculoskeletal System. 1998;6(1):616. Exclusion reason: Ineligible population

1289. Parr AT, Manchikanti L, Hameed H, et al. Caudal epidural injections in the management of chronic low back pain: a systematic appraisal of the literature. Pain Physician. 2012 May-Jun;15(3):E159-98. PMID: 22622911. Exclusion reason: Publication used as source document

1290. Patanwala AE, Jarzyna DL, Miller MD, et al. Comparison of opioid requirements and analgesic response in opioid-tolerant versus opioid-naive patients after total knee arthroplasty. Pharmacotherapy:The Journal of Human Pharmacology \& Drug Therapy. 2008 Dec;28(12):1453-60. doi: 10.1592/phco.28.12.1453. PMID: 19025426. Exclusion reason: Ineligible study design

1291. Patel HD, Srivastava A, Patel ND, et al. A Prospective Cohort Study of Postdischarge Opioid Practices After Radical Prostatectomy: The ORIOLES Initiative. Eur Urol. 2019 02;75(2):215-8. doi: 10.1016/j.eururo.2018.10.013. PMID: 30352714. Exclusion reason: Ineligible comparator
1292. Patel HD, Uppin RB, Naidu AR, et al. Efficacy and Safety of Combination of NSAIDs and Muscle Relaxants in the Management of Acute Low Back Pain. Pain Ther. 2019 PMID: 30652262. Exclusion reason: Ineligible comparator

1293. Patel VD, Eapen C, Ceepee Z, et al. Effect of muscle energy technique with and without strain-counterstrain technique in acute low back pain-A randomized clinical trial. Hong Kong Physiotherapy Journal. 2018;38(1):41-51. doi: 10.1142/S1013702518500051. PMID: 30930578. Exclusion reason: Ineligible comparator

1294. Pathan SA, Mitra B, Cameron PA. A Systematic Review and Meta-analysis Comparing the Efficacy of Nonsteroidal Anti-inflammatory Drugs, Opioids, and Paracetamol in the Treatment of Acute Renal Colic. Eur Urol. 2018 04;73(4):58395. doi: https://dx.doi.org/10.1016/j.eururo.2017.11. 001. PMID: 29174580. Exclusion reason: Publication used as source document

1295. Pathan SA, Mitra B, Romero L, et al. What is the best analgesic option for patients presenting with renal colic to the emergency department? Protocol for a systematic review and meta-analysis. BMJ Open. 2017 05 04;7(4):e015002. doi: https://dx.doi.org/10.1136/BMJopen-2016015002. PMID: 28473517. Exclusion reason: Publication used as source document

1296. Pathiraja C, Silva N, Sharpe G, et al. Is continuous infiltration of local anaesthetic/PCA an acceptable alternate pain management strategy in morbidly obese patients undergoing gastric bypass surgery? A retrospective comparison with epidural analgesia. Sri Lankan Journal of Anaesthesiology. 2010;18(2):66-71. doi: 10.4038/slja.v18i2.2440. Exclusion reason: Ineligible intervention

1297. Patil P, Chandu B, Metgud S, et al. Effectiveness of muscle energy technique on quadratus lumborum in acute low back painrandomized controlled trial. Ndian J Physiother Occup Ther. 2010;4(1):54-8. Exclusion reason: Ineligible comparator 
1298. Patnaik S, Acharya S, Kumar S, et al. Effect of Cold Compression Therapy on Pain, Swelling \& Trismus Following Surgical Removal of Impacted Mandibular Third Molars: A Clinical Research. Int J Dent Med Res| MAR-APR. 2015;1(6):18. Exclusion reason: Ineligible study design

1299. Pearson JW, Lasagna L, Laird RD. Analgesic activity of oral and intramuscular profadol. Clinical Pharmacology and Therapeutics. 1971;12(4):683-90. doi: 10.1002/cpt1971124683. PMID: 4936041. Exclusion reason: Ineligible comparator

1300. Pei Q, Wu B, Tang Y, et al. Repetitive Transcranial Magnetic Stimulation at Different Frequencies for Postherpetic Neuralgia: A Double-Blind, ShamControlled, Randomized Trial. Pain Physician. 2019 07;22(4):E303-E13. PMID: 31337172. Exclusion reason: Ineligible population

1301. Peloso MP, Gross A, Haines T, et al. Medicinal and injection therapies for mechanical neck disorders. Cochrane Database Syst Rev. 2015(5)doi: 10.1002/14651858.CD000319.pub4. PMID: 17636629. Exclusion reason: Publication used as source document

1302. Pengel LH, Refshauge KM, Maher CG, et al. Physiotherapist-directed exercise, advice, or both for subacute low back pain: a randomized trial. Ann Intern Med. 2007 Jun 5;146(11):787-96. doi: 10.7326/0003-4819146-11-200706050-00007. PMID: 17548410. Exclusion reason: Ineligible population

1303. Perez-Urizar J, Martinez-Rider R, TorresRoque I, et al. Analgesic efficacy of lysine clonixinate plus tramadol versus tramadol in multiple doses following impacted third molar surgery. Int J Oral Maxillofac Surg. 2014 Mar;43(3):348-54. doi: 10.1016/j.ijom.2013.08.003. PMID: 24042066. Exclusion reason: Ineligible comparator

1304. Perlin E, Finke H, Castro O, et al. Infusional/patient-controlled analgesia in sickle-cell vaso-occlusive crises. Pain Clinic. 1993;6(2):113-9. Exclusion reason: Ineligible comparator
1305. Perlman KM, Myers-Phariss S, Rhodes JC. A shift from demerol (meperidine) to dilaudid (hydromorphone) improves pain control and decreases admissions for patients in sickle cell crisis. J Emerg Nurs. 2004 Oct;30(5):439-46. PMID: 15452522. Exclusion reason: Ineligible study design

1306. Perna A, Ricciardi L, Barone G, et al. Medical management of acute non-specific low back pain: comparison of different medical treatments, one center's retrospective analysis. Journal of Biological Regulators \& Homeostatic Agents. 2018 Nov-Dec;32(6 Suppl. 1):121-9. PMID: 30644292. Exclusion reason: Ineligible publication type

1307. Persson NH, Bergqvist D, Melander A, et al. Comparison of a narcotic (oxicone) and a non-narcotic anti-inflammatory analgesic (indoprofen) in the treatment of renal colic. Acta Chir Scand. 1985;151(2):105-8. PMID: 3890435. Exclusion reason: Ineligible intervention

1308. Perterson CD. A pilot randomized controlled trial evaluating three treatments for pregnancy-related low back pain: exercise, spinal manipulation, and neuro emotional technique. J Midwifery Womens Health. 2012;57(5):537. Exclusion reason: Ineligible publication type

1309. Petersen GL, Finnerup NB, Norskov KN, et al. Placebo manipulations reduce hyperalgesia in neuropathic pain. Pain. 2012 Jun;153(6):1292-300. doi: 10.1016/j.pain.2012.03.011. PMID: 22503337. Exclusion reason: Ineligible population

1310. Peters-Lawrence MH, Bell MC, Hsu LL, et al. Clinical trial implementation and recruitment: lessons learned from the early closure of a randomized clinical trial. Contemporary Clinical Trials. 2012 Mar;33(2):291-7. doi: https://dx.doi.org/10.1016/j.cct.2011.11.018. PMID: 22155024. Exclusion reason: Background information only 
1311. Peterson CK, Bolton J, Humphreys BK. Predictors of improvement in patients with acute and chronic low back pain undergoing chiropractic treatment. J Manipulative Physiol Ther. 2012 Sep;35(7):525-33. doi: 10.1016/j.jmpt.2012.06.003. PMID: 22858233. Exclusion reason: Ineligible population

1312. Petersson A, Eriksson L, Lundh H. No short-term difference in outcome after temporomandibular joint arthrography alone or with immediate lavage. Oral Surg Oral Med Oral Pathol. 1994 Apr;77(4):322-6. PMID: 8015793. Exclusion reason: Ineligible intervention

1313. Pfeifer M, Begerow B, Minne HW. Effects of a new spinal orthosis on posture, trunk strength, and quality of life in women with postmenopausal osteoporosis: a randomized trial. Am J Phys Med Rehabil. 2004

Mar;83(3):177-86. doi: 10.1097/01.phm.0000113403.16617.93. PMID: 15043351. Exclusion reason: Ineligible population

1314. Pfeifer M, Kohlwey L, Begerow B, et al. Effects of two newly developed spinal orthoses on trunk muscle strength, posture, and quality-of-life in women with postmenopausal osteoporosis: a randomized trial. Am J Phys Med Rehabil. 2011 Oct;90(10):805-15. doi: 10.1097/PHM.0b013e31821f6df3. PMID: 21681065. Exclusion reason: Ineligible population

1315. Pham H, Pickell M, Yagnatovsky M, et al. The Utility of Oral Nonsteroidal Antiinflammatory Drugs Compared With Standard Opioids Following Arthroscopic Meniscectomy: A Prospective Observational Study. Arthroscopy. 2019 03;35(3):86470.e1. doi: https://dx.doi.org/10.1016/j.arthro.2018.09.0 18. PMID: 30733030. Exclusion reason: Ineligible outcome

1316. Pickett C, Clum GA. Comparative treatment strategies and their interaction with locus of control in the reduction of postsurgical pain and anxiety. J Consult Clin Psychol. 1982 Jun;50(3):439-41. doi: 10.1037//0022006x.50.3.439. PMID: 7047601. Exclusion reason: Ineligible intervention
1317. Pikula JR. The effect of spinal manipulative therapy (SMT) on pain reduction and range of motion in patients with acute unilateral neck pain: a pilot study. J Can Chiropr Assoc. 1999;43(2):111-9. Exclusion reason: Ineligible duration

1318. Pirbudak L, Cicek H, Isik M, et al. The effect of tramadol and tramadol + gabapentin combination in patients with lumbar disc herniation after epidural steroid injection. Turk J Med Sci. 2015;45(6):12149. PMID: 26775373. Exclusion reason: Ineligible comparator

1319. Pitangui AC, Araujo RC, Bezerra MJ, et al. Low and high-frequency TENS in postepisiotomy pain relief: a randomized, double-blind clinical trial. Braz J Phys Ther. 2014 Jan-Feb;18(1):72-8. PMID: 24675915. Exclusion reason: Ineligible population

1320. Pittler MH, Brown EM, Ernst E. Static magnets for reducing pain: systematic review and meta-analysis of randomized trials. CMAJ Can Med Assoc J. 2007 Sep 25;177(7):736-42. PMID: 17893349. Exclusion reason: Publication used as source document

1321. Pizzi LJ, Bates M, Chelly JE, et al. A Prospective Randomized Trial of an Oral Patient-Controlled Analgesia Device Versus Usual Care Following Total Hip Arthroplasty. Orthop Nurs. 2020 Jan/Feb;39(1):37-46. doi: https://dx.doi.org/10.1097/NOR.000000000 0000624. PMID: 31977740. Exclusion reason: Ineligible intervention

1322. Plapler PG, Scheinberg MA, Ecclissato Cda C, et al. Double-blind, randomized, doubledummy clinical trial comparing the efficacy of ketorolac trometamol and naproxen for acute low back pain. Drug Des Devel Ther. 2016;10:1987-93. doi: 10.2147/DDDT.S97756. PMID: 27382251. Exclusion reason: Ineligible comparator

1323. Plewa MC. Analgesic prescriptions for ED patients with pelvic or dental pain. Am J Emerg Med. 1997 May;15(3):326-8. PMID: 9149004. Exclusion reason: Ineligible publication type 
1324. Plunkett A, McCoart A, Howard RS, et al. A randomized, single-blind, prospective trial of auricular 'battlefield' acupuncture for the reduction of postoperative tonsillectomy pain in adults. Pain Manag 2018 Jul 01;8(4):287-95. doi: 10.2217/pmt-20180007. PMID: 29898645. Exclusion reason: Ineligible population

1325. Poflee VW, Gupta OP, Jain AP, et al. Haemorheological treatment of painful sickle cell crises. Use of pentoxifylline. J Assoc Physicians India. 1991 Aug;39(8):608-9. PMID: 1814876. Exclusion reason: Ineligible intervention

1326. Pohjolainen T, Jekunen A, Autio L, et al. Treatment of acute low back pain with the COX-2-selective anti- inflammatory drug nimesulide. Spine. 2000;25(12):1579-85. PMID: 10851109. Exclusion reason: Ineligible comparator

1327. Pohjolainen T, Jekunen A, Autio L, et al. Treatment of acute low back pain with the COX-2-selective anti-inflammatory drug nimesulide: results of a randomized, doubleblind comparative trial versus ibuprofen. Spine. 2000 Jun 15;25(12):1579-85. PMID: 10851109. Exclusion reason: Ineligible intervention

1328. Polanco-Garcia M, Garcia-Lopez J, Fabregas N, et al. Postoperative Pain Management in Spanish Hospitals: A Cohort Study Using the PAIN-OUT Registry. J Pain. 2017 10;18(10):1237-52. doi: 10.1016/j.jpain.2017.05.006. PMID: 28619696. Exclusion reason: Ineligible study design

1329. Pope MH, Phillips RB, Haugh LD, et al. A prospective randomized three-week trial of spinal manipulation, transcutaneous muscle stimulation, massage and corset in the treatment of subacute low back pain. Spine (Phila Pa 1976). 1994 Nov 15;19(22):25717. doi: 10.1097/00007632-19941100100013. PMID: 7855683. Exclusion reason: Ineligible population
1330. Pope N, Tallon M, McConigley R, et al. Experiences of acute pain in children who present to a healthcare facility for treatment: a systematic review of qualitative evidence. JBI Database System Rev Implement Rep. 2017 06;15(6):1612-44. doi: https://dx.doi.org/10.11124/JBISRIR-2016003029. PMID: 28628521. Exclusion

reason: Publication used as source document

1331. Pope N, Tallon M, McConigley R, et al. The experiences of acute non-surgical pain of children who present to a healthcare facility for treatment: a systematic review protocol. JBI Database System Rev Implement Rep. 2015 Oct;13(10):12-20. doi: https://dx.doi.org/10.11124/jbisrir-20152466. PMID: 26571278. Exclusion reason: Publication used as source document

1332. Pope RO, Corcoran S, McCaul K, et al. Continuous passive motion after primary total knee arthroplasty. Does it offer any benefits? J Bone Joint Surg Br. 1997 Nov;79(6):914-7. PMID: 9393903. Exclusion reason: Ineligible outcome

1333. Popp JE, Sanko WA, Sinha AK, et al. A comparison of ketorolac tromethamine/oxycodone versus patientcontrolled analgesia with morphine in anterior cruciate ligament reconstruction patients. Arthroscopy. 1998 NovDec;14(8):816-9. PMID: 9848591. Exclusion reason: Ineligible population

1334. Poquet N, Lin CW, Heymans MW, et al. Back schools for acute and subacute nonspecific low-back pain. Cochrane Database Syst Rev. 2016 Apr 26;4:CD008325. doi: https://dx.doi.org/10.1002/14651858.CD008 325.pub2. PMID: 27113258. Exclusion reason: Publication used as source document

1335. Porela-Tiihonen S, Kokki M, Kokki H. Sufentanil sublingual formulation for the treatment of acute, moderate to severe postoperative pain in adult patients. Expert Rev Neurother. 2017 02;17(2):101-11. doi: https://dx.doi.org/10.1080/14737175.2017.1 260005. PMID: 27835931. Exclusion reason: Background information only 
1336. Pornrattanamaneewong C, Ruangsomboon $\mathrm{P}$, Chareancholvanich $\mathrm{K}$, et al. Modified Robert Jones bandage can not reduce invisible blood loss after total knee arthroplasty: a randomized-controlled trial. Arch Orthop Trauma Surg. 2018 Aug;138(8):1151-7. doi: 10.1007/s00402018-2978-x. PMID: 29931392. Exclusion reason: Ineligible intervention

1337. Portenoy RK, Farrar JT, Backonja MM, et al. Long-term use of controlled-release oxycodone for noncancer pain: results of a 3-year registry study. Clin J Pain. 2007 May;23(4):287-99. PMID: 17449988.

Exclusion reason: Ineligible population

1338. Portenoy RK, Taylor D, Messina J, et al. A Randomized, Placebo-controlled Study of Fentanyl Buccal Tablet for Breakthrough Pain in Opioid-treated Patients with Cancer. Clin J Pain. 2006 Nov-Dec;22(9):805-11. doi: 10.1097/01.ajp.0000210932.27945.4a. PMID: 17057563. Exclusion reason: Ineligible comparator

1339. Porter SB, McClain RL, Howe BL, et al. Perioperative ketamine for acute postoperative analgesia: the Mayo ClinicFlorida experience. J Perianesth Nurs. 2015 Jun;30(3):189-95. doi: https://dx.doi.org/10.1016/j.jopan.2015.01.0 10. PMID: 26003764. Exclusion reason: Background information only

1340. Postlethwaite R, Stirling G, Peck CL. Stress inoculation for acute pain: a clinical trial. J Behav Med. 1986 Apr;9(2):219-27. doi: 1007/bf00848480. PMID: 3519976.

Exclusion reason: Ineligible intervention

1341. Potnuru P, Dudaryk R, Gebhard RE, et al. Opioid prescriptions for acute pain after outpatient surgery at a large public university-affiliated hospital: Impact of state legislation in Florida. Surgery. 2019 09;166(3):375-9. doi: https://dx.doi.org/10.1016/j.surg.2019.04.02 2. PMID: 31196705. Exclusion reason: Ineligible comparator
1342. Pouchain EC, Costa FW, Bezerra TP, et al. Comparative efficacy of nimesulide and ketoprofen on inflammatory events in third molar surgery: a split-mouth, prospective, randomized, double-blind study. Int J Oral Maxillofac Surg. 2015 Jul;44(7):876-84. doi: 10.1016/j.ijom.2014.10.026. PMID: 25847016. Exclusion reason: Ineligible comparator

1343. Pourmand A, Davis S, Marchak A, et al. Virtual Reality as a Clinical Tool for Pain Management. Curr Pain Headache Rep. 2018 Jun 15;22(8):53. doi: https://dx.doi.org/10.1007/s11916-0180708-2. PMID: 29904806. Exclusion reason: Ineligible study design

1344. Poursalehan S, Nesioonpour S, Akhondzadeh R, et al. The effect of lowlevel laser on postoperative pain after elective cesarean section. Anesth Pain Med. 2018;8(6)doi: 10.5812/aapm.84195. PMID: 30719420. Exclusion reason: Ineligible intervention

1345. Powell-Cope G, Quigley PA, BestermanDahan K, et al. Perceived benefits of group exercise among individuals with peripheral neuropathy. West J Nurs Res. 2014

Aug;36(7):855-74. doi:

10.1177/0193945914523493. PMID: 24622155. Exclusion reason: Ineligible population

1346. Poylin V, Quinn J, Messer K, et al. Gabapentin significantly decreases posthemorrhoidectomy pain: a prospective study. Int J Colorectal Dis. 2014 Dec;29(12):1565-9. doi: 10.1007/s00384014-2018-4. PMID: 25269619. Exclusion reason: Ineligible study design

1347. Prabhu M, Dubois H, James K, et al. Implementation of a quality improvement initiative to decrease opioid prescribing after cesarean delivery. Obstet Gynecol. 2018;132(3):631-6. doi: 10.1097/AOG.0000000000002789. PMID: 30095765. Exclusion reason: Ineligible study design 
1348. Prabhu M, McQuaid-Hanson E, Hopp S, et al. A Shared Decision-Making Intervention to Guide Opioid Prescribing After Cesarean Delivery. Obstet Gynecol. 2017

07;130(1):42-6. doi: https://dx.doi.org/10.1097/AOG.000000000 0002094. PMID: 28594762. Exclusion reason: Ineligible study design

1349. Predel HG, Ebel-Bitoun C, Lange R, et al. A randomized, placebo- and active-controlled, multicountry, multi-center parallel group trial to evaluate the efficacy and safety of a fixed-dose combination of $400 \mathrm{mg}$ ibuprofen and $100 \mathrm{mg}$ caffeine compared with ibuprofen $400 \mathrm{mg}$ and placebo in patients with acute lower back or neck pain. J Pain Res. 2019;12:2771-83. doi:

10.2147/JPR.S217045. Exclusion reason: Ineligible intervention

1350. Predel HG, Ebel-Bitoun C, Lange R, et al. A randomized, placebo- and active-controlled, multi-country, multi-center parallel group trial to evaluate the efficacy and safety of a fixed-dose combination of $400 \mathrm{mg}$ ibuprofen and $100 \mathrm{mg}$ caffeine compared with ibuprofen $400 \mathrm{mg}$ and placebo in patients with acute lower back or neck pain. J Pain Res. 2019;12:2771-83. doi: https://dx.doi.org/10.2147/JPR.S217045. PMID: 31576162. Exclusion reason: Ineligible comparator

1351. Predel HG, Giannetti B, Burnett I, et al. Efficacy and safety of diclofenac diethylamine $1.16 \%$ gel in the treatment of acute neck pain: A randomized, doubleblind, placebo-controlled study. Ann Rheum Dis. 2012 PMID: 23964752. Exclusion reason: Ineligible comparator

1352. Premkumar A, Lovecchio FC, Stepan JG, et al. Characterization of opioid consumption and disposal patterns after total knee arthroplasty. Bone Joint J. 2019 Jul;101B(7_Supple_C):98-103. doi: https://dx.doi.org/10.1302/0301620X.101B7.BJJ-2018-1518.R1. PMID: 31256646. Exclusion reason: Background information only
1353. Przkora R, Kinsky MP, Fisher SR, et al. Functional Improvements Utilizing the Short Physical Performance Battery (SPPB) in the Elderly after Epidural Steroid Injections. Curr Pain Headache Rep. 2019 Feb 22;23(2):14. doi: https://dx.doi.org/10.1007/s11916-0190748-2. PMID: 30796532. Exclusion reason: Background information only

1354. Puentedura EJ, Landers MR, Cleland JA, et al. Thoracic spine thrust manipulation versus cervical spine thrust manipulation in patients with acute neck pain: a randomized clinical trial. J Orthop Sports Phys Ther. 2011 Apr;41(4):208-20. doi: 10.2519/jospt.2011.3640. PMID: 21335931. Exclusion reason: Ineligible comparator

1355. Punt IM, Ziltener JL, Monnin D, et al. Wii Fit $^{\mathrm{TM}}$ exercise therapy for the rehabilitation of ankle sprains: Its effect compared with physical therapy or no functional exercises at all. Scand J Med Sci Sports. 2016 Jul;26(7):816-23. doi: 10.1111/sms.12509. PMID: 26076737. Exclusion reason: Ineligible comparator

1356. Puri L, Nottage KA, Hankins JS, et al. State of the Art Management of Acute Vasoocclusive Pain in Sickle Cell Disease. Paediatr Drugs. 2018 Feb;20(1):29-42. doi: https://dx.doi.org/10.1007/s40272-0170263-z. PMID: 28853040. Exclusion reason: Background information only

1357. Puzio T, Mangat S, Strassle P, et al. Lidocaine Infusion as an Adjunct to Narcotics in Trauma Patients with Rib Fractures-A Pilot Study. Am Surg. 2019 Apr 01;85(4):e187-e8. PMID: 31043209. Exclusion reason: Ineligible intervention

1358. Pyati S, Gan TJ. Perioperative pain management. CNS Drugs. 2007;21(3):185211. doi: http://dx.doi.org/10.2165/00023210200721030-00002. PMID: 17338592.

Exclusion reason: Background information only

1359. Qari MH, Aljaouni SK, Alardawi MS, et al. Reduction of painful vaso-occlusive crisis of sickle cell anaemia by tinzaparin in a double-blind randomized trial. Thromb Haemost. 2007 Aug;98(2):392-6. PMID: 17721622. Exclusion reason: Ineligible intervention 
1360. Qaseem A, Wilt TJ, McLean RM, et al. Noninvasive Treatments for Acute, Subacute, and Chronic Low Back Pain: A Clinical Practice Guideline From the American College of Physicians. Ann Intern Med. 2017 Apr 04;166(7):514-30. doi: https://dx.doi.org/10.7326/M16-2367. PMID: 28192789. Exclusion reason: Background information only

1361. Quilez C, Perez-Mateo M, Hernandez P, et al. [Usefulness of a non-steroid antiinflammatory, sodium diclofenac, in the treatment of renal colic. Comparative study with a spasmolytic and an opiate analgesic]. Med Clin (Barc). 1984 May 5;82(17):754-5. PMID: 6738191. Exclusion reason: Not in English

1362. Quinlan P, Davis J, Fields K, et al. Effects of Localized Cold Therapy on Pain in Postoperative Spinal Fusion Patients: A Randomized Control Trial. Orthop Nurs. 2017 Sep/Oct;36(5):344-9. doi: https://dx.doi.org/10.1097/NOR.000000000 0000382. PMID: 28930903. Exclusion reason: Ineligible setting

1363. Raab MG, Rzeszutko D, O'Connor W, et al. Early results of continuous passive motion after rotator cuff repair: a prospective, randomized, blinded, controlled study. Am J Orthop (Belle Mead NJ). 1996

Mar;25(3):214-20. PMID: 8775698. Exclusion reason: Ineligible comparator

1364. Radkowski CA, Pietrobon R, Vail TP, et al. Cryotherapy temperature differences after total knee arthroplasty: a prospective randomized trial. J Surg Orthop Adv. 2007 Summer;16(2):67-72. PMID: 17592713. Exclusion reason: Ineligible comparator

1365. Rafiq S, Steinbruchel DA, Wanscher MJ, et al. Multimodal analgesia versus traditional opiate based analgesia after cardiac surgery, a randomized controlled trial. J Cardiothorac Surg. 2014 Mar 20;9:52. doi: 10.1186/17498090-9-52. PMID: 24650125. Exclusion reason: Ineligible setting

1366. Ragonese J. A randomized trial comparing manual physical therapy to therapeutic exercises, to a combination of therapies, for the treatment of cervical radiculotherapy. Orthop Pract. 2009;21(3):71-7. doi: 10.1186/1471-2474-10-115. Exclusion reason: Ineligible population
1367. Rahimi M, Kazemeini AR, Pourtabatabaei $\mathrm{N}$, et al. Comparison of topical anesthetic cream (EMLA) and diclofenac suppository for pain relief after hemorrhoidectomy: a randomized clinical trial. Surg Today. 2012 Dec;42(12):1201-5. doi: 10.1007/s00595012-0222-9. PMID: 22711185. Exclusion reason: Ineligible comparator

1368. Rainov NG, Heidecke V, Albertz C, et al. Transcutaneous electrical nerve stimulation (TENS) for acute postoperative pain after spinal surgery. Eur J Pain (London, England). 1994;15(2-3):44-9. Exclusion reason: Ineligible setting

1369. Raja SN, Haythornthwaite JA, Pappagallo $\mathrm{M}$, et al. Opioids versus antidepressants in postherpetic neuralgia: a randomized, placebo-controlled trial. Neurology. 2002 Oct 8;59(7):1015-21. doi: 10.1212/wnl.59.7.1015. PMID: 12370455. Exclusion reason: Ineligible population

1370. Rajpal N, Aurora M, Chauhan V. A study on efficacy of Pilates \& Pilates \& McKenzie exercise in postural low back pain - a rehabilitative protocol. Physiotherapy and Occupational Therapy Journal. 2008;1(1):33-56. Exclusion reason: Ineligible publication type

1371. Ramanathan D, Saleh A, Klika AK, et al. The Use of Transcutaneous Electrical Nerve Stimulation After Total Knee Arthroplasty: A Prospective Randomized Controlled Trial. Surg Technol Int. 2017 Jul 25;30:425-34. PMID: 28537354. Exclusion reason: Ineligible comparator

1372. Ramaseshan AS, Tunitsky-Bitton E, O'Sullivan DM, et al. Predictive Factors of Postdischarge Narcotic Use After Female Pelvic Reconstructive Surgery. Female Pelvic Med Reconstr Surg. 2019 Mar/Apr;25(2):e18-e22. doi: 10.1097/SPV.0000000000000686. PMID: 30807430. Exclusion reason: Ineligible study design

1373. Ramia E, Nasser SC, Salameh P, et al. Patient Perception of Acute Pain Management: Data from Three Tertiary Care Hospitals. Pain Res Manag. 2017;2017:7459360. doi: https://dx.doi.org/10.1155/2017/7459360. PMID: 28458592. Exclusion reason: Background information only 
1374. Ranieri M, Sciuscio M, Cortese AM, et al. The use of alpha-lipoic acid (ALA), gamma linolenic acid (GLA) and rehabilitation in the treatment of back pain: effect on healthrelated quality of life. Int J Immunopathol Pharmacol. 2009 Jul-Sep;22(3 Suppl):45-50. PMID: 19887043. Exclusion reason: Ineligible intervention

1375. Rao R, Panghate A, Chandanwale A, et al. Clinical Comparative Study: efficacy and Tolerability of Tolperisone and Thiocolchicoside in Acute Low Back Pain and Spinal Muscle Spasticity. Asian Spine J. 2012;6(2):115-22. doi:

10.4184/asj.2012.6.2.115. PMID: 22708015

Exclusion reason: Ineligible intervention

1376. Rapp SE, Ready LB, Nessly ML. Acute pain management in patients with prior opioid consumption: a case-controlled retrospective review. Pain. 1995 May;61(2):195-201. PMID: 7659429. Exclusion reason: Ineligible study design

1377. Rasmussen G. Manipulation in treatment of low back pain: a randomized clinical trial. Man and Med. 1979;1:8-10. Exclusion reason: Ineligible comparator

1378. Rasmussen-Barr E, Held U, Grooten WJ, et al. Non-steroidal anti-inflammatory drugs for sciatica. Cochrane Database Syst Rev. 2016 Oct 15;10:CD012382. PMID: 27743405. Exclusion reason: Publication used as source document

1379. Rasmussen-Barr E, Held U, Grooten WJ, et al. Nonsteroidal Anti-inflammatory Drugs for Sciatica: An Updated Cochrane Review. Spine. 2017 Apr 15;42(8):586-94. doi: 10.1097/BRS.0000000000002092. PMID: 28399072. Exclusion reason: Publication used as source document

1380. Rawal N, Gupta A, Helsing M, et al. Pain relief following breast augmentation surgery: a comparison between incisional patient-controlled regional analgesia and traditional oral analgesia. Eur J Anaesthesiol. 2006 Dec;23(12):1010-7. PMID: 16780618. Exclusion reason: Ineligible comparator
1381. Rawal N, Kroner K, Simin-Geertsen M, et al. Safety of lornoxicam in the treatment of postoperative pain: a post-marketing study of analgesic regimens containing lornoxicam compared with standard analgesic treatment in 3752 day-case surgery patients. Clin Drug Investig 2010;30(10):687-97. doi: 10.2165/11538860-000000000-00000. PMID: 20701400. Exclusion reason: Ineligible comparator

1382. Rayburn KS. A Model for Integrative Transformation: A Back Pain Group Appointment Program. Altern Ther Health Med. 2017 Jan;23(1):8-13. PMID: 28160758. Exclusion reason: Ineligible population

1383. Reagan KML, O'Sullivan DM, Gannon R, et al. Decreasing postoperative narcotics in reconstructive pelvic surgery: a randomized controlled trial. Am J Obstet Gynecol. 2017 09;217(3):325.e1-.e10. doi: 10.1016/j.ajog.2017.05.041. PMID: 28551445. Exclusion reason: Ineligible intervention

1384. Reardon CE, Kane-Gill SL, Smithburger PL, et al. Sufentanil Sublingual Tablet: A New Option for Acute Pain Management. Ann Pharmacother. 2019 12;53(12):1220-6. doi: https://dx.doi.org/10.1177/10600280198631 44. PMID: 31280585. Exclusion reason: Ineligible comparator

1385. Recart A, Duchene D, White PF, et al. Efficacy and safety of fast-track recovery strategy for patients undergoing laparoscopic nephrectomy. J Endourol. 2005 Dec;19(10):1165-9. PMID: 16359206. Exclusion reason: Ineligible intervention

1386. Reddington M, Walters SJ, Cohen J, et al. Does early intervention improve outcomes in the physiotherapy management of lumbar radicular syndrome? Results of the POLAR pilot randomised controlled trial. BMJ Open. 201807 28;8(7):e021631. doi: 10.1136/BMJopen-2018-021631. PMID: 30056385. Exclusion reason: Ineligible population

1387. Reeser JC, Fischer V, Liu K. Acute low back pain treated with physical therapy versus a self-care program: results of a prospective randomized outcomes study. (Poster Session). Arch Phys Med Rehabil. 2002;83(11):1665. Exclusion reason: Ineligible publication type 
1388. Rehmani R. A Randomized Controlled Trial of Paracetamol Versus Morphine for the Treatment of Acute Painful Crisis of Sickle Cell Disease: 361. Acad Emerg Med. 2013;20. Exclusion reason: Ineligible publication type

1389. Reid DBC, Shah KN, Ruddell JH, et al. Effect of narcotic prescription limiting legislation on opioid utilization following lumbar spine surgery. Spine J. 2019 04;19(4):717-25. doi: https://dx.doi.org/10.1016/j.spinee.2018.09. 007. PMID: 30223089. Exclusion reason: Ineligible intervention

1390. Reines HD, Adams D, Hawkes T, et al. Evaluation of oral ketorolac, oxycodone plus acetaminophen, and placebo for pain relief after orthopedic surgery. Curr Ther Res Clin Exp. 1994;55(6):615-24. Exclusion reason: Ineligible population

1391. Relja M. The role of tramadol in the treatment of acute low back pain. J Neurol Sci. 1990;98(Suppl):334. Exclusion reason: Ineligible publication type

1392. Reuben SS, Ekman EF. The effect of initiating a preventive multimodal analgesic regimen on long-term patient outcomes for outpatient anterior cruciate ligament reconstruction surgery. Anesth Analg. 2007 Jul;105(1):228-32. doi: 10.1213/01.ane.0000265443.20919.c8. PMID: 17578979. Exclusion reason: Ineligible comparator

1393. Reuben SS, Ekman EF, Charron D. Evaluating the analgesic efficacy of administering celecoxib as a component of multimodal analgesia for outpatient anterior cruciate ligament reconstruction surgery. Anesth Analg. 2007 Jul;105(1):222-7. PMID: 17578978. Exclusion reason: Ineligible comparator

1394. Rice D, Rodriguez-Restrepo A, Mena G, et al. Matched Pairs Comparison of an Enhanced Recovery Pathway Versus Conventional Management on Opioid Exposure and Pain Control in Patients Undergoing Lung Surgery. Ann Surg. 2020 Mar 30;30:30. doi: https://dx.doi.org/10.1097/SLA.0000000000 003587. PMID: 32229762. Exclusion

reason: Ineligible intervention
1395. Richards P, Gimbel JS, Minkowitz HS, et al. Comparison of the efficacy and safety of dual-opioid treatment with morphine plus oxycodone versus oxycodone/acetaminophen for moderate to severe acute pain after total knee arthroplasty. Clin Ther. 2013 Apr;35(4):498511. doi: 10.1016/j.clinthera.2013.03.002. PMID: 23545428. Exclusion reason: Ineligible intervention

1396. Richards P, Riff D, Kelen R, et al. Analgesic and adverse effects of a fixed-ratio morphine-oxycodone combination (MoxDuo) in the treatment of postoperative pain. J Opioid Manag. 2011 MayJun;7(3):217-28. PMID: 21823552. Exclusion reason: Ineligible comparator

1397. Richardson E, Bedson J, Chen Y, et al. Increased risk of reproductive dysfunction in women prescribed long-term opioids for musculoskeletal pain: A matched cohort study in the Clinical Practice Research Datalink. Eur J Pain. 2018 10;22(9):1701-8. doi: 10.1002/ejp.1256. PMID: 29873872. Exclusion reason: Ineligible study design

1398. Richmond JC, Gladstone J, MacGillivray J. Continuous passive motion after arthroscopically assisted anterior cruciate ligament reconstruction: comparison of short- versus long-term use. Arthroscopy. 1991;7(1):39-44. doi: 10.1016/07498063(91)90076-a. PMID: 2009118. Exclusion reason: Ineligible comparator

1399. Rickard C, O'Meara P, McGrail M, et al. A randomized controlled trial of intranasal fentanyl vs intravenous morphine for analgesia in the prehospital setting. Am J Emerg Med. 2007 Oct;25(8):911-7. PMID: 17920976. Exclusion reason: Ineligible comparator

1400. Riddell M, Ospina M, Holroyd-Leduc JM. Use of Femoral Nerve Blocks to Manage Hip Fracture Pain among Older Adults in the Emergency Department: A Systematic Review. CJEM. 2016 Jul;18(4):245-52. doi: https://dx.doi.org/10.1017/cem.2015.94. PMID: 26354332. Exclusion reason: Publication used as source document 
1401. Ridderikhof ML, Lirk P, Schep NW, et al. The PanAM study: a multi-center, doubleblinded, randomized, non-inferiority study of paracetamol versus non-steroidal antiinflammatory drugs in treating acute musculoskeletal trauma. BMC Emerg Med. 2013 Nov 20;13:19. doi: 10.1186/1471227X-13-19. PMID: 24256450. Exclusion reason: Ineligible study design

1402. Ridderikhof ML, Saanen J, Goddijn H, et al. Paracetamol versus other analgesia in adult patients with minor musculoskeletal injuries: a systematic review. Emerg Med J. 2019 Aug;36(8):493-500. doi: https://dx.doi.org/10.1136/emermed-2019208439. PMID: 31230039. Exclusion reason: Publication used as source document

1403. Riediger C, Haschke M, Bitter C, et al. The analgesic effect of combined treatment with intranasal S-ketamine and intranasal midazolam compared with morphine patient-controlled analgesia in spinal surgery patients: A pilot study. J Pain Res. 2015;8:87-94. doi: 10.2147/JPR.S75928. PMID: 25709497. Exclusion reason: Ineligible intervention

1404. Riley SP, Bialosky J, Cote MP, et al. Thoracic spinal manipulation for musculoskeletal shoulder pain: Can an instructional set change patient expectation and outcome? Man Ther. 2015 Jun;20(3):469-74. doi: 10.1016/j.math.2014.11.011. PMID: 25543999. Exclusion reason: Ineligible outcome

1405. Rimmer A. Intracanal medications and antibiotics in the control of interappointment flare-ups. Quintessence Int. 1991 Dec;22(12):997-1005. PMID: 1813918. Exclusion reason: Ineligible population

1406. Rindos NB, Mansuria SM, Ecker AM, et al. Intravenous acetaminophen vs saline in perioperative analgesia with laparoscopic hysterectomy. Am J Obstet Gynecol. 2019 04;220(4):373.e1-.e8. doi: https://dx.doi.org/10.1016/j.ajog.2019.01.21 2. PMID: 30682359. Exclusion reason: Ineligible comparator
1407. Rio E, Kidgell D, Purdam C, et al. Isometric exercise induces analgesia and reduces inhibition in patellar tendinopathy. Br J Sports Med. 2015 Oct;49(19):1277-83. doi: 10.1136/bjsports-2014-094386. PMID: 25979840. Exclusion reason: Ineligible duration

1408. Riopelle J, Lopez-Anaya A, Cork RC, et al. Treatment of the cutaneous pain of acute herpes zoster with 9\% lidocaine (base) in petrolatum/paraffin ointment. J Am Acad Dermatol. 1994 May;30(5 Pt 1):757-67. PMID: 8176016. Exclusion reason: Background information only

1409. Rivera JJ, Villecco DJ, Dehner BK, et al. The efficacy of ketorolac as an adjunct to the Bier block for controlling postoperative pain following nontraumatic hand and wrist surgery. AANA J. 2008 Oct;76(5):341-5. PMID: 18947161. Exclusion reason: Ineligible intervention

1410. Roberts L, Little P, Chapman J, et al. The back home trial: general practitionersupported leaflets may change back pain behavior. Spine. 2002 Sep 01;27(17):18218. PMID: 12221342. Exclusion reason: Ineligible outcome

1411. Rodgers-Melnick SN, Matthie N, Jenerette C, et al. The Effects of a Single Electronic Music Improvisation Session on the Pain of Adults with Sickle Cell Disease: A Mixed Methods Pilot Study. J Music Ther. 2018 Jun 07;55(2):156-85. doi: 10.1093/jmt/thy004. PMID: 29796596. Exclusion reason: Ineligible duration

1412. Rodriguez-Huguet M, Gil-Salu JL, Rodriguez-Huguet P, et al. Effects of Myofascial Release on Pressure Pain Thresholds in Patients With Neck Pain: A Single-Blind Randomized Controlled Trial. Am J Phys Med Rehabil. 2018 Jan;97(1):1622. doi: 10.1097/PHM.0000000000000790. PMID: 28678033. Exclusion reason: Ineligible population

1413. Roelofs PD, Deyo RA, Koes BW, et al. Non-steroidal anti-inflammatory drugs for low back pain. Cochrane Database Syst Rev. 2008 Jan 23(1):CD000396. doi: 10.1002/14651858.CD000396.pub3. PMID: 18253976. Exclusion reason: Ineligible comparator 
1414. Rolim CSPL, da Silva MKE, Flumignan LGR, et al. Acetyl-L-carnitine for the treatment of diabetic peripheral neuropathy. Cochrane Database Syst Rev. 2019(6).

Exclusion reason: Ineligible population

1415. Rollings H. Management of acute musculoskeletal conditions-thoracolumbar strain or sprain : a double-blind evaluation comparing the efficacy and safety of carisoprodol with cyclobenzaprine hydrochloride. Curr Ther Res. 1983 1983;34:917-28. Exclusion reason: Ineligible comparator

1416. Rood JP, Coulthard P, Snowdon AT, et al. Safety and efficacy of levobupivacaine for postoperative pain relief after the surgical removal of impacted third molars: a comparison with lignocaine and adrenaline. British J Oral Maxillofac Surg. 2002 Dec;40(6):491-6. PMID: 12464207. Exclusion reason: Ineligible intervention

1417. Rouhani A, Tabrizi A, Ghavidel E. Effects of non-steroidal anti-inflammatory drugs on flexor tendon rehabilitation after repair. Arch Bone Jt Surg. 2013;1(1):28-30. PMID: 25207280 Exclusion reason: Ineligible comparator

1418. Rovetta G, Maggiani G, Molfetta L, et al. One-month follow-up of patients treated by intravenous clodronate for acute pain induced by osteoporotic vertebral fracture. Drugs Exp Clin Res. 2001;27(2):77-81. PMID: 11392057. Exclusion reason: Background information only

1419. Rowbotham MC, Reisner LA, Davies PS, et al. Treatment response in antidepressantnaive postherpetic neuralgia patients: Double-blind, randomized trial. J Pain. 2005 Nov;6(11):741-6. doi: 10.1016/j.jpain.2005.07.001. PMID: 16275598 Exclusion reason: Ineligible population

1420. Roy RA, Boucher JP, Comtois AS. Paraspinal cutaneous temperature modification after spinal manipulation at L5. J Manipulative Physiol Ther. 2010 May;33(4):308-14. doi: 10.1016/j.jmpt.2010.03.001. PMID: 20534318. Exclusion reason: Ineligible outcome
1421. Rudrappa GH, Chakravarthi PT, Benny IR. Efficacy of high-dissolution turmericsesame formulation for pain relief in adult subjects with acute musculoskeletal pain compared to acetaminophen: A randomized controlled study. Medicine. $2020 \mathrm{Jul}$ 10;99(28):e20373. doi: https://dx.doi.org/10.1097/MD.0000000000 020373. PMID: 32664057. Exclusion reason: Ineligible comparator

1422. Ruedy J, Bentley KC. Comparison of glaphenine and codeine in postoperative dental pain. Clin Pharmacol Ther. 1970 SepOct;11(5):718-23. PMID: 4917092.

Exclusion reason: Ineligible intervention

1423. Ruetzler K, Blome CJ, Nabecker S, et al. A randomised trial of oral versus intravenous opioids for treatment of pain after cardiac surgery. J Anesth. 2014 Aug;28(4):580-6. doi: 10.1007/s00540-013-1770-x. PMID: 24375220. Exclusion reason: Ineligible setting

1424. Ruffilli A, Buda R, Castagnini F, et al. Temperature-controlled continuous cold flow device versus traditional icing regimen following anterior cruciate ligament reconstruction: a prospective randomized comparative trial. Arch Orthop Trauma Surg. 2015 Oct;135(10):1405-10. doi: 10.1007/s00402-015-2273-z. PMID: 26141535. Exclusion reason: Ineligible comparator

1425. Rusinyol FC, Pericé RV, Boronat ER, et al. Effects of two different doses of eperisone in the treatment of acute low back pain. $\mathrm{J}$ Appl Res. 2009;9(1-2):23-9. Exclusion reason: Ineligible comparator

1426. Rutgers MJ, Dirksen R. The prevention of postherpetic neuralgia: a retrospective view of patients treated in the acute phase of herpes zoster. Br J Clin Pract. 1988 Oct;42(10):412-4. PMID: 3255420. Exclusion reason: Ineligible intervention

1427. Rzewuska M, Ferreira M, McLachlan AJ, et al. The efficacy of conservative treatment of osteoporotic compression fractures on acute pain relief: a systematic review with metaanalysis. Eur Spine J. 2015 Apr;24(4):70214. doi: https://dx.doi.org/10.1007/s00586015-3821-5. PMID: 25725810. Exclusion reason: Publication used as source document 
1428. Saavedra-Hernandez M, Castro-Sanchez AM, Arroyo-Morales M, et al. Short-term effects of kinesio taping versus cervical thrust manipulation in patients with mechanical neck pain: a randomized clinical trial. J Orthop Sports Phys Ther. 2012 Aug;42(8):724-30. doi: 10.2519/jospt.2012.4086. PMID: 22523090. Exclusion reason: Ineligible population

1429. Saayman L, Hay C, Abrahamse H. Chiropractic manipulative therapy and lowlevel laser therapy in the management of cervical facet dysfunction: a randomized controlled study. J Manipulative Physiol Ther. 2011 Mar-Apr;34(3):153-63. doi: 10.1016/j.jmpt.2011.02.010. PMID: 21492750. Exclusion reason: Ineligible population

1430. Saban B, Deutscher D, Ziv T. Deep massage to posterior calf muscles in combination with neural mobilization exercises as a treatment for heel pain: a pilot randomized clinical trial. Man Ther. 2014

Apr;19(2):102-8. doi: https://dx.doi.org/10.1016/j.math.2013.08.00 1. PMID: 24090993. Exclusion reason: Ineligible population

1431. Sabatino MJ, Kunkel ST, Ramkumar DB, et al. Excess Opioid Medication and Variation in Prescribing Patterns Following Common Orthopaedic Procedures. J Bone Joint Surg. 2018 Feb 07;100(3):180-8. doi: https://dx.doi.org/10.2106/JBJS.17.00672. PMID: 29406338. Exclusion reason: Background information only

1432. Saco M, Golda N. Postoperative Pain Management in Dermatologic Surgery: A Systematic Review. Dermatol Clin. 2019 Jul;37(3):341-8. doi: https://dx.doi.org/10.1016/j.det.2019.03.004. PMID: 31084728. Exclusion reason: Publication used as source document

1433. Sadaf D. Limited Quality Evidence Suggests that Application of Cryotherapy May Be Helpful in Reducing Postoperative Pain in Root Canal Therapy in Patients With Symptomatic Apical Periodontitis. J Evid Based Dent Pract. 2019 06;19(2):195-7. doi: https://dx.doi.org/10.1016/j.jebdp.2019.05.0 05. PMID: 31326055. Exclusion reason: Ineligible publication type
1434. Sadove MS, Balagot RC. Pentazocine--a New Nonaddicting Analgesic. A DoubleBlind Evaluation in Postoperative Pain. JAMA. 1965 Sep 13;193:887-92. PMID: 14341131. Exclusion reason: Ineligible intervention

1435. Safavi M, Honarmand A, Ghaedi F. Preincisional analgesia with subcutaneous administration of tramadol reduces postoperative pain in patients after open urologic surgeries: A randomized, doubleblind, placebo-controlled study. Pak J Med Sci. 2012;28(2):267-72. Exclusion reason: Ineligible intervention

1436. Safdar B, Degutis LC, Landry K, et al. Intravenous morphine plus ketorolac is superior to either drug alone for treatment of acute renal colic. Ann Emerg Med. 2006 Aug;48(2):173-81, 81.e1. doi: 10.1016/j.annemergmed.2006.03.013. PMID: 16953530. Exclusion reason: Ineligible duration

1437. Sahin N, Albayrak I, Ugurlu H. Effect of different transcutaneous electrical stimulation modalities on cervical myofascial pain syndrome. J Musculoskelet Pain. 2011 Jan;19(1):18-23. doi: 10.3109/10582452.2010.538825. Exclusion reason: Ineligible population

1438. Sahmeddini MA, Farbood A, Ghafaripuor S. Electro-acupuncture for pain relief after nasal septoplasty: a randomized controlled study. Journal of Alternative \& Complementary Medicine. 2010 Jan;16(1):53-7. doi: 10.1089/acm.2009.0288. PMID: 20001536. Exclusion reason: Ineligible intervention

1439. Sainoh T, Orita S, Miyagi M, et al. Single intradiscal injection of the interleukin-6 receptor antibody tocilizumab provides short-term relief of discogenic low back pain; prospective comparative cohort study. J Orthop Sci. 2016 Jan;21(1):2-6. doi: 10.1016/j.jos.2015.10.005. PMID: 26755382. Exclusion reason: Ineligible intervention 
1440. Sakamoto A, Nakagawa H, Nakagawa H, et al. Effects of exercises with a pelvic realignment device on low-back and pelvic girdle pain after childbirth: A randomized control study. J Rehabil Med. 2018 Nov 07;50(10):914-9. doi: 10.2340/165019772487. PMID: 30264849. Exclusion reason: Ineligible population

1441. Salameh S, Hiller N, Antopolsky M, et al. Diclofenac versus Tramadol in the Treatment of Renal Colic: A Prospective, Randomized Trial. The Open Emerg Med J. 2011;4:9-13. Exclusion reason: Ineligible duration

1442. Salaudeen GO, Afuwape OO, Eyelade OR, et al. Effectiveness of postoperative analgesia in the management of acute pain in day-case surgeries. Ann Afr Med. 2018 Jul-Sep;17(3):140-4. doi: https://dx.doi.org/10.4103/aam.aam_53_17. PMID: 30185683. Exclusion reason: Ineligible study design

1443. Salazar L, Findlay L. Implementation and Evaluation of an Opioid Risk Questionnaire in the Outpatient Plastic Surgery Setting. Plast Surg Nurs. 2019 Oct/Dec;39(4):13641. doi: https://dx.doi.org/10.1097/PSN.0000000000 000278. PMID: 31790042. Exclusion reason: Ineligible outcome

1444. Salo DF, Lavery R, Varma V, et al. A randomized, clinical trial comparing oral celecoxib $200 \mathrm{mg}$, celecoxib $400 \mathrm{mg}$, and ibuprofen $600 \mathrm{mg}$ for acute pain. Acad Emerg Med. 2003 Jan;10(1):22-30. doi: 10.1111/j.1553-2712.2003.tb01972.x. PMID: 12511311. Exclusion reason: Ineligible comparator

1445. Salottolo K, Peck L, Tanner Ii A, et al. The grass is not always greener: a multiinstitutional pilot study of marijuana use and acute pain management following traumatic injury. Patient Saf Surg. 2018;12:16. doi: 10.1186/s13037-018-0163-3. PMID: 29946360. Exclusion reason: Ineligible study design

1446. Salvador D, Neto PD, Ferrari FP. Application of muscle energy technique in garbage collectors with acute mechanical lumbar pain. Fisioterapia e pesquisa. 2005;2005 May-Aug; 12(2):20-7. Exclusion reason: Not in English
1447. Samimi Sede S, Davari Tanha F, Valadan $\mathrm{M}$, et al. Comparison between preoperative rectal diclofenac plus paracetamol and diclofenac alone for postoperative pain of hysterectomy. J Family Reprod Health. 2014;8(3):91-5. PMID: 25628716. Exclusion reason: Ineligible intervention

1448. Samuel N, Steiner IP, Shavit I. Prehospital pain management of injured children: a systematic review of current evidence. Am J Emerg Med. 2015 Mar;33(3):451-4. doi: https://dx.doi.org/10.1016/j.ajem.2014.12.01 2. PMID: 25572641. Exclusion reason: Publication used as source document

1449. Sanchez-Carpena J, Sesma-Sanchez J, Sanchez-Juan C, et al. Comparison of dexketoprofen trometamol and dipyrone in the treatment of renal colic. Clin Drug Investig. 2003;23(3):139-52. doi: 10.2165/00044011-200323030-00001. PMID: 23340921. Exclusion reason: Ineligible intervention

1450. Sandborgh M, Lindberg P, Denison E. The Pain Belief Screening Instrument (PBSI): predictive validity for disability status in persistent musculoskeletal pain. Disabil Rehabil. 2008;30(15):1123-30. PMID: 19230133. Exclusion reason: Ineligible population

1451. Sanders C, Liegey-Dougall A, Haggard R, et al. Temporomandibular Disorder Diagnostic Groups Affect Outcomes Independently of Treatment in Patients at Risk for Developing Chronicity: A 2-Year Follow-Up Study. J Oral Facial Pain Headache. 2016 2016;30(3):187-202. doi: 10.11607/ofph.1613. PMID: 27472521. Exclusion reason: Ineligible study design

1452. Sanders G, Tepe R, Maloney P, et al. The effect of spinal adjustive manipulation on subjects with acute low back pain: a comparison of visual analog pain scores and serum beta endorphin levels. Symposium of the Pacific Consortium for Chiropractic Research, Monterey, California; July 2, 1989. J Manipulative Physiol Ther. 1990;1(13):58. Exclusion reason: Ineligible publication type 
1453. Sanders GE, Reinert O, Tepe R, et al. Chiropractic adjustive manipulation on subjects with acute low back pain: visual analog pain scores and plasma betaendorphin levels. J Manipulative Physiol Ther. 1990 Sep;13(7):391-5. PMID: 2145384. Exclusion reason: Ineligible duration

1454. Sanger N, Bhatt M, Singhal N, et al. Adverse Outcomes Associated with Prescription Opioids for Acute Low Back Pain: A Systematic Review and MetaAnalysis. Pain Physician. 2019 03;22(2):119-38. PMID: 30921976. Exclusion reason: Publication used as source document

1455. Santiesteban AJ. Comparison of electroacupuncture and selected physical therapy for acute spine pain. Am J Acupunct. 1984;12(3):257-61. Exclusion reason: Ineligible duration

1456. Santos J, Jones S, Wakefield D, et al. Patient Controlled Analgesia for Adults with Sickle Cell Disease Awaiting Admission from the Emergency Department. Pain Res Manag. 2016;2016:3218186. doi: 10.1155/2016/3218186. PMID: 27445606. Exclusion reason: Ineligible study design

1457. Santos TS, Osborne PR, Jacob ES, et al. Effects of Water-Circulating Cooling Mask on Postoperative Outcomes in Orthognathic Surgery and Facial Trauma. J Craniofac Surg. 2020 Jun 19;19:19. doi: https://dx.doi.org/10.1097/SCS.0000000000 006624. PMID: 32604311. Exclusion reason: Publication used as source document

1458. Sanzone AG. Use of Nonopioid Analgesics and the Impact on Patient Outcomes. J Orthop Trauma. 2016 May;30 Suppl 1:S125. doi: https://dx.doi.org/10.1097/BOT.0000000000 000563. PMID: 27101320. Exclusion reason: Background information only
1459. Saporito A, Aguirre J, Borgeat A, et al. Persistent postdischarge pain and chronic postoperative pain after breast cancer surgery under general anesthesia and singleshot paravertebral block: Incidence, characteristics and impact on quality of life and healthcare costs. J Pain Res. 2019;12:1193-9. doi: 10.2147/JPR.S195702. PMID: 31114301. Exclusion reason: Background information only

1460. Saporito A, Anselmi L, Sturini E, et al. Is outpatient continuous regional analgesia more effective and equally safe than singleshot peripheral nerve blocks after ambulatory orthopedic surgery? Minerva Anestesiol. 2017 Sep;83(9):972-81. doi: https://dx.doi.org/10.23736/S03759393.17.11643-3. PMID: 28497931. Exclusion reason: Ineligible intervention

1461. Saragiotto BT, Machado GC, Ferreira ML, et al. Paracetamol for low back pain. Cochrane Database Syst Rev. 2016 Jun 07(6):CD012230. doi: https://dx.doi.org/10.1002/14651858.CD012 230. PMID: 27271789. Exclusion reason: Ineligible comparator

1462. Saragiotto BT, Machado GC, Ferreira ML, et al. Paracetamol for low back pain [Systematic Review]. Cochrane Database Syst Rev. 2019;1:1. PMID: 27271789. Exclusion reason: Publication used as source document

1463. Satpute KH, Bhandari P, Hall T. Efficacy of Hand Behind Back Mobilization With Movement for Acute Shoulder Pain and Movement Impairment: A Randomized Controlled Trial. J Manipulative Physiol Ther. 2015 Jun;38(5):324-34. doi: 10.1016/j.jmpt.2015.04.003. PMID: 26099206. Exclusion reason: Ineligible comparator

1464. Savolainen A, Ahlberg J, Nummila H, et al. Active or passive treatment for neckshoulder pain in occupational health care? A randomized controlled trial. Occup Med (Lond). 2004 Sep;54(6):422-4. doi: 10.1093/occmed/kqh070. PMID: 15358840. Exclusion reason: Ineligible population 
1465. Sayer JM, Kinsella RM, Cary BA, et al. Advanced musculoskeletal physiotherapists are effective and safe in managing patients with acute low back pain presenting to emergency departments. Aust Health Rev. 2018 Jun;42(3):321-6. doi: https://dx.doi.org/10.1071/AH16211. PMID: 28538139. Exclusion reason: Ineligible study design

1466. Scarcella JB, Cohn BT. The effect of cold therapy on the postoperative course of total hip and knee arthroplasty patients. Am J Orthop (Belle Mead NJ). 1995 Nov;24(11):847-52. PMID: 8581442. Exclusion reason: Ineligible setting

1467. Schattenkirchner M, Milachowski KA. A double-blind, multicentre, randomised clinical trial comparing the efficacy and tolerability of aceclofenac with diclofenac resinate in patients with acute low back pain. Clin Rheumatol. 2003 May;22(2):127-35. doi: 10.1007/s10067-003-0710-9. PMID: 12740678. Exclusion reason: Ineligible intervention

1468. Schiller J, Korallus C, Bethge M, et al. Effects of acupuncture on quality of life and pain in patients with osteoporosis-a pilot randomized controlled trial. Arch Osteoporos. 2016 12;11(1):34. PMID: 27766596. Exclusion reason: Ineligible population

1469. Schlaeger JM, Molokie RE, Yao Y, et al. Management of Sickle Cell Pain Using Pregabalin: A Pilot Study. Pain Manag Nurs. 2017 12;18(6):391-400. doi: 10.1016/j.pmn.2017.07.003. PMID: 28843636. Exclusion reason: Ineligible comparator

1470. Schmidt WK, Patou G, Joshi GP. Evaluating therapeutic benefit in postsurgical analgesia requires global assessment: an example from liposome bupivacaine in hemorrhoidectomy. Hosp Pract (1995). 2012 Feb;40(1):160-5. doi: 10.3810/hp.2012.02.956. PMID: 22406891. Exclusion reason: Ineligible comparator

1471. Schneider BJ, Huynh L, Levin J, et al. Does Immediate Pain Relief After an Injection into the Sacroiliac Joint with Anesthetic and Corticosteroid Predict Subsequent Pain Relief? Pain Med. 201802 01;19(2):244-51. doi: 10.1093/pm/pnx104. PMID: 28521006. Exclusion reason: Ineligible population
1472. Schneider M. Mechanical vs manual manipulation for low back pain purpose and study design: prospective cohort study to explore the clinical treatment effect of mechanical vs manual manipulation for acute low back pain. J Chiropr Educ. 2009;23(1):93. Exclusion reason: Ineligible publication type

1473. Schneider M, Haas M, Glick R, et al. Comparison of spinal manipulation methods and usual medical care for acute and subacute low back pain: a randomized clinical trial. Spine. 2015 Feb 15;40(4):20917. doi: $10.1097 /$ BRS.0000000000000724. PMID: 25423308. Exclusion reason: Ineligible population

1474. Schneider MJ. Comparison of mechanical vs. manual manipulation methods for low back pain. University of Pittsburgh. 2008doi: 10.1016/j.jmpt.2010.01.010. PMID: 20350672 Exclusion reason: Ineligible study design

1475. Schneiders AG, Zusman M, Singer KP. Exercise therapy compliance in acute low back pain patients. Man Ther. 1998;3(3):147-52. Exclusion reason: Ineligible outcome

1476. Scholz A, Bothmer J, Kok M, et al. Cebranopadol: A Novel, First-in-Class, Strong Analgesic: Results from a Randomized Phase IIa Clinical Trial in Postoperative Acute Pain. Pain Physician. 2018 05;21(3):E193-E206. PMID: 29871387. Exclusion reason: Ineligible intervention

1477. Schomacher J. The effect of an analgesic mobilization technique when applied at symptomatic or asymptomatic levels of the cervical spine in subjects with neck pain: a randomized controlled trial. J Man Manip Ther. 2009;17(2):101-8. doi: 10.1179/106698109790824758. PMID: 20046552. Exclusion reason: Ineligible population

1478. Schopf S, von Ahnen M, von Ahnen T, et al. Effect of local anesthesia and COX-2 inhibitors after thyroid resection on postoperative pain. Results of two consecutive randomized controlled monocenter studies. J Pain Manag. 2012;5(3):279-87. Exclusion reason: Ineligible intervention 
1479. Schreijenberg M, Chiarotto A, Mauff KAL, et al. Inferential reproduction analysis demonstrated that "paracetamol for acute low back pain" trial conclusions were reproducible. J Clin Epidemiol. 2020 May;121:45-54. doi: https://dx.doi.org/10.1016/j.jclinepi.2020.01. 010. PMID: 31982540. Exclusion reason: Ineligible study design

1480. Schreijenberg M, Luijsterburg PA, Van Trier YD, et al. Efficacy of paracetamol, diclofenac and advice for acute low back pain in general practice: design of a randomized controlled trial (PACE Plus). BMC Musculoskelet Disord. 201702 01;18(1):56. doi: https://dx.doi.org/10.1186/s12891-0171432-5. PMID: 28143496. Exclusion reason: Background information only

1481. Schroder D, Passler HH. Combination of cold and compression after knee surgery. A prospective randomized study. Knee Surg Sports Traumatol Arthrosc. 1994;2(3):15865. doi: 10.1007/bf01467918. PMID: 7584198. Exclusion reason: Ineligible comparator

1482. Schroer WC, Diesfeld PG, LeMarr AR, et al. Does Extended-Release Liposomal Bupivacaine Better Control Pain Than Bupivacaine After Total Knee Arthroplasty (TKA)? A Prospective, Randomized Clinical Trial. J Arthroplasty. 2015 Sep;30(9 Suppl):64-7. doi: 10.1016/j.arth.2015.01.059. PMID: 26117072. Exclusion reason: Ineligible comparator

1483. Schuermans Y, Rauis A. Comparative clinical trial of two injectable NSAIDs, tiaprofenic acid and alclofenac, in acute sciatica. Drugs. 1988;35 Suppl 1:83-5. PMID: 2896114. Exclusion reason: Ineligible comparator

1484. Schug SA, Sidebotham DA, McGuinnety M, et al. Acetaminophen as an adjunct to morphine by patient-controlled analgesia in the management of acute postoperative pain. Anesth Analg. 1998 Aug;87(2):368-72. PMID: 9706932. Exclusion reason: Ineligible comparator
1485. Schug SA, Torrie JJ. Safety assessment of postoperative pain management by an acute pain service. Pain. 1993 Dec;55(3):387-91. PMID: 8121701. Exclusion reason: Ineligible comparator

1486. Schulte H, Segerdahl M, Graven-Nielsen T, et al. Reduction of human experimental muscle pain by alfentanil and morphine. Eur J Pain. 2006 Nov;10(8):733-41. PMID: 16414295. Exclusion reason: Ineligible population

1487. Schwartzberg RS, Reuss BL, Rust R. Efficacy of continuous subacromial bupivacaine infusion for pain control after arthroscopic rotator cuff repair. J Shoulder Elbow Surg. 2013 Oct;22(10):1320-4. doi: 10.1016/j.jse.2013.03.016. PMID: 23668921. Exclusion reason: Ineligible intervention

1488. Schwarzkopf R, Drexler M, Ma MW, et al. Is There a Benefit for Liposomal Bupivacaine Compared to a Traditional Periarticular Injection in Total Knee Arthroplasty Patients With a History of Chronic Opioid Use? J Arthroplasty. 2016 08;31(8):1702-5. doi:

https://dx.doi.org/10.1016/j.arth.2016.01.03 7. PMID: 26897490. Exclusion reason: Background information only

1489. Scolari G, Lazzarin F, Fornaseri C, et al. A comparison of nimesulide beta cyclodextrin and nimesulide in postoperative dental pain. Int J Clin Pract. 1999 Jul-Aug;53(5):345-8. PMID: 10695097. Exclusion reason: Ineligible intervention

1490. Scopp IW, Morgan FH, Gillette WB, et al. Dialog. A double-blind clinical study of dialog, darvon and a placebo in the management of postoperative dental pain. $\mathrm{J}$ Oral Ther Pharmacol. 1967 Sep;4(2):123-7. PMID: 4863139. Exclusion reason: Ineligible intervention

1491. Scott LJ. Fentanyl Iontophoretic Transdermal System: A Review in Acute Postoperative Pain. Clin Drug Investig 2016 Apr;36(4):321-30. doi: https://dx.doi.org/10.1007/s40261-0160387-x. PMID: 26968174. Exclusion reason: Background information only 
1492. Scrimshaw SV, Maher CG. Randomized controlled trial of neural mobilization after spinal surgery. Spine (Phila Pa 1976). 2001 Dec 15;26(24):2647-52. doi: 10.1097/00007632-200112150-00002. PMID: 11740347. Exclusion reason: Ineligible setting

1493. Scuderi GR. The Challenges of Perioperative Pain Management in Total Joint Arthroplasty. Am J Orthop (Chatham, Nj). 2015 Oct;44(10 Suppl):S2-4. PMID: 26447428. Exclusion reason: Background information only

1494. Seers K, Crichton N, Tutton L, et al. Effectiveness of relaxation for postoperative pain and anxiety: Randomized controlled trial. J Adv Nurs. 2008 Jun;62(6):681-8. doi: 10.1111/j.1365-2648.2008.04642.x. PMID: 18503652. Exclusion reason: Ineligible duration

1495. Seferlis T, Nemeth G, Carlsson AM, et al. Conservative treatment in patients sicklisted for acute low-back pain: a prospective randomised study with 12 months' followup. Eur Spine J. 1998;7(6):461-70. doi: 10.1007/s005860050109. PMID: 9883955. Exclusion reason: Ineligible comparator

1496. Sekiguchi H, Inoue G, Nakazawa T, et al. Loxoprofen sodium and celecoxib for postoperative pain in patients after spinal surgery: a randomized comparative study. J Orthop Sci. 2015 Jul;20(4):617-23. doi: 10.1007/s00776-015-0726-4. PMID: 25911562. Exclusion reason: Ineligible comparator

1497. Selkow NM, Grindstaff TL, Cross KM, et al. Short-term effect of muscle energy technique on pain in individuals with nonspecific lumbopelvic pain: a pilot study. J Man Manip Ther. 2009;17(1):E14-8. doi: 10.1179/jmt.2009.17.1.14E. PMID: 20046557. Exclusion reason: Ineligible population

1498. Sener M, Ozgur Pektas Z, Yilmaz I, et al. Comparison of preemptive analgesic effects of a single dose of nonopioid analgesics for pain management after ambulatory surgery: A prospective, randomized, single-blind studyin Turkish patients. Curr Ther Res Clin Exp. 2005;66(6):541-51. doi: 10.1016/j.curtheres.2005.12.001. PMID: 24678075. Exclusion reason: Ineligible intervention
1499. Serpell M, Ratcliffe S, Hovorka J, et al. A double-blind, randomized, placebocontrolled, parallel group study of THC/CBD spray in peripheral neuropathic pain treatment. Eur J Pain. 2014 Aug;18(7):999-1012. doi: 10.1002/j.15322149.2013.00445.x. PMID: 24420962

Exclusion reason: Ineligible population

1500. Sertel S, Herrmann S, Greten HJ, et al. Additional use of acupuncture to NSAID effectively reduces post-tonsillectomy pain. Eur Arch Otorhinolaryngol. 2009 Jun;266(6):919-25. doi: 10.1007/s00405008-0851-1. PMID: 18982338. Exclusion reason: Ineligible setting

1501. Sezen CB, Akboga SA, Celik A, et al. Transcutaneous electrical nerve stimulation effect on postoperative complications. Asian Cardiovasc Thorac Ann. 2017 May;25(4):276-80. doi: 10.1177/0218492317703838. PMID: 28350210. Exclusion reason: Ineligible setting

1502. Shah N, Rollins M, Landi D, et al. Differences in pain management between hematologists and hospitalists caring for patients with sickle cell disease hospitalized for vasoocclusive crisis. Clin J Pain. 2014 Mar;30(3):266-8. doi: 10.1097/AJP.0b013e318295ec04. PMID: 23669451. Exclusion reason: Ineligible outcome

1503. Shah NA, Jain NP, Panchal KA. Adductor Canal Blockade Following Total Knee Arthroplasty-Continuous or Single Shot Technique? Role in Postoperative Analgesia, Ambulation Ability and Early Functional Recovery: A Randomized Controlled Trial. J Arthroplasty. 2015 Aug;30(8):1476-81. doi: 10.1016/j.arth.2015.03.006. PMID: 25824025. Exclusion reason: Ineligible study design

1504. Shah SP, Twilla JD, Kemp L, et al. Comparison of Parenteral Opioid Dosing in Adult Sickle Cell Disease Patients With Vaso-occlusive Crisis. J Pain Palliat Care Pharmacother. 2018 Dec;32(4):201-7. doi: https://dx.doi.org/10.1080/15360288.2019.1 577938. PMID: 30896312. Exclusion reason: Ineligible study design 
1505. Shaheen A, Alam SM, Ahmad A, et al. Clinical efficacy and tolerability of gabapentinoid with current prescription patterns in patients with neuropathic pain. Pak J Med Sci. 2019;35(6):1505-10. doi: 10.12669/pjms.35.6.652. Exclusion reason: Ineligible population

1506. Shahraki AD, Jabalameli M, Ghaedi S. Pain relief after cesarean section: Oral methadone vs. intramuscular pethidine. J Res Med Sci. 2012;17(2):143-7. PMID: 23264787.

Exclusion reason: Ineligible population

1507. Shakya H, Wang D, Zhou K, et al. Prospective randomized controlled study on improving sleep quality and impact of zolpidem after total hip arthroplasty. J Orthop Surg. 2019 Sep 03;14(1):289. doi: https://dx.doi.org/10.1186/s13018-0191327-2. PMID: 31481074. Exclusion reason: Ineligible intervention

1508. Shamliyan TA, Staal JB, Goldmann D, et al. Epidural steroid injections for radicular lumbosacral pain: a systematic review. Phys Med Rehabil Clin N Am. 2014 May;25(2):471-89.e1-50. doi: https://dx.doi.org/10.1016/j.pmr.2014.02.00 1. PMID: 24787344. Exclusion reason: Publication used as source document

1509. Shanks P, Curran M, Fletcher P, et al. The effectiveness of therapeutic ultrasound for musculoskeletal conditions of the lower limb: A literature review. Foot. 2010 Dec;20(4):133-9. doi: https://dx.doi.org/10.1016/j.foot.2010.09.00 6. PMID: 20961748. Exclusion reason: Publication used as source document

1510. Shanthanna H, Paul J, Lovrics P, et al. Satisfactory analgesia with minimal emesis in day surgeries: a randomised controlled trial of morphine versus hydromorphone. $\mathrm{Br}$ J Anaesth. 2019 Jun;122(6):e107-e13. doi: 10.1016/j.bja.2019.03.036. PMID: 31027915. Exclusion reason: Ineligible duration
1511. Sharma AK, Vorobeychik Y, Wasserman R, et al. The Effectiveness and Risks of Fluoroscopically Guided Lumbar Interlaminar Epidural Steroid Injections: A Systematic Review with Comprehensive Analysis of the Published Data. Pain Med. 201702 01;18(2):239-51. doi: https://dx.doi.org/10.1093/pm/pnw131. PMID: 28204730. Exclusion reason: Publication used as source document

1512. Sharpe L, Ianiello M, Dear BF, et al. Is there a potential role for attention bias modification in pain patients? Results of 2 randomised, controlled trials. Pain. 2012 Mar;153(3):722-31. doi: 10.1016/j.pain.2011.12.014. PMID: 22281100. Exclusion reason: Ineligible intervention

1513. Shertzer KE, Keck JF. Music and the PACU environment. J Perianesth Nurs. 2001 Apr;16(2):90-102. PMID: 11290990. Exclusion reason: Ineligible study design

1514. Shifman AC. The clinical response of 328 private patients to acupuncture therapy. Am J Chin Med. 1975 Apr;3(2):165-79. PMID: 123703. Exclusion reason: Ineligible study design

1515. Shin BC, Kim SD, Lee MS. Comparison between the effects of Chuna manipulation therapy and cervical traction treatment on pain in patients with herniated cervical disc: a randomized clinical pilot trial. Am J Chin Med. 2006;34(5):923-5. doi: 10.1142/s0192415x06004399. PMID: 20037928. Exclusion reason: Ineligible publication type

1516. Shin HJ, Kim EY, Na HS, et al. Magnesium sulphate attenuates acute postoperative pain and increased pain intensity after surgical injury in staged bilateral total knee arthroplasty: a randomized, double-blinded, placebo-controlled trial. Br J Anaesth. 2016 Oct;117(4):497-503. doi: 10.1093/bja/aew227. PMID: 28077538. Exclusion reason: Ineligible comparator 
1517. Shin JS, Ha IH, Lee J, et al. Effects of motion style acupuncture treatment in acute low back pain patients with severe disability: a multicenter, randomized, controlled, comparative effectiveness trial. Pain. 2013 Jul;154(7):1030-7. doi: 10.1016/j.pain.2013.03.013. PMID: 23639822. Exclusion reason: Ineligible comparator

1518. Shin JS, Ha IH, Lee TG, et al. Motion style acupuncture treatment (MSAT) for acute low back pain with severe disability: a multicenter, randomized, controlled trial protocol. BMC Complement Altern Med. 2011 Dec 13;11:127. doi: https://dx.doi.org/10.1186/1472-6882-11127. PMID: 22151475. Exclusion reason: Background information only

1519. Shirazi M, Salehipour M, Afrasiabi MA, et al. Analgesic effects and safety of desmopressin, tramadol and indomethacin in patients with acute renal colic; A randomized clinical trial. Bull Emerg Trauma. 2015;3(2):41-5. PMID: 27162901. Exclusion reason: Ineligible duration

1520. Shires A, Sharpe L, Davies JN, et al. The efficacy of mindfulness based interventions in acute pain: a systematic review and metaanalysis. Pain. 2020 Jun 18;18:18. doi: https://dx.doi.org/10.1097/j.pain.000000000 0001877. PMID: 32569085. Exclusion reason: Publication used as source document

1521. Shiri R, Martimo KP, Miranda H, et al. The effect of workplace intervention on pain and sickness absence caused by upper-extremity Musculoskelet Disord. Scand J Work Environ Health. 2011 Mar;37(2):120-8. doi: 10.5271/sjweh.3141. PMID: 21218270.

Exclusion reason: Ineligible intervention

1522. Shlay JC, Chaloner K, Max MB, et al. Acupuncture and amitriptyline for pain due to HIV-related peripheral neuropathy: a randomized controlled trial. Terry Beirn Community Programs for Clinical Research on AIDS. JAMA. 1998 Nov 11;280(18):1590-5. PMID: 9820261. Exclusion reason: Ineligible population
1523. Shoag JE, Patel N, Posada L, et al. Kidney Stones and Risk of Narcotic Use. J Urol. 2019 Jul;202(1):114-8. doi: 10.1097/ju.0000000000000197. PMID: 30829133. Exclusion reason: Ineligible outcome

1524. Shord SS, Chew L, Villano J. Evaluation of opioid induced nausea and vomiting in sickle cell disease. Am J Hematol. 2008 Mar;83(3):196-9. PMID: 17724690.

Exclusion reason: Ineligible outcome

1525. Shrestha R, Silwal P, Basnet N, et al. A Prospective Study of Commonly Prescribed Drugs in the Management of Neuropathic Pain and its Medication Adherence Pattern. Kathmandu Univ Med J (KUMJ). 2016 JanMar;14(53):47-53. PMID: 27892441.

Exclusion reason: Background information only

1526. Silva de Oliveira JC, Grossi de Oliveira GA, Bassi AP. Comparative Assessment of the Effect of Ibuprofen and Etodolac on Edema, Trismus, and Pain in Lower Third Molar Surgery: A Randomized Clinical Trial. J Oral Maxillofac Surg. 2016

Aug;74(8):1524-30. doi: 10.1016/j.joms.2016.04.003. PMID: 27160363. Exclusion reason: Ineligible population

1527. Sim DT. Effectiveness of Transcutaneous Electrical Nerve Stimulation Following Cholecystectomy. Physiotherapy. 1991 1991/10/10/;77(10):715-22. doi: https://doi.org/10.1016/S00319406(10)60447-6. Exclusion reason: Ineligible setting

1528. Simpson EL, Duenas A, Holmes MW, et al. Spinal cord stimulation for chronic pain of neuropathic or ischaemic origin: systematic review and economic evaluation. Health Technol Assess. 2009 Mar;13(17):iii, ix-x, 1-154. doi: https://dx.doi.org/10.3310/hta13170. PMID: 19331797. Exclusion reason: Publication used as source document

1529. Sin B, Wai M, Tatunchak T, et al. The Use of Intravenous Acetaminophen for Acute Pain in the Emergency Department. Acad Emerg Med. 2016 05;23(5):543-53. doi: https://dx.doi.org/10.1111/acem.12921. PMID: 26824905. Exclusion reason: Publication used as source document 
1530. Sin B, Wiafe J, Ciaramella C, et al. The use of intranasal analgesia for acute pain control in the emergency department: A literature review. Am J Emerg Med. 2018

Feb;36(2):310-8. doi: 10.1016/j.ajem.2017.11.043. PMID: 29239753. Exclusion reason: Publication used as source document

1531. Sin WM, Chow KM. Effect of Music Therapy on Postoperative Pain Management in Gynecological Patients: A Literature Review. Pain Manag Nurs. 2015 Dec;16(6):978-87. doi: https://dx.doi.org/10.1016/j.pmn.2015.06.00 8. PMID: 26697822. Exclusion reason: Publication used as source document

1532. Singelyn FJ, Gouverneur JM. Postoperative analgesia after total hip arthroplasty: i.v. PCA with morphine, patient-controlled epidural analgesia, or continuous "3-in-1" block?: a prospective evaluation by our acute pain service in more than 1,300 patients. J Clin Anesth. 1999 Nov;11(7):550-4. PMID: 10624638. Exclusion reason: Ineligible comparator

1533. Singh K, Bohl DD, Ahn J, et al. Multimodal Analgesia Versus Intravenous PatientControlled Analgesia for Minimally Invasive Transforaminal Lumbar Interbody Fusion Procedures. Spine. 2017 Aug 01;42(15):1145-50. doi: 10.1097/BRS.0000000000001992. PMID: 27879573. Exclusion reason: Ineligible population

1534. Singla N, Minkowitz HS, Soergel DG, et al. A randomized, phase IIb study investigating oliceridine (TRV130), a novel $\mu$-receptor Gprotein pathway selective ( $\mu$-GPS) modulator, for the management of moderate to severe acute pain following abdominoplasty. J Pain Res. 2017;10:241324. doi: 10.2147/JPR.S137952. PMID: 29062240. Exclusion reason: Ineligible intervention

1535. Singla N, Pong A, Newman K, et al. Combination oxycodone $5 \mathrm{mg} /$ ibuprofen $400 \mathrm{mg}$ for the treatment of pain after abdominal or pelvic surgery in women: a randomized, double-blind, placebo- and active-controlled parallel-group study. Clin Ther. 2005 Jan;27(1):45-57. PMID: 15763605. Exclusion reason: Ineligible setting
1536. Siqueira LTD, Ribeiro VV, Moreira PAM, et al. Effects of transcutaneous electrical nervous stimulation (TENS) associated with vocal therapy on musculoskeletal pain of women with behavioral dysphonia: A randomized, placebo-controlled doubleblind clinical trial. J Commun Disord. 2019 Jul 30;82:105923. doi: 10.1016/j.jcomdis.2019.105923. PMID: 31382210. Exclusion reason: Ineligible population

1537. Sjodin JG. Effects of intravenous indomethacin during acute ureteral obstruction. Experimental studies and studies in patients with pain due to ureteral stone obstruction. Scand J Urol Nephrol Suppl. 1981;66:1-43. PMID: 6758111. Exclusion reason: Ineligible study design

1538. Skargren EI, Carlsson PG, Oberg BE. Oneyear follow-up comparison of the cost and effectiveness of chiropractic and physiotherapy as primary management for back pain. Subgroup analysis, recurrence, and additional health care utilization. Spine. 1998 Sep 01;23(17):1875-83; discussion 84. PMID: 9762745. Exclusion reason: Ineligible study design

1539. Skargren EI, Oberg BE, Carlsson PG, et al. Cost and effectiveness analysis of chiropractic and physiotherapy treatment for low back and neck pain. Six-month followup. Spine (Phila Pa 1976). 1997 Sep 15;22(18):2167-77. doi: 10.1097/00007632199709150-00015. PMID: 9322328.

Exclusion reason: Ineligible population

1540. Skjelbred P, Album B, Lokken P. Acetylsalicylic acid vs paracetamol: effects on post-operative course. Eur J Clin Pharmacol. 1977 Dec 2;12(4):257-64. doi: 10.1007/bf00607424. PMID: 338309.

Exclusion reason: Ineligible intervention

1541. Skraastad E, Raeder J, Dahl V, et al. Development and validation of the Efficacy Safety Score (ESS), a novel tool for postoperative patient management. BMC Anesthesiol. 201703 28;17(1):50. doi: 10.1186/s12871-017-0344-0. PMID: 28351349. Exclusion reason: Ineligible study design

1542. Smally AJ. Analgesia in renal colic. Ann Emerg Med. 1997 Feb;29(2):296; author reply 7-9. PMID: 9018200. Exclusion reason: Ineligible publication type 
1543. Smedley F, Taube M, Wastell C.

Transcutaneous electrical nerve stimulation for pain relief following inguinal hernia repair: a controlled trial. Eur Surg Res. 1988;20(4):233-7. doi: 10.1159/000128766. PMID: 3262516. Exclusion reason: Ineligible setting

1544. Smith AR, Jr. Manual therapy: the historical, current, and future role in the treatment of pain. The Scientific World Journal. 2007 Feb 02;7:109-20. PMID: 17334604. Exclusion reason: Background information only

1545. Smith CM, Guralnick MS, Gelfand MM, et al. The effects of transcutaneous electrical nerve stimulation on post-cesarean pain. Pain. 1986 Nov;27(2):181-93. doi: 10.1016/0304-3959(86)90209-5. PMID: 3540813. Exclusion reason: Ineligible setting

1546. Smith I, Walley G, Bridgman S. Omitting fentanyl reduces nausea and vomiting, without increasing pain, after sevoflurane for day surgery. Eur J Anaesthesiol. 2008 Oct;25(10):790-9. doi: 10.1017/S026502150800464X. PMID: 18544179. Exclusion reason: Ineligible comparator

1547. Smith J, Stevens J, Taylor M, et al. A randomized, controlled trial comparing compression bandaging and cold therapy in postoperative total knee replacement surgery. Orthop Nurs. 2002 MarApr;21(2):61-6. doi: 10.1097/00006416200203000-00009. PMID: 11949239.

Exclusion reason: Ineligible comparator

1548. Smith WR, Ballas SK, McCarthy WF, et al. The association between hydroxyurea treatment and pain intensity, analgesic use, and utilization in ambulatory sickle cell anemia patients. Pain Med. 2011 May;12(5):697-705. doi: 10.1111/j.15264637.2011.01096.x. PMID: 21481164. Exclusion reason: Ineligible intervention

1549. Snir N, Moskovitz B, Nativ O, et al. Papaverine hydrochloride for the treatment of renal colic: an old drug revisited. A prospective, randomized study. J Urol. 2008 Apr;179(4):1411-4. doi: 10.1016/j.juro.2007.11.053. PMID: 18289563. Exclusion reason: Ineligible duration
1550. Snyder MB, Bregmen DB. SPRIX (ketorolac tromethamine) nasal spray: a novel nonopioid alternative for managing moderate to moderately severe dental pain. Compend Contin Educ Dent. 2012 Feb;33 Spec No 1(1):2-11. PMID: 22428363. Exclusion reason: Ineligible publication type

1551. Soave G, Lavezzari M, Ferrati G, et al. Indoprofen and pentazocine in posttraumatic pain. A double-blind, parallelgroup comparative trial. J Int Med Res. 1983;11(6):354-8. PMID: 6360751.

Exclusion reason: Ineligible setting

1552. Soderlund A, Olerud C, Lindberg P. Acute whiplash-associated disorders (WAD): the effects of early mobilization and prognostic factors in long-term symptomatology. Clin Rehabil. 2000 Oct;14(5):457-67. PMID: 11043871. Exclusion reason: Ineligible comparator

1553. Solomon DH, Bates DW, Horsky J, et al. Development and validation of a patient satisfaction scale for musculoskeletal care. Arthritis Care Res. 1999 Apr;12(2):96-100. PMID: 10513497. Exclusion reason: Ineligible intervention

1554. Solomon DH, Rassen JA, Glynn RJ, et al. The comparative safety of opioids for nonmalignant pain in older adults. Arch Intern Med. 2010 Dec 13;170(22):1979-86. doi: 10.1001/archinternmed.2010.450. PMID: 21149754. Exclusion reason: Ineligible population

1555. Solomon LR. Treatment and prevention of pain due to vaso-occlusive crises in adults with sickle cell disease: an educational void. Blood. 2008 Feb 01;111(3):997-1003. PMID: 17940207. Exclusion reason: Background information only

1556. Sommer P, Kromann-Andersen B, Lendorf A, et al. Analgesic effect and tolerance of Voltaren and Ketogan in acute renal or ureteric colic. Br J Urol. 1989 Jan;63(1):4-6. doi: 10.1111/j.1464-410x.1989.tb05112.x. PMID: 2645969. Exclusion reason: Ineligible comparator 
1557. Song D, He A, Xu R, et al. Efficacy of Pain Relief in Different Postherpetic Neuralgia Therapies: A Network Meta-Analysis. Pain Physician. 2018 01;21(1):19-32. PMID: 29357328. Exclusion reason: Publication used as source document

1558. Song L, Qiu P, Xu J, et al. The Effect of Combination Pharmacotherapy on Low Back Pain: A Meta-analysis. Clin J Pain. 2018 11;34(11):1039-46. doi: https://dx.doi.org/10.1097/AJP.0000000000 000622. PMID: 29727303. Exclusion reason: Publication used as source document

1559. Southerst D, Marchand AA, Cote P, et al. The effectiveness of noninvasive interventions for musculoskeletal thoracic spine and chest wall pain: a systematic review by the Ontario Protocol for Traffic Injury Management (OPTIMa) collaboration. J Manipulative Physiol Ther. 2015 Sep;38(7):521-31. doi: https://dx.doi.org/10.1016/j.jmpt.2015.06.00 1. PMID: 26141077. Exclusion reason: Publication used as source document

1560. Spagnoli AM, Rizzo MI, Palmieri A, et al. A single blind controlled comparison of tramadol/paracetamol combination and paracetamol in hand and foot surgery. A prospective study. In Vivo. 2011 MarApr;25(2):291-5. PMID: 21471550. Exclusion reason: Ineligible intervention

1561. Spijker-Huiges A, Vermeulen K, Winters JC, et al. Costs and cost-effectiveness of epidural steroids for acute lumbosacral radicular syndrome in general practice: an economic evaluation alongside a pragmatic randomized control trial. Spine. $2014 \mathrm{Nov}$ 15;39(24):2007-12. doi: 10.1097/BRS.0000000000000597. PMID: 25202937. Exclusion reason: Ineligible outcome

1562. Spivakovsky S, Spivakovsky Y. Reliable evidence for efficacy of single dose oral analgesics. Evid Based Dent. 2016 06;17(2):60-1. doi: 10.1038/sj.ebd.6401175. PMID: 27339244. Exclusion reason: Ineligible publication type
1563. Sproviero E, Albamonte E, Costantino C, et al. Efficacy and safety of a fixed combination of intramuscular diclofenac 75 $\mathrm{mg}+$ thiocolchicoside $4 \mathrm{mg}$ in the treatment of acute low back pain: a phase III, randomized, double blind, controlled trial. Eur J Phys Rehabil Med. 2018 Oct;54(5):654-62. doi: 10.23736/S19739087.17.04923-1. PMID: 29265793.

Exclusion reason: Ineligible comparator

1564. St John Dixon A. Cold-sensitive, nonspecific, low back pain : a comparative trial of treatment. Clin Trials J. 1972 1972;4:16-21. Exclusion reason: Ineligible population

1565. Stam C, Bonnet MS, van Haselen RA. The efficacy and safety of a homeopathic gel in the treatment of acute low back pain: a multi-centre, randomised, double-blind comparative clinical trial. Br Homeopath J. 2001 Jan;90(1):21-8. PMID: 11212085.

Exclusion reason: Ineligible intervention

1566. Stamenkovic DM, Mladenovic K, Rancic N, et al. Effect of Transcranial Direct Current Stimulation Combined With PatientControlled Intravenous Morphine Analgesia on Analgesic Use and Post-Thoracotomy Pain. A Prospective, Randomized, DoubleBlind, Sham-Controlled, Proof-of-Concept Clinical Trial. Front Pharmacol. 2020;11:125. doi: https://dx.doi.org/10.3389/fphar.2020.00125 . PMID: 32161547. Exclusion reason: Ineligible intervention

1567. Stamos A, Drum M, Reader A, et al. An Evaluation of Ibuprofen Versus Ibuprofen/Acetaminophen for Postoperative Endodontic Pain in Patients With Symptomatic Irreversible Pulpitis and Symptomatic Apical Periodontitis. Anesth Prog. 2019;66(4):192-201. doi: https://dx.doi.org/10.2344/anpr-66-03-06. PMID: 31891295. Exclusion reason: Ineligible comparator

1568. Standaert CJ. Bed rest or continuation of activity for acute low back pain? Clin J Sport Med. 2003 Jul;13(4):275. PMID: 12918494. Exclusion reason: Ineligible study design 
1569. Stankov G, Schmieder G, Zerle G, et al. Double-blind study with dipyrone versus tramadol and butylscopolamine in acute renal colic pain. World J Urol. 1994;12(3):155-61. PMID: 7951343.

Exclusion reason: Ineligible intervention

1570. Stankovic R, Johnell O. Conservative treatment of acute low-back pain. A prospective randomized trial: McKenzie method of treatment versus patient education in "mini back school". Spine. 1990 Feb;15(2):120-3. PMID: 2139241. Exclusion reason: Ineligible comparator

1571. Stankovic R, Johnell O. Conservative treatment of acute low back pain. A 5-year follow-up study of two methods of treatment. Spine. 1995 Feb 15;20(4):469-72. doi: 10.1097/00007632-199502001-00010. PMID: 7747231. Exclusion reason: Ineligible outcome

1572. Starkweather AR, Coyne P, Lyon DE, et al. Decreased low back pain intensity and differential gene expression following Calmare: results from a double-blinded randomized sham-controlled study. Res Nurs Health. 2015 Feb;38(1):29-38. doi: 10.1002/nur.21632. PMID: 25572279.

Exclusion reason: Ineligible population

1573. Stegmann JU, Weber H, Steup A, et al. The efficacy and tolerability of multiple-dose tapentadol immediate release for the relief of acute pain following orthopedic (bunionectomy) surgery. Curr Med Res Opin. 2008 Nov;24(11):3185-96. doi: https://dx.doi.org/10.1185/03007990802448 056. PMID: 18851776. Exclusion reason: Ineligible setting

1574. Stehman M. Double-blind clinical study of Idrocilamide ointment in the treatment of acute low back pain. Acta belgica medica physica. 1990;13(1):29-32. Exclusion reason: Not in English

1575. Stein D, Peri T, Edelstein E, et al. The efficacy of amitriptyline and acetaminophen in the management of acute low back pain. Psychosomatics. 1996 Jan-Feb;37(1):63-70. doi: 10.1016/S0033-3182(96)71600-6. PMID: 8600497. Exclusion reason: Ineligible population
1576. Steinberg PL, Nangia AK, Curtis K. A standardized pain management protocol improves timeliness of analgesia among emergency department patients with renal colic. Qual Manag Health Care. 2011 JanMar;20(1):30-6. doi: 10.1097/QMH.0b013e31820429d9. PMID: 21192205. Exclusion reason: Ineligible study design

1577. Steiner TJ, Voelker M. Gastrointestinal tolerability of aspirin and the choice of overthe-counter analgesia for short-lasting acute pain. J Clin Pharm Ther. 2009 Apr;34(2):177-86. doi: 10.1111/j.13652710.2008.00989.x. PMID: 19250138. Exclusion reason: Ineligible study design

1578. Sterling M, Jull G, Wright A. Cervical mobilisation: concurrent effects on pain, sympathetic nervous system activity and motor activity. Man Ther. 2001 May;6(2):72-81. PMID: 11414776. Exclusion reason: Ineligible population

1579. Sterling M, Smeets R, Keijzers G, et al. Physiotherapist-delivered stress inoculation training integrated with exercise versus physiotherapy exercise alone for acute whiplash-associated disorder (StressModex): a randomised controlled trial of a combined psychological/physical intervention. Br J Sports Med. 2019 Jan 19;19:19. doi: 10.1136/bjsports-2018-100139. PMID: 30661011. Exclusion reason: Ineligible comparator

1580. Stessel B, Boon M, Joosten EA, et al. Metamizole versus ibuprofen at home after day surgery: study protocol for a randomised controlled trial. Trials. 2016;17(1)doi: 10.1186/s13063-016-1586-8. PMID: 27669689. Exclusion reason: Ineligible intervention

1581. Stessel B, Boon M, Pelckmans C, et al. Metamizole vs. ibuprofen at home after day case surgery: a double-blind randomised controlled noninferiority trial. Eur J Anaesthesiol. 2019;36(5):351-9. doi: 10.1097/EJA.0000000000000972. PMID: 30946703. Exclusion reason: Ineligible intervention 
1582. Stessel B, Theunissen M, Fiddelers AA, et al. Controlled-Release Oxycodone Versus Naproxen at Home After Ambulatory Surgery: a Randomized Controlled Trial. Curr Ther Res clinical and experimental. 2014;76:120-5. doi:

10.1016/j.curtheres.2014.10.001. PMID: 25516773. Exclusion reason: Ineligible comparator

1583. Stevens ML, Lin CC, de Carvalho FA, et al. Advice for acute low back pain: a comparison of what research supports and what guidelines recommend. Spine J. 2017 10;17(10):1537-46. doi: https://dx.doi.org/10.1016/j.spinee.2017.05. 030. PMID: 28713052. Exclusion reason: Background information only

1584. Stevensen C. The psychophysiological effects of aromatherapy massage following cardiac surgery. Complement Ther Med. 1994 1994/01/01/;2(1):27-35. doi: https://doi.org/10.1016/09652299(94)90156-2. Exclusion reason: Ineligible setting

1585. Stewart AM, Polak E, Young R, et al. Injured workers' construction of expectations of return to work with subacute back pain: the role of perceived uncertainty. J Occup Rehabil. 2012 Mar;22(1):1-14. doi: 10.1007/s10926-0119312-6. PMID: 21656253. Exclusion reason: Ineligible population

1586. Stocchero M, Gobbato L, De Biagi M, et al. Pulsed electromagnetic fields for postoperative pain: a randomized controlled clinical trial in patients undergoing mandibular third molar extraction. Oral Surg Oral Med Oral Pathol and Oral Radiology. 2015 Mar;119(3):293-300. doi: 10.1016/j.oooo.2014.11.017. PMID: 25660828. Exclusion reason: Ineligible intervention

1587. Stochkendahl MJ, Christensen HW, Vach $\mathrm{W}$, et al. A randomized clinical trial of chiropractic treatment and self-management in patients with acute musculoskeletal chest pain: 1-year follow-up. J Manipulative Physiol Ther. 2012 May;35(4):254-62. doi: 10.1016/j.jmpt.2012.04.003. PMID: 22632585. Exclusion reason: Ineligible intervention
1588. Stochkendahl MJ, Christensen HW, Vach $\mathrm{W}$, et al. Chiropractic treatment vs selfmanagement in patients with acute chest pain: a randomized controlled trial of patients without acute coronary syndrome. J Manipulative Physiol Ther. 2012 Jan;35(1):7-17. doi: 10.1016/j.jmpt.2010.11.004. PMID: 22185955. Exclusion reason: Ineligible intervention

1589. Stoerkel E, Bellanti D, Paat C, et al. Effectiveness of a Self-Care Toolkit for Surgical Breast Cancer Patients in a Military Treatment Facility. Journal of Alternative \& Complementary Medicine. 2018 Sep/Oct;24(9-10):916-25. doi: 10.1089/acm.2018.0069. PMID: 30247967. Exclusion reason: Ineligible intervention

1590. Stoicea N, Costa A, Periel L, et al. Current perspectives on the opioid crisis in the US healthcare system: A comprehensive literature review. Medicine. 2019 May;98(20):e15425. doi: https://dx.doi.org/10.1097/MD.0000000000 015425. PMID: 31096439. Exclusion reason: Background information only

1591. Storheim K, Brox JI, Holm I, et al. Intensive group training versus cognitive intervention in sub-acute low back pain: short-term results of a single-blind randomized controlled trial. J Rehabil Med. 2003 May;35(3):132-40. doi: 10.1080/16501970310010484. PMID: 12809196. Exclusion reason: Ineligible population

1592. Straube S, Derry S, Moore AR, et al. Single dose oral gabapentin for established acute postoperative pain in adults. Cochrane Database Syst Rev. 2019(5) PMID: 20464764. Exclusion reason: Ineligible comparator

1593. Stumbo SP, Yarborough BJH, McCarty D, et al. Patient-reported pathways to opioid use disorders and pain-related barriers to treatment engagement. J Subst Abuse Treat. 2017 Feb;73:47-54. doi: 10.1016/j.jsat.2016.11.003. PMID: 28017184. Exclusion reason: Ineligible population 
1594. Sturesson L, Falk AC, Castren M, et al. Mandatory documentation of pain in the emergency department increases analgesic administration but does not improve patients' satisfaction of pain management. Scandinavian J Pain. 2016 10;13:32-5. doi: https://dx.doi.org/10.1016/j.sjpain.2016.06.0 06. PMID: 28850532. Exclusion reason: Ineligible population

1595. Su EP, Perna M, Boettner F, et al. A prospective, multi-center, randomised trial to evaluate the efficacy of a cryopneumatic device on total knee arthroplasty recovery. J Bone Joint Surg Br. 2012 Nov;94(11 Suppl A):153-6. doi: 10.1302/0301620X.94B11.30832. PMID: 23118406. Exclusion reason: Ineligible comparator

1596. Su JT, Zhou QH, Li R, et al. Immediate analgesic effect of wrist-ankle acupuncture for acute lumbago: a randomized controlled trial. Zhongguo zhen jiu= Chinese Acupuncture \& Moxibustion. 2010;30(8):617-22. PMID: 20942274.

Exclusion reason: Not in English

1597. Sugioka Y. Multicenter clinical evaluation of piroxicam gel vs. indomethacin gel in the treatment of non-traumatic diseases of tendon or muscle. Japanese Pharmacology and Therapeutics. 1984;12:139-53.

Exclusion reason: Not in English

1598. Sullivan D, Lyons M, Montgomery R, et al. Exploring Opioid-Sparing Multimodal Analgesia Options in Trauma: A Nursing Perspective. J Trauma Nurs. 2016 Nov/Dec;23(6):361-75. PMID: 27828892. Exclusion reason: Ineligible publication type

1599. Sultan A, McQuay HJ, Moore RA, et al. Single dose oral flurbiprofen for acute postoperative pain in adults. Cochrane Database Syst Rev. 2009 Jul 08(3):CD007358. doi: 10.1002/14651858.CD007358.pub2. PMID: 19588427. Exclusion reason: Ineligible publication type
1600. Sun BC, Charlesworth CJ, Lupulescu-Mann N, et al. Effect of Automated Prescription Drug Monitoring Program Queries on Emergency Department Opioid Prescribing. Ann Emerg Med. 2018 Mar;71(3):33747.e6. doi: 10.1016/j.annemergmed.2017.10.023. PMID: 29248333. Exclusion reason: Ineligible population

1601. Sun EC, Darnall BD, Baker LC, et al. Incidence of and Risk Factors for Chronic Opioid Use Among Opioid-Naive Patients in the Postoperative Period. JAMA Intern Med. 2016 Sep 1;176(9):1286-93. doi: 10.1001/jamainternmed.2016.3298. PMID: 27400458. Exclusion reason: Ineligible population

1602. Sun K, Xing T, Zhang F, et al. Perioperative transcutaneous electrical acupoint stimulation for postoperative pain relief following laparoscopic surgery: A randomized controlled trial. Clin J Pain. 2017 Apr;33(4):340-7. doi: 10.1097/AJP.000000000000400. PMID: 27437568. Exclusion reason: Ineligible intervention

1603. Sun N, Shi GX, Tu JF, et al. Traditional Chinese acupuncture versus minimal acupuncture for mild-to-moderate knee osteoarthritis: a protocol for a randomised, controlled pilot trial. BMJ Open. 201612 13;6(12):e013830. doi: 10.1136/BMJopen2016-013830. PMID: 27965256. Exclusion reason: Ineligible population

1604. Sun Y, Gan TJ, Dubose JW, et al. Acupuncture and related techniques for postoperative pain: a systematic review of randomized controlled trials. Br J Anaesth. 2008 Aug;101(2):151-60. doi: https://dx.doi.org/10.1093/bja/aen146. PMID: 18522936. Exclusion reason: Publication used as source document

1605. Sung W, Abraham M, Plastaras C, et al. Trunk motor control deficits in acute and subacute low back pain are not associated with pain or fear of movement. Spine J. 2015 Aug 01;15(8):1772-82. doi: 10.1016/j.spinee.2015.04.010. PMID: 25862508. Exclusion reason: Ineligible study design 
1606. Sunshine A, Olson NZ, Colon A, et al. Analgesic efficacy of controlled-release oxycodone in postoperative pain. J Clin Pharmacol. 1996 Jul;36(7):595-603. doi: 10.1002/j.1552-4604.1996.tb04223.x. PMID: 8844441. Exclusion reason: Ineligible comparator

1607. Sunshine A, Olson NZ, O'Neill E, et al. Analgesic efficacy of a hydrocodone with ibuprofen combination compared with ibuprofen alone for the treatment of acute postoperative pain. J Clin Pharmacol. 1997 Oct;37(10):908-15. PMID: 9505982. Exclusion reason: Ineligible setting

1608. Sunshine A, Slafta J, Gruber C, Jr. A comparative analgesic study of propoxyphene, fenoprofen, the combination of propoxyphene and fenoprofen, aspirin, and placebo. J Clin Pharmacol. 1978 NovDec;18(11-12):556-63. PMID: 363751.

Exclusion reason: Ineligible intervention

1609. Sunshine A, Zighelboim I, Olson NZ, et al. A comparative oral analgesic study of indoprofen, aspirin, and placebo in postpartum pain. J Clin Pharmacol. 1985 Jul-Aug;25(5):374-80. PMID: 3897296.

Exclusion reason: Ineligible intervention

1610. Suputtitada A, Wacharapreechanont T, Chaisayan P. Effect of the "sitting pelvic tilt exercise" during the third trimester in primigravidas on back pain. J Med Assoc Thai. 2002 Jun;85 Suppl 1:S170-9. PMID: 12188409. Exclusion reason: Ineligible population

1611. Suter E, McMorland G, Herzog W, et al. Decrease in quadriceps inhibition after sacroiliac joint manipulation in patients with anterior knee pain. J Manipulative Physiol Ther. 1999 Mar-Apr;22(3):149-53. PMID: 10220713. Exclusion reason: Ineligible population

1612. Sutlive TG, Mabry LM, Easterling EJ, et al. Comparison of short-term response to two spinal manipulation techniques for patients with low back pain in a military beneficiary population. Mil Med. 2009 Jul;174(7):7506. PMID: 19685848. Exclusion reason: Ineligible comparator
1613. Suzan E, Pud D, Eisenberg E. A crucial administration timing separates between beneficial and counterproductive effects of opioids on postoperative pain. Pain. 2018 Aug;159(8):1438-40. doi: http://dx.doi.org/10.1097/j.pain.0000000000 001200. PMID: 29521812. Exclusion reason: Background information only

1614. Swait G, Finch R. What are the risks of manual treatment of the spine? A scoping review for clinicians. Chiropr Man Therap. 2017;25:37. doi: https://dx.doi.org/10.1186/s12998-0170168-5. PMID: 29234493. Exclusion reason: Publication used as source document

1615. Swarup A, Mathis KA, Hill MV, et al. Patterns of opioid use and prescribing for outpatient anorectal operations. J Surg Res. 2018 09;229:283-7. doi: https://dx.doi.org/10.1016/j.jss.2018.04.005. PMID: 29937003. Exclusion reason: Background information only

1616. Sweetman BJ. A randomized controlled trial of exercises, short wave diathermy, and traction for low back pain, with evidence of diagnosis-related response to treatment. J Orthop Rheumatol. 1993 1993;6:159-66. Exclusion reason: Ineligible population

1617. Sweetman BJ, Baig A, Parsons DL. Mefenamic acid, chlormezanoneparacetamol, ethoheptazine-aspirinmeprobamate: a comparative study in acute low back pain. Br J Clin Pract. 1987 Feb;41(2):619-24. PMID: 2960369. Exclusion reason: Ineligible comparator

1618. Swislocki A, Orth M, Bales M, et al. A randomized clinical trial of the effectiveness of photon stimulation on pain, sensation, and quality of life in patients with diabetic peripheral neuropathy. J Pain Symptom Manage. 2010 Jan;39(1):88-99. doi: 10.1016/j.jpainsymman.2009.05.021. PMID: 19896325. Exclusion reason: Ineligible population

1619. Szpalski MHJ. Interest of NSAID's in the treatment of acute low back pain. A double blind study with objective assessment of function. J Bone Joint Surg. 1993;British volume. 75-B(Suppl 3):272. Exclusion reason: Ineligible publication type 
1620. Tabassi KT, Amini P, Mohammadi S, et al. The effect of acupuncture on pain score after open kidney surgery. Journal of Complementary \& Integrative Medicine. 2015 Sep;12(3):241-4. doi: https://dx.doi.org/10.1515/jcim-2014-0071. PMID: 25924232. Exclusion reason: Ineligible setting

1621. Tabatabaiee A, Ebrahimi-Takamjani I, Ahmadi A, et al. Comparison of pressure release, phonophoresis and dry needling in treatment of latent myofascial trigger point of upper trapezius muscle. J Back Musculoskelet Rehabil. 2019;32(4):587-94. doi: https://dx.doi.org/10.3233/BMR181302. PMID: 30584120. Exclusion reason: Ineligible population

1622. Takamoto K, Bito I, Urakawa S, et al. Effects of compression at myofascial trigger points in patients with acute low back pain: A randomized controlled trial. Eur J Pain. 2015 Sep;19(8):1186-96. doi: https://dx.doi.org/10.1002/ejp.694. PMID: 25808188. Exclusion reason: Ineligible comparator

1623. Takase H, Sakata T, Yamano T, et al. Advantage of early induction of opioid to control pain induced by irradiation in head and neck cancer patients. Auris, Nasus, Larynx. 2011 Aug;38(4):495-500. doi: 10.1016/j.anl.2010.12.012. PMID: 21277720. Exclusion reason: Ineligible population

1624. Takeda Y, Fukunishi S, Nishio S, et al. Evaluating the Effect of Intravenous Acetaminophen in Multimodal Analgesia After Total Hip Arthroplasty: A Randomized Controlled Trial. J Arthroplasty. 2019 06;34(6):1155-61. doi: https://dx.doi.org/10.1016/j.arth.2019.02.03 3. PMID: 30898388. Exclusion reason: Ineligible intervention

1625. Takla MKN. Low-frequency high-intensity versus medium-frequency low-intensity combined therapy in the management of active myofascial trigger points: A randomized controlled trial. Physiother Res Int. 2018 Oct;23(4):e1737. doi: 10.1002/pri.1737. PMID: 30095858. Exclusion reason: Ineligible population
1626. Takla MKN, Rezk-Allah SS. Immediate Effects of Simultaneous Application of Transcutaneous Electrical Nerve Stimulation and Ultrasound Phonophoresis on Active Myofascial Trigger Points: A Randomized Controlled Trial. Am J Phys Med Rehabil. 2018 05;97(5):332-8. doi: 10.1097/PHM.0000000000000876. PMID: 29206666. Exclusion reason: Ineligible population

1627. Tammachote N, Seangleulur A, Kanitnate S. Lumbar epidural corticosteroid injection reduces subacute pain and improves knee function in the first six weeks after total knee arthroplasty: A double-blinded randomized trial. J Bone Joint Surg American Volume. 2018;100(11):950-7. doi: 10.2106/JBJS.17.00578. PMID: 29870446. Exclusion reason: Ineligible intervention

1628. Tammimaki A, Mannisto PT. Catechol-Omethyltransferase gene polymorphism and chronic human pain: a systematic review and meta-analysis. Pharmacogenet Genomics. 2012 Sep;22(9):673-91. doi: https://dx.doi.org/10.1097/FPC.0b013e3283 560c46. PMID: 22722321. Exclusion reason: Publication used as source document

1629. Tan P, Martin MS, Shank N, et al. A Comparison of 4 Analgesic Regimens for Acute Postoperative Pain Control in Breast Augmentation Patients. Ann Plast Surg. 2017 Jun;78(6S Suppl 5):S299-S304. doi: 10.1097/SAP.0000000000001132. PMID: 28459704. Exclusion reason: Ineligible study design

1630. Tanabe P, Myers R, Zosel A, et al. Emergency department management of acute pain episodes in sickle cell disease. Acad Emerg Med. 2007 May;14(5):419-25. PMID: 17389246. Exclusion reason: Ineligible study design

1631. Tanabe P, Paice JA, Stancati J, et al. How do emergency department patients store and dispose of opioids after discharge? A pilot study. J Emerg Nurs. 2012 May;38(3):2739. doi: 10.1016/j.jen.2011.09.023. PMID: 22204885. Exclusion reason: Ineligible outcome 
1632. Tanabe P, Silva S, Bosworth HB, et al. A randomized controlled trial comparing two vaso-occlusive episode (VOE) protocols in sickle cell disease (SCD). Am J Hematol. 2018 02;93(2):159-68. doi:

10.1002/ajh.24948. PMID: 29047145.

Exclusion reason: Ineligible comparator

1633. Tanabe P, Thomas R, Paice J, et al. The effect of standard care, ibuprofen, and music on pain relief and patient satisfaction in adults with musculoskeletal trauma. J Emerg Nurs. 2001 Apr;27(2):124-31. PMID: 11275859. Exclusion reason: Ineligible study design

1634. Tanaka S, Yoshida A, Kono S, et al. Effectiveness of monotherapy and combined therapy with calcitonin and minodronic acid hydrate, a bisphosphonate, for early treatment in patients with new vertebral fractures: An open-label, randomized, parallel-group study. J Orthop Sci. 2017 May;22(3):536-41. doi:

10.1016/j.jos.2016.12.021. PMID: 28108224. Exclusion reason: Ineligible intervention

1635. Tanasescu R, Rog D, Constantinescu CS. A drug discovery case history of 'delta-9tetrahydrocannabinol, cannabidiol.' Expert Opin Drug Discov. 2011;6(4):437-52. doi: 10.1517/17460441.2011.560935. Exclusion reason: Background information only

1636. Taylor AG, West BA, Simon B, et al. How effective is TENS for acute pain? Am J Nurs. 1983 Aug;83(8):1171-4. PMID: 6603794. Exclusion reason: Ineligible setting

1637. Taylor LK, Kuttler KL, Parks TA, et al. The effect of music in the postanesthesia care unit on pain levels in women who have had abdominal hysterectomies. J Perianesth Nurs. 1998 Apr;13(2):88-94. doi: 10.1016/s1089-9472(98)80091-9. PMID: 9592448. Exclusion reason: Ineligible setting
1638. Taylor R, Jr., Gan TJ, Raffa RB, et al. A randomized, double-blind comparison shows the addition of oxygenated glycerol triesters to topical mentholated cream for the treatment of acute musculoskeletal pain demonstrates incremental benefit over time. Pain Pract. 2012 Nov;12(8):610-9. doi: 10.1111/j.1533-2500.2012.00529.x. PMID: 22304620. Exclusion reason: Ineligible comparator

1639. Teeley AM, Soltani M, Wiechman SA, et al. Virtual reality hypnosis pain control in the treatment of multiple fractures: a case series. Am J Clin Hypn. 2012 Jan;54(3):184-94. PMID: 22443021. Exclusion reason: Background information only

1640. Telles S, Dash M, Naveen KV. Effect of yoga on musculoskeletal discomfort and motor functions in professional computer users. Work. 2009;33(3):297-306. doi: 10.3233/WOR-2009-0877. PMID: 19759428. Exclusion reason: Ineligible population

1641. Terrell KM, Hui SL, Castelluccio P, et al. Analgesic prescribing for patients who are discharged from an emergency department. Pain Med. 2010 Jul;11(7):1072-7. doi: https://dx.doi.org/10.1111/j.15264637.2010.00884.x. PMID: 20642733. Exclusion reason: Background information only

1642. Tervo T, Petaja L, Lepisto P. A controlled clinical trial of a muscle relaxant analgesic combination in the treatment of acute lumbago. Br J Clin Pract. 1976 Mar;30(3):62-4. PMID: 130920. Exclusion reason: Ineligible study design

1643. Teuscher T, Von Der Ahe CW, Baillod P, et al. Double-blind randomised clinical trial of pentoxiphyllin in vaso-occlusive sickle cell crisis. Trop Geogr Med. 1989 Oct;41(4):320-5. PMID: 2635446.

Exclusion reason: Ineligible intervention

1644. Thackeray A, Fritz JM, Brennan GP, et al. A pilot study examining the effectiveness of physical therapy as an adjunct to selective nerve root block in the treatment of lumbar radicular pain from disk herniation: a randomized controlled trial. Phys Ther 2010 Dec;90(12):1717-29. doi: 10.2522/ptj.20090260. PMID: 20864600. Exclusion reason: Ineligible intervention 
1645. Thacoor A, Sandiford NA. Cryotherapy following total knee arthroplasty: What is the evidence? J Orthop Surg. 2019 JanApr;27(1):2309499019832752. doi: https://dx.doi.org/10.1177/23094990198327 52. PMID: 30827174. Exclusion reason: Publication used as source document

1646. Thagaard KS, Jensen HH, Raeder J. Analgesic and antiemetic effect of ketorolac vs. betamethasone or dexamethasone after ambulatory surgery. Acta Anaesthesiol Scand. 2007 Mar;51(3):271-7. PMID: 17257175. Exclusion reason: Ineligible comparator

1647. Thienpont E. Does Advanced Cryotherapy Reduce Pain and Narcotic Consumption After Knee Arthroplasty? Clinical Orthopaedics and Related Research. 2014 2014/11/01;472(11):3417-23. doi: 10.1007/s11999-014-3810-8. PMID: 25059851. Exclusion reason: Ineligible comparator

1648. Thomas C. Psychoeducational dvd intervention for acute low back pain. Diss Abstr Int. 2010;70(9-B):5850. Exclusion reason: Ineligible publication type

1649. Thomas IL, Tyle V, Webster J, et al. An evaluation of transcutaneous electrical nerve stimulation for pain relief in labour. Aust N Z J Obstet Gynaecol. 1988 Aug;28(3):182-9. PMID: 3266072. Exclusion reason: Ineligible population

1650. Thomas LS, Stephenson N, Swanson M, et al. A pilot study: the effect of healing touch on anxiety, stress, pain, pain medication usage, and physiological measures in hospitalized sickle cell disease adults experiencing a vaso-occlusive pain episode. J Holist Nurs. 2013 Dec;31(4):234-47. doi: 10.1177/0898010113491631. PMID: 23817144. Exclusion reason: Ineligible intervention

1651. Thomas V, Rose F, Heath M, et al. A multidimensional comparison of nurse and patient controlled analgesia in the management of acute postsurgical pain. Med Sci Res. 1993 May;21(10):379-81.

Exclusion reason: Ineligible intervention
1652. Thomas VJ, Dixon AL, Milligan P, et al. Cognitive-behaviour therapy for the management of sickle cell disease pain: An evaluation of a community-based intervention. Br J Health Psychol. 1999 1999/09/01;4(3):209-29. doi: 10.1348/135910799168588. Exclusion reason: Ineligible population

1653. Thompson JF, Pike JM, Chumas PD, et al. Rectal diclofenac compared with pethidine injection in acute renal colic. BMJ. 1989 Nov 4;299(6708):1140-1. doi: 10.1136/BMJ.299.6708.1140. PMID: 2513026. Exclusion reason: Ineligible intervention

1654. Tick H, Nielsen A, Pelletier KR, et al. Evidence-Based Nonpharmacologic Strategies for Comprehensive Pain Care: The Consortium Pain Task Force White Paper. Explore (NY). 2018 May Jun;14(3):177-211. doi: https://dx.doi.org/10.1016/j.explore.2018.02. 001. PMID: 29735382. Exclusion reason: Background information only

1655. Ting JZR, Chen X, Johnston V. WorkplaceBased Exercise Intervention Improves Work Ability in Office Workers: A Cluster Randomised Controlled Trial. Int J Environ Res Public Health. 2019 Jul 24;16(15):24. doi: 10.3390/ijerph16152633. PMID: 31344787. Exclusion reason: Ineligible outcome

1656. Tisdale SA, Jr., Ervin DK. A controlled study of methocarbamol (Robaxin) in acute painful musculoskeletal conditions. Curr Ther Res Clin Exp. 1975 Jun;17(6):525-30. PMID: 808374. Exclusion reason: Ineligible comparator

1657. Titler MG, Herr K, Schilling ML, et al. Acute pain treatment for older adults hospitalized with hip fracture: current nursing practices and perceived barriers. Appl Nurs Res. 2003 Nov;16(4):211-27. PMID: 14608555. Exclusion reason: Background information only

1658. Titlic M, Jukic I, Tonkic A, et al. Lamotrigine therapy for resistant pain in radicular lesions of cervical segments C4-C5 and C5-C6: a case report. Acta Clinica Croatica. 2009 Jun;48(2):157-60. PMID: 19928414. Exclusion reason: Ineligible population 
1659. Tivendale L, Scott J, Ternan A. Pressure support and elevation following the removal of a radial artery for coronary artery bypass grafting. Aust Crit Care. 2000

Nov;13(4):153-8. PMID: 16948207.

Exclusion reason: Ineligible intervention

1660. To TP, Lim TC, Hill ST, et al. Gabapentin for neuropathic pain following spinal cord injury. Spinal Cord. 2002 Jun;40(6):282-5. PMID: 12037709. Exclusion reason: Ineligible population

1661. Tokuda M, Tabira K, Masuda T, et al. Effect of modulated-frequency and modulatedintensity transcutaneous electrical nerve stimulation after abdominal surgery: A randomized controlled trial. Clin J Pain. 2014 Jul;30(7):565-70. doi: 10.1097/AJP.0b013e31829ea151. PMID: 24901753. Exclusion reason: Ineligible setting

1662. Tomruk M, Gelecek N, Basci O, et al. Effects of early manual therapy on functional outcomes after volar plating of distal radius fractures: A randomized controlled trial. Hand Surg Rehabil. 2020 May;39(3):178-85. doi: https://dx.doi.org/10.1016/j.hansur.2019.12. 002. PMID: 32070793. Exclusion reason: Ineligible outcome

1663. Toms L, Derry S, Moore AR, et al. Single dose oral paracetamol (acetaminophen) with codeine for postoperative pain in adults. Cochrane Database Syst Rev. 2019(5)doi: 10.1002/14651858.CD001547.pub2. PMID: 19160199. Exclusion reason: Publication used as source document

1664. Toms L, McQuay HJ, Derry S, et al. Single dose oral paracetamol (acetaminophen) for postoperative pain in adults. Cochrane Database Syst Rev. 2019(5) PMID:

18843665. Exclusion reason: Publication used as source document

1665. Tool AL, Kammerer-Doak DN, Nguyen $\mathrm{CM}$, et al. Postoperative pain relief following laparoscopic tubal sterilization with silastic bands. Obstet Gynecol. 1997 Nov;90(5):731-4. PMID: 9351754.

Exclusion reason: Ineligible intervention
1666. Traeger AC, Hubscher M, Henschke N, et al. Effect of Primary Care-Based Education on Reassurance in Patients With Acute Low Back Pain: Systematic Review and Metaanalysis. JAMA Intern Med. 2015 May;175(5):733-43. doi: https://dx.doi.org/10.1001/jamainternmed.20 15.0217. PMID: 25799308. Exclusion reason: Publication used as source document

1667. Tran L, Barthelemy M, Boileau P, et al. Sciatic nerve block or not for outpatient total knee arthroplasty? Study protocol for a randomized controlled trial. Trials [Electronic Resource]. 2019 Jan 08;20(1):30. doi: 10.1186/s13063-018-31421. PMID: 30621742. Exclusion reason: Ineligible intervention

1668. Trinh K, Graham N, Irnich D, et al. WITHDRAWN: Acupuncture for neck disorders. Cochrane Database Syst Rev. 201611 17;11:CD004870. PMID: 27852100. Exclusion reason: Background information only

1669. Trinh K, Graham N, Irnich D, et al. Acupuncture for neck disorders. Cochrane Database Syst Rev. 2016 May 04(5):CD004870. doi: https://dx.doi.org/10.1002/14651858.CD004 870.pub4. PMID: 27145001. Exclusion reason: Ineligible population

1670. Troullos ES, Hargreaves KM, Butler DP, et al. Comparison of nonsteroidal antiinflammatory drugs, ibuprofen and flurbiprofen, with methylprednisolone and placebo for acute pain, swelling, and trismus. J Oral Maxillofac Surg. 1990 Sep;48(9):945-52. PMID: 2395047. Exclusion reason: Ineligible comparator

1671. Tsang CC, Giudice MG. Nabilone for the Management of Pain. Pharmacotherapy: The Journal of Human Pharmacology \& Drug Therapy. 2016 Mar;36(3):273-86. doi: https://dx.doi.org/10.1002/phar.1709. PMID: 26923810. Exclusion reason: Ineligible population

1672. Tsang J, Brush B. Patient-controlled analgesia in postoperative cardiac surgery. Anaesth Intensive Care. 1999 Oct;27(5):464-70. PMID: 10520385.

Exclusion reason: Ineligible duration 
1673. Tsang RC, Tsang PL, Ko CY, et al. Effects of acupuncture and sham acupuncture in addition to physiotherapy in patients undergoing bilateral total knee arthroplasty-a randomized controlled trial. Clin Rehabil. 2007 Aug;21(8):719-28. doi: 10.1177/0269215507077362. PMID: 17846072. Exclusion reason: Ineligible intervention

1674. Tsang SMH, So BCL, Lau RWL, et al. Comparing the effectiveness of integrating ergonomics and motor control to conventional treatment for pain and functional recovery of work-related neckshoulder pain: A randomized trial. Eur J Pain. 2019 Jul;23(6):1141-52. doi: 10.1002/ejp.1381. PMID: 30793422.

Exclusion reason: Ineligible population

1675. Tsay SL, Chen HL, Chen SC, et al. Effects of reflexotherapy on acute postoperative pain and anxiety among patients with digestive cancer. Cancer Nurs. 2008 MarApr;31(2):109-15. doi: 10.1097/01.NCC.0000305694.74754.7b. PMID: 18490886. Exclusion reason: Ineligible setting

1676. Tsoumani AT, Asproudis IC, Damigos D. Tetracaine $0.5 \%$ eyedrops with or without lidocaine $2 \%$ gel in topical anesthesia for cataract surgery. 2010. p. 967-70. Exclusion reason: Ineligible intervention

1677. Tsuji M, Kobayashi N, Yukizawa Y, et al. Effect of Flurbiprofen and S-Flurbiprofen Patches on Multimodal Pain Management After Total Knee Arthroplasty: A Prospective Randomized Controlled Trial. J Arthroplasty. 2020 Apr 10;10:10. doi: https://dx.doi.org/10.1016/j.arth.2020.04.00 6. PMID: 32362479. Exclusion reason: Ineligible intervention

1678. Tsukayama H, Yamashita H, Amagai H, et al. Randomised controlled trial comparing the effectiveness of electroacupuncture and TENS for low back pain: a preliminary study for a pragmatic trial. Acupunct Med. 2002 Dec;20(4):175-80. doi: 10.1136/aim.20.4.175. PMID: 12512791. Exclusion reason: Ineligible population
1679. Tugay N, Akbayrak T, Demirturk F, et al. Effectiveness of transcutaneous electrical nerve stimulation and interferential current in primary dysmenorrhea. Pain Med. 2007 May-Jun;8(4):295-300. PMID: 17610451. Exclusion reason: Ineligible population

1680. Tungtrongjit Y, Weingkum P, Saunkool P. The effect of preoperative quadriceps exercise on functional outcome after total knee arthroplasty. J Med Assoc Thai. 2012 Oct;95 Suppl 10:S58-66. PMID: 23451440. Exclusion reason: Ineligible population

1681. Turan A, Karamanlioglu B, Memis D, et al. Analgesic effects of gabapentin after spinal surgery. Anesthesiology. 2004 Apr;100(4):935-8. PMID: 15087630.

Exclusion reason: Ineligible intervention

1682. Turan S, Ayik I, Öztürk D, et al. Effects of remifentanil and fentanyl on postoperative pain and recovery in fast-tract cardiac surgery. Gogus-Kalp-Damar Anestezi ve Yogun Bakim Dernegi Dergisi. 2012;18(4):80-7. doi: 10.5222/GKDAD.2012.087. Exclusion reason: Ineligible intervention

1683. Turek D, Reder R, Karpow S, et al. Pharmacokinetic/pharmacodynamic comparison of low and high dose hydromorphone and morphine oral solutions in acute postoperative pain. International J Clin Pharmacol and therapeutics. 1987;41(2):229. Exclusion reason: Ineligible publication type

1684. Turner DM, Warson JS, Wirt TC, et al. The use of ketorolac in lumbar spine surgery: a cost-benefit analysis. J Spinal Disord. 1995 Jun;8(3):206-12. PMID: 7670211.

Exclusion reason: Ineligible setting

1685. Turner GA, Anson N, Williamson R. A comparison of intramuscular ketorolac with indomethacin suppositories in the treatment of pain after oral surgery. Anaesth Intensive Care. 1996 Dec;24(6):665-8. doi: 10.1177/0310057X9602400605. PMID: 8971313. Exclusion reason: Ineligible comparator 
1686. Turner JA, Dworkin SF, Mancl L, et al. The roles of beliefs, catastrophizing, and coping in the functioning of patients with temporomandibular disorders. Pain. 2001 May;92(1-2):41-51. PMID: 11323125. Exclusion reason: Background information only

1687. Turturro MA, Paris PM, Seaberg DC. Intramuscular ketorolac versus oral ibuprofen in acute musculoskeletal pain. Ann Emerg Med. 1995 Aug;26(2):117-20. doi: 10.1016/s0196-0644(95)70138-9. PMID: 7618770. Exclusion reason: Ineligible comparator

1688. Turturro MA, Paris PM, Yealy DM, et al. Hydrocodone versus codeine in acute musculoskeletal pain. Ann Emerg Med. 1991 Oct;20(10):1100-3. PMID: 1928881. Exclusion reason: Ineligible comparator

1689. Tusek D, Church JM, Fazio VW. Guided imagery as a coping strategy for perioperative patients. AORN J. 1997 Oct;66(4):644-9. doi: 10.1016/s00012092(06)62917-7. PMID: 9337466.

Exclusion reason: Ineligible intervention

1690. Tusek DL. Guided imagery: a powerful tool to decrease length of stay, pain, anxiety, and narcotic consumption. J Invasive Cardiol. 1999 Apr;11(4):265-7. PMID: 10745528. Exclusion reason: Ineligible publication type

1691. Tusek DL, Cwynar R, Cosgrove DM. Effect of guided imagery on length of stay, pain and anxiety in cardiac surgery patients. J Cardiovasc Manag. 1999 Mar-Apr;10(2):228. PMID: 10557909. Exclusion reason: Ineligible setting

1692. Twersky RS, Lebovits A, Williams C, et al. Ketorolac versus fentanyl for postoperative pain management in outpatients. Clin J Pain. 1995 Jun;11(2):127-33. doi: 10.1097/00002508-199506000-00007. PMID: 7549168. Exclusion reason: Ineligible intervention

1693. Tyre CT. An effectiveness study of traditional and biopsychosocial treatment in temporomandibular joint pain. Diss Abstr Int. 1997 Jun;57(12-B):7745. Exclusion reason: Ineligible publication type
1694. Ucuzal M, Kanan N. Foot massage: effectiveness on postoperative pain in breast surgery patients. Pain Manag Nurs. 2014 Jun;15(2):458-65. doi: 10.1016/j.pmn.2012.03.001. PMID: 24882025. Exclusion reason: Ineligible setting

1695. Uden P, Rentzhog L, Berger T. A comparative study on the analgesic effects of indomethacin and hydromorphinechloride-atropine in acute, ureteral-stone pain. Acta Chir Scand. 1983;149(5):497-9. PMID: 6637313. Exclusion reason: Ineligible comparator

1696. Udezue E, Girshab AM. Observations on the management of acute pain crisis in adult sickle cell disease in eastern Saudi Arabia. Ann Saudi Med. 2005 Mar-Apr;25(2):115-9. PMID: 15977688. Exclusion reason: Background information only

1697. Udezue E, Herrera E. Pain management in adult acute sickle cell pain crisis: a viewpoint. West Afr J Med. 2007 JulSep;26(3):179-82. PMID: 18399330. Exclusion reason: Ineligible publication type

1698. Unlu Z, Tasci S, Tarhan S, et al. Comparison of 3 physical therapy modalities for acute pain in lumbar disc herniation measured by clinical evaluation and magnetic resonance imaging. J Manipulative Physiol Ther. 2008 Mar;31(3):191-8. doi: 10.1016/j.jmpt.2008.02.001. PMID: 18394495. Exclusion reason: Ineligible population

1699. Urbin Choffray D, Crielaard JM, Albert A, et al. Comparative study of high bioavailability glaphenine and paracetamol in cervical and lumbar arthrosis. Clin Rheumatol. 1987 Dec;6(4):518-25. doi: 10.1007/bf02330588. PMID: 2896557. Exclusion reason: Ineligible comparator

1700. Ursini T, Tontodonati M, Manzoli L, et al. Acupuncture for the treatment of severe acute pain in herpes zoster: results of a nested, open-label, randomized trial in the VZV Pain Study. BMC Complement Altern Med. 2011 Jun 05;11:46. doi: 10.1186/14726882-11-46. PMID: 21639941. Exclusion reason: Ineligible comparator 
1701. Usichenko TI, Dinse M, Hermsen M, et al. Auricular acupuncture for pain relief after total hip arthroplasty - a randomized controlled study. Pain. 2005

Apr;114(3):320-7. PMID: 15777857.

Exclusion reason: Ineligible setting

1702. Usichenko TI, Edinger H, Witstruck T, et al. Millimetre wave therapy for pain relief after total knee arthroplasty: a randomised controlled trial. Eur J Pain. 2008 Jul;12(5):617-23. PMID: 18042413. Exclusion reason: Ineligible intervention

1703. Usichenko TI, Kuchling S, Witstruck T, et al. Auricular acupuncture for pain relief after ambulatory knee surgery: a randomized trial. CMAJ. 2007 Jan 16;176(2):179-83. doi: 10.1503/cmaj.060875. PMID: 17224599. Exclusion reason: Ineligible intervention

1704. Uzture N, Ture H, Keskin O, et al. Comparison of Tramadol versus Tramadol with Paracetamol for efficacy of postoperative pain management in lumbar discectomy: a randomized controlled study. Int J Clin Pract. 2019 PMID: 31508863. Exclusion reason: Ineligible intervention

1705. Uzture N, Ture H, Keskin O, et al. Comparison of tramadol versus tramadol with paracetamol for efficacy of postoperative pain management in lumbar discectomy: A randomised controlled study. Int J Clin Pract. 2020 Jan;74(1):e13414. doi: https://dx.doi.org/10.1111/ijcp.13414. PMID: 31508863. Exclusion reason: Ineligible intervention

1706. Vaajoki A, Pietila AM, Kankkunen P, et al. Effects of listening to music on pain intensity and pain distress after surgery: an intervention. J Clin Nurs. 2012 Mar;21(56):708-17. doi: 10.1111/j.13652702.2011.03829.x. PMID: 21843204.

Exclusion reason: Ineligible setting

1707. Vadhanan P, Balakrishnan K. Comparison of postoperative analgesia with $0.8 \mathrm{mg}$ and $1.6 \mathrm{mg}$ intrathecal nalbuphine; A randomized controlled trial. Anaesthesia, Pain and Intensive Care. 2017;21(1):37-43. Exclusion reason: Ineligible intervention
1708. Valat JP, Accardo S, Reginster JY, et al. A comparison of the efficacy and tolerability of meloxicam and diclofenac in the treatment of patients with osteoarthritis of the lumbar spine. Inflamm Res. 2001 Mar;50 Suppl 1:S30-4. PMID: 11339519. Exclusion reason: Ineligible population

1709. Vallejo MC, Romeo RC, Davis DJ, et al. Propofol-ketamine versus propofol-fentanyl for outpatient laparoscopy: comparison of postoperative nausea, emesis, analgesia, and recovery. J Clin Anesth. 2002 Sep;14(6):426-31. PMID: 12393110. Exclusion reason: Ineligible intervention

1710. Valle-Jones JC, Walsh H, O'Hara J, et al. Controlled trial of a back support ('Lumbotrain') in patients with non-specific low back pain. Curr Med Res Opin. 1992;12(9):604-13. doi: 10.1185/03007999209111527. PMID: 1533832. Exclusion reason: Ineligible population

1711. van Beers EJ, van Tuijn CF, Nieuwkerk PT, et al. Patient-controlled analgesia versus continuous infusion of morphine during vaso-occlusive crisis in sickle cell disease, a randomized controlled trial. Am J Hematol. 2007 Nov;82(11):955-60. PMID: 17617790. Exclusion reason: Ineligible comparator

1712. van der Gaag WH, Roelofs PD, Enthoven WT, et al. Non-steroidal anti-inflammatory drugs for acute low back pain. Cochrane Database Syst Rev. 202004

16;4:CD013581. doi: https://dx.doi.org/10.1002/14651858.CD013 581. PMID: 32297973. Exclusion reason: Publication used as source document

1713. van der Roer N, Ostelo RW, Bekkering GE, et al. Minimal clinically important change for pain intensity, functional status, and general health status in patients with nonspecific low back pain. Spine. $2006 \mathrm{Mar}$ 01;31(5):578-82. PMID: 16508555.

Exclusion reason: Background information only

1714. van Duijvenbode I, Jellema P, van Poppel $\mathrm{M}$, et al. Lumbar supports for prevention and treatment of low back pain. Cochrane Database Syst Rev. 2011(2)doi: 10.1002/14651858.CD001823.pub3. PMID: 18425875. Exclusion reason: Publication used as source document 
1715. van Tilburg CW, Schuurmans FA, Stronks DL, et al. Randomized Sham-controlled Double-Blind Multicenter Clinical Trial to Ascertain the Effect of Percutaneous Radiofrequency Treatment for Sacroiliac Joint Pain: Three-month Results. Clin J Pain. 2016 11;32(11):921-6. PMID: 26889616. Exclusion reason: Ineligible population

1716. van Tulder MW, Furlan AD, Gagnier JJ. Complementary and alternative therapies for low back pain. Best Pract Res Clin Rheumatol. 2005 Aug;19(4):639-54. PMID: 15949781. Exclusion reason: Background information only

1717. van Tulder MW, Touray T, Furlan AD, et al. Muscle relaxants for non-specific low-back pain. Cochrane Database Syst Rev. 2017(3) PMID: 12804507. Exclusion reason: Publication used as source document

1718. VanDenKerkhof EG, Hopman WM, Towheed T, et al. Pain, health-related quality of life and health care utilization after inpatient surgery: a pilot study. Pain Res Manag. 2006;11(1):41-7. PMID: 16511613. Exclusion reason: Ineligible intervention

1719. VanderArk GD, McGrath KA. Transcutaneous electrical stimulation in treatment of postoperative pain. American Journal of Surgery. 1975 Sep;130(3):338-40. PMID: 1101719. Exclusion reason: Ineligible setting

1720. Varner C, McLeod S, Orkin A, et al. A blinded, randomized controlled trial of opioid analgesics for the management of acute fracture pain in older adults discharged from the emergency department. Canadian J Emerg Med. 2017;19:S63-S4. Exclusion reason: Ineligible publication type

1721. Vas J, Cintado MC, Aranda-Regules JM, et al. Effect of ear acupuncture on pregnancyrelated pain in the lower back and posterior pelvic girdle: A multicenter randomized clinical trial. Acta Obstet Gynecol Scand. 2019 10;98(10):1307-17. doi: https://dx.doi.org/10.1111/aogs.13635. PMID: 31034580. Exclusion reason: Ineligible population
1722. Vas J, Perea-Milla E, Mendez C, et al. Efficacy and safety of acupuncture for the treatment of non-specific acute low back pain: a randomised controlled multicentre trial protocol. BMC Complement Altern Med. 2006;6doi: 10.1186/1472-6882-6-14. PMID: 16630342. Exclusion reason: Ineligible publication type

1723. Vas J, Perea-Milla E, Mendez C, et al. Efficacy and safety of acupuncture for the treatment of non-specific acute low back pain: a randomised controlled multicentre trial protocol [ISRCTN65814467]. BMC Complement Altern Med. 2006 Apr 21;6:14. PMID: 16630342. Exclusion reason: Ineligible publication type

1724. Vase L, Baad-Hansen L, Pigg M. How May Placebo Mechanisms Influence Orofacial Neuropathic Pain? J Dent Res. 2019 Jul;98(8):861-9. doi: https://dx.doi.org/10.1177/00220345198487 19. PMID: 31084512. Exclusion reason: Background information only

1725. Vasseljen O, Woodhouse A, Bjorngaard JH, et al. Natural course of acute neck and low back pain in the general population: the HUNT study. Pain. 2013 Aug;154(8):123744. doi: https://dx.doi.org/10.1016/j.pain.2013.03.03 2. PMID: 23664654. Exclusion reason: Background information only

1726. Vernon WG. A double-blind evaluation of Parafon Forte in the treatment of musculoskeletal back conditions. Curr Ther Res Clin Exp. 1972 Dec;14(12):801-6. PMID: 4629819. Exclusion reason: Ineligible intervention

1727. Verwoerd AJ, Luijsterburg PA, Koes BW, et al. Does Kinesiophobia Modify the Effects of Physical Therapy on Outcomes in Patients With Sciatica in Primary Care? Subgroup Analysis From a Randomized Controlled Trial. Phys Ther 2015 Sep;95(9):1217-23. doi: 10.2522/ptj.20140458. PMID: 25929529. Exclusion reason: Ineligible outcome

1728. Vevelstad M, Pettersen S, Tallaksen C, et al. O-demethylation of codeine to morphine inhibited by low-dose levomepromazine. Eur J Clin Pharmacol. 2009 Aug;65(8):795801. doi: 10.1007/s00228-009-0640-9. PMID: 19308365. Exclusion reason: Ineligible comparator 
1729. Videman T, Heikkila J, Partanen T. Doubleblind parallel study of meptazinol versus diflunisal in the treatment of lumbago. Curr Med Res Opin. 1984;9(4):246-52. PMID: 6239753. Exclusion reason: Ineligible intervention

1730. Vieira ER, Brunt D. Does wearing unstable shoes reduce low back pain and disability in nurses? A randomized controlled pilot study. Clin Rehabil. 2016 Feb;30(2):167-73. doi: 10.1177/0269215515576812. PMID: 25780261. Exclusion reason: Ineligible population

1731. Vilkins AL, Bagley SM, Hahn KA, et al. Comparison of Post-Cesarean Section Opioid Analgesic Requirements in Women With Opioid Use Disorder Treated With Methadone or Buprenorphine. J Addict Med. 2017 Sep/Oct;11(5):397-401. doi: 10.1097/ADM.0000000000000339. PMID: 28727661. Exclusion reason: Ineligible study design

1732. Villafane JH, Perucchini D, Cleland JA, et al. The effectiveness of a cognitive behavioral exercise approach (CBEA) compared to usual care in patients with a Whiplash Associated Disorder: A quasiexperimental clinical trial. J Back Musculoskelet Rehabil. 2017 Sep 22;30(5):943-50. doi: 10.3233/BMR140162. PMID: 28453445. Exclusion reason: Ineligible population

1733. Viscusi ER, Grond S, Ding L, et al. A comparison of opioid-related adverse events with fentanyl iontophoretic transdermal system versus morphine intravenous patientcontrolled analgesia in acute postoperative pain. Pain Manag 2016;6(1):19-24. doi: 10.2217/pmt.15.49. PMID: 26376128.

Exclusion reason: Ineligible intervention

1734. Viscusi ER, Siccardi M, Damaraju CV, et al. The safety and efficacy of fentanyl iontophoretic transdermal system compared with morphine intravenous patientcontrolled analgesia for postoperative pain management: an analysis of pooled data from three randomized, active-controlled clinical studies. Anesth Analg. 2007 Nov;105(5):1428-36, table of contents. PMID: 17959978. Exclusion reason: Ineligible comparator
1735. Viscusi ER, Skobieranda F, Soergel DG, et al. APOLLO-1: a randomized placebo and activecontrolled phase iii study investigating oliceridine (TRV130), a G protein-biased ligand at the I1/4-opioid receptor, for management of moderateto-severe acute pain following bunionectomy. J Pain Res. 2019;12:927-43. PMID: 30881102.

Exclusion reason: Ineligible comparator

1736. Visser E, Schug SA. The role of ketamine in pain management. Biomed Pharmacother. 2006 Aug;60(7):341-8. PMID: 16854557. Exclusion reason: Background information only

1737. von Plato H, Kontinen V, Hamunen K. Efficacy and safety of epidural, continuous perineural infusion and adjuvant analgesics for acute postoperative pain after major limb amputation - a systematic review. Scandinavian J Pain. 201801 26;18(1):3-17. doi: https://dx.doi.org/10.1515/sjpain-20170170. PMID: 29794290. Exclusion reason: Publication used as source document

1738. Vorobeychik Y, Sharma A, Smith CC, et al. The Effectiveness and Risks of Non-ImageGuided Lumbar Interlaminar Epidural Steroid Injections: A Systematic Review with Comprehensive Analysis of the Published Data. Pain Med. 2016 12;17(12):2185-202. doi: https://dx.doi.org/10.1093/pm/pnw091. PMID: 28025354. Exclusion reason: Publication used as source document

1739. Vorsanger G, Xiang J, Biondi D, et al. Post hoc analyses of data from a 90-day clinical trial evaluating the tolerability and efficacy of tapentadol immediate release and oxycodone immediate release for the relief of moderate to severe pain in elderly and nonelderly patients. Pain Res Manag. 2011 Jul-Aug;16(4):245-51. PMID: 22059194. Exclusion reason: Ineligible population

1740. Vos C, Verhagen A, Passchier J, et al. Management of acute neck pain in general practice: a prospective study. Br J Gen Pract. 2007 Jan;57(534):23-8. PMID: 17244420. Exclusion reason: Ineligible study design 
1741. Wade AG, Ward PJ. A double-blind comparison of meptazinol versus paracetamol and placebo in acute and chronic painful conditions presented to the general practitioner. Curr Med Res Opin. 1982;8(3):191-6. PMID: 7128191. Exclusion reason: Ineligible population

1742. Walden M, Lahtinen J, Elvander E. Analgesic effect and tolerance of ketoprofen and diclofenac in acute ureteral colic. Scand J Urol Nephrol. 1993;27(3):323-5. doi: 10.3109/00365599309180441. PMID: 8290910. Exclusion reason: Ineligible comparator

1743. Waldfogel JM, Nesbit SA, Dy SM, et al. Pharmacotherapy for diabetic peripheral neuropathy pain and quality of life: A systematic review. Neurology. 2017 May 16;88(20):1958-67. doi: https://dx.doi.org/10.1212/WNL.000000000 0003882. PMID: 28341643. Exclusion reason: Publication used as source document

1744. Walker BF, Hebert JJ, Stomski NJ, et al. Short-term usual chiropractic care for spinal pain: a randomized controlled trial. Spine. 2013 Nov 15;38(24):2071-8. doi: 10.1097/01.brs.0000435032.73187.c7. PMID: 24026159. Exclusion reason: Ineligible population

1745. Walker MJ, Boyles RE, Young BA, et al. The effectiveness of manual physical therapy and exercise for mechanical neck pain: a randomized clinical trial. Spine. 2008 Oct 15;33(22):2371-8. doi: 10.1097/BRS.0b013e318183391e. PMID: 18923311. Exclusion reason: Ineligible population

1746. Walker RH, Morris BA, Angulo DL, et al. Postoperative use of continuous passive motion, transcutaneous electrical nerve stimulation, and continuous cooling pad following total knee arthroplasty. J Arthroplasty. 1991 Jun;6(2):151-6. PMID: 1875206. Exclusion reason: Ineligible setting
1747. Walsh DM, Howe TE, Johnson MI, et al. Transcutaneous electrical nerve stimulation for acute pain. Cochrane Database Syst Rev. 2009 Apr 15(2):CD006142. doi: https://dx.doi.org/10.1002/14651858.CD006 142.pub2. PMID: 19370629. Exclusion reason: Publication used as source document

1748. Walworth D, Rumana CS, Nguyen J, et al. Effects of live music therapy sessions on quality of life indicators, medications administered and hospital length of stay for patients undergoing elective surgical procedures for brain. J Music Ther. 2008;45(3):349-59. PMID: 18959455. Exclusion reason: Ineligible population

1749. Wan C, Dong DS, Song T. High-Voltage, Long-Duration Pulsed Radiofrequency on Gasserian Ganglion Improves Acute/Subacute Zoster-Related Trigeminal Neuralgia: A Randomized, Double-Blinded, Controlled Trial. Pain Physician. 2019 07;22(4):361-8. PMID: 31337167. Exclusion reason: Ineligible population

1750. Wand BM, Bird C, McAuley JH, et al. Early intervention for the management of acute low back pain: a single-blind randomized controlled trial of biopsychosocial education, manual therapy, and exercise. Spine. 2004 Nov 01;29(21):2350-6. doi: 10.1097/01.brs.0000143619.34308.b4. PMID: 15507794. Exclusion reason: Ineligible comparator

1751. Wang B, Tang J, White PF, et al. Effect of the intensity of transcutaneous acupoint electrical stimulation on the postoperative analgesic requirement. Anesth Analg. 1997 Aug;85(2):406-13. doi: 10.1097/00000539199708000-00029. PMID: 9249122.

Exclusion reason: Ineligible setting

1752. Wang BCM, Liu D, Furnback WE, et al. The Cost-Effectiveness of Pregabalin Versus Gabapentin for Peripheral Neuropathic Pain (pNeP) and Postherpetic Neuralgia (PHN) in China. Pain Ther. 2016;5(1):81-91. doi: 10.1007/s40122-016-0048-z. PMID: 26932262. Exclusion reason: Ineligible study design 
1753. Wang H, Gargano C, Lukac S, et al. An enhanced bunionectomy model as a potential tool for early decision-making in the development of new analgesics. Adv Ther. 2010 Dec;27(12):963-80. doi: 10.1007/s12325-010-0084-8. PMID: 21052881. Exclusion reason: Ineligible intervention

1754. Wang JX, Zhao WX, Zeng JC, et al. [Systematic review and sequential analysis on treatment of herpes zoster pain mainly by fire needle therapy]. Chen Tzu Yen Chiu Acupuncture Research. 2019 Sept 25;44(9):677-85. doi: https://dx.doi.org/10.13702/j.10000607.190004. PMID: 31532139. Exclusion reason: Not in English

1755. Wang L, Dong Y, Zhang J, et al. The efficacy of gabapentin in reducing pain intensity and postoperative nausea and vomiting following laparoscopic cholecystectomy: A meta-analysis. Medicine. 2017 Sep;96(37):e8007. doi: https://dx.doi.org/10.1097/MD.0000000000 008007. PMID: 28906382. Exclusion reason: Ineligible comparator

1756. Wang RI, Waite EM. The clinical analgesic efficacy of oral nefopam hydrochloride. J Clin Pharmacol. 1979 Jul;19(7):395-402. PMID: 479385. Exclusion reason: Ineligible comparator

1757. Wang RR, Tronnier V. Effect of acupuncture on pain management in patients before and after lumbar disc protrusion surgery--a randomized control study. Am J Chin Med. 2000;28(1):25-33. PMID: 10794114. Exclusion reason: Ineligible population

1758. Wang SL, Wang H, Nie HY, et al. The efficacy of pregabalin for acute pain control in herpetic neuralgia patients: A metaanalysis. Medicine. 2017 Dec;96(51):e9167. doi:

https://dx.doi.org/10.1097/MD.0000000000 009167. PMID: 29390451. Exclusion

reason: Background information only
1759. Wang SM, Dezinno P, Lin EC, et al. Auricular acupuncture as a treatment for pregnant women who have low back and posterior pelvic pain: a pilot study. Am J Obstet Gynecol. 2009 Sep;201(3):271.e1-9. doi: 10.1016/j.ajog.2009.04.028. PMID: 19560110. Exclusion reason: Ineligible population

1760. Wang TJ, Chang CF, Lou MF, et al. Biofeedback relaxation for pain associated with continuous passive motion in Taiwanese patients after total knee arthroplasty. Res Nurs Health. 2015 Feb;38(1):39-50. doi: 10.1002/nur.21633. PMID: 25557776. Exclusion reason: Ineligible setting

1761. Wang WC, George SL, Wilimas JA. Transcutaneous electrical nerve stimulation treatment of sickle cell pain crises. Acta Haematologica. 1988;80(2):99-102. PMID: 3138879. Exclusion reason: Ineligible population

1762. Wang X, Liu W, Xu Z, et al. Effect of Dexmedetomidine Alone for Intravenous Patient-Controlled Analgesia After Gynecological Laparoscopic Surgery: A Consort-Prospective, Randomized, Controlled Trial. Medicine. 2016 May;95(19):e3639. doi: 10.1097/MD.0000000000003639. PMID: 27175680. Exclusion reason: Ineligible setting

1763. Wang Y, Yang H, Shen C, et al. Morphine and pregabalin in the treatment of neuropathic pain. Exp Ther Med. 2017;13(4):1393-7. doi: 10.3892/etm.2017.4102. PMID: 28413483. Exclusion reason: Ineligible population

1764. Wanich T, Gelber J, Rodeo S, et al. Percutaneous neuromodulation pain therapy following knee replacement. J Knee Surg. 2011 Sep;24(3):197-202. PMID: 21980881. Exclusion reason: Ineligible setting

1765. Ward J, Coats J. Comparison of the BackJoy SitSmart Relief and Spine Buddy LT1 H/C Ergonomic Chair Supports on Short-Term Neck and Back Pain. J Manipulative Physiol Ther. 2017 01;40(1):41-9. doi: 10.1016/j.jmpt.2016.10.006. PMID: 27919432. Exclusion reason: Ineligible population 
1766. Ward JP, Albert DB, Altman R, et al. Are femoral nerve blocks effective for early postoperative pain management after hip arthroscopy? Arthroscopy. 2012

Aug;28(8):1064-9. doi: 10.1016/j.arthro.2012.01.003. PMID: 22498045. Exclusion reason: Ineligible intervention

1767. Warfield CA, Stein JM, Frank HA. The effect of transcutaneous electrical nerve stimulation on pain after thoracotomy. Ann Thorac Surg. 1985 May;39(5):462-5. doi: 10.1016/s0003-4975(10)61957-1. PMID: 2859841. Exclusion reason: Ineligible setting

1768. Wassilew S, Collaborative Brivudin PHNSG. Brivudin compared with famciclovir in the treatment of herpes zoster: effects in acute disease and chronic pain in immunocompetent patients. A randomized, double-blind, multinational study. J Eur Acad Dermatol Venereol. 2005 Jan;19(1):47-55. PMID: 15649191. Exclusion reason: Ineligible intervention

1769. Wassilew SW, Wutzler P, Brivddin Herpes Zoster Study G. Oral brivudin in comparison with acyclovir for herpes zoster: a survey study on postherpetic neuralgia. Antiviral Res. 2003 Jun;59(1):57-60. PMID: 12834861. Exclusion reason: Ineligible intervention

1770. Waterman B, Walker JJ, Swaims C, et al. The efficacy of combined cryotherapy and compression compared with cryotherapy alone following anterior cruciate ligament reconstruction. J Knee Surg. 2012 May;25(2):155-60. PMID: 22928433. Exclusion reason: Ineligible intervention

1771. Waterworth RF, Hunter IA. An open study of diflunisal, conservative and manipulative therapy in the management of acute mechanical low back pain. N Z Med J. 1985 May 22;98(779):372-5. PMID: 3157894. Exclusion reason: Ineligible comparator

1772. Weber H, Aasand G. The effect of phenylbutazone on patients with acute lumbago-sciatica. A double blind trial. J Oslo City Hosp. 1980 May;30(5):69-72. PMID: 6446598. Exclusion reason: Ineligible intervention
1773. Webster LR, Malan TP, Tuchman MM, et al. A multicenter, randomized, double-blind, controlled dose finding study of NGX-4010, a high-concentration capsaicin patch, for the treatment of postherpetic neuralgia. J Pain. 2010 Oct;11(10):972-82. doi: 10.1016/j.jpain.2010.01.270. PMID: 20655809. Exclusion reason: Ineligible comparator

1774. Webster LR, Tark M, Rauck R, et al. Effect of duration of postherpetic neuralgia on efficacy analyses in a multicenter, randomized, controlled study of NGX-4010, an $8 \%$ capsaicin patch evaluated for the treatment of postherpetic neuralgia. BMC Neurology. 2010 Oct 11;10:92. doi: 10.1186/1471-2377-10-92. PMID: 20937130. Exclusion reason: Ineligible comparator

1775. Weckwerth GM, Simoneti LF, ZupelariGoncalves P, et al. Efficacy of naproxen with or without esomeprazole for pain and inflammation in patients after bilateral third molar extractions: A double blinded crossover study. Medicina Oral, Patologia Oral y Cirugia Bucal. 2017 Jan 01;22(1):e122-e31. PMID: 27918744. Exclusion reason: Ineligible intervention

1776. Wedenberg KAJ, Moen B, Norling Å. A prospective randomized study comparing acupuncture with physiotherapy for lowback and pelvic pain in pregnancy. Acta Obstet Gynecol Scand. 2000 2000/05/01;79(5):331-5. doi: 10.1034/j.1600-0412.2000.079005331.x. PMID: 10830757. Exclusion reason: Ineligible population

1777. Weinbroum AA, Lalayev G, Yashar T, et al. Combined pre-incisional oral dextromethorphan and epidural lidocaine for postoperative pain reduction and morphine sparing: a randomised double-blind study on day-surgery patients. Anaesthesia. 2001 Jul;56(7):616-22. PMID: 11437760.

Exclusion reason: Ineligible intervention 
1778. Weiner SG, Griggs CA, Mitchell PM, et al. Clinician impression versus prescription drug monitoring program criteria in the assessment of drug-seeking behavior in the emergency department. Ann Emerg Med. 2013 Oct;62(4):281-9. doi:

10.1016/j.annemergmed.2013.05.025. PMID: 23849618. Exclusion reason: Ineligible population

1779. Weinheimer K, Michelotti B, Silver J, et al. A Prospective, Randomized, DoubleBlinded Controlled Trial Comparing Ibuprofen and Acetaminophen Versus Hydrocodone and Acetaminophen for Soft Tissue Hand Procedures. J Hand Surg Am. 2019 May;44(5):387-93. doi: https://dx.doi.org/10.1016/j.jhsa.2018.10.01 4. PMID: 30502019. Exclusion reason: Ineligible comparator

1780. Weiser T, Richter E, Hegewisch A, et al. Efficacy and safety of a fixed-dose combination of ibuprofen and caffeine in the management of moderate to severe dental pain after third molar extraction. Eur J Pain. 2018 01;22(1):28-38. doi: 10.1002/ejp.1068. PMID: 28805281. Exclusion reason: Ineligible intervention

1781. Weller WJ, Azzam MG, Smith RA, et al. Liposomal Bupivacaine Mixture Has Similar Pain Relief and Significantly Fewer Complications at Less Cost Compared to Indwelling Interscalene Catheter in Total Shoulder Arthroplasty. J Arthroplasty. 2017 11;32(11):3557-62. doi: 10.1016/j.arth.2017.03.017. PMID: 28390888. Exclusion reason: Ineligible intervention

1782. Wells N. The effect of relaxation on postoperative muscle tension and pain. Nurs Res. 1982 Jul-Aug;31(4):236-8. PMID: 7048260. Exclusion reason: Ineligible setting

1783. Werneke M, Hart DL. Centralization phenomenon as a prognostic factor for chronic low back pain and disability. Spine. 2001 Apr 01;26(7):758-64; discussion 65. doi: 10.1097/00007632-200104010-00012. PMID: 11295896. Exclusion reason: Ineligible intervention
1784. Westenberg RF, Zale EL, Heinhuis TJ, et al. Does a Brief Mindfulness Exercise Improve Outcomes in Upper Extremity Patients? A Randomized Controlled Trial. Clin Orthop Relat Res. 2018 04;476(4):790-8. doi: 10.1007/s11999.0000000000000086. PMID: 29480886. Exclusion reason: Ineligible population

1785. Westrom KK, Maiers MJ, Evans RL, et al. Individualized chiropractic and integrative care for low back pain: the design of a randomized clinical trial using a mixedmethods approach. Trials [Electronic Resource]. 2010 Mar 08;11:24. doi: 10.1186/1745-6215-11-24. PMID: 20210996. Exclusion reason: Ineligible population

1786. Whedon JM, Toler AWJ, Goehl JM, et al. Association Between Utilization of Chiropractic Services for Treatment of Low Back Pain and Risk of Adverse Drug Events. J Manipulative Physiol Ther. 2018;41(5):383-8. doi: 10.1016/j.jmpt.2018.01.004. PMID: 29843912. Exclusion reason: Ineligible population

1787. White CL, Pokrupa RP, Chan MH. An evaluation of the effectiveness of patientcontrolled analgesia after spinal surgery. J Neurosci Nurs. 1998 Aug;30(4):225-32. PMID: 9791777. Exclusion reason: Ineligible population

1788. White LD, Hodge A, Vlok R, et al. Efficacy and adverse effects of buprenorphine in acute pain management: systematic review and meta-analysis of randomised controlled trials. Br J Anaesth. 2018 Apr;120(4):66878. doi: https://dx.doi.org/10.1016/j.bja.2017.11.086. PMID: 29576108. Exclusion reason: Publication used as source document

1789. White PF. Management of postoperative pain and emesis. Can J Anaesth. 1995 Nov;42(11):1053-5. PMID: 8590497. Exclusion reason: Background information only 
1790. White PF, Tang J, Wender RH, et al. The effects of oral ibuprofen and celecoxib in preventing pain, improving recovery outcomes and patient satisfaction after ambulatory surgery. Anesth Analg. 2011 Feb;112(2):323-9. doi: 10.1213/ANE.0b013e3182025a8a. PMID: 21156974. Exclusion reason: Ineligible comparator

1791. Whitehurst DG, Lewis M, Yao GL, et al. A brief pain management program compared with physical therapy for low back pain: results from an economic analysis alongside a randomized clinical trial. Arthritis Rheum. 2007 Apr 15;57(3):466-73. PMID: 17394176. Exclusion reason: Ineligible population

1792. Whitelaw GP, DeMuth KA, Demos HA, et al. The use of the Cryo/Cuff versus ice and elastic wrap in the postoperative care of knee arthroscopy patients. Am J Knee Surg. 1995 Winter;8(1):28-30; discussion -1. PMID: 7866800. Exclusion reason: Ineligible outcome

1793. Wick EC, Grant MC, Wu CL. Postoperative Multimodal Analgesia Pain Management With Nonopioid Analgesics and Techniques: A Review. JAMA Surg. 201707 01;152(7):691-7. doi: https://dx.doi.org/10.1001/jamasurg.2017.08 98. PMID: 28564673. Exclusion reason: Ineligible publication type

1794. Wiesel SW, Cuckler JM, Deluca F, et al. Acute low-back pain: an objective analysis of conservative therapy. Spine. 1980;5(4):324-30. doi: 10.1097/00007632198007000-00006. PMID: 6450448 Exclusion reason: Ineligible study design

1795. Wiffen PJ, Xia J. Systematic review of topical diclofenac for the treatment of acute and chronic musculoskeletal pain. Curr Med Res Opin. 2020 Apr;36(4):637-50. doi: https://dx.doi.org/10.1080/03007995.2020.1 716703. PMID: 31944135. Exclusion reason: Ineligible comparator

1796. Wilder DG, Vining RD, Pohlman KA, et al. Effect of spinal manipulation on sensorimotor functions in back pain patients: study protocol for a randomised controlled trial. Trials [Electronic Resource]. 2011 Jun 28;12:161. doi: 10.1186/1745-6215-12-161. PMID: 21708042. Exclusion reason: Ineligible population
1797. Wilke J, Vogt L, Niederer D, et al. Shortterm effects of acupuncture and stretching on myofascial trigger point pain of the neck: a blinded, placebo-controlled RCT.

Complement Ther Med. 2014

Oct;22(5):835-41. doi:

10.1016/j.ctim.2014.09.001. PMID: 25440373. Exclusion reason: Ineligible duration

1798. Williams CM, Maher CG, Latimer J, et al. PACE--the first placebo controlled trial of paracetamol for acute low back pain: statistical analysis plan. Trials [Electronic Resource]. 2013 Aug 09;14:248. doi: 10.1186/1745-6215-14-248. PMID: 23937999. Exclusion reason: Ineligible study design

1799. Williams H, Tanabe P. Sickle Cell Disease: A Review of Nonpharmacological Approaches for Pain. J Pain Symptom Manage. 2016 Feb;51(2):163-77. doi: https://dx.doi.org/10.1016/j.jpainsymman.20 15.10.017. PMID: 26596876. Exclusion reason: Publication used as source document

1800. Williams NH, Wilkinson C, Russell I, et al. Randomized osteopathic manipulation study (ROMANS): pragmatic trial for spinal pain in primary care. Fam Pract. 2003 Dec;20(6):662-9. PMID: 14701889. Exclusion reason: Ineligible population

1801. Williamson E, Nichols V, Lamb SE. "If I can get over that, I can get over anything"-understanding how individuals with acute whiplash disorders form beliefs about pain and recovery: a qualitative study.

Physiotherapy. 2015 Jun;101(2):178-86. doi: 10.1016/j.physio.2014.06.001. PMID: 25062908. Exclusion reason: Ineligible study design

1802. Wilner AN, Sharma BK, Thompson AR, et al. Analgesic opioid use in a health-insured epilepsy population during 2012. Epilepsy Behav. 2016 Apr;57(Pt A):126-32. doi: https://dx.doi.org/10.1016/j.yebeh.2016.01.0 33. PMID: 26949154. Exclusion reason: Background information only 
1803. Wilsey B, Marcotte T, Tsodikov A, et al. A randomized, placebo-controlled, crossover trial of cannabis cigarettes in neuropathic pain. J Pain. 2008 Jun;9(6):506-21. doi: 10.1016/j.jpain.2007.12.010. PMID: 18403272. Exclusion reason: Ineligible comparator

1804. Wilson E, Payton O, Donegan-Shoaf L, et al. Muscle energy technique in patients with acute low back pain: a pilot clinical trial. J Orthop Sports Phys Ther. 2003

Sep;33(9):502-12. PMID: 14524509.

Exclusion reason: Ineligible population

1805. Wilson JL, Poulin PA, Sikorski R, et al. Opioid use among same-day surgery patients: Prevalence, management and outcomes. Pain Res Manag. 2015 NovDec;20(6):300-4. PMID: 26357683. Exclusion reason: Background information only

1806. Winkelman NW, Jr., Richards DJ. Doubleblind evaluation of an analgesic-tranquilizer combination for treating musculoskeletal pain associated with anxiety. Curr Ther Res Clin Exp. 1975 Apr;17(4):352-60. PMID: 804382. Exclusion reason: Ineligible intervention

1807. Wittig-Wells D, Johnson I, SammsMcPherson J, et al. Does the use of a brief cryotherapy intervention with analgesic administration improve pain management after total knee arthroplasty? Orthop Nurs. 2015 May-Jun;34(3):148-53. doi: 10.1097/NOR.0000000000000143. PMID: 25989123. Exclusion reason: Ineligible setting

1808. Wolford R, Kahler J, Mishra P, et al. A prospective comparison of transnasal butorphanol and acetaminophen with codeine for the relief of acute musculoskeletal pain. Am J Emerg Med. 1997 Jan;15(1):101-3. PMID: 9002589. Exclusion reason: Ineligible publication type
1809. Wong JJ, Shearer HM, Mior S, et al. Are manual therapies, passive physical modalities, or acupuncture effective for the management of patients with whiplashassociated disorders or neck pain and associated disorders? An update of the Bone and Joint Decade Task Force on Neck Pain and Its Associated Disorders by the OPTIMa collaboration. Spine J. 2016 12;16(12):1598630. doi:

https://dx.doi.org/10.1016/j.spinee.2015.08. 024. PMID: 26707074. Exclusion reason:

Publication used as source document

1810. Wong RH, Lee TW, Sihoe AD, et al. Analgesic effect of electroacupuncture in postthoracotomy pain: a prospective randomized trial. Ann Thorac Surg. 2006 Jun;81(6):2031-6. doi: 10.1016/j.athoracsur.2005.12.064. PMID: 16731125. Exclusion reason: Ineligible setting

1811. Wood MJ, Johnson RW, McKendrick MW, et al. A randomized trial of acyclovir for 7 days or 21 days with and without prednisolone for treatment of acute herpes zoster. N Engl J Med. 1994 Mar 31;330(13):896-900. PMID: 8114860. Exclusion reason: Ineligible intervention

1812. Wood TG, Colloca CJ, Matthews R. A pilot randomized clinical trial on the relative effect of instrumental (MFMA) versus manual (HVLA) manipulation in the treatment of cervical spine dysfunction. J Manipulative Physiol Ther. 2001 May;24(4):260-71. doi: 10.1067/mmt.2001.114365. PMID: 11353937. Exclusion reason: Ineligible population

1813. Woodhouse A, Hobbes AF, Mather LE, et al. A comparison of morphine, pethidine and fentanyl in the postsurgical patientcontrolled analgesia environment. Pain. 1996 Jan;64(1):115-21. PMID: 8867253. Exclusion reason: Ineligible comparator

1814. Woolf AD, Zeidler H, Haglund U, et al. Musculoskeletal pain in Europe: its impact and a comparison of population and medical perceptions of treatment in eight European countries. Ann Rheum Dis. 2004 Apr;63(4):342-7. PMID: 15020325. Exclusion reason: Background information only 
1815. Wreje U, Nordgren B, Aberg H. Treatment of pelvic joint dysfunction in primary care-a controlled study. Scand J Prim Health Care. 1992 Dec;10(4):310-5. PMID: 1480873. Exclusion reason: Ineligible population

1816. Wright A, Lloyd-Davies A, Williams S, et al. Individual active treatment combined with group exercise for acute and subacute low back pain. Spine. 2005 Jun 01;30(11):1235-41. doi: 10.1097/01.brs.0000164266.00150.b6. PMID: 15928545. Exclusion reason: Ineligible population

1817. Wright A, Sluka KA. Nonpharmacological treatments for musculoskeletal pain. Clin J Pain. 2001 Mar;17(1):33-46. PMID: 11289087. Exclusion reason: Publication used as source document

1818. Wu K, Zhu D, Gao F, et al. The immediate effect of acupuncturing Shu point combined with Mu point on acute discogenic back pain. Journal Emergency in Traditional Chinese Medicine [zhong guo zhong yi ji zheng]. 2015;24(4):583-5. Exclusion reason: Not in English

1819. Wu MS, Chen KH, Chen IF, et al. The Efficacy of Acupuncture in Post-Operative Pain Management: A Systematic Review and Meta-Analysis. PLoS ONE [Electronic Resource]. 2016;11(3):e0150367. doi: 10.1371/journal.pone.0150367. PMID: 26959661. Exclusion reason: Publication used as source document

1820. Wylde V, Dennis J, Beswick AD, et al. Systematic review of management of chronic pain after surgery. Br J Surg. 2017 Sep;104(10):1293-306. doi: https://dx.doi.org/10.1002/bjs.10601. PMID: 28681962. Exclusion reason: Publication used as source document

1821. X G, Xu S, Cheng C, et al. Local Administration of Methylcobalamin and Lidocaine for Acute Ophthalmic Herpetic Neuralgia: A Single-Center Randomized Controlled Trial. Pain Pract. 2016 09;16(7):869-81. doi: 10.1111/papr.12328. PMID: 26200815. Exclusion reason: Ineligible intervention
1822. Ximenes A, Robles M, Sands G, et al. Valdecoxib is as efficacious as diclofenac in the treatment of acute low back pain. Clin J Pain. 2007 Mar-Apr;23(3):244-50. PMID: 17314584. Exclusion reason: Ineligible comparator

1823. Xing XF, Zhou ZF, Zhang FJ, et al. The Effect of Early Use of Supplemental Therapy on Preventing Postherpetic Neuralgia: A Systematic Review and Metaanalysis. Pain Physician. 2017 09;20(6):47186. PMID: 28934778. Exclusion reason: Publication used as source document

1824. Xu G, Lv Z-W, Xu G, et al. Thiamine, cobalamin, locally injected alone or combination for herpetic itching: A singlecenter randomized controlled trial. Clin J Pain. 2014 Mar;30(3):269-78. doi: 10.1097/AJP.0b013e3182a0e085. PMID: 23887347 Exclusion reason: Ineligible intervention

1825. Xu H, Li H, Zuo Y, et al. A multicenter study of the analgesic effects of epidural chloroprocaine after lower limb orthopedic surgery. J Clin Anesth. 2016 Dec;35:313-20. doi: 10.1016/j.jclinane.2016.08.009. PMID: 27871549. Exclusion reason: Ineligible intervention

1826. Yajnik M, Hill JN, Hunter OO, et al. Patient education and engagement in postoperative pain management decreases opioid use following knee replacement surgery. Patient Educ Couns. 2019 02;102(2):383-7. doi: https://dx.doi.org/10.1016/j.pec.2018.09.001 . PMID: 30219634. Exclusion reason: Ineligible setting

1827. Yakaitis RW, Cooke JE, Redding JS. Selfadministered methoxyflurane for postoperative pain: effectiveness and patient acceptance. Anesth Analg. 1972 MarApr;51(2):208-12. PMID: 4551090.

Exclusion reason: Ineligible intervention

1828. Yakhno N, Guekht A, Skoromets A, et al. Analgesic efficacy and safety of lornoxicam quick-release formulation compared with diclofenac potassium: randomised, doubleblind trial in acute low back pain. Clin Drug Investig 2006;26(5):267-77. PMID: 17163260. Exclusion reason: Ineligible comparator 
1829. Yaman Aktas Y, Durgun H, Durhan R. Cold Therapy and the Effect on Pain and Physiological Parameters in Patients Recovering from Spine Surgery: A Randomized Prospective Study. Complementary Medical Research. $2020 \mathrm{Jul}$ 01:1-9. doi: https://dx.doi.org/10.1159/000508029. PMID: 32610330. Exclusion reason: Ineligible setting

1830. Yamashita A, Maeda T, Kita Y, et al. The impact of prehospital assessment and EMS transport of acute aortic syndrome patients. Am J Emerg Med. 2018 Jul;36(7):1188-94. doi: 10.1016/j.ajem.2017.12.005. PMID: 29276030. Exclusion reason: Ineligible population

1831. Yamashita Y, Sano N, Shimohira D, et al. A parallel-group comparison study of celecoxib with loxoprofen sodium in third mandibular molar extraction patients. Int J Oral Maxillofac Surg. 2014

Dec;43(12):1509-13. doi: 10.1016/j.ijom.2014.09.002. PMID: 25270186. Exclusion reason: Ineligible comparator

1832. Yamato TP, Maher CG, Saragiotto BT, et al. Pilates for Low Back Pain: Complete Republication of a Cochrane Review. Spine. 2016 Jun;41(12):1013-21. doi: https://dx.doi.org/10.1097/BRS.0000000000 001398. PMID: 26679894. Exclusion reason: Publication used as source document

1833. Yamato TP, Maher CG, Saragiotto BT, et al. Pilates for low back pain. Cochrane Database Syst Rev. 2015 Jul 02(7):CD010265. doi: 10.1002/14651858.CD010265.pub2. PMID: 26133923. Exclusion reason: Ineligible population

1834. Yan C, Ding N, Wei Y, et al. Effects of fentanyl and butorphanol on uterine contraction pain after induced abortion: A randomized controlled clinical trial. Int J Clin Exp Med. 2018;11(7):6454-64.

Exclusion reason: Ineligible setting
1835. Yao P, Hong T, Zhu YQ, et al. Efficacy and safety of continuous radiofrequency thermocoagulation plus pulsed radiofrequency for treatment of V1 trigeminal neuralgia: A prospective cohort study. Medicine. 2016 Nov;95(44):e5247. PMID: 27858881. Exclusion reason: Ineligible study design

1836. Yao-chi W, Bi-meng Z, Chong-miao W, et al. Observation on short-term and long-term therapeutic effects of electroacupuncture at Houxi (SI 3) on acute lumbar sprain. Zhongguo zhen jiu= Chinese Acupuncture \& Moxibustion. 2007;27(1):3-5. PMID: 17378192. Exclusion reason: Not in English

1837. Yawn BP, Buchanan GR, Afenyi-Annan AN, et al. Management of sickle cell disease: summary of the 2014 evidencebased report by expert panel members. JAMA. 2014 Sep 10;312(10):1033-48. doi: https://dx.doi.org/10.1001/jama.2014.10517. PMID: 25203083. Exclusion reason: Publication used as source document

1838. Yeh ML, Chung YC, Chen KM, et al. Pain reduction of acupoint electrical stimulation for patients with spinal surgery: a placebocontrolled study. Int J Nurs Stud. 2011 Jun;48(6):703-9. doi: 10.1016/j.ijnurstu.2010.10.009. PMID: 21122849. Exclusion reason: Ineligible population

1839. Yeh M-L, Tsou M-Y, Lee B-Y, et al. Effects of Auricular Acupressure on Pain Reduction in Patient-controlled Analgesia After Lumbar Spine Surgery. Acta Anaesthesiol Taiwan. 2010 2010/06/01/;48(2):80-6. doi: https://doi.org/10.1016/S18754597(10)60018-5. PMID: 20643366. Exclusion reason: Ineligible setting

1840. Yeying G, Liyong Y, Yuebo C, et al. Thoracic paravertebral block versus intravenous patient-controlled analgesia for pain treatment in patients with multiple rib fractures. J Int Med Res. 2017

Dec;45(6):2085-91. doi: 10.1177/0300060517710068. PMID: 28635359. Exclusion reason: Ineligible intervention 
1841. Yilmaz S, Yildizbas S, Guclu E, et al. Topical levobupivacaine efficacy in pain control after functional endoscopic sinus surgery. Otolaryngol Head Neck Surg. 2013 Nov;149(5):777-81. doi: 10.1177/0194599813502925. PMID: 24005134. Exclusion reason: Ineligible comparator

1842. Younes M, Nowakowski K, Didier-Laurent $B$, et al. Effect of spinal manipulative treatment on cardiovascular autonomic control in patients with acute low back pain. Chiropr Man Therap. 2017;25:33. doi: 10.1186/s12998-017-0167-6. PMID: 29214015. Exclusion reason: Ineligible outcome

1843. Young SE, Bothwell JD, Walsh RM. Safely Managing Acute Osteoarthritis in the Emergency Department: An EvidenceBased Review. J Emerg Med. 2016 Dec;51(6):648-57. doi: https://dx.doi.org/10.1016/j.jemermed.2016. 05.052. PMID: 27480348. Exclusion reason: Background information only

1844. Yousefi-Nooraie R, Schonstein E, Heidari $\mathrm{K}$, et al. Low level laser therapy for nonspecific low-back pain. Cochrane Database Syst Rev. 2007 Apr 18(2):CD005107. PMID: 17443572. Exclusion reason: Publication used as source document

1845. Yuan QL, Guo TM, Liu L, et al. Traditional Chinese medicine for neck pain and low back pain: a systematic review and metaanalysis. PLoS ONE [Electronic Resource]. 2015;10(2):e0117146. doi: 10.1371/journal.pone.0117146. PMID: 25710765. Exclusion reason: Publication used as source document

1846. Yuan QL, Wang P, Liu L, et al. Acupuncture for musculoskeletal pain: A meta-analysis and meta-regression of shamcontrolled randomized clinical trials. Sci Rep. 2016 Jul 29;6:30675. doi: 10.1038/srep30675. PMID: 27471137. Exclusion reason: Publication used as source document
1847. Yung E, Oh C, Wong M, et al. Non-thrust cervical manipulations reduce short-term pain and decrease systolic blood pressure during intervention in mechanical neck pain: a randomized clinical trial. J Man Manip Ther. 2019 Aug 04:1-12. doi: 10.1080/10669817.2019.1646985. PMID: 31379301. Exclusion reason: Ineligible population

1848. Yurkiw D, Mior S. Comparison of two chiropractic techniques on pain and lateral flexion in neck pain patients: a pilot study. Chiropractic Technique. 1996;8(4):155-62. Exclusion reason: Ineligible population

1849. Zachodnik J, Andersen JH, Geisler A. Barriers in pain treatment in the emergency and surgical department. Dan Med J. 2019 Feb;66(2) PMID: 30722824. Exclusion reason: Background information only

1850. Zaffagnini S, Iacono F, Petitto A, et al. Cryo/Cuff use after arthroscopic surgery: effect on knee joint temperature. Am J Knee Surg. 1998 Fall;11(4):203-7. PMID: 9853997. Exclusion reason: Ineligible outcome

1851. Zambito RF. Controlling acute pain in dentistry. Part I. N Y State Dent J. 1984 Aug-Sep;50(7):420-1. PMID: 6593648. Exclusion reason: Ineligible study design

1852. Zanjir M, Sgro A, Lighvan NL, et al. Efficacy and safety of post-operative medications in reducing pain following nonsurgical endodontic treatment: a systematic review and network meta-analysis. J Endod. 2020 Jul 12;12:12. doi: https://dx.doi.org/10.1016/j.joen.2020.07.00 2. PMID: 32668310. Exclusion reason: Publication used as source document

1853. Zarling BJ, Yokhana SS, Herzog DT, et al. Preoperative and Postoperative Opiate Use by the Arthroplasty Patient. J Arthroplasty. 2016 10;31(10):2081-4. doi: 10.1016/j.arth.2016.03.061. PMID: 27161903. Exclusion reason: Ineligible study design

1854. Zassman SM, Zamora FJ, Roberts JD. Inpatient pain management in sickle cell disease. Am J Health Syst Pharm. 2019 Oct 12;12:12. doi: https://dx.doi.org/10.1093/ajhp/zxz228. PMID: 31605120. Exclusion reason: Background information only 
1855. Zaveri S, Nobel TB, Khetan P, et al. Risk of Chronic Opioid Use in Opioid-Naïve and Non-Naïve Patients after Ambulatory Surgery. J Gastrointest Surg. 2020 Mar;24(3):688-94. doi: 10.1007/s11605019-04265-2. PMID: 31152348. Exclusion reason: Ineligible outcome

1856. Zencir G, Eser I. Effects of Cold Therapy on Pain and Breathing Exercises Among Median Sternotomy Patients. Pain Manag Nurs. 2016 12;17(6):401-10. doi: 10.1016/j.pmn.2016.05.006. PMID: 27746091. Exclusion reason: Ineligible setting

1857. Zeng AM, Nami NF, Wu CL, et al. The Analgesic Efficacy of Nonsteroidal Antiinflammatory Agents (NSAIDs) in Patients Undergoing Cesarean Deliveries: A MetaAnalysis. Reg Anesth Pain Med. 2016 Nov/Dec;41(6):763-72. PMID: 27755486. Exclusion reason: Publication used as source document

1858. Zeng Y, Li Y, Gao JH. Application of cold patch in relieving pain after transepithelial photorefractive keratectomy. Pain Res Manag. 2015 Jul-Aug;20(4):195-8. PMID: 25992866. Exclusion reason: Ineligible comparator

1859. Zhai L, Song Z, Liu K. The Effect of Gabapentin on Acute Postoperative Pain in Patients Undergoing Total Knee Arthroplasty: A Meta-Analysis. Medicine. 2016 May;95(20):e3673. doi: https://dx.doi.org/10.1097/MD.0000000000 003673. PMID: 27196473. Exclusion

reason: Ineligible comparator

1860. Zhang DDQ, Sussman J, Dossa F, et al. A Systematic Review of Behavioral Interventions to Decrease Opioid Prescribing After Surgery. Ann Surg. 2020 02;271(2):266-78. doi: https://dx.doi.org/10.1097/SLA.0000000000 003483. PMID: 31356268. Exclusion reason: Publication used as source document

1861. Zhang J, Enix D, Snyder B, et al. Effects of Biofreeze and chiropractic adjustments on acute low back pain: a pilot study Journal of chiropractic medicine. 2008;7(2):59-65. doi: 10.1016/j.jcme.2008.02.004. PMID: 19674721 Exclusion reason: Ineligible comparator
1862. Zhang S, Wang F, Lu ZD, et al. Effect of single-injection versus continuous local infiltration analgesia after total knee arthroplasty: a randomized, double-blind, placebo-controlled study. J Int Med Res. 2011;39(4):1369-80. PMID: 21986137. Exclusion reason: Ineligible comparator

1863. Zhang Y, Fatemi P, Medress Z, et al. A predictive-modeling based screening tool for prolonged opioid use after surgical management of low back and lower extremity pain. Spine J. 2020 Aug;20(8):1184-95. doi: https://dx.doi.org/10.1016/j.spinee.2020.05. 098. PMID: 32445803. Exclusion reason: Ineligible outcome

1864. Zhang Z, Xu H, Zhang Y, et al. Nonsteroidal anti-inflammatory drugs for postoperative pain control after lumbar spine surgery: A meta-analysis of randomized controlled trials. J Clin Anesth. 2017 Dec;43:84-9. doi: https://dx.doi.org/10.1016/j.jclinane.2017.08 .030. PMID: 29046234. Exclusion reason: Ineligible population

1865. Zhang Z, Yang Q, Xin W, et al. Comparison of local infiltration analgesia and sciatic nerve block as an adjunct to femoral nerve block for pain control after total knee arthroplasty: A systematic review and metaanalysis. Medicine. 2017 May;96(19):e6829. doi:

https://dx.doi.org/10.1097/MD.0000000000 006829. PMID: 28489762. Exclusion

reason: Publication used as source document

1866. Zhao CG, Sun W, Ju F, et al. Analgesic Effects of Directed Repetitive Transcranial Magnetic Stimulation in Acute Neuropathic Pain After Spinal Cord Injury. Pain Med. 2019 Nov 13;13:13. doi: https://dx.doi.org/10.1093/pm/pnz290. PMID: 31722404. Exclusion reason: Ineligible population

1867. Zheng YL, Wang XF, Chen BL, et al. Effect of 12-Week Whole-Body Vibration Exercise on Lumbopelvic Proprioception and Pain Control in Young Adults with Nonspecific Low Back Pain. Med Sci Monit. 2019 Jan 15;25:443-52. doi: 10.12659/MSM.912047. PMID: 30644383. Exclusion reason: Ineligible population 
1868. Zheng Z. Observations on the therapeutic effects of treating 90 cases of acute lumbar sprain by acupuncturing Xing Jian (LR2). Clin J Acupunct Moxibustion. 2005;2:38. Exclusion reason: Not in English

1869. Zhong Q, Wang D, Bai YM, et al. Effectiveness of Auricular Acupressure for Acute Postoperative Pain after Surgery: A Systematic Review and Meta-Analysis. Chin J Integr Med. 2019 Mar;25(3):225-32. doi: https://dx.doi.org/10.1007/s11655-0193063-1. PMID: 30815805. Exclusion reason: Publication used as source document

1870. Zhou J, Dan Y, Yixian Y, et al. Efficacy of Transcutaneous Electronic Nerve Stimulation in Postoperative Analgesia After Pulmonary Surgery: A Systematic Review and Meta-Analysis. Am J Phys Med Rehabil. 2020 03;99(3):241-9. doi: https://dx.doi.org/10.1097/PHM.000000000 0001312. PMID: 31498159. Exclusion reason: Ineligible population

1871. Zhou L, Huang J, Chen C. Most effective pain-control procedure for open liver surgery: a network meta-analysis. ANZ J Surg. 2018 12;88(12):1236-42. doi: https://dx.doi.org/10.1111/ans.14456. PMID: 29534349. Exclusion reason: Ineligible population

1872. Zhou TJ, Tang J, White PF. Propacetamol versus ketorolac for treatment of acute postoperative pain after total hip or knee replacement. Anesth Analg. 2001 Jun;92(6):1569-75. PMID: 11375848. Exclusion reason: Ineligible duration

1873. Zhu Q, Wang S, Chen R, et al. Continuous radiofrequency thermocoagulation under CT-guidance for glossopharyngeal neuralgia: Two case reports. Medicine. 2018 Jun;97(24):e11079. doi: 10.1097/MD.0000000000011079. PMID: 29901619. Exclusion reason: Ineligible study design

1874. Ziccardi VB, Desjardins PJ, Daly-DeJoy E, et al. Single-dose vicoprofen compared with acetaminophen with codeine and placebo in patients with acute postoperative pain after third molar extractions. J Oral Maxillofac Surg. 2000 Jun;58(6):622-8. PMID: 10847283. Exclusion reason: Ineligible comparator
1875. Ziegelmann MJ, Joseph JP, Glasgow AE, et al. Wide Variation in Opioid Prescribing After Urological Surgery in Tertiary Care Centers. Mayo Clin Proc. 2019 02;94(2):262-74. doi: https://dx.doi.org/10.1016/j.mayocp.2018.08 .035. PMID: 30711124. Exclusion reason: Background information only

1876. Ziehm S, Rosendahl J, Barth J, et al. Psychological interventions for acute pain after open heart surgery. Cochrane Database Syst Rev. 201707 12;7:CD009984. doi: https://dx.doi.org/10.1002/14651858.CD009 984.pub3. PMID: 28701028. Exclusion reason: Publication used as source document

1877. Zimmerman L, Nieveen J, Barnason S, et al. The effects of music interventions on postoperative pain and sleep in coronary artery bypass graft (CABG) patients. Scholarly Inquiry for Nursing Practice. 1996;10(2):153-70; discussion 71-4. PMID: 8826769. Exclusion reason: Ineligible population

1878. Zipple M, Braddock A. Success of Hospital Intervention and State Legislation on Decreasing and Standardizing Postoperative Opioid Prescribing Practices. J Am Coll Surg. 2019 Aug;229(2):158-63. doi: 10.1016/j.jamcollsurg.2019.02.049. PMID: 30880121. Exclusion reason: Ineligible comparator

1879. Zivi I, Maffia S, Ferrari V, et al. Effectiveness of aquatic versus land physiotherapy in the treatment of peripheral neuropathies: A randomized controlled trial. Clin Rehabil. 2018 May;32(5):663-70. doi: http://dx.doi.org/10.1177/026921551774671 6. PMID: 29232980. Exclusion reason: Ineligible population

1880. Zullo AR, Zhang T, Beaudoin FL, et al. Pain Treatments After Hip Fracture Among Older Nursing Home Residents. J Am Med Dir Assoc. 2018 02;19(2):174-6. doi: https://dx.doi.org/10.1016/j.jamda.2017.11.0 08. PMID: 29287695. Exclusion reason: Ineligible outcome 
1881. Zuniga JR, Papas AS, Daniels SE, et al.

Prevention of Opioid-Induced Nausea and

Vomiting During Treatment of Moderate to

Severe Acute Pain: A Randomized Placebo-

Controlled Trial Comparing CL-108

(Hydrocodone 7.5 mg/Acetaminophen 325

$\mathrm{mg}$ /Rapid-Release, Low-Dose Promethazine

$12.5 \mathrm{mg}$ ) with Conventional Hydrocodone

7.5 mg/Acetaminophen 325 mg. Pain Med. 2019 Jan 17;17:17. doi:

10.1093/pm/pny294. PMID: 30657996.

Exclusion reason: Ineligible intervention

1882. Zuniga JR, Phillips CL, Shugars D, et al. Analgesic safety and efficacy of diclofenac sodium softgels on postoperative third molar extraction pain. J Oral Maxillofac Surg. 2004 Jul;62(7):806-15. PMID: 15218558.

Exclusion reason: Ineligible comparator 